This is the peer reviewed version of the following article: Angew. Chem., Int. Ed. 2019, 58, 13880, which has been published in final form at https://onlinelibrary.wiley.com/doi/10.1002/anie.201907060. This article may be used for non-commercial purposes in accordance With Wiley-VCH Terms and Conditions for self-archiving

WILEY-VCH COMMUNICATION

\title{
1,3-Difunctionalization of Aminocyclopropanes via Dielectrophilic Intermediates
}

\author{
Ming-Ming Wang and Jérôme Waser*[a]
}

\begin{abstract}
We report an oxidative ring-opening strategy to transform acyl, sulfonyl or carbamate protected aminocyclopropanes into 1,3dielectrophilic carbon intermediates bearing a halide atom $(\mathrm{Br}, \mathrm{I})$ and a N,O-acetal. Replacing the alkoxy group of the N,O-acetal can be achieved under acidic conditions via an elimination-addition pathway, while substitution of the halides by nucleophiles can be done under basic conditions via a $S_{N} 2$ pathway, generating a wide range of 1,3difunctionalized propylamines. A proof of concept for asymmetric induction was realized using a chiral phosphoric acid (CPA) as catalyst, highlighting the potential of the method in enantioselective synthesis of important building blocks.
\end{abstract}

Aminocyclopropanes are important building blocks in synthetic chemistry. ${ }^{[1]}$ They can be incorporated into organic compounds simply by amidation or $S_{N} 2$ reaction. As structural units, they are found in potential lead compounds for new drugs and herbicides. ${ }^{[2]} \quad$ Although the biological activity of aminocyclopropanes is well recognized, their reactivity in ringopening reaction has been less explored. Our group, followed by others, showed that donor-acceptor substituted aminocyclopropanes (D-A aminocyclopropanes) are very useful intermediates, acting as zwitterionic nucleophile/electrophile synthons in the presence of Lewis acid catalysts (Scheme 1A). ${ }^{[3]}$ Although D-A aminocyclopropanes exhibit excellent reactivity, it usually takes extra steps to remove the electron-withdrawing groups or to transform them into other functionalities. For simple aminocyclopropanes lacking electron-withdrawing substituents at the vicinal position, current activation strategies focus mainly on: 1) oxidizing the amino group to a radical cation by strong oxidants or using photoredox chemistry; ${ }^{[4]}$ and 2) utilizing transition-metal catalysts to form metallocyclobutane intermediates. ${ }^{[5]}$ In both cases, the reported transformations were mostly limited to annulations. When considering the importance of 1,3difunctionalized propylamines in medicinal chemistry, with already commercialized drugs such as amprenavir (1) and proglumide (2) (Scheme 1B), the development of new synthetic methods starting from aminocyclopropanes appears therefore highly desirable. ${ }^{[6]}$

[a] Ming-Ming Wang and Prof. Dr. Jérôme Waser Laboratory of Catalysis and Organic Synthesis

Institute of Chemical Sciences and Engineering

Ecole Polytechnique Fédérale de Lausanne

EPFL SB ISIC LCSO, BCH 4306, 1015 Lausanne (CH)

Fax: (+)41216939700

E-mail: jerome.waser@epfl.ch

Homepage: http://lcso.epfl.ch/

Supporting information for this article is given via a link at the end of the document.
Inspired by the Hofmann-Löffler-Freytag (HLF) reaction and recent progress in the area (Scheme $1 \mathrm{C}),{ }^{[7]}$ we wondered whether generation of a $\mathrm{N}$-halogen bond on an aminocyclopropane followed by formation of the $\mathrm{N}$-centered radical could lead to $\mathrm{C}-\mathrm{C}$ bond activation instead of the hydrogen abstraction observed in the HLF reaction. Indeed, a single report on the photolysis of $\mathrm{N}$ bromo aminocyclopropanes indicated that such a process may be possible. ${ }^{[8]}$

Herein, we report that aminocyclopropanes can indeed be opened under mild conditions by reaction with halogenation reagents such as $\mathrm{N}$-bromo and iodo succinimide (NBS and NIS). The obtained $\mathrm{N}$-X cyclopropanes I then spontaneously open to give highly reactive 1,3-dielectrophilic imines II (Scheme 1D). ${ }^{[9]}$ Imines II can then either be isolated in high yield in the form of the more stable $\mathrm{N}, \mathrm{O}$-acetal, or reacted in a sequential nucleophilic addition and $S_{N} 2$ reaction, leading to a wide range of $\alpha, \gamma$ difunctionalized amines.

A. Previous work from our group involving D-A aminocyclopropanes

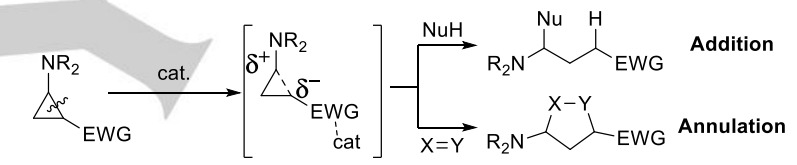

B. Drug molecules containing 1,3-difunctionalized propyl amines
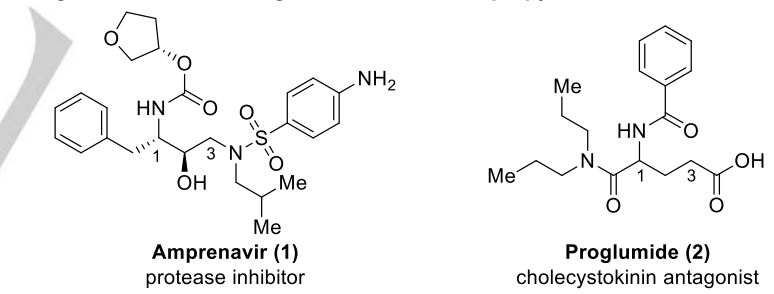

C. Hofmann-Löffler-Freytag reaction

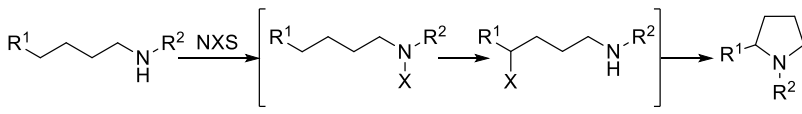

D. This work: 1,3-difunctionalization of aminocyclopropanes

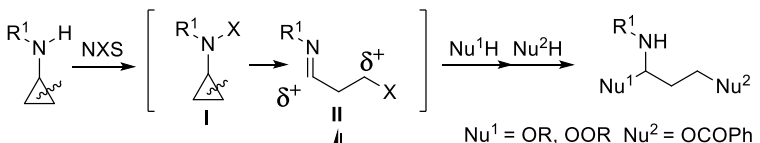

$$
\begin{aligned}
& -\mathrm{R}^{2} \mathrm{OH} \|+\mathrm{R}^{2} \mathrm{OH} \quad \mathrm{Nu}^{1}=\underset{\mathrm{NHMs}}{\mathrm{Or}} \mathrm{OOR} \mathrm{Nu}^{2}=\underset{\mathrm{OCOPh}}{\mathrm{OCH}} \\
& \text { 1-Naphthol NHPh } \\
& \mathrm{R}_{-\mathrm{NH}}^{1} \quad \mathrm{H} \quad \mathrm{CN} \\
& \mathrm{R}^{2} \mathrm{O} \mathrm{CN} \quad \mathrm{CH}\left(\mathrm{CO}_{2} \mathrm{Me}\right)_{2} \\
& \text { stable N,O-acetal indole } \quad \mathrm{NR}^{1} \text { (internal) }
\end{aligned}
$$

Scheme 1. Our previous work with D-A aminocyclopropanes (A). Bioactive compounds featuring $\alpha, \gamma$-difunctionalized amines (B). Hofmann-LöfflerFreytag reaction (C). And this work: oxidative ring opening strategy towards $\alpha, \gamma-$ difunctionalized amines (D). 
We started our investigation by reacting N-para-methoxybenzoyl protected aminocyclopropane $\mathbf{3 a}$ with a stoichiometric amount of NIS in $\mathrm{CDCl}_{3}$ (Table 1, entry 1). To our delight, formation of an imine intermediate II was observed by NMR, though accompanied by degradation. Due to the instability of this intermediate, it could not be isolated in pure form. Since N,O-acetals are stable precursors of imines, ${ }^{[10]}$ we then trapped II by adding methanol as nucleophile affording N,O-acetal $\mathbf{4 a}$ in $86 \%$ NMR yield (Table 1, entry 2). The yield could be further improved to $92 \%$ by adding 10 mol\% diphenyl phosphate and by performing the reaction in MeCN (Table 1, entry 3). N,O-acetal 4a was stable enough to allow isolation in $84 \%$ yield. NBS also promoted ring opening, giving 5 in $65 \%$ yield (Table 1, entry 4). In contrast, NCS (Nchloro-succinimide) and NFSI (N-fluorobenzenesulfonimide) failed to yield the ring-opening products 6 and 7 (Table 1, entries 5 and 6).

Table 1. Optimization of the ring-opening of aminocyclopropane $3 \mathrm{a}^{[a]}$

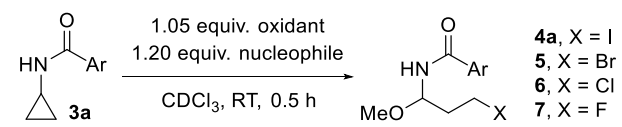

\begin{tabular}{cccc}
\hline Entry & Oxidant & Nucleophile & Yield $^{[\mathrm{b}]}$ \\
\hline 1 & NIS & - & $35 \%{ }^{[\mathrm{c}]}$ \\
2 & $\mathrm{NIS}$ & $\mathrm{MeOH}$ & $86 \%(74 \%)^{[\mathrm{d}]}$ \\
$3^{[\mathrm{e}]}$ & $\mathrm{NIS}$ & $\mathrm{MeOH}$ & $92 \%(84 \%)^{[\mathrm{d}]}$ \\
4 & $\mathrm{NBS}$ & $\mathrm{MeOH}$ & $65 \%$ \\
5 & $\mathrm{NCS}$ & $\mathrm{MeOH}$ & $0 \%$ \\
6 & NFSI & $\mathrm{MeOH}$ & $0 \%$ \\
\hline
\end{tabular}

[a] Reaction conditions: reactions were run at a $0.10 \mathrm{mmol}$ scale in $0.50 \mathrm{~mL}$ of $\mathrm{CDCl}_{3}$ at room temperature for $0.5 \mathrm{~h}$. $\mathrm{Ar}=p-\mathrm{MeO}-\mathrm{Ph}$. ${ }^{[\mathrm{b}]}$ The yield was determined by ${ }^{1} \mathrm{H}$ NMR using $\mathrm{CH}_{2} \mathrm{Br}_{2}$ as internal standard. [c]The ring-opening imine intermediate was formed. [d] Yield of isolated product. [e]Diphenyl phosphate $(10 \mathrm{~mol} \%)$ was added as additive, MeCN $(0.50 \mathrm{~mL})$ was used as solvent.

With the optimal conditions in hand, we then examined the scope of the reaction (Scheme 2). A series of benzoyl protected aminocyclopropanes with electron-withdrawing or electrondonating groups in the para position of the benzene ring all underwent the ring-opening well to give products $4 a-f$ in yields ranging between 70 and $85 \%$. Under these conditions, bromide 5 was isolated with an improved yield of $90 \%$. With substituents on the ortho, meta or 2,4,6-positions, no erosion of the yield was observed (products $\mathbf{4 g - i}$ ). Furyl-substituted amide $\mathbf{4 j}$ was obtained in $96 \%$ yield. With a cinnamoyl protected aminocyclopropane, N,O-acetal $\mathbf{4 k}$ was isolated in $80 \%$ yield with the double bond staying untouched. 2-Phenylacetoyl, naphthoyl and pivaloyl amides were also well tolerated and gave products 4I-n in 75-88\% yield. In addition to amides-, sulfonyl amides and carbamates-substituted cyclopropanes could also be used, giving the desired products $40-\mathbf{q}$ in good yields. N-alkyl substituted aminocyclopropanes were also converted to the corresponding products $\mathbf{4 r}$ and $\mathbf{4 s}$ in high yield. ${ }^{[11]}$ For non-protected aminocyclopropane (8), the ring was also opened but the imine was further oxidized to give cyanide 9 . Finally, more complex aminocyclopropanes were investigated. Aminocyclopropanes incorporated into an amino acid and the drugs isoxepac and indomethacin underwent the ring-opening well to give $\mathrm{N}, \mathrm{O}$ acetals $4 \mathbf{t}-\mathbf{v}$, showing the potential utility of this method in the latestage functionalization of bioactive compounds.

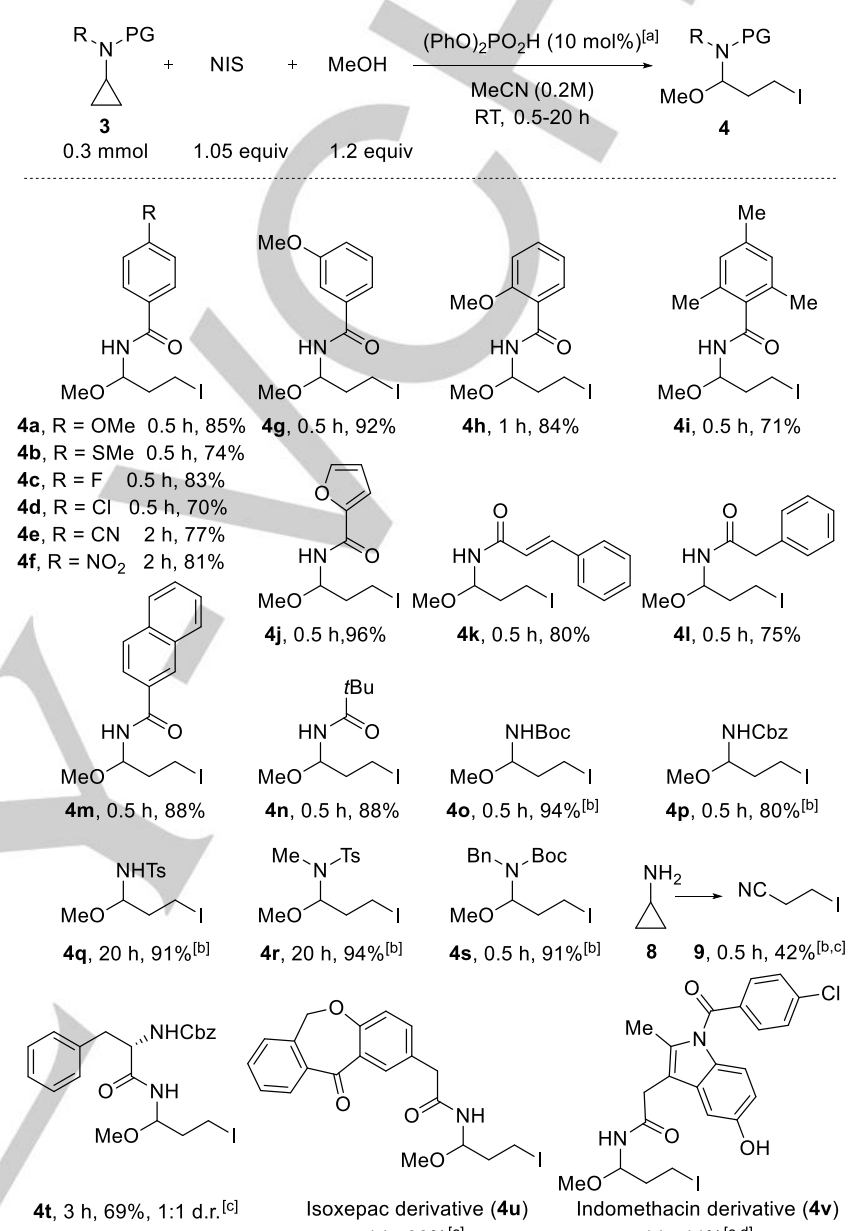

4t, 3 h, $69 \%, 1: 1$ d.r..$[c]$ $1 \mathrm{~h}, 88 \%{ }^{[\mathrm{c}]}$ $4 \mathrm{~h}, 44 \%[\mathrm{cod}]$

Scheme 2 Scope of aminocyclopropanes in the ring-opening reaction. [a] Reaction conditions: $3(0.30 \mathrm{mmol})$, NIS $(0.315 \mathrm{mmol}), \mathrm{MeOH}(0.36 \mathrm{mmol})$, diphenyl phosphate $(0.030 \mathrm{mmol})$ in $\mathrm{MeCN}(1.50 \mathrm{~mL})$ at room temperature. ${ }^{[\mathrm{b}]} \mathrm{No}$ diphenyl phosphate. ${ }^{[c]}$ Reaction scale: $0.10 \mathrm{mmol}$. ${ }^{[d]} \mathrm{NIS}$ (2.1 equiv).

We then explored the scope for the O-nucleophiles (Scheme 3). Apart from methanol, other primary alcohols (products 10a-c), secondary alcohols (products 10d-e) or even a diol (product 10f) were compatible with the reaction conditions. For optically pure menthol, the reaction proceeded with good yield albeit with poor diastereoselectivity to give product $10 \mathrm{e}$. For tert-butyl hydroperoxide (TBHP), molecular iodine was used as oxidant instead of NIS as it gave better yield, affording $\alpha$-amino peroxide $\mathbf{1 0 g}$. Peroxides are an important class of bioactive compounds..$^{[12]}$ The reaction also worked for hydrogen peroxide, though more equivalents of $\mathrm{H}_{2} \mathrm{O}_{2}$ were needed in order to obtain a good yield of $10 \mathrm{~h}$. 


$$
\text { (PIS + }
$$

Scheme 3: Scope of O-nucleophiles, $\mathrm{Ar}=p$-MeO-Ph. Reaction conditions: $3 \mathrm{a}$ $(0.30 \mathrm{mmol})$, NIS $(0.315 \mathrm{mmol}), \mathrm{ROH}(0.36 \mathrm{mmol})$, diphenyl phosphate $(0.030$ $\mathrm{mmol})$ in $\mathrm{MeCN}(1.50 \mathrm{~mL})$ at room temperature, $0.5-1$ h. $\left.{ }^{[\mathrm{a}]}\right]_{2}$ (1.1 equiv) was used instead of NIS. $\left.{ }^{[b]}\right]_{2}$ (1.1 equiv) was used instead of NIS with 2.0 equiv. of $\mathrm{H}_{2} \mathrm{O}_{2}$.

Our next objective was to exploit the full potential of our dielectrophilic synthons by adding a broad variety of other nucleophiles (Scheme 4). However, when other nucleophiles including TMSCN, $\mathrm{MsNH}_{2}$, 1-naphthol and indole derivatives were used instead of alcohols, very low yields were obtained, as side reactions between NIS and those nucleophiles were observed. Therefore, one-pot addition of nucleophiles to the N,O-acetals under acidic conditions was attempted. The addition of $\mathrm{MsNH}_{2}$, provided N,N-aminal $\mathbf{1 1}$ in $69 \%$ yield. The use of $\mathrm{C}$-nucleophiles was also possible, including cyanide, 2-naphthol and 3-methyl indole (products 12-14). When adding $\mathrm{NaBH}_{3} \mathrm{CN}, \mathrm{N}, \mathrm{O}$-acetal $4 \mathrm{a}$ was reduced to give $\mathbf{1 5}$.

Next, the iodide was replaced through an $\mathrm{S}_{\mathrm{N}} 2$ reaction either in a one-pot procedure after addition of a base and solvent switch, or in two steps after isolation of the N,O-acetal, depending on the strength of the nucleophile (Scheme 4B). For benzoic acid and benzyl thiol, no isolation of the N,O-acetal was necessary. Ester 16 and thioether 17 were isolated in $81 \%$ and $79 \%$ yield respectively. When using aniline as nucleophile, N,O-acetal 40 has to be isolated and purified, otherwise addition of succinimide originating from NIS rather than aniline was observed. Nevertheless, amine 18 was obtained in an excellent $86 \%$ yield over two steps. The same procedure was successful for malonate and $\mathrm{NaCN}$, giving products 19 and $\mathbf{2 0}$ in good yields. In absence of external nucleophiles, $\alpha$-methoxy azetidine 21 , which is an interesting building block for the synthesis of $\alpha$-substituted azetidine derivatives, ${ }^{[13]}$ was obtained in $25 \%$ yield. The low yield is due to the formation of a 6 -membered ring side product by attack of the oxygen of the carbamate. Finally, the one-pot addition of two different nucleophiles was investigated. Addition of 3-methyl-indole and a thiol or cyclopropylamine gave products 22 and $\mathbf{2 3}$ in $\mathbf{7 9 \%}$ and $55 \%$ yield respectively.
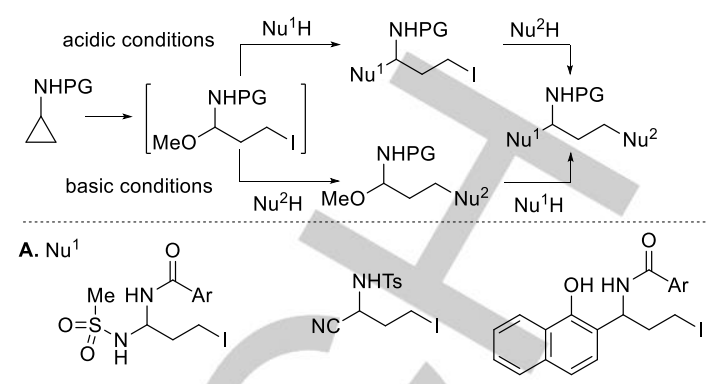

$11,69 \%$, one pot $12,65 \%$, one pot $13,53 \%$, one pot

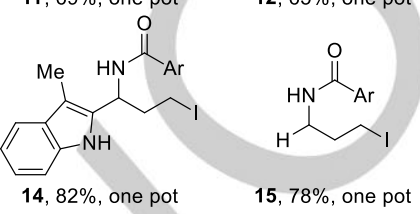

B. $\mathrm{Nu}^{2}$

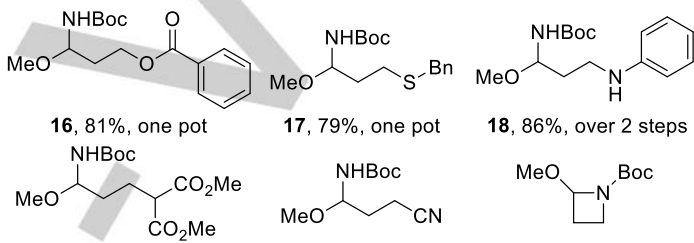

$\mathbf{1 9}, 80 \%$, over 2 steps $\quad \mathbf{2 0}, 94 \%$, over 2 steps $\quad \mathbf{2 1}, 25 \%$, one pot

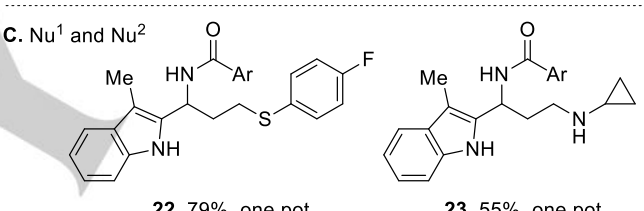
Scheme 4. Conversion of aminocyclopropanes into a variety of $\alpha, \gamma-$
difunctionalized amines. $\mathrm{Ar}=p-\mathrm{MeO}-\mathrm{Ph}$. See supporting Information for detailed reaction conditions.

Enantioselective addition of alcohols or peroxides to imines has been explored mainly by using two different types of catalysts: chiral phosphoric acids (CPA) and cinchona alkaloid derivatives. ${ }^{[14]}$ In order to obtain the product with enantiomeric excess, we utilized CPA 24 as catalyst (Scheme 5). N,O-acetal 4a could be obtained in $82 \%$ yield and $78 \%$ ee, which is highly promising for the future development of enantioselective transformations.

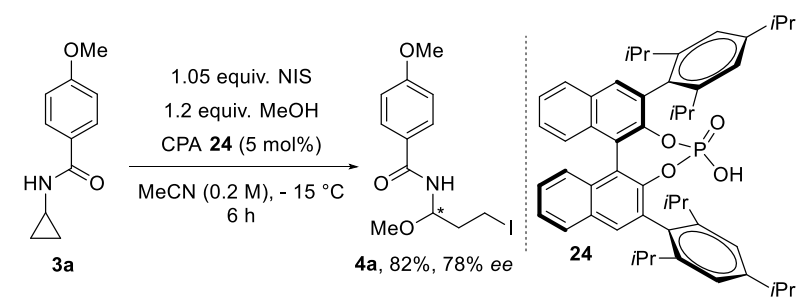

Scheme 5. Enantioselective ring-opening of cyclopropane 3a using CPA 24.

In order to understand the mechanism better, we performed several control experiments (Scheme 6). When mixing $3 q$ and 
$\mathrm{NXS}(\mathrm{X}=\mathrm{Br}, \mathrm{I})$ in $\mathrm{CDCl}_{3}$ for two hours, we observed formation of compounds $\mathbf{2 5}$ and $\mathbf{2 6}$, corresponding to the initially postulated intermediates I and II (Scheme 6, equation 1). In the case of NIS, compounds 25a was unstable and converted directly into imine $\mathbf{2 6 a}$. With NBS, only few conversion of $\mathbf{2 5 b}$ into $\mathbf{2 6}$ b was also observed after two hours. Longer reaction times led to nonreproducible results, with varying amounts of $26 \mathrm{~b}$ being formed. In order to get more reliable results, we synthesized $\mathrm{N}$-bromo cyclopropane 25b using a reported procedure. ${ }^{[8]}$ Pure 25b was converted quantitatively to $\mathbf{2 6 \mathrm { b }}$ in $6 \mathrm{~h}$ under ambient light (Scheme 6, equation 2). In contrast, nearly no conversion was observed in the dark. The fact that the reaction of the $\mathrm{N}$-bromo compound is mediated by light could rationalize the low reproducibility of the results obtained in the one-pot transformation. Imine $\mathbf{2 6 b}$ could then be transformed to N,O acetal $\mathbf{2 7}$ in the dark. The presence of methanol since the start of the reaction led to the same result, with only a small amount of competing debromination (Scheme 6, equation 3). Again, no reaction was observed in absence of light. Interestingly, when $\mathbf{2 5 b}$ was treated with NIS, the formation of both bromination and iodination products $\mathbf{2 6}$ b and $\mathbf{2 6 a}$ was observed, indicating that a cross-over pathway is operative (Scheme 6, equation 4). Finally, when using 2-methyl substituted aminocyclopropane 28 (d.r. = $4: 1$ ), difunctionalization product 29 was isolated as a mixture of two diastereoisomers in a 1:1 ratio after one-pot substitution of iodide with benzoate (Scheme 6, equation 5). ${ }^{[15]}$

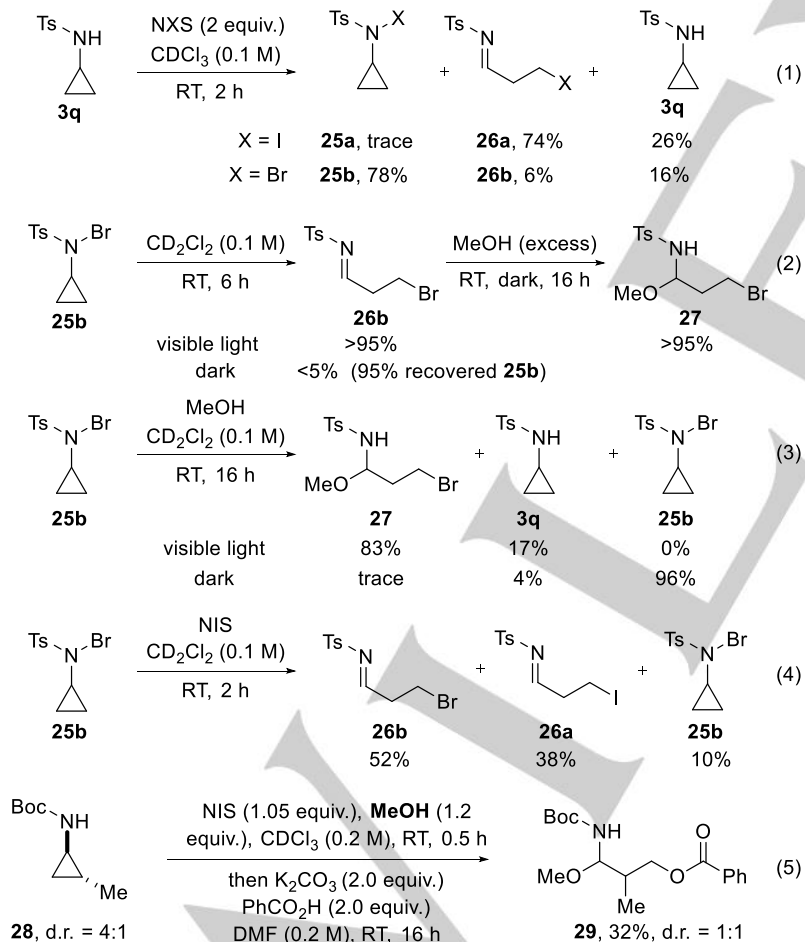

Scheme 6. Mechanistic investigations. NMR yields are reported, except for compound 29 (isolated yield).
Based on those results, we propose the mechanism depicted in Scheme 7. Halogenation of the aminocyclopropane by NXS provides $\mathrm{N}-\mathrm{X}$ cyclopropane I, which undergoes homolytic cleavage of the $\mathrm{N}-\mathrm{X}$ bond to form two radical species (III). Due to the ring strain of the cyclopropane, ring-opening occurs to form the primary alkyl radical which recombines with the halogen radical (IV) to give imine II. A radical chain process with the primary radical reacting with a further molecule of $\mathbf{I}$ can be envisaged. The incorporation of iodine in presence of NIS further support the presence of a primary radical such as IV. However, it is not possible at this stage to exclude other reaction mechanisms, such as direct halogenation of the $\mathrm{C}-\mathrm{C}$ bond or a concerted rearrangement of I to II. Further studies will be needed to better establish the reaction mechanism. In the presence of $\mathrm{MeOH}$, the $\mathrm{N}, \mathrm{O}$-acetal product is then formed. This last step can be accelerated by a Brønsted acid catalyst.

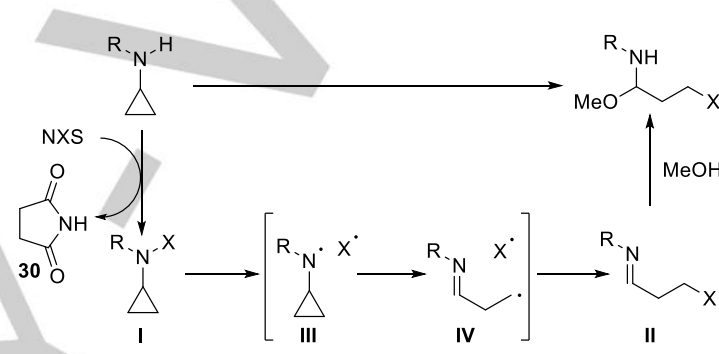

Scheme 7. Speculative reaction mechanism.

In summary, we have developed a practical and general strategy for the ring-opening and 1,3-difunctionalization of aminocyclopropanes. A series of stable 3-iodo N,O-acetal products were isolated. From these 1,3-dielectrophiles, a wide range of $\alpha, \gamma$-difunctionalized products were rapidly accessed in one pot or in two steps. By using a chiral phosphoric acid as catalyst, a proof-of-concept for asymmetric induction was achieved. Based on the simple reaction procedure and the structural diversity of nitrogen-containing building blocks obtained, we believe that this new methodology will be useful in synthetic and medicinal chemistry.

\section{Acknowledgements}

We thank the Swiss National Science Foundation (SNSF, grant nos. 200021_165788) and EPFL for financial support. We thank Dr. Hua Wu from Prof. Jieping Zhu's group (LSPN/ISIC/EPFL) for the generous donation of CPA catalyst.

Keywords: aminocyclopropanes • ring-opening • amines $\bullet$ 1,3dielectrophile intermediate $\cdot$ synthetic methodology

[1] a) E. Vilsmaier, The Chemistry of the Cyclopropyl Group, ed. Z. Rappoport, Wiley, New York, 1987, ch. 22, p. 1341. b) F. Brackmann, A de Meijere, Chem. Rev. 2007, 107, 4493; c) F. de Nanteuil, F. De Simone, 
R. Frei, F. Benfatti, E. Serrano, J. Waser, Chem. Commun. 2014, 50 10912; d) V. A. Rassadin, Y. Six, Tetrahedron 2016, 72, 4701.

[2] a) N. M. Aston, P. Bamborough, J. B. Buckton, C. D. Edwards, D. S. Holmes, K. L. Jones, V. K. Patel, P. A. Smee, D. O. Somers, G. Vitulli, A. L. Walker, J. Med. Chem. 2009, 52, 6257; b) T. Malcomson, K. Yelekci, M. T. Borrello, A. Ganesan, E. Semina, N. De Kimpe, S. Mangelinckx, R. R. Ramsay, FEBS Journal. 2015, 282, 3190; c) Y. Zheng, B. Liu, Z. Gou, Y. Li, X. Zhang, Y. Wang, S. Yu, Y. Li, D. Sun, Bioorg. Med. Chem. Lett. 2015, 25, 791; d) Q. Zheng, Z. Chen, H. Wan, S. Tang, Y. Ye, Y. Xu, L, Jiang, J. Ding, M. Geng, M. Huang, Y. Huang, Bioorg. Med. Chem. Lett 2018, 28, 3808;

[3] a) F. de Nanteuil, J. Waser, Angew. Chem. Int. Ed. 2011, 50, 12075 Angew. Chem. 2011, 123, 12281; b) F. Benfatti, F. de Nanteuil, J. Waser Org. Lett. 2012, 14, 386; c) F. Benfatti, F. de Nanteuil, J. Waser, Chem. Eur. J. 2012, 18, 4844; d) F. de Nanteuil, J. Loup, J. Waser, Org. Lett. 2013, 15, 3738; e) F. de Nanteuil, E. Serrano, D. Perrotta, J. Waser, J. Am. Chem. Soc. 2014, 136, 6239; f) S. Racine, F. de Nanteuil, E. Serrano, J. Waser, Angew. Chem. Int. Ed. 2014, 53, 8484; Angew. Chem. 2014, 126, 8624 ; g) E. Serrano, F. de Nanteuil, J. Waser, Synlett 2014, 25, 2285; h) S. Racine, B, Hegedus, R. Scopelliti, J. Waser, Chem. Eur. J. 2016, 22, 11997; i) J. Preindl, S, Chakrabarty, J. Waser, Chem. Sci. 2017 8, 7112; j) D. Perrotta, M.-M. Wang, J. Waser, Angew. Chem. Int. Ed. 2018, 57, 5120; Angew. Chem. 2018, 130, 5214; k) A. R. Rivero, I. Fernández, M. Á. Sierra, Org. Lett. 2013, 15, 4928; I) M. Zhu, D.-C. Wang, M.-S. Xie, G.-R. Qu, H.-M. Guo, Chem. Eur. J. 2018, 24, 15512; m) E. R. Hao, D.-D. Fu, D.-C. Wang, T. Zhang, G.-R. Qu, G.-X. Li, Y. Lan, H.M. Guo, Org. Chem. Front., 2019, 6, 863; n) For a general review on DA cyclopropanes, see: T. F. Schneider, J. Kaschel, D. B. Werz, Angew. Chem., Int. Ed. 2014, 53, 5504

[4] a) Y. Takemoto, S. Yamagata, S. Furuse, H. Hayase, T. Echigo, C. Iwata, Chem. Commun. 1998, 651; b) J. D. Ha, J. Lee, S. C. Blackstock, J. K. Cha, J. Org. Chem. 1998, 63, 8510; c) K. Wimalasena, H. B. Wickman, M. P. D. Mahindaratne, Eur. J. Org. Chem. 2001, 3811; d) N. Ouhamou, Y. Six, Org. Biomol. Chem. 2003, 1, 3007; e) C. Madelaine, Y. Six, O. Buriez, Angew. Chem. Int. Ed. 2007, 46, 8046; Angew. Chem. 2007, 119, 8192; f) S. Maity, M. Zhu, R. S. Shinabery, N. Zheng, Angew. Chem. Int Ed. 2012, 51, 222; Angew. Chem. 2012, 124, 226; g) T. H. Nguyen, S. Maity, N. Zheng, Beilstein J. Org. Chem. 2014, 10, 975.

[5] a) S. Rousseaux, B. Liégault, K. Fagnou, Chem. Sci. 2012, 3, 244; b) A. Dos Santos, L. El Kaïm, L. Grimaud, R. Ramozzi, Synlett 2012, 438; c) C. L. Ladd, D. S. Roman, A. B. Charette, Tetrahedron 2013, 69, 4479; d) M. H. Shaw, E. Y. Melikhova, D. P. Kloer, W. G. Wittingham, J. F. Bower, J. Am. Chem. Soc. 2013, 135, 4992; e) M. H. Shaw, N. G. McCreanor, W. G. Wittingham, J. F. Bower, J. Am. Chem. Soc. 2015, 137, 463; f) M. H. Shaw, R. A. Croft, W. G. Wittingham, J. F. Bower, J. Am. Chem. Soc 2015, 137, 8054; g) M. H. Shaw, W. G. Wittingham, J. F. Bower, Tetrahedron 2016, 72, 2731; h) N. G. McCreanor, S. Stanton, J. F. Bower J. Am. Chem. Soc. 2016, 138, 11465; i) G. W. Wang, J. F. Bower, J. Am. Chem. Soc. 2018, 140, 2743; j) H. Kondo, K. Itami, J. Yamaguchi, Chem. Sci. 2017, 8, 3799.

[6] To the best of our knowledge there is no report of a general synthesis of 1,3-difunctionalized non-cyclic propylamines starting from aminocyclopropanes. For examples of the synthesis of 1,3difunctionalized propylamines starting from other types of cyclopropanes, see: a) S. Das, C. G. Daniliuc, A. Studer, Angew. Chem. Int. Ed. 2017, 56, 11554; Angew. Chem. 2017, 129, 11712; b) S. M. Banik, K. M. Mennie, E. N. Jacobsen, J. Am. Chem. Soc. 2017, 139, 9152; c) J. Wallbaum, L. K. B. Garve, P. G. Jones, D. B. Werz, Org. Lett. 2017, 19, 98; d) D. B. Werz, A. U. Augustin, P. G. Jones, Chem. Eur. J. 2019, in press, DOI: 10.1002/chem.201902160.
[7] Selected reviews on Hofmann-Löffler-Freytag reaction, see: a) M. E. Wolff, Chem. Rev. 1963, 63, 55; b) R. S. Neale, Synthesis 1971, 1971, 1; c) L. Stella, Angew. Chem. Int. Ed. 1983, 22, 337; Angew. Chem. 1983, 95, 368; d) J. L. Jeffrey, R. Sarpong, Chem. Sci. 2013, 4, 4092. For selected examples of recent progress on the Hofmann-Löfler-Freytag reaction, see: e) C. Martinez, K. Muniz, Angew. Chem. Int. Ed. 2015, 54, 8287; Angew. Chem. 2015, 127, 8405; f) C. Q. O'Broin, P. Fernandez, C. Martínez, K. Muniz, Org. Lett. 2016, 18, 436; g) P. Becker, T. Duhamel, C. J. Stein, M. Reiher, K. Muñiz, Angew. Chem. Int. Ed. 2017, 56, 8004; Angew. Chem. 2017, 129, 8117; h) T. Duhamel, C. J. Stein, M. Reiher, K. Muñiz, ACS Catal. 2018, 8, 3918; i) P. Becker, T. Duhamel, C. Martínez, K. Muñiz, Angew. Chem. Int. Ed. 2018, 57, 5166; Angew. Chem. 2018, 130, 5262; j) Qin, Q.; Yu, S. Org. Lett. 2015, 17, 1894; k) N. R. Paz, D. Rodríguez-Sosa, H. Valdes, R. Marticorena, D. Melian, M. B. Copano, C. C. Gonzalez, A. Herrera, J. Org. Lett. 2015, 17, 2370; I) E. A. Wappes, S. C. Fosu, T. C. Chopko, D. A. Nagib, Angew. Chem. Int. Ed. 2016, 55, 9974; Angew. Chem. 2016, 128, 10128; m) E. A. Wappes, K. M. Nakafuku, D. A. Nagib, J. Am. Chem. Soc. 2017, 139, 10204; n) J. Long, X. Cao, L. Zhu, R. Qiu, C.-T. Au, S.-F. Yin, T. Iwasaki, N. Kambe, Org. Lett. 2017, 19, 2793; o) D. Zhang, H. Wang, H. Cheng, J. G. Hernandez, C. Bolm, Adv. Synth. Catal. 2017, 359, 4274; p) X.-Q. Mou, X.-Y.Chen, G. Chen, G. He, Chem. Commun. 2018, 54, 515; q) D. Chang, R. Zhao, C. Wei, Y. Yao, Y. Liu, L. Shi, J. Org. Chem. 2018, 83, 3305.

[8] E. E. J. Dekker, J. B. F. N. Engberts, Th. J. de. Boer, Recl. Trav. Chim. Pays-Bas, 1978, 97, 39. In this work, only the aldehyde resulting from hydrolysis of imines such as II could be isolated.

[9] Similar dielectrophilic synthons can be obtained from acrolein by condensation with an amine. However, they have not find widespread use in organic synthesis due to their low stability and difficult synthesis. See: M. Shimizu, I. Hachiya, I. Mizota, Chem. Commun. 2009, 874.

[10] For selected reviews on transformations of imines and N,O-acetals, see: a) S. Kobayashi, Y. Mori, J. S. Fossey, M. M. Salter, Chem. Rev. 2011, 111, 2626; b) Y.-Y. Huang, C. Cai, X. Yang, Z.-C. Lv, U. Schneider, ACS Catal. 2016, 6, 5747.

[11] For a single example of oxidative ring-opening of a tosyl/alkyl-substituted aminocyclopropane in the context of a total synthesis, see: N. C. Mancey, N, Sandon, A.-L. Auvidet, R. J. Butlin, W, Czechtizky, J. P. A. Harrity, Chem. Commun. 2011, 9804.

[12] D, A. Casteel, Nat. Prod. Rep. 1999, 16, 55.

[13] M. R. Faust, G. Hofner, J. Pabel, K. T. Wanner, Eur. J. Med. Chem. 2010, 45, 2453.

[14] a) G. Li, F. R. Fronczek, J. C. Antilla, J. Am. Chem. Soc. 2008, 130, 12216; b) W. Zheng, L. Wojtas, J. C. Antilla, Angew. Chem. Int. Ed. 2010 49, 6589; Angew. Chem. 2010, 122, 6739; c) S. Nakamura, S. Takahashi, Org. Lett. 2015, 17, 2590.

[15] For the ring-opening reaction, products involving C-C bond cleavage of the more and less substituted $C-C$ bonds were observed in a $1: 1$ ratio by ${ }^{1} \mathrm{H}$ NMR of the crude reaction mixture. The iodides were too unstable to be isolated in pure form. During the in situ $\mathrm{S}_{\mathrm{N}} 2$ reaction, the secondary iodide decomposed and only 29 could be isolated. The observed 1:1 ratio is surprising when considering the relative stability of secondary and primary radicals, but may be due to a very early transition state originating from high ring-strain. 


\section{COMMUNICATION}

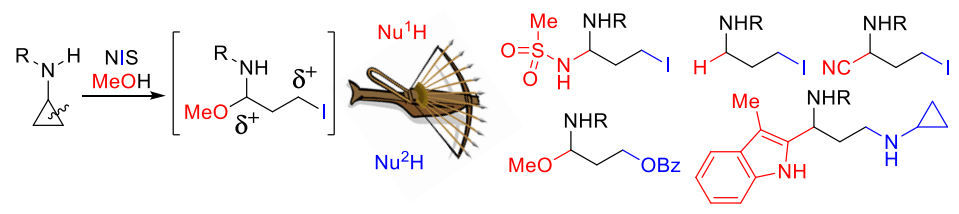

Choose your nucleophiles: We report an oxidative ring-opening strategy to transform aminocyclopropanes into 1,3-dielectrophilic halogenated N,O-acetals. Substitution of either the alkoxy group of the $\mathrm{N}, \mathrm{O}$-acetal or the halide by a series of nucleophiles then generates a broad range of 1,3-difunctionalized propylamines.
Ming-Ming Wang and Jérôme Waser*

Page No. - Page No.

1,3-Difunctionalization

of

Aminocyclopropanes via 


\section{Table of Contents}

1. General Methods $\quad$ S2

2. Preparation of starting materials $\quad$ S3

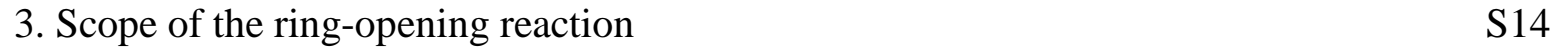

4. Flexible conversion of aminocyclopropanes $\quad$ S32

5. Mechanistic studies $\quad$ S43

6. Spectra for New Compounds $\quad$ S49 


\section{General Methods}

For quantitative flash chromatography, distilled technical grade solvents were used. THF, $\mathrm{Et}_{2} \mathrm{O}$, toluene, hexane and $\mathrm{CH}_{2} \mathrm{Cl}_{2}$ were dried by passage over activated alumina under nitrogen atmosphere $\left(\mathrm{H}_{2} \mathrm{O}\right.$ content $<7 \mathrm{ppm}$, Karl-Fischer titration). All chemicals were purchased and used as received unless stated otherwise. Chromatographic purification was performed as flash chromatography using Macherey-Nagel silica 40-63, $60 \AA$, or aluminium oxide, basic, Brockmann I, 50-200, $60 \AA$, using the solvents indicated as eluent with 0.1-0.5 bar pressure. TLC was performed on Merck silica gel 60 F254 TLC plastic or aluminium plates and visualized with UV light, permanganate CAN or panisaldehyde stains. Melting points were measured on a calibrated Büchi B-540 melting point apparatus using open glass capillaries. ${ }^{1} \mathrm{H}-\mathrm{NMR}$ spectra were recorded at room temperature on a Brucker DPX-400 $400 \mathrm{MHz}$ spectrometer in $\mathrm{CDCl}_{3}$, Acetone- $d_{6}, \mathrm{CD}_{3} \mathrm{CN}$ or $\mathrm{CD}_{3} \mathrm{OD}$, all signals are reported in ppm with the internal chloroform signal at $7.26 \mathrm{ppm}$, the internal acetone signal at 2.09 $\mathrm{ppm}$, the internal acetonitrile signal at $1.94 \mathrm{ppm}$ and the internal methanol signal at $3.34 \mathrm{ppm}$ as standard. The data is being reported as $(\mathrm{s}=$ singlet, $\mathrm{d}=$ doublet, $\mathrm{t}=$ triplet, $\mathrm{q}=$ quadruplet, $\mathrm{p}=$ quintet, $\mathrm{m}=$ multiplet or unresolved, $\mathrm{br}=$ broad signal, integration, coupling constant(s) in Hz, interpretation). ${ }^{13} \mathrm{C}-\mathrm{NMR}$ spectra were recorded with $1 \mathrm{H}$-decoupling on a Brucker DPX-400 $100 \mathrm{MHz}$ spectrometer in $\mathrm{CDCl}_{3}$, Acetone- $d_{6}, \mathrm{CD}_{3} \mathrm{CN}$ or $\mathrm{CD}_{3} \mathrm{OD}$, all signals are reported in ppm with the internal chloroform signal at $77.0 \mathrm{ppm}$. Acetone- $d_{6}$ signal at $29.8 \mathrm{ppm}, \mathrm{CD}_{3} \mathrm{CN}$ signal at $1.3 \mathrm{ppm}$ or $\mathrm{CD}_{3} \mathrm{OD}$ signal at 49.0 ppm as standard. Infrared spectra were recorded on a JASCO FT-IR B4100 or a Bruker Alpha-P spectrophotometer with an ATR device and a ZnSe prism and are reported as $\mathrm{cm}-1(\mathrm{w}=$ weak, $\mathrm{m}=$ medium, $\mathrm{s}=$ strong). High resolution mass spectrometric measurements were performed by the mass spectrometry service of ISIC at the EPFL on a MICROMASS (ESI) Q-TOF Ultima API. HPLC measurements were done on a Agilent 1260 Infinity autosampler using a CHIRALPAK IA, IB, IC or IF column from DAICEL Chemical. Optical rotations were measured on a polarimeter using a $10 \mathrm{~cm}$ cell with a $\mathrm{Na} 589 \mathrm{~nm}$ filter. The specific solvents and concentrations (in $\mathrm{g} / 100 \mathrm{~mL}$ ) are indicated. 


\section{$\underline{\text { 2. Preparation of starting materials }}$}

\subsection{Synthesis of the aminocyclopropanes}

\section{General Procedure A (GP A):}<smiles>[R]C(=O)NC1CC1</smiles>

Following a modified version of a reported procedure,$^{[1]}$ to a solution of cyclopropylamine $(0.70 \mathrm{~mL}$, $10 \mathrm{mmol}, 1.1$ equiv.) and triethylamine $(1.40 \mathrm{~mL}, 10.0 \mathrm{mmol}, 1.1$ equiv.) in dichloromethane $(10 \mathrm{~mL})$ was slowly added a solution of acyl chloride $(9.09 \mathrm{mmol}, 1.0$ equiv.) in dichloromethane $(10 \mathrm{~mL})$ at 0 ${ }^{\circ} \mathrm{C}$. The reaction mixture was stirred at room temperature usually for 16 hours. Upon completion, the mixture was quenched by the addition of $1 \mathrm{~N} \mathrm{HCl}(10 \mathrm{~mL})$. The aqueous layer was then extracted with dichloromethane. The organic extract was washed with brine $(10 \mathrm{~mL})$, dried over $\mathrm{Na}_{2} \mathrm{SO}_{4}$, filtered and concentrated in vacuo. In most cases, the crude product was pure enough to be used as such, without further purification.

\section{N-Cyclopropyl-4-methoxybenzamide (3a)}<smiles>COc1ccc(C(=O)NC2CC2)cc1</smiles>

Following GP A, using 4-methoxybenzoyl chloride (1.55 g, 9.09 mmol), N-cyclopropyl-4methoxybenzamide (3a) was obtained as a white solid (1.90 g, $8.99 \mathrm{mmol}, 99 \%)$.

${ }^{1} \mathrm{H}$ NMR $\left(400 \mathrm{MHz}, \mathrm{CDCl}_{3}\right) \delta 7.74-7.66(\mathrm{~d}, J=8.8 \mathrm{~Hz}, 2 \mathrm{H}, \operatorname{ArH}), 6.94-6.85(\mathrm{~d}, J=8.8 \mathrm{~Hz}, 2 \mathrm{H}, \operatorname{ArH})$, $6.21(\mathrm{~s}, 1 \mathrm{H}, \mathrm{NH}), 3.84\left(\mathrm{~s}, 3 \mathrm{H}, \mathrm{OCH}_{3}\right), 2.88(\mathrm{tq}, J=7.1,3.6 \mathrm{~Hz}, 1 \mathrm{H}, \mathrm{CH}), 0.85\left(\mathrm{td}, J=7.0,5.3 \mathrm{~Hz}, 2 \mathrm{H}, \mathrm{CH}_{2}\right)$, $0.65-0.55\left(\mathrm{~m}, 2 \mathrm{H}, \mathrm{CH}_{2}\right)$.

${ }^{1} \mathrm{H}$ NMR data correspond to the reported values. ${ }^{[1]}$

N-Cyclopropyl-4-(methylthio)benzamide (3b).

\footnotetext{
${ }^{1}$ Baburajan, P.; Elango, K. P. Tetrahedron Lett. 2014, 55, 1006-1010.
} 
<smiles>CSc1ccc(C(=O)NC2CC2)cc1</smiles>

Following GP A, using 4-(methylthio)benzoyl chloride $(1.70 \mathrm{~g}, 9.09 \mathrm{mmol}), \mathrm{N}$-cyclopropyl-4(methylthio)benzamide (3b) was obtained as an off-white solid (1.41 g, $6.80 \mathrm{mmol}, 75 \%$ ).

$\mathbf{R}_{\mathbf{f}}: 0.33$ (silica, pentanes:ethyl acetate $1: 1$ );

Mp: $158-160^{\circ} \mathrm{C}$;

${ }^{1} \mathrm{H}$ NMR $\left(400 \mathrm{MHz}, \mathrm{CDCl}_{3}\right): \delta=7.68-7.61(\mathrm{~m}, 2 \mathrm{H}, \mathrm{ArH}), 7.25-7.20(\mathrm{~m}, 2 \mathrm{H}, \mathrm{ArH}), 6.24(\mathrm{~s}, 1 \mathrm{H}, \mathrm{NH})$, $2.89(\mathrm{dq}, J=7.0,3.4 \mathrm{~Hz}, 1 \mathrm{H}, \mathrm{CH}), 2.50\left(\mathrm{~s}, 3 \mathrm{H}, \mathrm{SCH}_{3}\right), 0.91-0.80\left(\mathrm{~m}, 2 \mathrm{H}, \mathrm{CH}_{2}\right), 0.66-0.54\left(\mathrm{~m}, 2 \mathrm{H}, \mathrm{CH}_{2}\right)$; ${ }^{13} \mathrm{C}$ NMR $\left(101 \mathrm{MHz}, \mathrm{CDCl}_{3}\right): \delta=168.3,143.4,130.4,127.2,125.3,23.1,15.0,6.8$;

IR (film): $\tilde{v}=3271(\mathrm{~m}), 3006$ (w), 1622 (s), 1556 (s), $1486(\mathrm{~m}), 1315$ (m), 1121 (w), $840(\mathrm{~m}), 761$ (m); HRMS (ESI) calcd. for $\mathrm{C}_{11} \mathrm{H}_{13} \mathrm{NNaOS}^{+}[\mathrm{M}+\mathrm{Na}]^{+}$230.0610; Found 230.0613 .

N-Cyclopropyl-4-fluorobenzamide (3c).<smiles>O=C(NC1CC1)c1ccc(F)cc1</smiles>

Following GP A, using 4-fluorobenzoyl chloride (1.44 g, $9.09 \mathrm{mmol}), \mathrm{N}$-cyclopropyl-4-fluorobenzamide (3c) was obtained as a white solid $(1.50 \mathrm{~g}, 8.36 \mathrm{mmol}, 92 \%)$.

${ }^{1} \mathrm{H}$ NMR $\left(400 \mathrm{MHz}, \mathrm{CDCl}_{3}\right): \delta=7.79-7.70(\mathrm{~m}, 2 \mathrm{H}, \operatorname{ArH}), 7.12-7.01(\mathrm{~m}, 2 \mathrm{H}, \operatorname{Ar} H), 6.37(\mathrm{~s}, 1 \mathrm{H}, \mathrm{NH})$, $2.87(\mathrm{tq}, J=7.1,3.6 \mathrm{~Hz}, 1 \mathrm{H}, \mathrm{CH}), 0.84\left(\mathrm{td}, J=7.0,5.3 \mathrm{~Hz}, 2 \mathrm{H}, \mathrm{CH}_{2}\right), 0.65-0.56\left(\mathrm{~m}, 2 \mathrm{H}, \mathrm{CH}_{2}\right)$.

${ }^{1} \mathrm{H}$ NMR data correspond to the reported values. ${ }^{[2]}$

4-Chloro-N-cyclopropylbenzamide (3d).<smiles>O=C(NC1CC1)c1ccc(Cl)cc1</smiles>

\footnotetext{
${ }^{2}$ Kondo, H.; Itami, K.; Yamaguchi, J. Chem. Sci. 2017, 8, 3799-3803.
} 
Following GP A, using 4-chlorobenzoyl chloride (1.59 g, $9.09 \mathrm{mmol})$, 4-chloro- $\mathrm{N}$ cyclopropylbenzamide (3d) was obtained as a white solid (1.65 g, $8.43 \mathrm{mmol}, 93 \%)$.

$\mathbf{R}_{\mathbf{f}}: 0.59$ (silica, pentanes:ethyl acetate 2:3);

Mp: $133-135^{\circ} \mathrm{C}$;

${ }^{1}$ H NMR $\left(400 \mathrm{MHz}, \mathrm{CDCl}_{3}\right): \delta=7.70-7.64(\mathrm{~m}, 2 \mathrm{H}, \mathrm{ArH}), 7.41-7.35(\mathrm{~m}, 2 \mathrm{H}, \operatorname{ArH}), 6.27(\mathrm{~s}, 1 \mathrm{H}, \mathrm{NH})$, $2.88(\mathrm{tt}, J=7.1,3.5 \mathrm{~Hz}, 1 \mathrm{H}, \mathrm{CH}), 0.90-0.83\left(\mathrm{~m}, 2 \mathrm{H}, \mathrm{CH}_{2}\right), 0.66-0.55\left(\mathrm{~m}, 2 \mathrm{H}, \mathrm{CH}_{2}\right)$;

${ }^{13} \mathrm{C}$ NMR $\left(101 \mathrm{MHz}, \mathrm{CDCl}_{3}\right): \delta=167.8,137.7,132.7,128.8,128.3,23.2,6.8$;

IR (film): $\tilde{v}=3309$ (m), 1639 (s), 1528 (m), 1484 (m), 1312 (m), 1093 (m), 847 (m);

HRMS (ESI) calcd. for $\mathrm{C}_{10} \mathrm{H}_{10} \mathrm{CINNaO}^{+}[\mathrm{M}+\mathrm{Na}]^{+} 218.0343$; Found 218.0344.

\section{4-Cyano-N-cyclopropylbenzamide (3e).}<smiles>N#Cc1ccc(C(=O)NC2CC2)cc1</smiles>

Following GP A, using 4-cyanobenzoyl chloride (1.51 g, $9.09 \mathrm{mmol})$, 4-cyano-N-cyclopropylbenzamide (3e) was obtained as a white solid (1.60 g, $8.59 \mathrm{mmol}, 95 \%)$.

$\mathbf{R}_{\mathbf{f}}: 0.33$ (silica, pentanes:ethyl acetate $1: 1$ );

Mp: $159-161^{\circ} \mathrm{C}$;

${ }^{1}$ H NMR $\left(400 \mathrm{MHz}, \mathrm{CDCl}_{3}\right): \delta=7.84(\mathrm{~d}, J=8.1 \mathrm{~Hz}, 2 \mathrm{H}, \operatorname{ArH}), 7.77-7.64(\mathrm{~m}, 2 \mathrm{H}, \operatorname{ArH}), 6.34(\mathrm{~d}, J=38.4$ $\mathrm{Hz}, 1 \mathrm{H}, \mathrm{NH}), 2.91(\mathrm{tq}, J=7.0,3.6 \mathrm{~Hz}, 1 \mathrm{H}, \mathrm{CH}), 1.09-0.73\left(\mathrm{~m}, 2 \mathrm{H}, \mathrm{CH}_{2}\right), 0.70-0.52\left(\mathrm{~m}, 2 \mathrm{H}, \mathrm{CH}_{2}\right)$; ${ }^{13} \mathrm{C}$ NMR $\left(101 \mathrm{MHz}, \mathrm{CDCl}_{3}\right): \delta=167.0,138.3,132.4,127.6,118.0,115.1,23.3,6.8$;

IR (film): $\tilde{v}=3275$ (m), 3015 (w), $2230(\mathrm{~m}), 1632$ (s), 1532 (s), 1499 (m), $1313(\mathrm{~m}), 1284(\mathrm{~m}), 1018(\mathrm{w})$, $858(\mathrm{~m})$;

HRMS (ESI) calcd. for $\mathrm{C}_{11} \mathrm{H}_{11} \mathrm{~N}_{2} \mathrm{O}^{+}[\mathrm{M}+\mathrm{H}]^{+}$187.0866; Found 187.0862.

\section{N-Cyclopropyl-4-nitrobenzamide (3f).}<smiles>O=C(NC1CC1)c1ccc([N+](=O)[O-])cc1</smiles>

Following GP A, using 4-nitrobenzoyl chloride (1.69 g, $9.09 \mathrm{mmol}$ ), N-cyclopropyl-4-nitrobenzamide (3f) was obtained as a pale yellow solid $(1.71 \mathrm{~g}, 8.27 \mathrm{mmol}, 91 \%)$.

$\mathbf{R}_{\mathbf{f}}: 0.31$ (silica, pentanes:ethyl acetate 1:1);

Mp: $176-177^{\circ} \mathrm{C}$; 
${ }^{1} \mathrm{H}$ NMR $\left(400 \mathrm{MHz}, \mathrm{CDCl}_{3}\right): \delta=8.30-8.22(\mathrm{~m}, 2 \mathrm{H}, \mathrm{ArH}), 7.94-7.86(\mathrm{~m}, 2 \mathrm{H}, \operatorname{ArH}), 6.41(\mathrm{~s}, 1 \mathrm{H}, \mathrm{NH})$, $2.92(\mathrm{tq}, J=7.2,3.7 \mathrm{~Hz}, 1 \mathrm{H}, \mathrm{CH}), 0.91\left(\mathrm{td}, J=7.1,5.4 \mathrm{~Hz}, 2 \mathrm{H}, \mathrm{CH}_{2}\right), 0.70-0.59\left(\mathrm{~m}, 2 \mathrm{H}, \mathrm{CH}_{2}\right)$;

${ }^{13} \mathrm{C}$ NMR $\left(101 \mathrm{MHz}, \mathrm{CDCl}_{3}\right): \delta=166.8,149.6,139.9,128.0,123.8,23.4,6.8$;

IR (film): $\tilde{v}=3280$ (m), 1639 (s), 1597 (w), 1533 (m), 1514 (s), 1350 (m), 1308 (m);

HRMS (ESI) calcd. for $\mathrm{C}_{10} \mathrm{H}_{11} \mathrm{~N}_{2} \mathrm{O}_{3}{ }^{+}[\mathrm{M}+\mathrm{H}]^{+}$207.0764; Found 207.0761.

\section{$\mathrm{N}$-Cyclopropyl-3-methoxybenzamide (3g).}<smiles>COc1cccc(C(=O)NC2CC2)c1</smiles>

Following GP A, using 3-methoxybenzoyl chloride (1.55 g, $9.09 \mathrm{mmol})$, N-cyclopropyl-3methoxybenzamide $(\mathbf{g g})$ was obtained as a pale yellow solid $(1.88 \mathrm{~g}, 8.90 \mathrm{mmol}, 98 \%)$.

$\mathbf{R}_{\mathbf{f}}: 0.31$ (silica, pentanes:ethyl acetate 1:1);

Mp: $74-76^{\circ} \mathrm{C}$;

${ }^{1} \mathrm{H}$ NMR $\left(400 \mathrm{MHz}, \mathrm{CDCl}_{3}\right): \delta=7.29(\mathrm{~s}, 1 \mathrm{H}, \mathrm{ArH}), 7.23(\mathrm{~d}, J=7.7 \mathrm{~Hz}, 1 \mathrm{H}, \operatorname{ArH}), 7.19(\mathrm{~d}, J=7.7 \mathrm{~Hz}, 1 \mathrm{H}$, $\mathrm{ArH}), 6.96$ (ddd, $J=8.0,2.7,1.2 \mathrm{~Hz}, 1 \mathrm{H}, \mathrm{ArH}), 6.39(\mathrm{~s}, 1 \mathrm{H}, \mathrm{NH}), 3.78(\mathrm{~s}, 1 \mathrm{H}, \mathrm{OCH})_{3}, 2.84(\mathrm{tt}, J=7.2,3.5$ $\mathrm{Hz}, 1 \mathrm{H}, \mathrm{CH}), 0.92-0.69\left(\mathrm{~m}, 2 \mathrm{H}, \mathrm{CH}_{2}\right), 0.65-0.43\left(\mathrm{~m}, 2 \mathrm{H}, \mathrm{CH}_{2}\right)$;

${ }^{13} \mathrm{C}$ NMR $\left(101 \mathrm{MHz}, \mathrm{CDCl}_{3}\right): \delta=168.8,159.7,135.8,129.4,118.6,117.6,112.2,55.4,23.1,6.7$;

IR (film): $\tilde{v}=3295$ (w), 1638 (m), 1582 (m), 1527 (m), 1485 (m), 1286 (m), 1247 (m), 1040 (m), 732 (s); HRMS (ESI) calcd. for $\mathrm{C}_{11} \mathrm{H}_{13} \mathrm{NNaO}_{2}{ }^{+}[\mathrm{M}+\mathrm{Na}]^{+} 214.0838$; Found 214.0842 .

N-Cyclopropyl-2-methoxybenzamide (3h).<smiles>COc1ccccc1C(=O)NC1CC1</smiles>

Following GP A, using 2-methoxybenzoyl chloride (0.853 g, $5.00 \mathrm{mmol})$, N-cyclopropyl-3methoxybenzamide (3h) was obtained as a colorless solid $(0.586 \mathrm{~g}, 3.06 \mathrm{mmol}, 61 \%)$ after purification by column chromatography on silica using 1:1 pentanes:ethyl acetate as eluent.

$\mathbf{R}_{\mathbf{f}}: 0.36$ (silica, pentanes:ethyl acetate 1:1);

Mp: $56-58^{\circ} \mathrm{C}$;

${ }^{1} \mathrm{H}$ NMR $\left(400 \mathrm{MHz}, \mathrm{CDCl}_{3}\right): \delta=8.19(\mathrm{dq}, J=7.8,1.7 \mathrm{~Hz}, 1 \mathrm{H}, \mathrm{ArH}), 7.89(\mathrm{~s}, 1 \mathrm{H}, \mathrm{NH}), 7.40$ (ddq $J=8.7$, 7.3, 1.6 Hz, 1H, ArH), 7.04 (ddt, J = 8.9, 7.6, $1.5 \mathrm{~Hz}, 1 \mathrm{H}, \mathrm{ArH}), 6.92(\mathrm{dq}, J=8.3,1.2 \mathrm{~Hz}, 1 \mathrm{H}, \operatorname{ArH}), 3.91(\mathrm{~s}$, $\left.3 \mathrm{H}, \mathrm{OCH}_{3}\right), 2.91(\mathrm{tq}, J=7.4,3.8 \mathrm{~Hz}, 1 \mathrm{H}, \mathrm{CH}), 0.86-0.78\left(\mathrm{~m}, 2 \mathrm{H}, \mathrm{CH}_{2}\right), 0.61-0.52\left(\mathrm{~m}, 2 \mathrm{H}, \mathrm{CH}_{2}\right)$;

${ }^{13} \mathrm{C} \mathrm{NMR}\left(101 \mathrm{MHz}, \mathrm{CDCl}_{3}\right): \delta=166.5,157.3,132.6,132.1,121.3,121.2,111.2,55.8,22.7,6.7$;

IR (film): $\tilde{v}=3393$ (w), 3002 (w), 1651 (s), 1600 (m), 1525 (s), 1484 (s), 1295 (m), 1240 (s), 1020 (m), $756(\mathrm{~m})$;

HRMS (ESI) calcd. for $\mathrm{C}_{11} \mathrm{H}_{13} \mathrm{NNaO}_{2}^{+}[\mathrm{M}+\mathrm{Na}]^{+}$214.0838; Found 214.0843. 


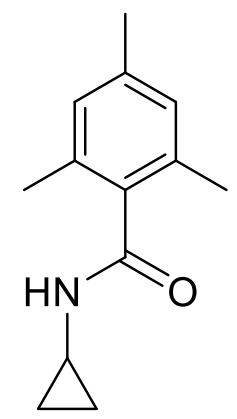

Following GP A, using 2,4,6-trimethylbenzoyl chloride (1.66 g, $9.09 \mathrm{mmol})$, N-cyclopropyl-2,4,6trimethylbenzamide (3i) was obtained as a white solid (1.70 g, $8.36 \mathrm{mmol}, 92 \%)$.

$\mathbf{R}_{\mathrm{f}} \mathbf{0 . 4 0}$ (silica, dichloromethane);

Mp: $133-135^{\circ} \mathrm{C}$;

${ }^{1} \mathrm{H}$ NMR $\left(400 \mathrm{MHz}, \mathrm{CDCl}_{3}\right): \delta=6.81(\mathrm{~s}, 2 \mathrm{H}, \mathrm{ArH}), 5.76(\mathrm{~s}, 1 \mathrm{H}, \mathrm{NH}), 2.90(\mathrm{dqd}, J=7.2,3.5,1.4 \mathrm{~Hz}, 1 \mathrm{H}$, $\mathrm{CH}), 2.25\left(\mathrm{~s}, 9 \mathrm{H}, \mathrm{CH}_{3}\right), 0.92-0.79\left(\mathrm{~m}, 2 \mathrm{H}, \mathrm{CH}_{2}\right), 0.63-0.52\left(\mathrm{~m}, 2 \mathrm{H}, \mathrm{CH}_{2}\right)$;

${ }^{13} \mathrm{C}$ NMR $\left(101 \mathrm{MHz}, \mathrm{CDCl}_{3}\right): \delta=172.0,138.4,134.6,134.1,128.1,22.6,21.0,19.0,6.8$;

IR (film): $\tilde{v}=3223$ (w), $1633(\mathrm{~m}), 1536(\mathrm{~m}), 1310$ (w), 1265 (m), 856 (m), 734 (s);

HRMS (ESI) calcd. for $\mathrm{C}_{13} \mathrm{H}_{17} \mathrm{NNaO}^{+}[\mathrm{M}+\mathrm{Na}]^{+} 226.1202$; Found 226.1206.

$\mathrm{N}$-Cyclopropylfuran-2-carboxamide (3j).

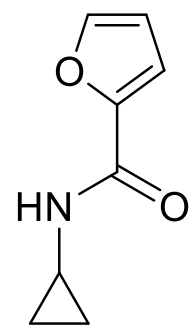

Following GP A, using 2-furoyl chloride (1.19 g, $9.09 \mathrm{mmol}$ ), N-cyclopropylfuran-2-carboxamide (3j) was obtained as an off-white solid (1.31 g, $8.67 \mathrm{mmol}, 95 \%)$.

${ }^{1} \mathrm{H}$ NMR $\left(400 \mathrm{MHz}, \mathrm{CDCl}_{3}\right): \delta=7.39(\mathrm{t}, J=3.1 \mathrm{~Hz}, 1 \mathrm{H}, \mathrm{ArH}), 7.13-7.03(\mathrm{~m}, 1 \mathrm{H}, \mathrm{ArH}), 6.53-6.40(\mathrm{~m}$, $2 \mathrm{H}, \mathrm{ArH}, \mathrm{NH}), 2.85(\mathrm{tq}, J=7.2,3.7 \mathrm{~Hz}, 1 \mathrm{H}, \mathrm{CH}), 0.84\left(\mathrm{tt}, J=6.7,4.1 \mathrm{~Hz}, 2 \mathrm{H}, \mathrm{CH}_{2}\right), 0.66-0.54(\mathrm{~m}, 2 \mathrm{H}$, $\mathrm{CH}_{2}$ ).

${ }^{1} \mathrm{H}$ NMR data correspond to the reported values. ${ }^{[2]}$

N-Cyclopropylcinnamamide (3k).<smiles>O=C(/C=C/c1ccccc1)NC1CC1</smiles>

Following GP A, using cinnamoyl chloride $(1.52 \mathrm{~g}, 9.09 \mathrm{mmol}), \mathrm{N}$-cyclopropylcinnamamide (3k) was obtained as a white solid (1.25 g, $6.68 \mathrm{mmol}, 73 \%)$ after purification by column chromatography on silica using 1:1 pentanes:ethyl acetate as eluent.

$\mathbf{R}_{\mathbf{f}}: 0.32$ (silica, pentanes:ethyl acetate 3:2); 
${ }^{1}$ H NMR $\left(400 \mathrm{MHz}, \mathrm{CDCl}_{3}\right): \delta=7.63(\mathrm{~d}, J=15.6 \mathrm{~Hz}, 1 \mathrm{H}$, vinylH), $7.51-7.45(\mathrm{~m}, 2 \mathrm{H}, \operatorname{ArH}), 7.38-7.31$ $(\mathrm{m}, 3 \mathrm{H}, \mathrm{ArH}), 6.35(\mathrm{~d}, J=15.6 \mathrm{~Hz}, 1 \mathrm{H}$, vinylH), 5.90 (brs, $1 \mathrm{H}, \mathrm{NH}), 2.86(\mathrm{tq}, J=7.1,3.6 \mathrm{~Hz}, 1 \mathrm{H}, \mathrm{CH}), 0.87$ $-0.80\left(\mathrm{~m}, 2 \mathrm{H}, \mathrm{CH}_{2}\right), 0.62-0.52\left(\mathrm{~m}, 2 \mathrm{H}, \mathrm{CH}_{2}\right)$.

${ }^{1} \mathrm{H}$ NMR data correspond to the reported values. ${ }^{[3]}$

N-Cyclopropyl-2-phenylacetamide (3I).<smiles>O=C(Cc1ccccc1)NC1CC1</smiles>

Following GP A, using 2-phenylacetyl chloride (1.41 g, $9.09 \mathrm{mmol}), \mathrm{N}$-cyclopropyl-2-phenylacetamide (3I) was obtained as a white solid $(1.52 \mathrm{~g}, 8.67 \mathrm{mmol}, 95 \%)$.

$\mathbf{R}_{\mathbf{f}}: 0.36$ (silica, dichloromethane:ethyl acetate 4:1);

${ }^{1} \mathrm{H}$ NMR $\left(400 \mathrm{MHz}, \mathrm{CDCl}_{3}\right): \delta=7.38-7.27(\mathrm{~m}, 3 \mathrm{H}, \mathrm{ArH}), 7.23(\mathrm{dd}, J=6.8,1.8 \mathrm{~Hz}, 2 \mathrm{H}, \operatorname{ArH}), 5.44(\mathrm{~s}, 1 \mathrm{H}$, $\mathrm{NH}), 3.54\left(\mathrm{~s}, 2 \mathrm{H}, \mathrm{CH}_{2}\right), 2.66(\mathrm{tq}, J=7.1,3.6 \mathrm{~Hz}, 1 \mathrm{H}, \mathrm{CH}), 0.75-0.67\left(\mathrm{~m}, 2 \mathrm{H}, \mathrm{CH}_{2}\right), 0.44-0.33(\mathrm{~m}, 2 \mathrm{H}$, $\mathrm{CH}_{2}$ ).

${ }^{1} \mathrm{H}$ NMR data correspond to the reported values. ${ }^{[4]}$

N-Cyclopropyl-2-naphthamide (3m).<smiles>O=C(NC1CC1)c1ccc2ccccc2c1</smiles>

Following GP A, using 2-naphthoyl chloride (1.73 g, $9.09 \mathrm{mmol}), \mathrm{N}$-cyclopropyl-2-naphthamide (3m) was obtained as a white solid $(1.90 \mathrm{~g}, 8.99 \mathrm{mmol}, 99 \%)$.

$\mathbf{R}_{\mathbf{f}}: 0.32$ (silica, dichloromethane);

Mp: $179-181^{\circ} \mathrm{C}$;

${ }^{1} \mathrm{H}$ NMR $\left(400 \mathrm{MHz}, \mathrm{CDCl}_{3}\right): \delta=8.24(\mathrm{~d}, J=1.7 \mathrm{~Hz}, 1 \mathrm{H}, \operatorname{ArH}), 7.92-7.83(\mathrm{~m}, 3 \mathrm{H}, \mathrm{ArH}), 7.80(\mathrm{dd}, J=8.5$, $1.8 \mathrm{~Hz}, 1 \mathrm{H}, \mathrm{ArH}), 7.54(\mathrm{pd}, J=6.9,1.6 \mathrm{~Hz}, 2 \mathrm{H}, \mathrm{ArH}), 6.45(\mathrm{~s}, 1 \mathrm{H}, \mathrm{NH}), 2.97$ (tq, $J=7.2,3.7 \mathrm{~Hz}, 1 \mathrm{H}, \mathrm{CH})$, $0.90\left(\mathrm{td}, J=7.0,5.2 \mathrm{~Hz}, 2 \mathrm{H}, \mathrm{CH}_{2}\right), 0.77-0.59\left(\mathrm{~m}, 2 \mathrm{H}, \mathrm{CH}_{2}\right)$;

${ }^{13} \mathrm{C}$ NMR $\left(101 \mathrm{MHz}, \mathrm{CDCl}_{3}\right): \delta=168.9,134.7,132.6,131.6,128.9,128.4,127.7,127.6,127.2,126.7$, 123.5, 23.2, 6.8;

IR (film): $\tilde{v}=3243$ (m), 3056 (w), 1623 (s), $1538(\mathrm{~s}), 1314(\mathrm{~m}), 1043(\mathrm{w}), 870(\mathrm{~m}), 782(\mathrm{~m})$;

HRMS (ESI) calcd. for $\mathrm{C}_{14} \mathrm{H}_{13} \mathrm{NNaO}^{+}[\mathrm{M}+\mathrm{Na}]^{+} 234.0889$; Found 234.0890.

\section{$\mathrm{N}$-Cyclopropylpivalamide (3n).}

\footnotetext{
${ }^{3}$ Zhang, B.; Feng, P.; Cui, Y.; Jiao, N. Chem. Commun. 2012, 48, 7280-7282.
}

${ }^{4}$ Tam, E.; Rita.; Liu, Y.; Chen, A. Eur. J. Org. Chem. 2015, 1100-1107. 


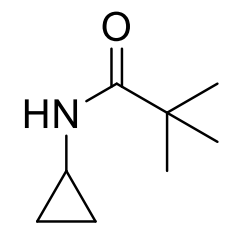

Following GP A, using pivaloyl chloride $(1.10 \mathrm{~g}, 9.09 \mathrm{mmol}), \mathrm{N}$-cyclopropylpivalamide (3n) was obtained as a white solid (1.22 g, $8.64 \mathrm{mmol}, 95 \%)$.

$\mathbf{R}_{\mathbf{f}}: 0.36$ (silica, pentanes:ethyl acetate $3: 2$ ) $\mathrm{KMnO}_{4}$;

${ }^{1} \mathrm{H}$ NMR $\left(400 \mathrm{MHz}, \mathrm{CDCl}_{3}\right): \delta=5.71(\mathrm{~s}, 1 \mathrm{H}, \mathrm{NH}), 2.74-2.59(\mathrm{~m}, 1 \mathrm{H}, \mathrm{CH}), 1.16\left(\mathrm{~s}, 9 \mathrm{H}, \mathrm{CH}_{3}\right), 0.83-0.65$ $\left(\mathrm{m}, 2 \mathrm{H}, \mathrm{CH}_{2}\right), 0.61-0.34\left(\mathrm{~m}, 2 \mathrm{H}, \mathrm{CH}_{2}\right)$.

${ }^{1} \mathrm{H}$ NMR data correspond to the reported values. ${ }^{[5]}$

tert-Butyl cyclopropylcarbamate (30).
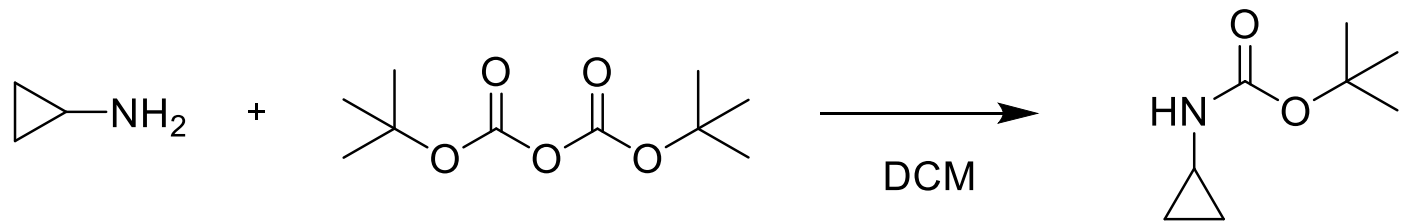

Following a modified version of a reported procedure ${ }^{[6]}$ to a solution of cyclopropylamine $(1.40 \mathrm{~mL}$, $20.0 \mathrm{mmol}, 1.0$ equiv.) in dichloromethane $(20 \mathrm{~mL})$ was slowly added a solution of di-tert-butyl dicarbonate ( $4.85 \mathrm{~g}, 22.0 \mathrm{mmol}, 1.1$ equiv.) in dichloromethane $(20 \mathrm{~mL})$ at $0{ }^{\circ} \mathrm{C}$. The reaction mixture was stirred at for 16 hours room temperature. Upon completion, the mixture was quenched by addition of $1 \mathrm{~N} \mathrm{HCl}(10 \mathrm{~mL})$. The aqueous layer was then extracted with dichloromethane. The organic layer was washed with brine $(10 \mathrm{~mL})$, and dried over $\mathrm{Na}_{2} \mathrm{SO}_{4}$, filtered and concentrated in vacuo. tert-Butyl cyclopropylcarbamate (3o) was obtained as a white solid (3.11 g, $19.8 \mathrm{mmol}, 99 \%)$, which was pure enough to be used without further purification.

${ }^{1} \mathrm{H}$ NMR $\left(400 \mathrm{MHz}, \mathrm{CDCl}_{3}\right): \delta=4.70$ (brs, $\left.1 \mathrm{H}, \mathrm{NH}\right), 2.57-2.47(\mathrm{~m}, 1 \mathrm{H}, \mathrm{CH}), 1.44\left(\mathrm{~s}, 9 \mathrm{H}, \mathrm{CH}_{3}\right), 0.72-$ $0.63\left(\mathrm{~m}, 2 \mathrm{H}, \mathrm{CH}_{2}\right), 0.53-0.39\left(\mathrm{~m}, 2 \mathrm{H}, \mathrm{CH}_{2}\right)$.

${ }^{1} \mathrm{H}$ NMR data correspond to the reported values. ${ }^{[6]}$

\section{Benzyl cyclopropylcarbamate (3p).}<smiles>O=C(NC1CC1)OCc1ccccc1</smiles>

Following GP A, using benzyl chloroformate (1.55 g, $9.09 \mathrm{mmol})$, benzyl cyclopropylcarbamate (3p) was obtained as a colorless solid (1.72 g, $9.00 \mathrm{mmol}, 99 \%)$.

${ }^{1} \mathrm{H}$ NMR $\left(400 \mathrm{MHz}, \mathrm{CDCl}_{3}\right): \delta=7.41-7.29(\mathrm{~m}, 5 \mathrm{H}, \mathrm{ArH}), 5.10\left(\mathrm{~s}, 2 \mathrm{H}, \mathrm{CH}_{2}\right), 5.01-4.80(\mathrm{~m}, 1 \mathrm{H}, \mathrm{NH})$, $2.60(\mathrm{ttd}, J=7.0,3.6,2.0 \mathrm{~Hz}, 1 \mathrm{H}, \mathrm{CH}), 0.76-0.69\left(\mathrm{~m}, 2 \mathrm{H}, \mathrm{CH}_{2}\right), 0.55-0.49\left(\mathrm{~m}, 2 \mathrm{H}, \mathrm{CH}_{2}\right)$.

\footnotetext{
${ }^{5}$ Zhou, L.; Lu, W. Org. Lett. 2014, 16, 508-511.

${ }^{6}$ Tars, K.; Leitan, J.; Kazaks, A.; Zelencova, D.; Liepinsh, E.; Kuka, J.; Makrecka, M.; Lola, D.; Andrianovs, V.; Gustina, D.; Grinberga, S.; Liepinsh, E.; Kalvinsh, I.; Dambrova, M.; Loza, E.; Pugovics, O. J. Med. Chem. 2014, 57, 2213-2236.
} 
${ }^{1} \mathrm{H}$ NMR data correspond to the reported values. ${ }^{[7]}$

\section{N-(1-Cyano-3-iodopropyl)-4-methylbenzenesulfonamide (3q).}<smiles>Cc1ccc(S(=O)(=O)NC2CC2)cc1</smiles>

Following GP A, using tosyl chloride (1.73 g, 9.09 mmol), N-(1-Cyano-3-iodopropyl)-4methylbenzenesulfonamide (3q) was obtained as a colorless solid (1.90 g, $9.00 \mathrm{mmol}, 99 \%)$.

${ }^{1} \mathrm{H}$ NMR $\left(400 \mathrm{MHz}, \mathrm{CDCl}_{3}\right): \delta=7.79(\mathrm{~d}, J=8.4 \mathrm{~Hz}, 2 \mathrm{H}, \operatorname{ArH}), 7.32(\mathrm{~d}, J=8.0 \mathrm{~Hz}, 2 \mathrm{H}, \operatorname{ArH}), 4.94(\mathrm{~s}, 1 \mathrm{H}$, $\mathrm{NH}), 2.43\left(\mathrm{~s}, 3 \mathrm{H}, \mathrm{CH}_{3}\right), 2.23(\mathrm{tt}, J=6.6,3.7 \mathrm{~Hz}, 1 \mathrm{H}, \mathrm{CH}), 0.65-0.52\left(\mathrm{~m}, 4 \mathrm{H}, \mathrm{CH}_{2}\right)$.

${ }^{1} \mathrm{H}$ NMR data correspond to the reported values. ${ }^{[8]}$

N-Cyclopropyl-N,4-dimethylbenzenesulfonamide (3r).<smiles>CNC1CC1</smiles><smiles>Cc1ccc(S(=O)(=O)Cl)cc1</smiles><smiles>CCN(C)CC</smiles><smiles>Cc1ccc(S(=O)(=O)N(C)C2CC2)cc1</smiles>

Following a modified version of a reported procedure, ${ }^{[1]}$ to a solution of $\mathrm{N}$-cyclopropyl-methylamine hydrochloride $(0.323 \mathrm{~g}, 3.00 \mathrm{mmol}, 1.1$ equiv.) and triethylamine $(0.84 \mathrm{~mL}, 6.0 \mathrm{mmol}, 2.2$ equiv.) in dichloromethane $(10 \mathrm{~mL})$ was slowly added a solution of tosyl chloride $(0.535 \mathrm{~g}, 2.80 \mathrm{mmol}, 1.0$ equiv.) in dichloromethane $(10 \mathrm{~mL})$ at $0{ }^{\circ} \mathrm{C}$. The reaction mixture was stirred for 16 hours at room temperature. Upon completion, the mixture was quenched by addition of $1 \mathrm{~N} \mathrm{HCl}(10 \mathrm{~mL})$. The aqueous layer was then extracted with dichloromethane. The organic layer was washed with brine $(10 \mathrm{~mL})$, and dried over $\mathrm{Na}_{2} \mathrm{SO}_{4}$, filtered and concentrated in vacuo. N-cyclopropyl-N,4dimethylbenzenesulfonamide ( $3 \mathrm{r})$ was obtained as a yellow solid $(0.580 \mathrm{~g}, 2.58 \mathrm{mmol}, 92 \%)$, which was pure enough to be used without further purification.

$\mathbf{R}_{\mathbf{f}}: 0.60$ (silica, pentanes:ethyl acetate 3:1);

Mp: $73-76^{\circ} \mathrm{C}$;

${ }^{1} \mathrm{H}$ NMR $\left(400 \mathrm{MHz}, \mathrm{CDCl}_{3}\right): \delta=7.73(\mathrm{~d}, J=8.3 \mathrm{~Hz}, 2 \mathrm{H}, \operatorname{ArH}), 7.34(\mathrm{~d}, J=7.7 \mathrm{~Hz}, 2 \mathrm{H}, \operatorname{ArH}), 2.73(\mathrm{~s}, 3 \mathrm{H}$, $\left.\mathrm{CH}_{3}\right), 2.44\left(\mathrm{~s}, 3 \mathrm{H}, \mathrm{CH}_{3}\right), 1.78(\mathrm{tt}, J=6.9,3.6 \mathrm{~Hz}, 1 \mathrm{H}, \mathrm{CH}), 0.90-0.83\left(\mathrm{~m}, 2 \mathrm{H}, \mathrm{CH}_{2}\right), 0.72-0.64(\mathrm{~m}, 2 \mathrm{H}$, $\mathrm{CH}_{2}$ );

${ }^{13} \mathrm{C}$ NMR $\left(101 \mathrm{MHz}, \mathrm{CDCl}_{3}\right): \delta=143.5,132.8,129.5,128.0,37.5,32.1,21.5,7.5$;

\footnotetext{
${ }^{7}$ Shaw, M. H.; McCreanor, N. G.; Whittingham, W. G.; Bower, J. F. J. Am. Chem. Soc. 2015, 137, 463-468

${ }^{8}$ O'Sullivan, S.; Doni, E.; Tuttle, T.; Murphy. J. A. Angew. Chem. Int. Ed. 2014, 53, 474-478.
} 
IR (film): $\tilde{v}=2963$ (w), 1596 (w), 1456 (m), 1365 (m), 1338 (s), 1151 (s), 1089 (s), 1034 (m), 817 (s), $691(\mathrm{~s})$;

HRMS (ESI) calcd. for $\mathrm{C}_{11} \mathrm{H}_{15} \mathrm{NNaO}_{2} \mathrm{~S}^{+}[\mathrm{M}+\mathrm{Na}]^{+}$248.0716; Found 248.0721 .

tert-Butyl benzyl(cyclopropyl)carbamate (3s).<smiles>CC(C)(C)OC(=O)NC1CC1</smiles>

Following a modified version of a reported procedure, ${ }^{[9]}$ to a solution of benzyl bromide $(0.616 \mathrm{~g}$, $3.60 \mathrm{mmol}, 1.2$ equiv.) and $\mathrm{NaH}$ (60\% dispersion in mineral oil, $156 \mathrm{mg}, 3.90 \mathrm{mmol}, 1.3$ equiv.) in DMF $(10 \mathrm{~mL})$ was slowly added a solution of tert-butyl cyclopropylcarbamate $(0.472 \mathrm{~g}, 3.00 \mathrm{mmol}$, 1.0 equiv.) in DMF $(5 \mathrm{~mL})$ at $0{ }^{\circ} \mathrm{C}$. The reaction mixture was stirred at room temperature for 16 hours. Upon completion, the mixture was quenched by addition of $1 \mathrm{~N} \mathrm{HCl}(10 \mathrm{~mL})$. The aqueous layer was then extracted with dichloromethane. The organic layer was washed with brine $(10 \mathrm{~mL})$, dried over $\mathrm{Na}_{2} \mathrm{SO}_{4}$, filtered and concentrated in vacuo. tert-Butyl benzyl(cyclopropyl)carbamate (3s) was obtained as a bright yellow oil $(0.540 \mathrm{~g}, 2.18 \mathrm{mmol}, 73 \%)$ after purification by column chromatography on silica using 8:1 pentanes:ethyl acetate as eluent.

${ }^{1} \mathrm{H}$ NMR $\left(400 \mathrm{MHz}, \mathrm{CDCl}_{3}\right): \delta=7.35-7.15(\mathrm{~m}, 5 \mathrm{H}, \mathrm{ArH}), 4.39\left(\mathrm{~s}, 2 \mathrm{H}, \mathrm{NCH}_{2}\right), 2.42(\mathrm{~s}, 1 \mathrm{H}, \mathrm{CH}), 1.42(\mathrm{~s}$, $\left.9 \mathrm{H}, \mathrm{CH}_{3}\right), 0.74-0.53\left(\mathrm{~m}, 4 \mathrm{H}, \mathrm{CH}_{2}\right)$.

${ }^{1} \mathrm{H}$ NMR data correspond to the reported values. ${ }^{[9]}$

Benzyl (S)-(1-(cyclopropylamino)-1-oxo-3-phenylpropan-2-yl)carbamate (3t).<smiles>CC(C(=O)N[C@@H](Cc1ccccc1)C(=O)N[C@@H](Cc1ccccc1)C(=O)N[C@H](Cc1ccccc1)C(=O)O)C(F)(F)F</smiles>

Following a modified version of a reported procedure, ${ }^{[10]}$ Boc-L-phenylalanine ( $898 \mathrm{mg}, 3.00 \mathrm{mmol}$, 1.0 equiv.) and CDI (486 mg, $3.00 \mathrm{mmol}, 1.0$ equiv.) were suspended in THF (15 mL). The resulting mixture was stirred for 2 hour before adding cyclopropylamine $(185 \mathrm{mg}, 3.30 \mathrm{mmol}, 1.1$ equiv.). The reaction mixture was stirred at room temperature for another 16 hours. Upon completion, the mixture was quenched by addition of $2 \mathrm{M} \mathrm{NaOH}(5 \mathrm{~mL} \times 2)$. The aqueous layer was then extracted with dichloromethane. The organic layer was washed with $1 \mathrm{~N} \mathrm{HCl}(10 \mathrm{~mL})$. The combined organic layers were washed with brine $(10 \mathrm{~mL})$, dried over $\mathrm{Na}_{2} \mathrm{SO}_{4}$, filtered and concentrated in vacuo. Benzyl (S)-(1-(cyclopropylamino)-1-oxo-3-phenylpropan-2-yl)carbamate (3t) was obtained as a white solid (480 mg, $1.44 \mathrm{mmol}, 48 \%$ ) after purification by column chromatography on silica using 1:1 pentanes:ethyl acetate as eluent.

$\mathbf{R}_{\mathbf{f}} \mathbf{0 . 3 5}$ (silica, pentane:ethyl acetate 1:1);

\footnotetext{
${ }^{9}$ Park, Y.; Beak, P. Tetrahedron 1996, 52, 12333-12350.

${ }^{10}$ Shang, M.; Wang, M.-M.; Saint-Denis, T. G.; Li, M.-H.; Dai, H.-X.; Yu, J.-Q. Angew. Chem. Int. Ed. 2017, 56, 5317-5321.
} 
Mp: $161-163^{\circ} \mathrm{C}$;

${ }^{1} \mathrm{H}$ NMR (400 MHz, CDCl $): \delta=7.38-7.22(\mathrm{~m}, 8 \mathrm{H}, \mathrm{ArH}), 7.17(\mathrm{~d}, J=7.2 \mathrm{~Hz}, 2 \mathrm{H}, \mathrm{ArH}), 5.69(\mathrm{~s}, 1 \mathrm{H}, \mathrm{NH})$, $5.43(\mathrm{~d}, J=8.1 \mathrm{~Hz}, 1 \mathrm{H}, \mathrm{NH}), 5.07\left(\mathrm{~s}, 2 \mathrm{H}, \mathrm{OCH}_{2}\right), 4.28(\mathrm{q}, J=7.6 \mathrm{~Hz}, 1 \mathrm{H}, \mathrm{CHN}), 3.11(\mathrm{dd}, J=13.6,6.1 \mathrm{~Hz}$, $\left.1 \mathrm{H}, \mathrm{CH}_{2}\right), 2.96\left(\mathrm{dd}, J=13.6,8.1 \mathrm{~Hz}, 1 \mathrm{H}, \mathrm{CH}_{2}\right), 2.58(\mathrm{tq}, J=7.1,3.6 \mathrm{~Hz}, 1 \mathrm{H}, \mathrm{CHN}), 0.72-0.60(\mathrm{~m}, 2 \mathrm{H}$, $\left.\mathrm{CH}_{2}\right), 0.34-0.19\left(\mathrm{~m}, 2 \mathrm{H}, \mathrm{CH}_{2}\right)$;

${ }^{13} \mathrm{C}$ NMR $\left(101 \mathrm{MHz}, \mathrm{CDCl}_{3}\right): \delta=171.9,155.8,136.5,136.1,129.3,128.7,128.5,128.2,128.0,127.1$, 67.1, 56.3, 38.9, 22.4, 6.5, 6.3;

IR (film): $\tilde{v}=3298(\mathrm{~m}), 1690$ (m), 1652 (s), 1533 (m), 1286 (w), 1261 (w), $1040(\mathrm{w}), 747(\mathrm{w}), 698(\mathrm{w})$;

HRMS (APPI) calcd. for $\mathrm{C}_{20} \mathrm{H}_{22} \mathrm{~N}_{2} \mathrm{NaO}_{3}{ }^{+}[\mathrm{M}+\mathrm{Na}]^{+}$361.1523; Found 361.1518.

N-Cyclopropyl-2-(11-oxo-6,11-dihydrodibenzo[b,e]oxepin-2-yl)acetamide (3u).
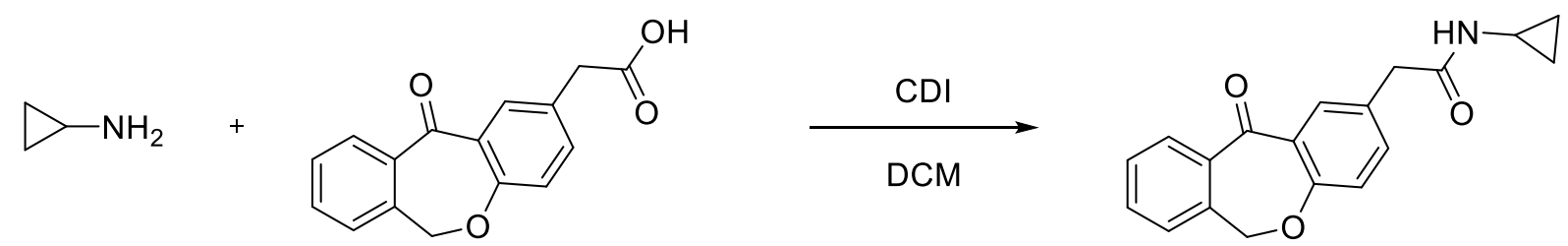

Following a modified version of a reported procedure, ${ }^{[10]}$ Isoxepac (536 mg, $2.00 \mathrm{mmol}, 1.0$ equiv.) and CDI (324 mg, $2.00 \mathrm{mmol}, 1.0$ equiv.) were suspended in DCM (10 mL). The resulting mixture was stirred for 1 hour before adding cyclopropylamine (123 mg, $2.20 \mathrm{mmol}, 1.1$ equiv.). The reaction mixture was stirred at room temperature for another 2 hours. Upon completion, the mixture was quenched by addition of $2 \mathrm{M} \mathrm{NaOH}(5 \mathrm{~mL} \times 2)$. The aqueous layer was then extracted with dichloromethane. The organic layer was washed with $1 \mathrm{~N} \mathrm{HCl}(10 \mathrm{~mL})$. The combined organic layers were washed with brine $(10 \mathrm{~mL})$, dried over $\mathrm{Na}_{2} \mathrm{SO}_{4}$, filtered and concentrated in vacuo. $\mathrm{N}$ Cyclopropyl-2-(11-oxo-6,11-dihydrodibenzo[b,e]oxepin-2-yl)acetamide (3u) was obtained as a white solid (550 mg, $1.79 \mathrm{mmol}, 90 \%$ ), which was found pure enough to be used without further purification.

$\mathbf{R}_{\mathbf{f}}: 0.35$ (silica, pentane:ethyl acetate 1:3);

Mp: $161-163^{\circ} \mathrm{C}$;

${ }^{1} \mathrm{H}$ NMR $\left(400 \mathrm{MHz}, \mathrm{CDCl}_{3}\right): \delta=8.05(\mathrm{~d}, J=2.3 \mathrm{~Hz}, 1 \mathrm{H}, \operatorname{ArH}), 7.88(\mathrm{~d}, J=7.6 \mathrm{~Hz}, 1 \mathrm{H}, \operatorname{ArH}), 7.57(\mathrm{t}, J=7.5$ $\mathrm{Hz}, 1 \mathrm{H}, \operatorname{ArH}), 7.48(\mathrm{t}, J=7.6 \mathrm{~Hz}, 1 \mathrm{H}, \operatorname{ArH}), 7.43(\mathrm{dd}, J=8.4,2.4 \mathrm{~Hz}, 1 \mathrm{H}, \operatorname{ArH}), 7.37(\mathrm{~d}, J=7.4 \mathrm{~Hz}, 1 \mathrm{H}$, $\operatorname{ArH}), 7.04(\mathrm{~d}, J=8.4 \mathrm{~Hz}, 1 \mathrm{H}, \mathrm{ArH}), 5.72(\mathrm{~s}, 1 \mathrm{H}, \mathrm{NH}), 5.19(\mathrm{~s}, 2 \mathrm{H}, \mathrm{OCH})_{2}, 3.53\left(\mathrm{~s}, 2 \mathrm{H}, \mathrm{CH}_{2}\right), 2.68(\mathrm{tq}, J=$ 7.3, 3.6 Hz, $1 \mathrm{H}, \mathrm{CH}), 0.73\left(\mathrm{td}, J=7.1,5.3 \mathrm{~Hz}, 2 \mathrm{H}, \mathrm{CH}_{2}\right), 0.52-0.35\left(\mathrm{~m}, 2 \mathrm{H}, \mathrm{CH}_{2}\right)$;

${ }^{13} \mathrm{C}$ NMR $\left(101 \mathrm{MHz}, \mathrm{CDCl}_{3}\right): \delta=190.8,172.1,160.5,140.3,136.3,135.4,132.9,132.3,129.4,129.3$, $128.6,127.9,125.2,121.5,73.6,42.5,22.9,6.6$;

IR (film): $\tilde{v}=3272(\mathrm{w}), 3057$ (w), 1643 (s), 1609 (m), $1541(\mathrm{~m}), 1487(\mathrm{~s}), 1412(\mathrm{~m}), 1300(\mathrm{~s}), 1138(\mathrm{~m})$, $1016(\mathrm{~m}), 801(\mathrm{w}), 735(\mathrm{~m})$;

HRMS (ESI) calcd. for $\mathrm{C}_{19} \mathrm{H}_{17} \mathrm{NNaO}_{3}{ }^{+}[\mathrm{M}+\mathrm{Na}]^{+} 330.1101$; Found 330.1104.

2-(1-(4-Chlorobenzoyl)-5-methoxy-2-methyl-1H-indol-3-yl)-N-cyclopropylacetamide (3v).

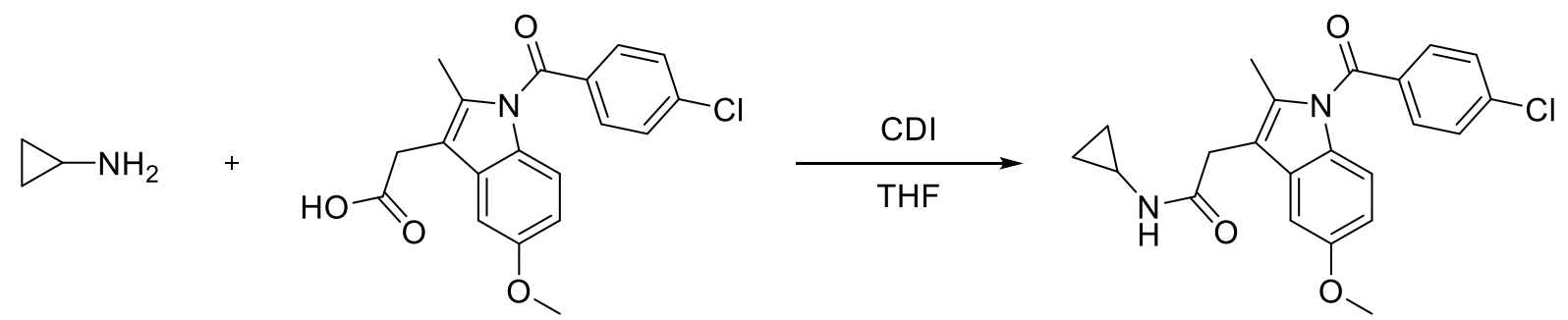

Following a modified version of a reported procedure, ${ }^{[10]}$ indomethacin ( $1.07 \mathrm{~g}, 3.00 \mathrm{mmol}, 1.0$ equiv.) and CDI (486 mg, $3.00 \mathrm{mmol}, 1.0$ equiv.) were suspended in THF (10 mL). The resulting mixture was 
stirred for 2 hours before adding cyclopropylamine (188 mg, $3.30 \mathrm{mmol}, 1.1$ equiv.). The reaction mixture was stirred at room temperature for another 2 hours. Upon completion, the mixture was quenched by addition of $2 \mathrm{M} \mathrm{NaOH}(5 \mathrm{~mL} \times 2)$. The aqueous layer was extracted with dichloromethane. The combined organic layers were then washed with $1 \mathrm{~N} \mathrm{HCl}(10 \mathrm{~mL})$ and extracted with dichloromethane. The combined organic extracts were washed with brine $(10 \mathrm{~mL})$, dried over $\mathrm{Na}_{2} \mathrm{SO}_{4}$, filtered and concentrated in vacuo. 2-(1-(4-Chlorobenzoyl)-5-methoxy-2-methyl$1 \mathrm{H}$-indol-3-yl)-N-cyclopropylacetamide (3v) was obtained as a yellow solid (1.14 g, $2.88 \mathrm{mmol}, 96 \%)$, which was found pure enough to be used without further purification.

$\mathbf{R}_{\mathbf{f}}: 0.20$ (silica, pentanes:ethyl acetate 3:7);

Mp: $166-168^{\circ} \mathrm{C}$;

${ }^{1} \mathrm{H}$ NMR $\left(400 \mathrm{MHz}, \mathrm{CDCl}_{3}\right): \delta=7.65(\mathrm{~d}, J=8.5 \mathrm{~Hz}, 2 \mathrm{H}, \operatorname{ArH}), 7.48(\mathrm{~d}, J=8.5 \mathrm{~Hz}, 2 \mathrm{H}, \operatorname{ArH}), 6.88-6.82$ $\left.(\mathrm{m}, 2 \mathrm{H}, \mathrm{ArH}), 6.69(\mathrm{dd}, J=9.0,2.5 \mathrm{~Hz}, 1 \mathrm{H}, \mathrm{ArH}), 5.70(\mathrm{~s}, 1 \mathrm{H}, \mathrm{NH}), 3.82(\mathrm{~s}, 3 \mathrm{H}, \mathrm{OCH})_{3}\right), 3.61\left(\mathrm{~s}, 2 \mathrm{H}, \mathrm{CH}_{2}\right)$, 2.65 (tq, J = 7.1, 3.6 Hz, 1H, CH), $2.36\left(\mathrm{~s}, 3 \mathrm{H}, \mathrm{CH}_{3}\right), 0.76-0.68\left(\mathrm{~m}, 2 \mathrm{H}, \mathrm{CH}_{2}\right), 0.42-0.33\left(\mathrm{~m}, 2 \mathrm{H}, \mathrm{CH}_{2}\right)$;

${ }^{13} \mathrm{C} \mathrm{NMR}\left(101 \mathrm{MHz}, \mathrm{CDCl}_{3}\right): \delta=171.3,168.3,156.3,139.6,136.2,133.5,131.2,130.8,130.2,129.2$, $115.1,112.8,112.3,100.7,55.7,32.2,22.7,13.2,6.7$;

IR (film): $\tilde{v}=3268$ (w), 3006 (w), 2929 (w), 1676 (s), 1644 (s), 1541 (m), 1476 (s), 1356 (s), 1314 (s), $1223(\mathrm{~s}), 1147(\mathrm{~m}), 1088(\mathrm{~m}), 908(\mathrm{~m}), 832(\mathrm{~m}), 730(\mathrm{~s})$;

HRMS (APPI) calcd. for $\mathrm{C}_{22} \mathrm{H}_{21} \mathrm{CIN}_{2} \mathrm{O}_{3}{ }^{+}[\mathrm{M}]^{+} 396.1235$; Found 396.1242.

tert-Butyl ((1S*,2S*)-2-methylcyclopropyl)carbamate (28).

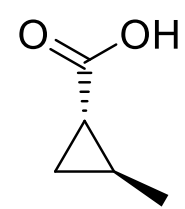

4:1 d.r.

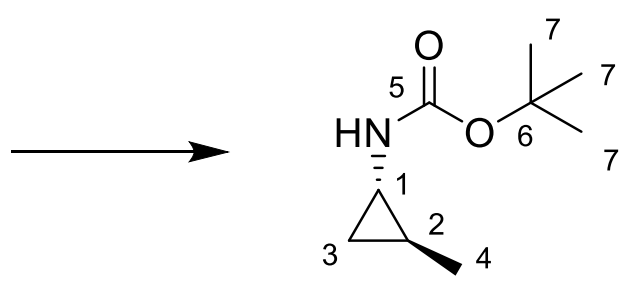

$4: 1$ d.r.

Following a reported procedure, ${ }^{[11]}$ to a solution of $\left(1 S^{*}, 2 S^{*}\right)-2$-methylcyclopropanecarboxylic acid $\left(1.00 \mathrm{~g}, 10.0 \mathrm{mmol}, 4: 1\right.$ d.r.) and $\mathrm{Et}_{3} \mathrm{~N}(1.40 \mathrm{~mL}, 10.0 \mathrm{mmol})$ in anhydrous $t-\mathrm{BuOH}(6 \mathrm{~mL})$ was added diphenylphosphoryl azide $(2.37 \mathrm{~mL}, 11.0 \mathrm{mmol})$ dropwise. The reaction was heated to $80{ }^{\circ} \mathrm{C}$ and stirred for $72 \mathrm{~h}$ before cooling to r.t. The reaction mixture was concentrated in vacuo and the residue was dissolved in $\mathrm{Et}_{2} \mathrm{O}(20 \mathrm{~mL})$. Water $(20 \mathrm{~mL})$ was added and the aqueous portion was extracted with $\mathrm{Et}_{2} \mathrm{O}(3 \times 20 \mathrm{~mL})$. The organics extracts were combined, washed with sat. aq. sodium bicarbonate ( 20 $\mathrm{mL}$ ) and brine $(20 \mathrm{~mL})$, dried over $\mathrm{Na}_{2} \mathrm{SO}_{4}$ and concentrated in vacuo. The residue was purified by column chromatography on silica using 10:1 pentanes:ethyl acetate as eluent. tert-Butyl ((1S*,2S*)-2methylcyclopropyl)carbamate (28) was obtained as a colorless oil (1.02 g, $5.96 \mathrm{mmol}, 60 \%$ ), which was a mixture of diastereisomers $A$ and $B$ in a 4:1 (A:B) ratio.

${ }^{1} \mathrm{H}$ NMR $\left(400 \mathrm{MHz}, \mathrm{CDCl}_{3}\right): \delta=4.68(2 \mathrm{H}, \mathrm{br} \mathrm{s}, \mathrm{N}-\mathrm{H}, \mathrm{A}+\mathrm{B}), 2.51(1 \mathrm{H}, \mathrm{br} \mathrm{m}, \mathrm{C} 1-\mathrm{H}, \mathrm{B}), 2.18(1 \mathrm{H}, \mathrm{br} \mathrm{m}, \mathrm{C} 1-\mathrm{H}$, A), $1.43\left(18 \mathrm{H}, \mathrm{br} \mathrm{s}, 3 \times \mathrm{C} 7-\mathrm{H}_{3}, \mathrm{~A}+\mathrm{B}\right), 1.09-1.07\left(6 \mathrm{H}, \mathrm{m}, \mathrm{C} 4-\mathrm{H}_{3}, \mathrm{~A}+\mathrm{B}\right), 0.91-0.83(3 \mathrm{H}, \mathrm{m}, \mathrm{C} 2-\mathrm{H}(\mathrm{A}+\mathrm{B})$ and 1 $\left.\times \mathrm{C}-\mathrm{H}_{2}(\mathrm{~B})\right), 0.60\left(1 \mathrm{H}, \mathrm{m}, \mathrm{C} 3-\mathrm{H}_{2}, \mathrm{~A}\right), 0.45\left(1 \mathrm{H}, \mathrm{m}, \mathrm{C} 3-\mathrm{H}_{2}, \mathrm{~A}\right), 0.09\left(1 \mathrm{H}, \mathrm{m}, \mathrm{C} 3-\mathrm{H}_{2}, \mathrm{~B}\right)$.

${ }^{1} \mathrm{H}$ NMR data correspond to the reported values. ${ }^{[11]}$

\footnotetext{
${ }^{11}$ Shaw, M. H.; Melikhova, E. Y.; Kloer, D. P.; Whittingham, W. G.; Bower, J. F. J. Am. Chem. Soc. 2013, 135, 4992-4995.
} 


\section{Scope of the ring-opening reaction}

\section{General Procedure B (GP B):}

In a glass vial, the correspondent aminocyclopropane $(0.300 \mathrm{mmol}, 1.0$ equiv. $), \mathrm{N}$-lodosuccinimide (70.8 mg, $0.315 \mathrm{mmol}, 1.05$ equiv.), methanol (14.4 $\mu \mathrm{L}, 0.360 \mathrm{mmol}, 1.2$ equiv.) and diphenyl phosphate $(7.5 \mathrm{mg}, 0.030 \mathrm{mmol}, 0.10$ equiv.) were dissolved in $1.5 \mathrm{~mL}$ of $\mathrm{MeCN}(0.20 \mathrm{M})$. The reaction mixture was stirred at room temperature for 30 minutes, if not specified otherwise. After the completion of the reaction, the crude product was directly submitted to column chromatography on silica using pentanes:ethyl acetate as eluent.

Note: The obtained products were found heat-sensitive; in order to avoid their degradation, they were concentrated maintaining the heating bath of the rotatory evaporator below $30{ }^{\circ} \mathrm{C}$ and they were stored at $-20{ }^{\circ} \mathrm{C}$ in the freezer. It is recommended that they are used for further transformations following a one-pot protocol, without isolation.

\section{N-(3-lodo-1-methoxypropyl)-4-methoxybenzamide (4a).}<smiles>COc1ccc(C(=O)NC(CCI)OC)cc1</smiles>

Following GP B, starting from N-cyclopropyl-4-methoxybenzamide $3 a(57.3 \mathrm{mg}, 0.300 \mathrm{mmol}), \mathrm{N}-(3-$ iodo-1-methoxypropyl)-4-methoxybenzamide $4 a(88.8 \mathrm{mg}, 0.255 \mathrm{mmol}, 85 \%)$ was obtained as a yellow solid after purification by column chromatography on silica using 2:1 pentanes:ethyl acetate as eluent.

For enantioselective version: in an oven dried microwave vial, $\mathrm{N}$-cyclopropyl-4-methoxybenzamide 3a (19.1 mg, $0.100 \mathrm{mmol}, 1.0$ equiv.), $\mathrm{N}$-lodosuccinimide (23.6 mg, $0.105 \mathrm{mmol}, 1.05$ equiv.), methanol ( $4.8 \mu \mathrm{L}, 0.12 \mathrm{mmol}, 1.2$ equiv.) and CPA 24 (3.8 $\mathrm{mg}, 0.0050 \mathrm{mmol}, 0.05$ equiv.) were dissolved in $0.5 \mathrm{~mL}$ of $\mathrm{MeCN}(0.20 \mathrm{M})$. The reaction mixture was stirred at $-15{ }^{\circ} \mathrm{C}$ in a cryostat for 6 hours. After the completion of the reaction, $\mathrm{N}$-(3-iodo-1-methoxypropyl)-4-methoxybenzamide 4a (28.6 $\mathrm{mg}, 0.0819 \mathrm{mmol}, 82 \%$ ) was obtained as a yellow solid after purification by column chromatography on silica using 2:1 pentanes:ethyl acetate as eluent. Chiral HPLC conditions: er = 88.9:11.1; Chiralpak IC 90:10 Hexane/iPrOH, $1.0 \mathrm{~mL} / \mathrm{min}, 31$ min. $\mathrm{t}_{\mathrm{r}}$ (minor) = $17.4 \mathrm{~min}$. and $\mathrm{t}_{\mathrm{r}}$ (major) $=19.9 \min . \lambda=260 \mathrm{~cm}^{-1}$.

$\mathbf{R}_{\mathbf{f}}: 0.30$ (silica, pentanes:ethyl acetate 3:1);

Mp: $92-95^{\circ} \mathrm{C}$;

${ }^{1} \mathrm{H}$ NMR $\left(400 \mathrm{MHz}, \mathrm{CDCl}_{3}\right): \delta=7.80-7.74(\mathrm{~m}, 2 \mathrm{H}, \mathrm{ArH}), 6.96-6.90(\mathrm{~m}, 2 \mathrm{H}, \mathrm{ArH}), 6.36(\mathrm{~d}, J=9.5 \mathrm{~Hz}$, $1 \mathrm{H}, \mathrm{NH}$ ), 5.44 (ddd, $J=9.6,7.0,5.3 \mathrm{~Hz}, 1 \mathrm{H}, \mathrm{CH}), 3.85\left(\mathrm{~s}, 3 \mathrm{H}, \mathrm{OCH}_{3}\right), 3.41\left(\mathrm{~s}, 3 \mathrm{H}, \mathrm{OCH}_{3}\right), 3.29-3.17(\mathrm{~m}$, $2 \mathrm{H}, \mathrm{CH}_{2} \mathrm{l}$ ), 2.25 (dddd, $J=17.7,12.0,7.4,3.2 \mathrm{~Hz}, 2 \mathrm{H}, \mathrm{CH}_{2} \mathrm{CH}_{2} \mathrm{l}$ );

${ }^{13} \mathrm{C}$ NMR $\left(101 \mathrm{MHz}, \mathrm{CDCl}_{3}\right): \delta=166.8,162.6,128.9,125.7,113.8,81.6,56.2,55.4,39.5,-0.9$;

IR (film): $\tilde{v}=3308$ (w), 2933 (w), 2836 (w), 1641 (s), 1606 (s), 1532 (m), 1502 (s), 1307 (m), 1255 (s), $1175(\mathrm{~s}), 1104(\mathrm{~m}), 844(\mathrm{~m}), 768(\mathrm{w})$;

HRMS (ESI) calcd. for $\mathrm{C}_{12} \mathrm{H}_{16} \mathrm{INNaO}_{3}{ }^{+}[\mathrm{M}+\mathrm{Na}]^{+} 372.0067$; Found 372.0066. 
N-(3-lodo-1-methoxypropyl)-4-(methylthio)benzamide (4b).<smiles>COC(CCI)NC(=O)c1ccc(SC)cc1</smiles>

Following GP B, starting from N-cyclopropyl-4-(methylthio)benzamide $3 b(62.1 \mathrm{mg}, 0.300 \mathrm{mmol}), \mathrm{N}$ (3-iodo-1-methoxypropyl)-4-methoxybenzamide $\mathbf{4 b}(81.4 \mathrm{mg}, 0.223 \mathrm{mmol}, 74 \%)$ was obtained as a yellow solid after purification by column chromatography on silica using 2:1 pentanes:ethyl acetate as eluent.

$\mathbf{R}_{\mathbf{f}}: 0.31$ (silica, pentanes:ethyl acetate 3:1);

Mp: $99-102^{\circ} \mathrm{C}$;

${ }^{1} \mathrm{H}$ NMR $\left(400 \mathrm{MHz}, \mathrm{CDCl}_{3}\right): \delta=7.71(\mathrm{~d}, J=8.2 \mathrm{~Hz}, 2 \mathrm{H}, \mathrm{ArH}), 7.27(\mathrm{~d}, J=8.0 \mathrm{~Hz}, 2 \mathrm{H}, \operatorname{ArH}), 6.32(\mathrm{~d}, J=9.6$ $\mathrm{Hz}, 1 \mathrm{H}, \mathrm{NH}), 5.45(\mathrm{dt}, J=9.7,6.2 \mathrm{~Hz}, 1 \mathrm{H}, \mathrm{CH}), 3.42\left(\mathrm{~s}, 3 \mathrm{H}, \mathrm{OCH}_{3}\right), 3.30-3.19\left(\mathrm{~m}, 2 \mathrm{H}, \mathrm{CH}_{2} \mathrm{l}\right), 2.51(\mathrm{~s}, 3 \mathrm{H}$, $\mathrm{SCH}_{3}$ ), 2.26 (ddd, $J=13.9,8.8,6.7 \mathrm{~Hz}, 2 \mathrm{H}, \mathrm{CH}_{2} \mathrm{CH}_{2} \mathrm{l}$ );

${ }^{13} \mathrm{C}$ NMR $\left(101 \mathrm{MHz}, \mathrm{CDCl}_{3}\right): \delta=166.8,144.3,129.5,127.4,125.4,81.7,56.3,39.5,15.0,-1.0$;

IR (film): $\tilde{v}=3317$ (w), 2928 (w), 1644 (s), 1596 (m), 1520 (s), 1488 (m), 1318 (w), 1107 (m), 837 (w);

HRMS (ESI) calcd. for $\mathrm{C}_{12} \mathrm{H}_{16} \mathrm{INNaO}_{2} \mathrm{~S}^{+}[\mathrm{M}+\mathrm{Na}]^{+}$387.9839; Found 387.9842.

4-Fluoro-N-(3-iodo-1-methoxypropyl)benzamide (4c).<smiles>COC(CCI)NC(=O)c1ccc(F)cc1</smiles>

Following GP B, starting from N-cyclopropyl-4-fluorobenzamide $3 \mathrm{c}(62.1 \mathrm{mg}, 0.300 \mathrm{mmol})$, 4-fluoro$\mathrm{N}$-(3-iodo-1-methoxypropyl)benzamide $4 \mathrm{c}(83.6 \mathrm{mg}, 0.248 \mathrm{mmol}, 83 \%)$ was obtained as a yellow solid after purification by column chromatography on silica using 3:1 pentanes:ethyl acetate as eluent.

$\mathbf{R}_{\mathbf{f}}: 0.48$ (silica, pentanes:ethyl acetate 3:1);

Mp: $78-81^{\circ} \mathrm{C}$;

${ }^{1} \mathrm{H}$ NMR $\left(400 \mathrm{MHz}, \mathrm{CDCl}_{3}\right): \delta=7.87-7.77(\mathrm{~m}, 2 \mathrm{H}, \operatorname{ArH}), 7.12(\mathrm{t}, J=8.6 \mathrm{~Hz}, 2 \mathrm{H}, \operatorname{ArH}), 6.48(\mathrm{~d}, J=9.5 \mathrm{~Hz}$, $1 \mathrm{H}, \mathrm{NH}), 5.43(\mathrm{ddd}, J=9.5,7.1,5.2 \mathrm{~Hz}, 1 \mathrm{H}, \mathrm{CH}), 3.41\left(\mathrm{~s}, 3 \mathrm{H}, \mathrm{OCH}_{3}\right), 3.30-3.15\left(\mathrm{~m}, 2 \mathrm{H}, \mathrm{CH}_{2} \mathrm{l}\right), 2.34-$ $2.16\left(\mathrm{~m}, 2 \mathrm{H}, \mathrm{CH}_{2} \mathrm{CH}_{2} \mathrm{l}\right)$;

${ }^{13} \mathrm{C}$ NMR $\left(101 \mathrm{MHz}, \mathrm{CDCl}_{3}\right): \delta=166.3,165.0(\mathrm{~d}, J=252.8 \mathrm{~Hz}), 129.7(\mathrm{~d}, J=3.1 \mathrm{~Hz}), 129.4(\mathrm{~d}, J=9.0 \mathrm{~Hz})$, $115.7(\mathrm{~d}, J=21.9 \mathrm{~Hz}), 81.8,56.3,39.4,-1.0$;

${ }^{19}$ F NMR $\left(376 \mathrm{MHz}, \mathrm{CDCl}_{3}\right) \delta=-107.1$; 
IR (film): $\tilde{v}=3292$ (w), 2933 (w), 1643 (s), 1602 (s), 1530 (m), 1496 (s), 1285 (m), 1233 (s), 1157 (s), $1100(\mathrm{~s}), 1064$ (s), $848(\mathrm{~s})$;

HRMS (ESI) calcd. for $\mathrm{C}_{11} \mathrm{H}_{13} \mathrm{FINNaO}_{2}^{+}[\mathrm{M}+\mathrm{Na}]^{+}$359.9867; Found 359.9868.

4-Chloro-N-(3-iodo-1-methoxypropyl)benzamide (4d).<smiles>COC(CCI)NC(=O)c1ccc(Cl)cc1</smiles>

Following GP B, starting from 4-chloro-N-cyclopropylbenzamide $3 \mathrm{~d}(58.5 \mathrm{mg}, 0.300 \mathrm{mmol})$, 4-chloro$\mathrm{N}$-(3-iodo-1-methoxypropyl)benzamide $4 \mathrm{~d}(74.5 \mathrm{mg}, 0.211 \mathrm{mmol}, 70 \%)$ was obtained as an off-white solid after purification by column chromatography on silica using 2:1 pentanes:ethyl acetate as eluent.

$\mathbf{R}_{\mathbf{f}}: 0.48$ (silica, pentanes:ethyl acetate $3: 1$ );

Mp: $110-114^{\circ} \mathrm{C}$;

${ }^{1}$ H NMR $\left(400 \mathrm{MHz}, \mathrm{CDCl}_{3}\right): \delta=7.75(\mathrm{~d}, J=8.5 \mathrm{~Hz}, 2 \mathrm{H}, \mathrm{ArH}), 7.44(\mathrm{dd}, J=8.5,1.9 \mathrm{~Hz}, 2 \mathrm{H}, \operatorname{ArH}), 6.29$ (d, J $=11.6 \mathrm{~Hz}, 1 \mathrm{H}, \mathrm{NH}), 5.50-5.38(\mathrm{~m}, 1 \mathrm{H}, \mathrm{CH}), 3.43\left(\mathrm{~d}, J=1.2 \mathrm{~Hz}, 3 \mathrm{H}, \mathrm{CH}_{3}\right), 3.32-3.15\left(\mathrm{~m}, 2 \mathrm{H}, \mathrm{CH}_{2}\right), 2.33$ $-2.18\left(\mathrm{~m}, 2 \mathrm{H}, \mathrm{CH}_{2}\right)$;

${ }^{13} \mathrm{C}$ NMR $\left(101 \mathrm{MHz}, \mathrm{CDCl}_{3}\right): \delta=166.3,138.4,132.0,129.0,128.5,81.8,56.4,39.4,-1.2$;

IR (film): $\tilde{v}=3209$ (w), 2930 (w), 1645 (s), 1594 (m), 1527 (s), 1485 (s), 1314 (m), 1092 (s), 1065 (m), $844(\mathrm{~m})$;

HRMS (ESI) calcd. for $\mathrm{C}_{11} \mathrm{H}_{13} \mathrm{ClINNaO}_{2}^{+}[\mathrm{M}+\mathrm{Na}]^{+}$375.9572; Found 375.9577.

4-Cyano-N-(3-iodo-1-methoxypropyl)benzamide (4e).<smiles>COC(CCI)NC(=O)c1ccc(C#N)cc1</smiles>

Following GP B, starting from 4-cyano-N-cyclopropylbenzamide $3 e(55.8 \mathrm{mg}, 0.300 \mathrm{mmol})$ and after stirring for 2 hours, $\mathrm{N}$-(3-iodo-1-methoxypropyl)-4-nitrobenzamide $4 \mathrm{e}$ (79.6 mg, $0.231 \mathrm{mmol}, 77 \%)$ was obtained as a yellow oil after purification by column chromatography on silica using 3:1 pentanes:ethyl acetate as eluent.

$\mathbf{R}_{\mathbf{f}}: 0.35$ (silica, pentanes:ethyl acetate 3:1);

${ }^{1}$ H NMR $\left(400 \mathrm{MHz}, \mathrm{CDCl}_{3}\right): \delta=7.91(\mathrm{~d}, J=8.4 \mathrm{~Hz}, 2 \mathrm{H}, \mathrm{ArH}), 7.77(\mathrm{~d}, J=8.4 \mathrm{~Hz}, 2 \mathrm{H}, \operatorname{ArH}), 6.44(\mathrm{~d}, J=9.5$ $\mathrm{Hz}, 1 \mathrm{H}, \mathrm{NH}), 5.45(\mathrm{ddd}, J=9.5,6.9,5.2 \mathrm{~Hz}, 1 \mathrm{H}, \mathrm{CH}), 3.44\left(\mathrm{~s}, 3 \mathrm{H}, \mathrm{OCH}_{3}\right), 3.31-3.18\left(\mathrm{~m}, 2 \mathrm{H}, \mathrm{CH}_{2} \mathrm{l}\right), 2.33$ $-2.20\left(\mathrm{~m}, 2 \mathrm{H}, \mathrm{CH}_{2} \mathrm{CH}_{2} \mathrm{l}\right)$; 
${ }^{13} \mathrm{C}$ NMR $\left(101 \mathrm{MHz}, \mathrm{CDCl}_{3}\right): \delta=165.6,137.5,132.6,127.7,117.8,115.6,82.0,56.6,39.2,-1.3$;

IR (film): $\tilde{v}=3307$ (m), 2927 (m), 2853 (w), 2231 (m), 1651 (s), 1531 (s), 1496 (s), 1312 (m), 1284 (m), $1104(\mathrm{~m}), 1065(\mathrm{~m}), 856(\mathrm{~m}), 764(\mathrm{~m})$;

HRMS (ESI) calcd. for $\mathrm{C}_{11} \mathrm{H}_{10} \mathrm{IN}_{2} \mathrm{O}^{+}\left[\mathrm{M}-\mathrm{OCH}_{3}\right]^{+}$312.9832; Found 312.9835 .

\section{N-(3-lodo-1-methoxypropyl)-4-nitrobenzamide (4f).}<smiles>COC(CCI)NC(=O)c1ccc([N+](=O)[O-])cc1</smiles>

Following GP B, starting from N-cyclopropyl-4-nitrobenzamide $\mathbf{3 f}(61.8 \mathrm{mg}, 0.300 \mathrm{mmol})$ and after stirring for 2 hours, $\mathrm{N}$-(3-iodo-1-methoxypropyl)-4-nitrobenzamide $4 \mathrm{f}(88.0 \mathrm{mg}, 0.242 \mathrm{mmol}, 81 \%)$ was obtained as a yellow solid after purification by column chromatography on silica using 3:1 pentanes:ethyl acetate as eluent.

$\mathbf{R}_{\mathbf{f}}: 0.50$ (silica, pentanes:ethyl acetate 3:1);

Mp: $65-68^{\circ} \mathrm{C}$;

${ }^{1} \mathrm{H}$ NMR $\left(400 \mathrm{MHz}, \mathrm{CDCl}_{3}\right): \delta=8.34-8.29(\mathrm{~m}, 2 \mathrm{H}, \mathrm{ArH}), 8.00-7.96(\mathrm{~m}, 2 \mathrm{H}, \operatorname{ArH}), 6.48(\mathrm{~d}, J=9.4 \mathrm{~Hz}$, $1 \mathrm{H}, \mathrm{NH}), 5.47(\mathrm{ddd}, J=9.4,6.9,5.2 \mathrm{~Hz}, 1 \mathrm{H}, \mathrm{CH}), 3.45\left(\mathrm{~s}, 3 \mathrm{H}, \mathrm{OCH}_{3}\right), 3.30-3.20\left(\mathrm{~m}, 2 \mathrm{H}, \mathrm{CH}_{2} \mathrm{l}\right), 2.33-$ $2.23\left(\mathrm{~m}, 2 \mathrm{H}, \mathrm{CH}_{2} \mathrm{CH}_{2} \mathrm{l}\right)$;

${ }^{13} \mathrm{C}$ NMR $\left(101 \mathrm{MHz}, \mathrm{CDCl}_{3}\right): \delta=165.3,149.9,139.1,128.3,123.9,82.1,56.7,39.2,-1.3$;

IR (film): $\tilde{v}=3293$ (w), 2930 (w), 1652 (m), 1601 (m), 1522 (s), 1487 (m), 1346 (s), 1105 (m), 1065 (m), $868(\mathrm{~m}), 838(\mathrm{~m})$;

HRMS (APCl) calcd. for $\mathrm{C}_{10} \mathrm{H}_{10} \mathrm{IN}_{2} \mathrm{O}_{3}{ }^{+}\left[\mathrm{M}-\mathrm{OCH}_{3}\right]^{+} 332.9731$; Found 332.9733.

\section{N-(3-lodo-1-methoxypropyl)-3-methoxybenzamide (4g).}<smiles>COc1cccc(C(=O)NC(CCI)OC)c1</smiles>

Following GP B, starting from N-cyclopropyl-3-methoxybenzamide $3 \mathrm{~g}(57.3 \mathrm{mg}, 0.300 \mathrm{mmol}), \mathrm{N}-(3-$ iodo-1-methoxypropyl)-3-methoxybenzamide $4 \mathrm{~g}(96.0 \mathrm{mg}, 0.275 \mathrm{mmol}, 92 \%)$ was obtained as a yellow oil after purification by column chromatography on silica using 2:1 pentanes:ethyl acetate as eluent.

$\mathbf{R}_{\mathbf{f}}: 0.36$ (silica, pentanes:ethyl acetate 3:1);

${ }^{1} \mathrm{H}$ NMR $\left(400 \mathrm{MHz}, \mathrm{CDCl}_{3}\right): \delta=7.38(\mathrm{~s}, 1 \mathrm{H}, \operatorname{ArH}), 7.32(\mathrm{~d}, J=4.9 \mathrm{~Hz}, 2 \mathrm{H}, \operatorname{ArH}), 7.04(\mathrm{td}, J=4.9,2.7 \mathrm{~Hz}$, $1 \mathrm{H}, \operatorname{ArH}), 6.61(\mathrm{~d}, J=9.5 \mathrm{~Hz}, 1 \mathrm{H}, \mathrm{NH}), 5.42(\mathrm{ddd}, J=9.5,7.1,5.2 \mathrm{~Hz}, 1 \mathrm{H}, \mathrm{CH}), 3.82\left(\mathrm{~s}, 3 \mathrm{H}, \mathrm{OCH}_{3}\right), 3.40$ $\left(\mathrm{s}, 3 \mathrm{H}, \mathrm{OCH}_{3}\right), 3.27-3.14\left(\mathrm{~m}, 2 \mathrm{H}, \mathrm{CH}_{2} \mathrm{l}\right), 2.30-2.17\left(\mathrm{~m}, 2 \mathrm{H}, \mathrm{CH}_{2} \mathrm{CH}_{2} \mathrm{l}\right)$; 
${ }^{13} \mathrm{C}$ NMR $\left(101 \mathrm{MHz}, \mathrm{CDCl}_{3}\right): \delta=167.3,159.8,135.0,129.6,118.7,118.1,112.5,81.7,56.2,55.4,39.3,-$ 1.0;

IR (film): $\tilde{v}=3297$ (w), 2934 (w), 2833 (w), 1645 (s), 1583 (s), 1524 (s), 1485 (s), 1358 (w), 1310 (m), $1290(\mathrm{~m}), 1251(\mathrm{~m}), 1103(\mathrm{~m}), 1145(\mathrm{~s}), 994(\mathrm{w}), 746(\mathrm{~m})$;

HRMS (ESI) calcd. for $\mathrm{C}_{12} \mathrm{H}_{16} \mathrm{INNaO}_{3}{ }^{+}[\mathrm{M}+\mathrm{Na}]^{+} 372.0067$; Found 372.0069 .

$\mathrm{N}$-(3-lodo-1-methoxypropyl)-2-methoxybenzamide (4h).<smiles>COc1ccccc1C(=O)NC(CCI)OC</smiles>

Following GP B, starting from N-cyclopropyl-2-methoxybenzamide $3 \mathrm{~h}(57.3 \mathrm{mg}, 0.300 \mathrm{mmol}$ ) and after stirring for 1 hour, $\mathrm{N}$-(3-iodo-1-methoxypropyl)-2-methoxybenzamide $4 \mathrm{~h}$ ( $87.5 \mathrm{mg}, 0.251 \mathrm{mmol}$, $84 \%$ ) was obtained as a yellow oil after purification by column chromatography on silica using 3:1 pentanes:ethyl acetate as eluent.

$\mathbf{R}_{\mathbf{f}}: 0.45$ (silica, pentanes:ethyl acetate 3:1);

${ }^{1}$ H NMR $\left(400 \mathrm{MHz}, \mathrm{CDCl}_{3}\right): \delta=8.19(\mathrm{dd}, J=7.8,1.9 \mathrm{~Hz}, 1 \mathrm{H}, \mathrm{ArH}), 7.99(\mathrm{~d}, J=9.4 \mathrm{~Hz}, 1 \mathrm{H}, \mathrm{ArH}), 7.48$ (ddd, $J=8.8,7.4,1.9 \mathrm{~Hz}, 1 \mathrm{H}, \operatorname{ArH}), 7.09(\mathrm{t}, J=7.6 \mathrm{~Hz}, 1 \mathrm{H}, \mathrm{ArH}), 6.99$ (d, J=8.3 Hz, 1H, NH), 5.47 (ddd, J $=9.3,7.0,5.3 \mathrm{~Hz}, 1 \mathrm{H}, \mathrm{CH}), 3.97\left(\mathrm{~s}, 3 \mathrm{H}, \mathrm{OCH}_{3}\right), 3.42\left(\mathrm{~s}, 3 \mathrm{H}, \mathrm{OCH}_{3}\right), 3.30-3.18\left(\mathrm{~m}, 2 \mathrm{H}, \mathrm{CH}_{2} \mathrm{l}\right), 2.34-2.19$ (m, $\left.2 \mathrm{H}, \mathrm{CH}_{2} \mathrm{CH}_{2} \mathrm{l}\right)$;

${ }^{13} \mathrm{C}$ NMR $\left(101 \mathrm{MHz}, \mathrm{CDCl}_{3}\right): \delta=165.5,157.5,133.3,132.5,121.4,120.8,111.4,81.5,56.2,56.0,39.9$, 0.9 ;

IR (film): $\tilde{v}=3376$ (w), 2932 (w), 2834 (w), 1656 (s), 1600 (m), 1516 (s), 1482 (s), 1464 (m), 1293 (m), $1237(\mathrm{~s}), 1098(\mathrm{~s}), 1019(\mathrm{~m}), 843(\mathrm{w}), 755(\mathrm{~s})$;

HRMS (ESI) calcd. for $\mathrm{C}_{12} \mathrm{H}_{16} \mathrm{INNaO}_{3}{ }^{+}[\mathrm{M}+\mathrm{Na}]^{+} 372.0067$; Found 372.0078.

N-(3-lodo-1-methoxypropyl)-2,4,6-trimethylbenzamide (4i).<smiles>COC(CCI)NC(=O)c1c(C)cc(C)cc1C</smiles>

Following GP B, starting from N-cyclopropyl-2,4,6-trimethylbenzamide $3 \mathbf{i}(61.0 \mathrm{mg}, 0.300 \mathrm{mmol})$ and after stirring for 1 hour, $\mathrm{N}$-(3-iodo-1-methoxypropyl)-2,4,6-trimethylbenzamide $4 \mathbf{i}$ (76.7 mg, 0.212 mmol, $71 \%$ ) was obtained as a pale yellow solid after purification by column chromatography on silica using 3:1 pentanes:ethyl acetate as eluent.

$\mathbf{R}_{\mathbf{f}}: 0.50$ (silica, pentanes:ethyl acetate 3:1);

Mp: $97-99^{\circ} \mathrm{C}$; 
${ }^{1} \mathrm{H}$ NMR $\left(400 \mathrm{MHz}, \mathrm{CDCl}_{3}\right): \delta=6.85(\mathrm{~s}, 2 \mathrm{H}, \mathrm{ArH}), 5.84(\mathrm{~d}, J=9.5 \mathrm{~Hz}, 1 \mathrm{H}, \mathrm{NH}), 5.40$ (ddd, $J=9.7,6.8,5.6$ $\mathrm{Hz}, 1 \mathrm{H}, \mathrm{CH}), 3.52\left(\mathrm{~s}, 3 \mathrm{H}, \mathrm{OCH}_{3}\right), 3.23\left(\mathrm{td}, \mathrm{J}=7.5,7.1,1.5 \mathrm{~Hz}, 2 \mathrm{H}, \mathrm{CH}_{2} \mathrm{l}\right), 2.31\left(\mathrm{~s}, 6 \mathrm{H}, \mathrm{CH}_{3}\right), 2.27(\mathrm{~s}, 3 \mathrm{H}$, $\mathrm{CH}_{3}$ ), 2.19 (qd, $J=7.4,2.0 \mathrm{~Hz}, 2 \mathrm{H}, \mathrm{CH}_{2} \mathrm{CH}_{2} \mathrm{l}$ );

${ }^{13} \mathrm{C}$ NMR $\left(101 \mathrm{MHz}, \mathrm{CDCl}_{3}\right): \delta=170.6,138.7,134.4,133.8,128.3,81.4,56.7,39.6,21.0,19.3,-1.2$;

IR (film): $\tilde{v}=3252$ (w), 2924 (m), 2854 (w), 1644 (s), 1523 (s), 1442 (m), 1310 (w), 1176 (m), 1099 (m), $1067(\mathrm{~m}), 847(\mathrm{w})$;

HRMS (ESI) calcd. for $\mathrm{C}_{14} \mathrm{H}_{20} \mathrm{INNaO}_{2}^{+}[\mathrm{M}+\mathrm{Na}]^{+} 384.0431$; Found 384.0436.

$\mathrm{N}$-(3-lodo-1-methoxypropyl)furan-2-carboxamide (4j).<smiles>COC(CCI)NC(=O)c1ccco1</smiles>

Following GP B, starting from N-cyclopropylfuran-2-carboxamide $3 \mathbf{j}(45.3 \mathrm{mg}, 0.300 \mathrm{mmol}), \mathrm{N}-(3-$ iodo-1-methoxypropyl)furan-2-carboxamide $4 \mathbf{j}(88.7 \mathrm{mg}, 0.287 \mathrm{mmol}, 96 \%)$ was obtained as a yellow oil after purification by column chromatography on silica using 2:1 pentanes:ethyl acetate as eluent.

$\mathbf{R}_{\mathbf{f}}: 0.29$ (silica, pentanes:ethyl acetate $3: 1$ );

${ }^{1} \mathrm{H}$ NMR $\left(400 \mathrm{MHz}, \mathrm{CDCl}_{3}\right): \delta=7.49-7.43(\mathrm{~m}, 1 \mathrm{H}, \operatorname{ArH}), 7.16(\mathrm{~d}, J=3.1 \mathrm{~Hz}, 1 \mathrm{H}, \operatorname{ArH}), 6.57(\mathrm{~d}, J=9.9 \mathrm{~Hz}$, $1 \mathrm{H}, \mathrm{NH}), 6.52$ (dd, $J=3.5,1.8 \mathrm{~Hz}, 1 \mathrm{H}, \mathrm{ArH}), 5.37$ (ddd, $J=9.8,7.2,5.2 \mathrm{~Hz}, 1 \mathrm{H}, \mathrm{CH}), 3.39\left(\mathrm{~s}, 3 \mathrm{H}, \mathrm{OCH}_{3}\right)$, $3.25-3.14\left(\mathrm{~m}, 2 \mathrm{H}, \mathrm{CH}_{2} \mathrm{l}\right), 2.33-2.15\left(\mathrm{~m}, 2 \mathrm{H}, \mathrm{CH}_{2} \mathrm{CH}_{2} \mathrm{l}\right)$;

${ }^{13} \mathrm{C} \mathrm{NMR}\left(101 \mathrm{MHz}, \mathrm{CDCl}_{3}\right): \delta=158.2,147.1,144.3,115.3,112.4,80.9,56.2,39.5,-1.1$;

IR (film): $\tilde{v}=3285$ (w), 2930 (w), 1650 (w), 1589 (s), 1512 (s), 1471 (s), 1310 (m), 1291 (m), 1174 (s), $1103(\mathrm{~s}), 1065$ (s), 1010 (s);

HRMS (ESI) calcd. for $\mathrm{C}_{9} \mathrm{H}_{12} \mathrm{INNaO}_{3}{ }^{+}[\mathrm{M}+\mathrm{Na}]^{+} 331.9754$; Found 331.9762 .

$\mathrm{N}$-(3-lodo-1-methoxypropyl)cinnamamide (4k).<smiles>COC(CCI)NC(=O)/C=C/c1ccccc1</smiles>

Following GP B, starting from N-cyclopropylcinnamamide $3 \mathbf{k}(56.2 \mathrm{mg}, 0.300 \mathrm{mmol}), \mathrm{N}$-(3-iodo-1methoxypropyl)cinnamamide $\mathbf{4 k}(83.1 \mathrm{mg}, 0.241 \mathrm{mmol}, 80 \%)$ was obtained as a yellow solid after purification by column chromatography on silica using 2:1 pentanes:ethyl acetate as eluent.

$\mathbf{R}_{\mathbf{f}}: 0.40$ (silica, pentanes:ethyl acetate 3:1);

Mp: $93-95^{\circ} \mathrm{C}$;

${ }^{1} \mathrm{H}$ NMR $\left(400 \mathrm{MHz}, \mathrm{CDCl}_{3}\right): \delta=7.69(\mathrm{~d}, J=15.6 \mathrm{~Hz}, 1 \mathrm{H}$, vinylH), $7.51(\mathrm{dd}, J=6.6,2.9 \mathrm{~Hz}, 2 \mathrm{H}, \operatorname{ArH}), 7.42$ $-7.33(\mathrm{~m}, 3 \mathrm{H}, \mathrm{ArH}), 6.41(\mathrm{~d}, J=15.6 \mathrm{~Hz}, 1 \mathrm{H}$, vinyl $H), 5.91(\mathrm{~d}, J=9.8 \mathrm{~Hz}, 1 \mathrm{H}, \mathrm{NH}), 5.38$ (ddd, $J=9.7,7.1$, $5.3 \mathrm{~Hz}, 1 \mathrm{H}, \mathrm{CH}), 3.41\left(\mathrm{~s}, 3 \mathrm{H}, \mathrm{OCH}_{3}\right), 3.27-3.16\left(\mathrm{~m}, 2 \mathrm{H}, \mathrm{CH}_{2} \mathrm{l}\right), 2.21$ (ddt, J = 14.3, 11.9, $6.7 \mathrm{~Hz}, 2 \mathrm{H}$, $\left.\mathrm{CH}_{2} \mathrm{CH}_{2} \mathrm{l}\right)$;

${ }^{13} \mathrm{C}$ NMR $\left(101 \mathrm{MHz}, \mathrm{CDCl}_{3}\right): \delta=165.9,142.6,134.4,130.0,128.9,127.9,119.8,81.3,56.3,39.6,-1.0$; IR (film): $\tilde{v}=3264$ (m), 3028 (w), 2926 (m), 2853 (w), 1657 (s), 1623 (s), 1530 (s), 1449 (m), 1358 (m), $1336(\mathrm{~m}), 1203(\mathrm{~s}), 1110(\mathrm{~s}), 1068(\mathrm{~s}), 976(\mathrm{~m}), 862(\mathrm{w}), 765(\mathrm{~m})$; 
HRMS (ESI) calcd. for $\mathrm{C}_{13} \mathrm{H}_{16} \mathrm{INNaO}_{2}^{+}[\mathrm{M}+\mathrm{Na}]^{+} 368.0118$; Found 368.0118.

\section{$\mathrm{N}$-(3-lodo-1-methoxypropyl)-2-phenylacetamide (4I).}<smiles>COC(CCI)NC(=O)Cc1ccccc1</smiles>

Following GP B, starting from N-cyclopropyl-2-phenylacetamide 31 ( $52.6 \mathrm{mg}, 0.300 \mathrm{mmol}$ ), N-(3-iodo1-methoxypropyl)-2-phenylacetamide $4 \mathbf{1}(75.0 \mathrm{mg}, 0.225 \mathrm{mmol}, 75 \%)$ was obtained as a yellow solid after purification by column chromatography on silica using 2:1 pentanes:ethyl acetate as eluent.

$\mathbf{R}_{\mathbf{f}}: 0.40$ (silica, pentanes:ethyl acetate $3: 1$ );

Mp: $84-87^{\circ} \mathrm{C}$;

${ }^{1}$ H NMR $\left(400 \mathrm{MHz}, \mathrm{CDCl}_{3}\right): \delta=7.40-7.34(\mathrm{~m}, 2 \mathrm{H}, \operatorname{ArH}), 7.33-7.28(\mathrm{~m}, 1 \mathrm{H}, \mathrm{ArH}), 7.26(\mathrm{dd}, J=6.1,2.3$ $\mathrm{Hz}, 2 \mathrm{H}, \mathrm{ArH}), 5.60(\mathrm{~d}, J=9.5 \mathrm{~Hz}, 1 \mathrm{H}, \mathrm{NH}), 5.19(\mathrm{ddd}, J=9.6,7.1,5.2 \mathrm{~Hz}, 1 \mathrm{H}, \mathrm{CH}), 3.61(\mathrm{~d}, J=3.7 \mathrm{~Hz}, 2 \mathrm{H}$, $\left.\mathrm{CH}_{2}\right), 3.29\left(\mathrm{~s}, 3 \mathrm{H}, \mathrm{OCH}_{3}\right), 3.08\left(\mathrm{td}, J=7.6,7.1,1.4 \mathrm{~Hz}, 2 \mathrm{H}, \mathrm{CH}_{2} \mathrm{l}\right), 2.10-1.97\left(\mathrm{~m}, 2 \mathrm{H}, \mathrm{CH}_{2} \mathrm{CH}_{2} \mathrm{l}\right)$;

${ }^{13} \mathrm{C}$ NMR $\left(101 \mathrm{MHz}, \mathrm{CDCl}_{3}\right): \delta=171.3,134.3,129.3,129.2,127.6,81.2,56.1,43.9,39.2,-1.3$;

IR (film): $\tilde{v}=3279(w), 3062$ (w), 3030 (w), 2930 (w), $1654(\mathrm{~s}), 1535(\mathrm{~m}), 1496(\mathrm{~m}), 1176(\mathrm{~m}), 1111(\mathrm{~m})$, $1068(\mathrm{~m}), 724(\mathrm{~m})$;

HRMS (ESI) calcd. for $\mathrm{C}_{12} \mathrm{H}_{16} \mathrm{INNaO}_{2}^{+}[\mathrm{M}+\mathrm{Na}]^{+}$356.0118; Found 356.0123 .

N-(3-lodo-1-methoxypropyl)-2-naphthamide (4m).<smiles>COC(CCI)NC(=O)c1ccc2ccccc2c1</smiles>

Following GP B, starting from N-cyclopropyl-2-naphthamide $3 \mathrm{~m}(63.4 \mathrm{mg}, 0.300 \mathrm{mmol}), \mathrm{N}$-(3-iodo-1methoxypropyl)-2-naphthamide $4 \mathrm{~m}(97.5 \mathrm{mg}, 0.264 \mathrm{mmol}, 88 \%)$ was obtained as a yellow solid after purification by column chromatography on silica using 3:1 pentanes:ethyl acetate as eluent.

$\mathbf{R}_{\mathbf{f}}: 0.58$ (silica, pentanes:ethyl acetate $3: 1$ );

Mp: $96-98^{\circ} \mathrm{C}$;

${ }^{1} \mathrm{H}$ NMR $\left(400 \mathrm{MHz}, \mathrm{CDCl}_{3}\right): \delta=8.32(\mathrm{~s}, 1 \mathrm{H}, \mathrm{ArH}), 7.95-7.83(\mathrm{~m}, 4 \mathrm{H}, \mathrm{ArH}), 7.57(\mathrm{pd}, J=6.9,1.5 \mathrm{~Hz}, 2 \mathrm{H}$, $\operatorname{Ar} H), 6.54(\mathrm{~d}, J=9.6 \mathrm{~Hz}, 1 \mathrm{H}, \mathrm{NH}), 5.52(\mathrm{ddd}, J=9.6,7.0,5.2 \mathrm{~Hz}, 1 \mathrm{H}, \mathrm{CH}), 3.47\left(\mathrm{~s}, 3 \mathrm{H}, \mathrm{OCH}_{3}\right), 3.34-$ $3.20\left(\mathrm{~m}, 2 \mathrm{H}, \mathrm{CH}_{2} \mathrm{l}\right), 2.39-2.24\left(\mathrm{~m}, 2 \mathrm{H}, \mathrm{CH}_{2} \mathrm{CH}_{2} \mathrm{l}\right)$;

${ }^{13} \mathrm{C}$ NMR $\left(101 \mathrm{MHz}, \mathrm{CDCl}_{3}\right): \delta=167.4,134.9,132.5,130.8,129.0,128.7,128.0,127.8,127.7,126.9$, 123.4, 81.8, 56.4, 39.6, -1.0;

IR (film): $\tilde{v}=3296$ (w), 3058 (w), 2925 (m), 2852 (w), 1645 (s), 1527 (s), 1503 (m), 1309 (m), 1066 (m), $863(w)$;

HRMS (ESI) calcd. for $\mathrm{C}_{15} \mathrm{H}_{16} \mathrm{INNaO}_{2}^{+}[\mathrm{M}+\mathrm{Na}]^{+}$392.0118; Found 392.0115. 
<smiles>COC(CCI)NC(=O)C(C)(C)C</smiles>

Following GP B, starting from N-cyclopropylpivalamide $3 \mathrm{n}(42.4 \mathrm{mg}, 0.300 \mathrm{mmol}), \mathrm{N}$-(3-iodo-1methoxypropyl)pivalamide $4 \mathrm{n}(78.6 \mathrm{mg}, 0.263 \mathrm{mmol}, 88 \%)$ was obtained as a dark yellow solid after purification by column chromatography on silica using 2:1 pentanes:ethyl acetate as eluent.

$\mathbf{R}_{\mathbf{f}}: 0.48$ (silica, pentanes:ethyl acetate $3: 1$ );

Mp: $76-78^{\circ} \mathrm{C}$;

${ }^{1} \mathrm{H}$ NMR (400 MHz, CDCl $): \delta=5.81(\mathrm{~d}, J=9.5 \mathrm{~Hz}, 1 \mathrm{H}, \mathrm{NH}), 5.23$ (ddd, $\left.J=9.5,7.1,5.2 \mathrm{~Hz}, 1 \mathrm{H}, \mathrm{CH}\right), 3.33$ (s, 3H, OCH 3$), 3.26-3.05\left(\mathrm{~m}, 2 \mathrm{H}, \mathrm{CH}_{2} \mathrm{l}\right), 2.21-2.08\left(\mathrm{~m}, 2 \mathrm{H}, \mathrm{CH}_{2} \mathrm{CH}_{2} \mathrm{l}\right), 1.22\left(\mathrm{~s}, 9 \mathrm{H}, \mathrm{CH}_{3}\right)$;

${ }^{13} \mathrm{C}$ NMR $\left(101 \mathrm{MHz}, \mathrm{CDCl}_{3}\right): \delta=178.7,81.1,56.0,39.5,38.9,27.5,-1.1$;

IR (film): $\tilde{v}=3329$ (w), 2961 (m), 2934 (m), 1650 (s), 1518 (s), 1367 (w), $1190(\mathrm{~m}), 1110(\mathrm{~m}), 1069$ (m);

HRMS (ESI) calcd. for $\mathrm{C}_{9} \mathrm{H}_{18} \mathrm{INNaO}_{2}^{+}[\mathrm{M}+\mathrm{Na}]^{+} 322.0274$; Found 322.0282 .

tert-Butyl (3-iodo-1-methoxypropyl)carbamate (40).<smiles>COC(CCI)NC(=O)OC(C)(C)C</smiles>

Following GP B, starting from tert-butyl cyclopropylcarbamate $30(47.2 \mathrm{mg}, 0.300 \mathrm{mmol})$ and without the addition of diphenyl phosphate, tert-butyl (3-iodo-1-methoxypropyl)carbamate $40(89.0 \mathrm{mg}$, $0.282 \mathrm{mmol}, 94 \%$ ) was obtained as a pale yellow oil after purification by column chromatography on silica using 4:1 pentanes:ethyl acetate as eluent. $\mathrm{K}_{2} \mathrm{CO}_{3}(0.300 \mathrm{mmol}, 1.00$ equiv. $)$ has to be added to the reaction mixture before purification.

$\mathbf{R}_{\mathbf{f}}: 0.45$ (silica, pentanes:ethyl acetate 6:1);

${ }^{1} \mathrm{H}$ NMR (400 MHz, Methanol- $\left.d_{4}\right): \delta=4.85-4.80(\mathrm{~m}, 2 \mathrm{H}, \mathrm{NH}+\mathrm{CH}), 3.27\left(\mathrm{~s}, 3 \mathrm{H}, \mathrm{OCH}_{3}\right), 3.16(\mathrm{td}, \mathrm{J}=$ 7.1, $\left.2.5 \mathrm{~Hz}, 2 \mathrm{H}, \mathrm{CH}_{2} \mathrm{l}\right), 2.12-2.03\left(\mathrm{~m}, 1 \mathrm{H}, \mathrm{CH}_{2} \mathrm{CH}_{2} \mathrm{l}\right), 1.95$ (dtd, J = 14.4, 7.4, $\left.5.7 \mathrm{~Hz}, 1 \mathrm{H}, \mathrm{CH}_{2} \mathrm{CH}_{2} \mathrm{l}\right), 1.42$ (s, 9H, $\mathrm{CH}_{3}$ );

${ }^{13} \mathrm{C}$ NMR $\left(101 \mathrm{MHz}\right.$, Methanol- $\left.d_{4}\right): \delta=157.9,84.2,80.5,55.5,39.7,28.6,0.4$;

IR (film): $\tilde{v}=3335$ (w), 2977 (w), 2931 (w), 2832 (w), 1698 (s), $1513(\mathrm{~m}), 1366$ (m), 1244 (s), 1161 (s), $1086(\mathrm{~m}), 994(\mathrm{~m}), 865(\mathrm{~m})$;

HRMS (ESI) calcd. for $\mathrm{C}_{9} \mathrm{H}_{18} \mathrm{INNaO}_{3}{ }^{+}[\mathrm{M}+\mathrm{Na}]^{+} 338.0224$; Found 338.0226.

Benzyl (3-iodo-1-methoxypropyl)carbamate (4p).<smiles>COC(CCI)NC(=O)OCc1ccccc1</smiles> 
Following GP B, starting from benzyl cyclopropylcarbamate $3 p(57.4 \mathrm{mg}, 0.300 \mathrm{mmol})$ and without the addition of diphenyl phosphate, benzyl (3-iodo-1-methoxypropyl)carbamate $4 p$ ( $84.0 \mathrm{mg}, 0.241$ mmol, $80 \%$ ) was obtained as a colorless oil after purification by column chromatography on silica using 4:1 pentanes:ethyl acetate as eluent. $\mathrm{K}_{2} \mathrm{CO}_{3}(0.300 \mathrm{mmol}, 1.00$ equiv. $)$ has to be added to the reaction mixture before purification.

$\mathbf{R}_{\mathbf{f}}: 0.40$ (silica, pentanes:ethyl acetate 4:1);

${ }^{1} \mathrm{H}$ NMR $\left(400 \mathrm{MHz}, \mathrm{CDCl}_{3}\right): \delta=7.48-7.28(\mathrm{~m}, 5 \mathrm{H}, \mathrm{ArH}), 5.13\left(\mathrm{~s}, 2 \mathrm{H}, \mathrm{OCH}_{2}\right), 5.07(\mathrm{~d}, J=10.0 \mathrm{~Hz}, 1 \mathrm{H}$, $\left.\mathrm{NH}), 4.99(\mathrm{td}, J=10.3,8.6,5.8 \mathrm{~Hz}, 1 \mathrm{H}, \mathrm{CH}), 3.38(\mathrm{~s}, 3 \mathrm{H}, \mathrm{OCH})_{3}\right), 3.17\left(\mathrm{t}, J=7.1 \mathrm{~Hz}, 2 \mathrm{H}, \mathrm{CH}_{2} \mathrm{l}\right), 2.14(\mathrm{tt}, J=$ $\left.14.1,7.8 \mathrm{~Hz}, 2 \mathrm{H}, \mathrm{CH}_{2} \mathrm{CH}_{2} \mathrm{l}\right)$;

${ }^{13} \mathrm{C}$ NMR $\left(101 \mathrm{MHz}^{\mathrm{CDCl}} 3\right): \delta=155.8,136.0,128.6,128.3,128.1,83.6,67.0,55.9,39.4,-0.7$;

IR (film): $\tilde{v}=3317$ (w), 3033 (w), 2934 (w), 2831 (w), 1698 (s), 1521 (s), $1453(\mathrm{~m}), 1227$ (s), 1200 (s), $1089(\mathrm{~s}), 1036(\mathrm{~s}), 979(\mathrm{~s}), 855(\mathrm{w}), 735(\mathrm{~m}), 696(\mathrm{~s})$;

HRMS (ESI) calcd. for $\mathrm{C}_{12} \mathrm{H}_{16} \mathrm{INNaO}_{3}{ }^{+}[\mathrm{M}+\mathrm{Na}]^{+} 372.0067$; Found 372.0078 .

N-(3-lodo-1-methoxypropyl)-4-methylbenzenesulfonamide (4q).<smiles>COC(CCI)NS(=O)(=O)c1ccc(C)cc1</smiles>

Following GP B, starting from N-cyclopropyl-4-methylbenzenesulfonamide $3 q$ ( $63.4 \mathrm{mg}, 0.300 \mathrm{mmol}$ ), without the addition of diphenyl phosphate. After stirring for 20 hours, $\mathrm{N}$-(3-iodo-1-methoxypropyl)4-methylbenzenesulfonamide $\mathbf{4 q}(101 \mathrm{mg}, 0.274 \mathrm{mmol}, 91 \%)$ was obtained as a yellow oil after purification by column chromatography on silica using 4:1 pentanes:ethyl acetate as eluent.

$\mathbf{R}_{\mathbf{f}}: 0.29$ (silica, pentanes:ethyl acetate 3:1);

${ }^{1}$ H NMR $\left(400 \mathrm{MHz}\right.$, Methanol- $\left.d_{4}\right): \delta=7.72(\mathrm{~d}, J=8.4 \mathrm{~Hz}, 2 \mathrm{H}, \operatorname{ArH}), 7.33(\mathrm{~d}, J=7.9 \mathrm{~Hz}, 2 \mathrm{H}, \operatorname{ArH}), 4.52$ (dd, J = 7.5, 4.9 Hz, $1 \mathrm{H}, \mathrm{CH}$ ), $3.17\left(\mathrm{~s}, 3 \mathrm{H}, \mathrm{OCH}_{3}\right), 3.09-2.96\left(\mathrm{~m}, 2 \mathrm{H}, \mathrm{CH}_{2} \mathrm{l}\right), 2.39\left(\mathrm{~s}, 3 \mathrm{H}, \mathrm{CH}_{3}\right), 2.00$ (dddd, $J=14.1,7.5,6.5,5.6 \mathrm{~Hz}, 1 \mathrm{H}, \mathrm{CH}_{2} \mathrm{CH}_{2} \mathrm{l}$ ), 1.71 (dddd, $J=14.5,8.3,7.0,5.0 \mathrm{~Hz}, 1 \mathrm{H}, \mathrm{CH}_{2} \mathrm{CH}_{2} \mathrm{l}$ ), $\mathrm{NH}$ signal was not resolved;

${ }^{13} \mathrm{C}$ NMR $\left(101 \mathrm{MHz}\right.$, Methanol- $\left.d_{4}\right): \delta=144.7,140.6,130.7,127.8,87.3,55.4,40.2,21.4,0.4$;

IR (film): $\tilde{v}=3272(\mathrm{~m}), 2932$ (w), 2832 (w), 1598 (w), 1495 (w), 1449 (m), 1324 (m), 1245 (w), 1155 (s), $1114(\mathrm{~m}), 1032(\mathrm{~m}), 985(\mathrm{~m}), 860(\mathrm{~m}), 812(\mathrm{~m})$;

HRMS (ESI) calcd. for $\mathrm{C}_{11} \mathrm{H}_{16} \mathrm{INNaO}_{3} \mathrm{~S}^{+}[\mathrm{M}+\mathrm{Na}]^{+}$391.9788; Found 391.9792.

$\mathrm{N}$-(3-lodo-1-methoxypropyl)-N,4-dimethylbenzenesulfonamide (4r). 
<smiles>COC(CCI)N(C)S(=O)(=O)c1ccc(C)cc1</smiles>

Following GP B, starting from N-cyclopropyl-N,4-dimethylbenzenesulfonamide 3r $(67.6 \mathrm{mg}, 0.300$ $\mathrm{mmol})$, without the addition of diphenyl phosphate. After stirring for 20 hours, N-(3-iodo-1methoxypropyl)-N,4-dimethylbenzenesulfonamide $4 \mathrm{r}$ (104 mg, $0.282 \mathrm{mmol}, 94 \%$ ) was obtained as a pale yellow oil after purification by column chromatography on silica using 4:1 pentanes:ethyl acetate as eluent.

$\mathbf{R}_{\mathbf{f}}: 0.50$ (silica, pentanes:ethyl acetate 6:1);

${ }^{1} \mathrm{H}$ NMR (400 MHz, Acetone- $\left.d_{6}\right): \delta=7.77(\mathrm{~d}, J=8.3 \mathrm{~Hz}, 2 \mathrm{H}, \mathrm{ArH}), 7.44(\mathrm{~d}, J=8.0 \mathrm{~Hz}, 2 \mathrm{H}, \mathrm{ArH}), 5.10$ (dd, $J=8.3,4.4 \mathrm{~Hz}, 1 \mathrm{H}, \mathrm{CH}), 3.21\left(\mathrm{~s}, 3 \mathrm{H}, \mathrm{OCH}_{3}\right), 3.19-3.13\left(\mathrm{~m}, 2 \mathrm{H}, \mathrm{CH}_{2} \mathrm{l}\right), 2.68\left(\mathrm{~s}, 3 \mathrm{H}, \mathrm{NCH}_{3}\right), 2.43(\mathrm{~s}$, $3 \mathrm{H}, \mathrm{ArCH}_{3}$ ), 2.12 (ddd, J = 14.2, 8.2, 5.9 Hz, $1 \mathrm{H}, \mathrm{CH}_{2} \mathrm{CH}_{2} \mathrm{l}$ ), 1.68 (dtd, J=14.6, 7.9, $4.4 \mathrm{~Hz}, 1 \mathrm{H}, \mathrm{CH}_{2} \mathrm{CH}_{2} \mathrm{l}$ );

${ }^{13} \mathrm{C}$ NMR $\left(101 \mathrm{MHz}\right.$, Acetone- $\left.d_{6}\right): \delta=144.4,138.0,130.6,127.9,89.3,56.0,37.4,27.5,21.4,1.3$;

IR (film): $\tilde{v}=2938$ (w), 2831 (w), 1597 (w), 1494 (w), 1449 (w), 1340 (s), 1216 (m), 1185 (m), 1153 (s), $1099(\mathrm{~m}), 932(\mathrm{~s}), 814(\mathrm{~m})$;

HRMS (ESI) calcd. for $\mathrm{C}_{12} \mathrm{H}_{18} \mathrm{INNaO} \mathrm{N}^{+}[\mathrm{M}+\mathrm{Na}]^{+}$405.9944; Found 405.9960.

tert-Butyl benzyl(3-iodo-1-methoxypropyl)carbamate (4s).<smiles>COC(CCI)N(Cc1ccccc1)C(=O)OC(C)(C)C</smiles>

Following GP B, starting from tert-butyl benzyl(cyclopropyl)carbamate $3 \mathrm{~s}(74.2 \mathrm{mg}, 0.300 \mathrm{mmol}$ ) and without the addition of diphenyl phosphate, tert-butyl benzyl(3-iodo-1-methoxypropyl)carbamate $4 \mathbf{s}$ (110 mg, $0.271 \mathrm{mmol}, 90 \%$ ) was obtained as a colorless oil after purification by column chromatography on silica using 6:1 pentanes:ethyl acetate as eluent. $\mathrm{K}_{2} \mathrm{CO}_{3}(0.300 \mathrm{mmol}, 1.00$ equiv.) has to be added to the reaction mixture before purification.

$\mathbf{R}_{\mathbf{f}}$ : 0.44 (silica, pentanes:ethyl acetate 8:1);

${ }^{1}$ H NMR (400 MHz, Acetone- $\left.d_{6}\right): \delta=7.47-7.14(\mathrm{~m}, 5 \mathrm{H}, \mathrm{ArH}), 5.61-5.34(\mathrm{~m}, 1 \mathrm{H}, \mathrm{CH}), 4.62-4.42(\mathrm{~m}$, $\left.1 \mathrm{H}, \mathrm{ArCH}_{2}\right), 4.24\left(\mathrm{~d}, \mathrm{~J}=16.2 \mathrm{~Hz}, 1 \mathrm{H}, \mathrm{ArCH}_{2}\right), 3.10\left(\mathrm{~m}, 5 \mathrm{H}, \mathrm{OCH}_{3}+\mathrm{CH}_{2} \mathrm{l}\right), 2.06-2.02\left(\mathrm{~m}, 1 \mathrm{H}, \mathrm{CH}_{2}\right), 1.97-$ $1.85\left(\mathrm{~m}, 1 \mathrm{H}, \mathrm{CH}_{2}\right), 1.59-1.31\left(\mathrm{~m}, 9 \mathrm{H}, \mathrm{CH}_{3}\right)$;

${ }^{13} \mathrm{C}$ NMR $\left(101 \mathrm{MHz}\right.$, Acetone- $\left.d_{6}\right): \delta={ }^{13} \mathrm{C}$ NMR $(101 \mathrm{MHz},) \delta 156.4,140.9,129.1,129.0,128.4,128.2$, $127.8,127.5,88.7,87.5,80.8,80.6,55.7,45.1,44.3,38.5,28.4,1.9,1.6$; Mixture of 2 rotamers with almost 1:1 ratio. They are not completely resolved.

IR (film): $\tilde{v}=2975$ (w), 2933 (w), 1695 (s), $1453(\mathrm{w}), 1403(\mathrm{~m}), 1379(\mathrm{~m}), 1366(\mathrm{~m}), 1252$ (m), $1158(\mathrm{~s})$, $1074(\mathrm{~m}), 956(\mathrm{w}), 889(\mathrm{w})$;

HRMS (ESI) calcd. for $\mathrm{C}_{16} \mathrm{H}_{24} \mathrm{INNaO}_{3}{ }^{+}[\mathrm{M}+\mathrm{Na}]^{+}$428.0693; Found 428.0700 . 
Benzyl ((2S)-1-((3-iodo-1-methoxypropyl)amino)-1-oxo-3-phenylpropan-2-yl)carbamate (4t).<smiles>COC(CCI)NC(=O)[C@H](Cc1ccccc1)NC(=O)OCc1ccccc1</smiles>

Following GP B, starting from benzyl (S)-(1-(cyclopropylamino)-1-oxo-3-phenylpropan-2-yl)carbamate 3t $(33.8 \mathrm{mg}, 0.100 \mathrm{mmol}), \mathrm{N}$-lodosuccinimide $(23.6 \mathrm{mg}, 0.105 \mathrm{mmol}, 1.05$ equiv.), methanol ( $4.8 \mu \mathrm{L}$, $0.120 \mathrm{mmol}, 1.2$ equiv.) and diphenyl phosphate $(2.5 \mathrm{mg}, 0.010 \mathrm{mmol}, 0.10$ equiv.) were dissolved in $0.5 \mathrm{~mL}$ of MeCN $(0.20 \mathrm{M})$. The reaction mixture was stirred at room temperature for 3 hours. Benzyl ((2S)-1-((3-iodo-1-methoxypropyl)amino)-1-oxo-3-phenylpropan-2-yl)carbamate 4t (34.2 mg, 0.0690 $\mathrm{mmol}, 69 \%)$ was obtained as a beige solid after purification by column chromatography on silica using 3:1 pentanes:ethyl acetate as eluent.

$\mathbf{R}_{\mathbf{f}}: 0.30$ (silica, pentanes:ethyl acetate 3:1);

Mp: $120-122^{\circ} \mathrm{C}$;

${ }^{1} \mathrm{H}$ NMR (400 MHz, Acetone- $d_{6}$; mixture of diastereoisomers in a $1: 1$ ratio: the signals corresponding to the two diastereoisomers are partially resolved): $\delta=7.56(\mathrm{~d}, J=9.4 \mathrm{~Hz}, 1 \mathrm{H}, \mathrm{NH}), 7.50(\mathrm{~d}, J=9.5 \mathrm{~Hz}$, $1 \mathrm{H}, \mathrm{NH}), 7.39-7.15(\mathrm{~m}, 10 \mathrm{H}, \mathrm{ArH}), 6.59(\mathrm{~d}, J=8.1 \mathrm{~Hz}, 1 \mathrm{H}, \mathrm{NH}), 6.53(\mathrm{~d}, J=8.4 \mathrm{~Hz}, 1 \mathrm{H}, \mathrm{NH}), 5.20-$ $5.08(\mathrm{~m}, 1 \mathrm{H}, \mathrm{CH}), 5.02\left(\mathrm{~s}, 2 \mathrm{H}, \mathrm{OCH}_{2}\right), 4.41(\mathrm{dtd}, \mathrm{J}=20.8,8.4,6.2 \mathrm{~Hz}, 1 \mathrm{H}, \mathrm{CH}), 3.28-3.17\left(\mathrm{~m}, 3 \mathrm{H}, \mathrm{OCH}_{3}\right)$, $3.18-3.10\left(\mathrm{~m}, 2 \mathrm{H}, \mathrm{PhCH}_{2}\right), 3.10-3.03\left(\mathrm{~m}, 1 \mathrm{H}, \mathrm{CH}_{2} \mathrm{l}\right), 3.02-2.93\left(\mathrm{~m}, 1 \mathrm{H}, \mathrm{CH}_{2} \mathrm{l}\right), 2.19-2.10(\mathrm{~m}, 1 \mathrm{H}$, $\left.\mathrm{CH}_{2} \mathrm{CH}_{2} \mathrm{l}\right), 2.09-2.07\left(\mathrm{~m}, 1 \mathrm{H}, \mathrm{CH}_{2} \mathrm{CH}_{2} \mathrm{l}\right), 1.97-1.85\left(\mathrm{~m}, 1 \mathrm{H}, \mathrm{CH}_{2} \mathrm{CH}_{2} \mathrm{l}\right)$;

${ }^{13} \mathrm{C}$ NMR (101 MHz, Acetone- $d_{6}$; mixture of diastereoisomers in a $1: 1$ ratio: the signals corresponding to the two diastereoisomers are partially resolved): $\delta=172.7,172.6,156.8,138.5,138.4,138.2$, $138.1,130.2,129.2,129.2$, 129.1, 128.6, 128.6, 128.6, 128.5, 127.4, 127.4, 81.9, 81.6, 66.7, 66.7, 57.7, $57.7,55.7,55.7,40.0,39.8,38.8,38.7,1.0,0.9$;

IR (film): $\tilde{v}=3289$ (m), 3030 (w), 2932 (w), 1664 (s), 1537 (s), 1256 (m), 1066 (m), 745 (m), 698(m); HRMS (APPI) calcd. for $\mathrm{C}_{21} \mathrm{H}_{25} \mathrm{IN}_{2} \mathrm{NaO}_{4}^{+}[\mathrm{M}+\mathrm{Na}]^{+}$519.0751; Found 519.0751.

N-(3-lodo-1-methoxypropyl)-2-(11-oxo-6,11-dihydrodibenzo[b,e]oxepin-2-yl)acetamide (4u).<smiles>COC(CCI)NC(=O)Cc1ccc2c(c1)C(=O)c1ccccc1CO2</smiles>

Following GP B, starting from N-cyclopropyl-2-(11-oxo-6,11-dihydrodibenzo[b,e]oxepin-2yl)acetamide $3 \mathrm{u}(30.7 \mathrm{mg}, 0.100 \mathrm{mmol}), \mathrm{N}$-lodosuccinimide $(23.6 \mathrm{mg}, 0.105 \mathrm{mmol}, 1.05$ equiv.), methanol ( $4.8 \mu \mathrm{L}, 0.120 \mathrm{mmol}, 1.2$ equiv.) and diphenyl phosphate $(2.5 \mathrm{mg}, 0.010 \mathrm{mmol}, 0.10$ equiv.) were dissolved in $0.5 \mathrm{~mL}$ of $\mathrm{MeCN}(0.20 \mathrm{M})$. The reaction mixture was stirred at room temperature for 1 hour. N-(3-lodo-1-methoxypropyl)-2-(11-oxo-6,11-dihydrodibenzo[b,e]oxepin-2-yl)acetamide 4u $(40.7 \mathrm{mg}, 0.0880 \mathrm{mmol}, 88 \%)$ was obtained as a yellow oil after purification by column chromatography on silica, using 1:1 pentanes:ethyl acetate as eluent. 
$\mathbf{R}_{\mathbf{f}}: 0.50$ (silica, pentanes:ethyl acetate $1: 1$ );

${ }^{1} \mathrm{H}$ NMR $\left(400 \mathrm{MHz}\right.$, Acetone- $\left.d_{6}\right): \delta=8.09(\mathrm{~s}, 1 \mathrm{H}, \operatorname{ArH}), 7.84(\mathrm{~d}, J=7.6 \mathrm{~Hz}, 1 \mathrm{H}, \operatorname{Ar} H), 7.69-7.58(\mathrm{~m}, 2 \mathrm{H}$, $\left.\mathrm{ArH}), 7.58-7.49(\mathrm{~m}, 3 \mathrm{H}, \mathrm{ArH}+\mathrm{NH}), 7.04(\mathrm{~d}, J=8.4 \mathrm{~Hz}, 1 \mathrm{H}, \mathrm{ArH}), 5.29(\mathrm{~s}, 2 \mathrm{H}, \mathrm{OCH})_{2}\right), 5.18(\mathrm{dt}, J=9.4$, $6.3 \mathrm{~Hz}, 1 \mathrm{H}, \mathrm{CH}), 3.61\left(\mathrm{~d}, J=2.4 \mathrm{~Hz}, 2 \mathrm{H}, \mathrm{CH}_{2}\right), 3.31-3.16\left(\mathrm{~m}, 5 \mathrm{H}, \mathrm{OCH}_{3}+\mathrm{CH}_{2} \mathrm{l}\right), 2.17(\mathrm{dd}, J=14.0,6.9 \mathrm{~Hz}$, $\left.1 \mathrm{H}, \mathrm{CH}_{2} \mathrm{CH}_{2} \mathrm{l}\right), 2.11-2.06\left(\mathrm{~m}, 1 \mathrm{H}, \mathrm{CH}_{2} \mathrm{CH}_{2} \mathrm{l}\right)$;

${ }^{13} \mathrm{C}$ NMR $\left(101 \mathrm{MHz}\right.$, Acetone- $\left.d_{6}\right): \delta=190.8,171.6,161.1,141.4,137.2,137.1,133.7,132.7,130.8$, $129.9,129.9,128.9,125.9,121.5,81.7,74.1,55.8,42.7,40.0,1.0$;

IR (film): $\tilde{v}=3292(\mathrm{w}), 2930(\mathrm{w}), 1647$ (s), 1609 (m), 1530 (m), 1487 (s), 1378 (m), 1299 (s), 1253 (m), $1120(\mathrm{~m}), 1069(\mathrm{~m}), 1015(\mathrm{~m}), 831(\mathrm{w}), 760(\mathrm{~m})$;

HRMS (APCl) calcd. for $\mathrm{C}_{20} \mathrm{H}_{20} \mathrm{INNaO}_{4}{ }^{+}[\mathrm{M}+\mathrm{Na}]^{+}$488.0329; Found 488.0335 .

\section{2-(1-(4-Chlorobenzoyl)-5-methoxy-2-methyl-1H-indol-3-yl)-N-(3-iodo-1-methoxypropyl)acetamide} $(4 v)$.

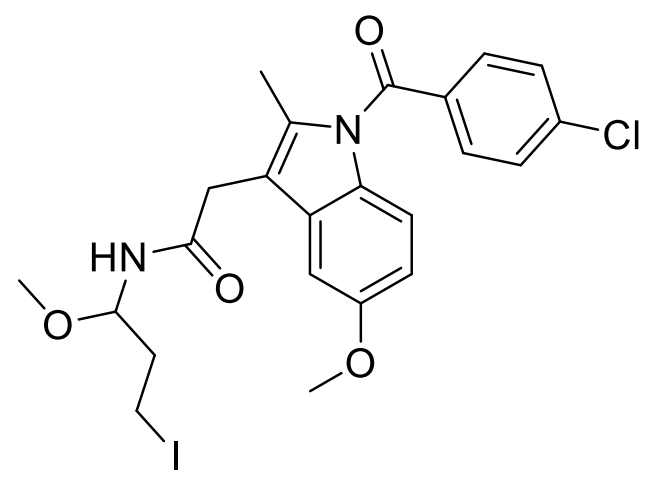

Following GP B, starting from 2-(1-(4-chlorobenzoyl)-5-methoxy-2-methyl-1H-indol-3-yl)-Ncyclopropylacetamide $3 \mathrm{v}(39.6 \mathrm{mg}, 0.100 \mathrm{mmol}), \mathrm{N}$-lodosuccinimide $(47.3 \mathrm{mg}, 0.210 \mathrm{mmol}, 2.1$ equiv.), methanol ( $4.8 \mu \mathrm{L}, 0.120 \mathrm{mmol}, 1.2$ equiv.) and diphenyl phosphate $(2.5 \mathrm{mg}, 0.010 \mathrm{mmol}, 0.10$ equiv.) were dissolved in $0.5 \mathrm{~mL}$ of $\operatorname{MeCN}(0.20 \mathrm{M})$. The reaction mixture was stirred at room temperature for 4 hours. 2-(1-(4-Chlorobenzoyl)-5-methoxy-2-methyl-1H-indol-3-yl)-N-(3-iodo-1methoxypropyl)acetamide $4 \mathbf{v}(24.4 \mathrm{mg}, 0.0440 \mathrm{mmol}, 44 \%)$ was obtained as a pale yellow solid after purification by column chromatography on silica using 2:1 pentanes:ethyl acetate as eluent.

$\mathbf{R}_{\mathbf{f}}: 0.30$ (silica, pentanes:ethyl acetate 2:1);

Mp: $135-138^{\circ} \mathrm{C}$;

${ }^{1} \mathrm{H}$ NMR $\left(400 \mathrm{MHz}\right.$, Acetone- $\left.d_{6}\right): \delta=7.77-7.72(\mathrm{~m}, 2 \mathrm{H}, \operatorname{ArH}), 7.66-7.61(\mathrm{~m}, 2 \mathrm{H}, \operatorname{ArH}), 7.47(\mathrm{~d}, J=$ $10.0 \mathrm{~Hz}, 1 \mathrm{H}, \mathrm{NH}), 7.14(\mathrm{~d}, J=2.6 \mathrm{~Hz}, 1 \mathrm{H}, \mathrm{ArH}), 7.02(\mathrm{~d}, J=9.0 \mathrm{~Hz}, 1 \mathrm{H}, \operatorname{ArH}), 6.71(\mathrm{dd}, J=9.0,2.6 \mathrm{~Hz}, 1 \mathrm{H}$, $\operatorname{ArH}), 5.24-5.14(\mathrm{~m}, 1 \mathrm{H}, \mathrm{CH}), 3.81\left(\mathrm{~s}, 3 \mathrm{H}, \mathrm{OCH}_{3}\right), 3.68\left(\mathrm{~s}, 2 \mathrm{H}, \mathrm{ArCH}_{2}\right), 3.25\left(\mathrm{~s}, 3 \mathrm{H}, \mathrm{OCH}_{3}\right), 3.20(\mathrm{t}, J=7.2$ $\left.\mathrm{Hz}, 2 \mathrm{H}, \mathrm{CH}_{2} \mathrm{l}\right), 2.33\left(\mathrm{~s}, 3 \mathrm{H}, \mathrm{CH}_{3}\right), 2.12\left(\mathrm{dt}, \mathrm{J}=13.8,7.0 \mathrm{~Hz}, 1 \mathrm{H}, \mathrm{CH}_{2} \mathrm{CH}_{2} \mathrm{l}\right), 2.03-1.97\left(\mathrm{~m}, 1 \mathrm{H}, \mathrm{CH}_{2} \mathrm{CH}_{2} \mathrm{l}\right)$;

${ }^{13} \mathrm{C}$ NMR $\left(101 \mathrm{MHz}\right.$, Acetone- $\left.d_{6}\right): \delta=171.0,168.8,157.1,139.2,136.6,135.6,132.1,131.9,131.8$, $129.9,115.7,114.6,112.5,102.2,81.8,55.9,55.8,39.9,32.5,13.7,1.0$;

IR (film): $\tilde{v}=3296$ (w), 2931 (w), 2832 (w), 1680 (s), 1591 (w), 1521 (w), 1477 (s), 1358 (m), 1321 (s), $1224(\mathrm{~m}), 1088(\mathrm{~m}), 835(\mathrm{w})$;

HRMS (APPI) calcd. for $\mathrm{C}_{23} \mathrm{H}_{24} \mathrm{ClIN}_{2} \mathrm{O}_{4}{ }^{+}[\mathrm{M}]^{+} 554.0464$; Found 554.0463.

$\mathrm{N}$-(3-Bromo-1-methoxypropyl)-4-methoxybenzamide (5). 
<smiles>COc1ccc(C(=O)NC(CCBr)OC)cc1</smiles>

In a glass vial, $\mathrm{N}$-cyclopropyl-4-methoxybenzamide $3 \mathrm{3a}(0.300 \mathrm{mmol}, 1.0$ equiv.), $\mathrm{N}$-bromosuccinimide (70.8 mg, $0.315 \mathrm{mmol}, 1.05$ equiv.), methanol (14.4 $\mathrm{LL}, 0.360 \mathrm{mmol}, 1.2$ equiv.) and diphenyl phosphate $(7.5 \mathrm{mg}, 0.030 \mathrm{mmol}, 0.10$ equiv.) were dissolved in $1.5 \mathrm{~mL}$ of $\mathrm{MeCN}(0.20 \mathrm{M})$. The reaction mixture was stirred at room temperature for 30 minutes. After the completion of the reaction, the crude was directly submitted to column chromatography on silica using 2:1 pentanes:ethyl acetate as eluent. N-(3-Bromo-1-methoxypropyl)-4-methoxybenzamide 5 (81.2 mg, $0.270 \mathrm{mmol}, 90 \%)$ was obtained as a white solid.

$\mathbf{R}_{\mathbf{f}}: 0.29$ (silica, pentanes:ethyl acetate 2:1);

Mp: $76-78{ }^{\circ} \mathrm{C}$;

${ }^{1} \mathrm{H}$ NMR $\left(400 \mathrm{MHz}, \mathrm{CDCl}_{3}\right): \delta=7.78(\mathrm{~d}, J=8.8 \mathrm{~Hz}, 2 \mathrm{H}, \operatorname{ArH}), 6.94(\mathrm{~d}, J=8.8 \mathrm{~Hz}, 2 \mathrm{H}, \operatorname{ArH}), 6.42(\mathrm{~d}, J=9.6$ $\mathrm{Hz}, 1 \mathrm{H}, \mathrm{NH}), 5.53(\mathrm{ddd}, J=9.6,6.7,5.3 \mathrm{~Hz}, 1 \mathrm{H}, \mathrm{CH}), 3.85\left(\mathrm{~s}, 3 \mathrm{H}, \mathrm{OCH}_{3}\right), 3.54-3.46\left(\mathrm{~m}, 2 \mathrm{H}, \mathrm{CH}_{2} \mathrm{Br}\right)$, $3.42\left(\mathrm{~s}, 3 \mathrm{H}, \mathrm{OCH}_{3}\right), 2.34-2.20\left(\mathrm{~m}, 2 \mathrm{H}, \mathrm{CH}_{2} \mathrm{CH}_{2} \mathrm{Br}\right)$;

${ }^{13} \mathrm{C}$ NMR $(101 \mathrm{MHz}, \mathrm{CDCl} 3): \delta=166.8,162.6,128.9,125.8,113.9,80.0,56.2,55.4,38.6,27.9$;

IR (film): $\tilde{v}=3304$ (w), 2936 (w), 2837 (w), 1641 (m), 1605 (s), 1532 (m), 1501 (s), 1310 (m), 1252 (s), $1177(\mathrm{~m}), 1106(\mathrm{~m}), 1030(\mathrm{~m}), 844(\mathrm{~m}), 769(\mathrm{~m})$;

HRMS (ESI) calcd. for $\mathrm{C}_{12} \mathrm{H}_{16} \mathrm{BrNNaO}_{3}{ }^{+}[\mathrm{M}+\mathrm{Na}]^{+} 324.0206$; Found 324.0206 .

\section{3-lodopropanenitrile (9).}

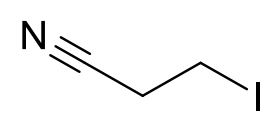

Following GP B, starting from cyclopropyl amine $(7.0 \mu \mathrm{L}, 0.10 \mathrm{mmol})$, N-lodosuccinimide $(23.6 \mathrm{mg}$, $0.105 \mathrm{mmol}, 1.05$ equiv.) and methanol $(4.8 \mu \mathrm{L}, 0.12 \mathrm{mmol}, 1.2$ equiv.) were dissolved in $0.5 \mathrm{~mL}$ of $\operatorname{MeCN}(0.20 \mathrm{M})$. The reaction mixture was stirred at room temperature for 0.5 hour. 3lodopropanenitrile $9(7.6 \mathrm{mg}, 0.042 \mathrm{mmol}, 42 \%)$ was obtained as a pale yellow oil after purification by column chromatography on silica, using 4:1 pentanes:ethyl acetate as eluent.

$\mathbf{R}_{\mathbf{f}}: 0.40$ (silica, pentanes:ethyl acetate $4: 1$ );

${ }^{1} \mathrm{H}$ NMR $\left(400 \mathrm{MHz}, \mathrm{CDCl}_{3}\right): \delta=3.28\left(\mathrm{t}, J=7.1 \mathrm{~Hz}, 2 \mathrm{H}, \mathrm{CH}_{2} \mathrm{l}\right), 3.01\left(\mathrm{t}, J=7.1 \mathrm{~Hz}, 2 \mathrm{H}, \mathrm{CH}_{2} \mathrm{CN}\right)$;

${ }^{13} \mathrm{C}$ NMR (101 MHz, $\left.\mathrm{CDCl}_{3}\right): \delta=118.1,22.5,-6.9$;

IR (film): $\tilde{v}=2957$ (w), 2921 (m), 2852 (w), 2252 (w), 1413 (s), 1250 (s), 1174 (s), 883 (s);

${ }^{13} \mathrm{C}$ NMR and IR data correspond to the reported values. ${ }^{[12]}$

\section{General Procedure C (GP C):}

In a glass vial, N-cyclopropyl-4-methoxybenzamide $3 a(57.3 \mathrm{mg}, 0.300 \mathrm{mmol}, 1.0$ equiv.), $\mathrm{N}$ lodosuccinimide ( $70.8 \mathrm{mg}, 0.315 \mathrm{mmol}, 1.05$ equiv.), alcohol $(0.360 \mathrm{mmol}, 1.2$ equiv.) and diphenyl phosphate $(7.5 \mathrm{mg}, 0.030 \mathrm{mmol}, 0.10$ equiv.) were dissolved in $1.5 \mathrm{~mL}$ of $\mathrm{MeCN}(0.20 \mathrm{M})$. The

${ }^{12}$ Irifune, S.; Kibayashi, T.; Ishii, Y.; Ogawa, M. Synthesis 1998, 5, 366-369. 
reaction mixture was stirred at room temperature for 30 minutes, if not specified otherwise. After the completion of the reaction, the crude product was directly submitted to column chromatography on silica using pentanes:ethyl acetate as eluent.

\section{N-(1-(Benzyloxy)-3-iodopropyl)-4-methoxybenzamide (10a).}<smiles>COc1ccc(C(=O)NC(CCI)OCc2ccccc2)cc1</smiles>

Following GP C, using from phenylmethanol (38.9 mg, $0.360 \mathrm{mmol}, 1.2$ equiv.), $\mathrm{N}$-(1-(benzyloxy)-3iodopropyl)-4-methoxybenzamide $10 \mathrm{a}(120 \mathrm{mg}, 0.282 \mathrm{mmol}, 94 \%)$ was obtained as a yellow oil after purification by column chromatography on silica using 3:1 pentanes:ethyl acetate as eluent.

$\mathbf{R}_{\mathbf{f}}: 0.33$ (silica, pentanes:ethyl acetate 3:1);

${ }^{1}$ H NMR $\left(400 \mathrm{MHz}, \mathrm{CDCl}_{3}\right): \delta=7.74(\mathrm{~d}, J=8.8 \mathrm{~Hz}, 2 \mathrm{H}, \mathrm{ArH}), 7.40-7.26(\mathrm{~m}, 5 \mathrm{H}, \mathrm{ArH}), 6.94(\mathrm{~d}, J=8.8$ $\mathrm{Hz}, 2 \mathrm{H}, \mathrm{ArH}), 6.36(\mathrm{~d}, J=9.7 \mathrm{~Hz}, 1 \mathrm{H}, \mathrm{NH}), 5.68$ (ddd, J = 9.7, 7.3, $4.9 \mathrm{~Hz}, 1 \mathrm{H}, \mathrm{CH}), 4.72-4.60(\mathrm{~m}, 2 \mathrm{H}$, $\left.\mathrm{CH}_{2}\right), 3.86\left(\mathrm{~s}, 3 \mathrm{H}, \mathrm{OCH}_{3}\right), 3.32-3.13\left(\mathrm{~m}, 2 \mathrm{H}, \mathrm{CH}_{2} \mathrm{l}\right), 2.40-2.21\left(\mathrm{~m}, 2 \mathrm{H}, \mathrm{CH}_{2} \mathrm{CH}_{2} \mathrm{l}\right)$;

${ }^{13} \mathrm{C}$ NMR $\left(101 \mathrm{MHz}, \mathrm{CDCl}_{3}\right): \delta=166.7,162.6,137.8,128.9,128.5,127.9,127.8,125.7,113.9,80.1$, 70.8, 55.5, 39.8, -0.8;

IR (film): $\tilde{v}=3306$ (w), 3030 (w), 2938 (w), 2837 (w), 1640 (s), 1606 (s), 1532 (m), 1501 (s), 1307 (m), $1255(\mathrm{~s}), 1177(\mathrm{~s}), 1100(\mathrm{~m}), 1063(\mathrm{~m}), 1028(\mathrm{~s}), 844(\mathrm{~m}), 737(\mathrm{~m})$;

HRMS (ESI) calcd. for $\mathrm{C}_{18} \mathrm{H}_{20} \mathrm{INNaO}_{3}{ }^{+}[\mathrm{M}+\mathrm{Na}]^{+} 448.0380$; Found 448.0384.

N-(3-lodo-1-(prop-2-yn-1-yloxy)propyl)-4-methoxybenzamide (10b).<smiles>C#CCOC(CCI)NC(=O)c1ccc(OC)cc1</smiles>

Following GP C, using from prop-2-yn-1-ol (20.2 mg, $0.360 \mathrm{mmol}, 1.2$ equiv.) and after stirring for 1 hour, N-(3-iodo-1-(prop-2-yn-1-yloxy)propyl)-4-methoxybenzamide 10b (91.2 mg, 0.244 mmol, 81\%) was obtained as a yellow solid after purification by column chromatography on silica using 3:1 pentanes:ethyl acetate as eluent.

$\mathbf{R}_{\mathbf{f}}: 0.40$ (silica, pentanes:ethyl acetate $3: 1$ );

Mp: $78-80^{\circ} \mathrm{C}$; 
${ }^{1} \mathrm{H}$ NMR $\left(400 \mathrm{MHz}, \mathrm{CDCl}_{3}\right): \delta=7.77(\mathrm{~d}, J=8.8 \mathrm{~Hz}, 2 \mathrm{H}, \mathrm{ArH}), 6.94(\mathrm{~d}, J=8.8 \mathrm{~Hz}, 2 \mathrm{H}, \operatorname{ArH}), 6.39(\mathrm{~d}, J=9.6$ $\mathrm{Hz}, 1 \mathrm{H}, \mathrm{NH}), 5.67(\mathrm{ddd}, J=9.6,7.3,5.0 \mathrm{~Hz}, 1 \mathrm{H}, \mathrm{CH}), 4.34-4.24\left(\mathrm{~m}, 2 \mathrm{H}, \mathrm{CH}_{2}\right), 3.86\left(\mathrm{~s}, 3 \mathrm{H}, \mathrm{OCH}_{3}\right), 3.33-$ $3.16\left(\mathrm{~m}, 2 \mathrm{H}, \mathrm{CH}_{2} \mathrm{l}\right), 2.43\left(\mathrm{t}, \mathrm{J}=2.4 \mathrm{~Hz}, 1 \mathrm{H}\right.$, alkynylH), $2.38-2.22\left(\mathrm{~m}, 2 \mathrm{H}, \mathrm{CH}_{2} \mathrm{CH}_{2} \mathrm{l}\right)$;

${ }^{13} \mathrm{C} \mathrm{NMR}\left(101 \mathrm{MHz}, \mathrm{CDCl}_{3}\right): \delta=166.8,162.7,129.0,125.5,113.9,80.1,79.7,74.4,56.3,55.5,39.5$, 1.3;

IR (film): $\tilde{v}=3291$ (w), 2957 (w), 2924 (w), 2854 (w), 1642 (m), 1605 (s), 1530 (m), 1498 (s), 1307 (m), $1253(\mathrm{~s}), 1176(\mathrm{~s}), 1062(\mathrm{~s}), 1029(\mathrm{~s}), 909(\mathrm{w}), 843(\mathrm{~m}), 607(\mathrm{~m})$;

HRMS (ESI) calcd. for $\mathrm{C}_{14} \mathrm{H}_{16} \mathrm{INNaO}_{3}{ }^{+}[\mathrm{M}+\mathrm{Na}]^{+} 396.0067$; Found 396.0077.

N-(1-(Allyloxy)-3-iodopropyl)-4-methoxybenzamide (10c).<smiles>C=CCOC(CCI)NC(=O)c1ccc(OC)cc1</smiles>

Following GP C, using from prop-2-en-1-ol $(20.9 \mathrm{mg}, 0.360 \mathrm{mmol}, 1.2$ equiv.) and after stirring for 1 hour, N-(1-(allyloxy)-3-iodopropyl)-4-methoxybenzamide 10c $(71.1 \mathrm{mg}, 0.189 \mathrm{mmol}, 63 \%)$ was obtained as a yellow oil after purification by column chromatography on silica using 3:1 pentanes:ethyl acetate as eluent.

$\mathbf{R}_{\mathbf{f}}$ : 0.41 (silica, pentanes:ethyl acetate $3: 1$ );

${ }^{1} \mathrm{H}$ NMR $\left(400 \mathrm{MHz}, \mathrm{CDCl}_{3}\right): \delta=7.76(\mathrm{~d}, J=8.9 \mathrm{~Hz}, 2 \mathrm{H}, \mathrm{ArH}), 6.94(\mathrm{~d}, J=8.9 \mathrm{~Hz}, 2 \mathrm{H}, \operatorname{ArH}), 6.38(\mathrm{~d}, J=9.6$ $\mathrm{Hz}, 1 \mathrm{H}, \mathrm{NH}), 5.91$ (ddt, $J=17.2,10.4,5.7 \mathrm{~Hz}, 1 \mathrm{H}$, vinylH), 5.58 (ddd, $J=9.6,7.2,5.1 \mathrm{~Hz}, 1 \mathrm{H}, \mathrm{CH}), 5.29$ (dq, $J=17.2,1.7 \mathrm{~Hz}, 1 \mathrm{H}$, vinyl $\mathrm{H}$ ), $5.18\left(\mathrm{dq}, J=10.4,1.4 \mathrm{~Hz}, 1 \mathrm{H}\right.$, vinylH), $4.21-4.05\left(\mathrm{~m}, 2 \mathrm{H}, \mathrm{CH}_{2}\right), 3.85$ (s, $\left.3 \mathrm{H}, \mathrm{OCH}_{3}\right), 3.33-3.14\left(\mathrm{~m}, 2 \mathrm{H}, \mathrm{CH}_{2} \mathrm{l}\right), 2.41-2.14\left(\mathrm{~m}, 2 \mathrm{H}, \mathrm{CH}_{2} \mathrm{CH}_{2} \mathrm{l}\right)$;

${ }^{13} \mathrm{C}$ NMR $\left(101 \mathrm{MHz}, \mathrm{CDCl}_{3}\right): \delta=166.7,162.6,134.1,128.9,125.7,117.4,113.9,79.9,69.5,55.4,39.7$, 0.9 ;

IR (film): $\tilde{v}=3305$ (w), 2933 (w), 2839 (w), 1640 (s), 1606 (s), 1532 (m), 1501 (s), 1308 (m), 1255 (s), $1178(\mathrm{~s}), 1032(\mathrm{~s}), 927(\mathrm{w}), 844(\mathrm{~m}), 768(\mathrm{w})$;

HRMS (ESI) calcd. for $\mathrm{C}_{14} \mathrm{H}_{18} \mathrm{INNaO}_{3}{ }^{+}[\mathrm{M}+\mathrm{Na}]^{+} 398.0224$; Found 398.0224.

N-(3-lodo-1-isopropoxypropyl)-4-methoxybenzamide (10d).<smiles>COc1ccc(C(=O)NC(CCI)OC(C)C)cc1</smiles>

Following GP C, using from propan-2-ol (21.6 mg, $0.360 \mathrm{mmol}, 1.2$ equiv.), N-(3-iodo-1isopropoxypropyl)-4-methoxybenzamide $10 \mathrm{~d}(94.6 \mathrm{mg}, 0.251 \mathrm{mmol}, 84 \%)$ was obtained as a yellow oil after purification by column chromatography on silica using 2:1 pentanes:ethyl acetate as eluent. 
$\mathbf{R}_{\mathbf{f}}: 0.40$ (silica, pentanes:ethyl acetate 3:1);

${ }^{1} \mathrm{H}$ NMR $\left(400 \mathrm{MHz}, \mathrm{CDCl}_{3}\right): \delta=7.75(\mathrm{~d}, J=8.8 \mathrm{~Hz}, 2 \mathrm{H}, \mathrm{ArH}), 6.92(\mathrm{~d}, J=8.9 \mathrm{~Hz}, 2 \mathrm{H}, \mathrm{ArH}), 6.40$ (d, $J=9.3$ $\mathrm{Hz}, 1 \mathrm{H}, \mathrm{NH}), 5.60$ (ddd, J=9.4, 7.4, $5.0 \mathrm{~Hz}, 1 \mathrm{H}, \mathrm{CH}), 3.90(\mathrm{p}, J=6.1 \mathrm{~Hz}, 1 \mathrm{H}, \mathrm{CH}), 3.84\left(\mathrm{~s}, 3 \mathrm{H}, \mathrm{OCH}_{3}\right)$, $3.29-3.14\left(\mathrm{~m}, 2 \mathrm{H}, \mathrm{CH}_{2} \mathrm{l}\right), 2.22\left(\mathrm{qd}, J=7.4,5.3 \mathrm{~Hz}, 2 \mathrm{H}, \mathrm{CH}_{2} \mathrm{CH}_{2} \mathrm{l}\right), 1.22\left(\mathrm{~d}, J=6.1 \mathrm{~Hz}, 3 \mathrm{H}, \mathrm{CH}_{3}\right), 1.13(\mathrm{~d}, J$ $\left.=6.2 \mathrm{~Hz}, 3 \mathrm{H}, \mathrm{CH}_{3}\right)$;

${ }^{13} \mathrm{C}$ NMR $\left(101 \mathrm{MHz}, \mathrm{CDCl}_{3}\right): \delta=166.4,162.5,128.8,125.9,113.8,78.1,69.5,55.4,40.0,23.4,21.8,-$ 0.4 ;

IR (film): $\tilde{v}=3303$ (w), 2969 (w), 2933 (w), 2838 (w), 1638 (s), 1606 (s), 1532 (m), 1501 (s), 1442 (w), $1336(\mathrm{w}), 1255(\mathrm{~s}), 1175(\mathrm{~s}), 1033(\mathrm{~s}), 844(\mathrm{~m})$;

HRMS (ESI) calcd. for $\mathrm{C}_{14} \mathrm{H}_{20} \mathrm{INNaO}_{3}{ }^{+}[\mathrm{M}+\mathrm{Na}]^{+} 400.0380$; Found 400.0388 .

N-(3-lodo-1-(((1R,2S,5R)-2-isopropyl-5-methylcyclohexyl)oxy)propyl)-4-methoxybenzamide (10e).<smiles>COc1ccc(C(=O)NC(CCI)O[C@@H]2C[C@H](C)CC[C@H]2C(C)C)cc1</smiles>

Following GP C, using from (-)-menthol (56.3 mg, $0.360 \mathrm{mmol}, 1.2$ equiv.), $\mathrm{N}$-(3-iodo-1-(((1R,2S,5R)-2isopropyl-5-methylcyclohexyl)oxy)propyl)-4-methoxybenzamide $10 \mathrm{e}(120 \mathrm{mg}, 0.253 \mathrm{mmol}, 84 \%)$ was obtained as an off-white solid after purification by column chromatography on silica using 3:1 pentanes:ethyl acetate as eluent.

$\mathbf{R}_{\mathbf{f}}: 0.45$ (silica, pentanes:ethyl acetate $4: 1$ );

Mp: 89-91 ${ }^{\circ} \mathrm{C}$;

${ }^{1} \mathrm{H}$ NMR (400 MHz, $\mathrm{CDCl}_{3}$; mixture of diastereoisomers in a $1.5: 1$ ratio: the signals corresponding to the two distereoisomers are partially resolved): $\delta=7.79-7.70(\mathrm{~m}, 4 \mathrm{H}, \mathrm{ArH}$, major+minor), $6.99-$ $6.86(\mathrm{~m}, 4 \mathrm{H}, \mathrm{ArH}$, major+minor), $6.31(\mathrm{~d}, J=9.4 \mathrm{~Hz}, 1 \mathrm{H}, \mathrm{NH}$ minor), $6.25(\mathrm{~d}, J=9.4 \mathrm{~Hz}, 1 \mathrm{H}, \mathrm{NH}$ major), $5.70-5.52\left(\mathrm{~m}, 2 \mathrm{H}, \mathrm{CH}\right.$, major+minor), $3.85\left(\mathrm{~d}, J=1.2 \mathrm{~Hz}, 6 \mathrm{H}, \mathrm{OCH}_{3}\right.$, major+minor), 3.48 (td, $J=10.5$, $4.3 \mathrm{~Hz}, 1 \mathrm{H}, \mathrm{OCH}$ ring minor), 3.36 (td, $J=10.5,4.3 \mathrm{~Hz}, 1 \mathrm{H}, \mathrm{OCH}$ ring major), $3.29-3.09\left(\mathrm{~m}, 4 \mathrm{H}, \mathrm{CH}_{2} \mathrm{l}\right.$, major+minor), $2.40-2.07\left(\mathrm{~m}, 8 \mathrm{H}, \mathrm{CH}_{2} \mathrm{CH}_{2} \mathrm{l}\right.$ major+minor + $\mathrm{CH}_{2}$ ring, major+minor $), 2.01-1.93(\mathrm{~m}, 1 \mathrm{H}$, $\mathrm{CH}$ ring minor), $1.69-1.55\left(\mathrm{~m}, 6 \mathrm{H}, \mathrm{CH}_{2}\right.$ ring, major+minor $+\mathrm{CH}_{2}$ ring minor), $1.44-1.27(\mathrm{~m}, 3 \mathrm{H}, \mathrm{CH}$ major+minor $+\mathrm{CH}$ ring major), $1.22-1.12\left(\mathrm{~m}, 2 \mathrm{H}, \mathrm{CH}_{2}\right.$ ring major $), 1.00-0.94(\mathrm{~m}, 2 \mathrm{H}, \mathrm{CH}$ major+minor), $0.92\left(\mathrm{~d}, J=6.0 \mathrm{~Hz}, 6 \mathrm{H}, \mathrm{CH}_{3}\right.$ major+minor), 0.88 (d, $J=7.1 \mathrm{~Hz}, 6 \mathrm{H}, \mathrm{CH}_{3}$, major+minor), 0.80 (d, $J=6.5 \mathrm{~Hz}, 3 \mathrm{H}, \mathrm{CH}_{3}$ minor), 0.62 (d, $J=6.9 \mathrm{~Hz}, 3 \mathrm{H}, \mathrm{CH}_{3}$ major);

${ }^{13} \mathrm{C}$ NMR (101 MHz, CDCl 3 ; mixture of diastereoisomers in a $1.5: 1$ ratio: the signals corresponding to the two distereoisomers are partially resolved): $\delta=166.4,166.0,162.5,128.8,126.2,125.9,113.9$, $113.8,81.0,79.5,75.7,55.4,55.4,49.1,47.8,43.3,40.9,40.7,40.3,34.4,34.2,31.6,31.3,25.5,25.4$, $22.9,22.8,22.3,21.3,21.1,16.1,15.9,-0.5,-0.9$;

IR (film): $\tilde{v}=3317$ (w), $2953(\mathrm{~m}), 2922(\mathrm{~m}), 2868(\mathrm{w}), 1636(\mathrm{~m}), 1606(\mathrm{~s}), 1501(\mathrm{~m}), 1257(\mathrm{~s}), 1178(\mathrm{~m})$, $1026(\mathrm{~m}), 844(\mathrm{~m})$;

HRMS (ESI) calcd. for $\mathrm{C}_{21} \mathrm{H}_{32} \mathrm{INNaO}_{3}{ }^{+}[\mathrm{M}+\mathrm{Na}]^{+}$496.1319; Found 496.1323.

$\mathrm{N}$-(1-(2-Hydroxyethoxy)-3-iodopropyl)-4-methoxybenzamide (10f). 
<smiles>COc1ccc(C(=O)NC(CCI)OCCO)cc1</smiles>

Following GP C, using from ethane-1,2-diol $(22.3 \mathrm{mg}, 0.360 \mathrm{mmol}, 1.2$ equiv.) and after stirring for 1 hour, N-(1-(2-hydroxyethoxy)-3-iodopropyl)-4-methoxybenzamide $10 \mathrm{f}(65.0 \mathrm{mg}, 0.171 \mathrm{mmol}, 57 \%)$ was obtained as a yellow oil after purification by column chromatography on silica using 2:3 pentanes:ethyl acetate as eluent.

$\mathbf{R}_{\mathbf{f}}: 0.22$ (silica, pentanes:ethyl acetate 2:3);

${ }^{1} \mathrm{H}$ NMR $\left(400 \mathrm{MHz}\right.$, Acetone- $\left.d_{6}\right): \delta=7.98-7.81(\mathrm{~m}, 3 \mathrm{H}, \mathrm{ArH}+\mathrm{NH}), 7.05-6.93(\mathrm{~m}, 2 \mathrm{H}, \mathrm{ArH}), 5.55$ (ddd, J = 9.2, 7.2, $5.4 \mathrm{~Hz}, 1 \mathrm{H}, \mathrm{CH}), 3.86\left(\mathrm{~s}, 3 \mathrm{H}, \mathrm{OCH}_{3}\right), 3.70-3.59\left(\mathrm{~m}, 4 \mathrm{H}, \mathrm{OCH}_{2} \mathrm{CH}_{2} \mathrm{O}\right), 3.35(\mathrm{t}, J=7.2 \mathrm{~Hz}$, $\left.2 \mathrm{H}, \mathrm{CH}_{2} \mathrm{l}\right), 2.35\left(\mathrm{dq}, J=13.9,6.9 \mathrm{~Hz}, 1 \mathrm{H}, \mathrm{CH}_{2} \mathrm{CH}_{2} \mathrm{l}\right), 2.26-2.16\left(\mathrm{~m}, 1 \mathrm{H}, \mathrm{CH}_{2} \mathrm{CH}_{2} \mathrm{l}\right)$;

${ }^{13} \mathrm{C}$ NMR (101 MHz, Acetone- $\left.d_{6}\right): \delta=167.3,163.4,130.2,127.3,114.3,81.3,70.6,62.0,55.8,40.1,1.5$; IR (film): $\tilde{v}=3307$ (w), 2934 (w), $2872(w), 2839(w), 1708(\mathrm{~m}), 1640(\mathrm{~m}), 1605$ (s), 1534 (m), 1501 (s), $1255(\mathrm{~s}), 1177(\mathrm{~s}), 1106(\mathrm{~m}), 1027(\mathrm{~s}), 844(\mathrm{~m}), 768(\mathrm{w})$;

HRMS (ESI) calcd. for $\mathrm{C}_{13} \mathrm{H}_{18} \mathrm{INNaO}_{4}{ }^{+}[\mathrm{M}+\mathrm{Na}]^{+}$402.0173; Found 402.0181.

N-(1-(tert-Butylperoxy)-3-iodopropyl)-4-methoxybenzamide (10g).<smiles>COc1ccc(C(=O)NC(CCI)OOC(C)(C)C)cc1</smiles>

In a glass vial, $\mathrm{N}$-cyclopropyl-4-methoxybenzamide $3 \mathrm{a}(0.300 \mathrm{mmol}, 1.0$ equiv.), iodine $(83.8 \mathrm{mg}$, $0.330 \mathrm{mmol}, 1.1$ equiv.), a tert-butyl hydroperoxide solution, $5.0-6.0 \mathrm{M}$ in decane $(72.0 \mu \mathrm{L}, 0.360$ mmol, 1.2 equiv.) and diphenyl phosphate ( $7.5 \mathrm{mg}, 0.030 \mathrm{mmol}, 0.10$ equiv.) were dissolved in 1.5 $\mathrm{mL}$ of $\mathrm{MeCN}(0.20 \mathrm{M})$. The reaction mixture was stirred at room temperature for 30 minutes. After the completion of the reaction, the crude product was directly submitted to column chromatography on silica using 4:1 pentanes:ethyl acetate as eluent. N-(1-(tert-Butylperoxy)-3-iodopropyl)-4methoxybenzamide $10 \mathrm{~g}(83.2 \mathrm{mg}, 0.204 \mathrm{mmol}, 68 \%)$ was obtained as a yellow oil.

$\mathbf{R}_{\mathbf{f}}: 0.38$ (silica, pentanes:ethyl acetate $4: 1$ );

${ }^{1} \mathrm{H}$ NMR $\left(400 \mathrm{MHz}, \mathrm{CDCl}_{3}\right): \delta=7.77(\mathrm{~d}, J=8.8 \mathrm{~Hz}, 2 \mathrm{H}, \mathrm{ArH}), 6.93(\mathrm{~d}, J=8.8 \mathrm{~Hz}, 2 \mathrm{H}, \mathrm{ArH}), 6.58(\mathrm{~d}, J=8.8$ $\mathrm{Hz}, 1 \mathrm{H}, \mathrm{NH}), 5.91$ (ddd, $J=8.9,7.1,5.7 \mathrm{~Hz}, 1 \mathrm{H}, \mathrm{CH}), 3.85\left(\mathrm{~s}, 3 \mathrm{H}, \mathrm{OCH}_{3}\right), 3.30-3.16\left(\mathrm{~m}, 2 \mathrm{H}, \mathrm{CH}_{2} \mathrm{l}\right), 2.51$ $-2.39\left(\mathrm{~m}, 1 \mathrm{H}, \mathrm{CH}_{2} \mathrm{CH}_{2} \mathrm{l}\right), 2.35-2.24\left(\mathrm{~m}, 1 \mathrm{H}, \mathrm{CH}_{2} \mathrm{CH}_{2} \mathrm{l}\right), 1.23\left(\mathrm{~s}, 9 \mathrm{H}, \mathrm{CH}_{3}\right)$;

${ }^{13} \mathrm{C}$ NMR $\left(101 \mathrm{MHz}, \mathrm{CDCl}_{3}\right): \delta=166.4,162.5,129.0,126.0,113.8,83.7,80.9,55.4,37.2,26.4,-1.6$; 
IR (film): $\tilde{v}=3312$ (w), 2976 (m), 2933 (w), 2838 (w), 1647 (s), 1606 (s), 1534 (m), 1503 (s), 1363 (m), $1255(\mathrm{~s}), 1177$ (s), $1031(\mathrm{~m}), 845(\mathrm{~m})$;

HRMS (ESI) calcd. for $\mathrm{C}_{15} \mathrm{H}_{22} \mathrm{INNaO}_{4}{ }^{+}[\mathrm{M}+\mathrm{Na}]^{+}$430.0486; Found 430.0483 .

N-(1-Hydroperoxy-3-iodopropyl)-4-methoxybenzamide (10h).<smiles>COc1ccc(C(=O)NC(CCI)OO)cc1</smiles>

In a glass vial, $\mathrm{N}$-cyclopropyl-4-methoxybenzamide $3 \mathrm{a}(57.3 \mathrm{mg}, 0.300 \mathrm{mmol}, 1.0$ equiv.), iodine (83.8 $\mathrm{mg}, 0.330 \mathrm{mmol}, 1.1$ equiv.), a hydrogen peroxide $30 \%$ aqueous solution $(61.3 \mu \mathrm{L}, 0.600 \mathrm{mmol}, 2.0$ equiv.) and diphenyl phosphate ( $7.5 \mathrm{mg}, 0.030 \mathrm{mmol}, 0.10$ equiv.) were dissolved in $1.5 \mathrm{~mL}$ of $\mathrm{MeCN}$ $(0.20 \mathrm{M})$. The reaction mixture was stirred at room temperature for 30 minutes. After the completion of the reaction, the crude product was directly submitted to column chromatography on silica using 3:2 pentanes:ethyl acetate as eluent. $\mathrm{N}$-(1-Hydroperoxy-3-iodopropyl)-4-methoxybenzamide $10 \mathrm{~h}$ (94.6 $\mathrm{mg}, 0.269 \mathrm{mmol}, 90 \%$ ) was obtained as a grey solid.

$\mathbf{R}_{\mathbf{f}}: 0.30$ (silica, pentanes:ethyl acetate 3:2);

${ }^{1} \mathrm{H}$ NMR $\left(400 \mathrm{MHz}\right.$, Acetone- $\left.d_{6}\right): \delta=10.94(\mathrm{~s}, 1 \mathrm{H}, \mathrm{OOH}), 8.08(\mathrm{~d}, J=8.9 \mathrm{~Hz}, 1 \mathrm{H}, \mathrm{NH}), 7.93(\mathrm{~d}, J=8.8 \mathrm{~Hz}$, $\left.2 \mathrm{H}, \mathrm{ArH}), 6.99(\mathrm{~d}, J=8.8 \mathrm{~Hz}, 2 \mathrm{H}, \mathrm{ArH}), 5.90(\mathrm{dt}, J=8.8,6.7 \mathrm{~Hz}, 1 \mathrm{H}, \mathrm{CH}), 3.86(\mathrm{~s}, 3 \mathrm{H}, \mathrm{OCH})_{3}\right), 3.34(\mathrm{t}, J=$ $7.2 \mathrm{~Hz}, 2 \mathrm{H}, \mathrm{CH}_{2} \mathrm{l}$ ), 2.52 (dq, J = 14.0, 7.0 Hz, $1 \mathrm{H}, \mathrm{CH}_{2} \mathrm{CH}_{2} \mathrm{l}$ ), 2.27 (dq, J = 14.2, 7.5 Hz, $1 \mathrm{H}, \mathrm{CH}_{2} \mathrm{CH}_{2} \mathrm{l}$ );

${ }^{13} \mathrm{C}$ NMR $\left(101 \mathrm{MHz}\right.$, Acetone- $\left.d_{6}\right): \delta=167.0,163.4,130.3,127.4,114.3,85.5,55.8,36.8,0.9$;

IR (film): $\tilde{v}=3280$ (w), 2963 (w), 2837 (w), 1635 (m), 1605 (s), 1500 (s), 1254 (s), 1176 (s), 1027 (m), $842(\mathrm{~m}), 768(\mathrm{~m})$;

HRMS (ESI) calcd. for $\mathrm{C}_{11} \mathrm{H}_{14} \mathrm{INNaO}_{4}{ }^{+}[\mathrm{M}+\mathrm{Na}]^{+} 373.9860$; Found 373.9857 . 


\section{$\underline{\text { 4. Flexible conversion of aminocyclopropanes }}$}

N-(3-lodo-1-(methylsulfonamido)propyl)-4-methoxybenzamide (11).<smiles>COc1ccc(C(=O)NC2CC2)cc1</smiles>

$$
\underset{\text { NIS, } \mathrm{MeOH},(\mathrm{PhO})_{2} \mathrm{PO}_{2} \mathrm{H}, \mathrm{MeCN}}{\text { then } \mathrm{MsNH}_{2}}
$$<smiles>COc1ccc(C(=O)NC(CCI)NS(C)(=O)=O)cc1</smiles>

In a glass vial, N-cyclopropyl-4-methoxybenzamide 3a $(57.3 \mathrm{mg}, 0.300 \mathrm{mmol}, 1.0$ equiv.), $\mathrm{N}$ iodosuccinimide ( $70.8 \mathrm{mg}, 0.315 \mathrm{mmol}, 1.05$ equiv.), diphenyl phosphate $(7.5 \mathrm{mg}, 0.030 \mathrm{mmol}, 0.1$ equiv.), and methanol (14.4 $\mu \mathrm{L}, 0.360 \mathrm{mmol}, 1.2$ equiv.) were dissolved in $1.5 \mathrm{~mL}$ of $\mathrm{MeCN}(0.20 \mathrm{M})$. The reaction mixture was stirred at room temperature for 30 minutes. Methanesulfonamide (85.6 $\mathrm{mg}, 0.900 \mathrm{mmol}, 3.0$ equiv.) was then added and the reaction mixture was stirred at room temperature for another 10 minutes. After the completion of the reaction, the crude product was directly submitted to column chromatography on silica using 2:3 pentanes:Et ${ }_{2} \mathrm{O}$ as eluent. $\mathrm{N}$-(3-lodo1-(methylsulfonamido)propyl)-4-methoxybenzamide 11 (84.9 mg, $0.206 \mathrm{mmol}, 69 \%)$ was obtained as a pale yellow solid.

$\mathbf{R}_{\mathbf{f}}: 0.26$ (silica, pentanes:diethyl ether 2:3);

Mp: $102-105^{\circ} \mathrm{C}$;

${ }^{1} \mathrm{H}$ NMR $\left(400 \mathrm{MHz}, \mathrm{CDCl}_{3}\right): \delta=7.86-7.65(\mathrm{~m}, 2 \mathrm{H}, \mathrm{ArH}), 7.03-6.83(\mathrm{~m}, 2 \mathrm{H}, \mathrm{ArH}), 6.24(\mathrm{~d}, J=9.6 \mathrm{~Hz}$, $1 \mathrm{H}, \mathrm{NH}), 5.45(\mathrm{ddd}, J=9.6,7.0,5.3 \mathrm{~Hz}, 1 \mathrm{H}, \mathrm{CH}), 3.86\left(\mathrm{~s}, 3 \mathrm{H}, \mathrm{OCH}_{3}\right), 3.42\left(\mathrm{~s}, 3 \mathrm{H}, \mathrm{CH}_{3}\right), 3.32-3.15(\mathrm{~m}$, $\left.2 \mathrm{H}, \mathrm{CH}_{2} \mathrm{l}\right), 2.37-2.14\left(\mathrm{~m}, 2 \mathrm{H}, \mathrm{CH}_{2} \mathrm{CH}_{2} \mathrm{l}\right) ; 1 \mathrm{NH}$ is not resolved.

${ }^{13} \mathrm{C}$ NMR $\left(101 \mathrm{MHz}, \mathrm{CDCl}_{3}\right): \delta=166.8,162.6,128.9,125.8,113.9,81.6,56.3,55.5,39.6,-1.0$;

IR (film): $\tilde{v}=3308$ (w), 2933 (w), 2835 (w), 1642 (s), 1606 (s), 1532 (m), 1502 (s), 1255 (s), 1176 (m), $1104(\mathrm{~m}), 1066(\mathrm{~m}), 844(\mathrm{~m})$;

HRMS (ESI) calcd. for $\mathrm{C}_{12} \mathrm{H}_{17} / \mathrm{N}_{2} \mathrm{NaO}_{4} \mathrm{~S}^{+}[\mathrm{M}+\mathrm{Na}]^{+} 434.9846$; Found 434.9855.

N-(1-Cyano-3-iodopropyl)-4-methylbenzenesulfonamide (12).<smiles>Cc1ccc(S(=O)(=O)NC2CC2)cc1</smiles>

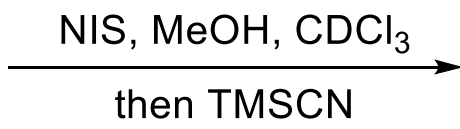<smiles>Cc1ccc(S(=O)(=O)NC(C#N)CCI)cc1</smiles>

Starting from $\mathrm{N}$-cyclopropyl-4-methylbenzenesulfonamide $3 q$ ( $21.1 \mathrm{mg}, 0.100 \mathrm{mmol}, 1.0$ equiv.), $\mathrm{N}$ iodosuccinimide ( $23.6 \mathrm{mg}, 0.105 \mathrm{mmol}, 1.05$ equiv.) and methanol ( $4.8 \mu \mathrm{L}, 0.12 \mathrm{mmol}, 1.2$ equiv.) were dissolved in $0.5 \mathrm{~mL}$ of $\mathrm{CDCl}_{3}(0.20 \mathrm{M})$. After stirring for 20 hours at room temperature, TMSCN (18.8 $\mu \mathrm{L}, 0.150 \mathrm{mmol}, 1.5$ equiv.) was added to the reaction crude and kept stirring at room 
temperature for another 20 hours. N-(1-Cyano-3-iodopropyl)-4-methylbenzenesulfonamide 12 (23.5 $\mathrm{mg}, 0.0650 \mathrm{mmol}, 65 \%)$ was obtained as a beige solid after purification by preparative TLC on silica using 4:1 pentanes:ethyl acetate as eluent.

Note: It was later found that $\mathrm{N}$-(1-cyano-3-iodopropyl)-4-methylbenzenesulfonamide $\mathbf{1 2}$ can be synthesized simply by replacing methanol with TMSCN. Starting from N-cyclopropyl-4methylbenzenesulfonamide 3a $(84.4 \mathrm{mg}, 0.400 \mathrm{mmol}, 1.0$ equiv.), NIS (108 mg, $0.480 \mathrm{mmol}, 1.2$ equiv.) and TMSCN (75.1 $\mu \mathrm{L}, 0.600 \mathrm{mmol}, 1.5$ equiv.) were dissolved in $2.0 \mathrm{~mL}$ of $\mathrm{CHCl}_{3}(0.20 \mathrm{M})$. After stirring at room temperature for 24 hours, N-(1-cyano-3-iodopropyl)-4methylbenzenesulfonamide 12 (109 mg, $0.300 \mathrm{mmol}, 75 \%$ ) was obtained as a beige solid after purification by column chromatography on silica using 3:1 pentanes:ethyl acetate as eluent.

$\mathbf{R}_{\mathbf{f}}: 0.25$ (silica, pentanes:ethyl acetate 4:1);

Mp: $132-133^{\circ} \mathrm{C}$;

${ }^{1} \mathrm{H}$ NMR (400 MHz, CDCl 3$): \delta=7.80(\mathrm{~d}, J=8.4 \mathrm{~Hz}, 2 \mathrm{H}, \operatorname{ArH}), 7.38(\mathrm{~d}, J=8.0 \mathrm{~Hz}, 2 \mathrm{H}, \operatorname{ArH}), 5.49(\mathrm{~d}, J=9.8$ $\mathrm{Hz}, 1 \mathrm{H}, \mathrm{NH}), 4.40(\mathrm{dt}, J=9.9,7.4 \mathrm{~Hz}, 1 \mathrm{H}, \mathrm{CH}), 3.21\left(\mathrm{t}, J=6.7 \mathrm{~Hz}, 2 \mathrm{H}, \mathrm{CH}_{2} \mathrm{l}\right), 2.45\left(\mathrm{~s}, 3 \mathrm{H}, \mathrm{CH}_{3}\right), 2.29(\mathrm{q}, J=$ $\left.6.9 \mathrm{~Hz}, 2 \mathrm{H}, \mathrm{CH}_{2} \mathrm{CH}_{2} \mathrm{l}\right)$;

${ }^{13} \mathrm{C}$ NMR $\left(101 \mathrm{MHz}, \mathrm{CDCl}_{3}\right): \delta=144.9,135.6,130.2,127.3,116.6,45.1,37.2,21.7,-2.5$; IR (film): $\tilde{v}=3267$ (w), 1436 (w), 1337 (m), 1164 (s), 1090 (m), 936 (w), 819 (w), 757 (m);

HRMS (ESI) calcd. for $\mathrm{C}_{11} \mathrm{H}_{13} \mathrm{IN}_{2} \mathrm{NaO}_{2} \mathrm{~S}^{+}[\mathrm{M}+\mathrm{Na}]^{+} 386.9635$; Found 386.9633 .

N-(1-(1-Hydroxynaphthalen-2-yl)-3-iodopropyl)-4-methoxybenzamide (13).<smiles>COc1ccc(C(=O)NC2CC2)cc1</smiles><smiles>COc1ccc([N+](=O)[O-])cc1</smiles><smiles>COc1ccc(C(=O)NC(CCI)c2ccc3ccccc3c2O)cc1</smiles>

In a glass vial, $\mathrm{N}$-cyclopropyl-4-methoxybenzamide $3 a(57.3 \mathrm{mg}, 0.300 \mathrm{mmol}, 1.0$ equiv.), diphenyl phosphate ( $7.5 \mathrm{mg}, 0.030 \mathrm{mmol}, 0.1$ equiv.), $\mathrm{N}$-iodosuccinimide ( $70.8 \mathrm{mg}, 0.315 \mathrm{mmol}, 1.05$ equiv.) and methanol (14.4 $\mu \mathrm{L}, 0.360 \mathrm{mmol}, 1.2$ equiv.) were dissolved in $1.5 \mathrm{~mL}$ of $\mathrm{CHCl}_{3}(0.20 \mathrm{M})$. The reaction mixture was stirred at room temperature for 30 minutes. 1-Naphthol $(47.2 \mathrm{mg}, 0.360 \mathrm{mmol}$, 1.2 equiv.) and TFA ( $23.0 \mu \mathrm{L}, 0.300 \mathrm{mmol}, 1.0$ equiv.) were added and the reaction mixture was stirred at room temperature for another 1 hour. After the completion of the reaction, the crude product was directly submitted to column chromatography on silica using 2:1 pentanes: ethyl acetate as eluent. $\mathrm{N}$-(3-lodo-1-(3-methyl-1H-indol-2-yl)propyl)-4-methoxybenzamide 13 (73.0 mg, 0.159 $\mathrm{mmol}, 53 \%)$ was obtained as a yellow oil.

$\mathbf{R}_{\mathbf{f}}: 0.44$ (silica, pentanes:ethyl acetate 2:1);

${ }^{1} \mathrm{H}$ NMR $\left(400 \mathrm{MHz}\right.$, Acetone- $\left.d_{6}\right): \delta=10.64(\mathrm{~s}, 1 \mathrm{H}, \mathrm{OH}), 8.61(\mathrm{~d}, J=8.1 \mathrm{~Hz}, 1 \mathrm{H}, \mathrm{NH}), 8.43-8.25(\mathrm{~m}, 1 \mathrm{H}$, $\operatorname{ArH}), 7.92(\mathrm{~d}, J=8.9 \mathrm{~Hz}, 2 \mathrm{H}, \operatorname{ArH}), 7.82-7.72(\mathrm{~m}, 1 \mathrm{H}, \operatorname{ArH}), 7.50-7.36(\mathrm{~m}, 4 \mathrm{H}, \operatorname{ArH}), 6.98(\mathrm{~d}, J=8.9$ $\mathrm{Hz}, 2 \mathrm{H}, \mathrm{ArH}), 5.65(\mathrm{td}, J=8.5,6.3 \mathrm{~Hz}, 1 \mathrm{H}, \mathrm{CH}), 3.83\left(\mathrm{~s}, 3 \mathrm{H}, \mathrm{OCH}_{3}\right), 3.51-3.29\left(\mathrm{~m}, 2 \mathrm{H}, \mathrm{CH}_{2} \mathrm{l}\right), 2.98-2.86$ ( $\left.m, 1 \mathrm{H}, \mathrm{CH}_{2} \mathrm{CH}_{2} \mathrm{l}\right), 2.64$ (dq, J = 14.1, 7.0 Hz, $1 \mathrm{H}, \mathrm{CH}_{2} \mathrm{CH}_{2} \mathrm{l}$ ); 
${ }^{13} \mathrm{C}$ NMR $\left(101 \mathrm{MHz}\right.$, Acetone- $\left.d_{6}\right): \delta=169.7,163.7,152.5,135.2,130.4,128.1,127.4,127.2,126.0$, $125.9,124.8,123.9,121.9,120.5,114.5,55.8,50.0,37.5,3.2$;

IR (film): $\tilde{v}=3253$ (brs), 3055 (w), 1695 (w), 1605 (s), 1500 (s), 1257 (s), 1178 (s), 1028 (m), 843 (w);

HRMS (ESI) calcd. for $\mathrm{C}_{21} \mathrm{H}_{21} \mathrm{INO}_{3}{ }^{+}[\mathrm{M}+\mathrm{H}]^{+} 462.0561$; Found 462.0557 .

N-(3-lodo-1-(3-methyl-1H-indol-2-yl)propyl)-4-methoxybenzamide (14).<smiles>COc1ccc(C(=O)NC2CC2)cc1</smiles>

$$
\underset{\text { then TFA, 3-Methylindole }}{\stackrel{\mathrm{NIS}, \mathrm{MeOH},(\mathrm{PhO})_{2} \mathrm{PO}_{2} \mathrm{H}, \mathrm{CHCl}_{3}}{\longrightarrow}}
$$

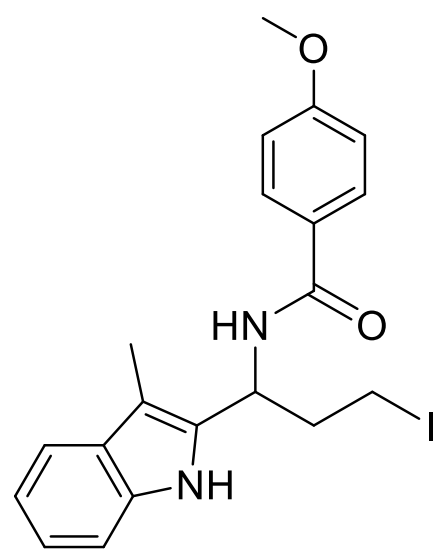

In a glass vial, N-cyclopropyl-4-methoxybenzamide $3 a(57.3 \mathrm{mg}, 0.300 \mathrm{mmol}, 1.0$ equiv.), diphenyl phosphate ( $7.5 \mathrm{mg}, 0.030 \mathrm{mmol}, 0.1$ equiv.), $\mathrm{N}$-iodosuccinimide ( $70.8 \mathrm{mg}, 0.315 \mathrm{mmol}, 1.05$ equiv.) and methanol (14.4 $\mu \mathrm{L}, 0.360 \mathrm{mmol}, 1.2$ equiv.) were dissolved in $1.5 \mathrm{~mL}$ of $\mathrm{CHCl}_{3}(0.20 \mathrm{M})$. The reaction mixture was stirred at room temperature for 30 minutes. 3-Methylindole $(47.2 \mathrm{mg}, 0.360$ mmol, 1.2 equiv.) and TFA ( $23.0 \mu \mathrm{L}, 0.300 \mathrm{mmol}, 1.0$ equiv.) were added and the reaction mixture was stirred at room temperature for another 20 minutes. After the completion of the reaction, the crude product was directly submitted to column chromatography on silica using 2:1 pentanes: ethyl acetate as eluent. N-(3-lodo-1-(3-methyl-1H-indol-2-yl)propyl)-4-methoxybenzamide 14 (110 mg, $0.246 \mathrm{mmol}, 82 \%$ ) was obtained as a dark yellow solid.

$\mathbf{R}_{\mathbf{f}}: 0.33$ (silica, pentanes:ethyl acetate 2:1);

Mp: $83-86^{\circ} \mathrm{C}$;

${ }^{1} \mathrm{H}$ NMR (400 MHz, Acetone- $\left.d_{6}\right): \delta=9.99(\mathrm{~s}, 1 \mathrm{H}$, indole- $\mathrm{NH}), 8.04(\mathrm{~d}, J=7.6 \mathrm{~Hz}, 1 \mathrm{H}, \mathrm{NH}), 7.91-7.83$ $(\mathrm{m}, 2 \mathrm{H}, \operatorname{ArH}), 7.47(\mathrm{~d}, J=7.8 \mathrm{~Hz}, 1 \mathrm{H}, \operatorname{ArH}), 7.30(\mathrm{~d}, J=8.0 \mathrm{~Hz}, 1 \mathrm{H}, \operatorname{ArH}), 7.08-7.03(\mathrm{~m}, 1 \mathrm{H}, \operatorname{ArH}), 7.02-$ $6.97(\mathrm{~m}, 1 \mathrm{H}, \mathrm{ArH}), 6.96-6.89(\mathrm{~m}, 2 \mathrm{H}, \mathrm{ArH}), 5.63(\mathrm{q}, J=7.7 \mathrm{~Hz}, 1 \mathrm{H}, \mathrm{CH}), 3.81(\mathrm{~s}, 3 \mathrm{H}, \mathrm{OCH})_{3}, 3.30$ (ddd, J $\left.=9.8,7.6,6.5 \mathrm{~Hz}, 1 \mathrm{H}, \mathrm{CH}_{2} \mathrm{l}\right), 3.21\left(\mathrm{dt}, J=9.7,7.2 \mathrm{~Hz}, 1 \mathrm{H}, \mathrm{CH}_{2} \mathrm{l}\right), 2.65(\mathrm{ddt}, J=26.6,14.3,6.9 \mathrm{~Hz}, 2 \mathrm{H}$, $\left.\mathrm{CH}_{2} \mathrm{CH}_{2} \mathrm{I}\right), 2.39\left(\mathrm{~s}, 3 \mathrm{H}, \mathrm{CH}_{3}\right)$;

${ }^{13} \mathrm{C}$ NMR $\left(101 \mathrm{MHz}\right.$, Acetone- $\left.d_{6}\right): \delta=166.7,163.1,136.9,135.0,130.0,129.8,127.7,122.2,119.5$, $119.2,114.3,111.7,108.5,55.8,48.0,39.5,9.0,2.9$;

IR (film): $\tilde{v}=3303$ (m), 2959 (w), 2924 (m), 2854 (w), 1606 (s), $1539(\mathrm{~m}), 1504$ (s), $1460(\mathrm{~m}), 1302(\mathrm{~m})$, $1256(\mathrm{~s}), 1177(\mathrm{~m}), 1130(\mathrm{~m}), 907(\mathrm{~m}), 842(\mathrm{~m})$;

HRMS (ESI) calcd. for $\mathrm{C}_{20} \mathrm{H}_{21} \mathrm{IN}_{2} \mathrm{NaO}_{2}^{+}[\mathrm{M}+\mathrm{Na}]^{+}$471.0540; Found 471.0546.

N-(3-lodopropyl)-4-methoxybenzamide (15). 
<smiles>COc1ccc(C(=O)NC2CC2)cc1</smiles>

NIS, MeOH, Acetic acid

then $\mathrm{NaBH}_{3} \mathrm{CN}$<smiles>COc1ccc(C(=O)NCCCI)cc1</smiles>

In a glass vial, $\mathrm{N}$-cyclopropyl-4-methoxybenzamide $3 a(57.3 \mathrm{mg}, 0.300 \mathrm{mmol}, 1.0$ equiv.), $\mathrm{N}$ iodosuccinimide ( $70.8 \mathrm{mg}, 0.315 \mathrm{mmol}, 1.05$ equiv.) and methanol ( $14.4 \mu \mathrm{L}, 0.360 \mathrm{mmol}, 1.2$ equiv.) were dissolved in $1.5 \mathrm{~mL}$ of acetic acid $(0.20 \mathrm{M})$. The reaction mixture was stirred at room temperature for 30 minutes. Sodium cyanoborohydride $(28.3 \mathrm{mg}, 0.450 \mathrm{mmol}, 1.5$ equiv.) was added and the reaction mixture was stirred at room temperature for another 30 minutes. After the completion of the reaction, the crude product was directly submitted to column chromatography on silica using 1:1 pentanes:ethyl acetate as eluent. N-(3-lodopropyl)-4-methoxybenzamide $\mathbf{1 5}$ (75.0 mg, $0.235 \mathrm{mmol}, 78 \%$ ) was obtained as a pale yellow solid.

$\mathbf{R}_{\mathbf{f}}: 0.34$ (silica, pentanes:ethyl acetate 1:1);

Mp: $84-86^{\circ} \mathrm{C}$;

${ }^{1} \mathrm{H}$ NMR $\left(400 \mathrm{MHz}, \mathrm{CDCl}_{3}\right): \delta=7.73(\mathrm{~d}, J=8.8 \mathrm{~Hz}, 2 \mathrm{H}, \operatorname{ArH}), 6.92(\mathrm{~d}, J=8.8 \mathrm{~Hz}, 2 \mathrm{H}, \operatorname{ArH}), 6.27(\mathrm{~s}, 1 \mathrm{H}$, $\mathrm{NH}), 3.85\left(\mathrm{~s}, 3 \mathrm{H}, \mathrm{OCH}_{3}\right), 3.54\left(\mathrm{q}, J=6.4 \mathrm{~Hz}, 2 \mathrm{H}, \mathrm{CH}_{2}\right), 3.24\left(\mathrm{t}, J=6.8 \mathrm{~Hz}, 2 \mathrm{H}, \mathrm{CH}_{2}\right), 2.15(\mathrm{p}, J=6.7 \mathrm{~Hz}, 2 \mathrm{H}$, $\mathrm{CH}_{2}$ );

${ }^{13} \mathrm{C}$ NMR $\left(101 \mathrm{MHz}, \mathrm{CDCl}_{3}\right): \delta=167.2,162.2,128.7,126.5,113.8,55.4,40.5,32.9,3.4$;

IR (film): $\tilde{v}=3289(\mathrm{w}), 2932(\mathrm{w}), 1633(\mathrm{~m}), 1606$ (s), $1543(\mathrm{~m}), 1504(\mathrm{~s}), 1299(\mathrm{~m}), 1254(\mathrm{~s}), 1179(\mathrm{~m})$, $1029(w), 844(w)$;

HRMS (APCl) calcd. for $\mathrm{C}_{11} \mathrm{H}_{15} \mathrm{INO}_{2}{ }^{+}[\mathrm{M}+\mathrm{H}]^{+}$320.0142; Found 320.0138 .

3-((tert-Butoxycarbonyl)amino)-3-methoxypropyl benzoate (16).<smiles>CC(C)(C)OC(=O)NC1CC1</smiles><smiles>COc1ccccc1</smiles><smiles>COC(CCOC(=O)c1ccccc1)NC(=O)OC(C)(C)C</smiles>

In a glass vial, tert-butyl cyclopropylcarbamate $30(47.1 \mathrm{mg}, 0.300 \mathrm{mmol}, 1.0$ equiv.), Niodosuccinimide ( $70.8 \mathrm{mg}, 0.315 \mathrm{mmol}, 1.05$ equiv.) and methanol ( $14.4 \mu \mathrm{L}, 0.360 \mathrm{mmol}, 1.2$ equiv.) were dissolved in $1.5 \mathrm{~mL}$ of $\mathrm{MeCN}(0.20 \mathrm{M})$. The reaction mixture was stirred at room temperature for 30 minutes. After the completion of the reaction, $\mathrm{K}_{2} \mathrm{CO}_{3}(82.8 \mathrm{mg}, 0.600 \mathrm{mmol}, 2.0$ equiv.) was added to the reaction mixture and then solvent was removed under reduced pressure. The crude was dissolved in $1.5 \mathrm{~mL}$ of DMF, and benzoic acid ( $73.2 \mathrm{mg}, 0.600 \mathrm{mmol}, 2.0$ equiv.) was added. The resulting mixture was stirred at room temperature for 16 hours and then diluted with brine $(10.0 \mathrm{~mL})$. The aqueous layer was extracted with EtOAc $(10.0 \mathrm{~mL} \times 2)$. The combined organic layers were dried over $\mathrm{Na}_{2} \mathrm{SO}_{4}$ and concentrated under reduced pressure. The crude residue was purified by column 
chromatography on silica using 3:1 pentanes:ethyl acetate as eluent. 3-((tert-Butoxycarbonyl)amino)3-methoxypropyl benzoate $16(75.1 \mathrm{mg}, 0.243 \mathrm{mmol}, 81 \%)$ was obtained as a yellow oil.

$\mathbf{R}_{\mathbf{f}}: 0.33$ (silica, pentanes:ethyl acetate 4:1);

${ }^{1} \mathrm{H}$ NMR $\left(400 \mathrm{MHz}, \mathrm{CDCl}_{3}\right): \delta=8.03(\mathrm{~d}, J=7.4 \mathrm{~Hz}, 2 \mathrm{H}, \operatorname{ArH}), 7.55(\mathrm{t}, J=7.4 \mathrm{~Hz}, 1 \mathrm{H}, \operatorname{ArH}), 7.43(\mathrm{t}, J=7.6$ $\mathrm{Hz}, 2 \mathrm{H}, \mathrm{ArH}), 5.12-4.83(\mathrm{~m}, 2 \mathrm{H}, \mathrm{NH}+\mathrm{CH}), 4.42\left(\mathrm{t}, J=6.2 \mathrm{~Hz}, 2 \mathrm{H}, \mathrm{OCH}_{2}\right), 3.36\left(\mathrm{~s}, 3 \mathrm{H}, \mathrm{OCH}_{3}\right), 2.08-$ $2.04\left(\mathrm{~m}, 2 \mathrm{H}, \mathrm{CH}_{2} \mathrm{CH}_{2} \mathrm{O}\right), 1.43\left(\mathrm{~s}, 9 \mathrm{H}, \mathrm{CH}_{3}\right)$;

${ }^{13} \mathrm{C}$ NMR $\left(101 \mathrm{MHz}, \mathrm{CDCl}_{3}\right): \delta=166.4,155.3,133.0,130.1,129.6,128.4,80.4,79.9,61.1,55.5,34.6$, 28.2;

IR (film): $\tilde{v}=3354$ (w), 2977 (w), 2933 (w), 1716 (s), 1513 (m), 1367 (m), 1274 (s), 1167 (s), 1114 (m), $861(\mathrm{w}), 712(\mathrm{~s})$;

HRMS (APCl) calcd. for $\mathrm{C}_{16} \mathrm{H}_{23} \mathrm{NNaO}_{5}{ }^{+}[\mathrm{M}+\mathrm{Na}]^{+} 332.1468$; Found 332.1469.

tert-Butyl (3-(benzylthio)-1-methoxypropyl)carbamate (17).<smiles>CC(C)(C)OC(=O)NC1CC1</smiles><smiles>COc1ccccc1</smiles><smiles>COC(CCSCc1ccccc1)NC(=O)OC(C)(C)C</smiles>

In a glass vial, tert-butyl cyclopropylcarbamate $30(47.1 \mathrm{mg}, 0.300 \mathrm{mmol}, 1.0$ equiv.), $\mathrm{N}$ iodosuccinimide ( $70.8 \mathrm{mg}, 0.315 \mathrm{mmol}, 1.05$ equiv.) and methanol ( $14.4 \mu \mathrm{L}, 0.360 \mathrm{mmol}, 1.2$ equiv.) were dissolved in $1.5 \mathrm{~mL}$ of $\mathrm{MeCN}(0.20 \mathrm{M})$. The reaction mixture was stirred at room temperature for 30 minutes. After the completion of the reaction, $\mathrm{K}_{2} \mathrm{CO}_{3}(82.8 \mathrm{mg}, 0.600 \mathrm{mmol}, 2.0$ equiv.) was added to the reaction mixture and then solvent was removed under reduced pressure. The crude was dissolved in $1.5 \mathrm{~mL}$ of DMF, and phenylmethanethiol (52.7 $\mu \mathrm{L}, 0.450 \mathrm{mmol}, 1.5$ equiv.) was added. The resulting mixture was stirred at room temperature for 3 hours and then diluted with brine $(10.0 \mathrm{~mL})$. The aqueous layer was extracted with EtOAc $(10.0 \mathrm{~mL} \times 2)$. The combined organic layers were dried over $\mathrm{Na}_{2} \mathrm{SO}_{4}$ and concentrated under reduced pressure. The crude residue was purified by column chromatography on silica using 4:1 pentanes:ethyl acetate as eluent. tert-Butyl (3(benzylthio)-1-methoxypropyl)carbamate $17(74.1 \mathrm{mg}, 0.238 \mathrm{mmol}, 79 \%)$ was obtained as a colorless oil.

$\mathbf{R}_{\mathbf{f}}: 0.37$ (silica, pentanes:ethyl acetate 6:1);

${ }^{1} \mathrm{H}$ NMR $\left(400 \mathrm{MHz}\right.$, Acetonitrile- $\left.d_{3}\right): 7.33-7.26(\mathrm{~m}, 4 \mathrm{H}, \operatorname{ArH}), 7.23(\mathrm{~h}, J=4.3 \mathrm{~Hz}, 1 \mathrm{H}, \operatorname{ArH}), 5.61(\mathrm{~d}, J=$ $10.0 \mathrm{~Hz}, 1 \mathrm{H}, \mathrm{NH}), 4.78(\mathrm{dt}, J=10.2,6.4 \mathrm{~Hz}, 1 \mathrm{H}, \mathrm{CH}), 3.69\left(\mathrm{~s}, 2 \mathrm{H}, \mathrm{PhCH}_{2}\right), 3.19\left(\mathrm{~s}, 3 \mathrm{H}, \mathrm{OCH}_{3}\right), 2.40(\mathrm{t}, J=$ $\left.7.4 \mathrm{~Hz}, 2 \mathrm{H}, \mathrm{SCH}_{2}\right), 1.81\left(\mathrm{dt}, J=14.0,7.0 \mathrm{~Hz}, 1 \mathrm{H}, \mathrm{CH}_{2} \mathrm{CH}_{2} \mathrm{~S}\right), 1.69\left(\mathrm{dq}, J=14.0,7.3 \mathrm{~Hz}, 1 \mathrm{H}, \mathrm{CH}_{2} \mathrm{CH}_{2} \mathrm{~S}\right), 1.40$ $\left(\mathrm{s}, 9 \mathrm{H}, \mathrm{CH}_{3}\right)$;

${ }^{13} \mathrm{C}$ NMR $\left(101 \mathrm{MHz}\right.$, Acetonitrile- $\left.d_{3}\right): \delta=156.5,139.9,129.8,129.4,127.8,82.7,79.7,55.2,36.5,35.5$, 28.5, 27.5;

IR (film): $\tilde{v}=3337$ (w), 2977 (w), 2930 (w), 1703 (s), 1495 (s), 1453 (m), 1366 (s), 1248 (m), 1163 (s), $1047(\mathrm{~m}), 700(\mathrm{~m})$;

HRMS (ESI) calcd. for $\mathrm{C}_{16} \mathrm{H}_{25} \mathrm{NNaO}_{3} \mathrm{~S}^{+}[\mathrm{M}+\mathrm{Na}]^{+} 334.1447$; Found 334.1455.

tert-Butyl (1-methoxy-3-(phenylamino)propyl)carbamate (18). 

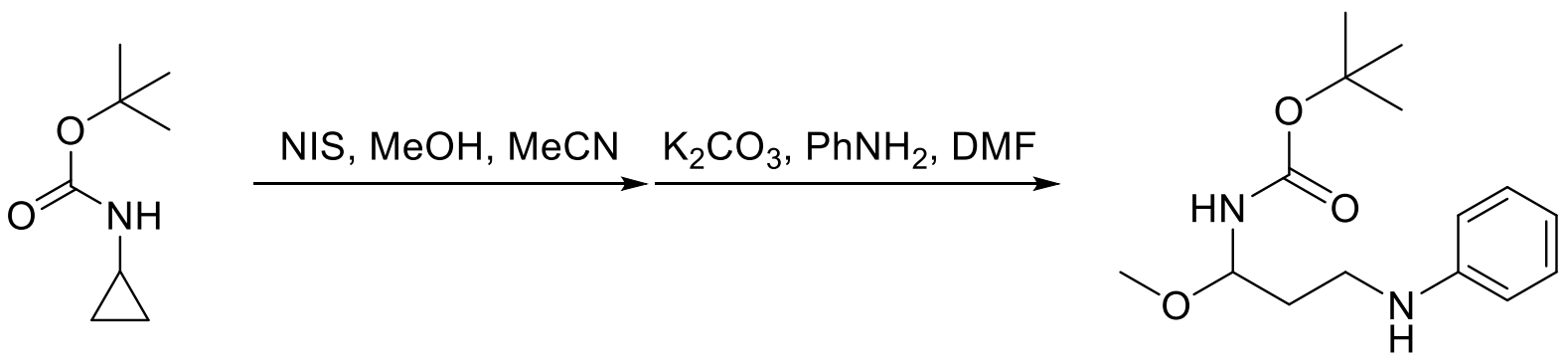

In a glass vial, tert-butyl cyclopropylcarbamate $30(47.1 \mathrm{mg}, 0.300 \mathrm{mmol}, 1.0$ equiv.), Niodosuccinimide ( $70.8 \mathrm{mg}, 0.315 \mathrm{mmol}, 1.05$ equiv.) and methanol ( $14.4 \mu \mathrm{L}, 0.360 \mathrm{mmol}, 1.2$ equiv.) were dissolved in $1.5 \mathrm{~mL}$ of $\mathrm{MeCN}(0.20 \mathrm{M})$. The reaction mixture was stirred at room temperature for 30 minutes. After the completion of the reaction, $\mathrm{K}_{2} \mathrm{CO}_{3}(41.4 \mathrm{mg}, 0.300 \mathrm{mmol}, 1.0$ equiv.) was added to the mixture before purification by column chromatography on silica using $4: 1$ pentanes:ethyl acetate as eluent. The obtained tert-Butyl (3-iodo-1-methoxypropyl)carbamate 40 was then dissolved in $1.5 \mathrm{~mL}$ of DMF, $\mathrm{K}_{2} \mathrm{CO}_{3}(82.8 \mathrm{mg}, 0.600 \mathrm{mmol}, 2.0$ equiv.) and aniline $(54.8 \mu \mathrm{L}$, $0.600 \mathrm{mmol}, 2.0$ equiv.) were added. The resulting mixture was stirred at room temperature for 16 hours and then diluted with brine $(10.0 \mathrm{~mL})$. The aqueous layer was extracted with EtOAc $(10.0 \mathrm{~mL} x$ 2). The combined organic layers were dried over $\mathrm{Na}_{2} \mathrm{SO}_{4}$ and concentrated under reduced pressure. The crude residue was purified by column chromatography on silica using 3:1 pentanes:ethyl acetate as eluent. tert-Butyl (1-methoxy-3-(phenylamino)propyl)carbamate 18 (72.1 mg, 0.258 mmol, 86\%) was obtained as a pale yellow oil.

$\mathbf{R}_{\mathbf{f}}: 0.45$ (silica, pentanes:ethyl acetate $3: 1$ );

${ }^{1} \mathrm{H}$ NMR $\left(400 \mathrm{MHz}, \mathrm{CDCl}_{3}\right): \delta=7.18(\mathrm{t}, J=7.8 \mathrm{~Hz}, 2 \mathrm{H}, \mathrm{ArH}), 6.70$ (t, $\left.J=7.3 \mathrm{~Hz}, 1 \mathrm{H}, \operatorname{ArH}\right), 6.62$ (d, $J=8.0$ $\mathrm{Hz}, 2 \mathrm{H}, \mathrm{ArH}), 5.06-4.80(\mathrm{~m}, 2 \mathrm{H}, \mathrm{NH}+\mathrm{CH}), 3.94(\mathrm{~s}, 1 \mathrm{H}, \mathrm{NH}), 3.36\left(\mathrm{~s}, 3 \mathrm{H}, \mathrm{OCH}_{3}\right), 3.25(\mathrm{td}, J=6.5,3.4$ $\left.\mathrm{Hz}, 2 \mathrm{H}, \mathrm{NCH}_{2}\right), 1.99-1.82\left(\mathrm{~m}, 2 \mathrm{H}, \mathrm{CH}_{2} \mathrm{CH}_{2} \mathrm{~N}\right), 1.46\left(\mathrm{~s}, 9 \mathrm{H}, \mathrm{CH}_{3}\right)$;

${ }^{13} \mathrm{C} \mathrm{NMR}\left(101 \mathrm{MHz}, \mathrm{CDCl}_{3}\right): \delta=155.4,148.1,129.2,117.4,112.9,81.8,80.0,55.5,40.1,35.1,28.3$;

IR (film): $\tilde{v}=3377$ (w), 2977 (w), 2932 (w), 2832 (w), 1699 (s), 1603 (s), 1504 (s), 1366 (m), 1251 (m), $1168(\mathrm{~s}), 1080(\mathrm{~m}), 749(\mathrm{~m})$;

HRMS (APCl) calcd. for $\mathrm{C}_{15} \mathrm{H}_{24} \mathrm{~N}_{2} \mathrm{NaO}_{3}{ }^{+}[\mathrm{M}+\mathrm{Na}]^{+}$303.1679; Found 303.1677.

Dimethyl 2-(3-((tert-butoxycarbonyl)amino)-3-methoxypropyl)malonate (19).

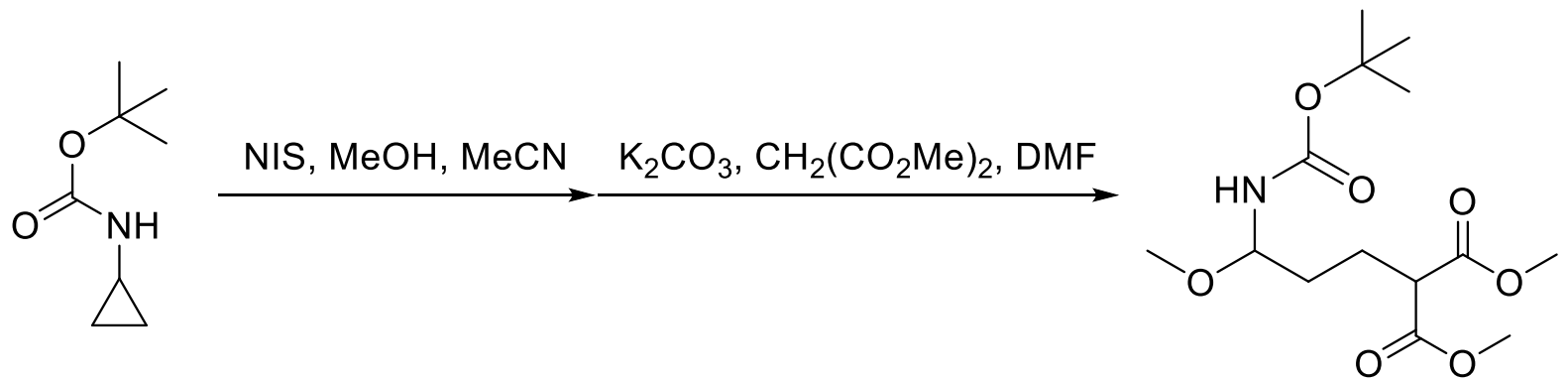

In a glass vial, tert-butyl cyclopropylcarbamate $30(47.1 \mathrm{mg}, 0.300 \mathrm{mmol}, 1.0$ equiv.), Niodosuccinimide ( $70.8 \mathrm{mg}, 0.315 \mathrm{mmol}, 1.05$ equiv.) and methanol (14.4 $\mu \mathrm{L}, 0.360 \mathrm{mmol}, 1.2$ equiv.) were dissolved in $1.5 \mathrm{~mL}$ of $\mathrm{MeCN}(0.20 \mathrm{M})$. The reaction mixture was stirred at room temperature for 30 minutes. After the completion of the reaction, $\mathrm{K}_{2} \mathrm{CO}_{3}(41.4 \mathrm{mg}, 0.300 \mathrm{mmol}, 1.0$ equiv.) was added to the mixture before purification by column chromatography on silica using $4: 1$ pentanes:ethyl acetate as eluent. The obtained tert-butyl (3-iodo-1-methoxypropyl)carbamate 40 was then dissolved in $1.5 \mathrm{~mL}$ of DMF, $\mathrm{K}_{2} \mathrm{CO}_{3}(82.8 \mathrm{mg}, 0.600 \mathrm{mmol}, 2.0$ equiv.) and dimethyl malonate ( $70.0 \mu \mathrm{L}, 0.600 \mathrm{mmol}, 2.0$ equiv.) were added. The resulting mixture was stirred at room temperature 
for 16 hours and then diluted with brine $(10.0 \mathrm{~mL})$. The aqueous layer was extracted with EtOAc (10.0 $\mathrm{mL} \times 2$ ). The combined organic layers were dried over $\mathrm{Na}_{2} \mathrm{SO}_{4}$ and concentrated under reduced pressure. The crude residue was purified by column chromatography on silica using 3:1 pentanes:ethyl acetate as eluent. Dimethyl 2-(3-((tert-butoxycarbonyl)amino)-3methoxypropyl)malonate $19(76.6 \mathrm{mg}, 0.240 \mathrm{mmol}, 80 \%)$ was obtained as a pale yellow oil.

$\mathbf{R}_{\mathbf{f}}: 0.35$ (silica, pentanes:ethyl acetate $\left.3: 1\right) \mathrm{KMnO}_{4}$;

${ }^{1} \mathrm{H}$ NMR $\left(400 \mathrm{MHz}\right.$, Acetone- $\left.d_{6}\right): \delta=6.33(\mathrm{~d}, J=9.9 \mathrm{~Hz}, 1 \mathrm{H}, \mathrm{NH}), 4.78(\mathrm{dt}, J=10.0,6.4 \mathrm{~Hz}, 1 \mathrm{H}, \mathrm{CH})$, $3.69\left(\mathrm{~s}, 6 \mathrm{H}, \mathrm{CO}_{2} \mathrm{CH}_{3}\right), 3.45(\mathrm{t}, J=7.5 \mathrm{~Hz}, 1 \mathrm{H}, \mathrm{CH}), 3.25(\mathrm{~s}, 3 \mathrm{H}, \mathrm{OCH}), 1.97-1.85\left(\mathrm{~m}, 2 \mathrm{H}, \mathrm{CH}_{2}\right), 1.75-$ $1.65\left(\mathrm{~m}, 1 \mathrm{H}, \mathrm{CH}_{2}\right), 1.62-1.54(\mathrm{~m}, 1 \mathrm{H}, \mathrm{CH}), 1.42\left(\mathrm{~s}, 9 \mathrm{H}, \mathrm{CH}_{3}\right)$;

${ }^{13} \mathrm{C}$ NMR (101 MHz, Acetone- $\left.d_{6}\right): \delta=170.3,170.2,156.5,83.3,79.1,55.0,52.6,51.7,33.3,28.5,25.5$; IR (film): $\tilde{v}=3367$ (w), 2977 (w), 2955 (w), 2833 (w), 1733 (s), 1717 (s), $1511(\mathrm{~m}), 1437(\mathrm{~m}), 1366(\mathrm{~m})$, $1245(\mathrm{~m}), 1162(\mathrm{~m}), 1110(\mathrm{~m}), 1048(\mathrm{~m}), 863(\mathrm{w})$;

HRMS (ESI) calcd. for $\mathrm{C}_{14} \mathrm{H}_{25} \mathrm{NNaO}_{7}^{+}[\mathrm{M}+\mathrm{Na}]^{+} 342.1523$; Found 342.1530 .

tert-Butyl (3-cyano-1-methoxypropyl)carbamate (20).<smiles>CC(C)(C)OC(=O)NC1CC1</smiles>

$$
\stackrel{\mathrm{NIS}, \mathrm{MeOH}, \mathrm{MeCN}}{\longrightarrow} \mathrm{K}_{2} \mathrm{CO}_{3}, \mathrm{NaCN}, \mathrm{DMF}
$$<smiles>COC(CCC#N)NC(=O)OC(C)(C)C</smiles>

In a glass vial, tert-butyl cyclopropylcarbamate $30(47.1 \mathrm{mg}, 0.300 \mathrm{mmol}, 1.0$ equiv.), $\mathrm{N}$ iodosuccinimide ( $70.8 \mathrm{mg}, 0.315 \mathrm{mmol}, 1.05$ equiv.) and methanol ( $14.4 \mu \mathrm{L}, 0.360 \mathrm{mmol}, 1.2$ equiv.) were dissolved in $1.5 \mathrm{~mL}$ of $\mathrm{MeCN}(0.20 \mathrm{M})$. The reaction mixture was stirred at room temperature for 30 minutes. After the completion of the reaction, $\mathrm{K}_{2} \mathrm{CO}_{3}(41.4 \mathrm{mg}, 0.300 \mathrm{mmol}, 1.0$ equiv.) was added to the mixture before purification by column chromatography on silica using $4: 1$ pentanes:ethyl acetate as eluent. The obtained tert-butyl (3-iodo-1-methoxypropyl)carbamate 40 was then dissolved in $1.5 \mathrm{~mL}$ of DMF. $\mathrm{K}_{2} \mathrm{CO}_{3}(82.8 \mathrm{mg}, 0.600 \mathrm{mmol}, 2.0$ equiv.) and $\mathrm{NaCN}(29.4 \mathrm{mg}$, $0.600 \mathrm{mmol}, 2.0$ equiv.) were added. The resulting mixture was stirred at room temperature for 2 hours and then diluted with EtOAc $(10.0 \mathrm{~mL})$ and extracted with brine $(10.0 \mathrm{~mL} \times 6)$. The combined organic layers were dried over $\mathrm{Na}_{2} \mathrm{SO}_{4}$ and concentrated under reduced pressure. tert-Butyl (3cyano-1-methoxypropyl)carbamate 20 (60.4 mg, $0.282 \mathrm{mmol}, 94 \%)$ was obtained as a colorless oil without any further purification.

$\mathbf{R}_{\mathbf{f}}: 0.48$ (silica, pentanes:ethyl acetate 2:1) $\mathrm{KMnO}_{4}$;

Mp: $49-52{ }^{\circ} \mathrm{C}$;

${ }^{1} \mathrm{H}$ NMR $\left(400 \mathrm{MHz}, \mathrm{CDCl}_{3}\right): \delta=4.90(\mathrm{dt}, J=12.6,6.1 \mathrm{~Hz}, 1 \mathrm{H}, \mathrm{CH}), 4.80(\mathrm{~d}, J=10.5 \mathrm{~Hz}, 1 \mathrm{H}, \mathrm{NH}), 3.35(\mathrm{~s}$, $3 \mathrm{H}, \mathrm{OCH}_{3}$ ), 2.44 (td, J = 7.4, $4.6 \mathrm{~Hz}, 2 \mathrm{H}, \mathrm{CH}_{2} \mathrm{CN}$ ), 1.93 (q, J = 7.1 Hz, 2H, $\mathrm{CH}_{2} \mathrm{CH}_{2} \mathrm{CN}$ ), $1.46\left(\mathrm{~s}, 9 \mathrm{H}, \mathrm{CH}_{3}\right.$ );

${ }^{13} \mathrm{C}$ NMR $\left(101 \mathrm{MHz}, \mathrm{CDCl}_{3}\right): \delta=155.1,119.0,81.3,80.3,55.6,31.4,28.2,13.3$;

IR (film): $\tilde{v}=3341$ (w), 2979 (w), 2936 (w), 2834 (w), 2247 (w), 1700 (s), 1515 (s), 1367 (s), 1214 (m), $1169(\mathrm{~s}), 1050(\mathrm{~s}), 892(\mathrm{w})$;

HRMS (APCl) calcd. for $\mathrm{C}_{10} \mathrm{H}_{18} \mathrm{~N}_{2} \mathrm{NaO}_{3}{ }^{+}[\mathrm{M}+\mathrm{Na}]^{+}$237.1210; Found 237.1214.

tert-Butyl 2-methoxyazetidine-1-carboxylate (21). 

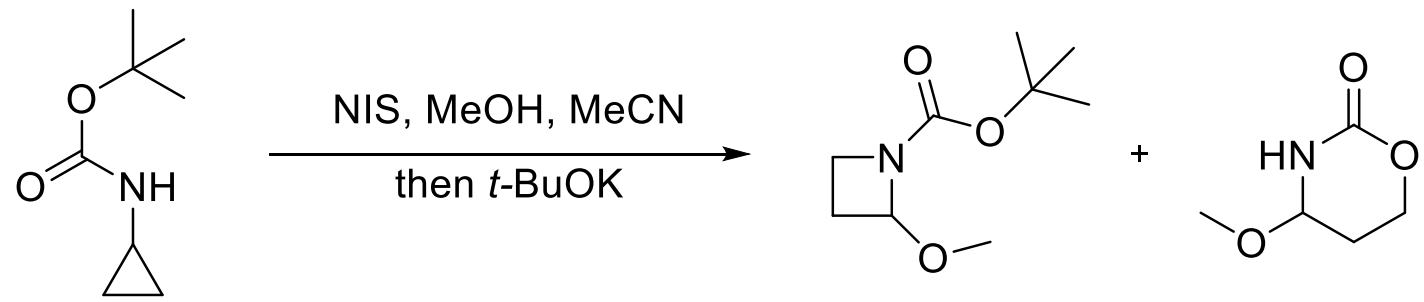

In a glass vial, tert-butyl cyclopropylcarbamate $30(47.1 \mathrm{mg}, 0.300 \mathrm{mmol}, 1.0$ equiv.), Niodosuccinimide ( $70.8 \mathrm{mg}, 0.315 \mathrm{mmol}, 1.05$ equiv.) and methanol (14.4 $\mu \mathrm{L}, 0.360 \mathrm{mmol}, 1.2$ equiv.) were dissolved in $1.5 \mathrm{~mL}$ of $\mathrm{MeCN}(0.20 \mathrm{M})$. The reaction mixture was stirred at room temperature for 30 minutes. After the completion of the reaction, $t$-BuOK (101 mg, $0.900 \mathrm{mmol}, 3.0$ equiv.) was added. The resulting mixture was stirred at room temperature for 16 hours and then diluted with brine $(10.0 \mathrm{~mL})$. The aqueous layer was extracted with $\mathrm{DCM}(10.0 \mathrm{~mL} \times 2)$. The combined organic layers were dried over $\mathrm{Na}_{2} \mathrm{SO}_{4}$ and concentrated under reduced pressure. The crude product was purified by column chromatography on basic aluminium oxide. tert-Butyl 2-methoxyazetidine-1carboxylate 21 (14.1 mg, $0.075 \mathrm{mmol}, 25 \%)$ was obtained as a pale yellow oil by using dichloromethane as eluent. 4-Methoxy-1,3-oxazinan-2-one $21^{\prime}$ (11.8 mg, $\left.0.090 \mathrm{mmol}, 30 \%\right)$ was obtained as a yellow oil by using 20:1 dichloromethane:methanol as eluent.

tert-Butyl 2-methoxyazetidine-1-carboxylate (21).

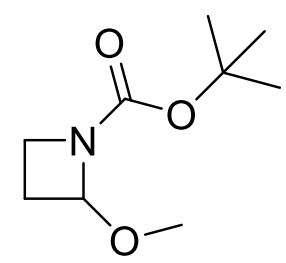

$\mathbf{R}_{\mathbf{f}}: 0.50$ (silica, pentanes:ethyl acetate $6: 1$ );

${ }^{1} \mathrm{H}$ NMR $\left(400 \mathrm{MHz}, \mathrm{CDCl}_{3}\right): \delta=5.17(\mathrm{dd}, J=6.8,4.0 \mathrm{~Hz}, 1 \mathrm{H}, \mathrm{CH}), 3.74\left(\mathrm{dt}, J=8.7,4.3 \mathrm{~Hz}, 1 \mathrm{H}, \mathrm{NCH}_{2}\right)$, $3.64\left(\mathrm{td}, J=8.5,6.2 \mathrm{~Hz}, 1 \mathrm{H}, \mathrm{NCH}_{2}\right.$ ), $3.49\left(\mathrm{~s}, 3 \mathrm{H}, \mathrm{OCH}_{3}\right), 2.42$ (dddd, $J=12.2,8.9,6.8,5.4 \mathrm{~Hz}, 1 \mathrm{H}, \mathrm{CH}_{2}$ ), $2.17-2.08\left(\mathrm{~m}, 1 \mathrm{H}, \mathrm{CH}_{2}\right), 1.46\left(\mathrm{~s}, 9 \mathrm{H}, \mathrm{CH}_{3}\right)$;

${ }^{13} \mathrm{C}$ NMR $\left(101 \mathrm{MHz}, \mathrm{CDCl}_{3}\right): \delta=156.4,91.2,80.0,56.2,43.5,28.3,24.8$;

IR (film): $\tilde{v}=$ 2974(w), 2931 (w), 2900 (w), 1702 (s), 1456 (w), 1381 (m), 1349 (m), 1257 (w), $1154(\mathrm{~m})$, $1092(\mathrm{~s}), 999(\mathrm{w}), 925(\mathrm{w}), 853(\mathrm{w})$;

HRMS (ESI) calcd. for $\mathrm{C}_{9} \mathrm{H}_{17} \mathrm{NNaO}_{3}{ }^{+}[\mathrm{M}+\mathrm{Na}]^{+} 210.1101$; Found 210.1107 .

4-Methoxy-1,3-oxazinan-2-one (21')<smiles>COC1CCOC(=O)N1</smiles>

$\mathbf{R}_{\mathbf{f}}: 0.37$ (silica, dichloromethane:methanol 20:1);

${ }^{1} \mathrm{H}$ NMR $\left(400 \mathrm{MHz}, \mathrm{CDCl}_{3}\right): \delta=7.70(\mathrm{~s}, 1 \mathrm{H}, \mathrm{NH}), 4.57$ (q, $\left.J=3.6 \mathrm{~Hz}, 1 \mathrm{H}, \mathrm{CH}\right), 4.49$ (ddd, $J=12.6,10.9$, $\left.2.9 \mathrm{~Hz}, 1 \mathrm{H}, \mathrm{OCH}_{2}\right), 4.26$ (dddd, $\left.J=10.9,4.8,2.5,1.1 \mathrm{~Hz}, 1 \mathrm{H}, \mathrm{OCH}_{2}\right), 3.36\left(\mathrm{~s}, 3 \mathrm{H}, \mathrm{OCH}_{3}\right), 2.05-1.99(\mathrm{~m}$, $\left.1 \mathrm{H}, \mathrm{CH}_{2}\right), 1.97-1.90\left(\mathrm{~m}, 1 \mathrm{H}, \mathrm{CH}_{2}\right)$;

${ }^{13} \mathrm{C}$ NMR $\left(101 \mathrm{MHz}, \mathrm{CDCl}_{3}\right): \delta=153.8,79.8,63.1,54.6,26.9$;

IR (film): $\tilde{v}=3269$ (w), 2939 (w), 2182 (w), 1698 (s), 1284 (s) 1087 (s);

HRMS (ESI) calcd. for $\mathrm{C}_{5} \mathrm{H}_{10} \mathrm{NO}_{3}{ }^{+}[\mathrm{M}+\mathrm{H}]^{+} 132.0655$; Found 132.0651 .

$\mathrm{N}$-(3-((4-Fluorophenyl)thio)-1-(3-methyl-1H-indol-2-yl)propyl)-4-methoxybenzamide (22). 

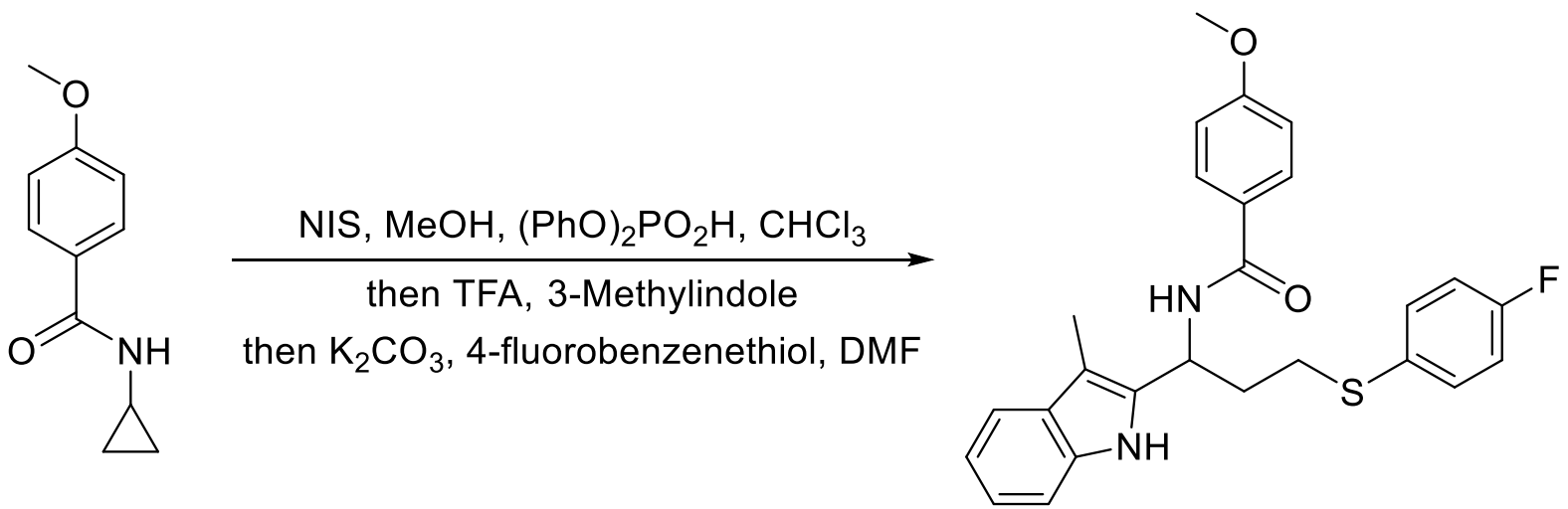

In a glass vial, N-cyclopropyl-4-methoxybenzamide 3 a $(57.3 \mathrm{mg}, 0.300 \mathrm{mmol}, 1.0$ equiv.), diphenyl phosphate ( $7.5 \mathrm{mg}, 0.030 \mathrm{mmol}, 0.1$ equiv.), $\mathrm{N}$-iodosuccinimide (70.8 mg, $0.315 \mathrm{mmol}, 1.05$ equiv.) and methanol (14.4 $\mu \mathrm{L}, 0.360 \mathrm{mmol}, 1.2$ equiv.) were dissolved in $1.5 \mathrm{~mL}$ of $\mathrm{CHCl}_{3}(0.20 \mathrm{M})$. The reaction mixture was stirred at room temperature for 30 minutes. 3-Methylindole $(47.2 \mathrm{mg}, 0.360$ mmol, 1.2 equiv.) and TFA ( $23.0 \mu \mathrm{L}, 0.300 \mathrm{mmol}, 1.0$ equiv.) were added to the reaction mixture and the reaction mixture was stirred at room temperature for another 20 minutes. Then the solvent was removed under reduced pressure. $\mathrm{K}_{2} \mathrm{CO}_{3}(124 \mathrm{mg}, 0.900 \mathrm{mmol}, 3.0$ equiv.), 4-fluorobenzenethiol (64.0 $\mu \mathrm{L}, 0.600 \mathrm{mmol}, 2.0$ equiv.) and $1.5 \mathrm{~mL}$ of DMF were added to the residue. After stirring at room temperature for 16 hours, the crude product was diluted with DCM $(20.0 \mathrm{~mL})$ and extracted with brine $(20.0 \mathrm{~mL} \times 3)$. The combined organic layers were dried over $\mathrm{Na}_{2} \mathrm{SO}_{4}$ and concentrated under reduced pressure. The crude residue was purified by column chromatography on silica using 2:1 pentanes:ethyl acetate as eluent. N-(3-((4-Fluorophenyl)thio)-1-(3-methyl-1H-indol-2-yl)propyl)4-methoxybenzamide 22 (106 $\mathrm{mg}, 0.240 \mathrm{mmol}, 79 \%$ ) was obtained as a pale yellow solid.

$\mathbf{R}_{\mathbf{f}}: 0.41$ (silica, pentanes:ethyl acetate 2:1);

Mp: $143-146^{\circ} \mathrm{C}$;

${ }^{1} \mathrm{H}$ NMR $\left(400 \mathrm{MHz}, \mathrm{CDCl}_{3}\right): \delta=9.01(\mathrm{~s}, 1 \mathrm{H}$, indole-NH), $7.73-7.62(\mathrm{~m}, 2 \mathrm{H}, \operatorname{ArH}), 7.51(\mathrm{~d}, \mathrm{~J}=7.8 \mathrm{~Hz}, 1 \mathrm{H}$, $\operatorname{ArH}$ ), 7.30 (ddd, J = 8.7, 5.4, 2.7 Hz, 2H, ArH), $7.27-7.25(\mathrm{~m}, 1 \mathrm{H}, \mathrm{ArH}), 7.15$ (ddd, J = 8.1, 7.0, 1.3 Hz, $1 \mathrm{H}, \mathrm{ArH}), 7.11-7.06(\mathrm{~m}, 1 \mathrm{H}, \mathrm{ArH}), 6.99-6.91(\mathrm{~m}, 2 \mathrm{H}, \mathrm{ArH}), 6.89-6.83(\mathrm{~m}, 2 \mathrm{H}, \mathrm{ArH}), 6.69(\mathrm{~d}, J=7.4$ $\mathrm{Hz}, 1 \mathrm{H}, \mathrm{NH}), 5.28(\mathrm{q}, J=7.5 \mathrm{~Hz}, 1 \mathrm{H}, \mathrm{CH}), 3.81\left(\mathrm{~s}, 3 \mathrm{H}, \mathrm{OCH}_{3}\right), 2.86\left(\mathrm{t}, J=7.0 \mathrm{~Hz}, 2 \mathrm{H}, \mathrm{CH}_{2} \mathrm{~S}\right), 2.55(\mathrm{dq}, J=$ 14.3, 7.1 Hz, $\left.1 \mathrm{H}, \mathrm{CH}_{2} \mathrm{CH}_{2} \mathrm{~S}\right), 2.38$ (dt, $\left.J=14.0,7.3 \mathrm{~Hz}, 1 \mathrm{H}, \mathrm{CH}_{2} \mathrm{CH}_{2} \mathrm{~S}\right), 2.31\left(\mathrm{~s}, 3 \mathrm{H}, \mathrm{CH}_{3}\right)$;

${ }^{13} \mathrm{C}$ NMR $\left(101 \mathrm{MHz} \mathrm{CDCl}_{3}\right): \delta=167.6,162.4,161.9(\mathrm{~d}, \mathrm{~J}=246.7 \mathrm{~Hz}), 135.4,133.4,132.6(\mathrm{~d}, J=8.0 \mathrm{~Hz})$, $130.4(d, J=3.4 \mathrm{~Hz}), 128.8,128.6,126.2,122.1,118.9(\mathrm{~d}, J=51.0 \mathrm{~Hz}), 116.2,116.0,113.8,111.0$, 108.2, 55.4, 47.1, 33.1, 32.1, 8.8;

${ }^{19} \mathrm{~F}$ NMR $\left(376 \mathrm{MHz}^{\mathrm{CDCl}} \mathrm{CD}_{3}\right): \delta=-115.1$;

IR (film): $\tilde{v}=3313$ (m), 2924 (w), 2856 (w), 1606 (s), 1540 (m), 1490 (s), 1460 (m), 1306 (m), 1255 (s), $1178(\mathrm{~m}), 1029(\mathrm{~m}), 832(\mathrm{~m}), 734(\mathrm{~m})$;

HRMS (ESI) calcd. for $\mathrm{C}_{26} \mathrm{H}_{25} \mathrm{FN}_{2} \mathrm{NaO}_{2} \mathrm{~S}^{+}[\mathrm{M}+\mathrm{Na}]^{+} 471.1513$; Found 471.1505.

N-(3-(Cyclopropylamino)-1-(3-methyl-1H-indol-2-yl)propyl)-4-methoxybenzamide (23). 
<smiles>COc1ccc(C(=O)NC2CC2)cc1</smiles>
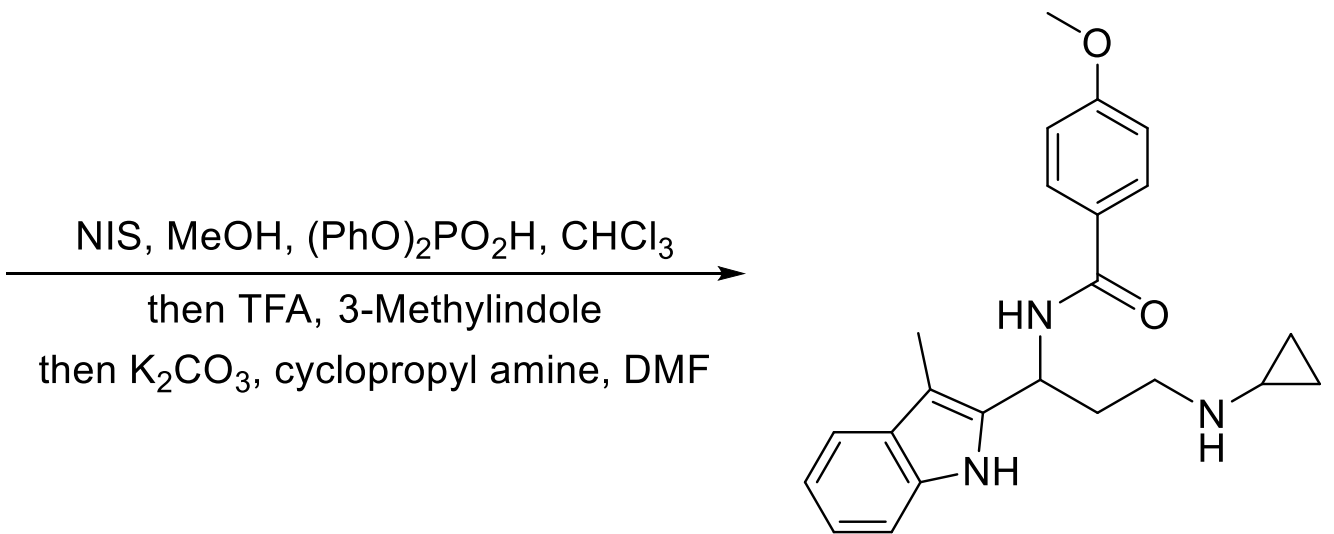

In a glass vial, N-cyclopropyl-4-methoxybenzamide $3 a(57.3 \mathrm{mg}, 0.300 \mathrm{mmol}, 1.0$ equiv.), diphenyl phosphate ( $7.5 \mathrm{mg}, 0.030 \mathrm{mmol}, 0.1$ equiv.), $\mathrm{N}$-iodosuccinimide ( $70.8 \mathrm{mg}, 0.315 \mathrm{mmol}, 1.05$ equiv.) and methanol (14.4 $\mu \mathrm{L}, 0.360 \mathrm{mmol}, 1.2$ equiv.) were dissolved in $1.5 \mathrm{~mL}$ of $\mathrm{CHCl}_{3}(0.20 \mathrm{M})$. The reaction mixture was stirred for 30 minutes. 3-Methylindole $(47.2 \mathrm{mg}, 0.360 \mathrm{mmol}, 1.2$ equiv.) and TFA $(23.0 \mu \mathrm{L}, 0.300 \mathrm{mmol}, 1.0$ equiv.) were added to the reaction mixture and the reaction mixture was stirred for another 20 minutes. Then the solvent was removed under reduced pressure, $\mathrm{K}_{2} \mathrm{CO}_{3}$ (124 mg, $0.900 \mathrm{mmol}, 3.0$ equiv.), cyclopropyl amine ( $42.0 \mu \mathrm{L}, 0.600 \mathrm{mmol}, 2.0$ equiv.) and $1.5 \mathrm{~mL}$ of DMF were added to the residue. After stirring at room temperature for $16 \mathrm{~h}$, the crude product was diluted with $\mathrm{DCM}(20.0 \mathrm{~mL})$ and extracted with brine $(20.0 \mathrm{~mL} \times 3)$. The combined organic layers were dried over $\mathrm{Na}_{2} \mathrm{SO}_{4}$ and concentrated under reduced pressure. The crude residue was purified by column chromatography on silica using 8:1 dichloromethane:methanol as eluent. N-(3(Cyclopropylamino)-1-(3-methyl-1H-indol-2-yl)propyl)-4-methoxybenzamide 23 (62.2 mg, 0.165 $\mathrm{mmol}, 55 \%)$ was obtained as a yellow solid.

$\mathbf{R}_{\mathbf{f}}: 0.28$ (silica, dichloromethane:methanol 8:1);

Mp: $177-179^{\circ} \mathrm{C}$;

${ }^{1} \mathrm{H}$ NMR $\left(400 \mathrm{MHz}\right.$, Acetone- $\left.d_{6}\right): \delta=10.19(\mathrm{~s}, 1 \mathrm{H}$, indole-NH), $8.45(\mathrm{~d}, J=7.6 \mathrm{~Hz}, 1 \mathrm{H}, \mathrm{NH}), 7.93-7.84$ $(\mathrm{m}, 2 \mathrm{H}, \mathrm{ArH}), 7.45(\mathrm{~d}, J=7.1 \mathrm{~Hz}, 1 \mathrm{H}, \mathrm{ArH}), 7.27(\mathrm{~d}, J=7.8 \mathrm{~Hz}, 1 \mathrm{H}, \mathrm{ArH}), 7.02(\mathrm{td}, J=8.0,7.5,1.4 \mathrm{~Hz}, 1 \mathrm{H}$, ArH), $6.99-6.96(\mathrm{~m}, 1 \mathrm{H}, \mathrm{ArH}), 6.96-6.92(\mathrm{~m}, 2 \mathrm{H}, \mathrm{ArH}), 5.67-5.54(\mathrm{~m}, 1 \mathrm{H}, \mathrm{CH}), 3.83\left(\mathrm{~s}, 3 \mathrm{H}, \mathrm{OCH}_{3}\right)$, $2.84\left(\mathrm{t}, J=6.3 \mathrm{~Hz}, 2 \mathrm{H}, \mathrm{CH}_{2} \mathrm{~N}\right), 2.31\left(\mathrm{~s}, 3 \mathrm{H}, \mathrm{CH}_{3}\right), 2.24\left(\mathrm{qd}, J=6.5,1.7 \mathrm{~Hz}, 2 \mathrm{H}, \mathrm{CH}_{2} \mathrm{CH}_{2} \mathrm{~N}\right), 2.21-2.15(\mathrm{~m}$, $1 \mathrm{H}, \mathrm{CH}), 0.47-0.33\left(\mathrm{~m}, 4 \mathrm{H}, \mathrm{CH}_{2}\right) ; 1 \mathrm{NH}$ was not resolved.

${ }^{13} \mathrm{C}$ NMR $\left(101 \mathrm{MHz}\right.$, Acetone- $\left.d_{6}\right): \delta=166.3,163.0,136.4,136.3,130.0,129.9,127.9,121.7,119.2$, $118.9,114.2,111.6,107.0,55.7,47.0,46.1,34.8,31.1,8.8,6.2,6.2$;

IR (film): $\tilde{v}=3296$ (m), 2933 (w), 1606 (s), 1504 (S), 1256 (s), 1179 (m), 1030 (m), 844 (m), 742 (m);

HRMS (ESI) calcd. for $\mathrm{C}_{23} \mathrm{H}_{28} \mathrm{~N}_{3} \mathrm{O}_{2}^{+}[\mathrm{M}+\mathrm{H}]^{+}$378.2176; Found 378.2171.

3-((tert-Butoxycarbonyl)amino)-3-methoxy-2-methylpropyl benzoate (29).<smiles>CC1CC1NC(=O)OC(C)(C)C</smiles>

d.r. $4: 1$
NIS, MeOH, DCM<smiles>O=C(OCc1ccccc1)OCc1ccccc1</smiles><smiles>COC(NC(=O)OC(C)(C)C)C(C)COC(=O)c1ccccc1</smiles>

d.r. $1: 1$ 
In a glass vial, tert-butyl (2-methylcyclopropyl)carbamate $28(17.1 \mathrm{mg}, 0.100 \mathrm{mmol}, 1.0$ equiv.), Niodosuccinimide ( $23.6 \mathrm{mg}, 0.105 \mathrm{mmol}, 1.05$ equiv.) and methanol ( $4.8 \mu \mathrm{L}, 0.12 \mathrm{mmol}, 1.2$ equiv.) were dissolved in $0.5 \mathrm{~mL}$ of dichloromethane $(0.20 \mathrm{M})$. The reaction mixture was stirred for 30 minutes. After the completion of the reaction, $\mathrm{K}_{2} \mathrm{CO}_{3}(27.6 \mathrm{mg}, 0.200 \mathrm{mmol}, 2.0$ equiv.) was added to the reaction mixture and then solvent was removed under reduced pressure. The crude was dissolved in $0.5 \mathrm{~mL}$ of DMF, and benzoic acid ( $24.4 \mathrm{mg}, 0.200 \mathrm{mmol}, 2.0$ equiv.) was added. The resulting mixture was stirred at room temperature for 16 hours. The crude was then diluted with brine $(5.0 \mathrm{~mL})$. The aqueous layer was extracted with EtOAc $(5.0 \mathrm{~mL} \times 2)$. The combined organic layers were dried over $\mathrm{Na}_{2} \mathrm{SO}_{4}$ and concentrated under reduced pressure. The crude residue was purified by preparative TLC on silica using 3:1 pentanes:ethyl acetate as eluent. 3-((tertButoxycarbonyl)amino)-3-methoxy-2-methylpropyl benzoate 29 (10.3 mg, $0.032 \mathrm{mmol}, 32 \%)$ was obtained as a colorless oil.

$\mathbf{R}_{\mathbf{f}}: 0.30$ (silica, pentanes:ethyl acetate 6:1);

${ }^{1} \mathbf{H}$ NMR (400 MHz, $\mathrm{CDCl}_{3}$; mixture of two diastereoisomers in a 1:1 ratio: the signals corresponding to the two diastereoisomers are not resolved): $\delta=8.09-7.99(\mathrm{~m}, 2 \mathrm{H}, \mathrm{ArH}), 7.60-7.53(\mathrm{~m}, 1 \mathrm{H}, \mathrm{ArH})$, 7.45 (dd, $J=8.4,7.0 \mathrm{~Hz}, 2 \mathrm{H}, \mathrm{ArH}$ ), 5.09 (dd, $J=20.7,10.4 \mathrm{~Hz}, 1 \mathrm{H}, \mathrm{NH}$ ), 4.89 (ddd, $J=13.2,10.4,5.3 \mathrm{~Hz}$, $1 \mathrm{H}, \mathrm{CH}), 4.41-4.22\left(\mathrm{~m}, 2 \mathrm{H}, \mathrm{OCH}_{2}\right), 3.35\left(\mathrm{~d}, J=1.0 \mathrm{~Hz}, 3 \mathrm{H}, \mathrm{OCH}_{3}\right), 2.19(\mathrm{dq}, J=13.2,6.4 \mathrm{~Hz}, 1 \mathrm{H}$, $\left.\mathrm{CHCH}_{3}\right), 1.45\left(\mathrm{~d}, J=2.6 \mathrm{~Hz}, 9 \mathrm{H}, \mathrm{CH}_{3}\right), 1.07$ (dd, $J=7.0,1.8 \mathrm{~Hz}, 3 \mathrm{H}, \mathrm{CH}_{3}$ );

${ }^{13} \mathrm{C}$ NMR (101 MHz, $\mathrm{CDCl}_{3}$; mixture of two diastereoisomers in a 1:1 ratio: the signals corresponding to the two diastereoisomers are partially resolved): $\delta=166.4,155.6,133.0,130.2,130.1,129.5$, $128.4,84.1,83.8,79.8,66.0,55.8,55.7,38.0,37.7,28.3,12.8,12.2$;

IR (film): $\tilde{v}=3355$ (w), 2977 (w), 2932 (w), 1718 (s), 1508 (m), 1367 (m), 1273 (s), 1170 (s), 1083 (m), $712(\mathrm{~m})$;

HRMS (ESI) calcd. for $\mathrm{C}_{17} \mathrm{H}_{25} \mathrm{NNaO}_{5}{ }^{+}[\mathrm{M}+\mathrm{Na}]^{+} 346.1625$; Found 346.1634. 


\section{Mechanistic studies}

\subsection{In-situ analysis of reaction intermediates}

$\mathrm{N}$-cyclopropyl-4-methylbenzenesulfonamide $3 \mathrm{q}(21.1 \mathrm{mg}, 0.100 \mathrm{mmol}, 1.0$ equiv.) and NXS (0.200 mmol, 2.0 equiv.) were dissolved in $0.5 \mathrm{~mL}$ of $\mathrm{CDCl}_{3}(0.20 \mathrm{M})$. After stirring for 2 hours at room temperature, the reaction crude mixture was submitted to ${ }^{1} \mathrm{H}$ NMR analysis directly.

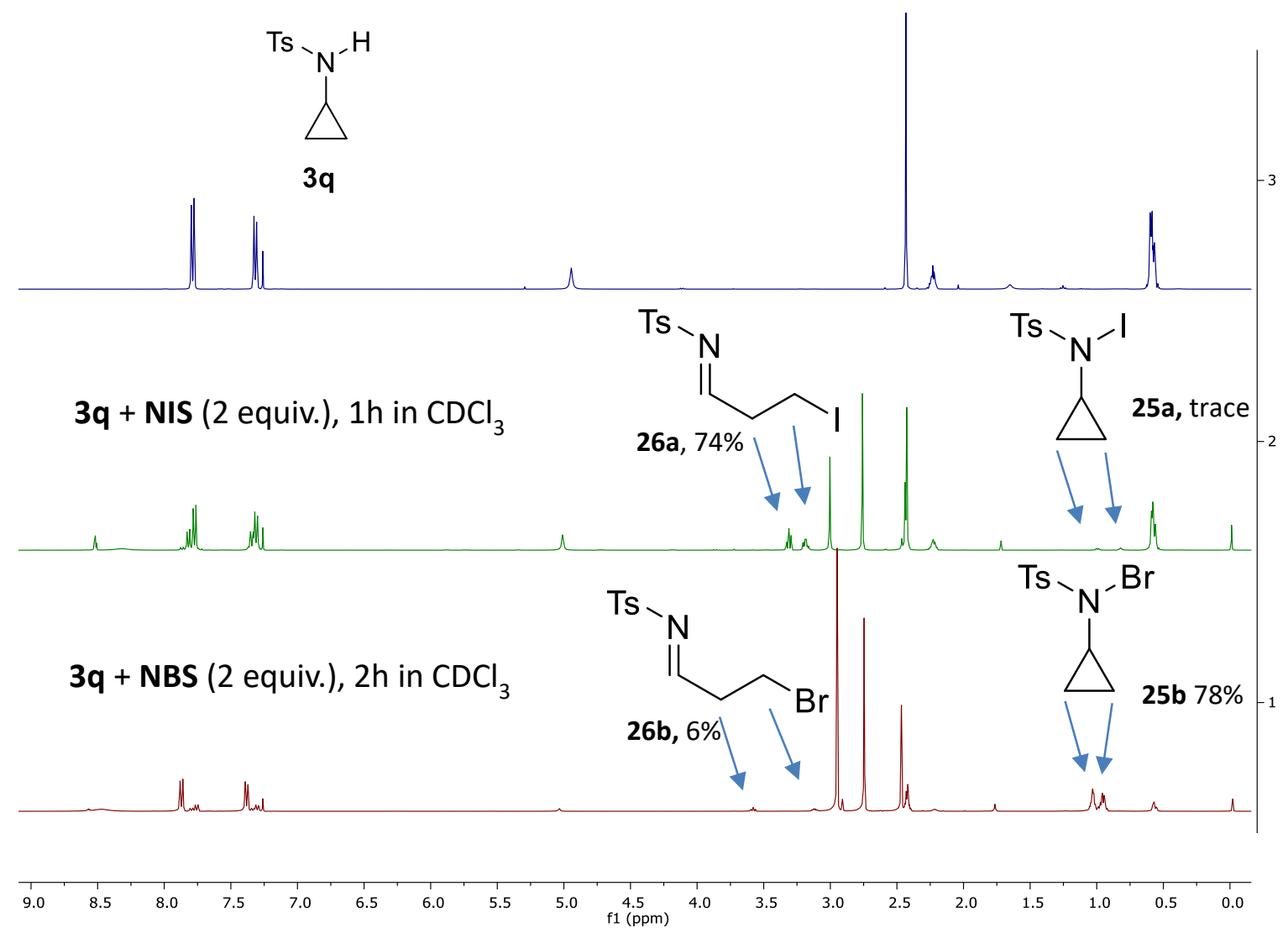

25a was not isolated because of low yield as well as low stability.

Due to the stability issue, we were not able to isolate $\mathbf{2 6 a}$ and its structure was only assumed based on the crude ${ }^{1} \mathrm{H}$ NMR spectra.

26a ${ }^{1} \mathrm{H}$ NMR (400 MHz, CDCl $): \delta=8.51(\mathrm{t}, J=3.5 \mathrm{~Hz}, 1 \mathrm{H}, \mathrm{CH}), 7.81(\mathrm{~d}, J=8.3 \mathrm{~Hz}, 2 \mathrm{H}, \mathrm{ArH}), 7.34(\mathrm{~d}, J=$ $8.0 \mathrm{~Hz}, 2 \mathrm{H}, \mathrm{ArH}), 3.34-3.26\left(\mathrm{~m}, 2 \mathrm{H}, \mathrm{CH}_{2} \mathrm{l}\right), 3.22-3.14\left(\mathrm{~m}, 2 \mathrm{H}, \mathrm{CH}_{2}\right), 2.43\left(\mathrm{~s}, 3 \mathrm{H}, \mathrm{CH}_{3}\right)$.

\subsection{Efforts towards the isolation of intermediate $25 b$}

In 5.1 experiment part, $\mathbf{2 5 b}$ was isolated with $80 \%$ purity, accompanied by $\mathbf{2 6 b}$ as a major impurity. 


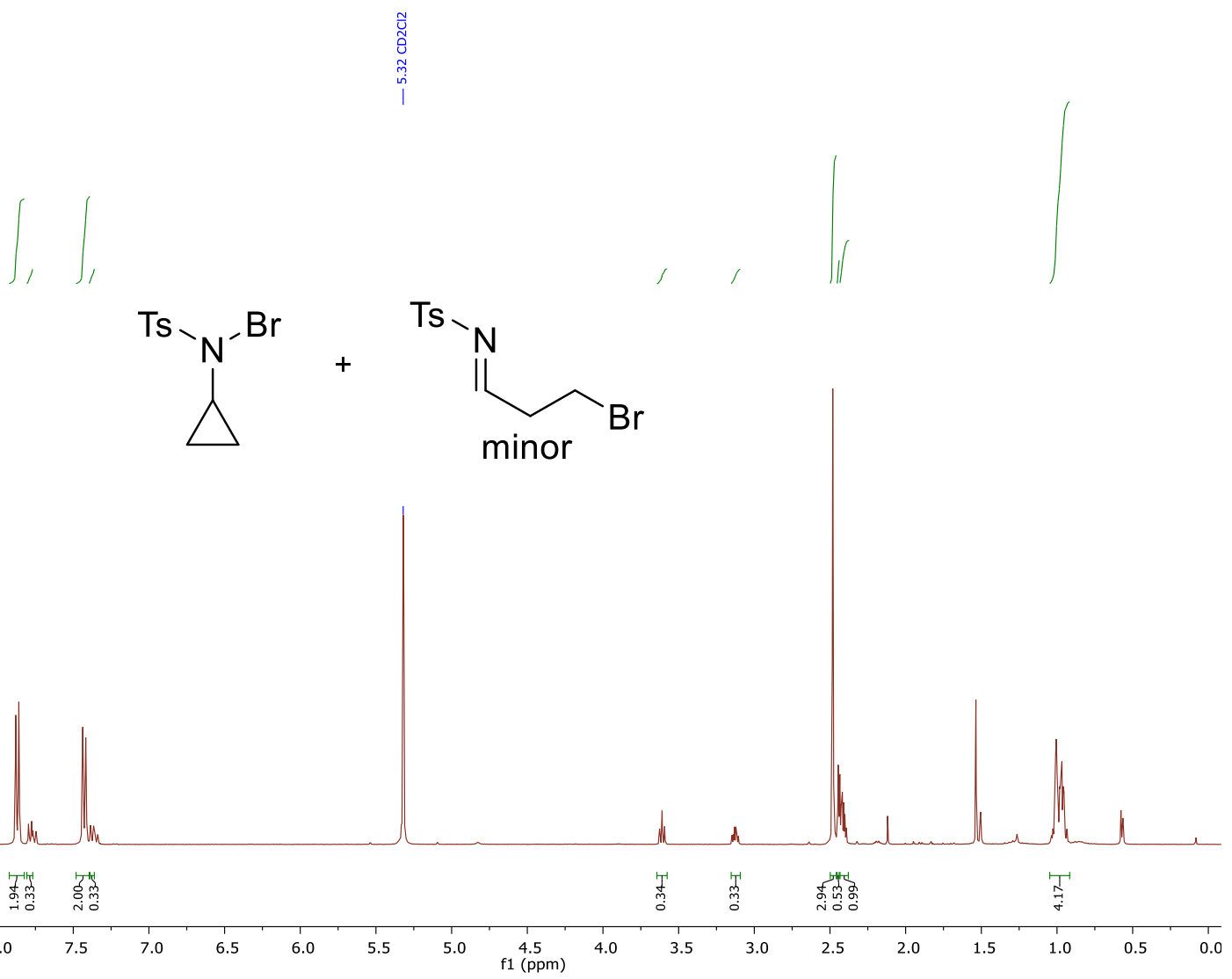

25b ${ }^{1} \mathrm{H}$ NMR $\left(400 \mathrm{MHz}, \mathrm{CD}_{2} \mathrm{Cl}_{2}\right): \delta=7.92-7.82(\mathrm{~m}, 2 \mathrm{H}, \operatorname{ArH}), 7.43(\mathrm{~d}, \mathrm{~J}=8.0 \mathrm{~Hz}, 2 \mathrm{H}, \operatorname{ArH}), 2.48(\mathrm{~s}, 3 \mathrm{H}$, $\left.\mathrm{CH}_{3}\right), 2.43-2.38(\mathrm{~m}, 1 \mathrm{H}, \mathrm{CH}), 1.04-0.93\left(\mathrm{~m}, 4 \mathrm{H}, \mathrm{CH}_{2}\right)$.

26 ${ }^{1}{ }^{1}$ NMR $\left(400 \mathrm{MHz}, \mathrm{CD}_{2} \mathrm{Cl}_{2}\right): \delta=8.53(\mathrm{t}, J=3.7 \mathrm{~Hz}, 1 \mathrm{H}, \mathrm{CH}), 7.81-7.77(\mathrm{~m}, 2 \mathrm{H}, \operatorname{ArH}), 7.38(\mathrm{~d}, J=$ $8.0 \mathrm{~Hz}, 2 \mathrm{H}, \mathrm{ArH}), 3.61\left(\mathrm{t}, J=6.6 \mathrm{~Hz}, 2 \mathrm{H}, \mathrm{CH}_{2} \mathrm{Br}\right), 3.13\left(\mathrm{td}, J=6.6,3.7 \mathrm{~Hz}, 2 \mathrm{H}, \mathrm{CH}_{2}\right), 2.45\left(\mathrm{~s}, 3 \mathrm{H}, \mathrm{CH}_{3}\right)$.

In fact, 25b was a reported compound but the spectra was not given in the original paper. ${ }^{[13]}$ Therefore, we synthesized $\mathbf{2 5 b}$ by following the modified reported protocol and found that in these two cases, the ${ }^{1} \mathrm{H}$ NMR data corresponds well to each other.

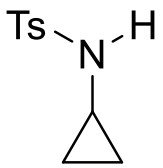

$3 q$

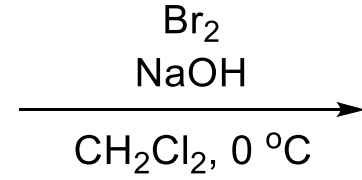

$\mathrm{CH}_{2} \mathrm{Cl}_{2}, 0^{\circ} \mathrm{C}$

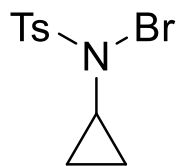

25b

A solution of $2 \mathrm{mmol}$ of $\mathbf{3 q}$ in $3 \mathrm{ml}$ dichloromethane was added to a $25 \mathrm{~mL}$ flask covered by foil paper, then bromine $(0.4 \mathrm{~mL})$ in $10 \mathrm{~mL}$ of $2 \mathrm{~N} \mathrm{NaOH}(\mathrm{aq})$ was added drop by drop to the solution above. The temperature was kept at $0{ }^{\circ} \mathrm{C}$ and the reaction mixture was stirred for an additional 2 hours. The organic layer was washed twice with ice water. The organic layer was dried over $\mathrm{Na}_{2} \mathrm{SO}_{4}$ at $-20{ }^{\circ} \mathrm{C}$, after which the $\mathrm{Na}_{2} \mathrm{SO}_{4}$ was filtered off. The crude product was obtained after concentration under reduced pressure at room temperature, covered by foil paper. Beige solid was obtained as product albeit with some dichloromethane.

${ }^{13}$ Dekker, E. E. J.; Engberts, J. B. F. N.; de Boer, Th. J. Recl. Trav. Chim. Pays-Bas, 1978, 97, 39-41. 


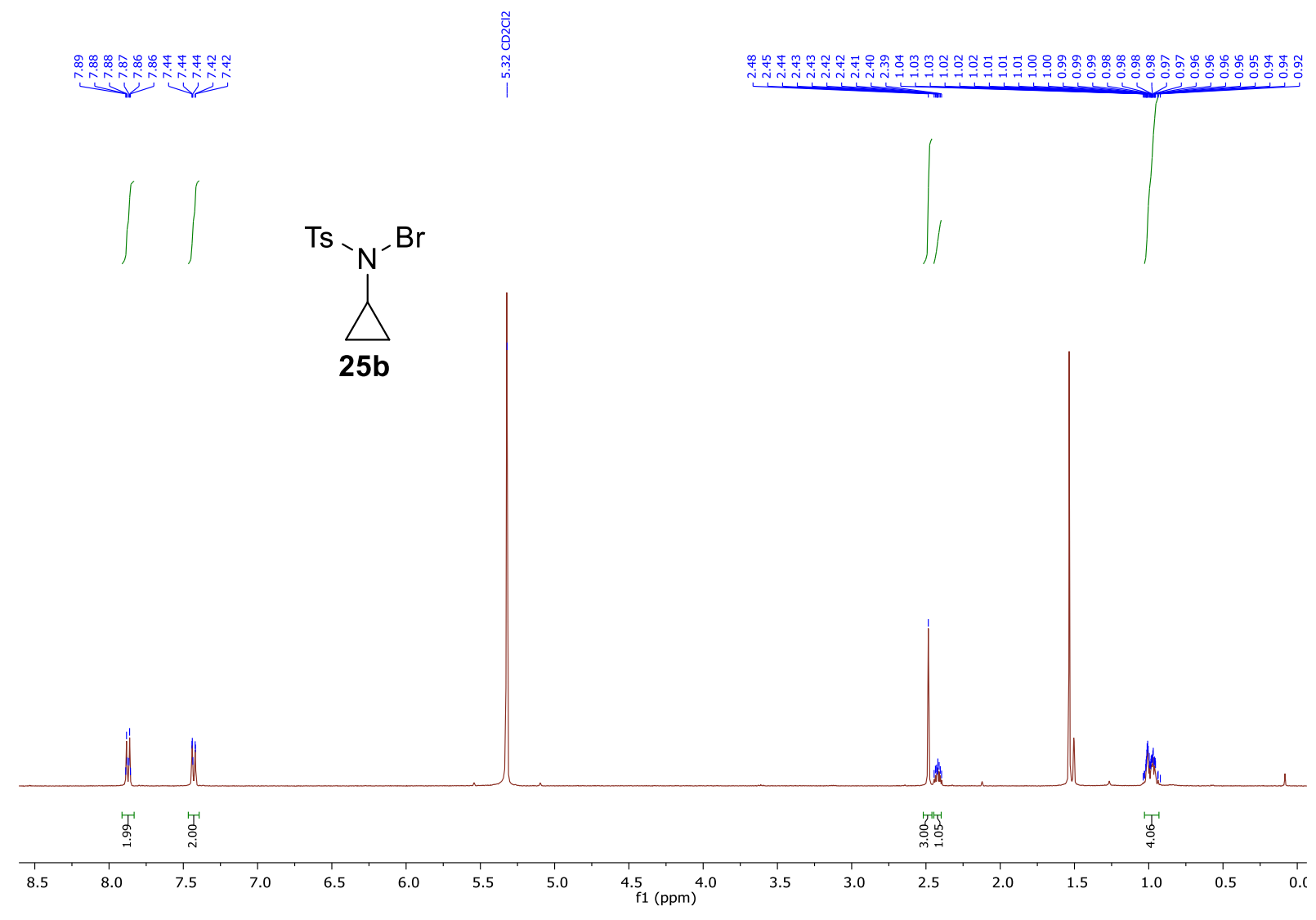

\subsection{Reactivity of intermediate $25 \mathrm{~b}$ and $26 \mathrm{~b}$}

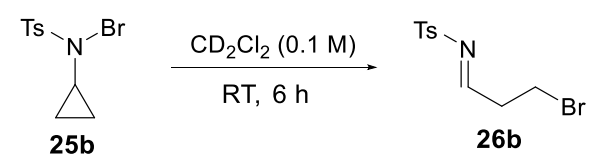

$\mathrm{N}$-bromo-N-cyclopropyl-4-methylbenzenesulfonamide $25 \mathrm{~b}$ (14.5 mg, $0.050 \mathrm{mmol}, 1.0$ equiv.) was added in $0.5 \mathrm{~mL}$ of $\mathrm{CD}_{2} \mathrm{Cl}_{2}(0.10 \mathrm{M})$. After stirring for 6 hours at room temperature, the reaction crude was submitted to ${ }^{1} \mathrm{H}$ NMR analysis directly. In this case, full conversion of $\mathbf{2 5 b}$ was observed and the structure of $\mathbf{2 6 b}$ was assumed based on the crude ${ }^{1} \mathrm{H}$ NMR spectra as it is not stable enough to be isolated. Note: under the same conditions, no conversion was observed when the vial was covered with foil paper. 

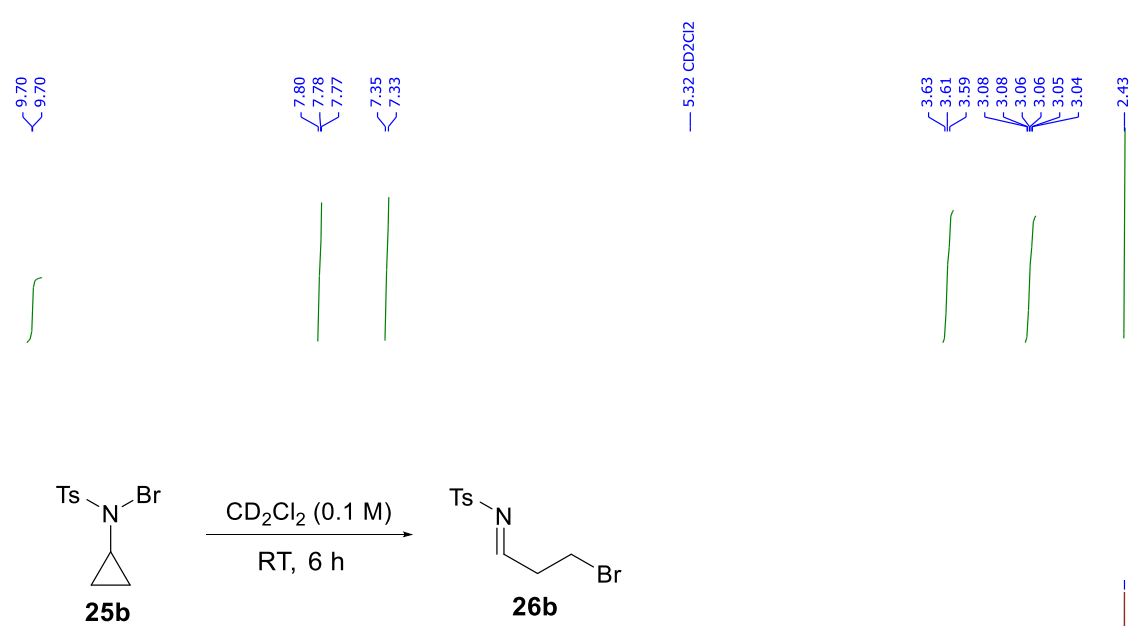

26b

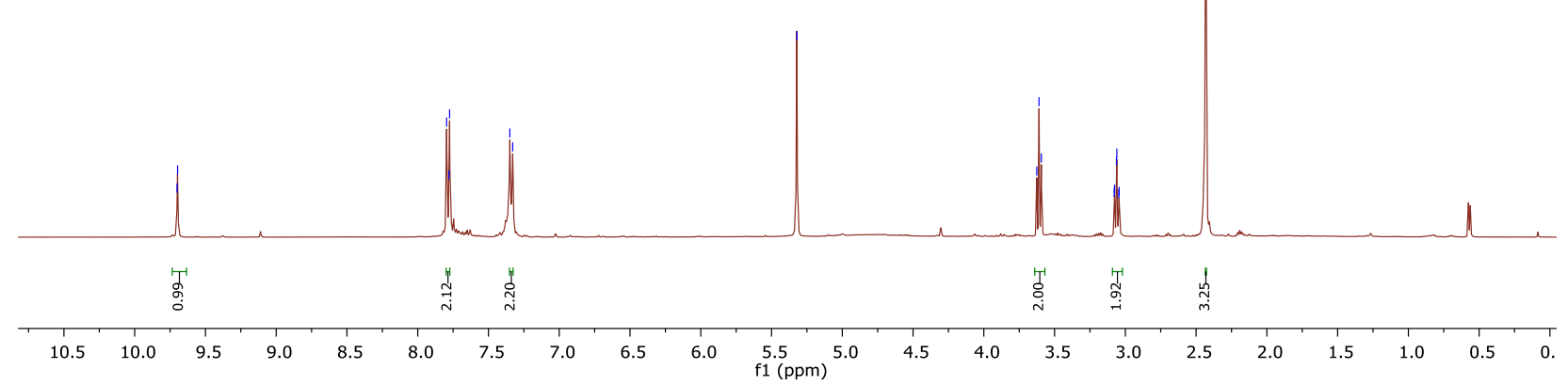

To the crude solution of $\mathbf{2 6 \mathbf { b }}$ in $\mathrm{CD}_{2} \mathrm{Cl}_{2}$ was added $\mathrm{MeOH}(8.1 \mu \mathrm{L}, 0.200 \mathrm{mmol}, 4.0$ equiv.), then the vial was covered with foil paper to exclude any role of light in this reaction. After stirring for 16 hours at room temperature, the reaction crude was submitted to ${ }^{1} \mathrm{H}$ NMR and HRMS analysis directly. In this case, full conversion of $\mathbf{2 6} \mathbf{b}$ was observed and the structure of $\mathbf{2 7}$ was assumed based on the crude ${ }^{1} \mathrm{H}$ NMR spectra as this Ts protected $\mathrm{N}, \mathrm{O}$-acetal is not stable.

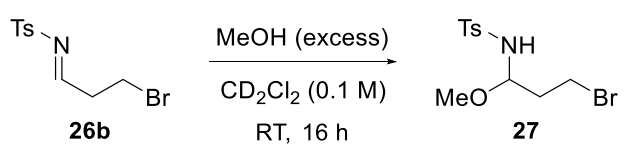




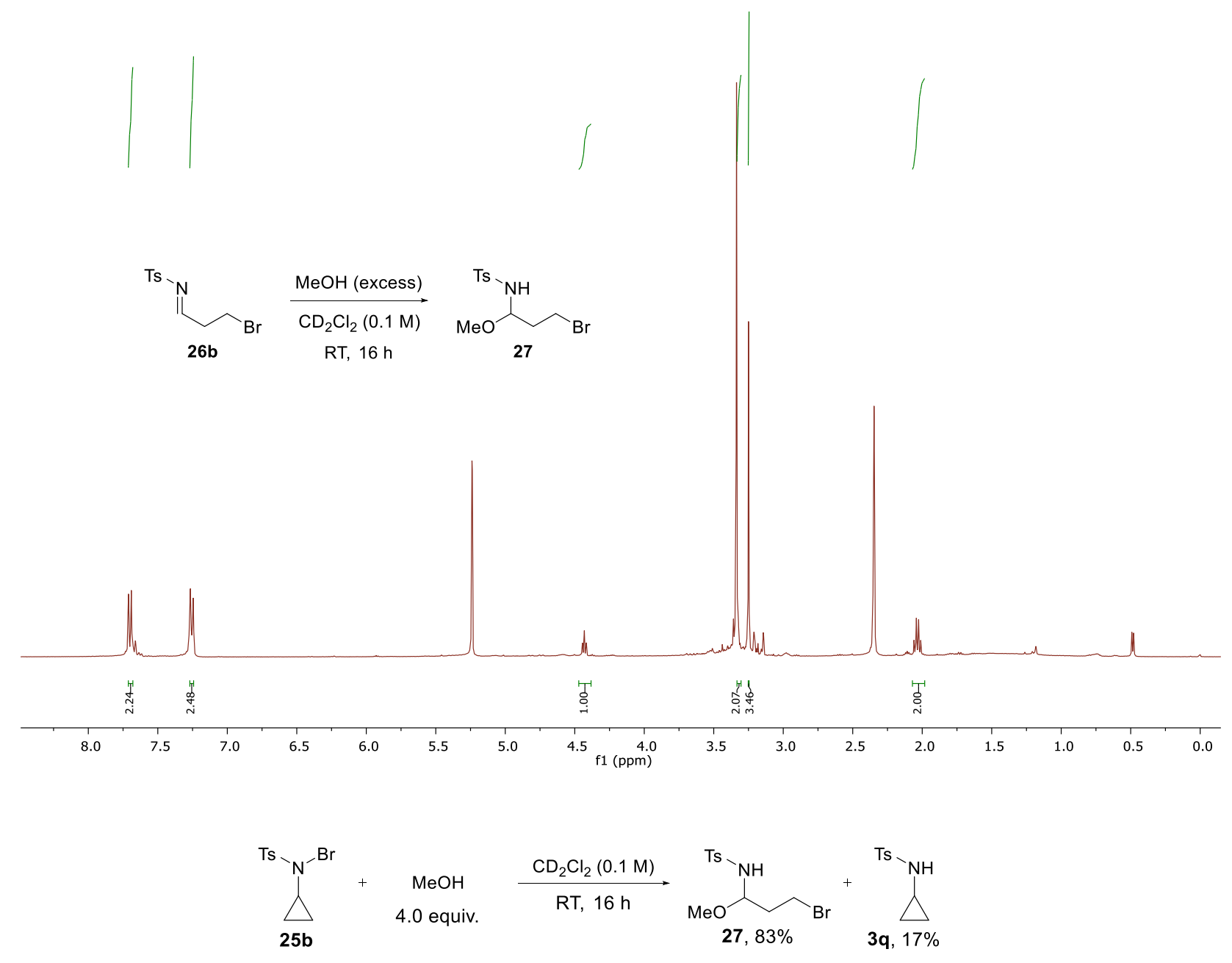

Methanol ( $8.1 \mu \mathrm{L}, 0.200 \mathrm{mmol}, 4.0$ equiv.) was added to the solution of $\mathrm{N}$-bromo-N-cyclopropyl-4methylbenzenesulfonamide $25 \mathrm{~b}$ ( $14.5 \mathrm{mg}, 0.050 \mathrm{mmol}, 1.0$ equiv.) in $0.5 \mathrm{~mL}$ of $\mathrm{CD}_{2} \mathrm{Cl}_{2}$ (0.10 M). After stirring for 16 hours under ambient light at room temperature, the reaction crude was submitted to ${ }^{1} \mathrm{H}$ NMR and HRMS analysis directly. . Note: under the same conditions, no conversion was observed when the vial was covered with foil paper.

$27^{1} \mathrm{H}$ NMR $\left(400 \mathrm{MHz}, \mathrm{CD}_{2} \mathrm{Cl}_{2}\right): \delta=7.75(\mathrm{dd}, J=8.4,1.9 \mathrm{~Hz}, 2 \mathrm{H}, \mathrm{ArH}), 7.35-7.31(\mathrm{~m}, 2 \mathrm{H}, \mathrm{ArH}), 5.51(\mathrm{~d}$, $J=9.7 \mathrm{~Hz}, 1 \mathrm{H}, \mathrm{NH}$, note: this proton is not resolved sometimes), 4.66 (ddd, $J=9.7,7.5,4.9 \mathrm{~Hz}, 1 \mathrm{H}$, $\mathrm{CH}), 3.37-3.27\left(\mathrm{~m}, 2 \mathrm{H}, \mathrm{CH}_{2} \mathrm{Br}\right), 3.22\left(\mathrm{~s}, 3 \mathrm{H}, \mathrm{OCH}_{3}\right), 2.13-2.05\left(\mathrm{~m}, 1 \mathrm{H}, \mathrm{CH}_{2}\right), 1.92-1.85\left(\mathrm{~m}, 1 \mathrm{H}, \mathrm{CH}_{2}\right)$.

HRMS (ESI) calcd. for $\mathrm{C}_{11} \mathrm{H}_{16} \mathrm{BrNNaO}_{3} \mathrm{~S}^{+}[\mathrm{M}+\mathrm{Na}]^{+}$343.9926; Found 343.9920 . 


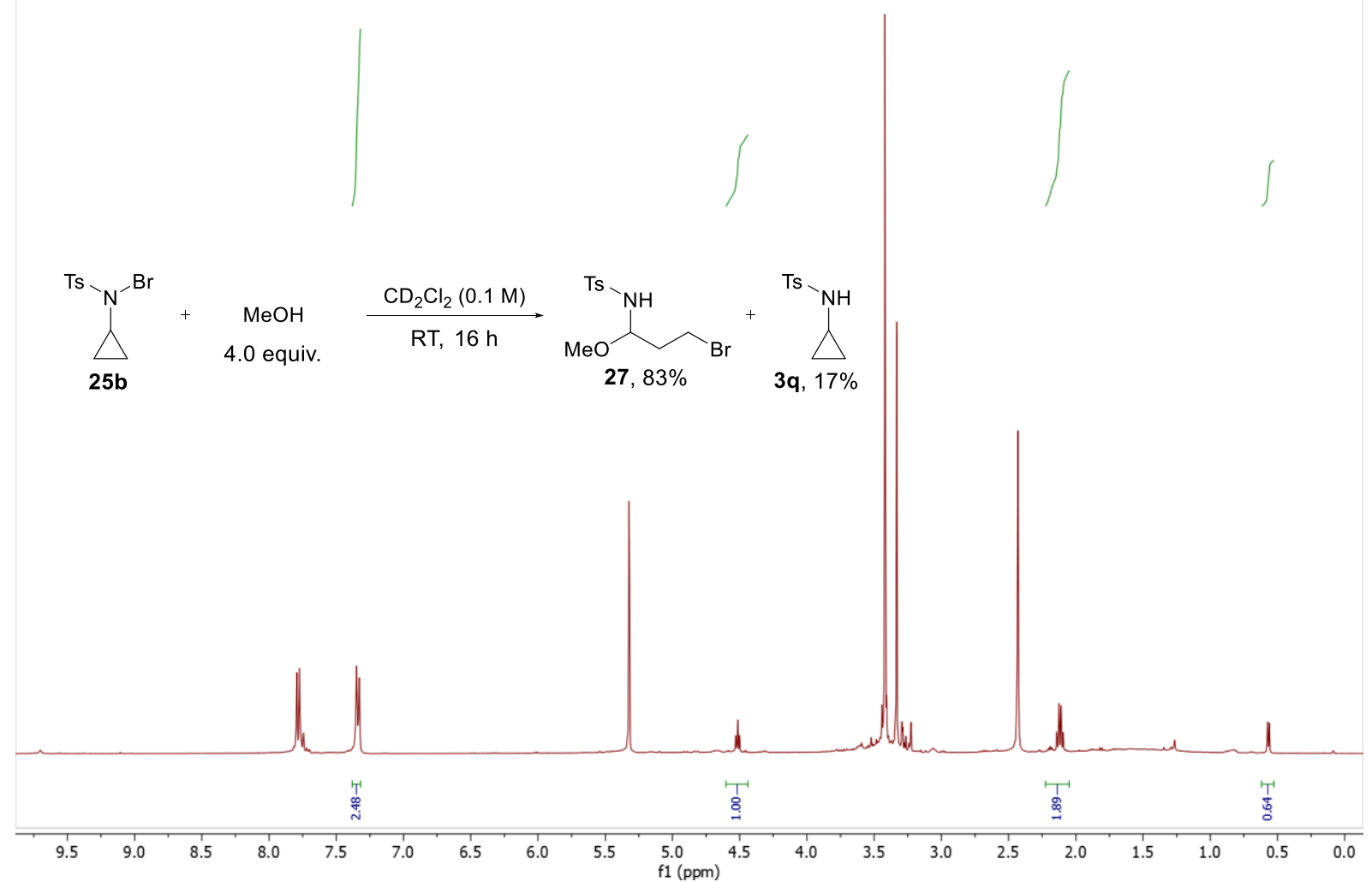




\subsection{Crossover experiment}

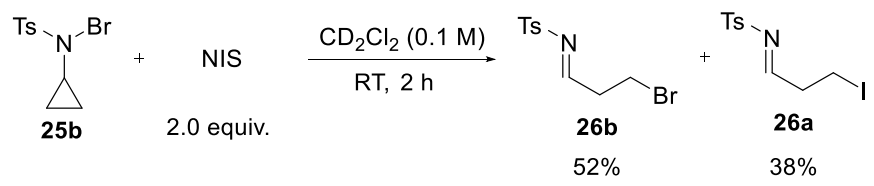

$\mathrm{N}$-bromo-N-cyclopropyl-4-methylbenzenesulfonamide $25 \mathrm{~b}(14.5 \mathrm{mg}, 0.050 \mathrm{mmol}, 1.0$ equiv.) and NIS (22.5 mg, $0.100 \mathrm{mmol}, 2.0$ equiv.) was added in $0.5 \mathrm{~mL}$ of $\mathrm{CD}_{2} \mathrm{Cl}_{2}(0.10 \mathrm{M})$. After stirring for 2 hours at room temperature, the reaction crude was submitted to ${ }^{1} \mathrm{H}$ NMR analysis directly.
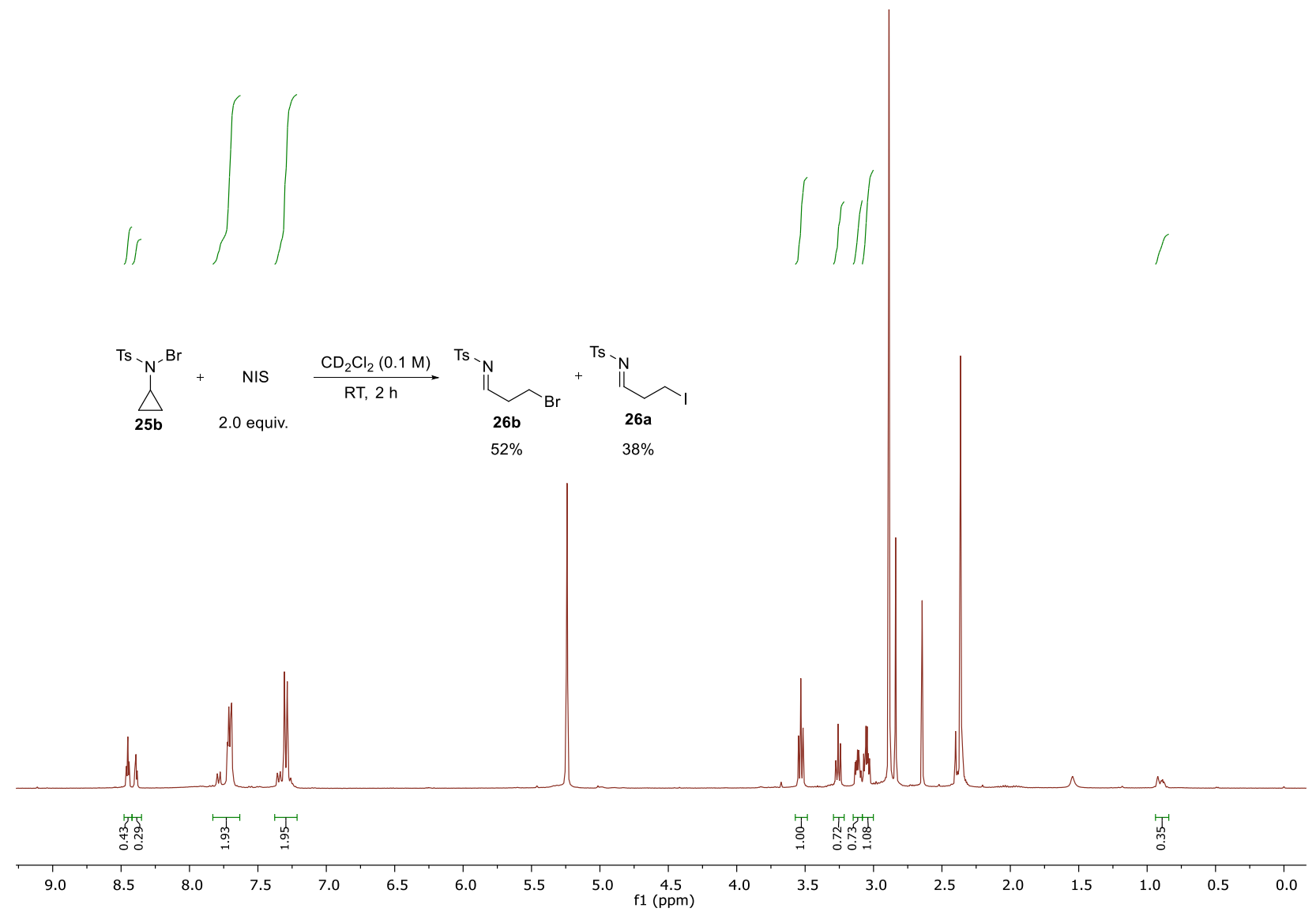

\section{Spectra of new compounds}

N-Cyclopropyl-4-(methylthio)benzamide (3b)

${ }^{1}$ H-NMR (400 MHz, CDCl3) 


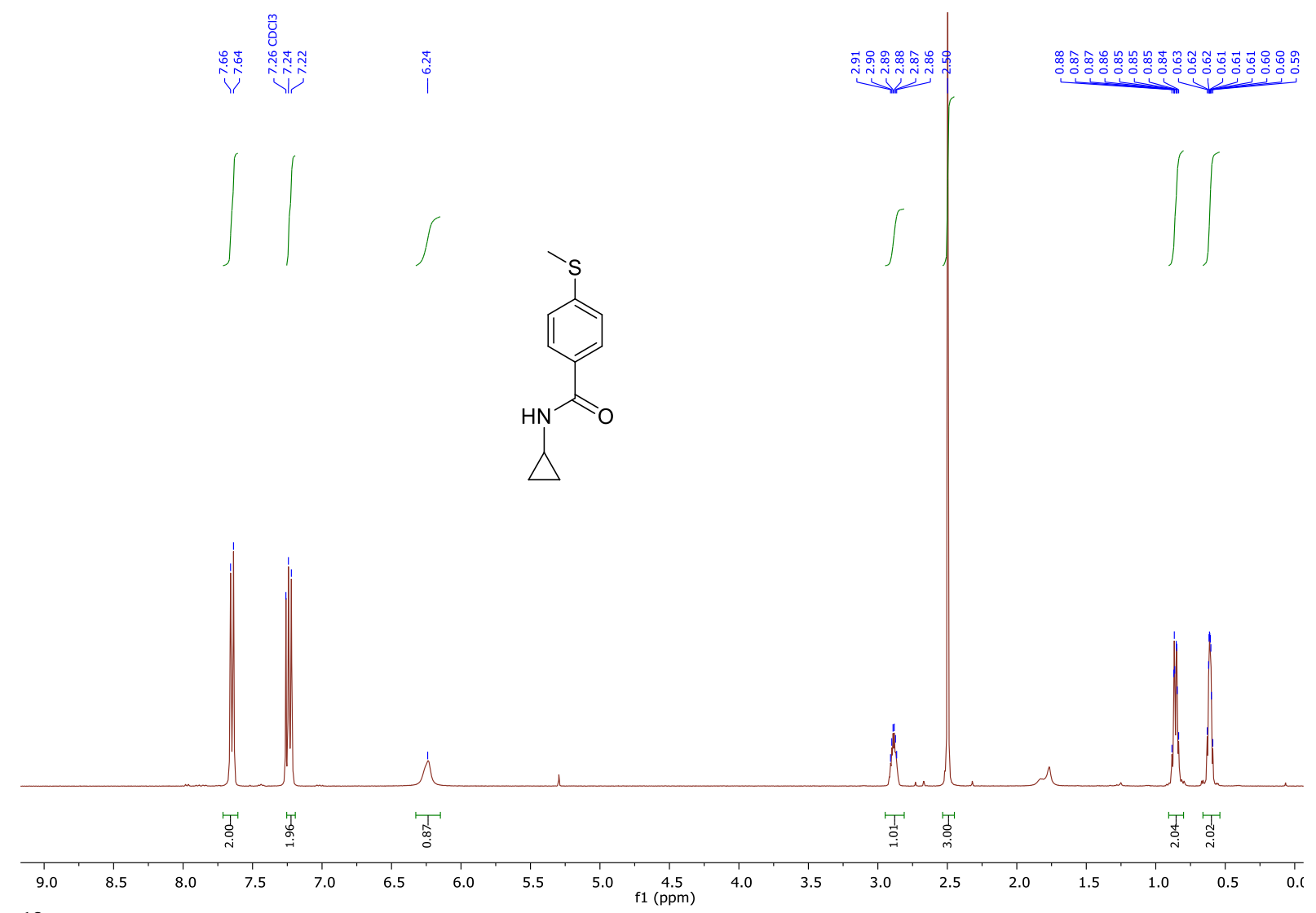

${ }^{13} \mathrm{C}$-NMR (101 MHz, CDCl3)
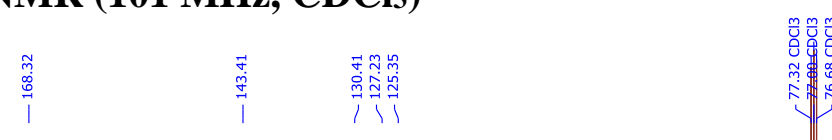<smiles>CSc1ccc(C(=O)NC2CC2)cc1</smiles>
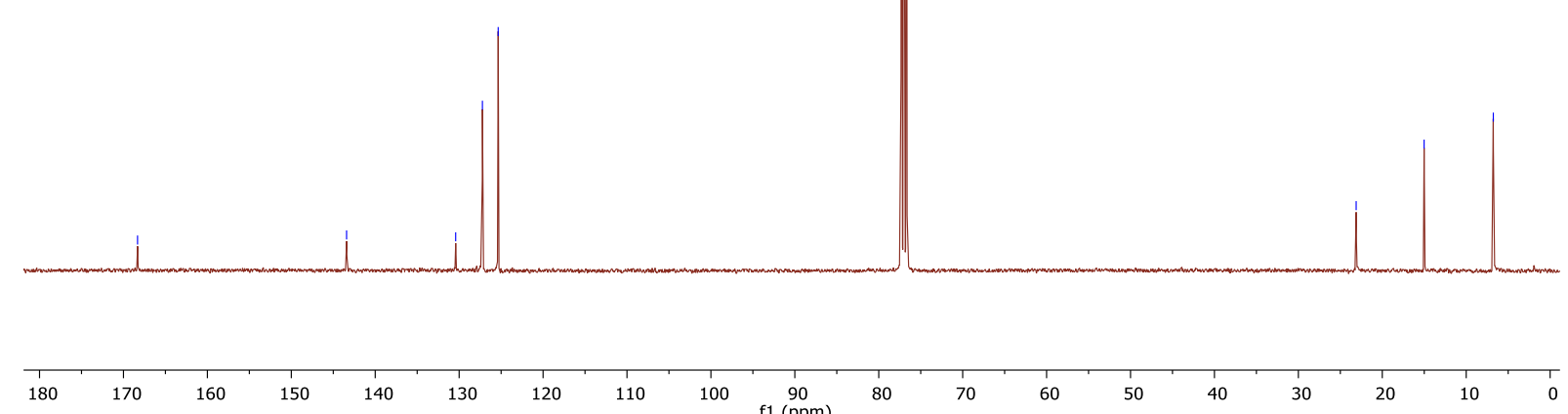

IR 


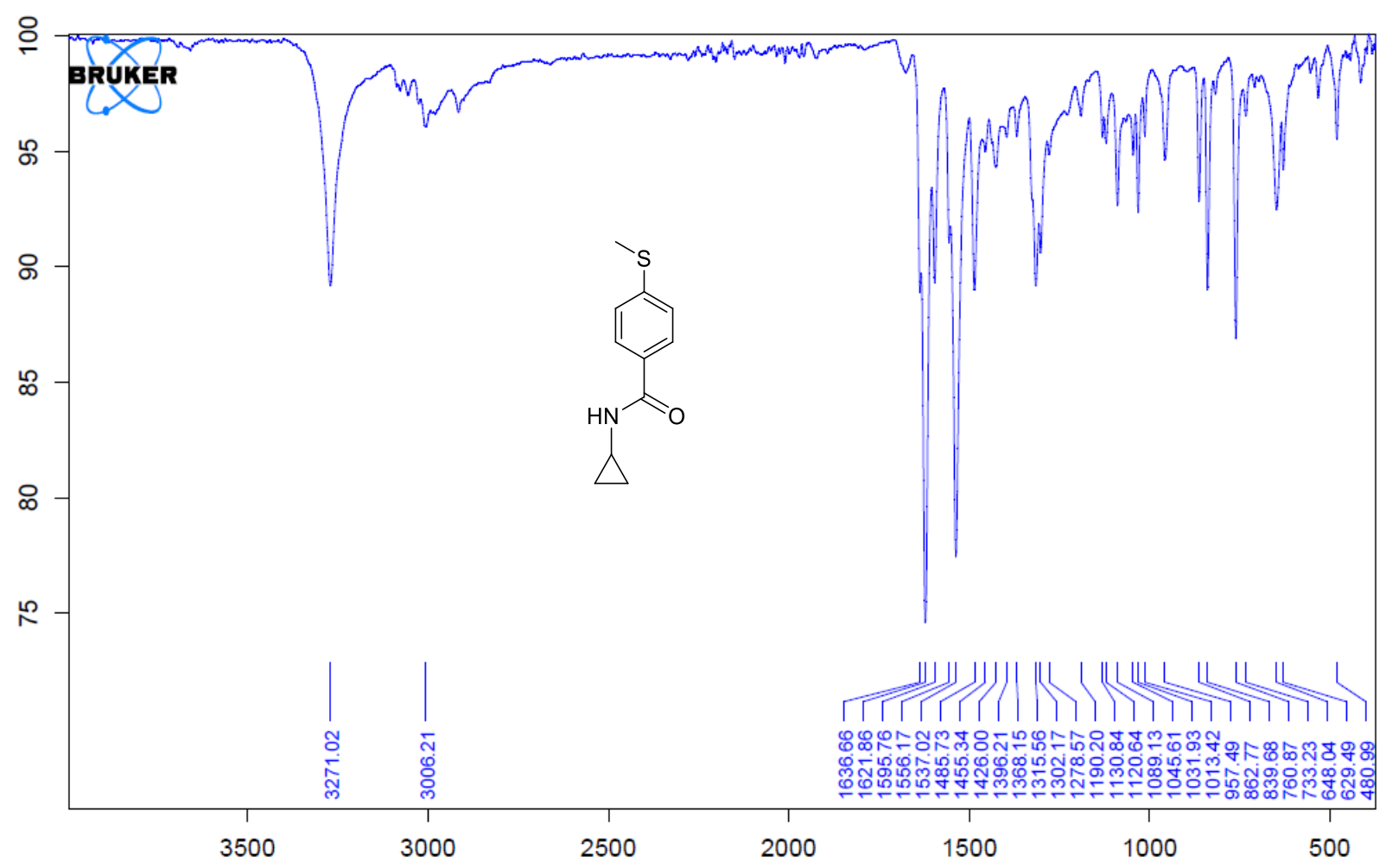

4-Chloro-N-cyclopropylbenzamide (3d)

${ }^{1}$ H-NMR (400 MHz, CDCl 3 )

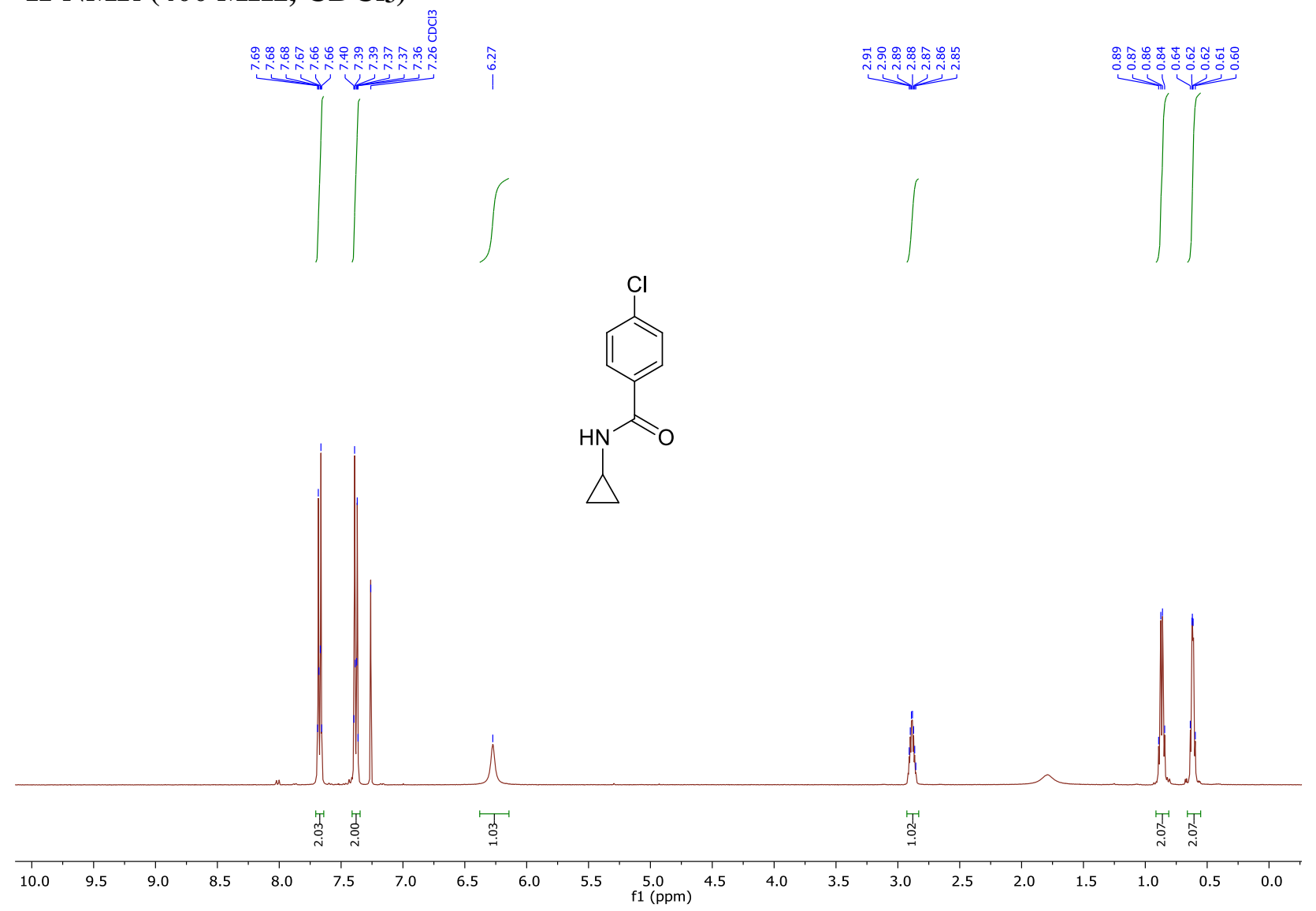

${ }^{13} \mathrm{C}-\mathrm{NMR}$ (101 MHz, $\left.\mathrm{CDCl}_{3}\right)$ 

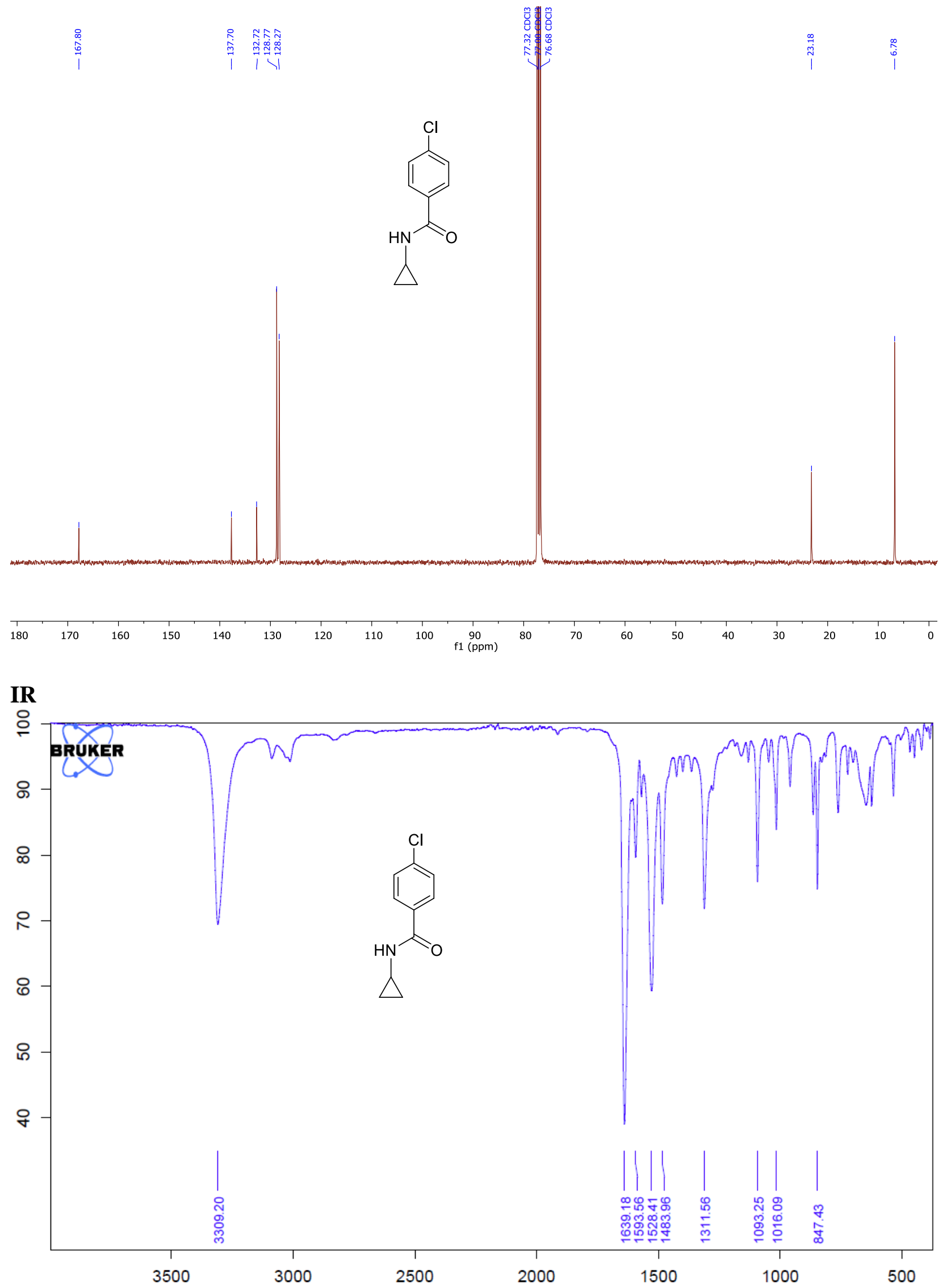

4-Cyano-N-cyclopropylbenzamide (3e) 
${ }^{1} \mathrm{H}-\mathrm{NMR}$ (400 MHz, $\mathrm{CDCl}_{3}$ )

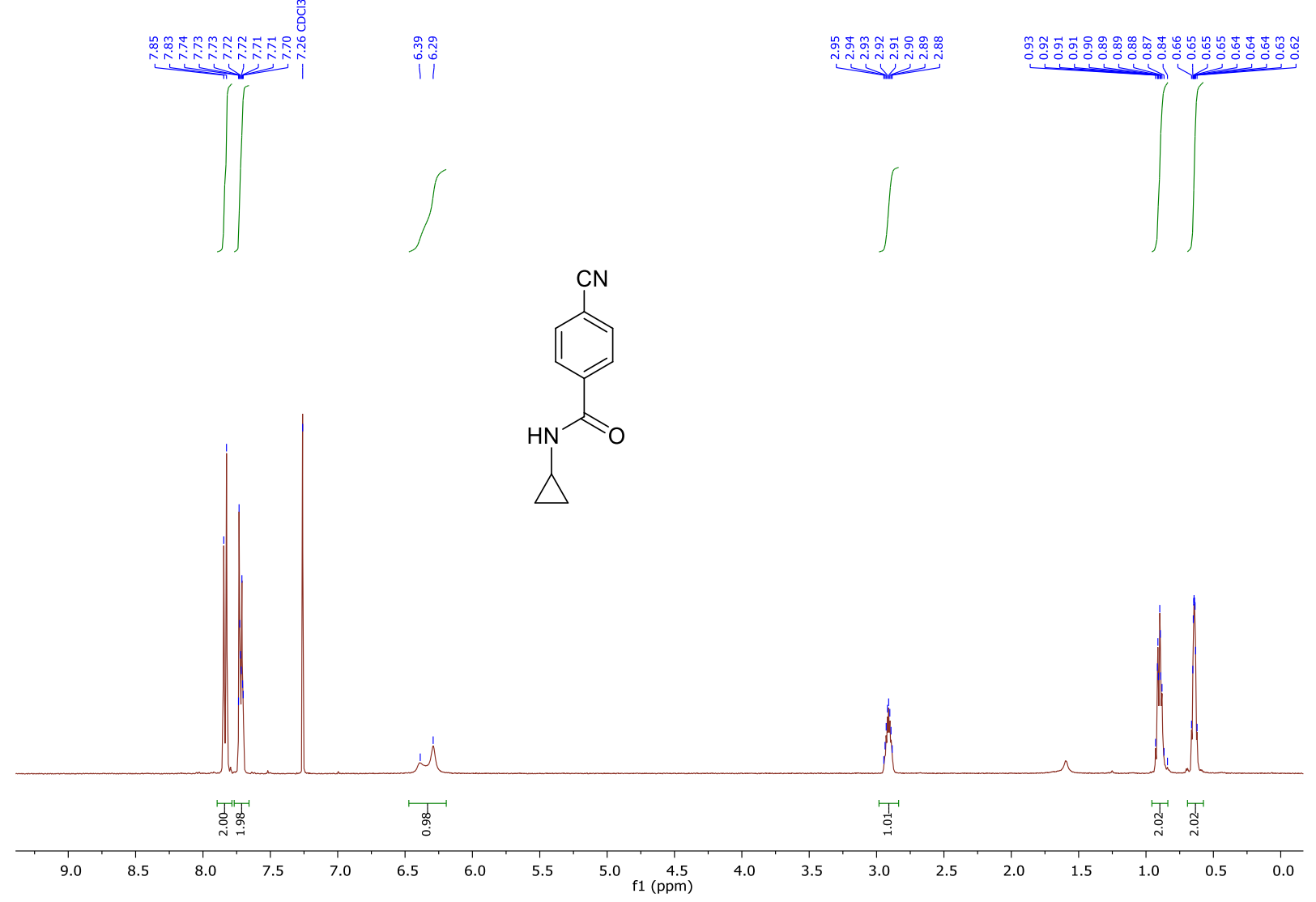

${ }^{13} \mathrm{C}-\mathrm{NMR}$ (101 MHz, $\left.\mathrm{CDCl}_{3}\right)$

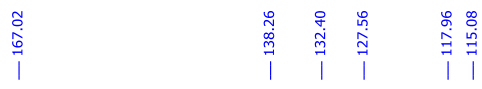
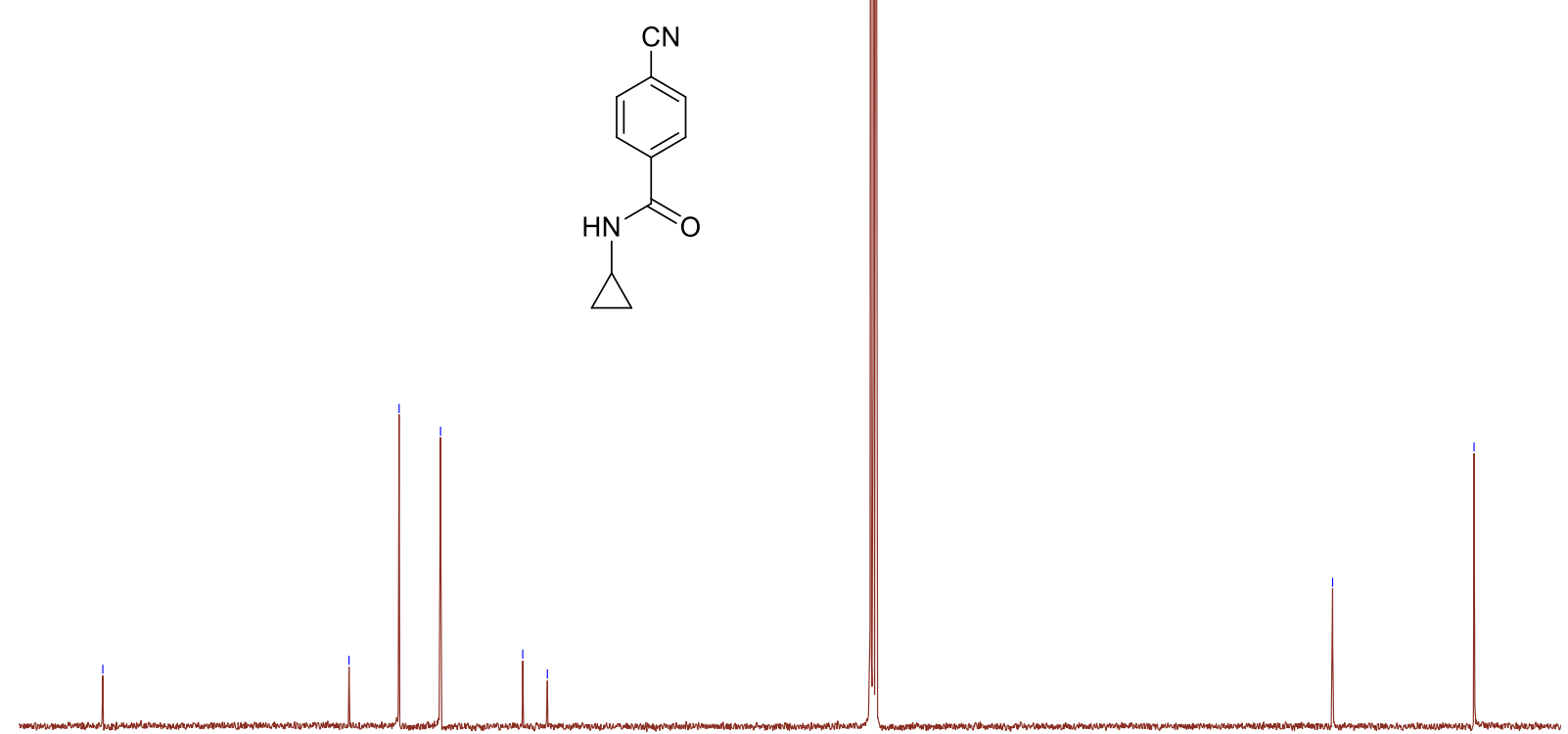

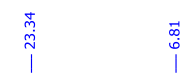

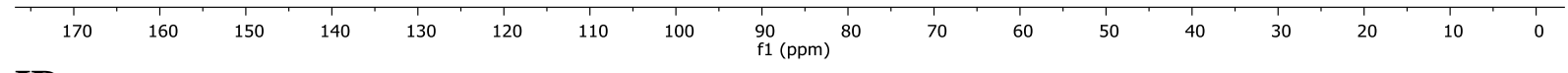

IR 


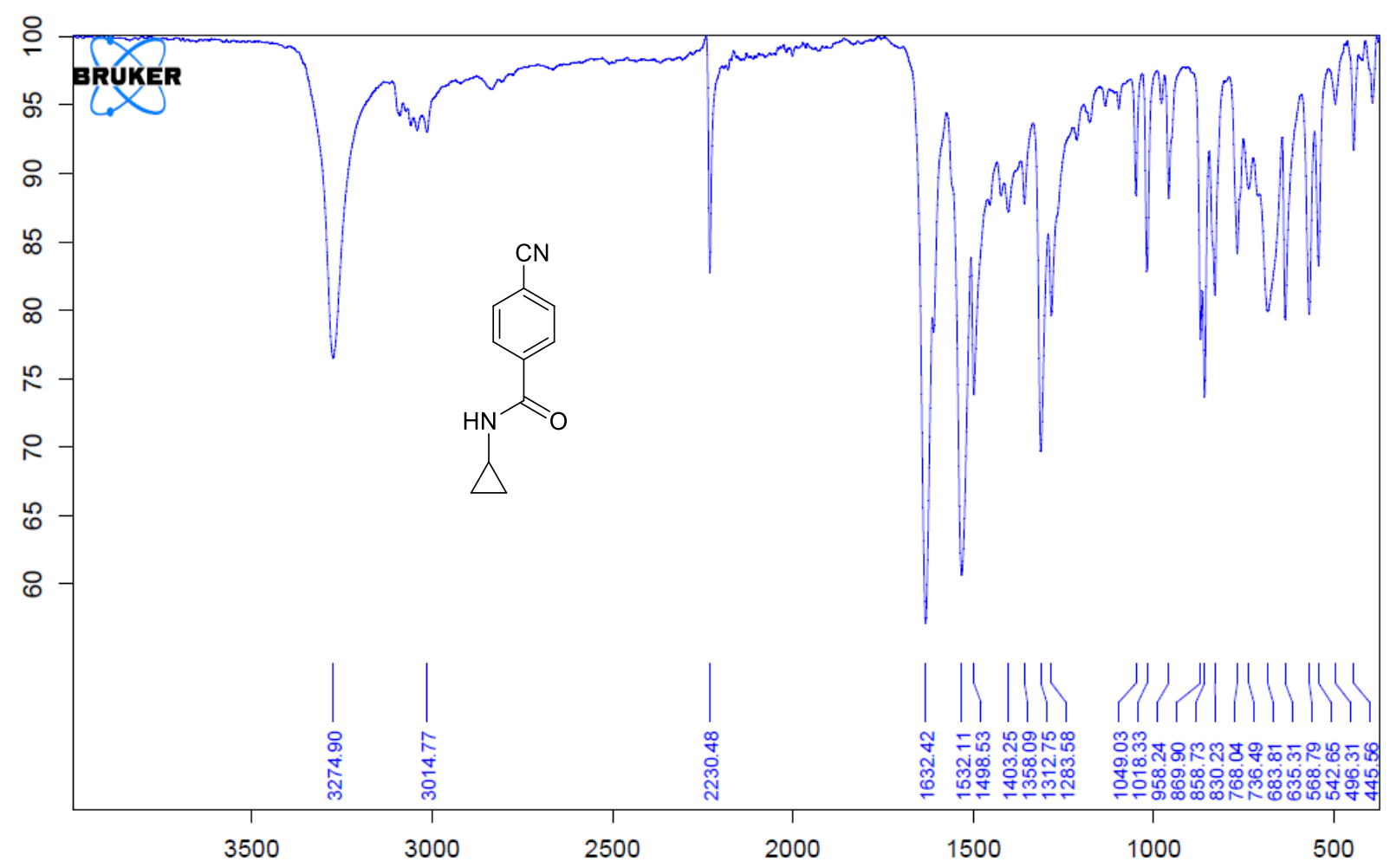

N-Cyclopropyl-4-nitrobenzamide (3f)

${ }^{1} \mathrm{H}-\mathrm{NMR}$ (400 MHz, $\mathrm{CDCl}_{3}$ )
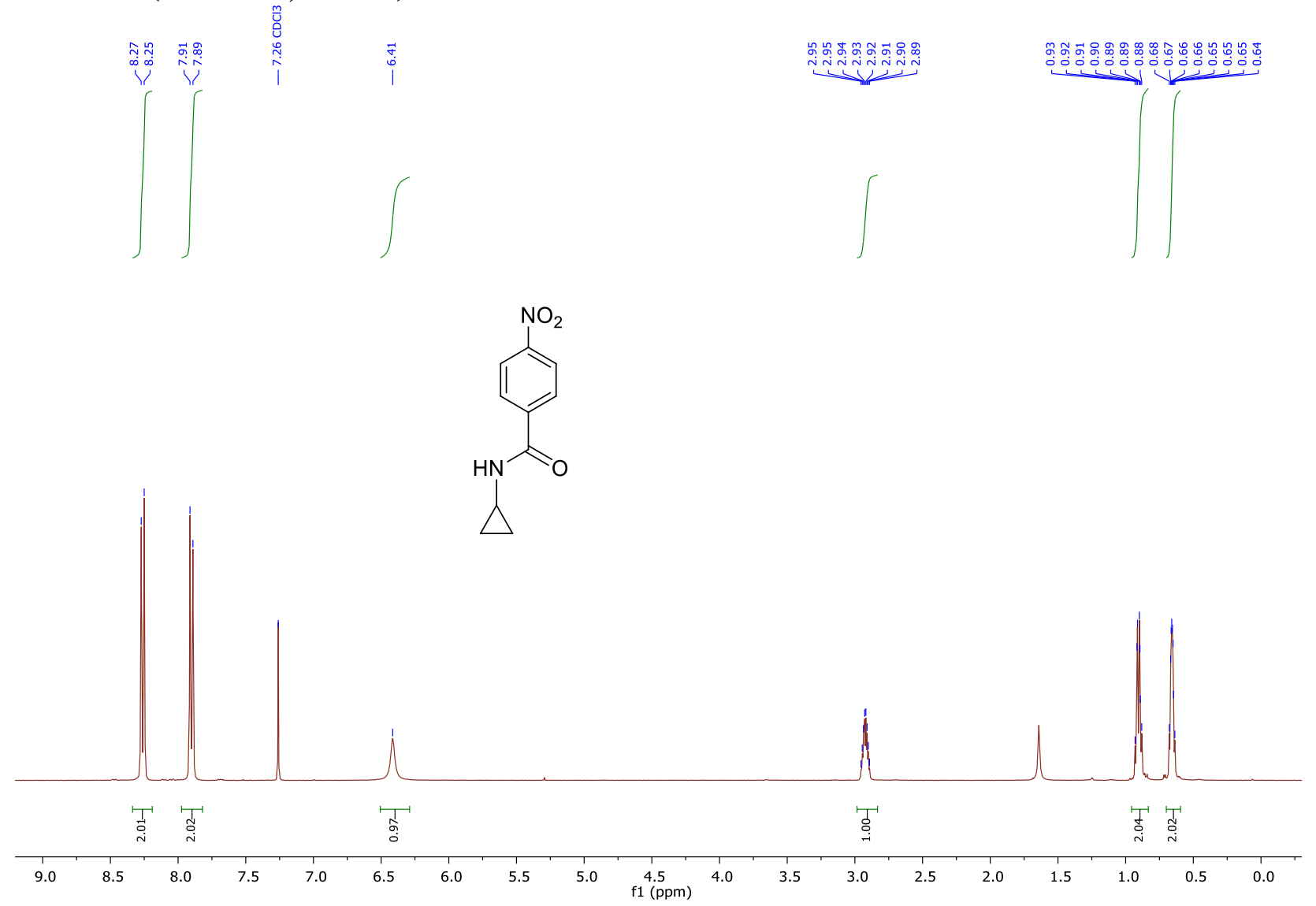

${ }^{13} \mathrm{C}-\mathrm{NMR}\left(101 \mathrm{MHz}, \mathrm{CDCl}_{3}\right)$ 

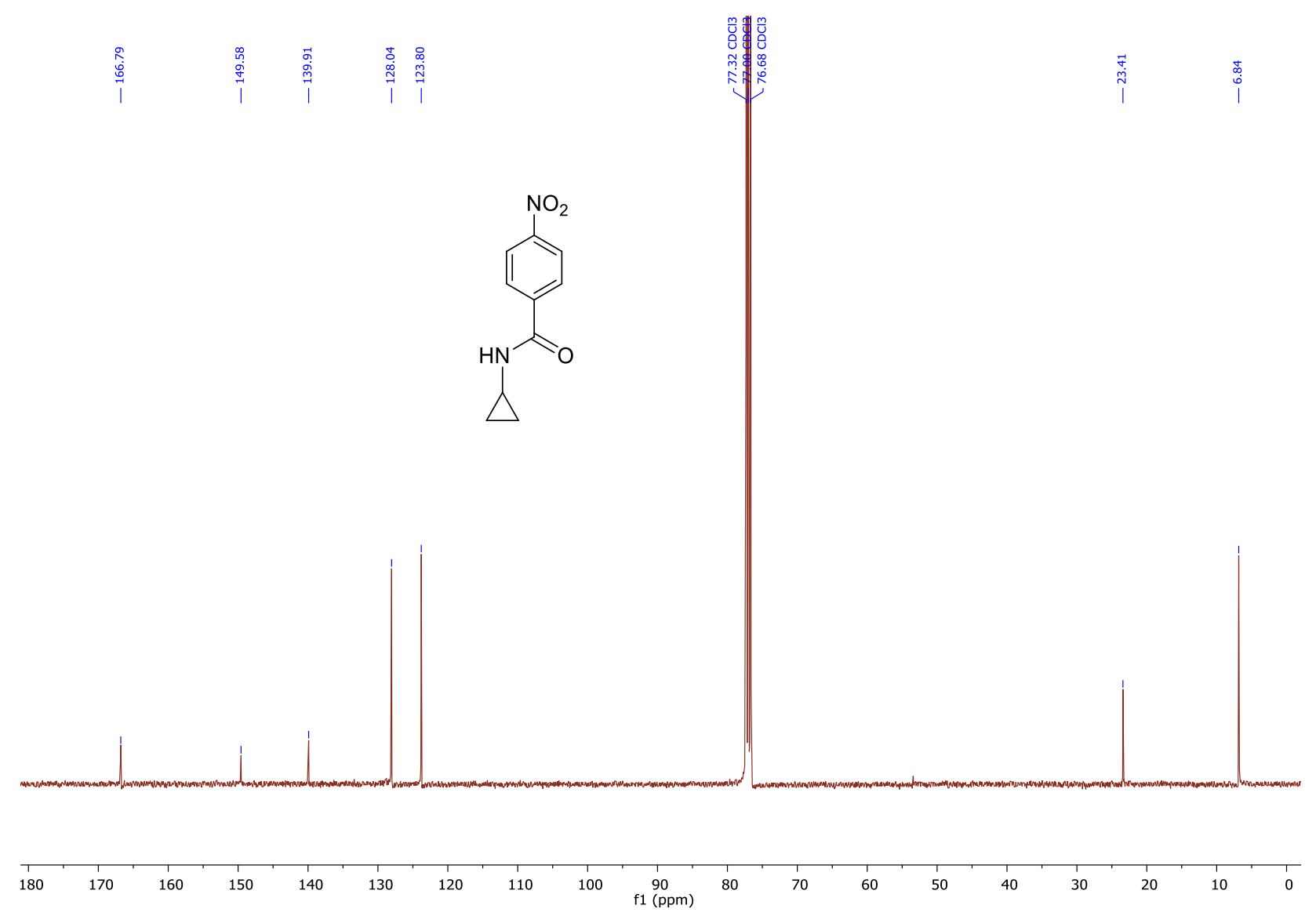

IR

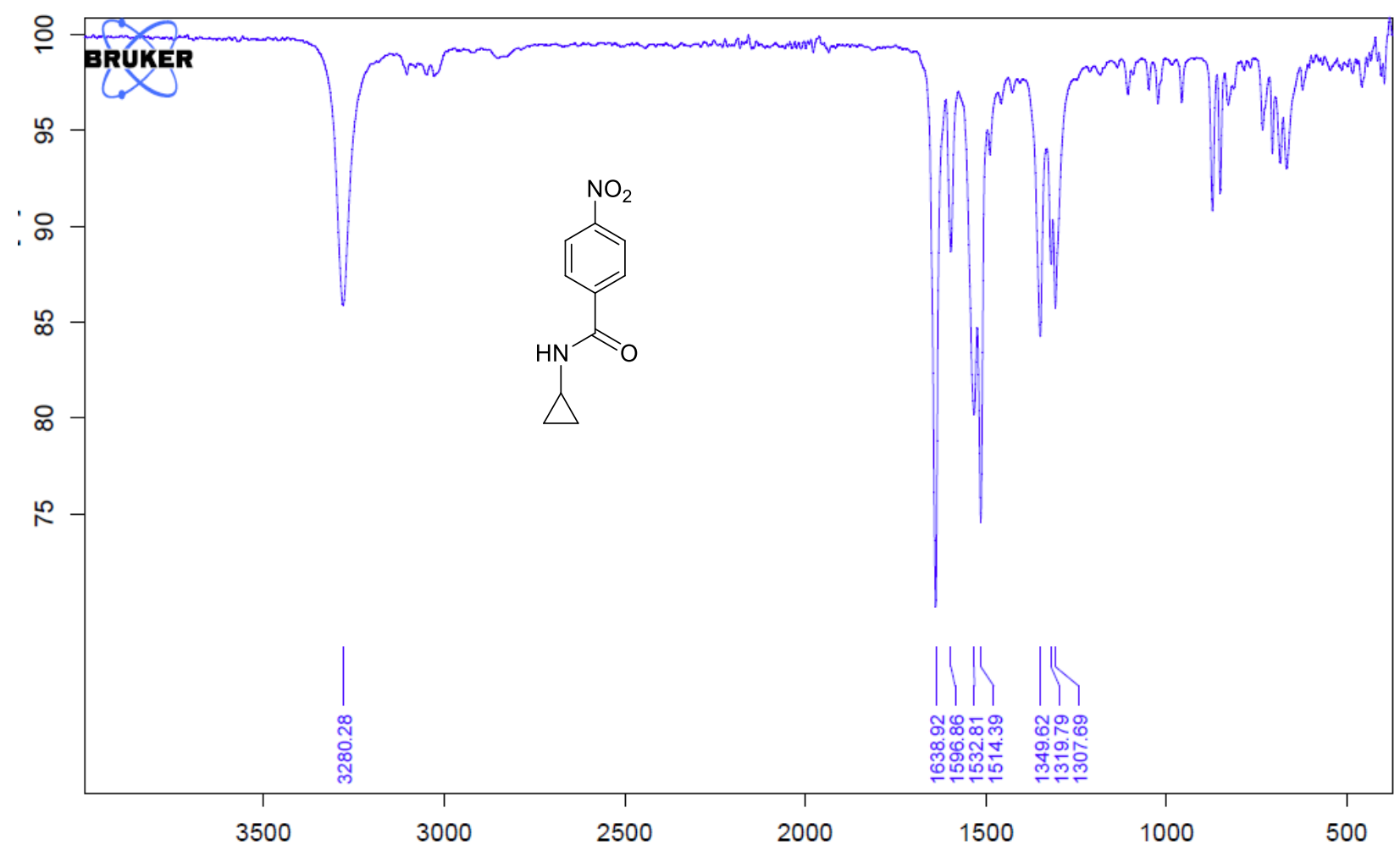

N-Cyclopropyl-3-methoxybenzamide (3g) 
${ }^{1} \mathrm{H}-\mathrm{NMR}$ (400 MHz, $\mathrm{CDCl}_{3}$ )

范

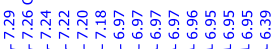

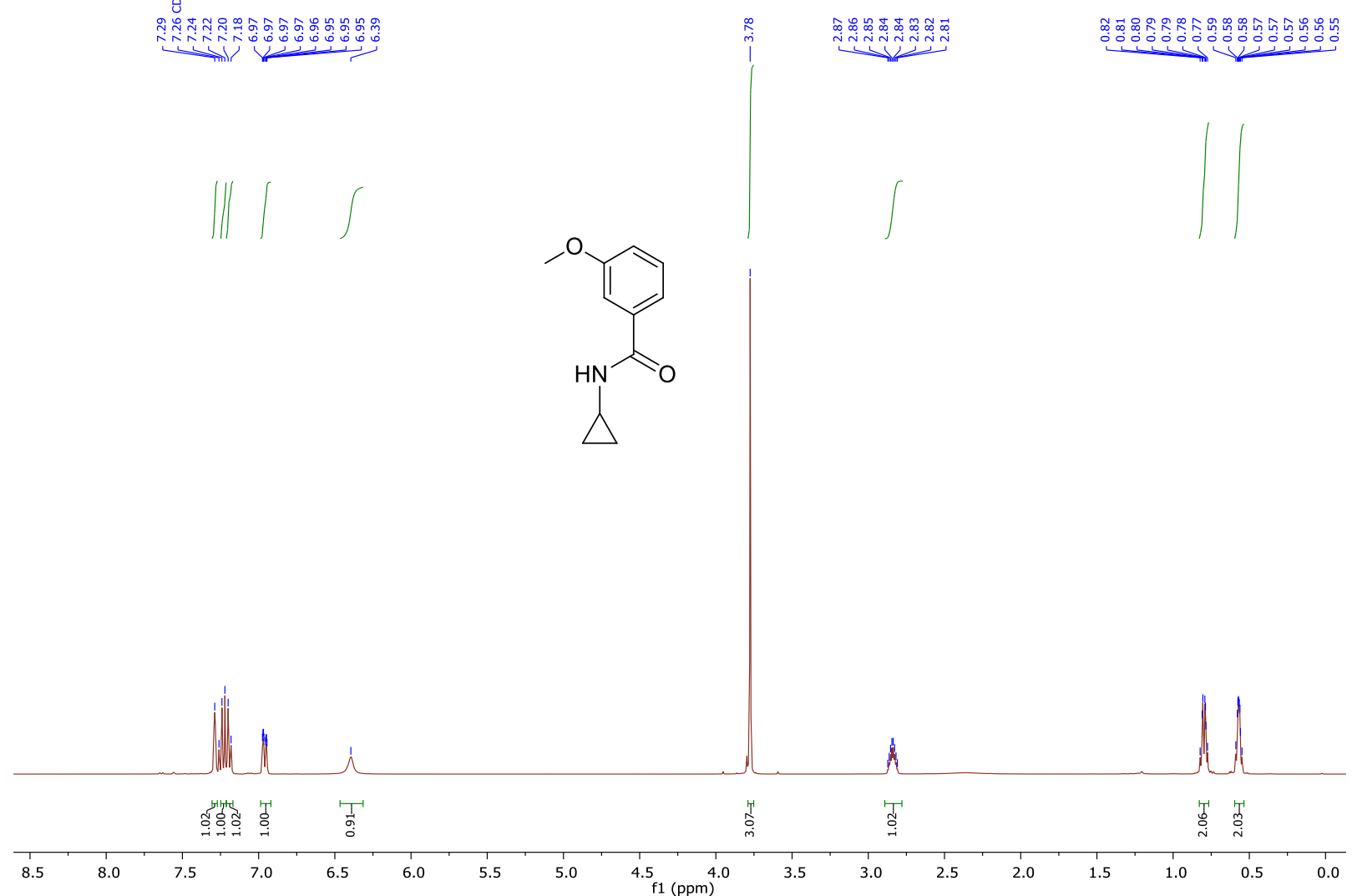

${ }^{13}$ C-NMR (101 MHz, CDCl $)$

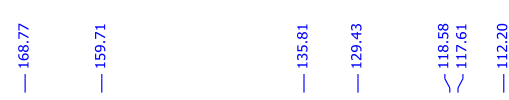
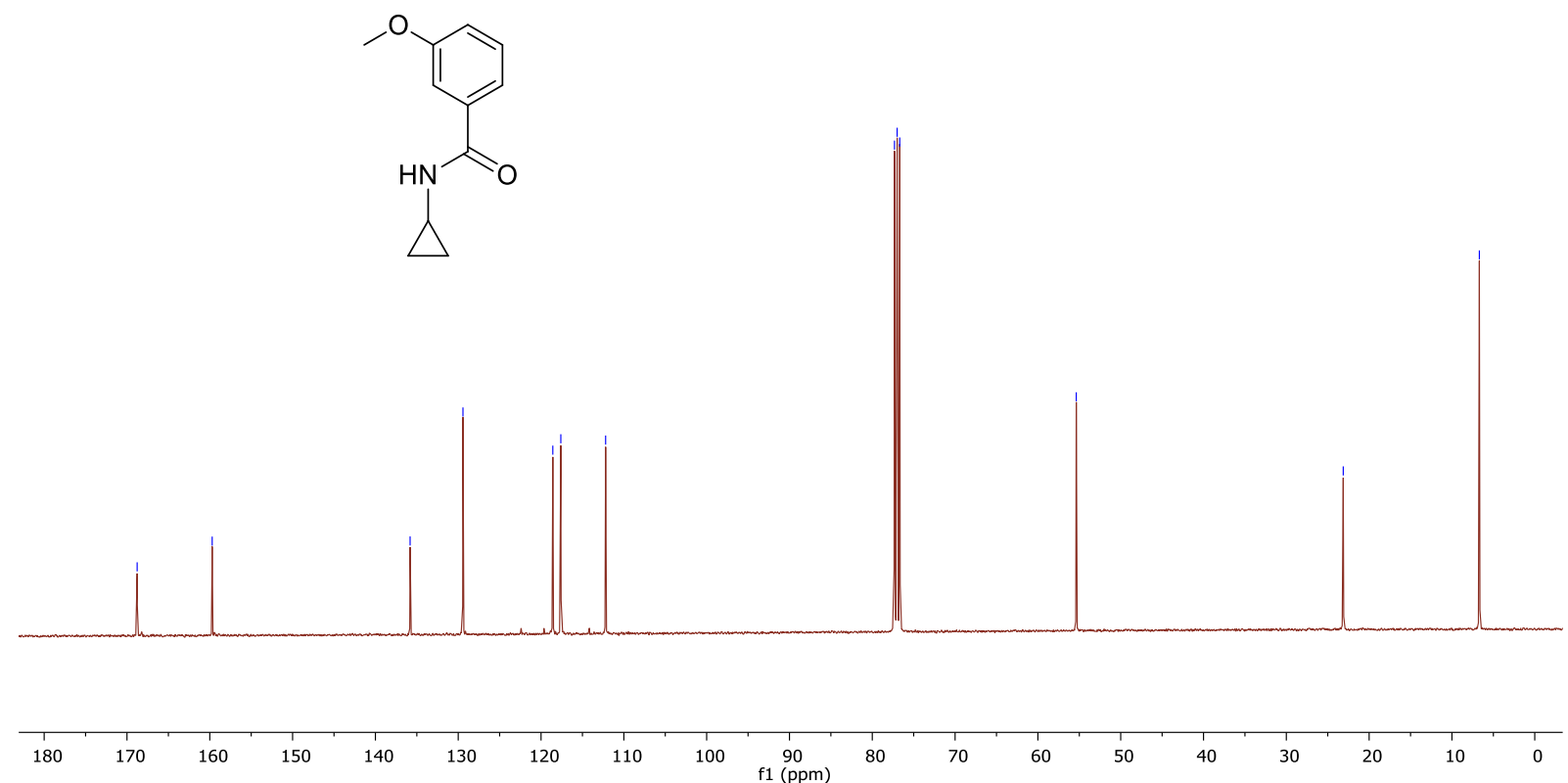

IR 


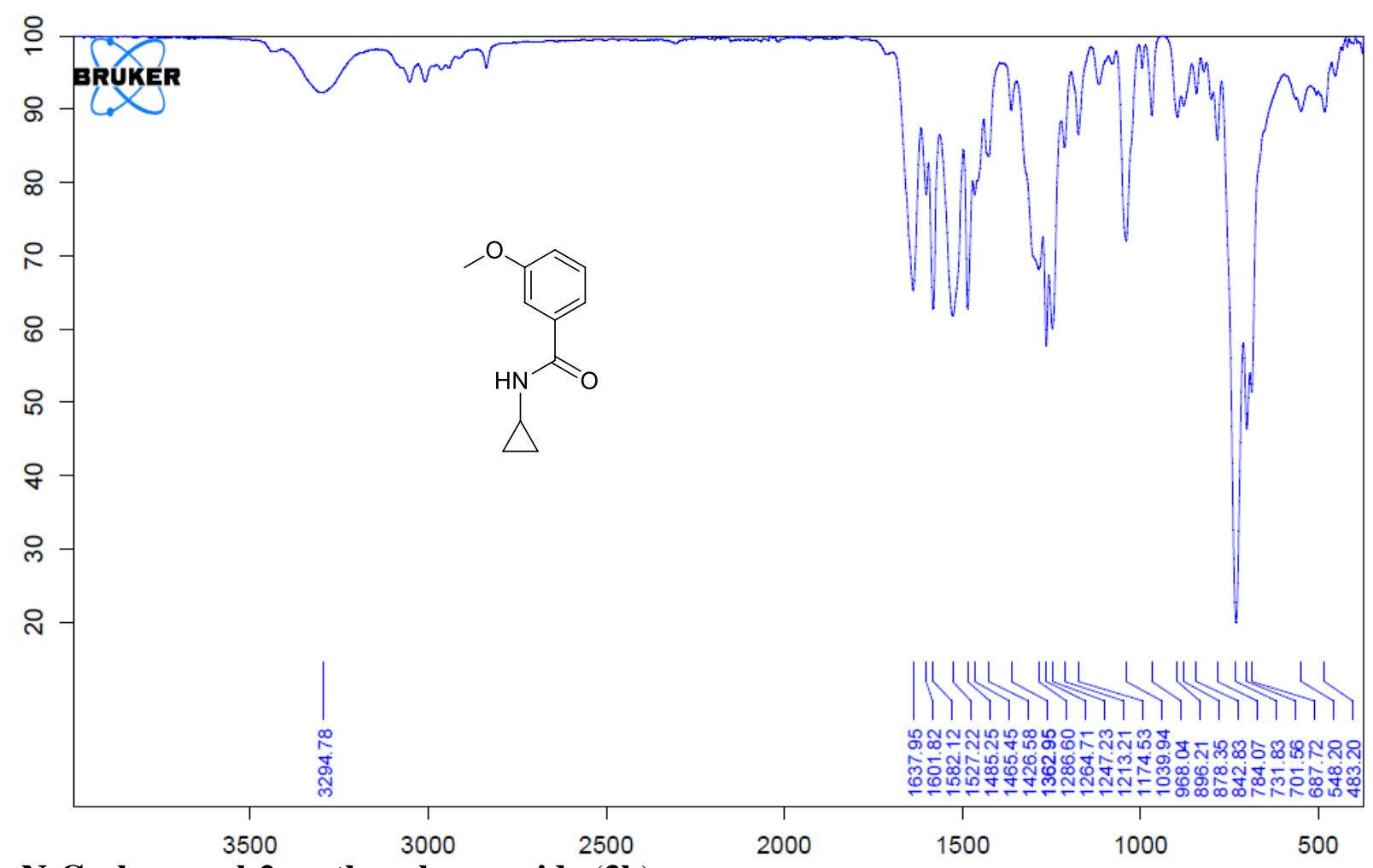

$\mathrm{N}$-Cyclopropyl-2-methoxybenzamide (3h)

${ }^{1} \mathrm{H}-\mathrm{NMR}$ (400 MHz, $\mathrm{CDCl}_{3}$ )

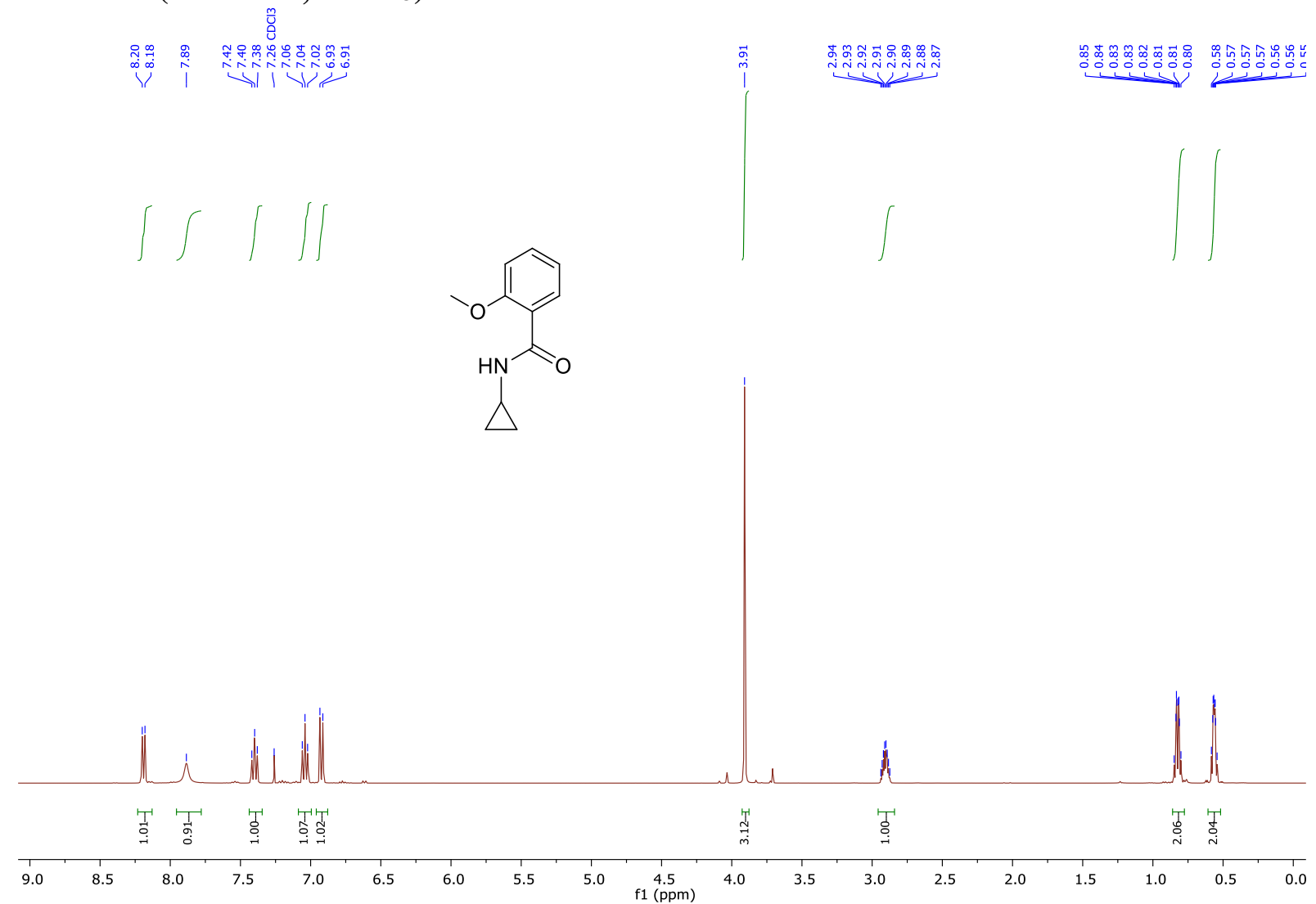

${ }^{13} \mathrm{C}$-NMR (101 MHz, $\left.\mathrm{CDCl}_{3}\right)$ 

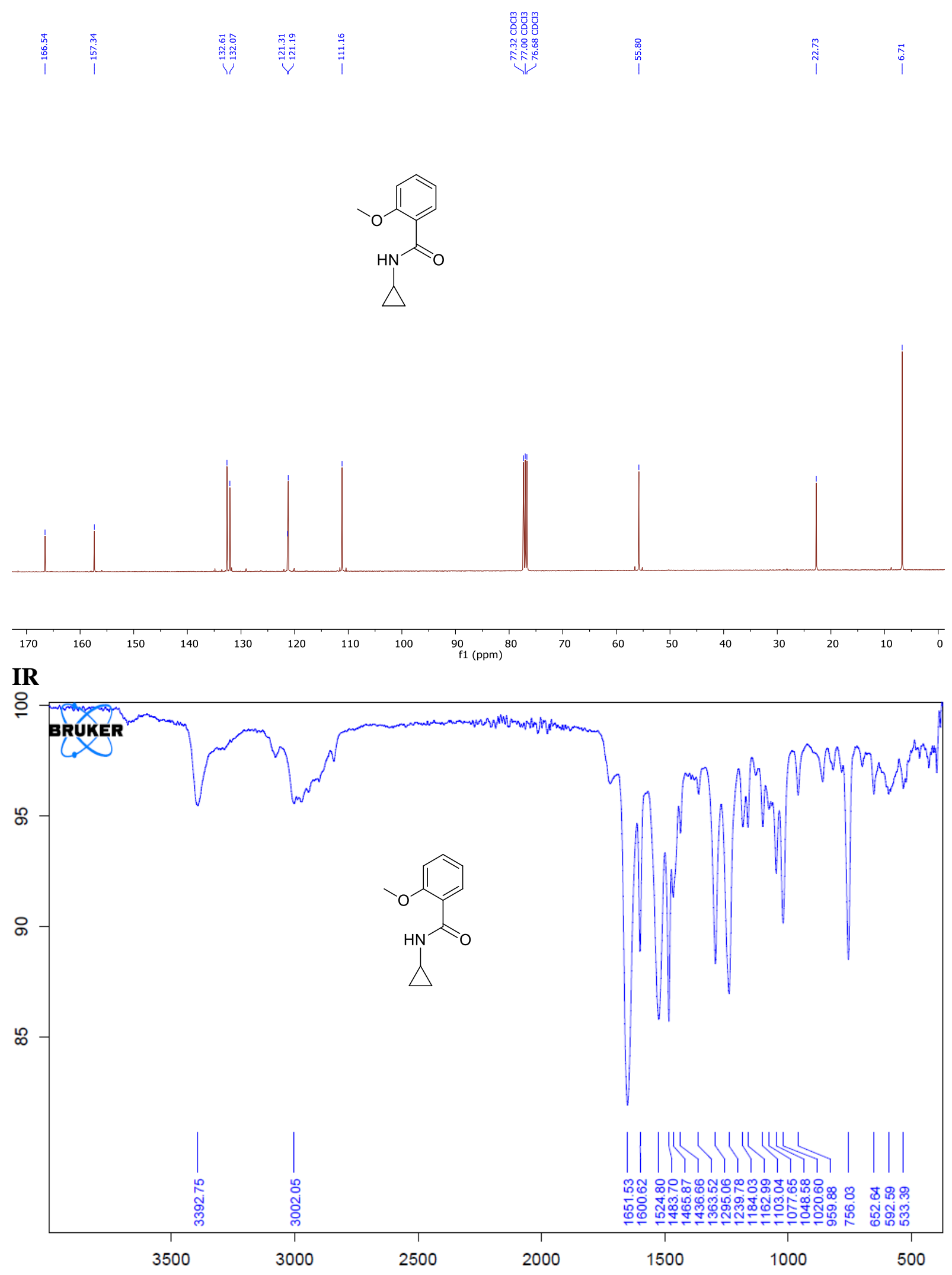

N-Cyclopropyl-2,4,6-trimethylbenzamide (3i) 
${ }^{1} \mathrm{H}-\mathrm{NMR}$ (400 MHz, CDCl 3 )

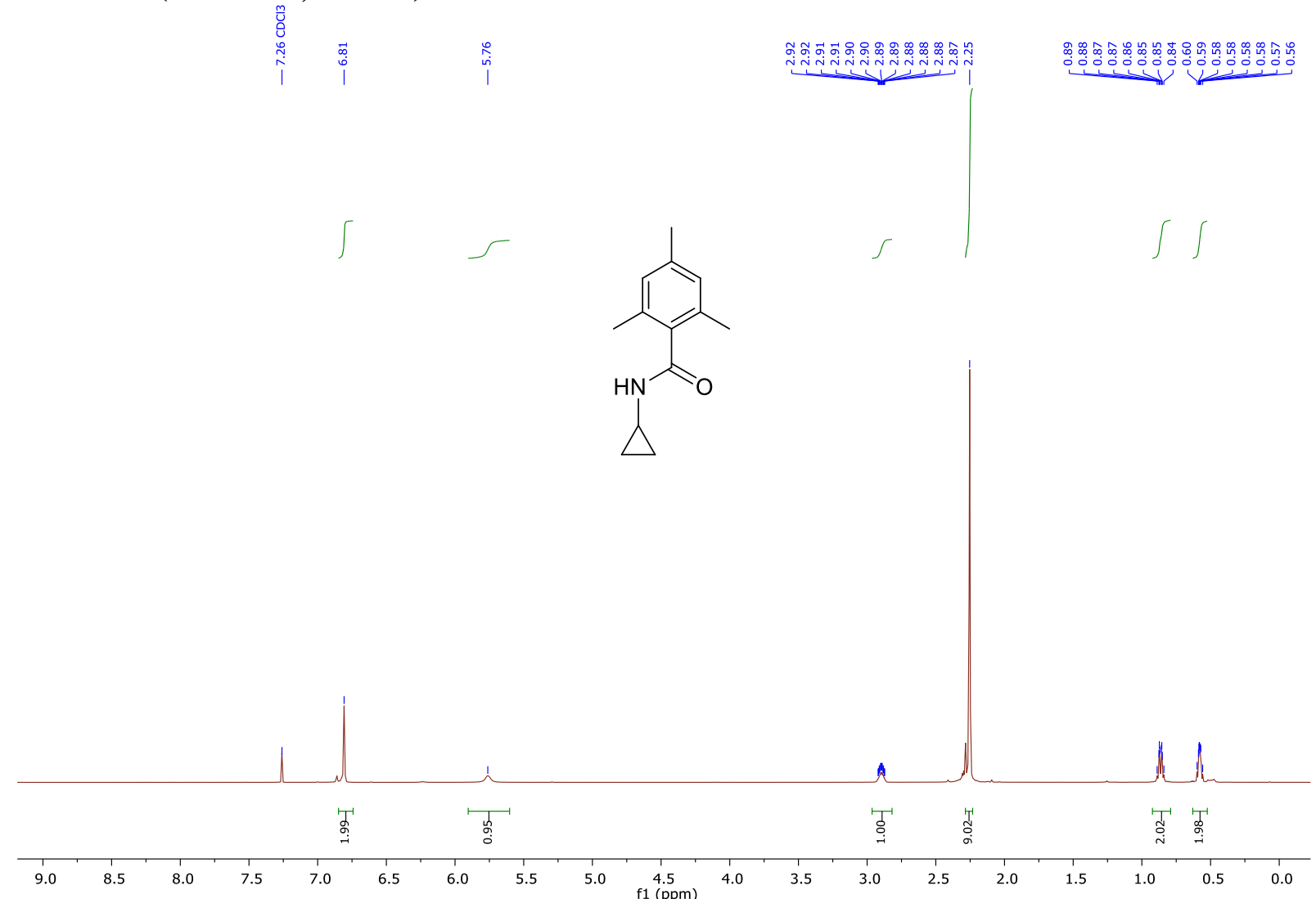

${ }^{13} \mathrm{C}$-NMR (101 MHz, CDCl3)

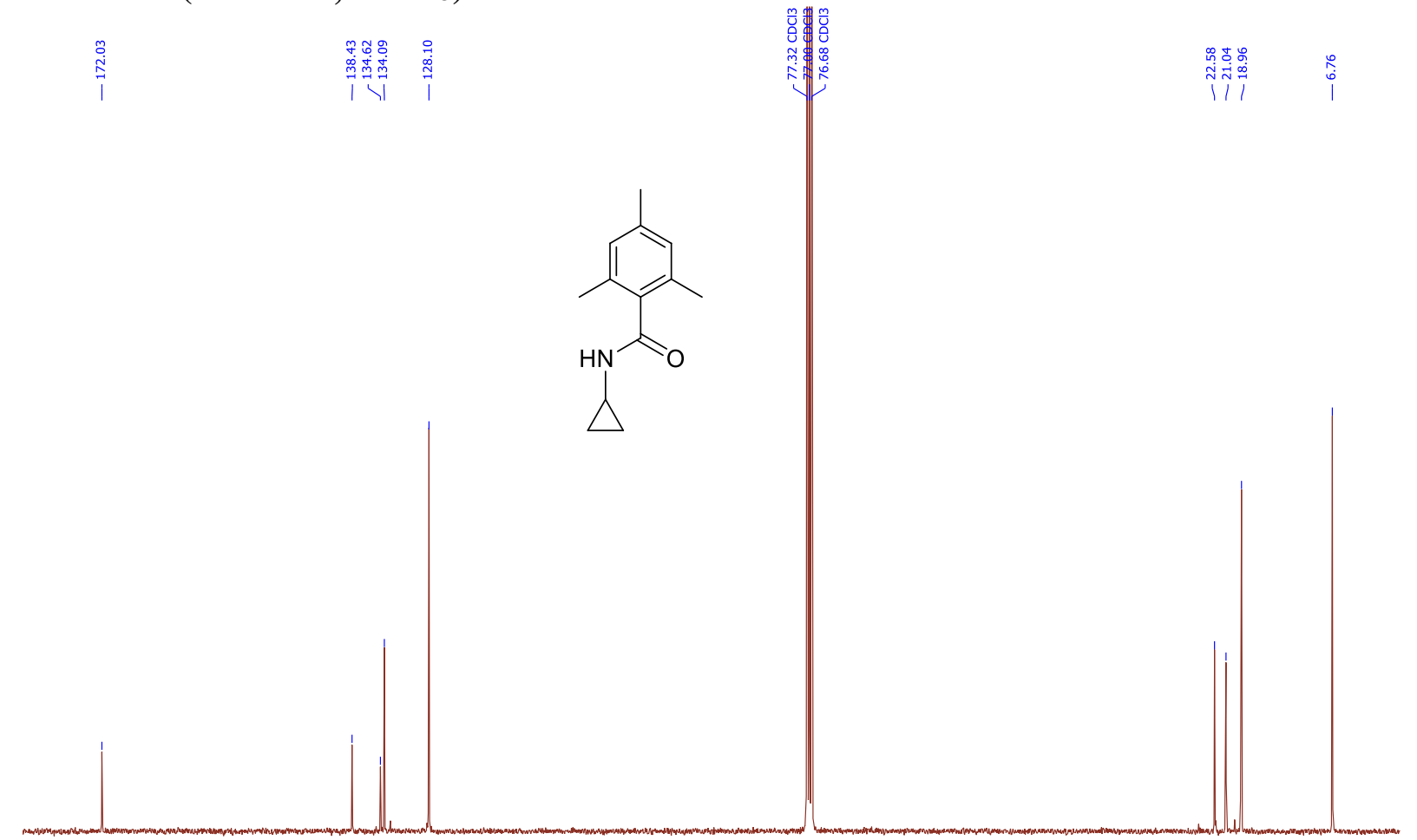

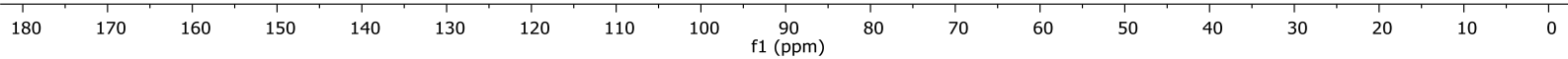

IR 


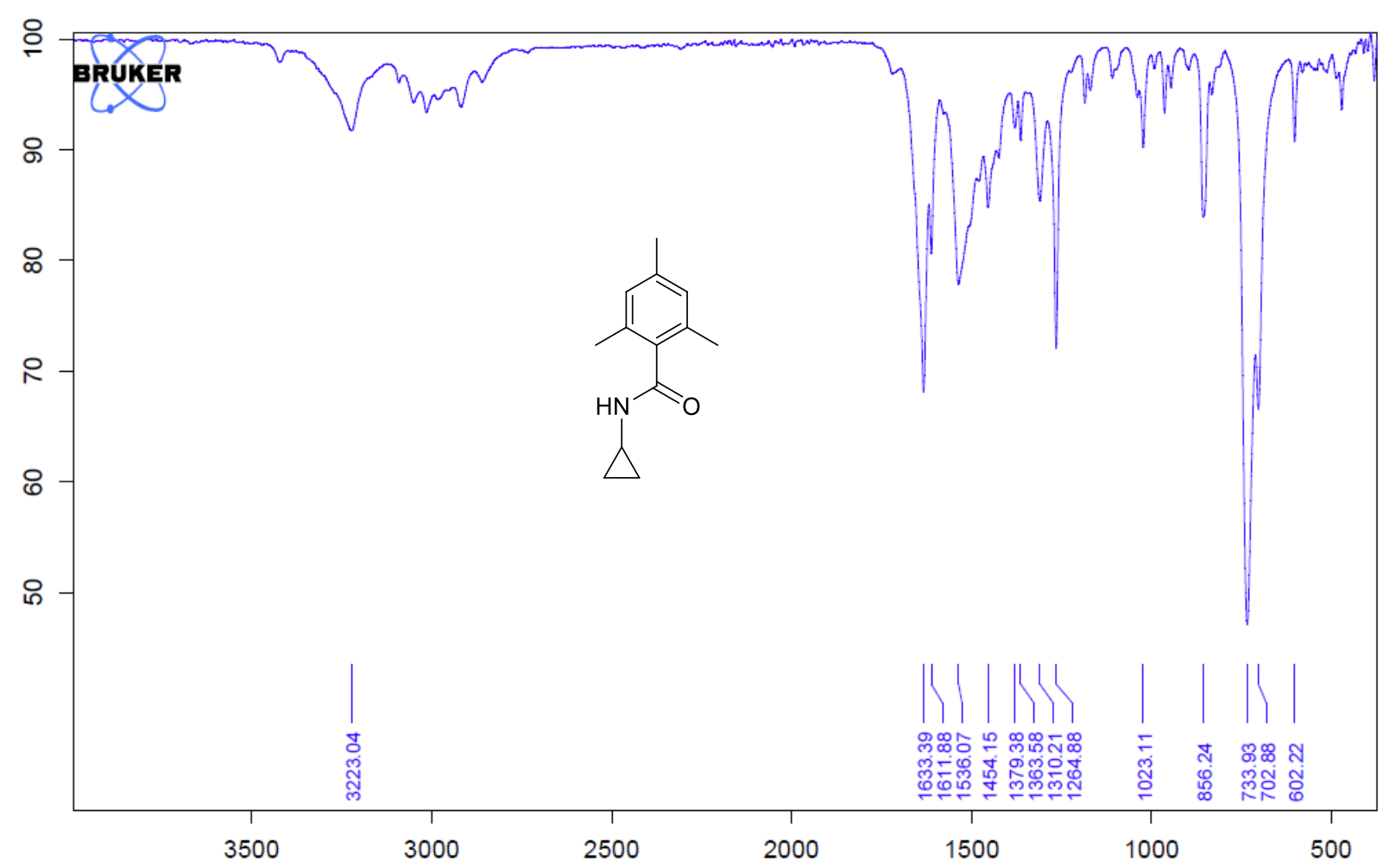

N-Cyclopropyl-2-naphthamide (3m)

${ }^{1} \mathrm{H}-\mathrm{NMR}$ (400 MHz, $\mathrm{CDCl}_{3}$ )

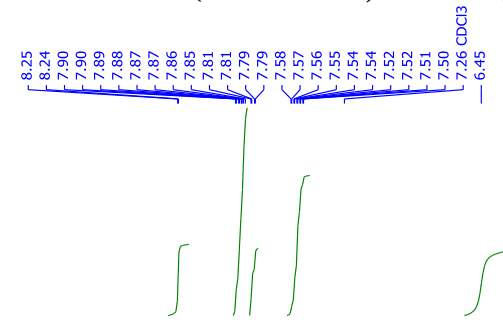<smiles>O=C(NC1CC1)c1ccc2ccccc2c1</smiles>

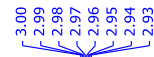

$\underbrace{3}$
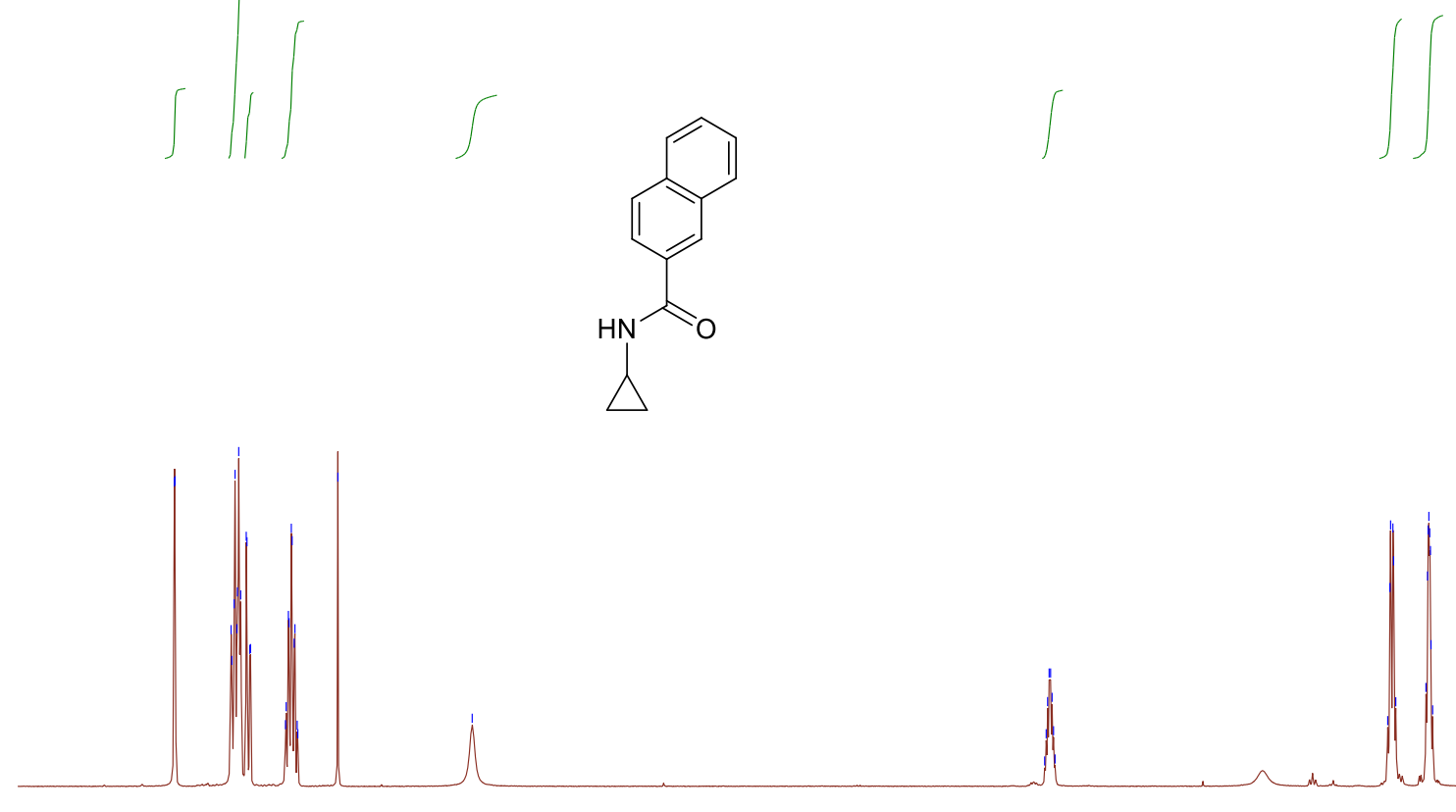

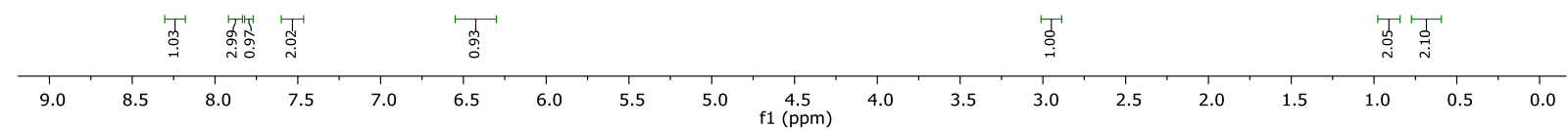


${ }^{13}$ C-NMR (101 MHz, CDCl 3$)$
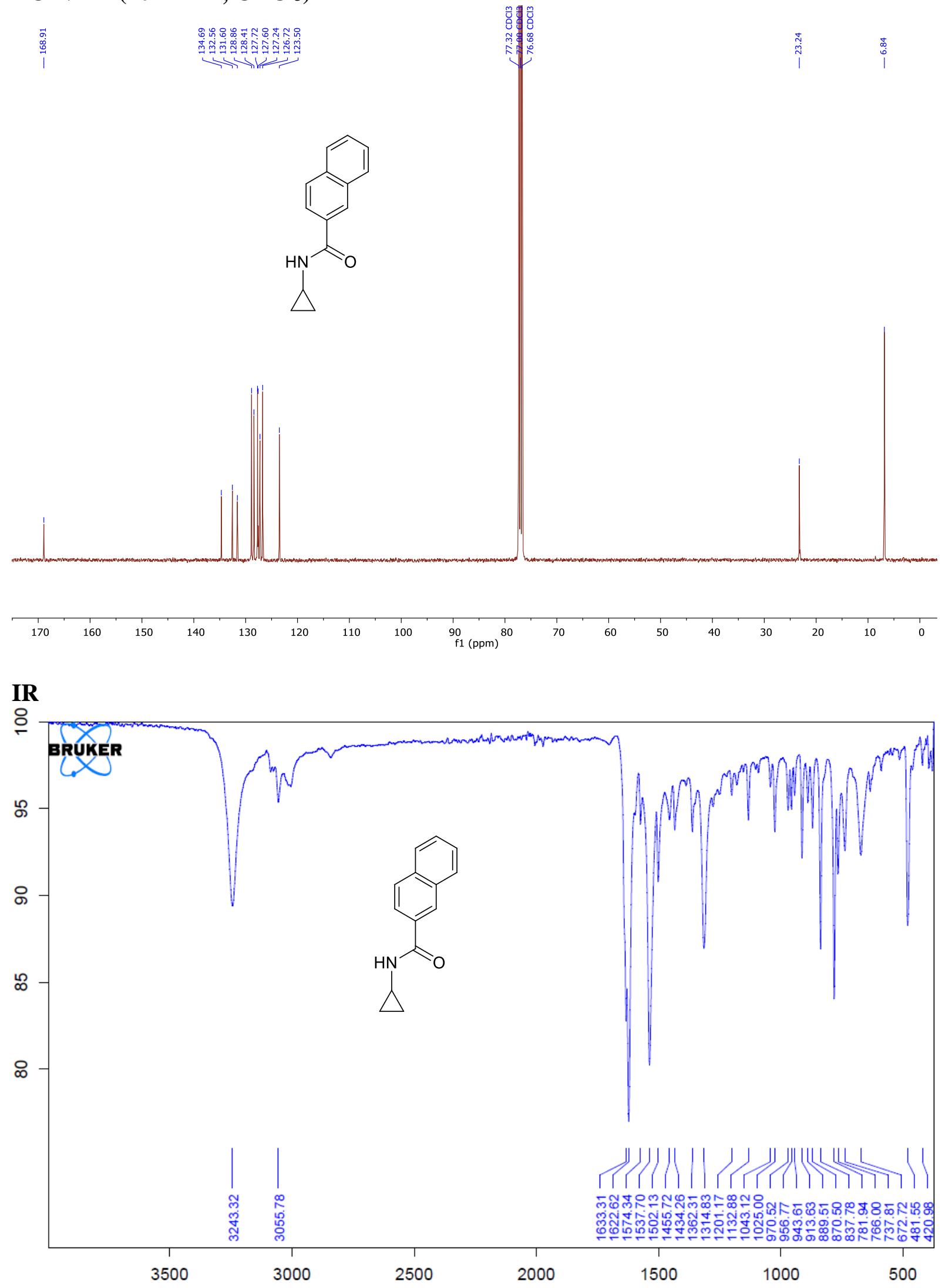
N-Cyclopropyl-N,4-dimethylbenzenesulfonamide (3r)

${ }^{1} \mathrm{H}-\mathrm{NMR}$ (400 MHz, CDCl 3$)$

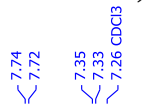

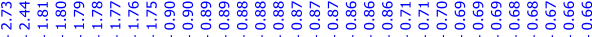
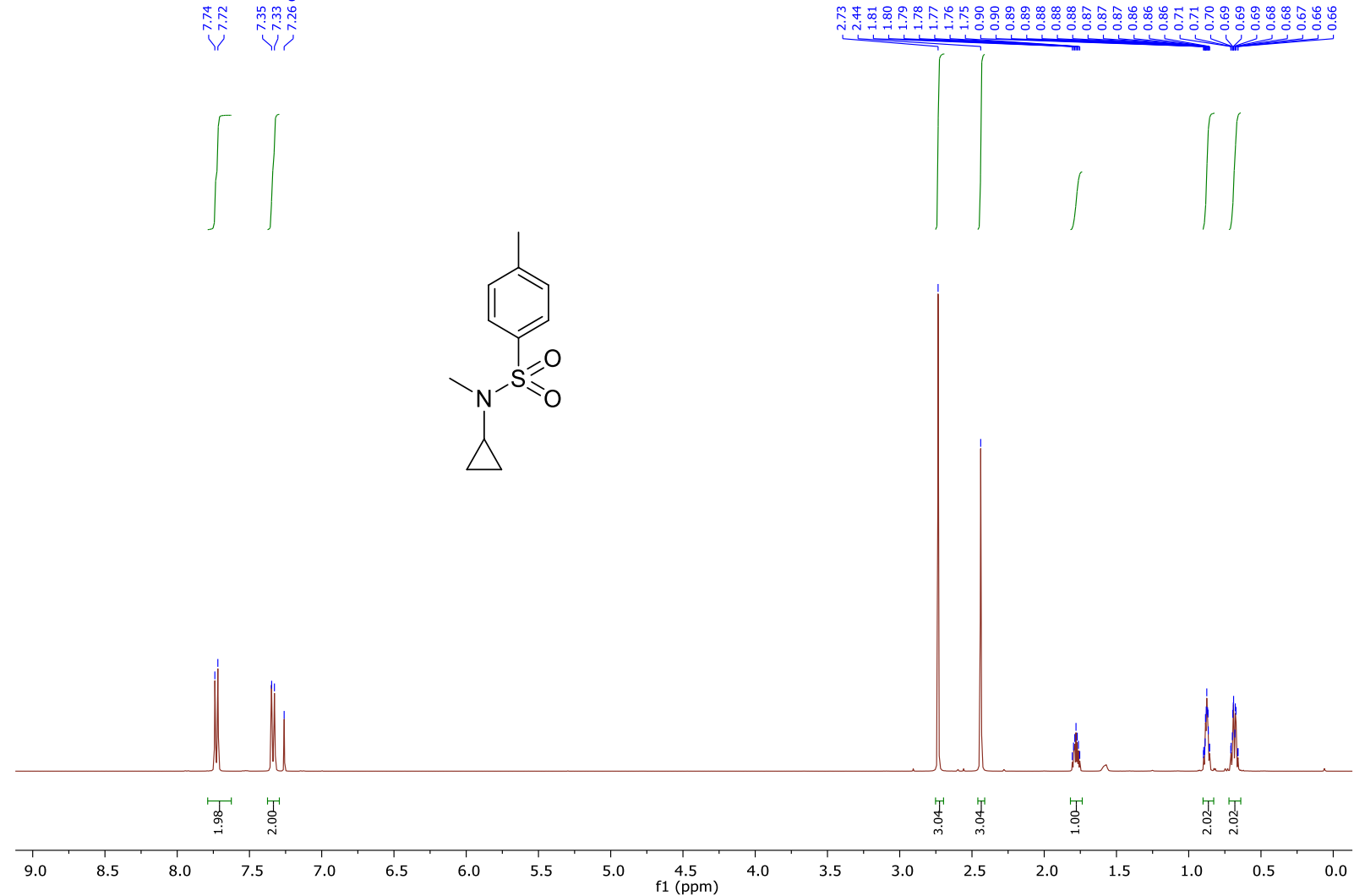

${ }^{13} \mathrm{C}-\mathrm{NMR}$ (101 MHz, CDCl3)
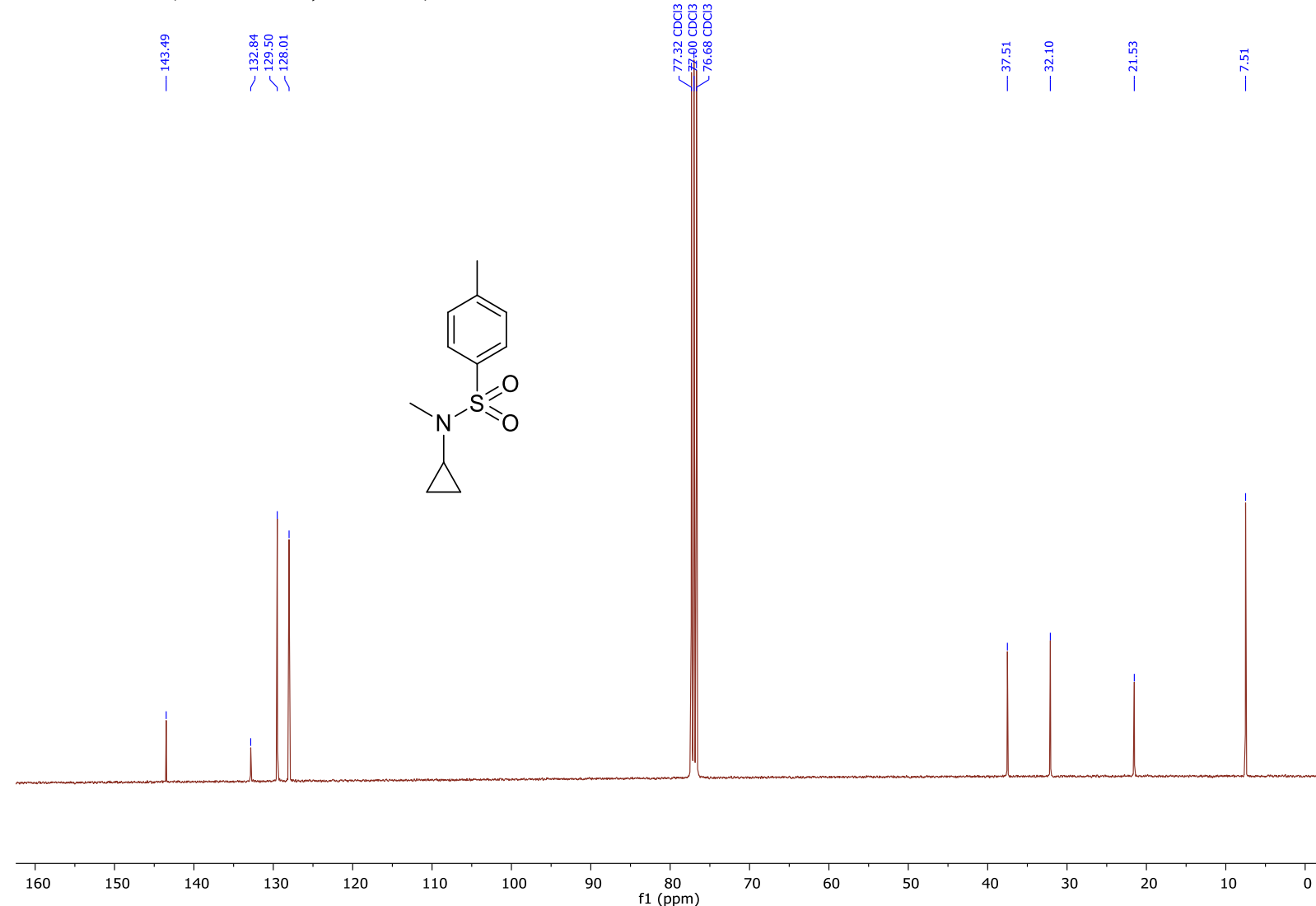


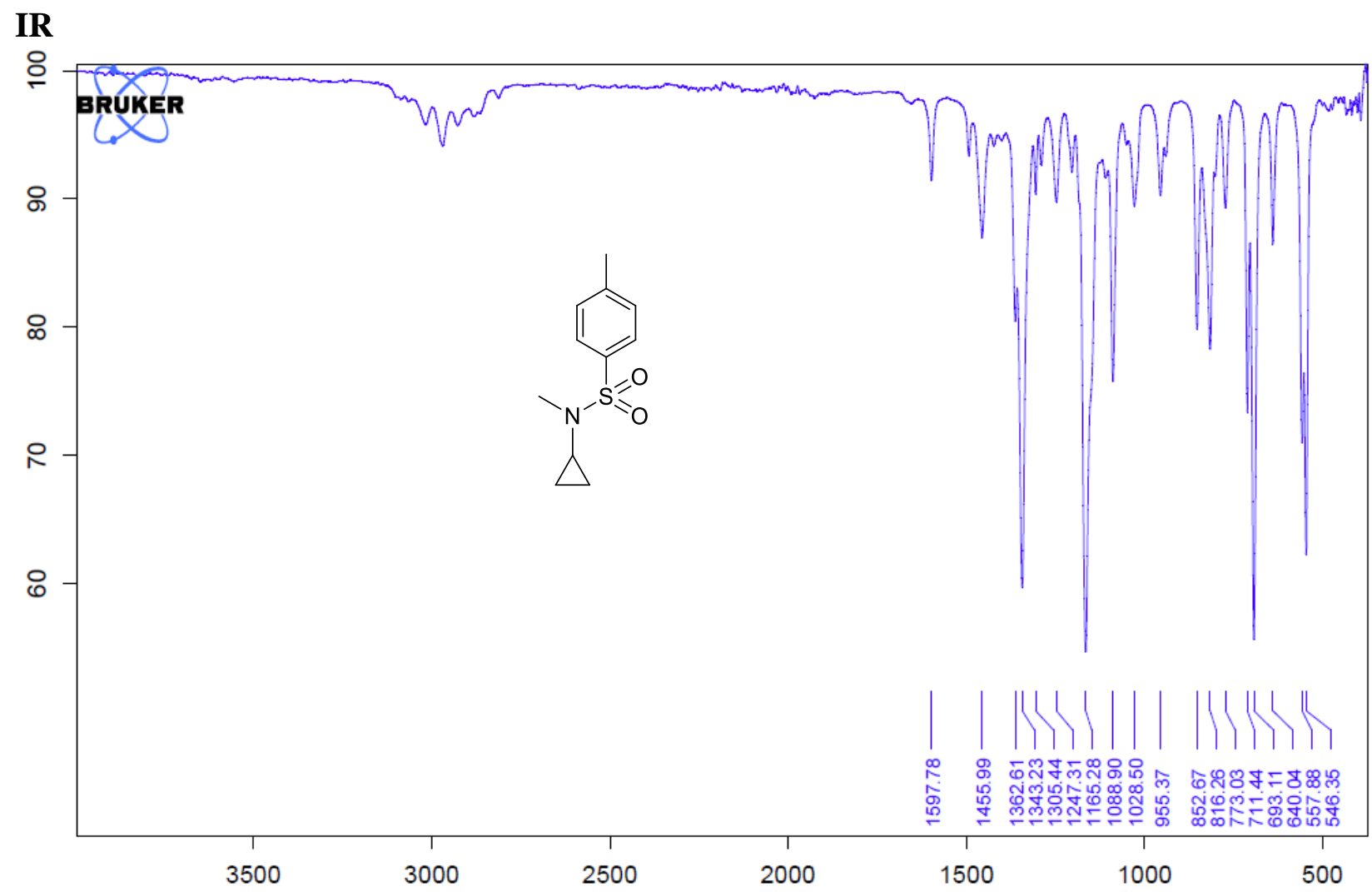

Benzyl (S)-(1-(cyclopropylamino)-1-oxo-3-phenylpropan-2-yl)carbamate (3t) ${ }^{1} \mathrm{H}-\mathrm{NMR}$ (400 $\left.\mathrm{MHz}, \mathrm{CDCl}_{3}\right)$

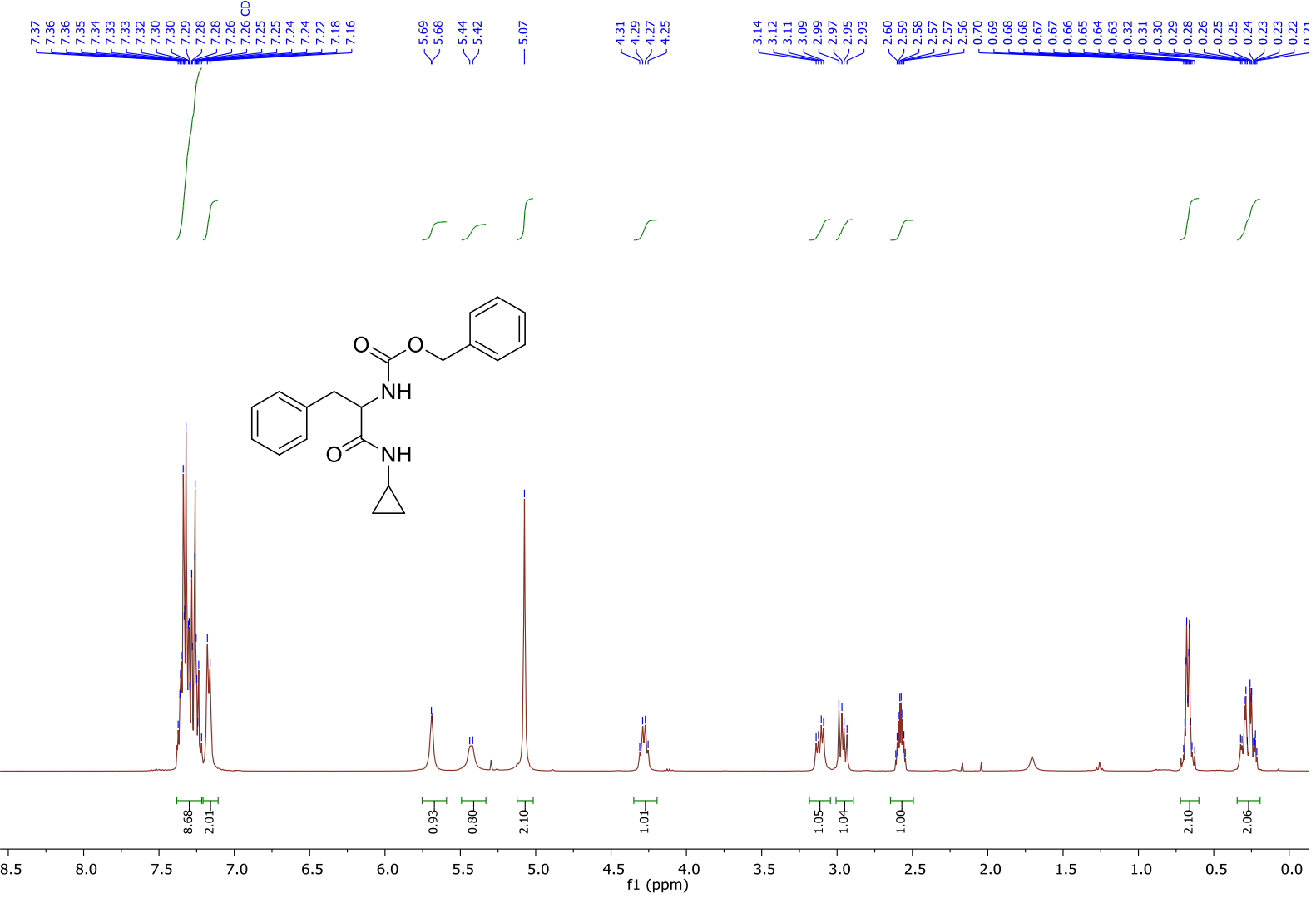


${ }^{13}$ C-NMR (101 MHz, CDCl 3 )

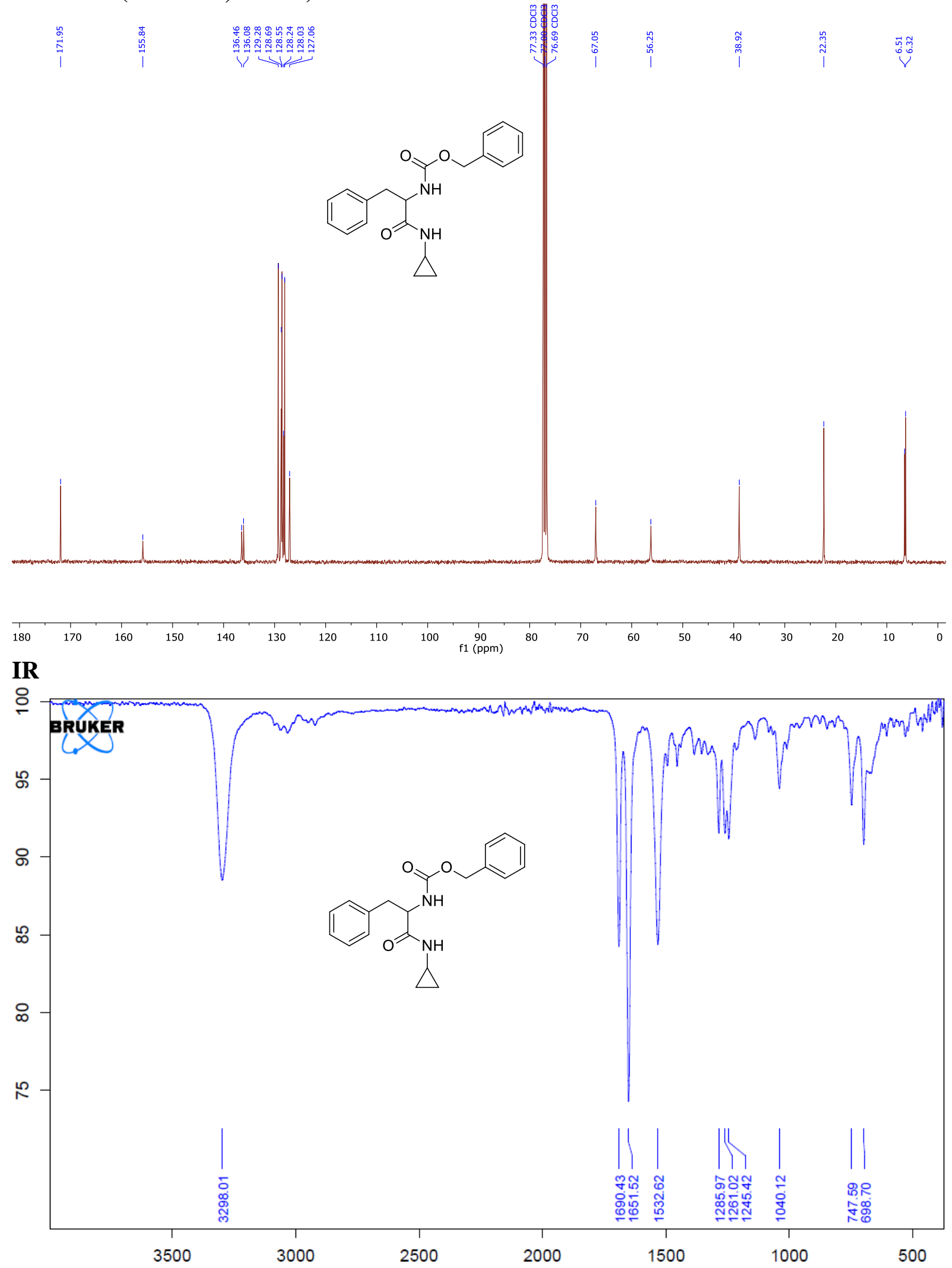


N-Cyclopropyl-2-(11-oxo-6,11-dihydrodibenzo[b,e]oxepin-2-yl)acetamide (3u)

${ }^{1} \mathrm{H}-\mathrm{NMR}\left(400 \mathrm{MHz}, \mathrm{CDCl}_{3}\right)$
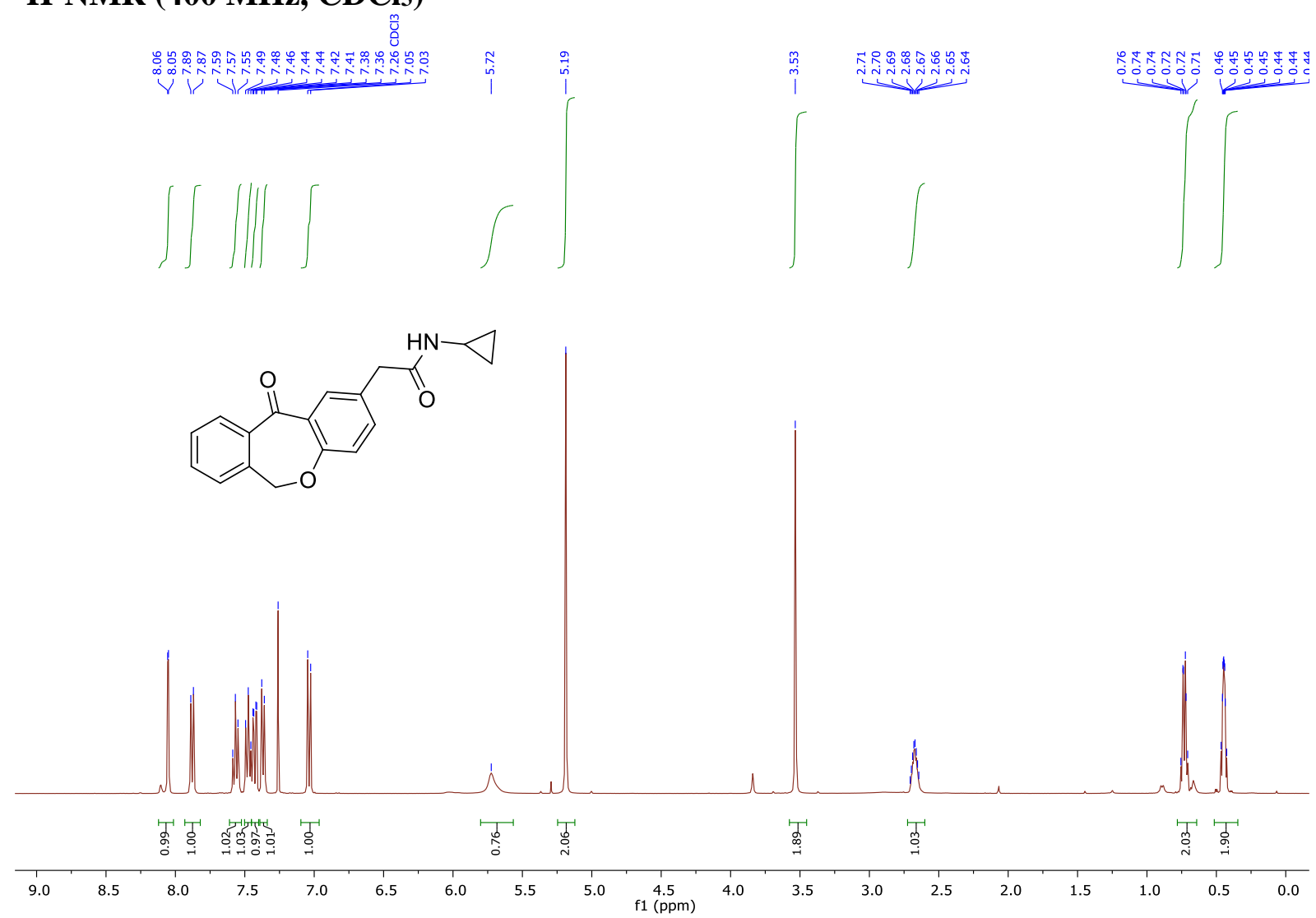

${ }^{13}$ C-NMR (101 MHz, CDCl3)

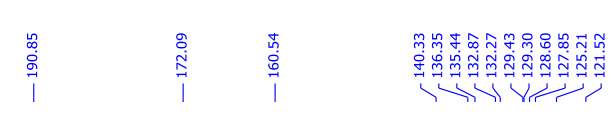<smiles>O=C(Cc1ccc2c(c1)C(=O)c1ccccc1CO2)NC1CC1</smiles>

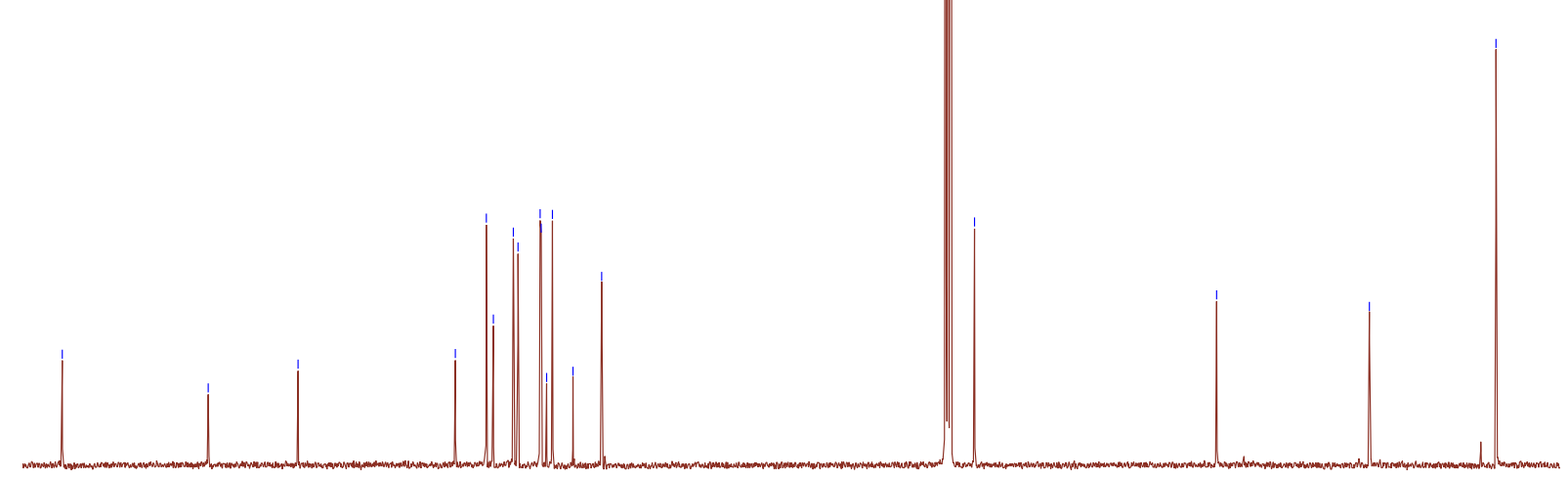

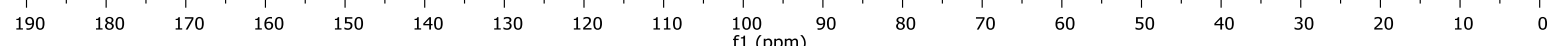




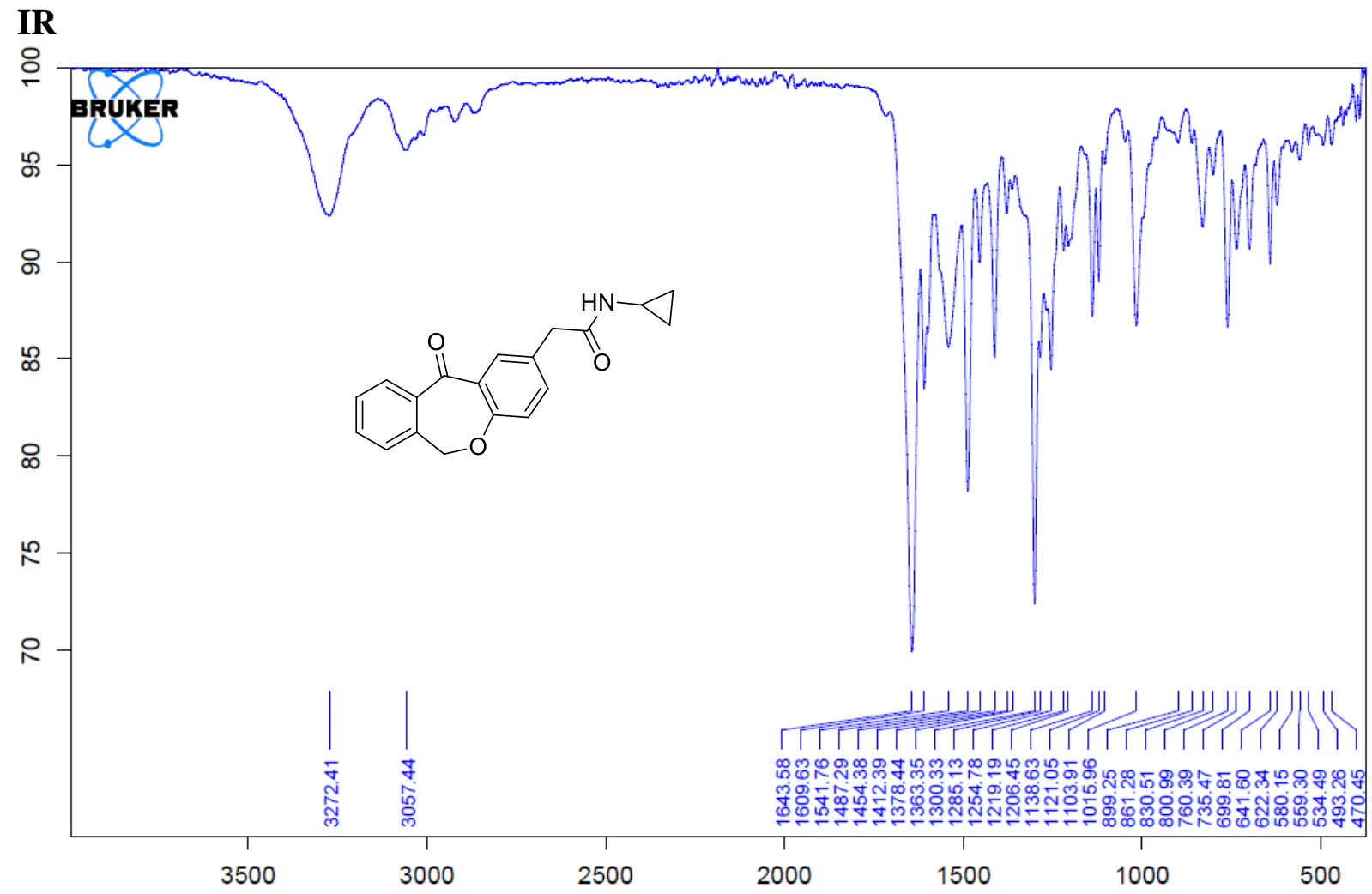

2-(1-(4-Chlorobenzoyl)-5-methoxy-2-methyl-1H-indol-3-yl)-N-cyclopropylacetamide (3v) ${ }^{1} \mathrm{H}-\mathrm{NMR}\left(400 \mathrm{MHz}, \mathrm{CDCl}_{3}\right)$

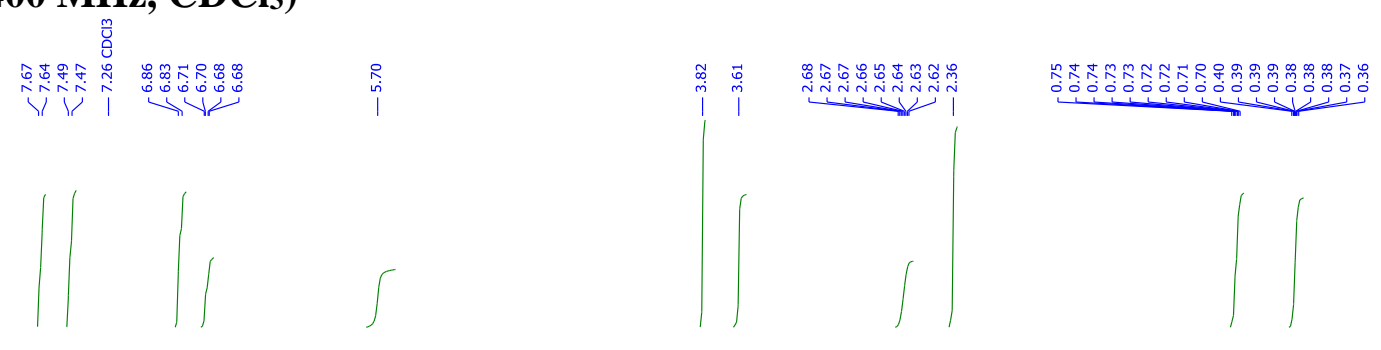<smiles>COc1ccc2c(c1)c(CC(=O)NC1CC1)c(C)n2C(=O)c1ccc(Cl)cc1</smiles>

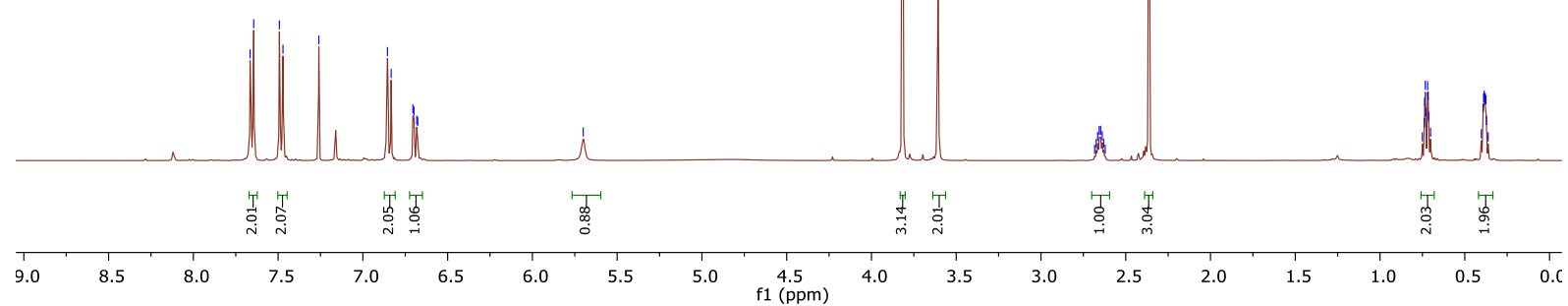


${ }^{13} \mathrm{C}-\mathrm{NMR}$ (101 MHz, $\left.\mathrm{CDCl}_{3}\right)$

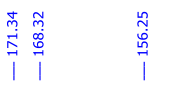<smiles>COc1ccc2c(c1)c(CC(=O)NC1CC1)c(C)n2C(=O)c1ccc(Cl)cc1</smiles>
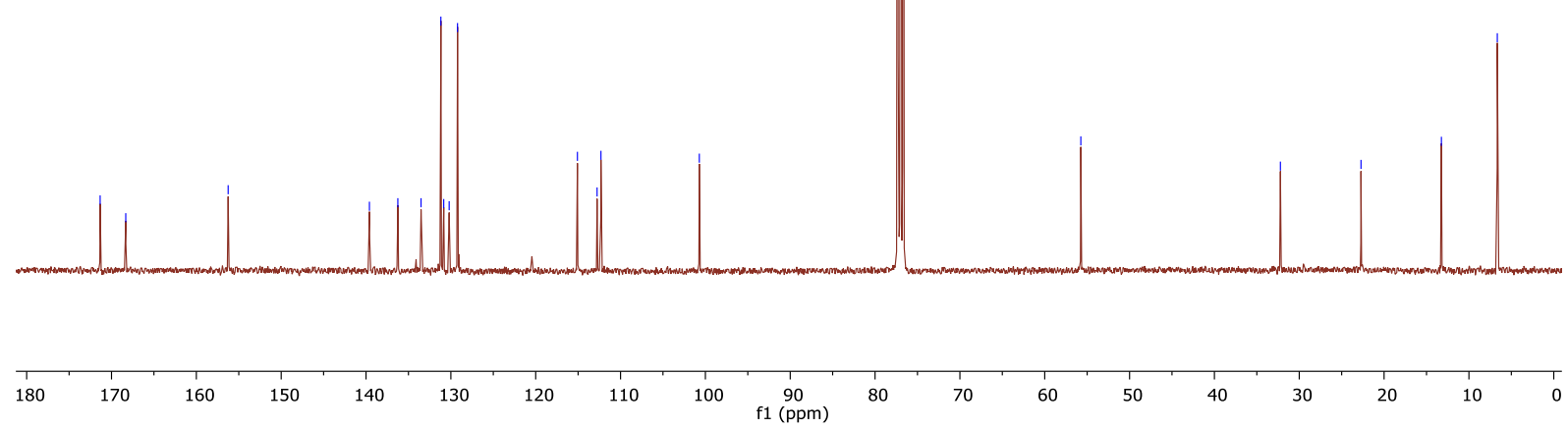

IR

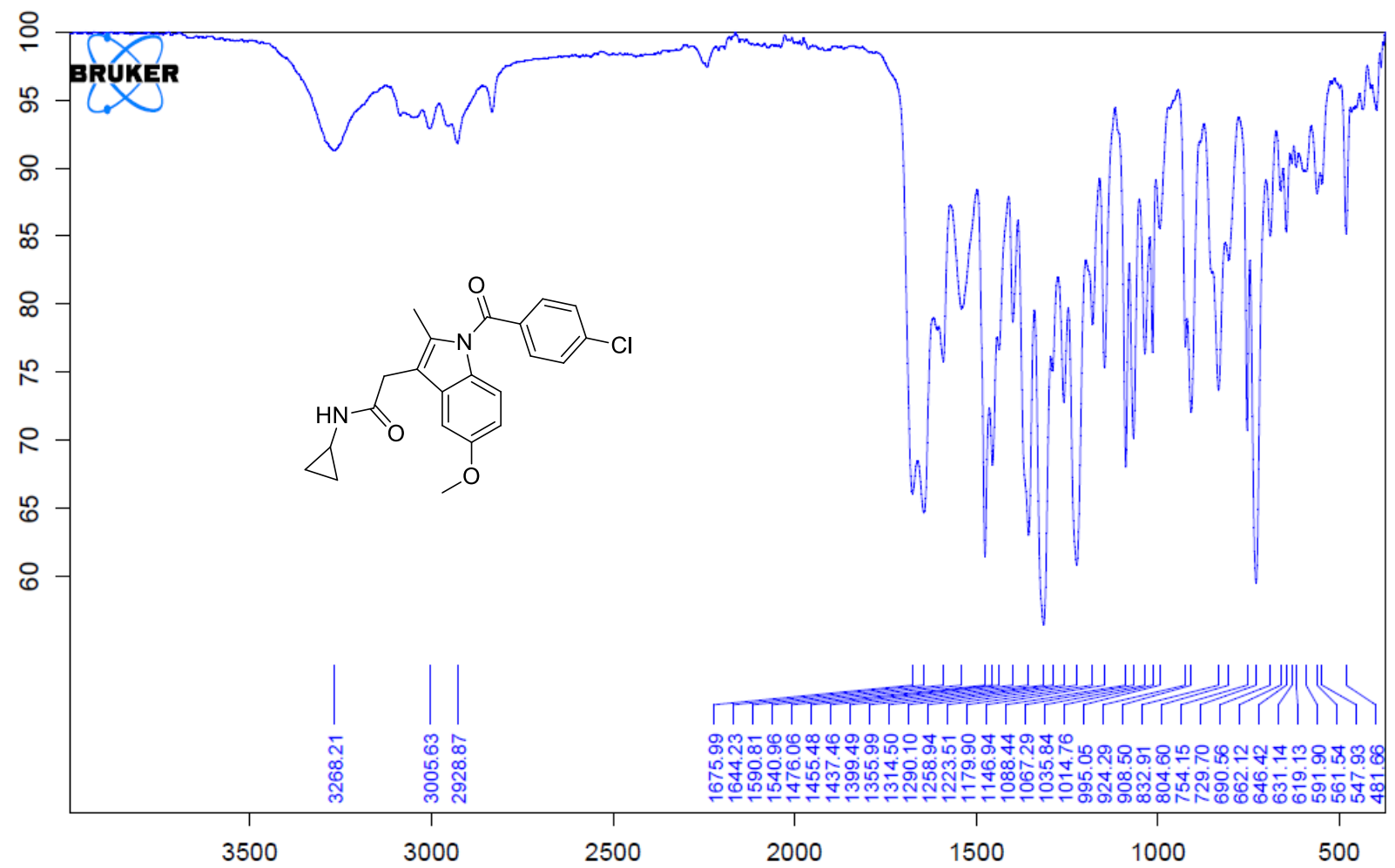


N-(3-Iodo-1-methoxypropyl)-4-methoxybenzamide (4a)

${ }^{1} \mathrm{H}-\mathrm{NMR}$ (400 MHz, $\mathrm{CDCl}_{3}$ )

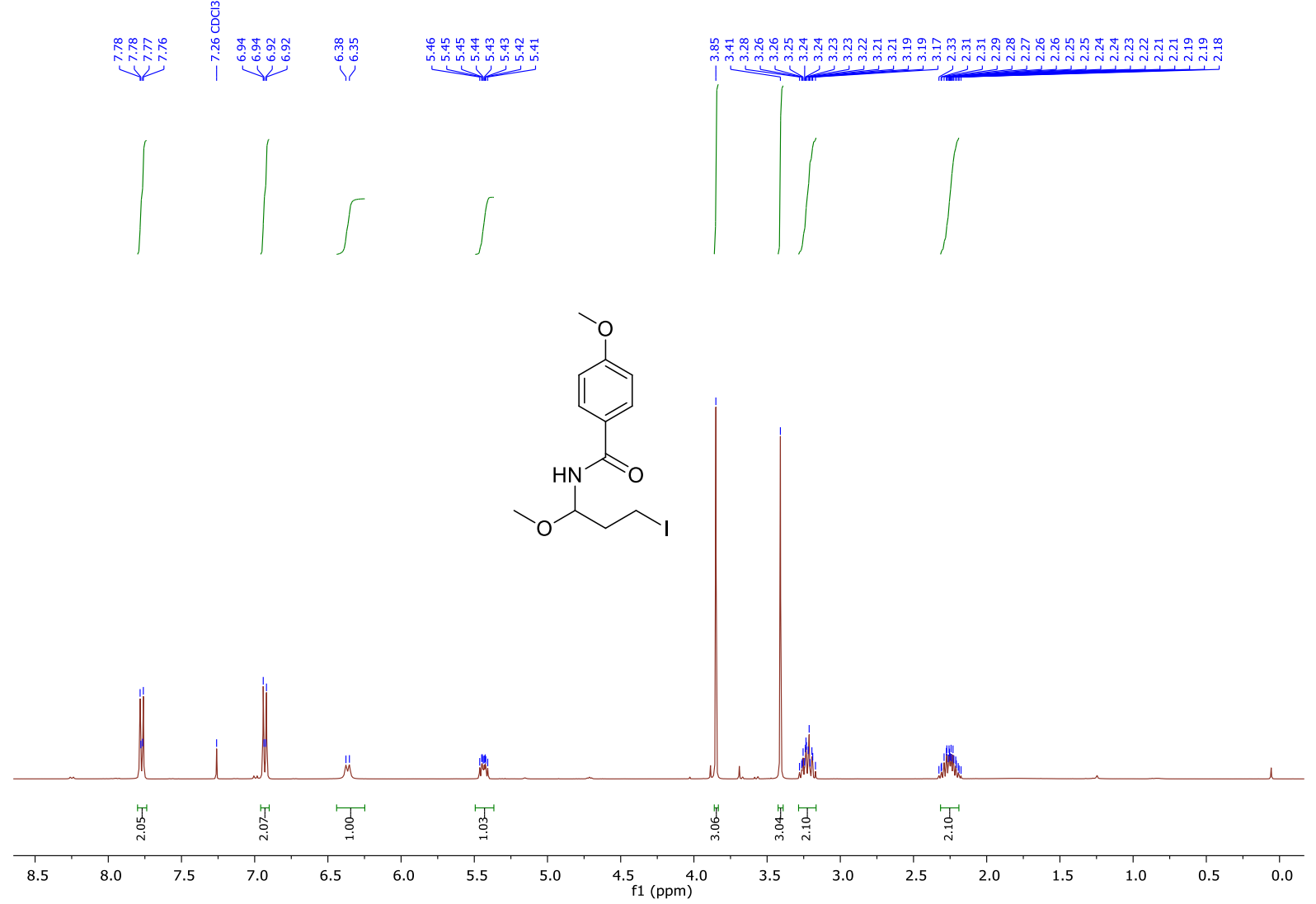

${ }^{13} \mathrm{C}-\mathrm{NMR}$ (101 MHz, CDCl 3$)$
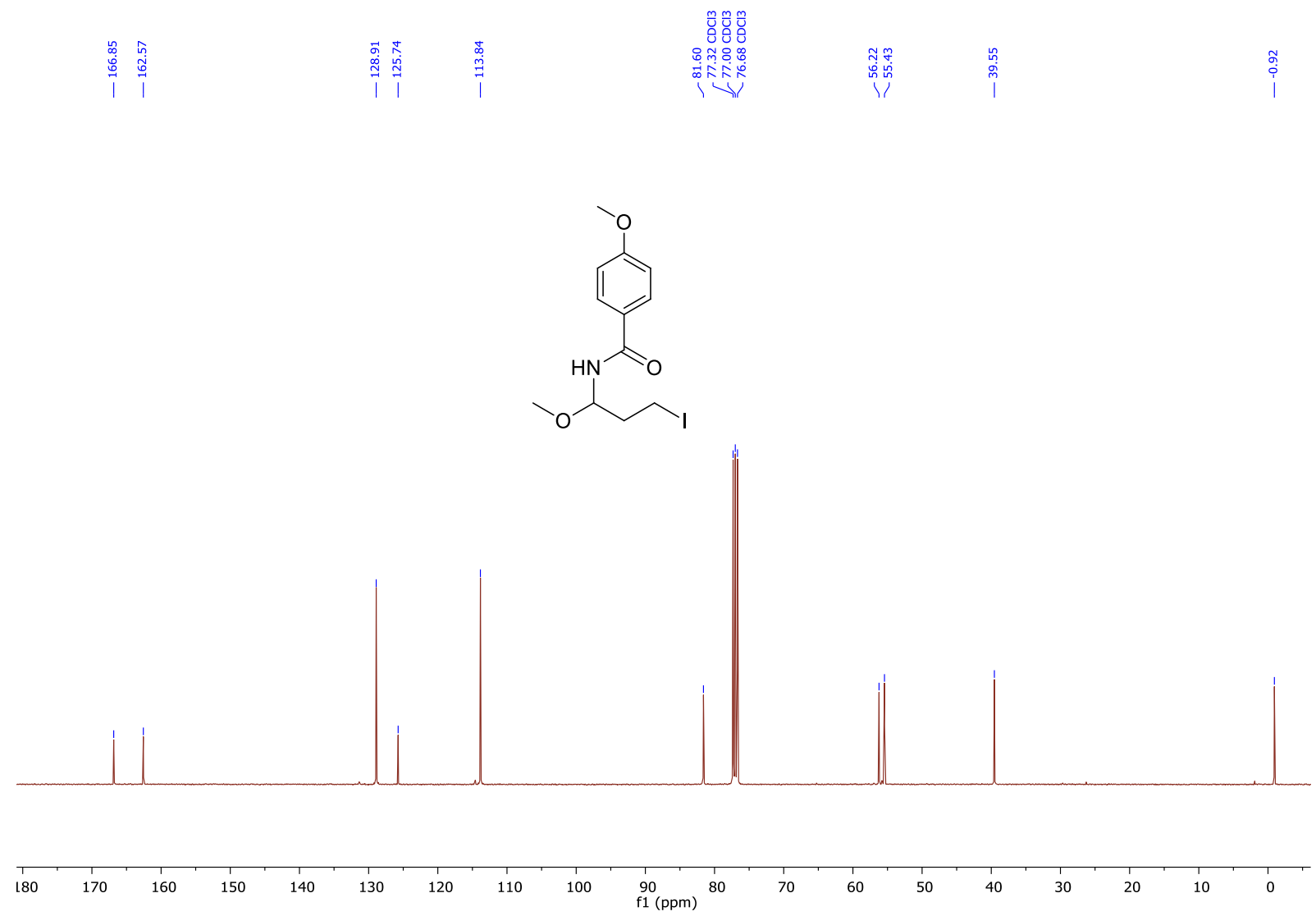
IR

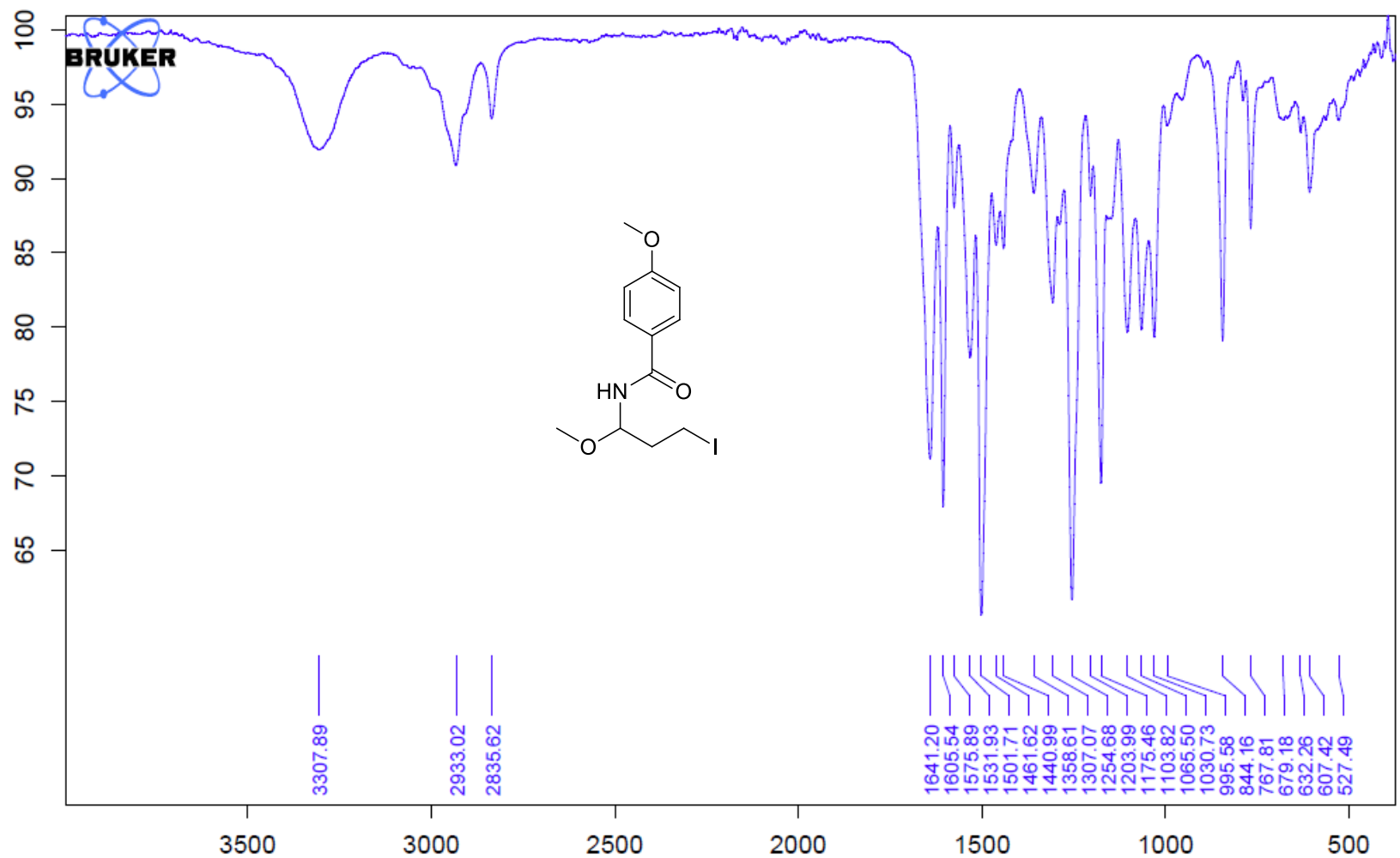

HPLC

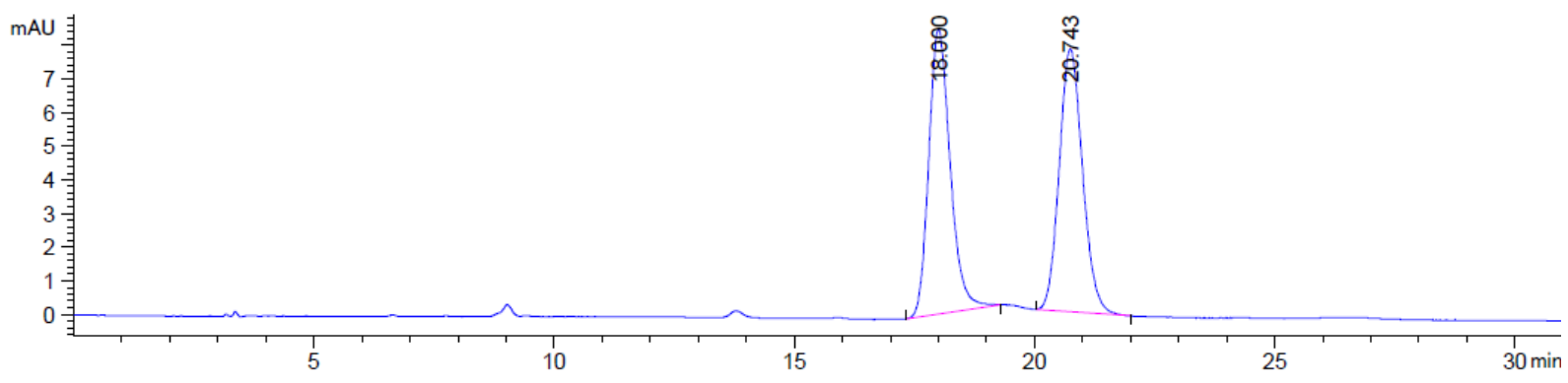

Peak RetTime Type width Area Height Area

\begin{tabular}{|c|c|c|c|c|c|}
\hline \# & [min] & [min] & [mAU*s] & [mAU] & $\%$ \\
\hline & & & 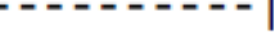 & & \\
\hline 1 & 18.000 вВ & 0.4742 & 262.42535 & 8.45173 & 49.8710 \\
\hline 2 & $20.743 \mathrm{BB}$ & 0.5242 & 263.78317 & 7.80741 & 50.1290 \\
\hline
\end{tabular}

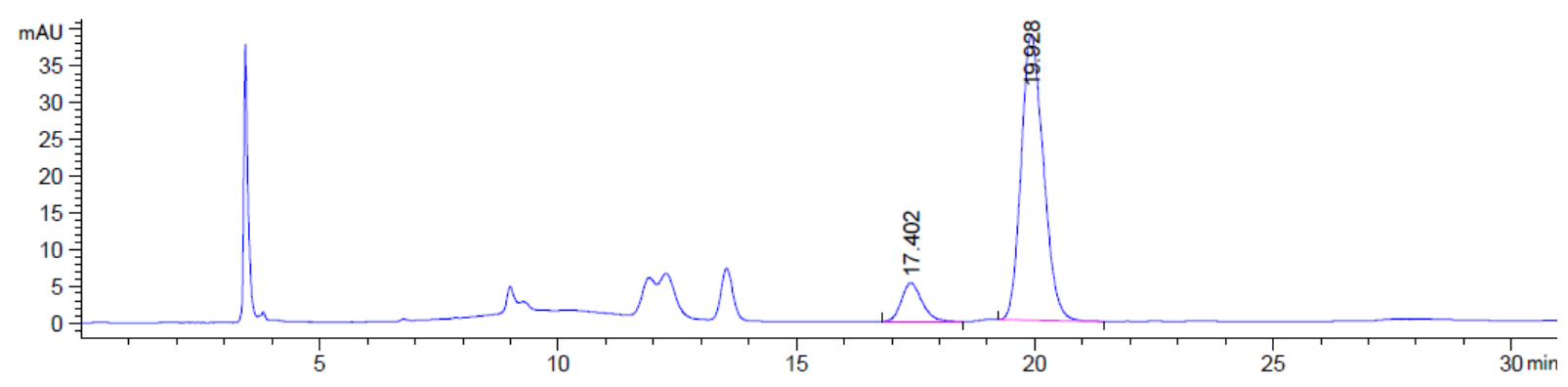




\begin{tabular}{|c|c|c|c|c|c|}
\hline $\begin{array}{c}\text { Peak } \\
\#\end{array}$ & $\begin{array}{l}\text { RetTime Type } \\
\text { [min] }\end{array}$ & $\begin{array}{l}\text { Width } \\
\text { [min] }\end{array}$ & $\begin{array}{c}\text { Area } \\
{\left[\mathrm{mAU}^{*} \mathrm{~s}\right]}\end{array}$ & $\begin{array}{l}\text { Height } \\
{[\mathrm{mAU}]}\end{array}$ & $\begin{array}{c}\text { Area } \\
\%\end{array}$ \\
\hline & & & & & \\
\hline & & & 157.17209 & & 308 \\
\hline 2 & $28 \mathrm{BB}$ & 57 & 1261.24243 & 38.95499 & 88.9192 \\
\hline
\end{tabular}


N-(3-Iodo-1-methoxypropyl)-4-(methylthio)benzamide (4b)

${ }^{1} \mathrm{H}-\mathrm{NMR}$ (400 MHz, $\mathrm{CDCl}_{3}$ )
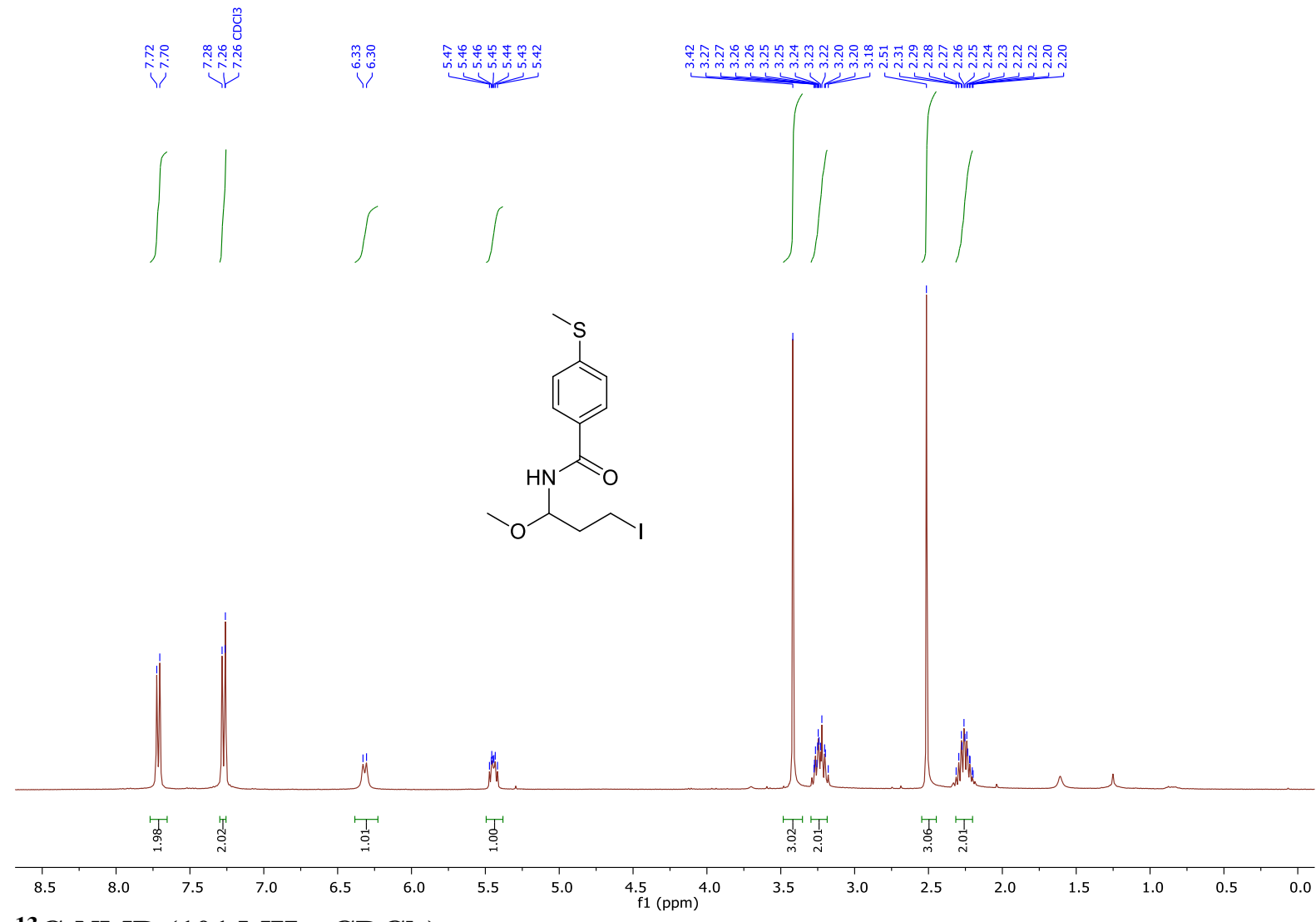

${ }^{13} \mathrm{C}-\mathrm{NMR}$ (101 MHz, $\left.\mathrm{CDCl}_{3}\right)$

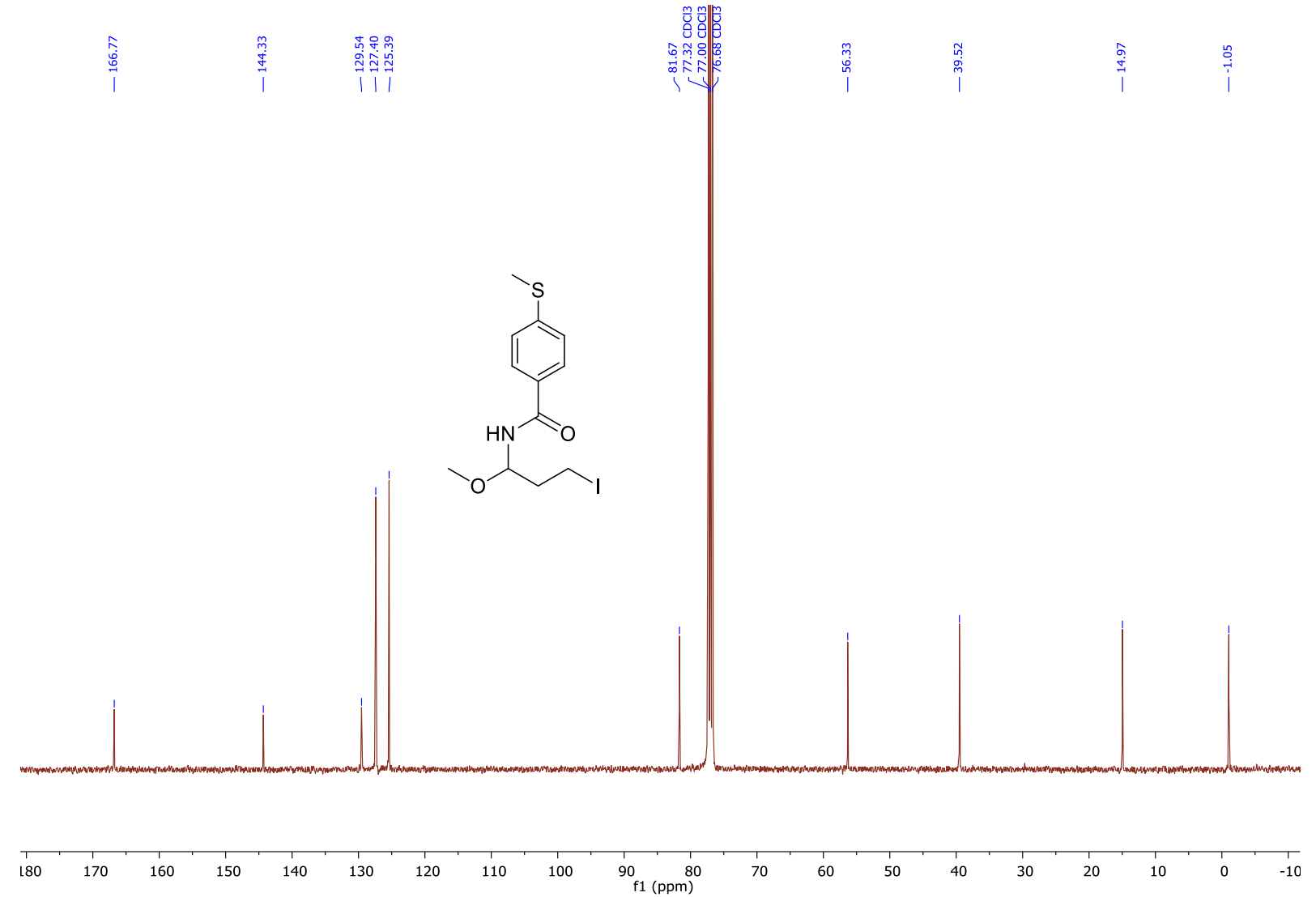


IR

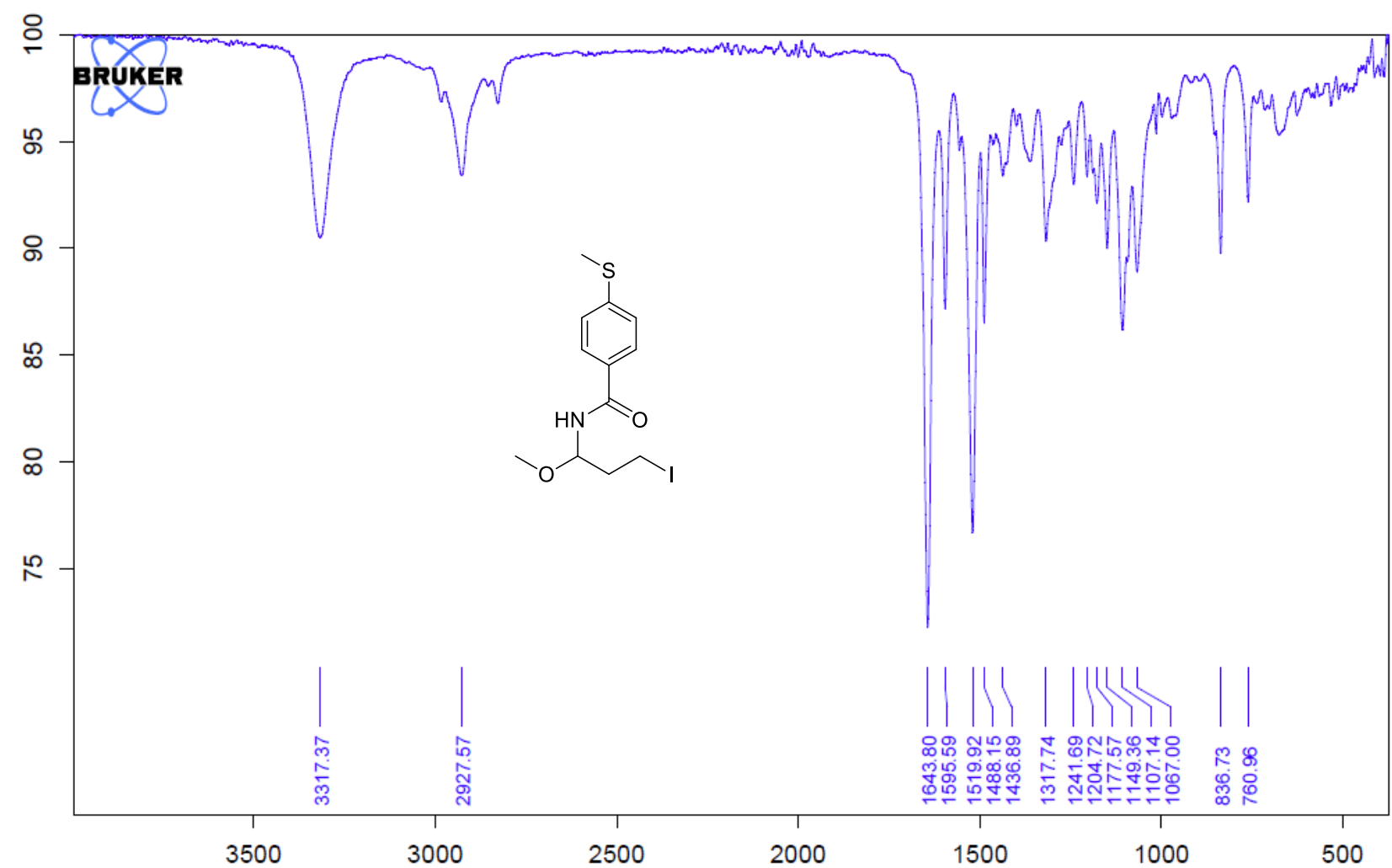

4-Fluoro-N-(3-iodo-1-methoxypropyl)benzamide (4c) ${ }^{1} \mathrm{H}-\mathrm{NMR}$ (400 MHz, $\left.\mathrm{CDCl}_{3}\right)$

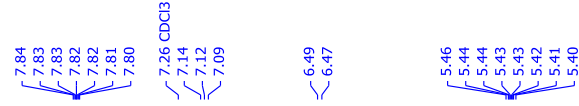

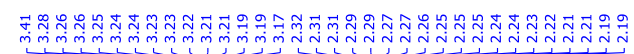
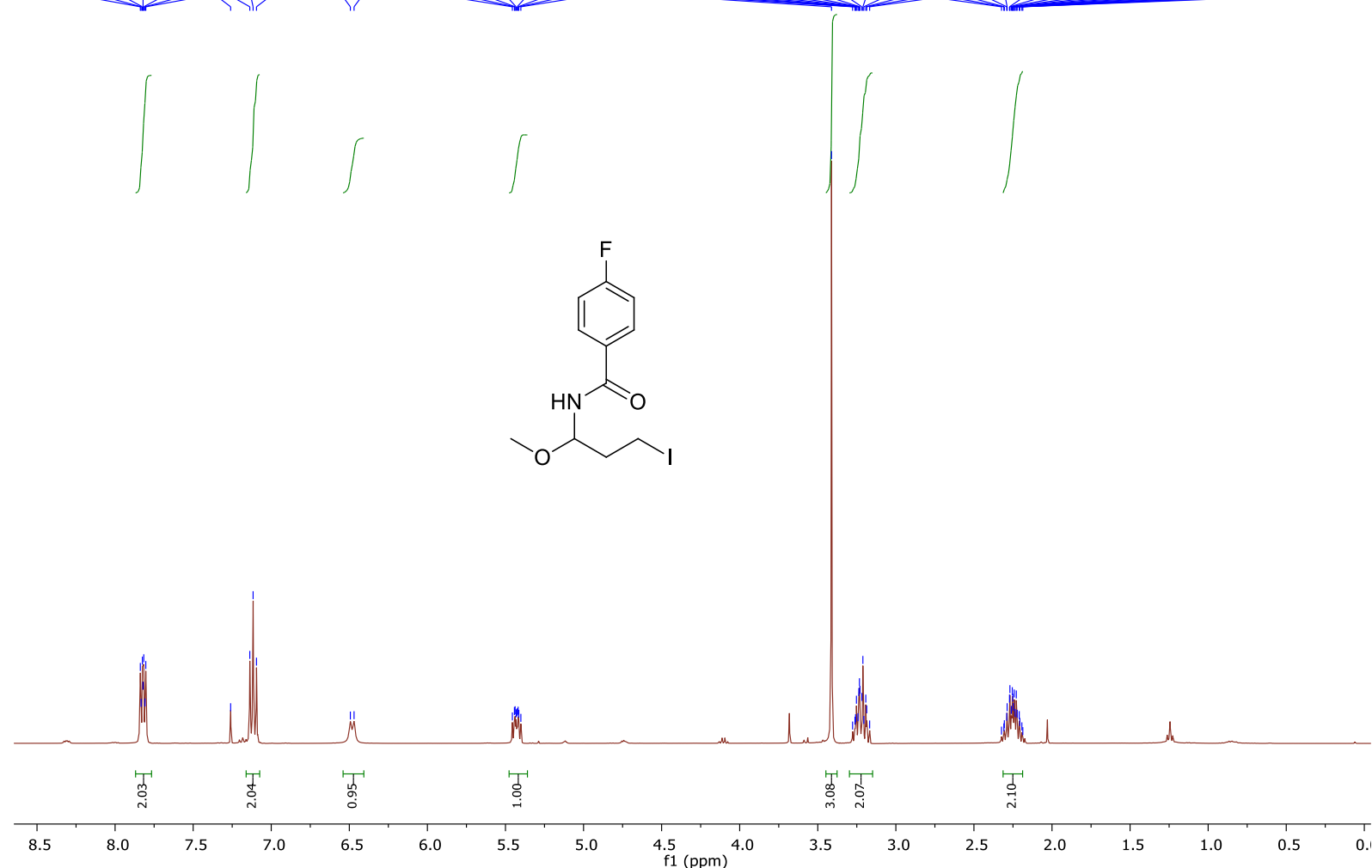
${ }^{13} \mathrm{C}$-NMR (101 MHz, CDCl3)

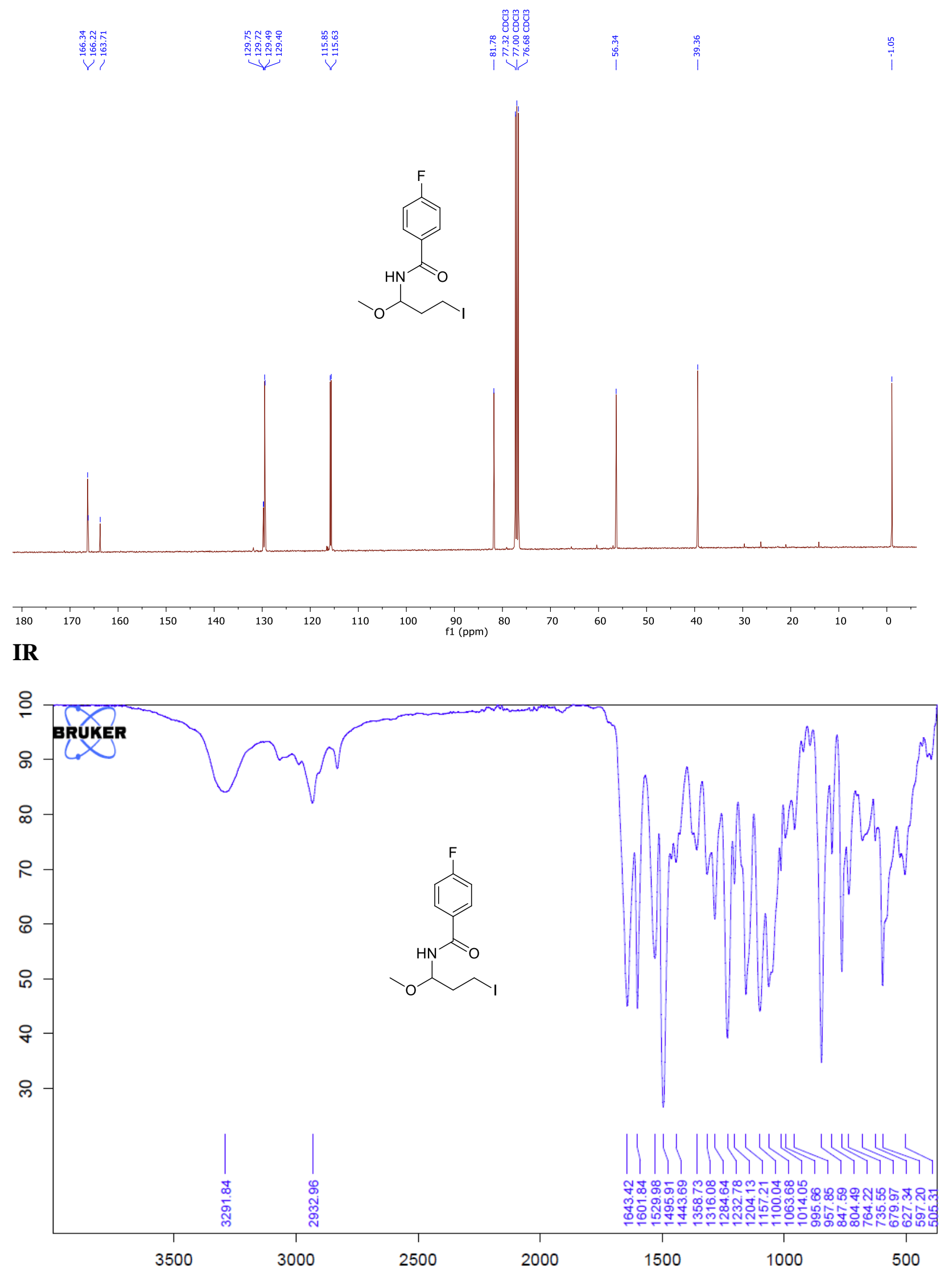


4-Chloro-N-(3-iodo-1-methoxypropyl)benzamide (4d)

${ }^{1} \mathrm{H}-\mathrm{NMR}$ (400 MHz, $\mathrm{CDCl}_{3}$ )

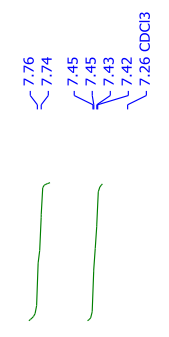

V|
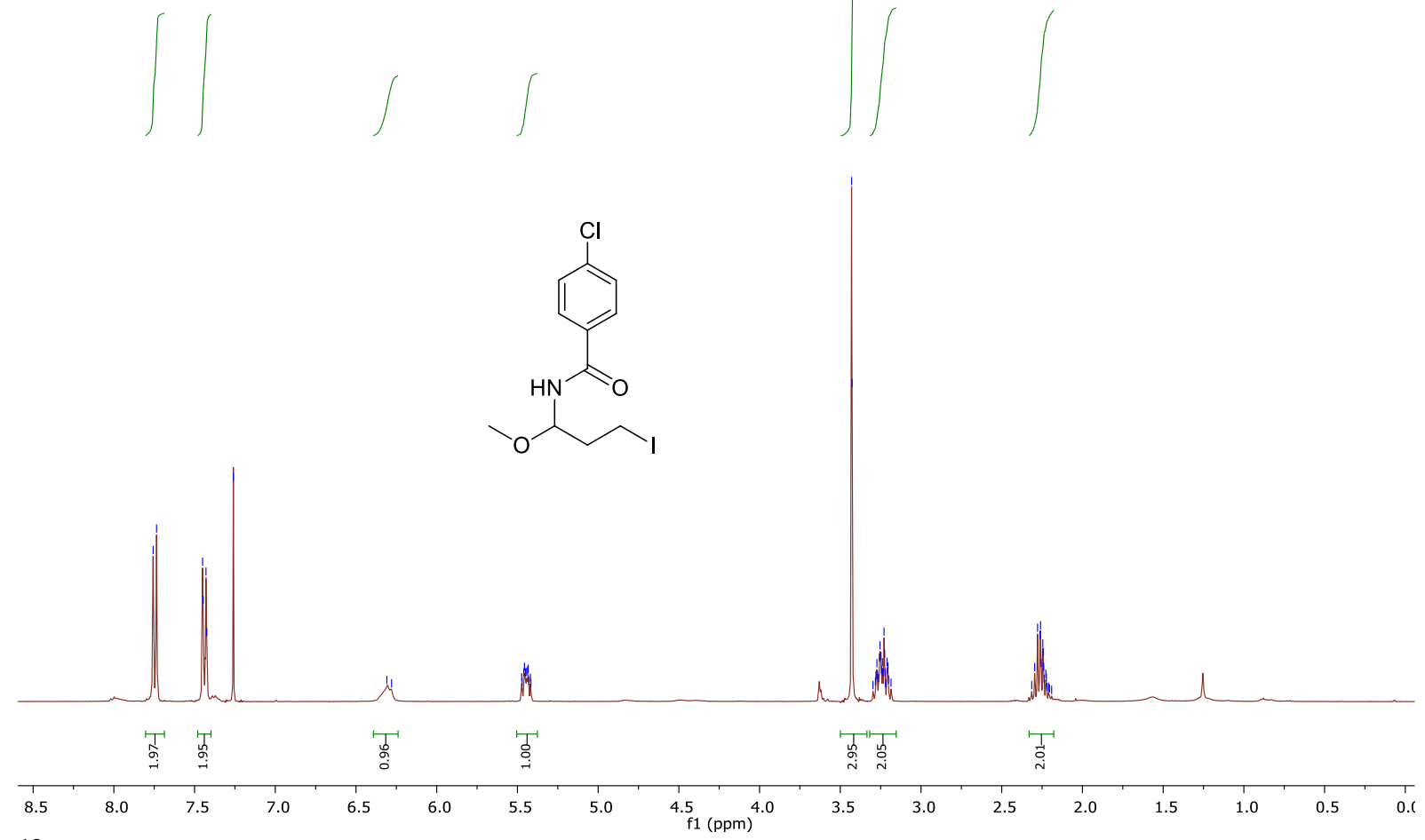

${ }^{13} \mathrm{C}-\mathrm{NMR}$ (101 MHz, $\left.\mathrm{CDCl}_{3}\right)$
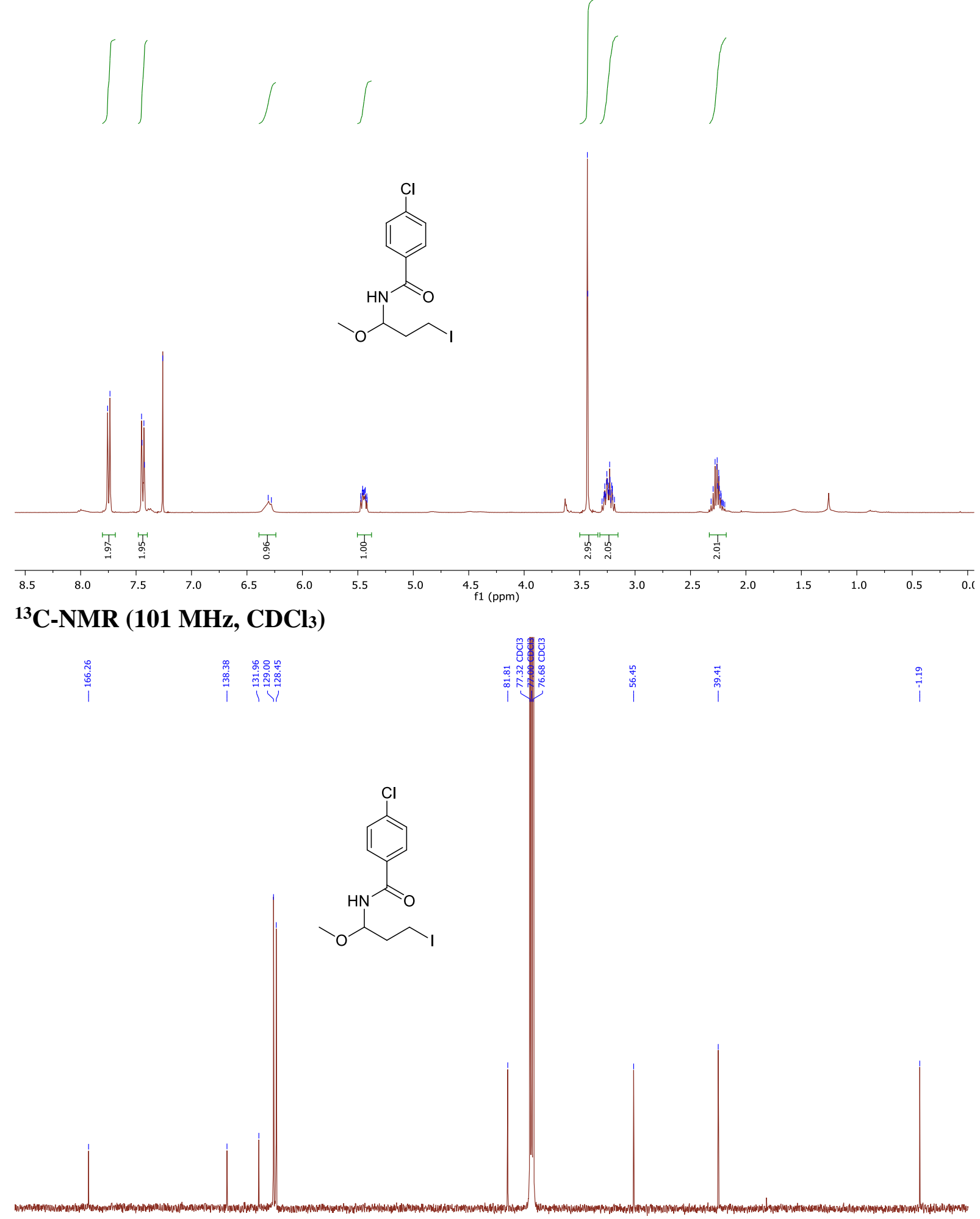

180
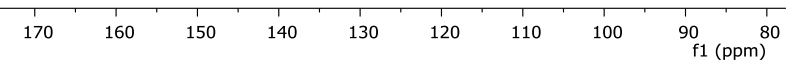
IR

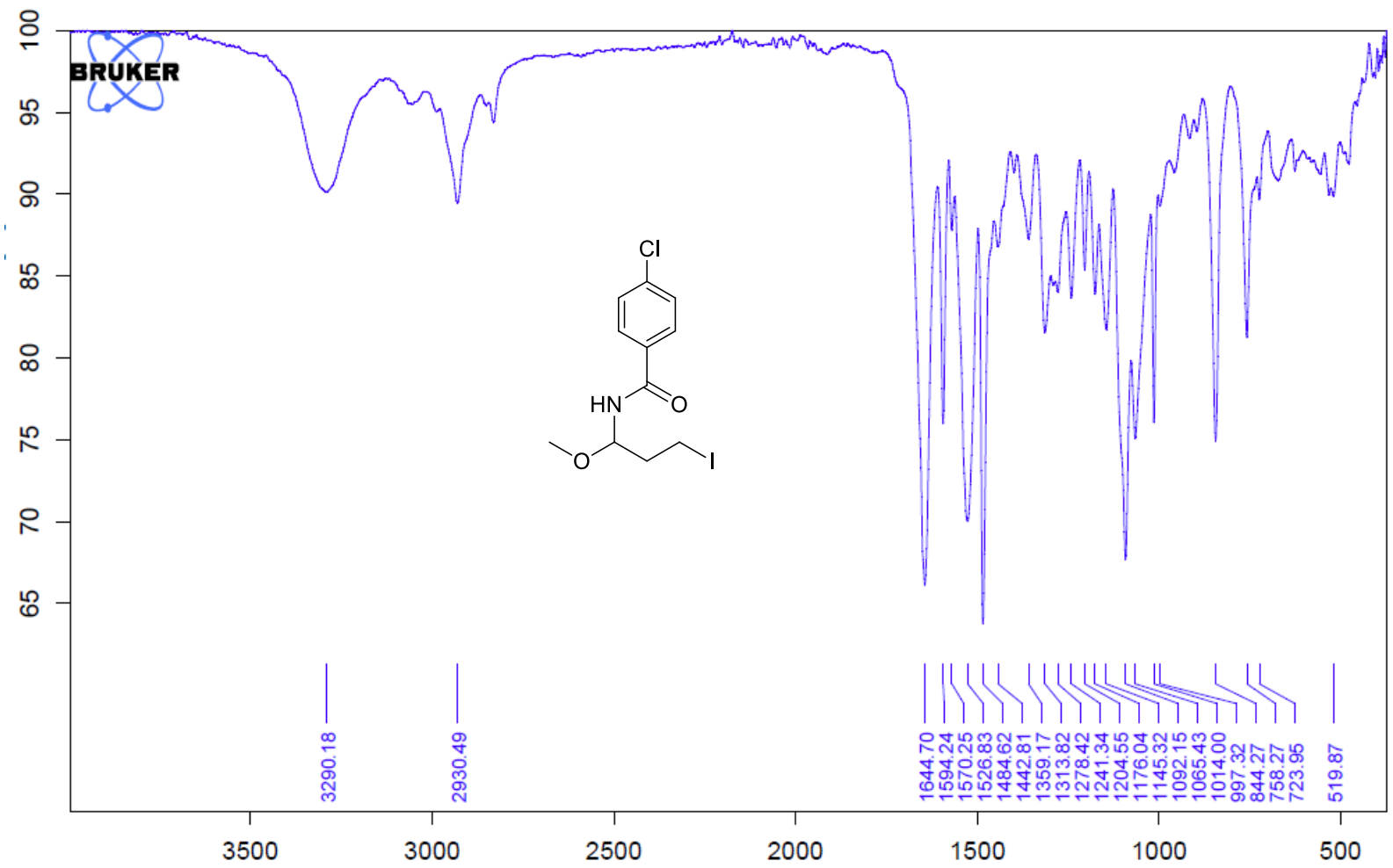

4-Cyano-N-(3-iodo-1-methoxypropyl)benzamide (4e)

${ }^{1} \mathrm{H}-\mathrm{NMR}$ (400 MHz, CDCl 3$)$
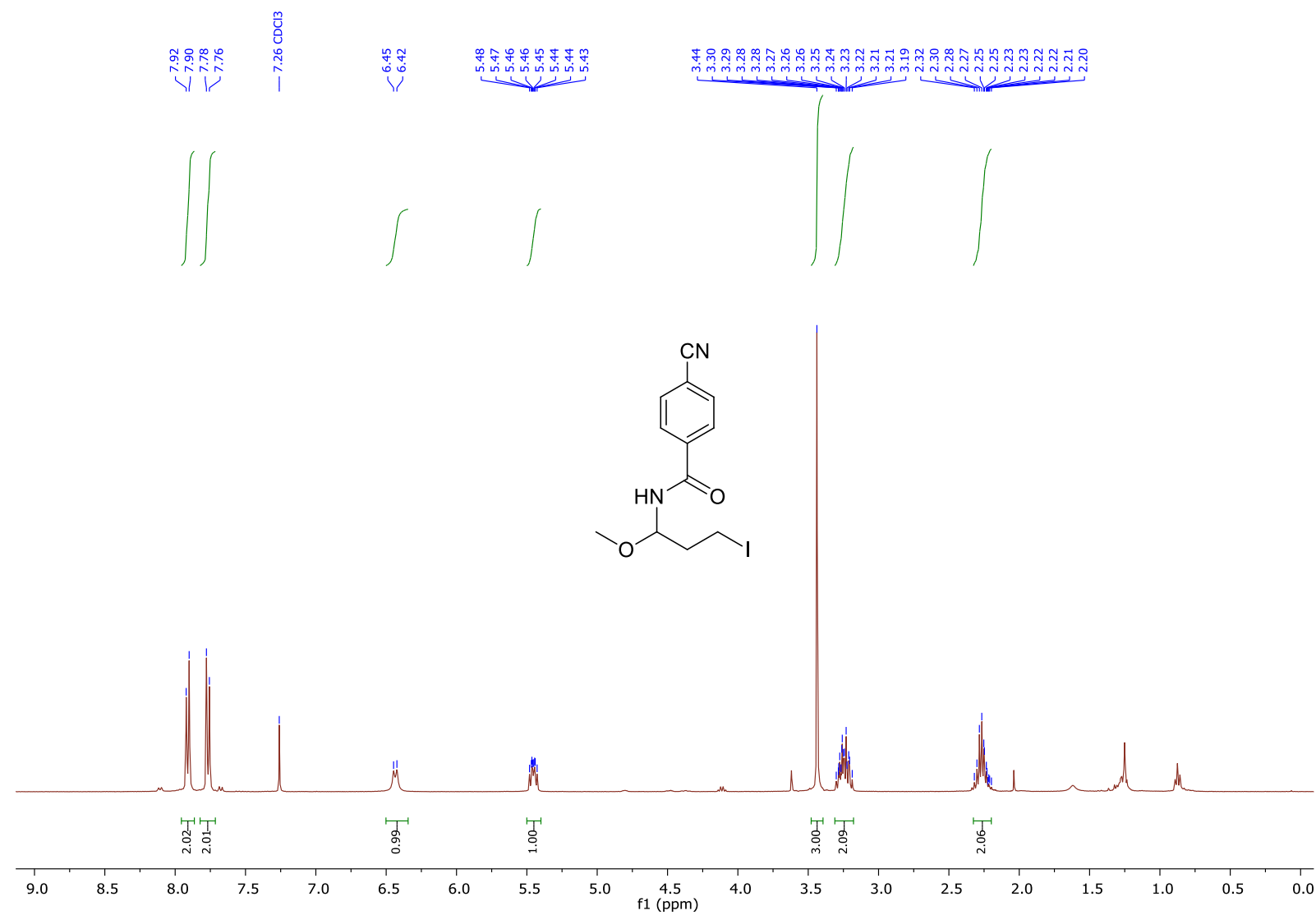
${ }^{13} \mathrm{C}$-NMR (101 MHz, CDCl3)
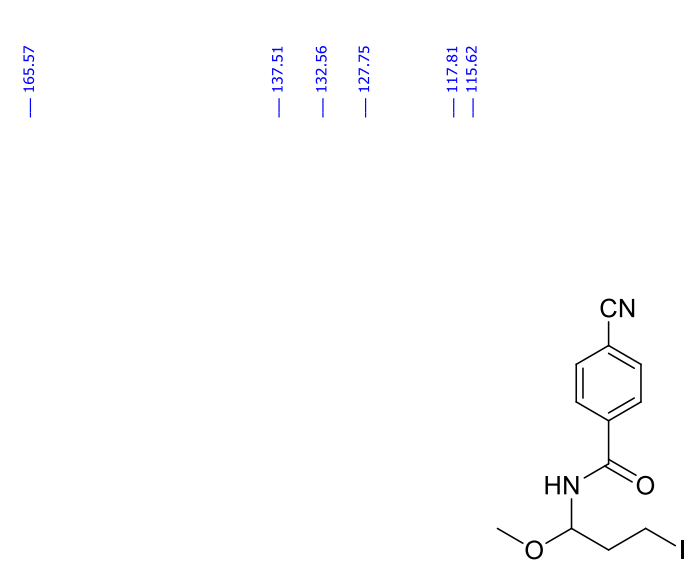

|

$$
\stackrel{8}{\substack{0 \\ \text { I }}}
$$
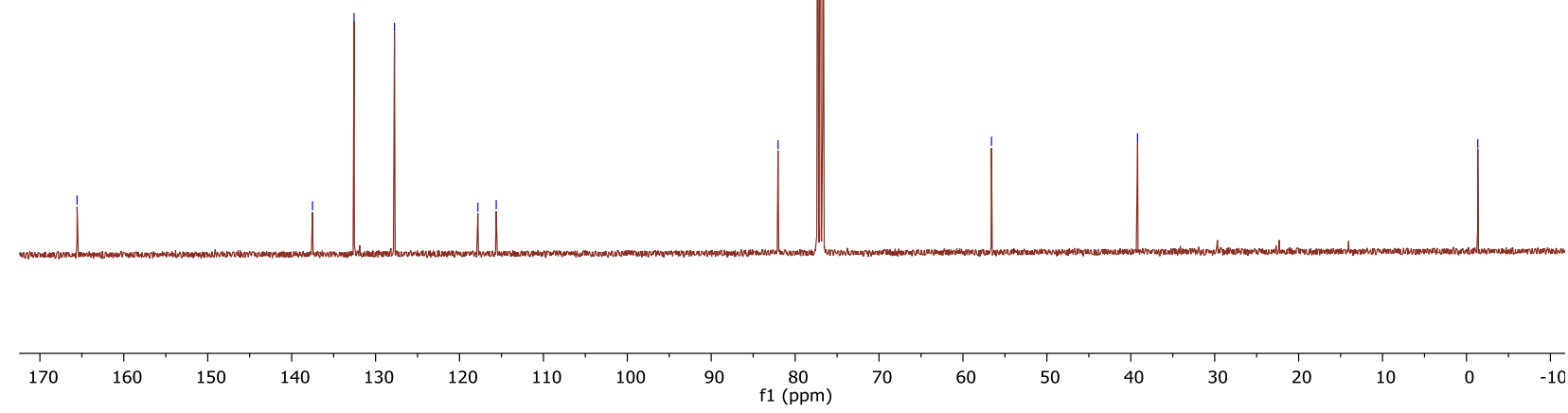

IR

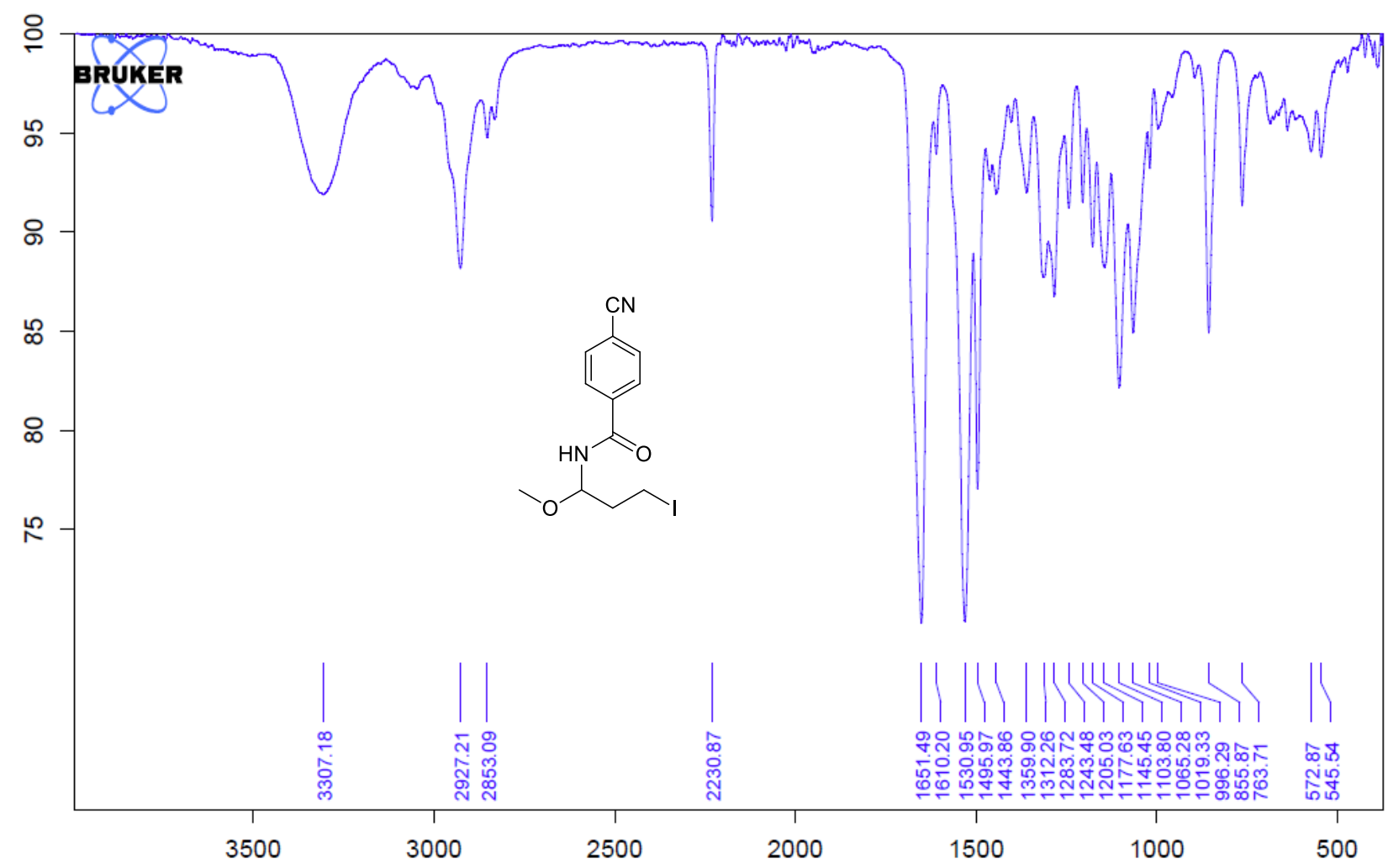


N-(3-Iodo-1-methoxypropyl)-4-nitrobenzamide (4f)

${ }^{1} \mathrm{H}-\mathrm{NMR}$ (400 MHz, CDCl 3$)$
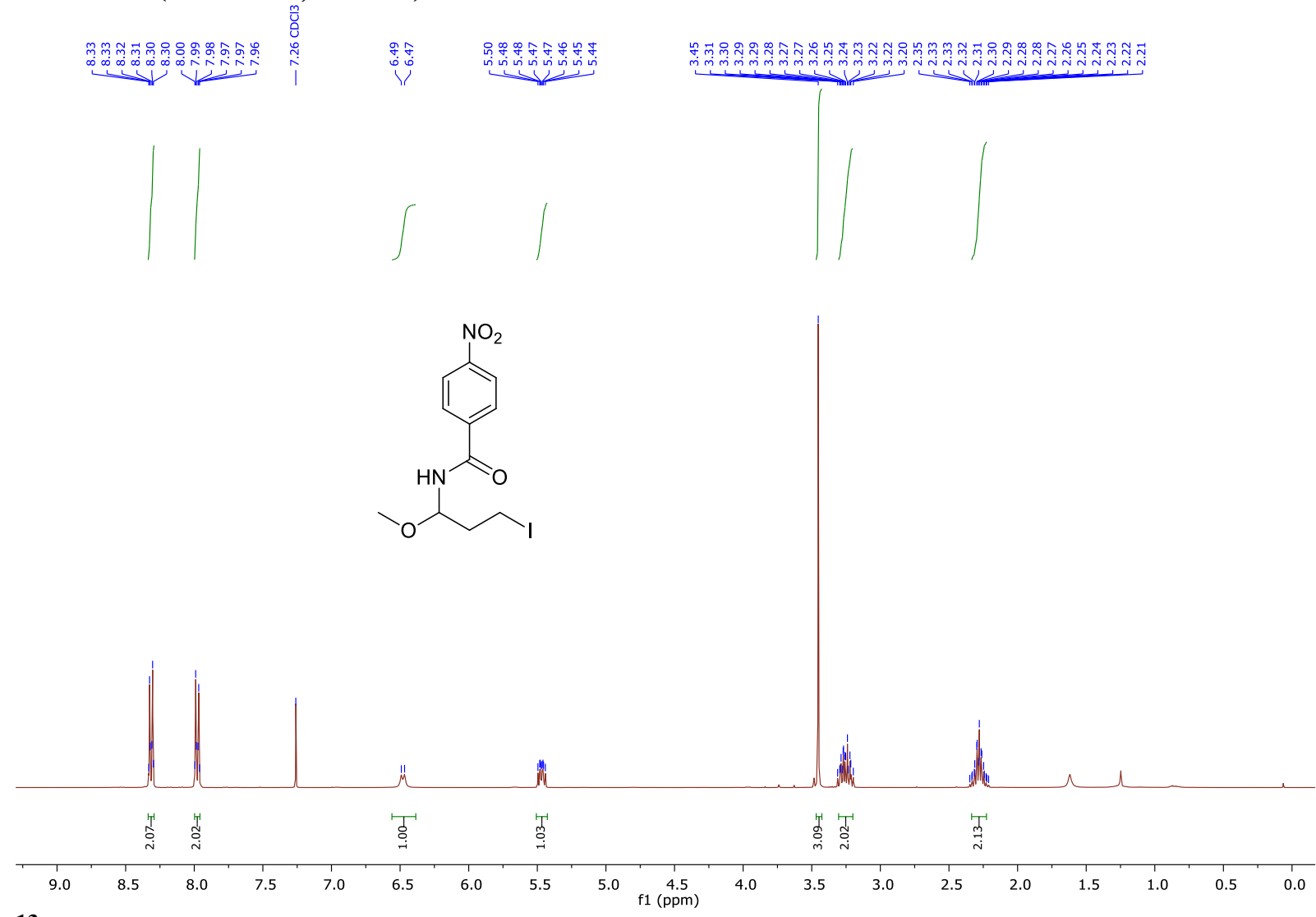

${ }^{13} \mathrm{C}-\mathrm{NMR}$ (101 MHz, CDCl3)

我
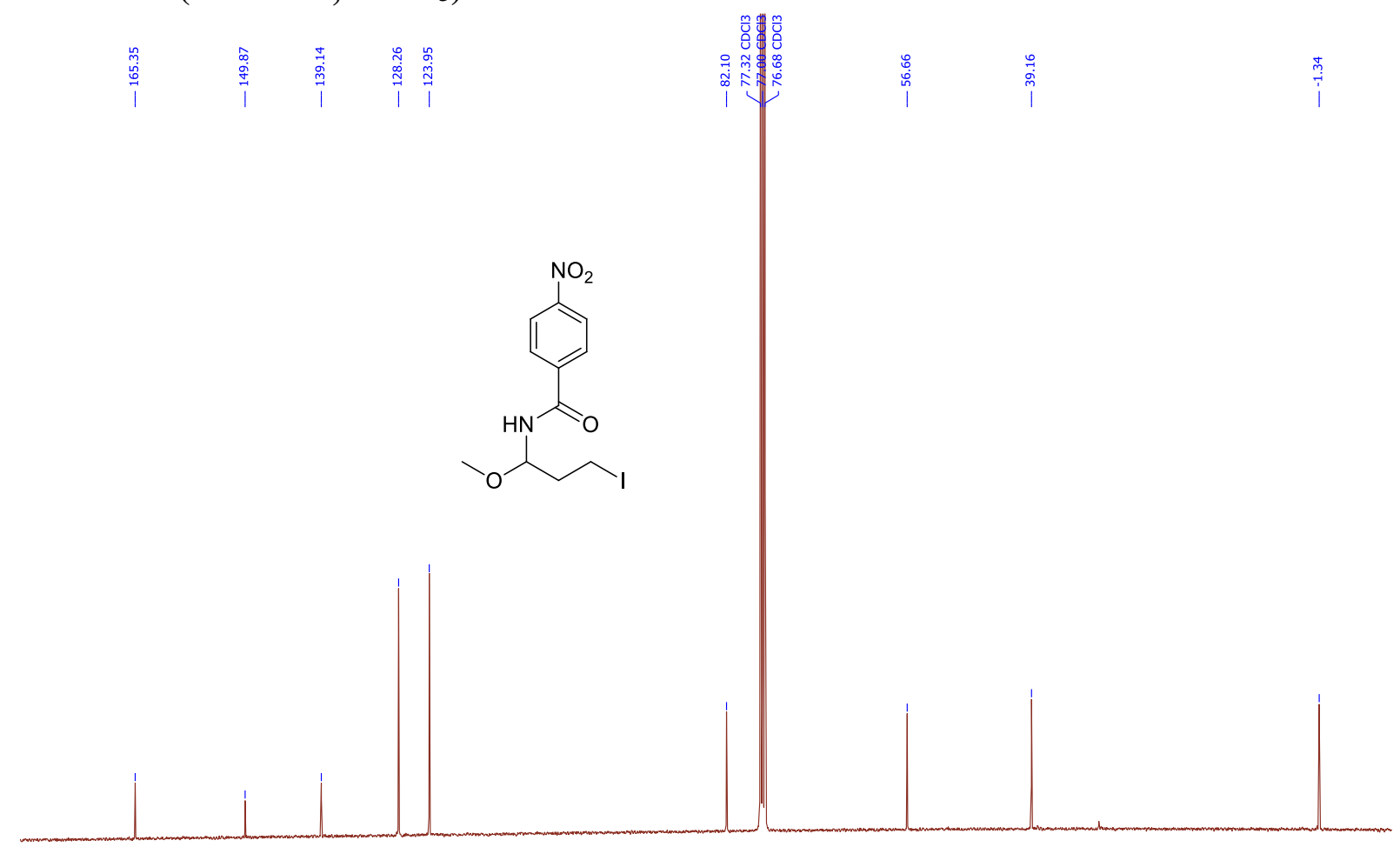
IR

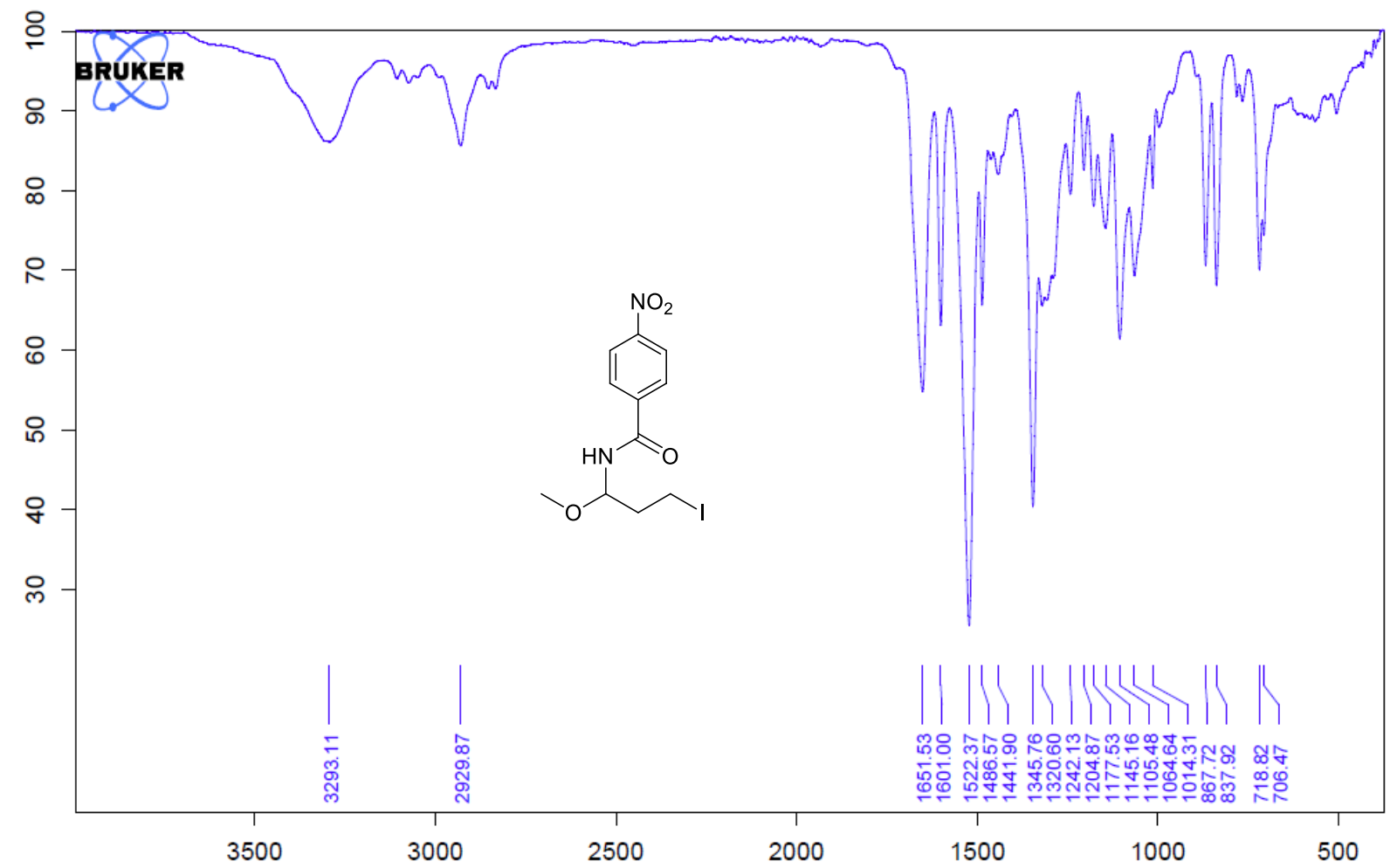

N-(3-Iodo-1-methoxypropyl)-3-methoxybenzamide (4g)

${ }^{1}$ H-NMR (400 MHz, CDCl $)$

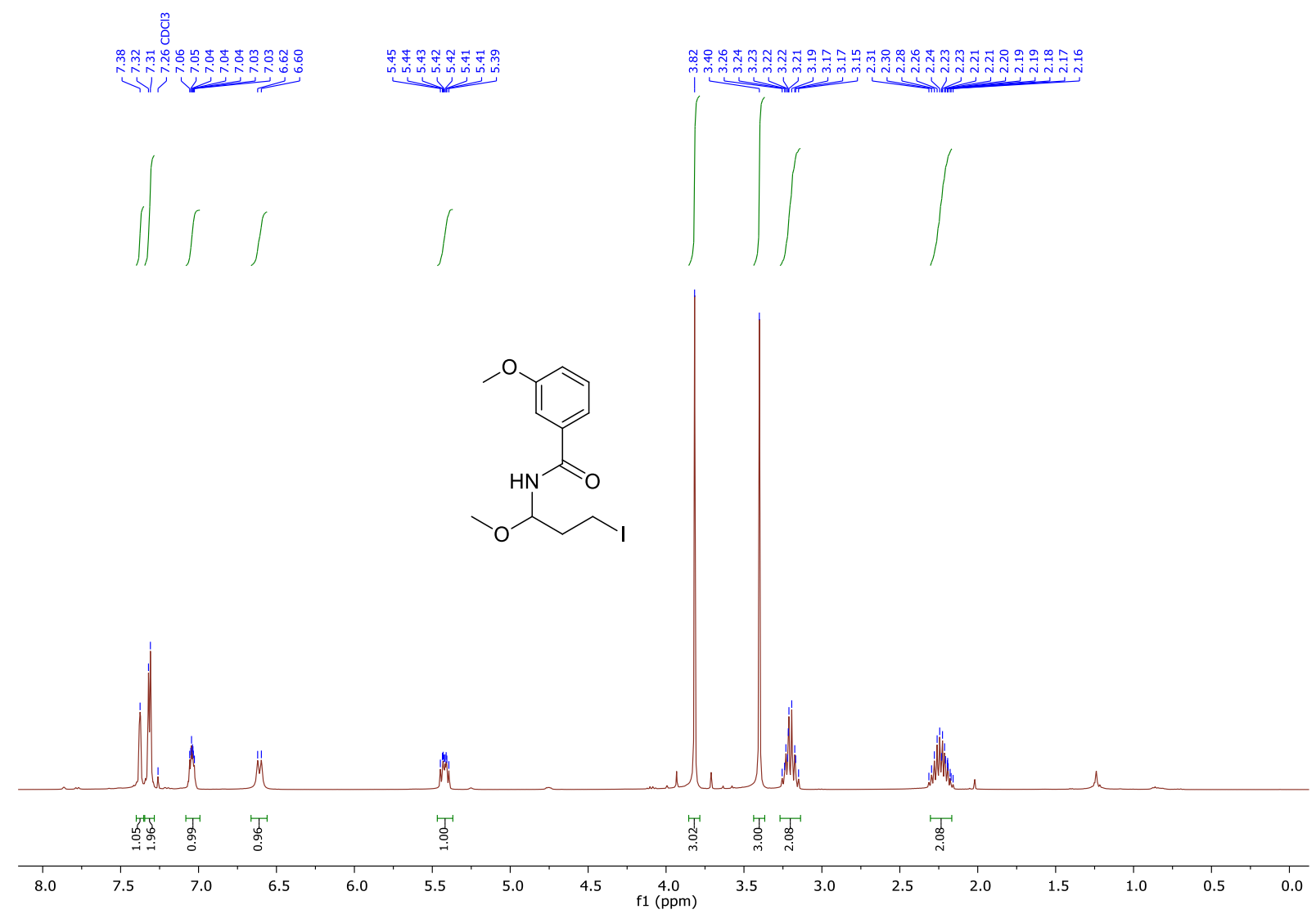


${ }^{13} \mathrm{C}-\mathrm{NMR}(101 \mathrm{MHz}, \mathrm{CDCl} 3)$

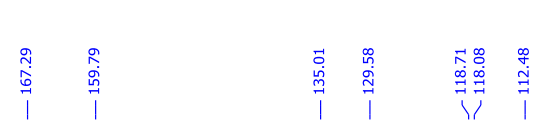<smiles>COc1cccc(C(=O)NC(CCI)OC)c1</smiles>

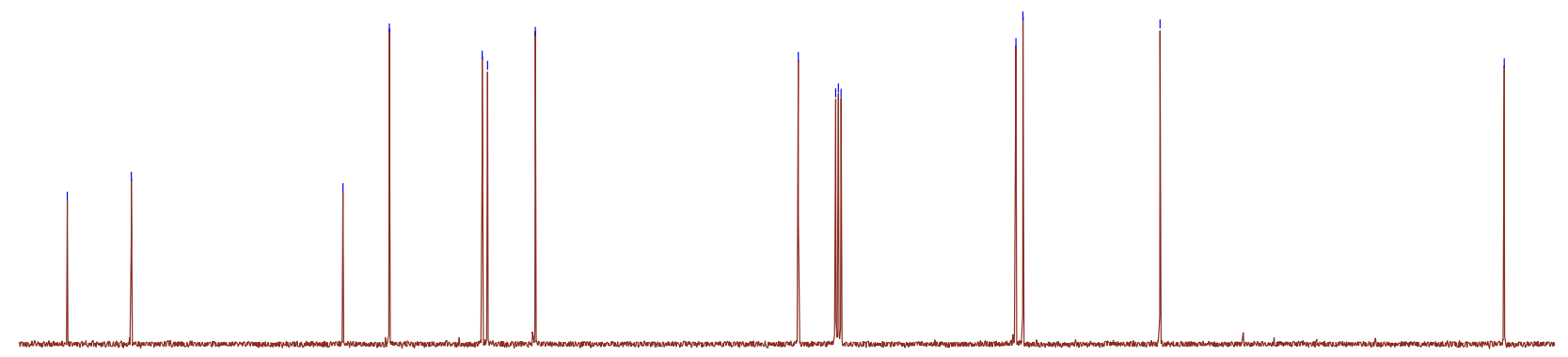

\begin{tabular}{|c|c|c|c|c|c|c|c|c|c|c|c|c|c|c|c|c|c|}
\hline 170 & 160 & 150 & 140 & 130 & 120 & 110 & 100 & 90 & $\begin{array}{r}80 \\
\text { f1 }(\mathrm{ppm})\end{array}$ & 70 & 60 & 50 & 40 & 30 & 20 & 10 & 0 \\
\hline
\end{tabular}

IR

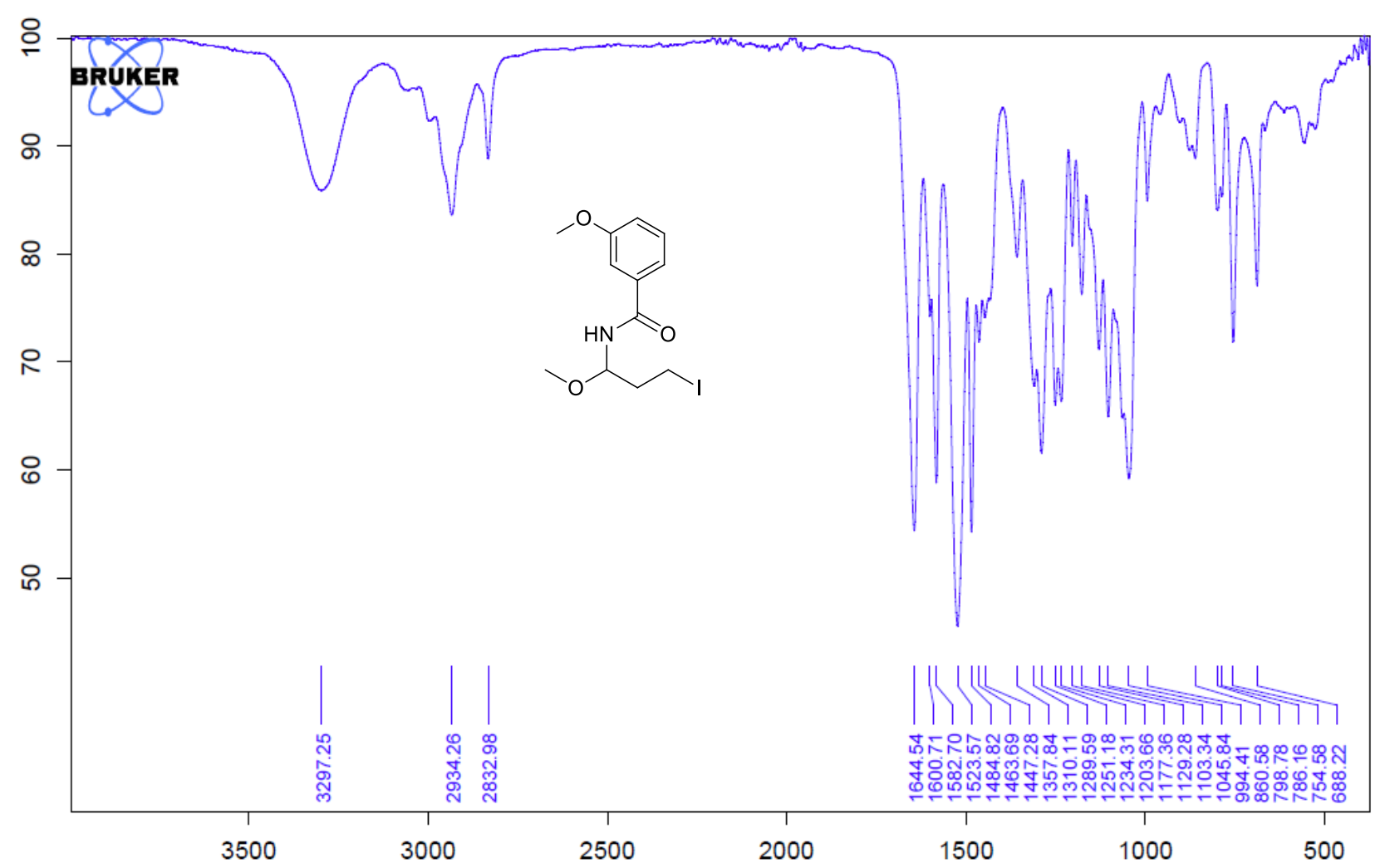


N-(3-Iodo-1-methoxypropyl)-2-methoxybenzamide (4h)

${ }^{1}$ H-NMR (400 MHz, CDCl $)$

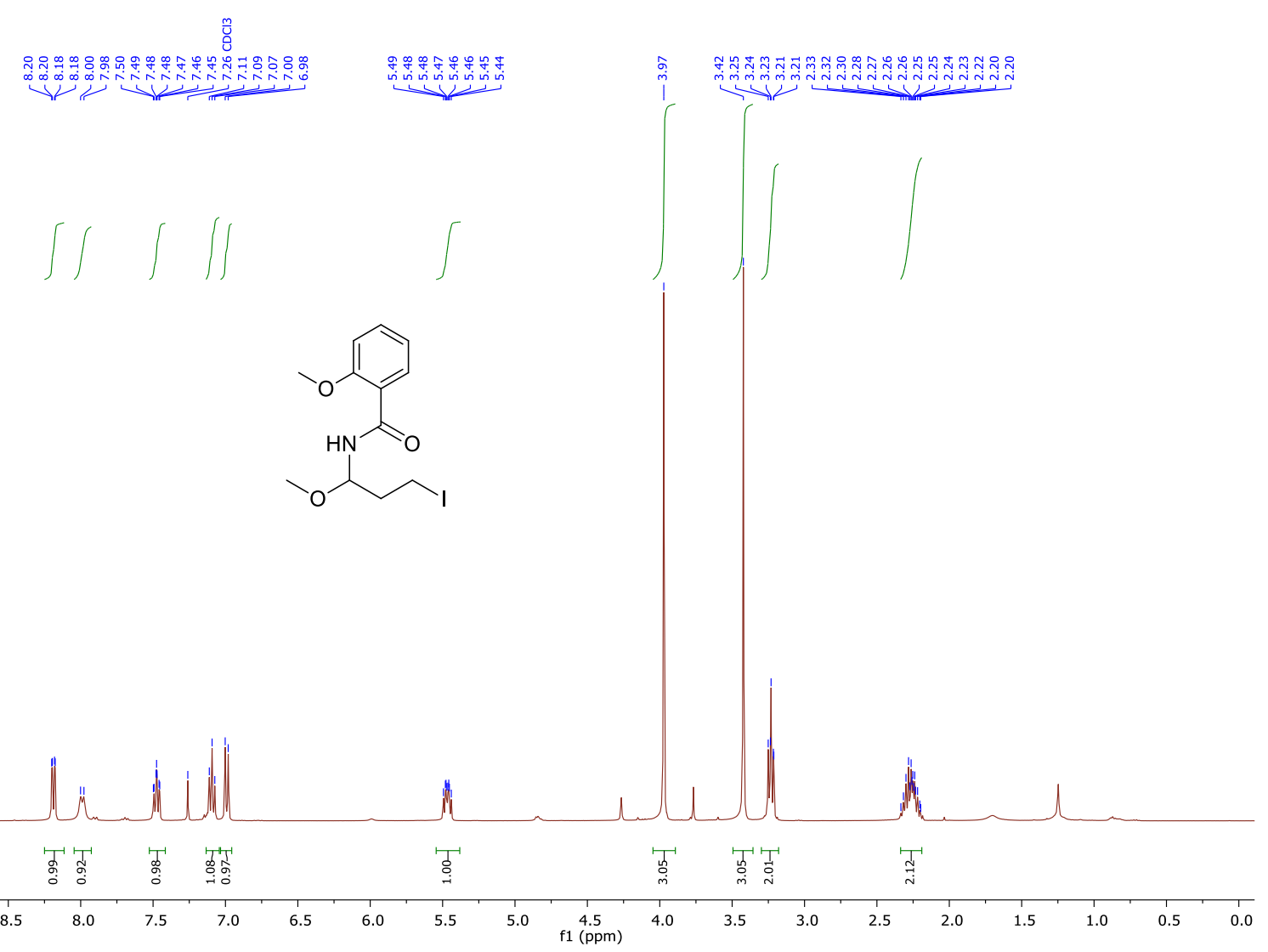

${ }^{13} \mathrm{C}-\mathrm{NMR}$ (101 MHz, CDCl3)
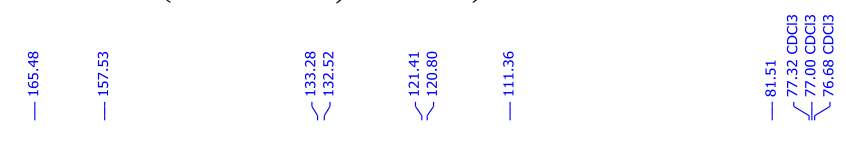

ข้ำ

|

임
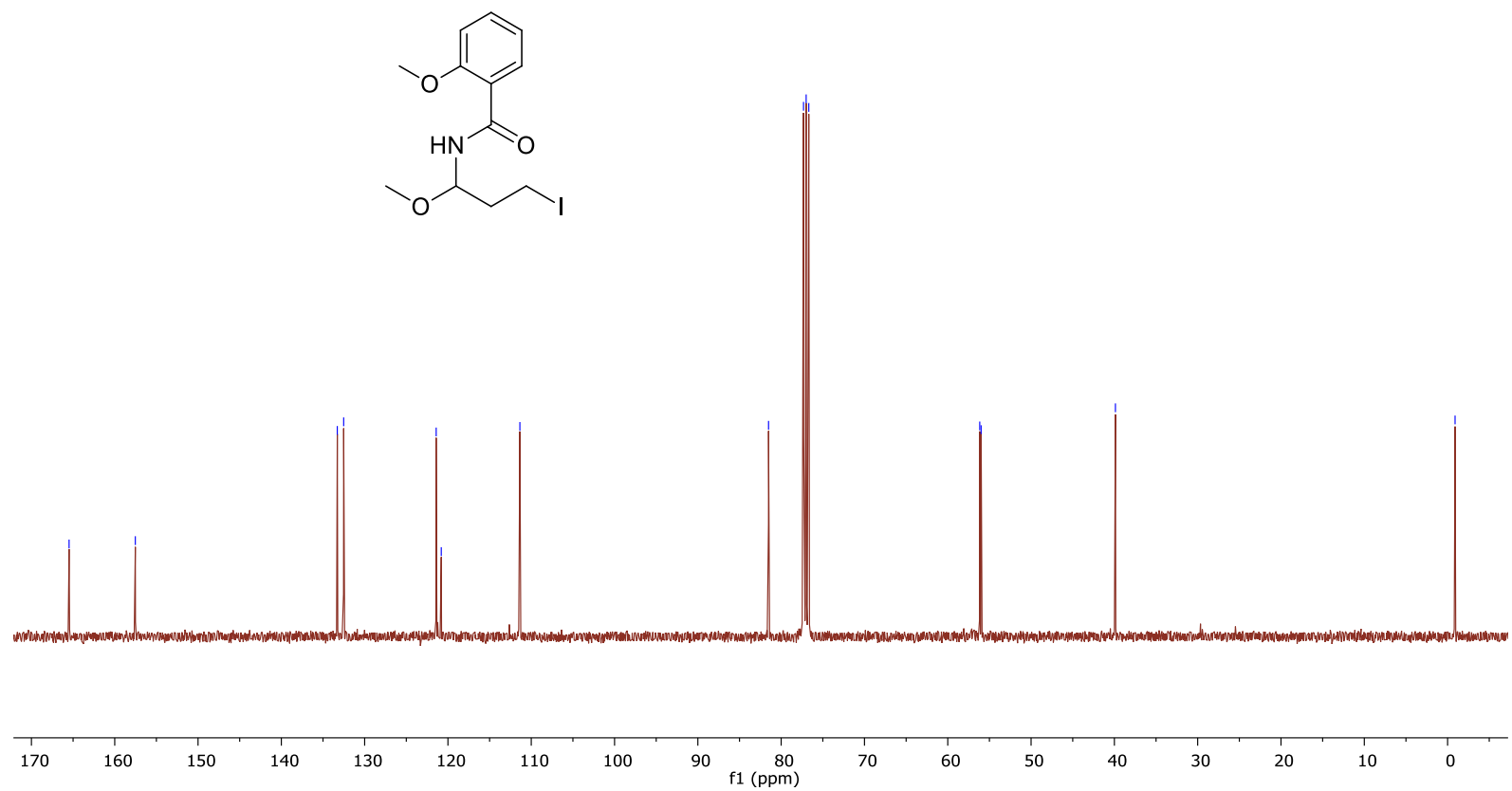

S80 
IR

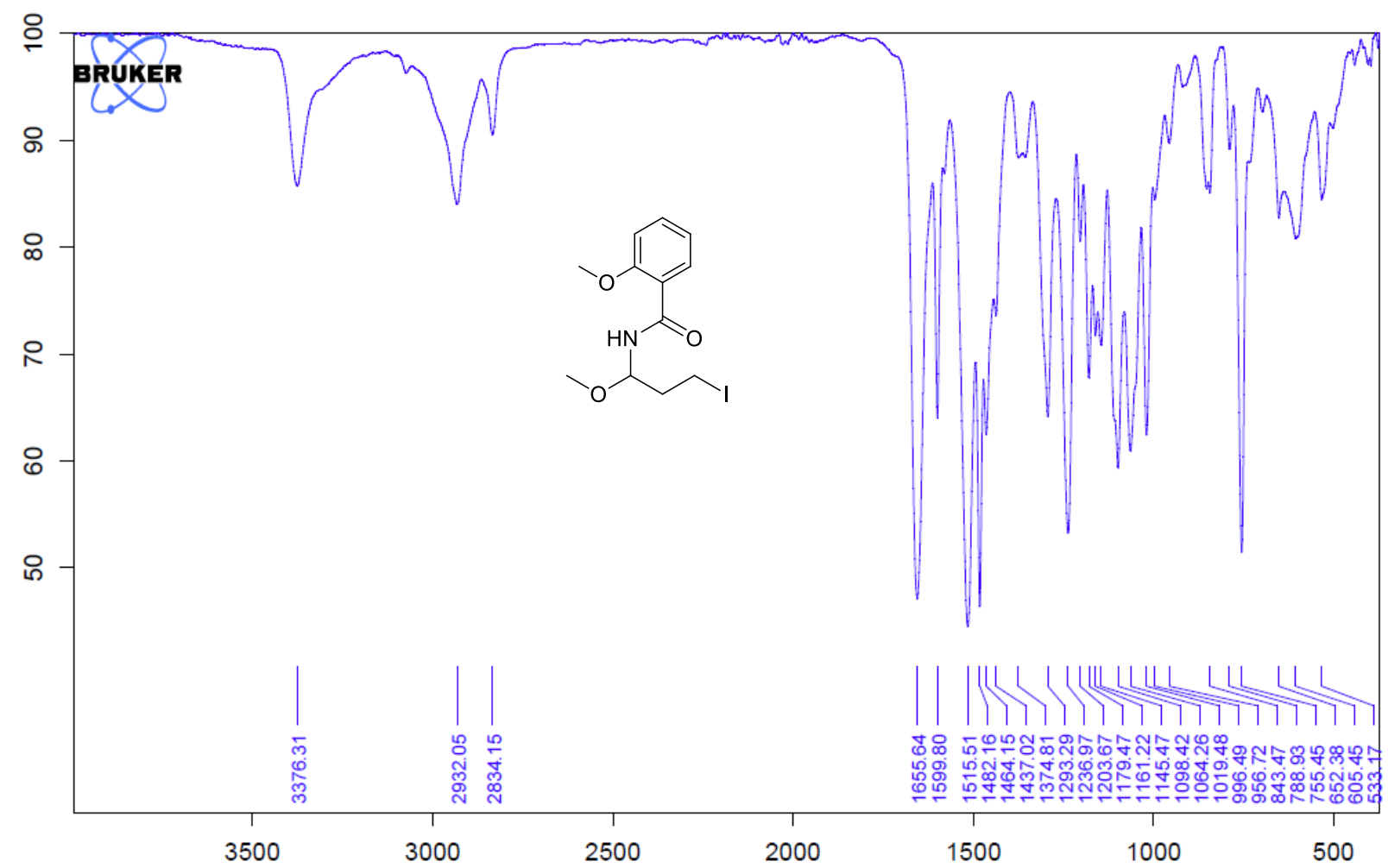

N-(3-Iodo-1-methoxypropyl)-2,4,6-trimethylbenzamide (4i)

${ }^{1}$ H-NMR (400 MHz, CDCl3)

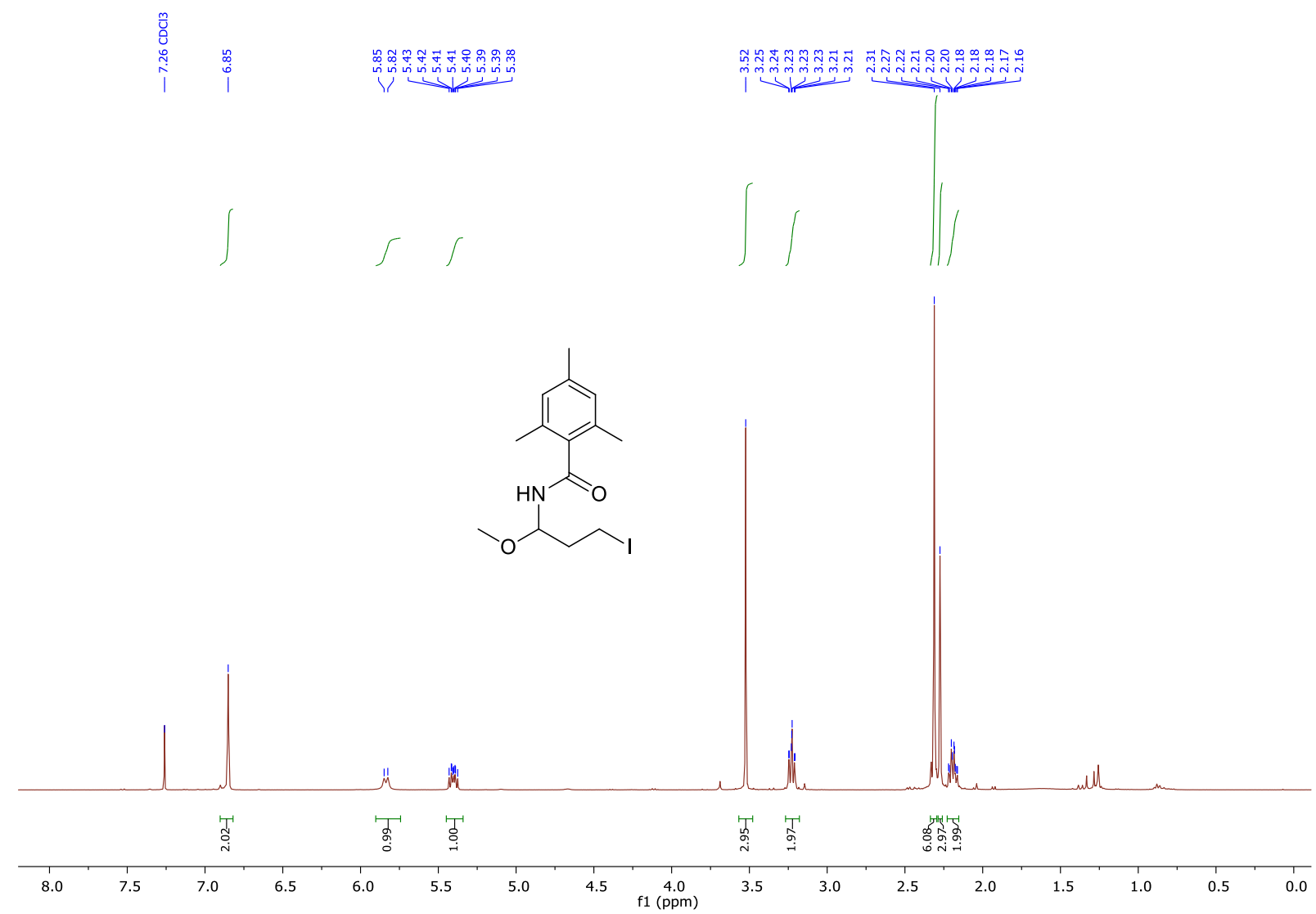


${ }^{13} \mathrm{C}$-NMR (101 MHz, CDCl3)
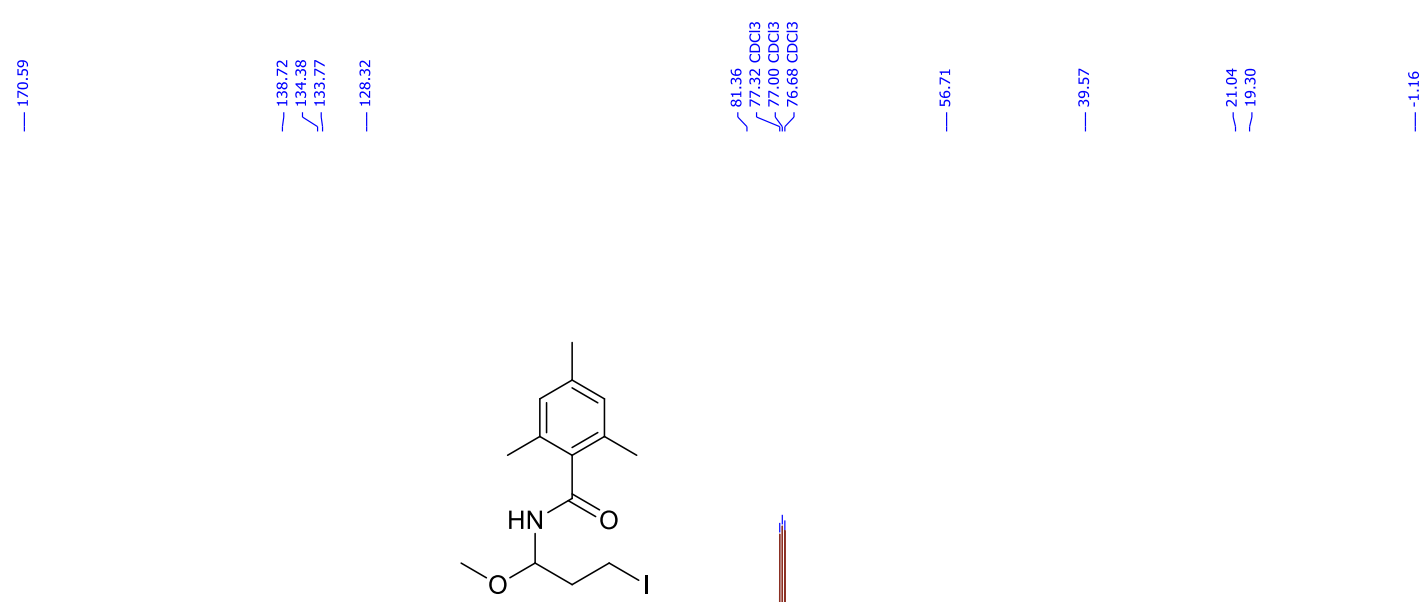

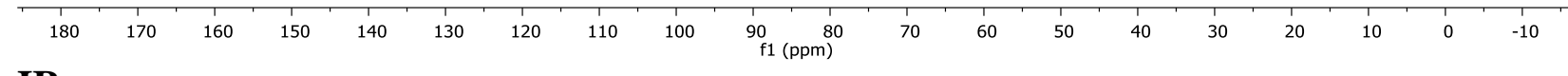

IR

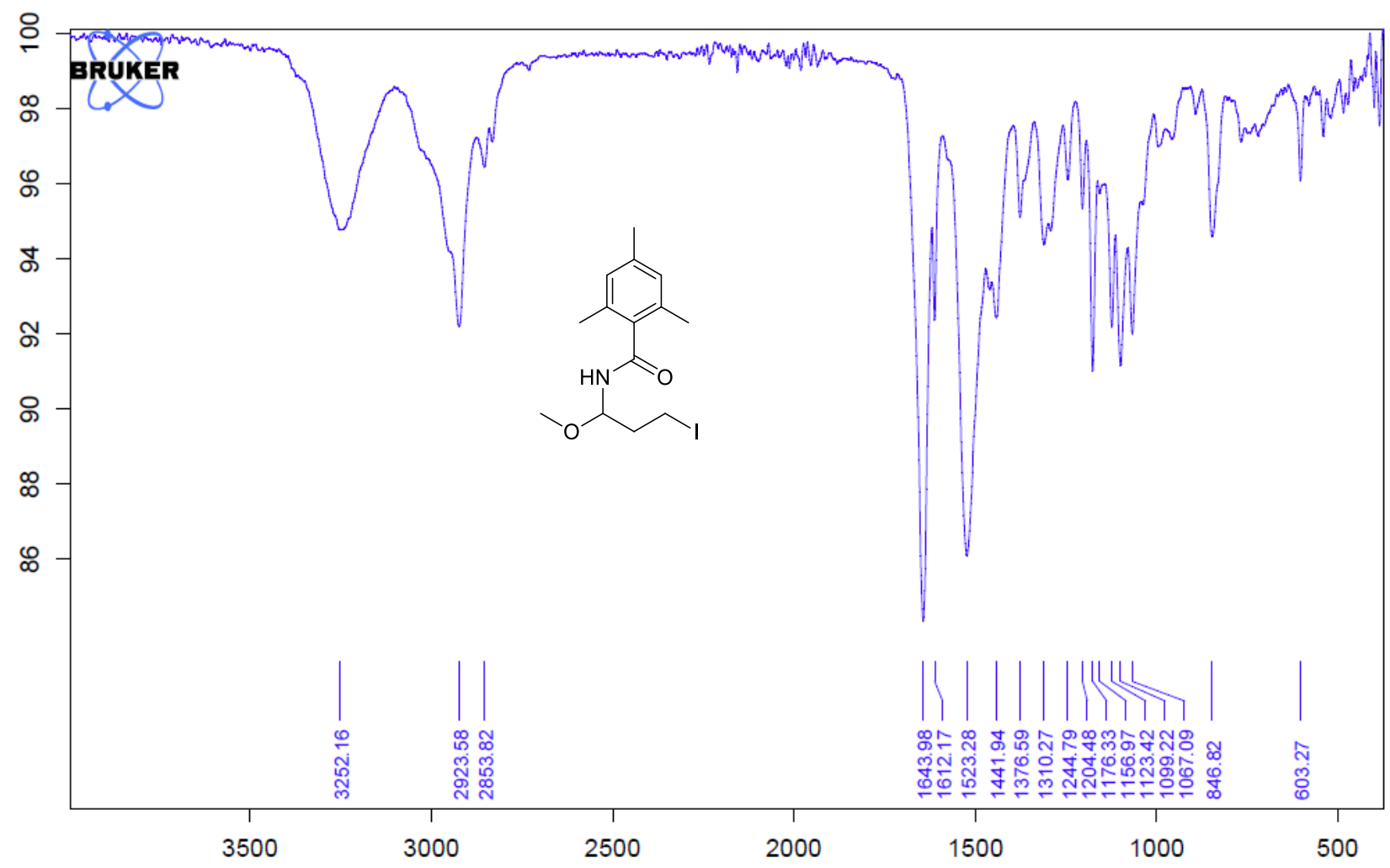


N-(3-Iodo-1-methoxypropyl)furan-2-carboxamide (4j)

${ }^{1} \mathrm{H}-\mathrm{NMR}$ (400 MHz, $\left.\mathrm{CDCl}_{3}\right)$

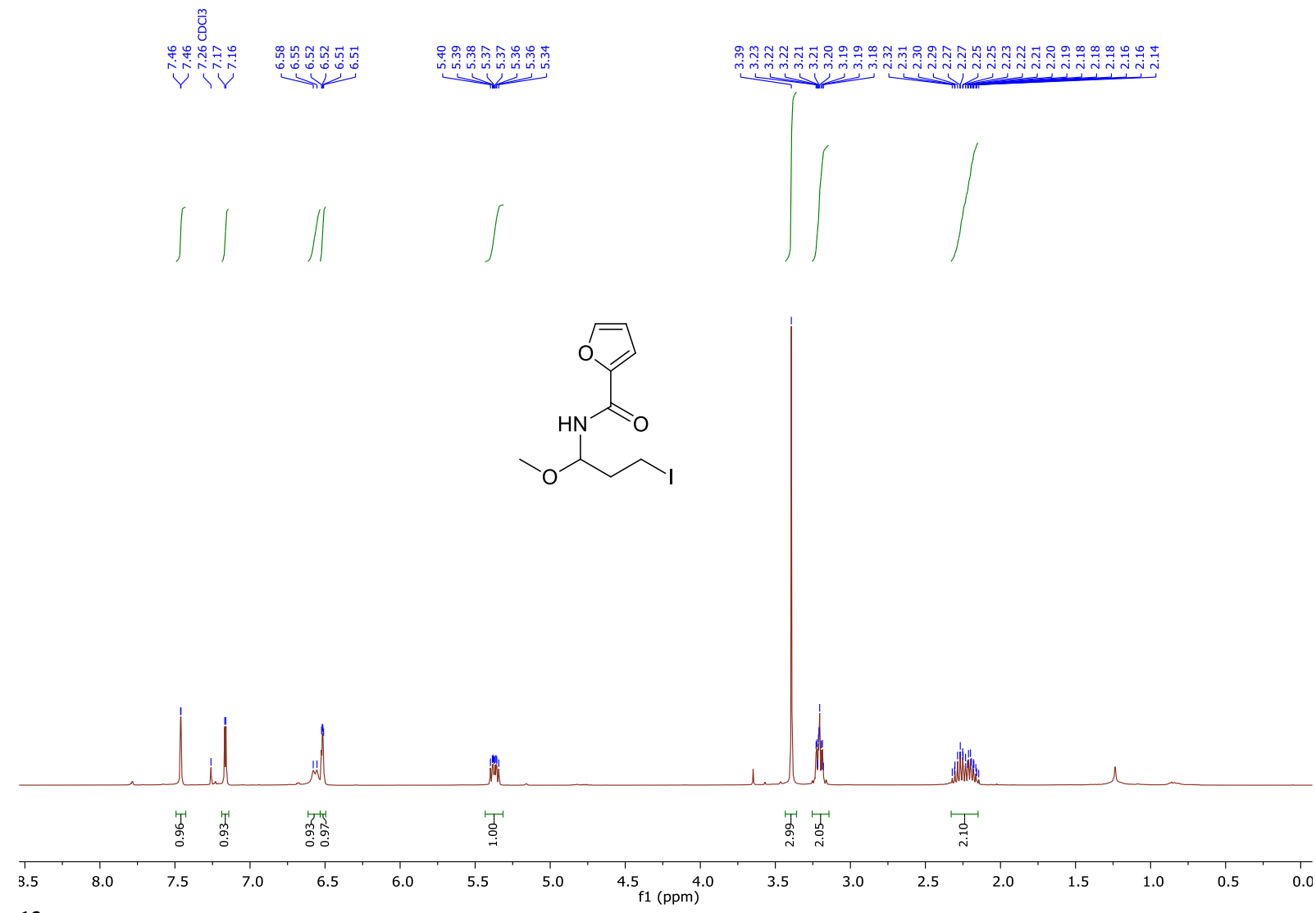

${ }^{13}$ C-NMR (101 MHz, CDCl3)

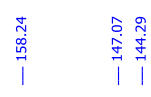

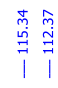

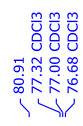

0
0
0

$\stackrel{\infty}{i}$

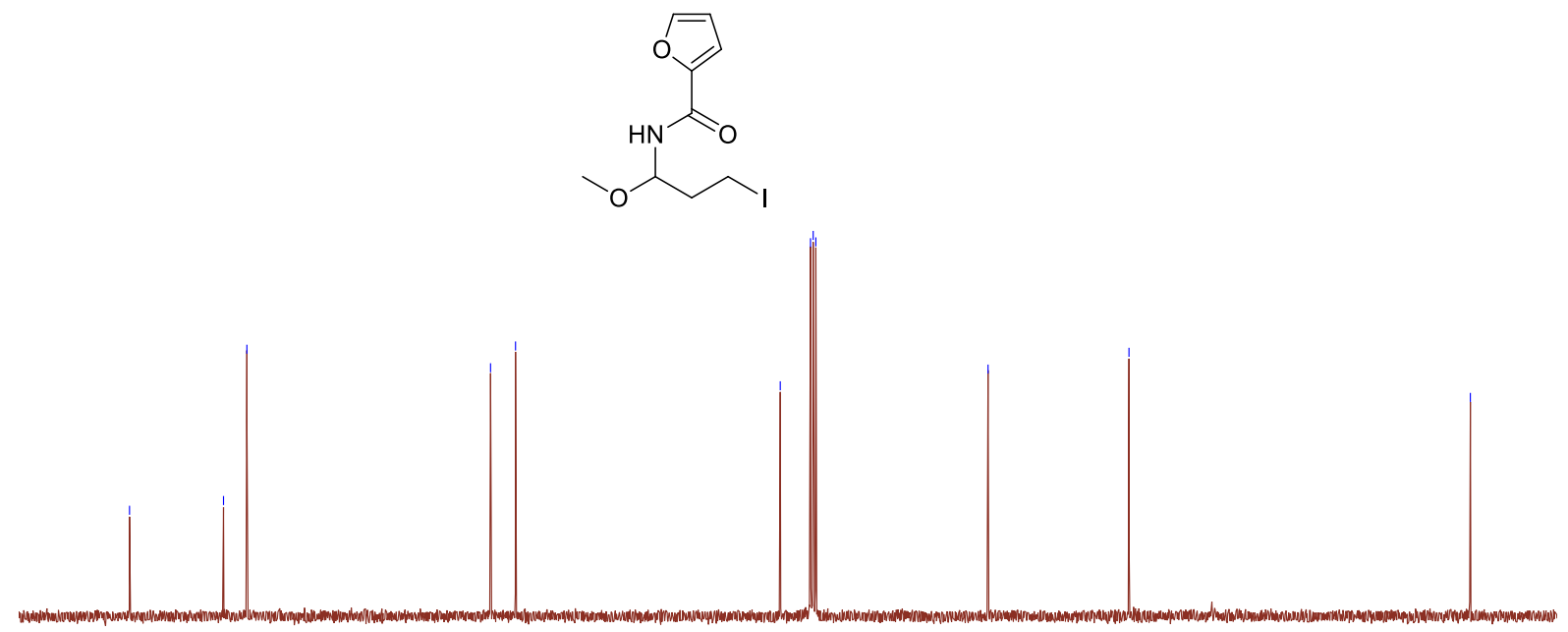

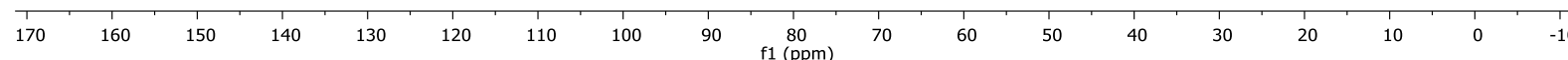


IR

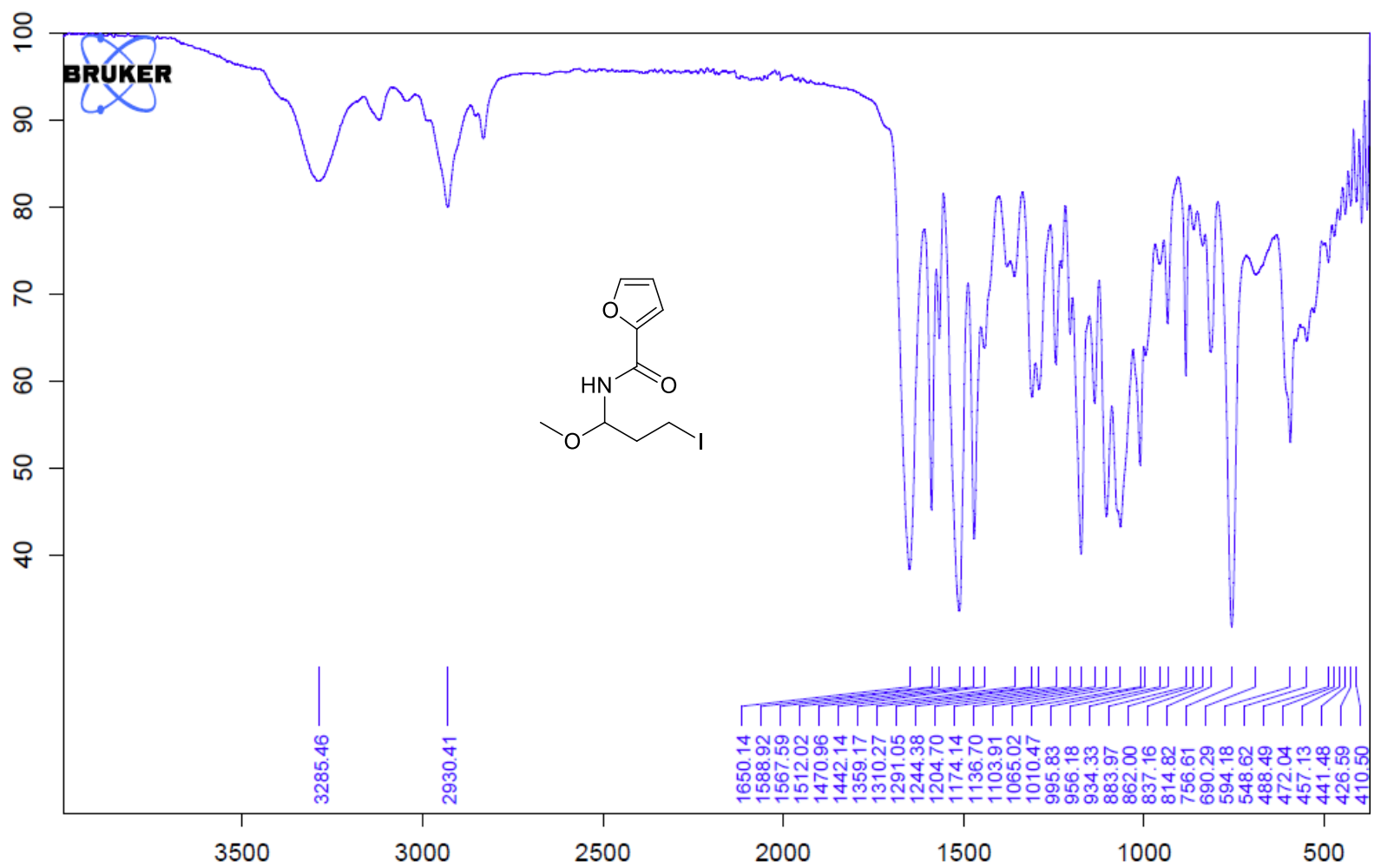

N-(3-Iodo-1-methoxypropyl)cinnamamide (4k)

${ }^{1} \mathrm{H}-\mathrm{NMR}$ (400 MHz, $\mathrm{CDCl}_{3}$ )

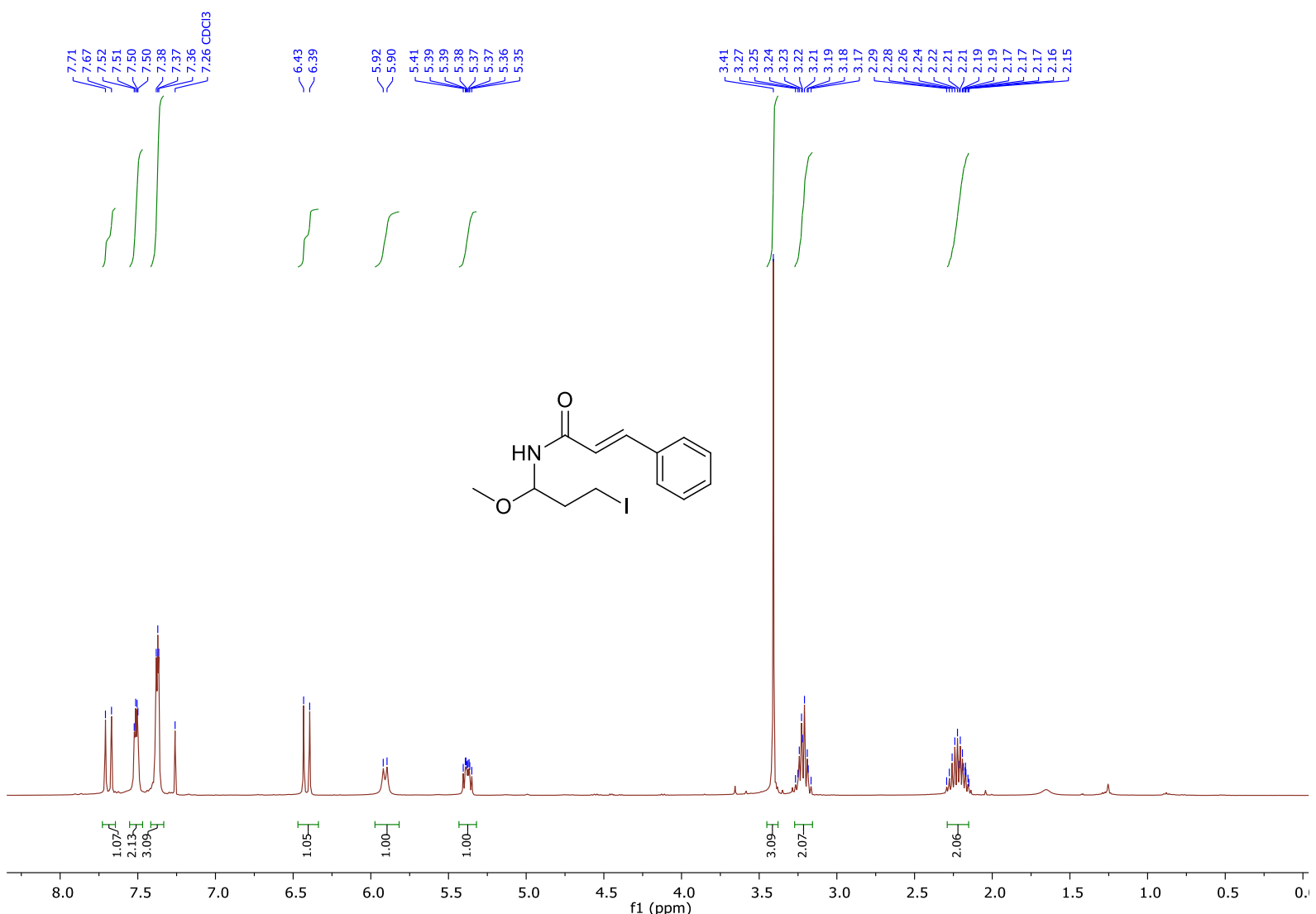


${ }^{13} \mathrm{C}$-NMR (101 MHz, CDCl3)

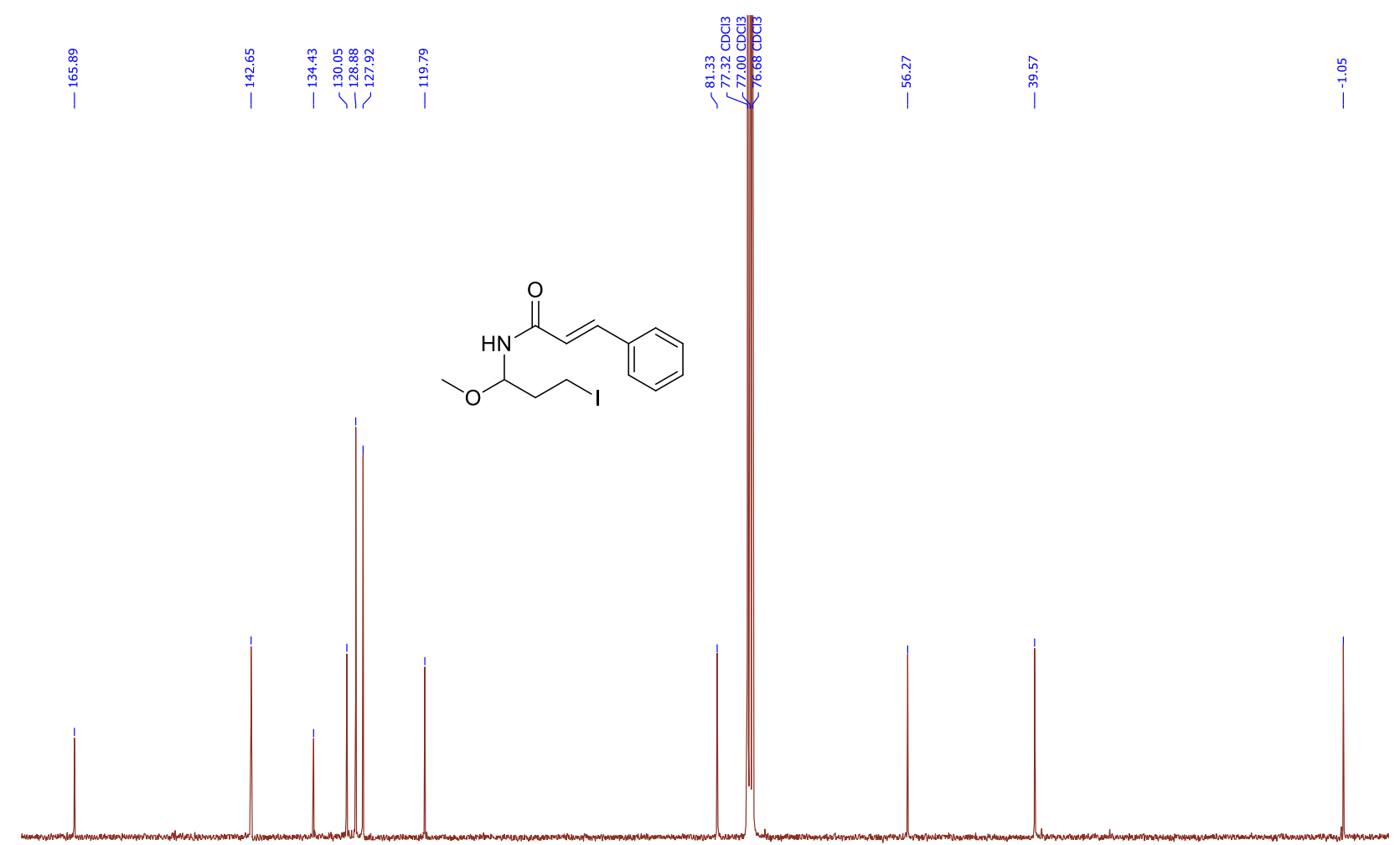

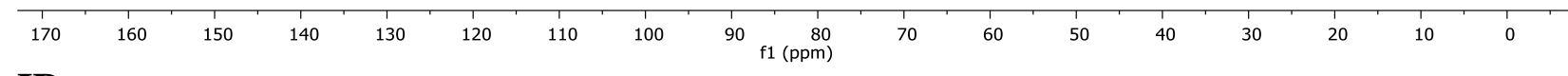

IR

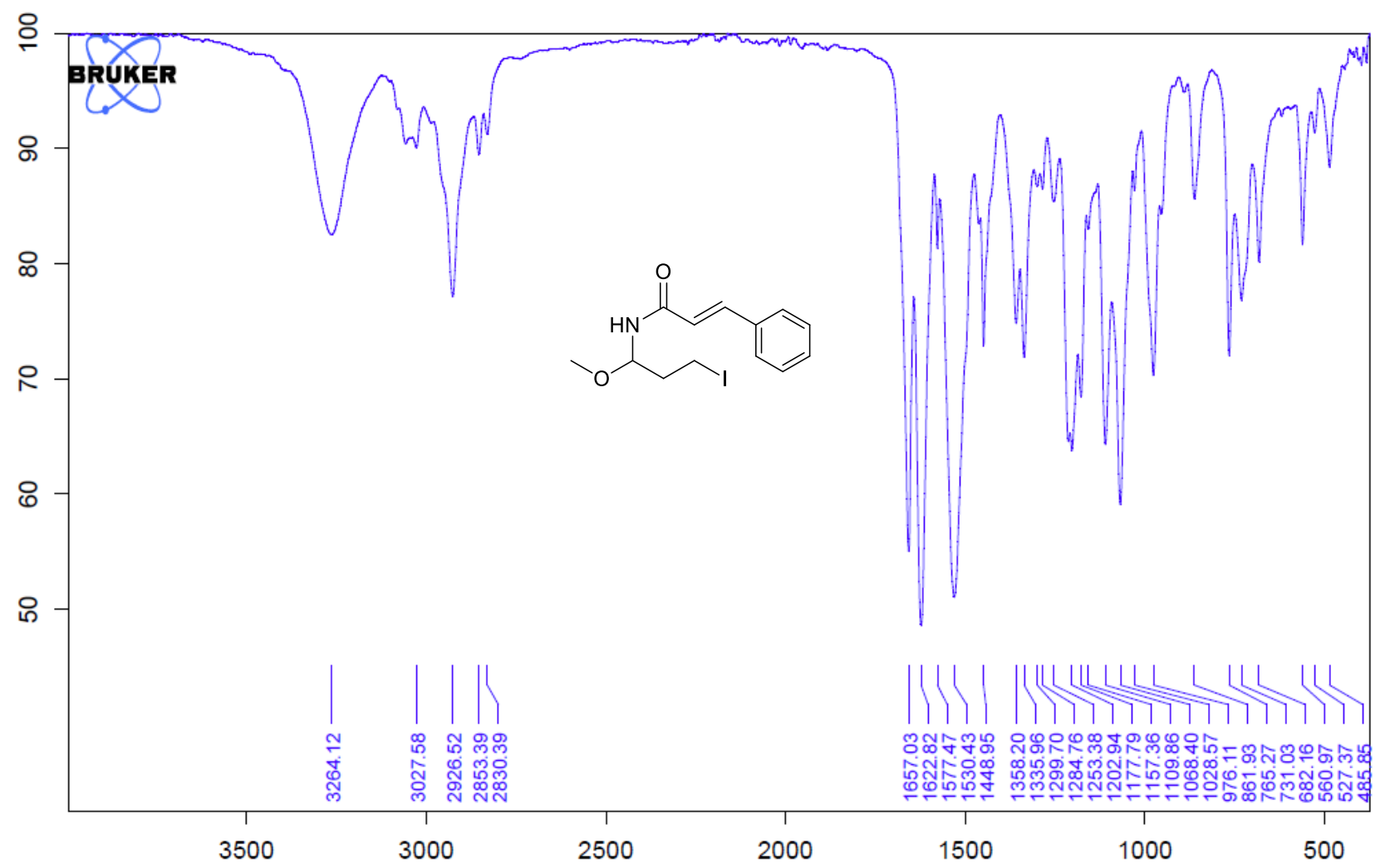


N-(3-Iodo-1-methoxypropyl)-2-phenylacetamide (4I)

${ }^{1} \mathrm{H}-\mathrm{NMR}$ (400 MHz, $\mathrm{CDCl}_{3}$ )

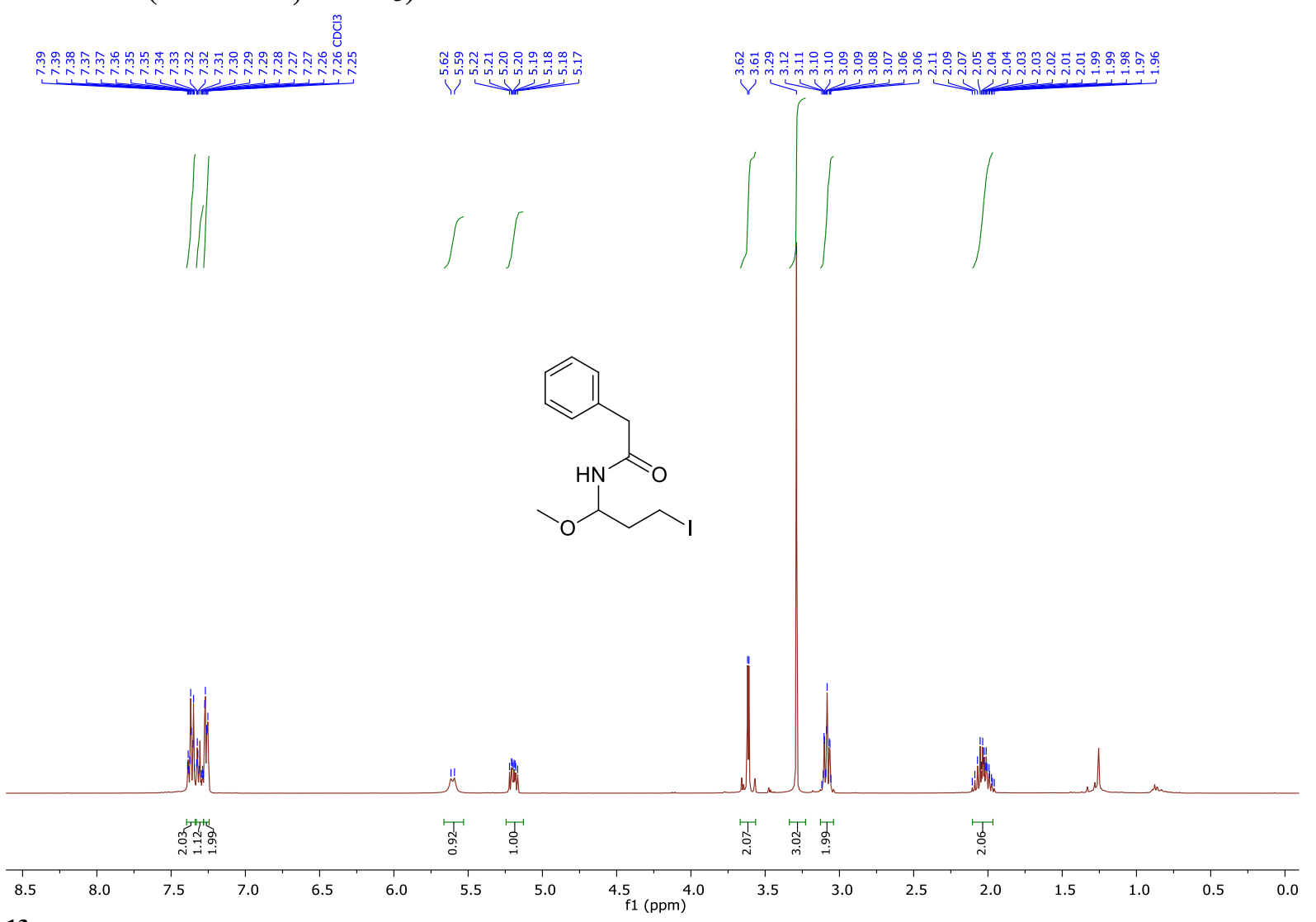

${ }^{13} \mathrm{C}-\mathrm{NMR}$ (101 MHz, CDCl3)
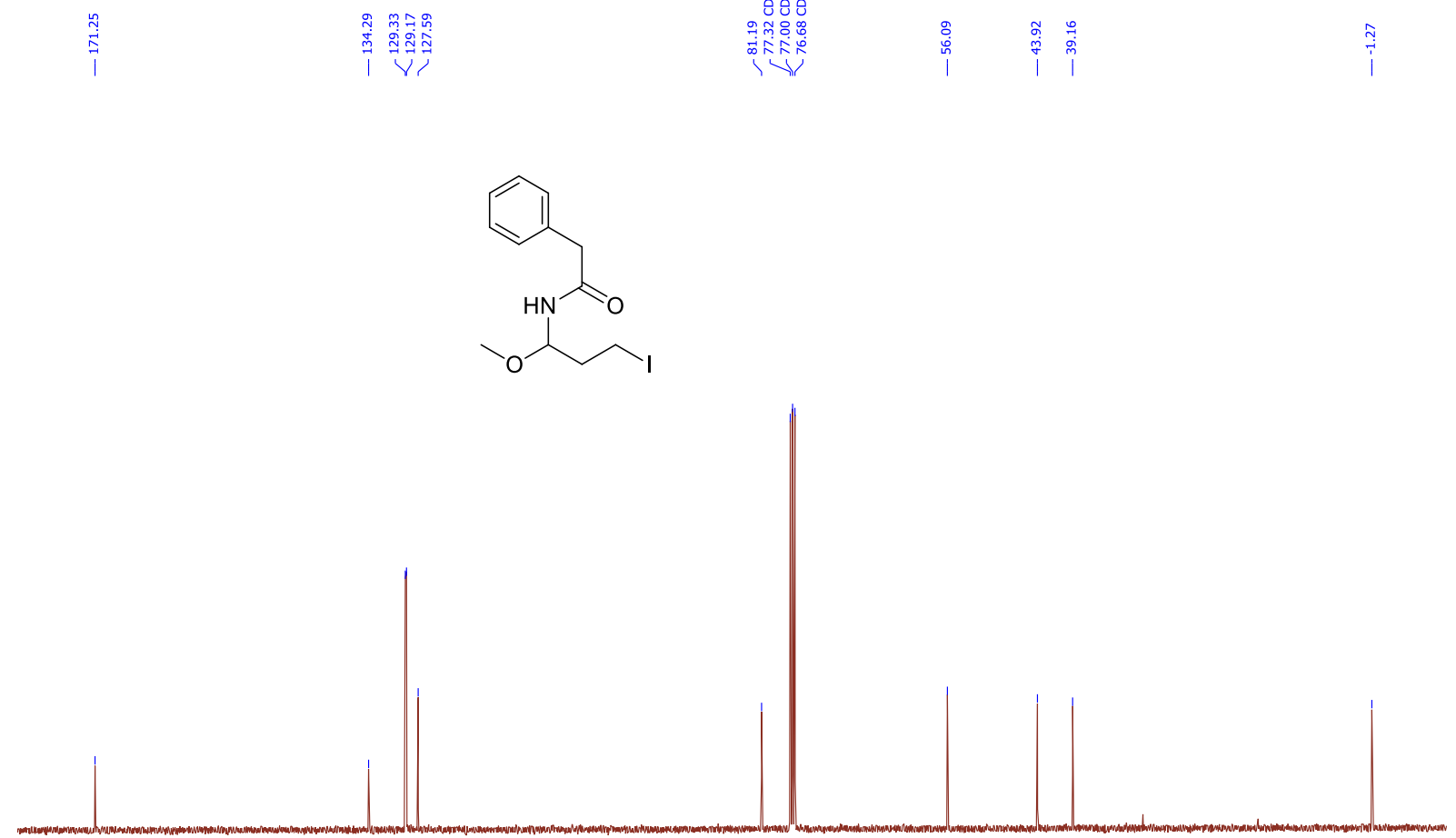


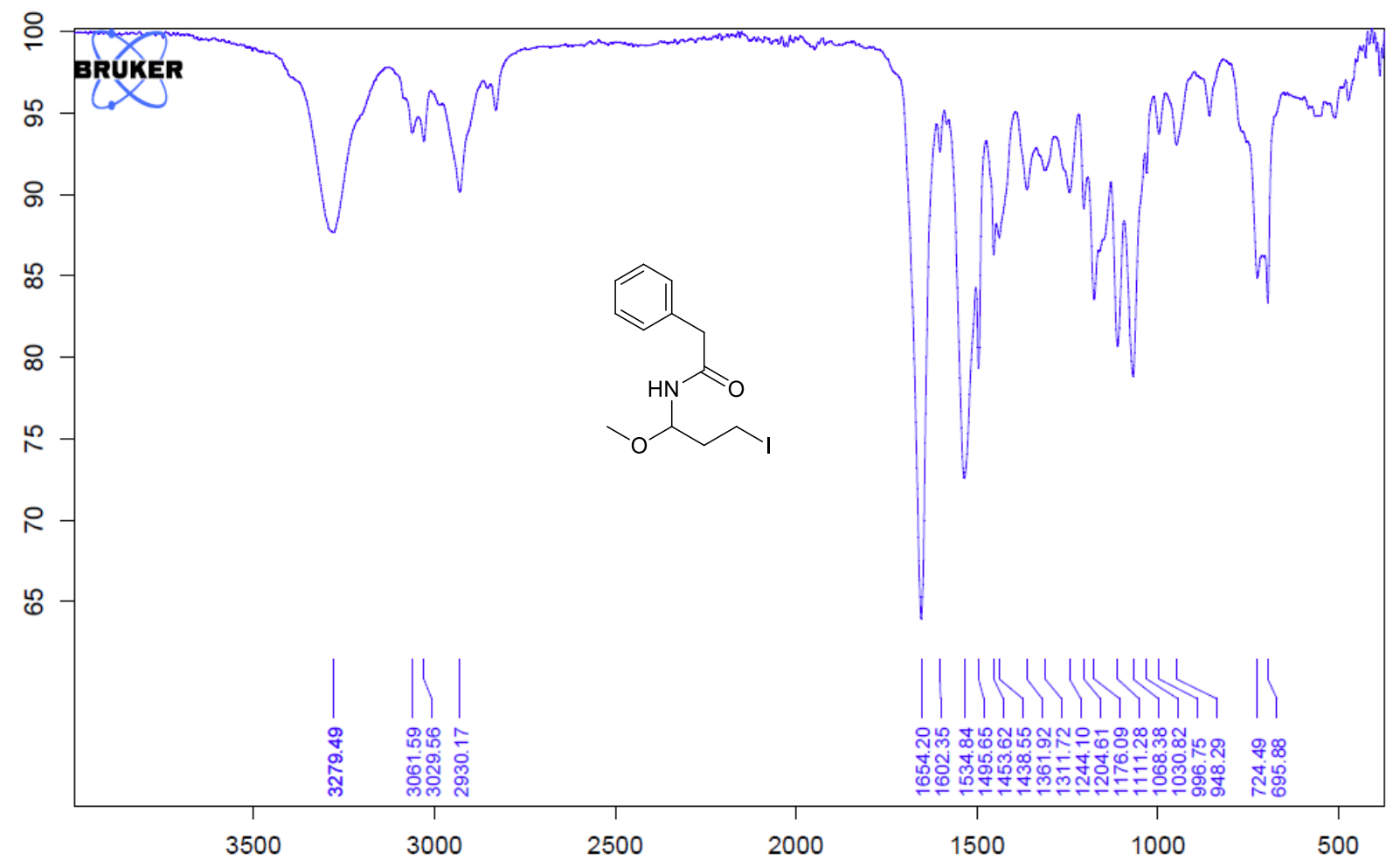

N-(3-Iodo-1-methoxypropyl)-2-naphthamide (4m)

${ }^{1}$ H-NMR (400 MHz, CDCl 3 )

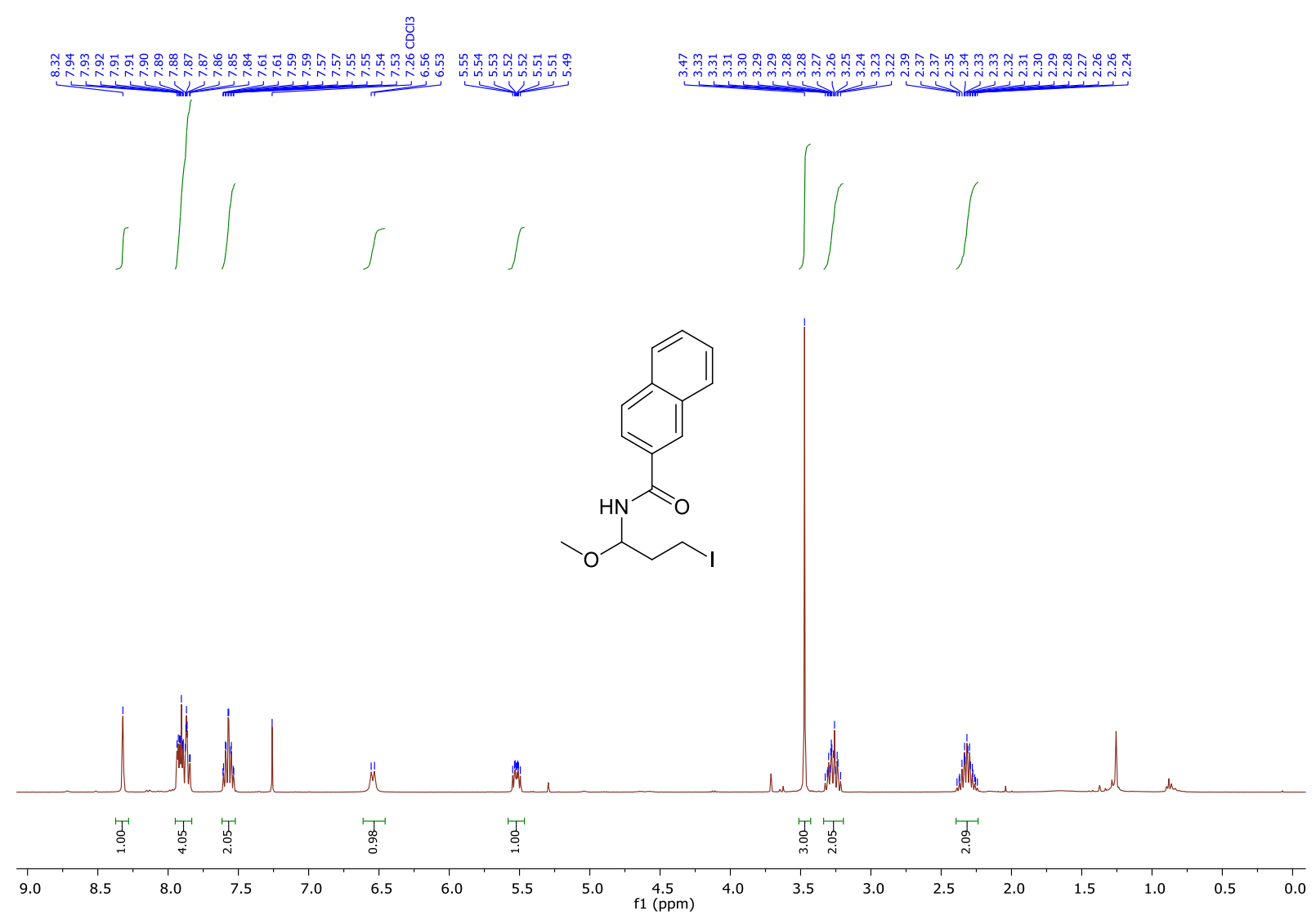


${ }^{13} \mathrm{C}$-NMR (101 MHz, CDCl3)
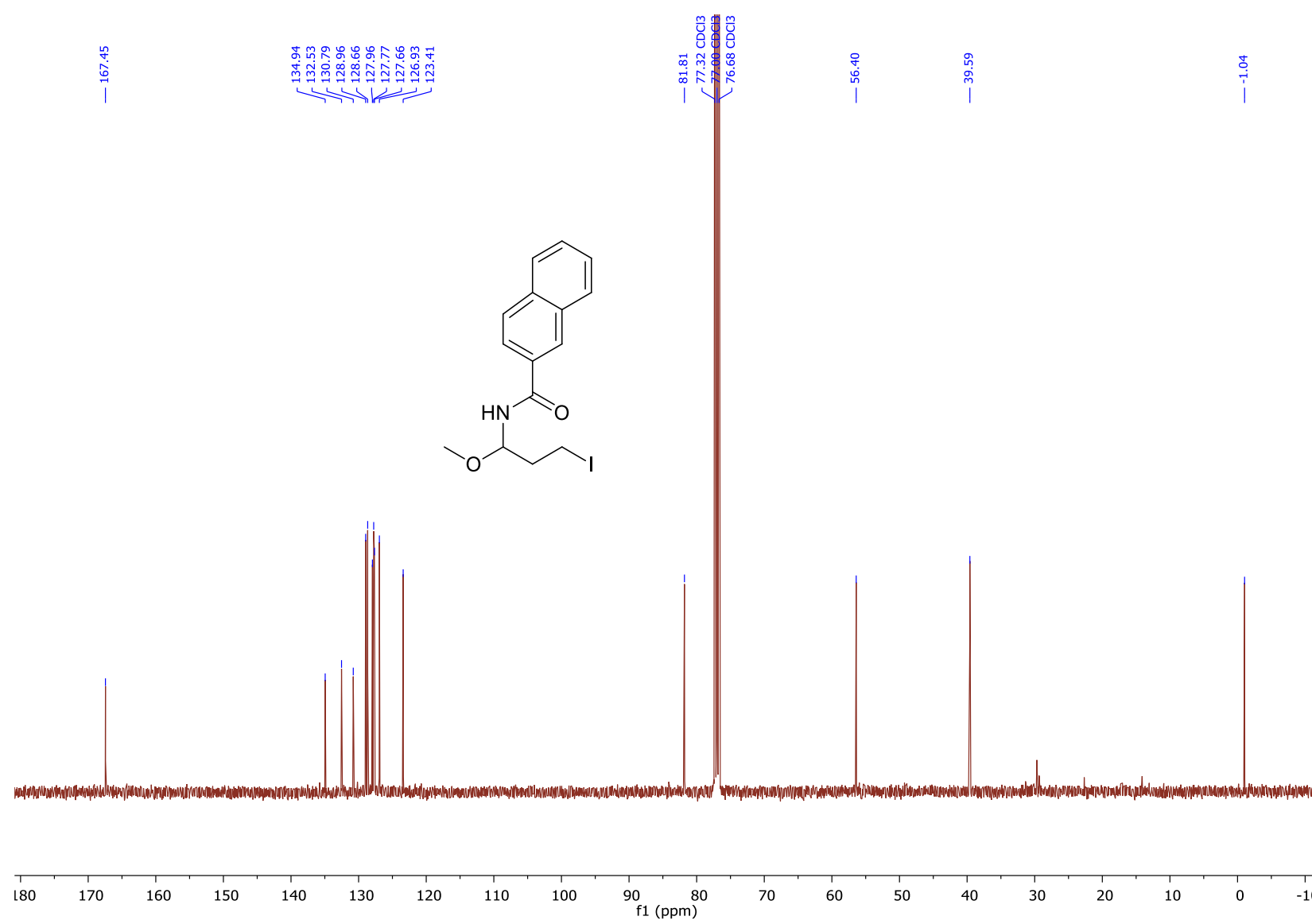

IR

음

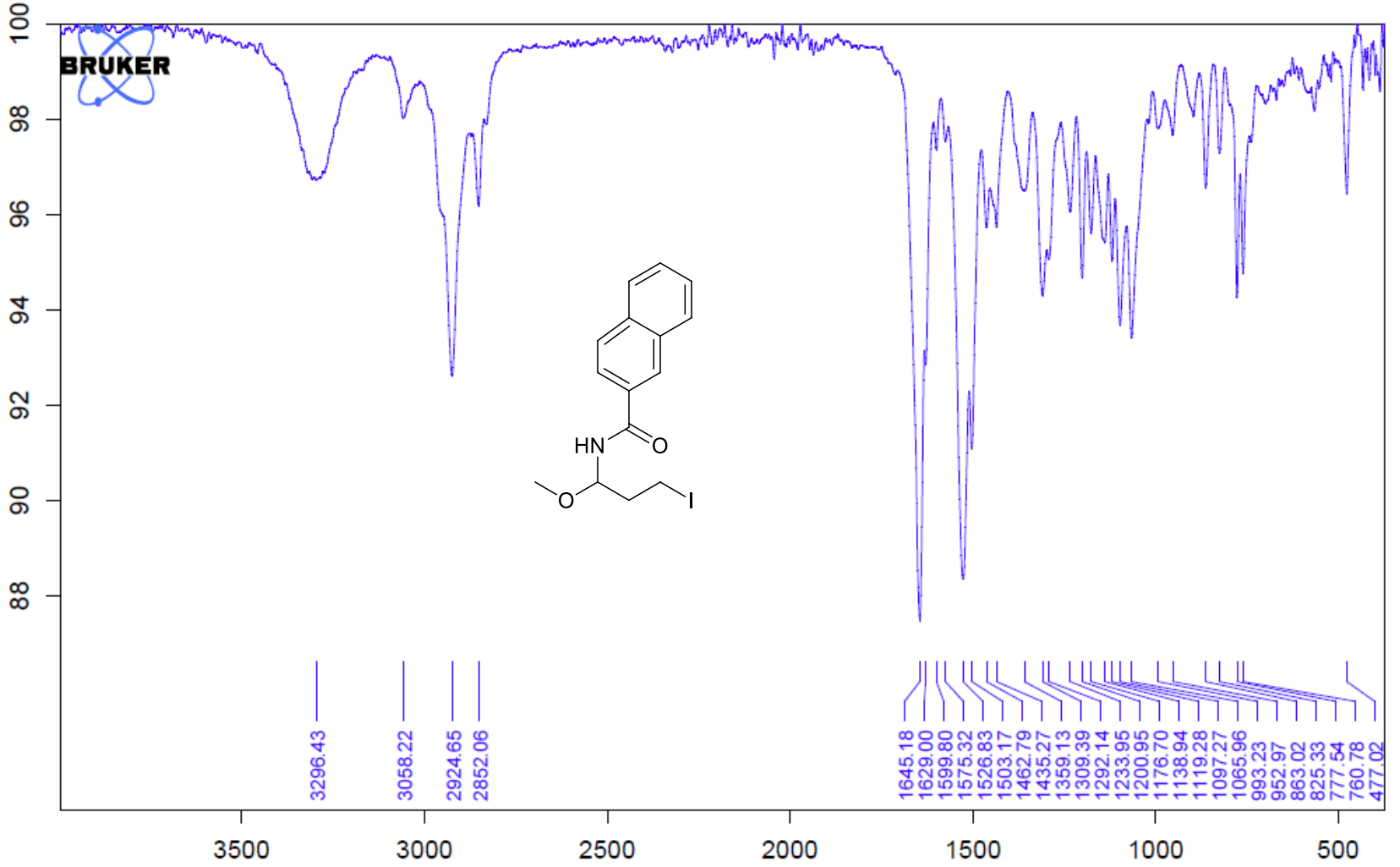


N-(3-Iodo-1-methoxypropyl)pivalamide (4n)

${ }^{1} \mathrm{H}-\mathrm{NMR}$ (400 MHz, CDCl 3$)$

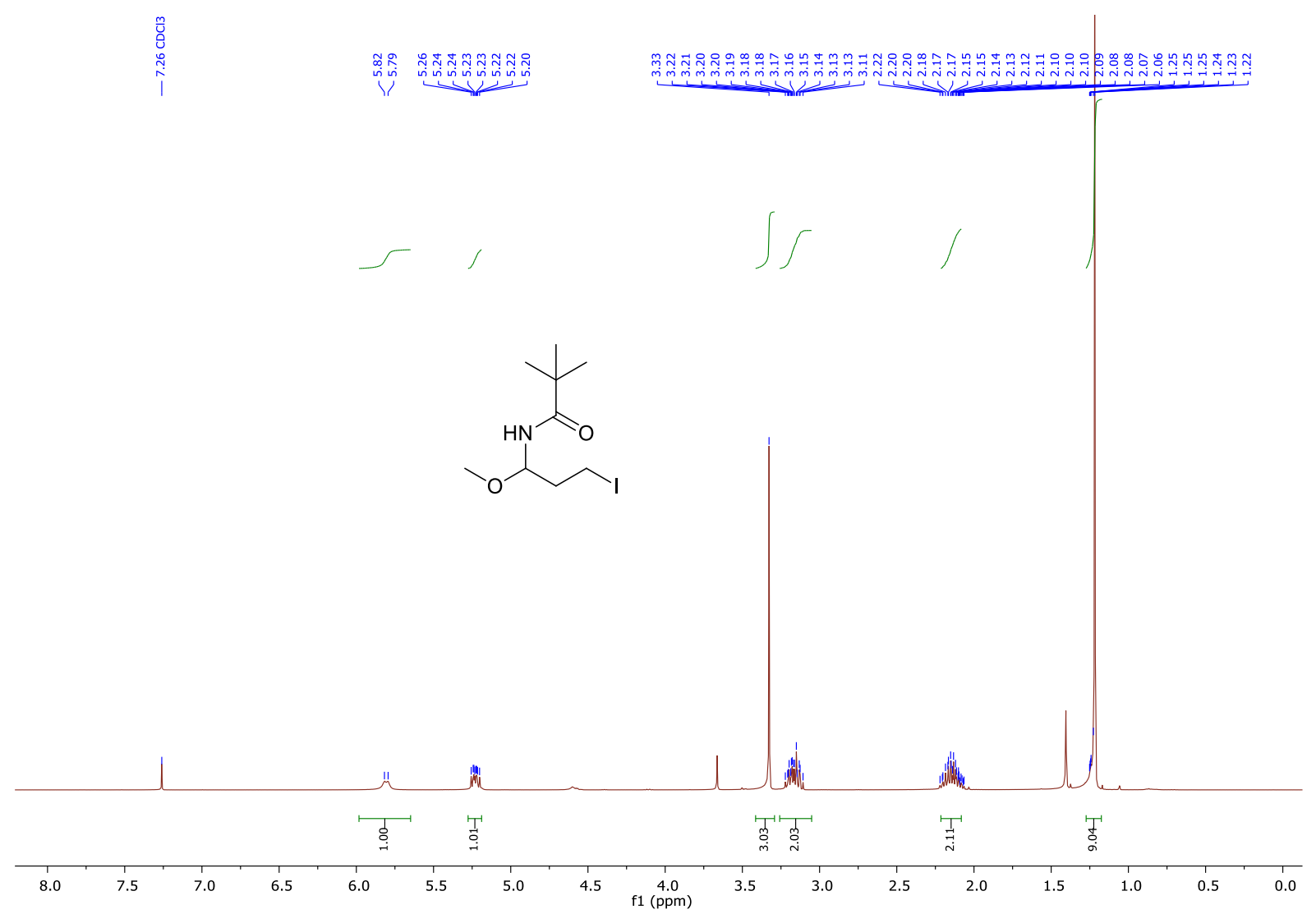

${ }^{13} \mathrm{C}-\mathrm{NMR}\left(101 \mathrm{MHz}, \mathrm{CDCl}_{3}\right)$

$\stackrel{\substack{+1 \\ \infty}}{\stackrel{5}{5}}$
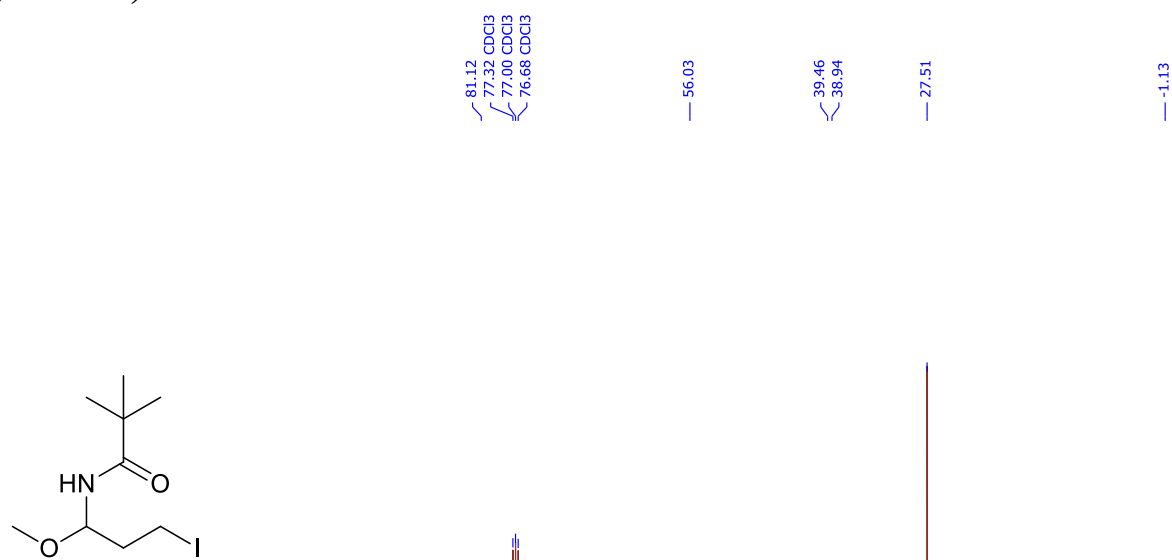

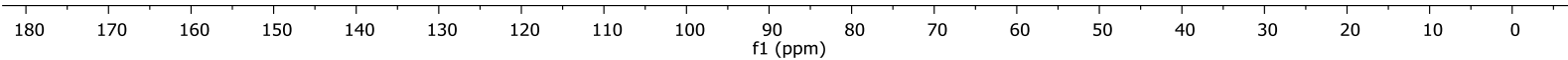


IR

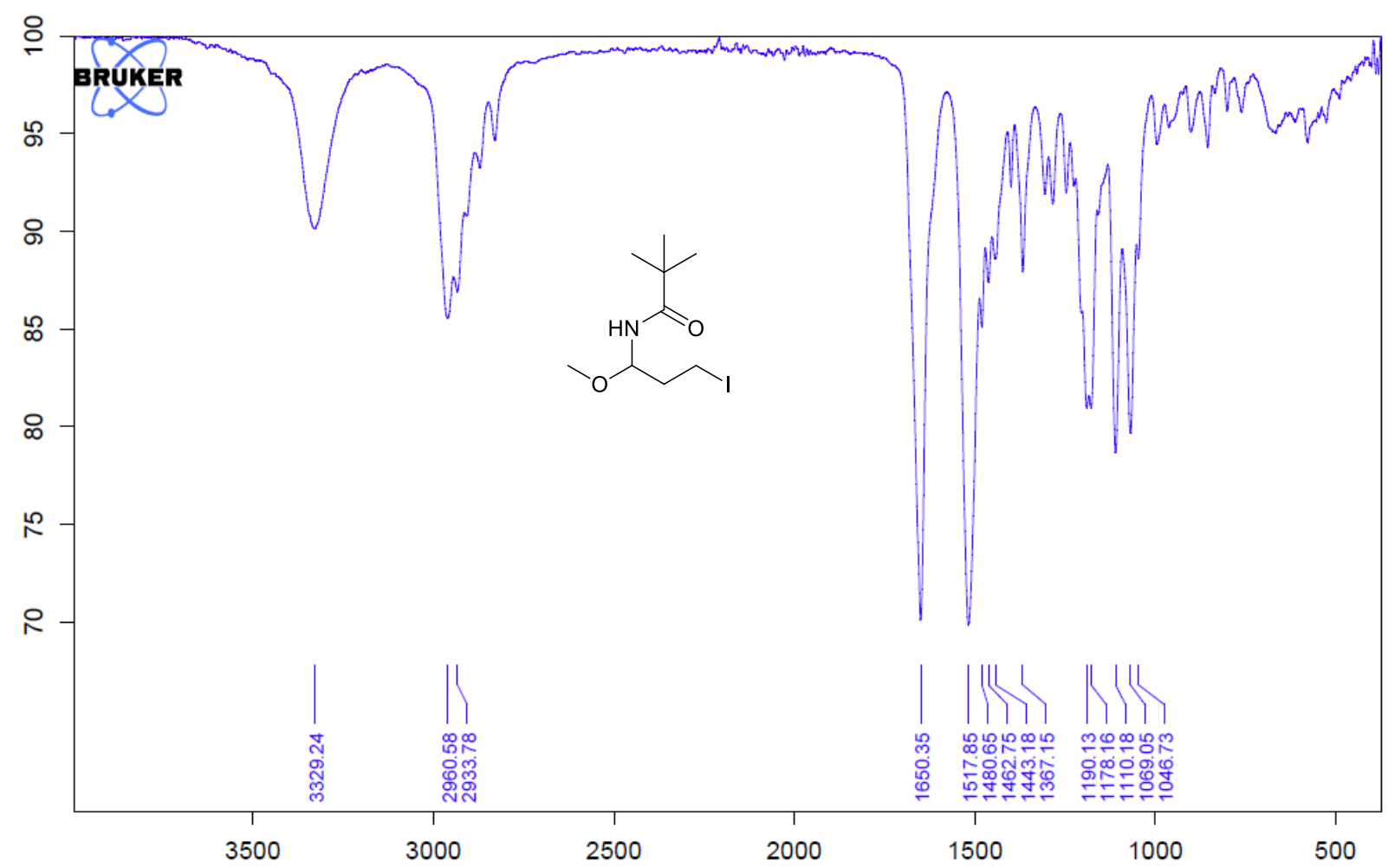

tert-Butyl (3-iodo-1-methoxypropyl)carbamate (4o)

${ }^{1}$ H-NMR (400 MHz, CD 3 OD)
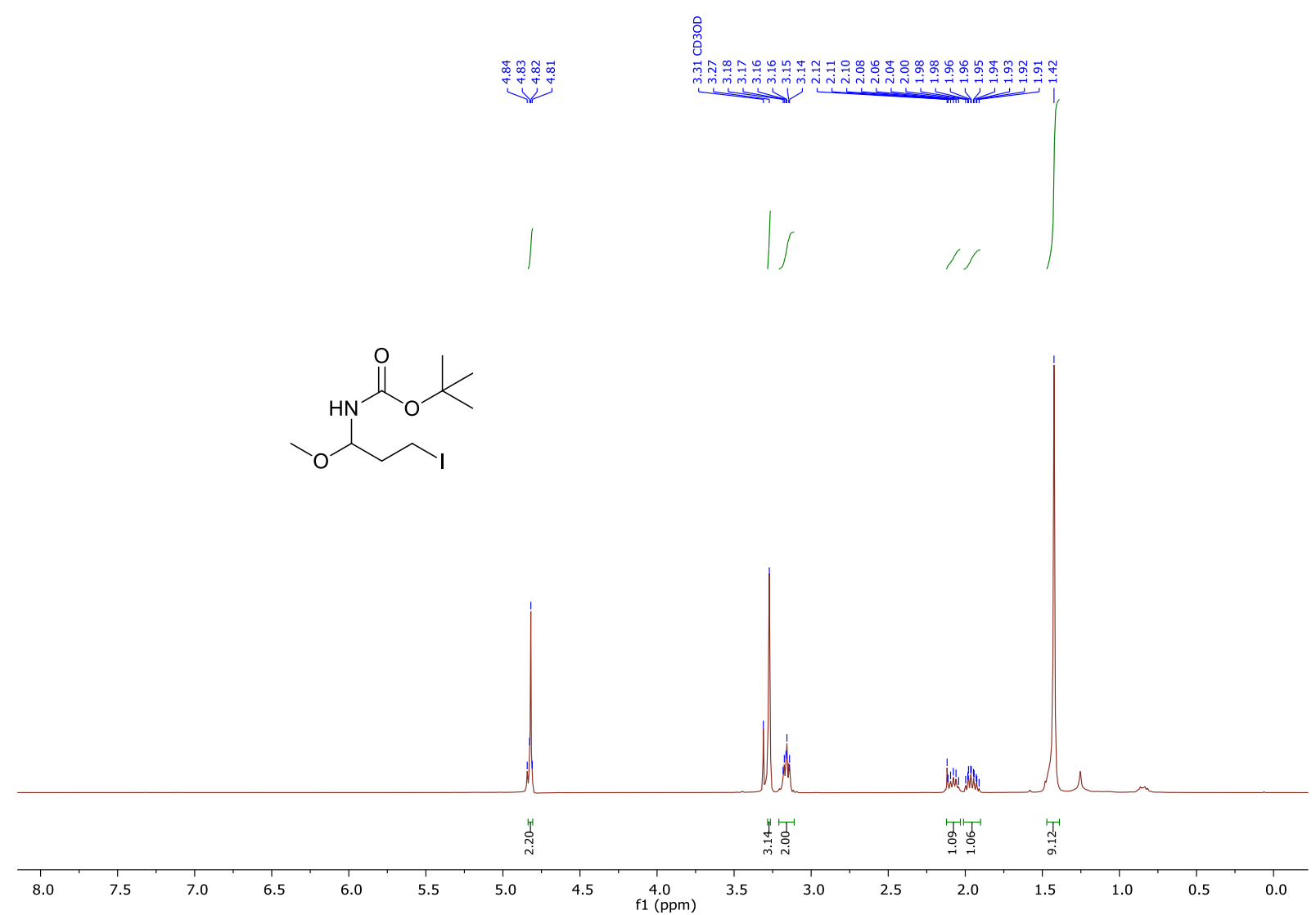
${ }^{13}$ C-NMR (101 MHz, CD3OD)
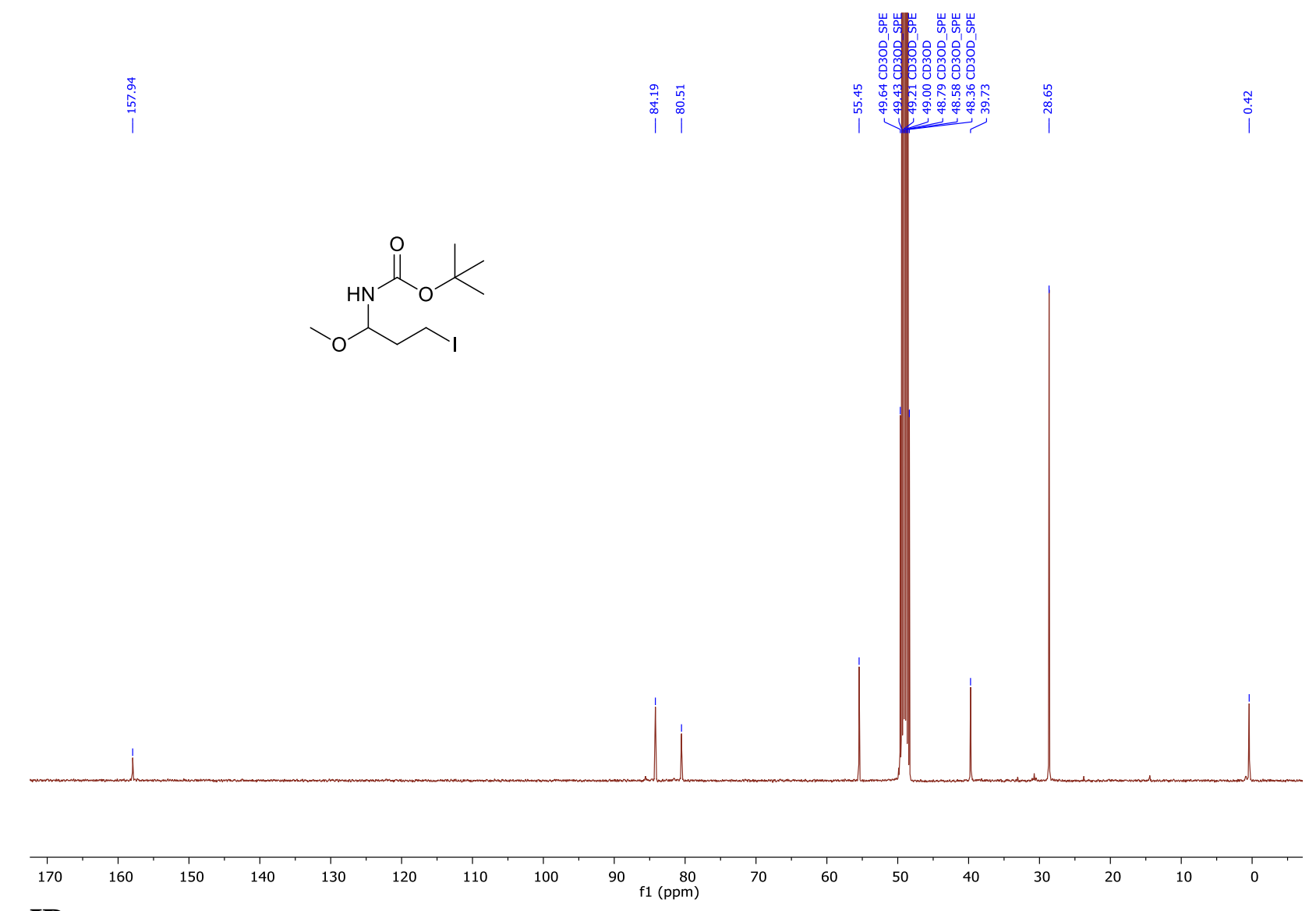

IR

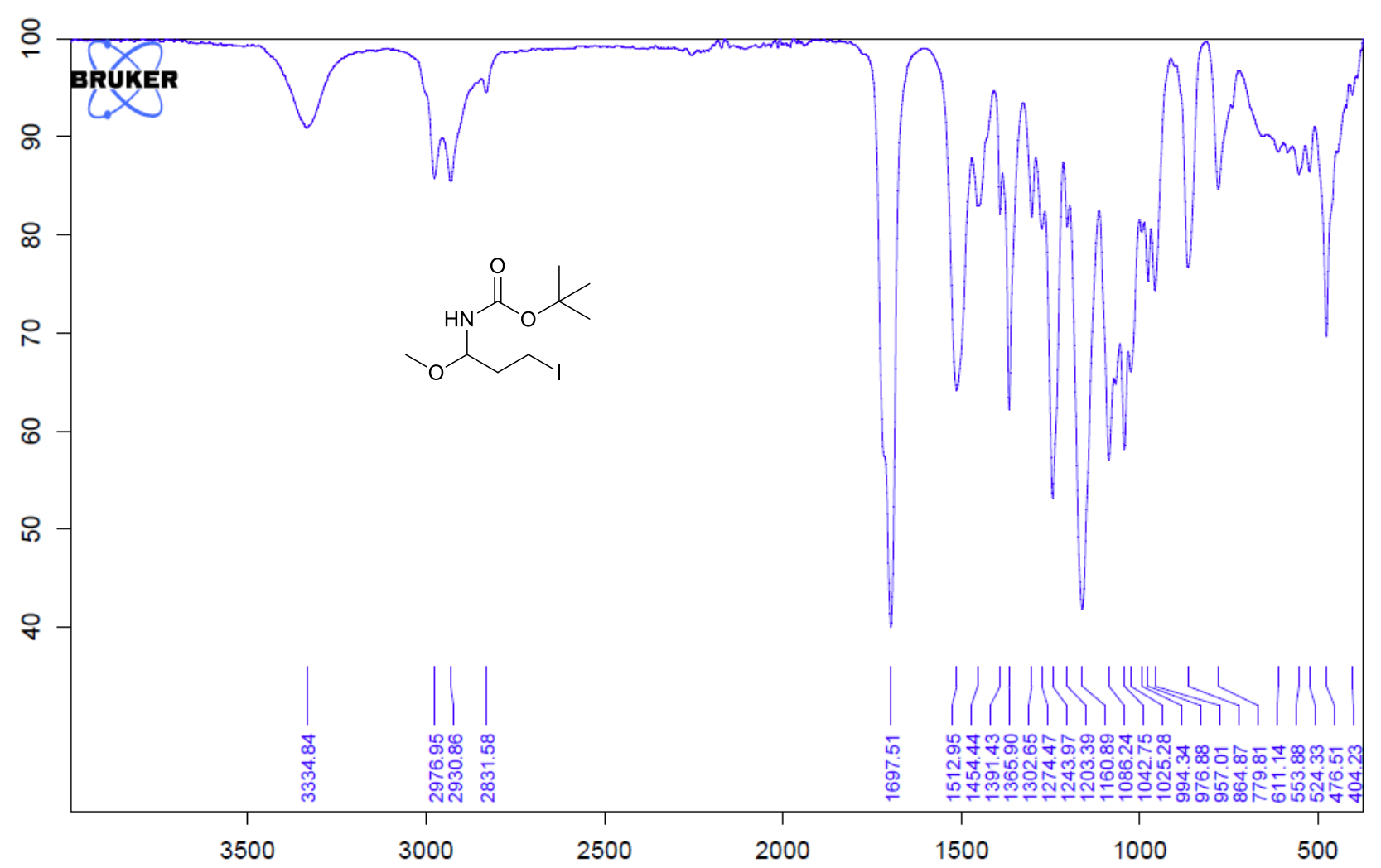


Benzyl (3-iodo-1-methoxypropyl)carbamate (4p)

${ }^{1}$ H-NMR (400 MHz, CDCl $)$
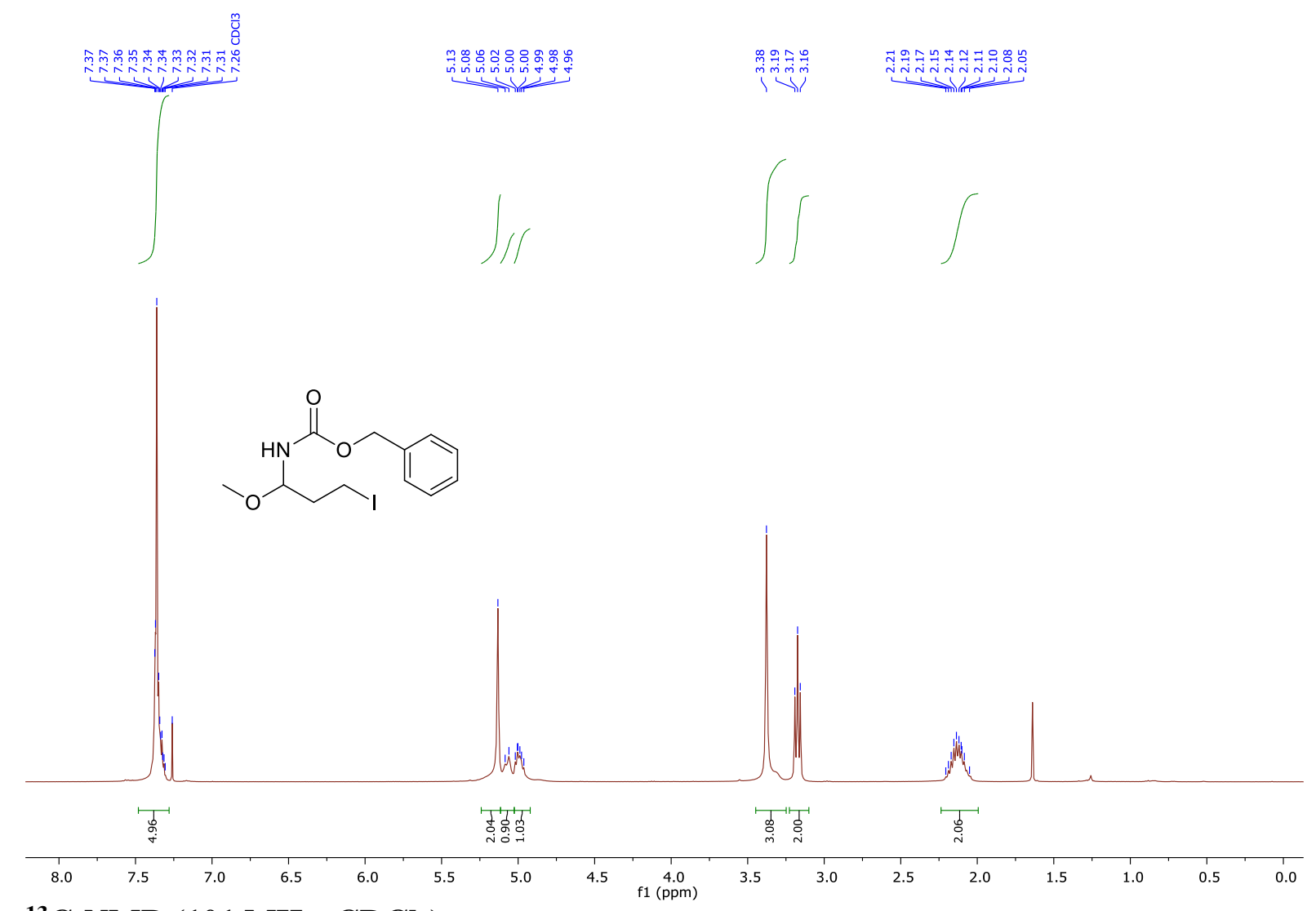

${ }^{13}$ C-NMR (101 MHz, CDCl3)
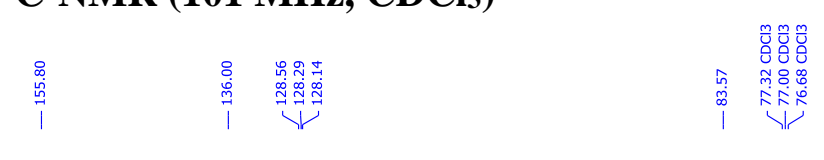

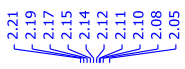

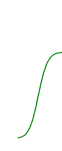

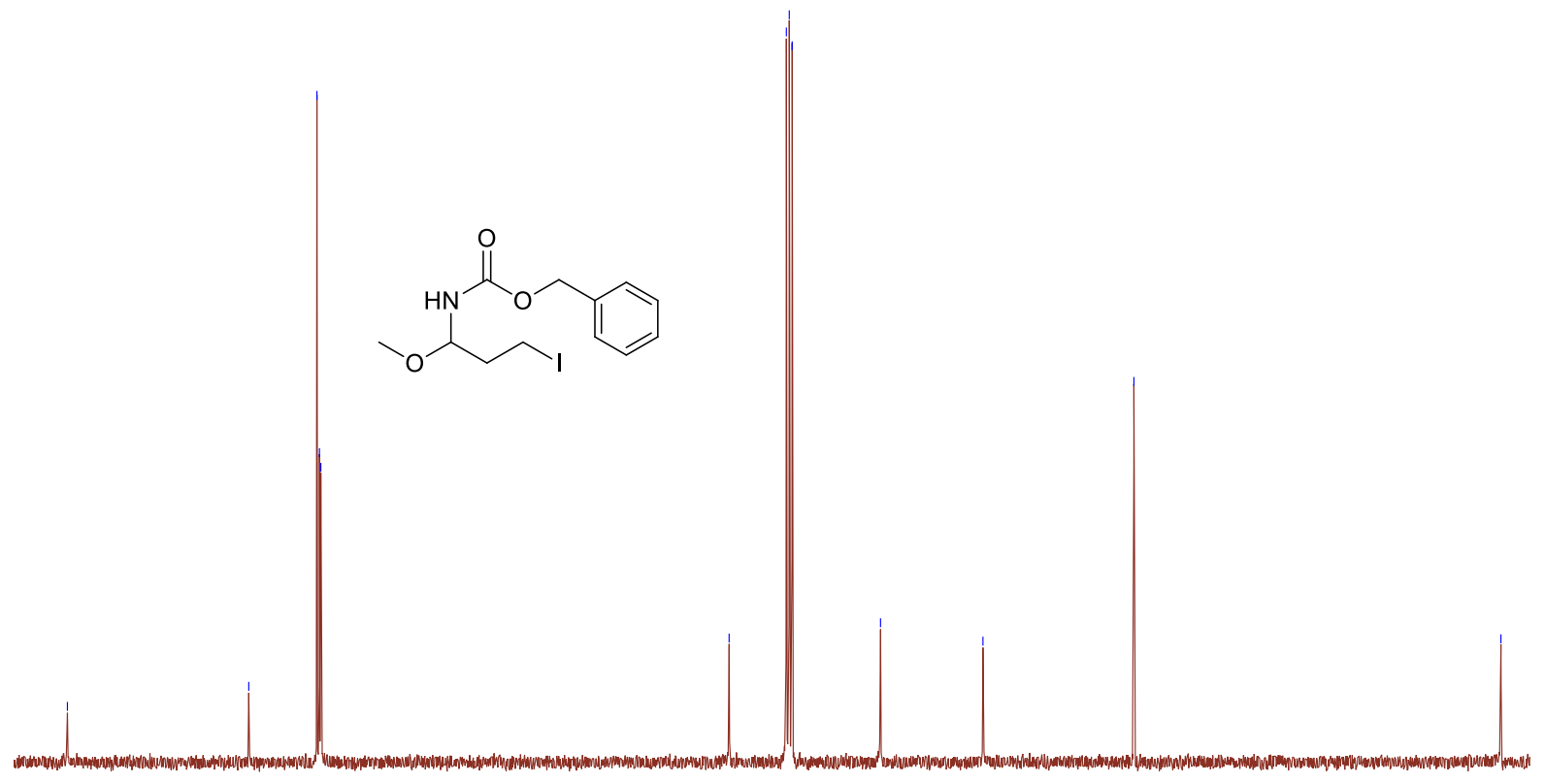

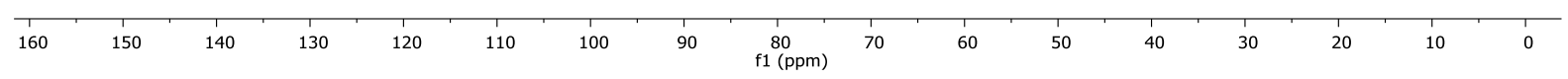

S92 
IR

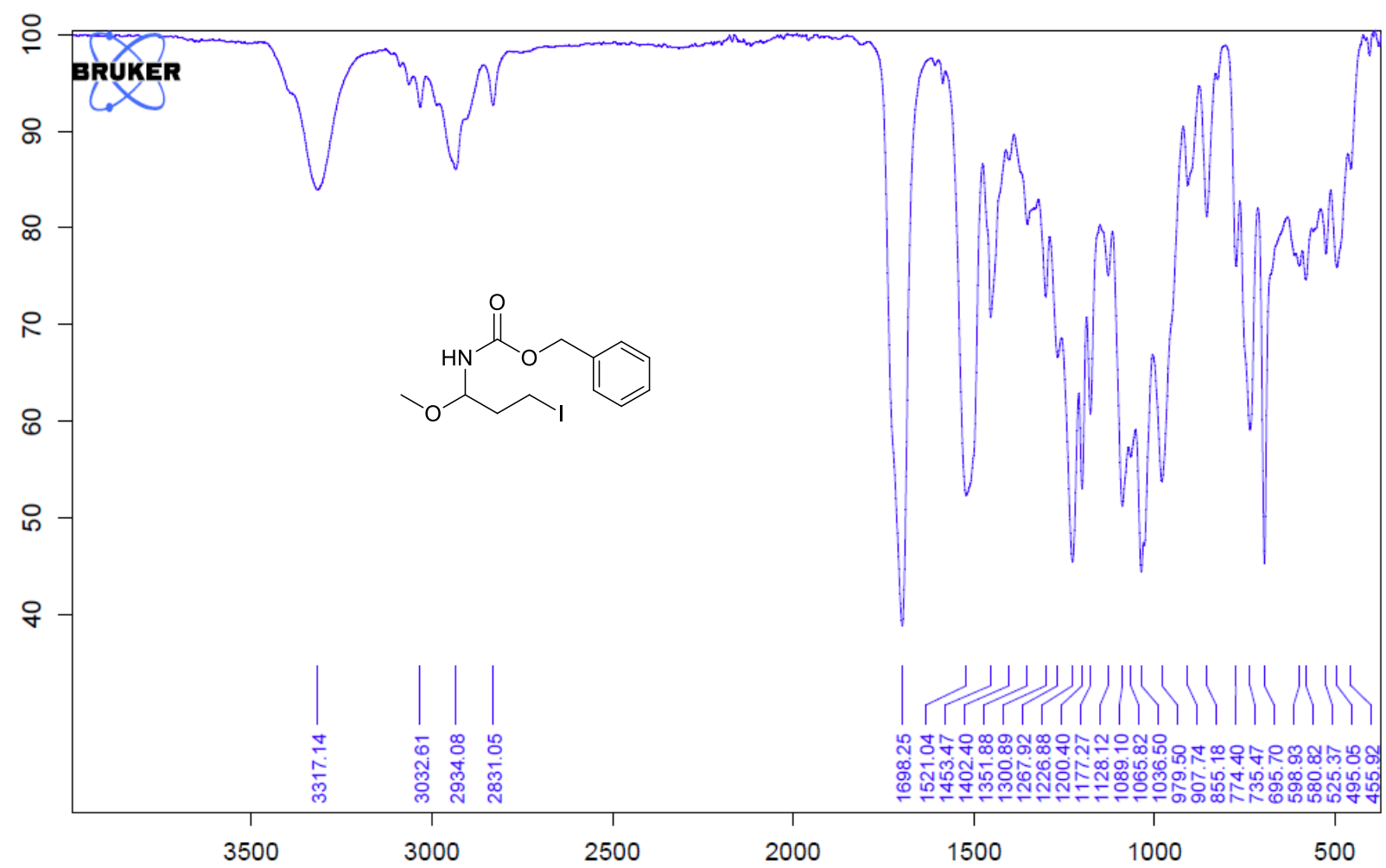

N-(3-Iodo-1-methoxypropyl)-4-methylbenzenesulfonamide (4q)

${ }^{1} \mathrm{H}-\mathrm{NMR}$ (400 MHz, CD3OD)

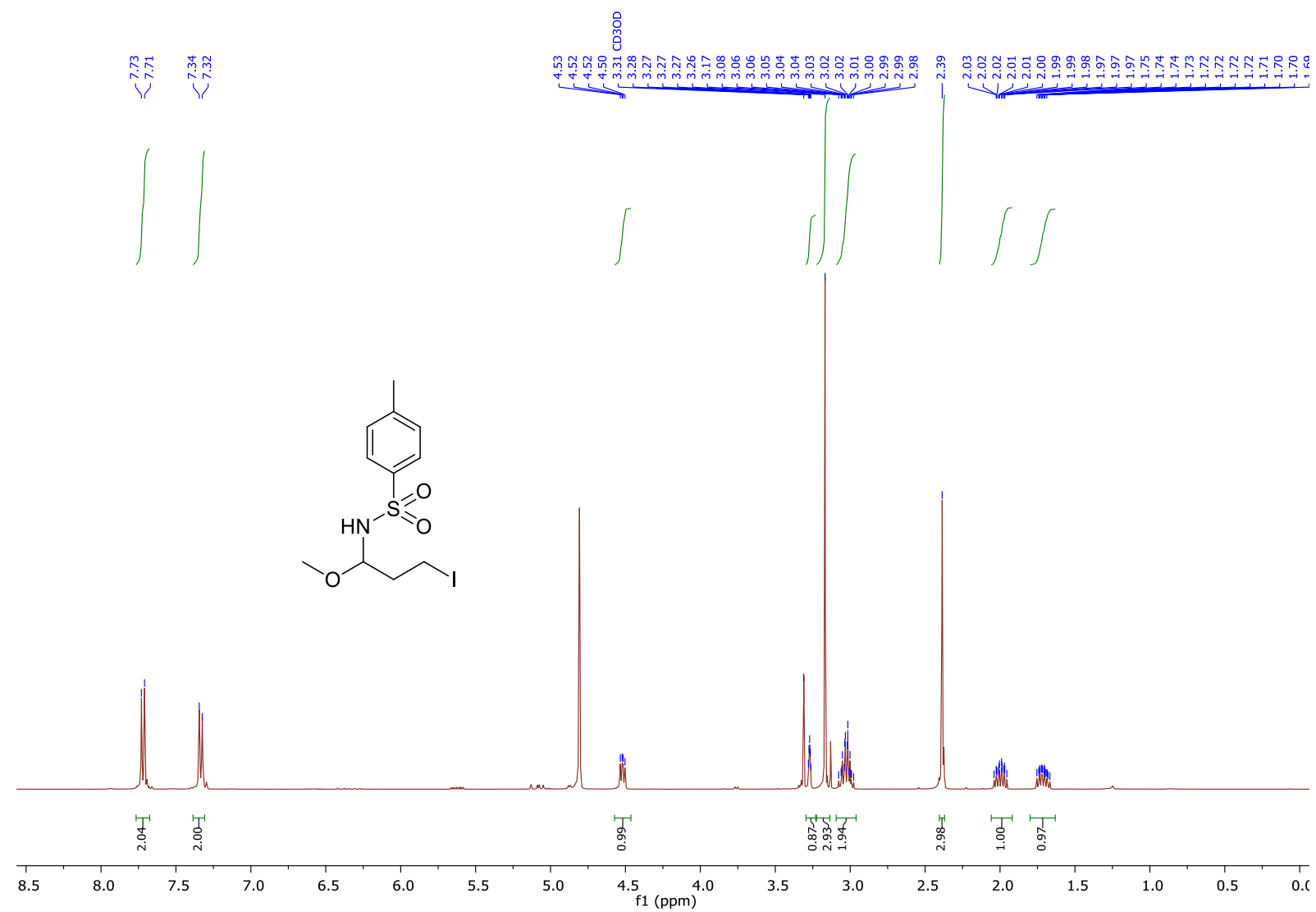


${ }^{13}$ C-NMR (101 MHz, CD3OD)

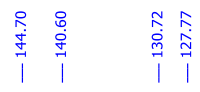
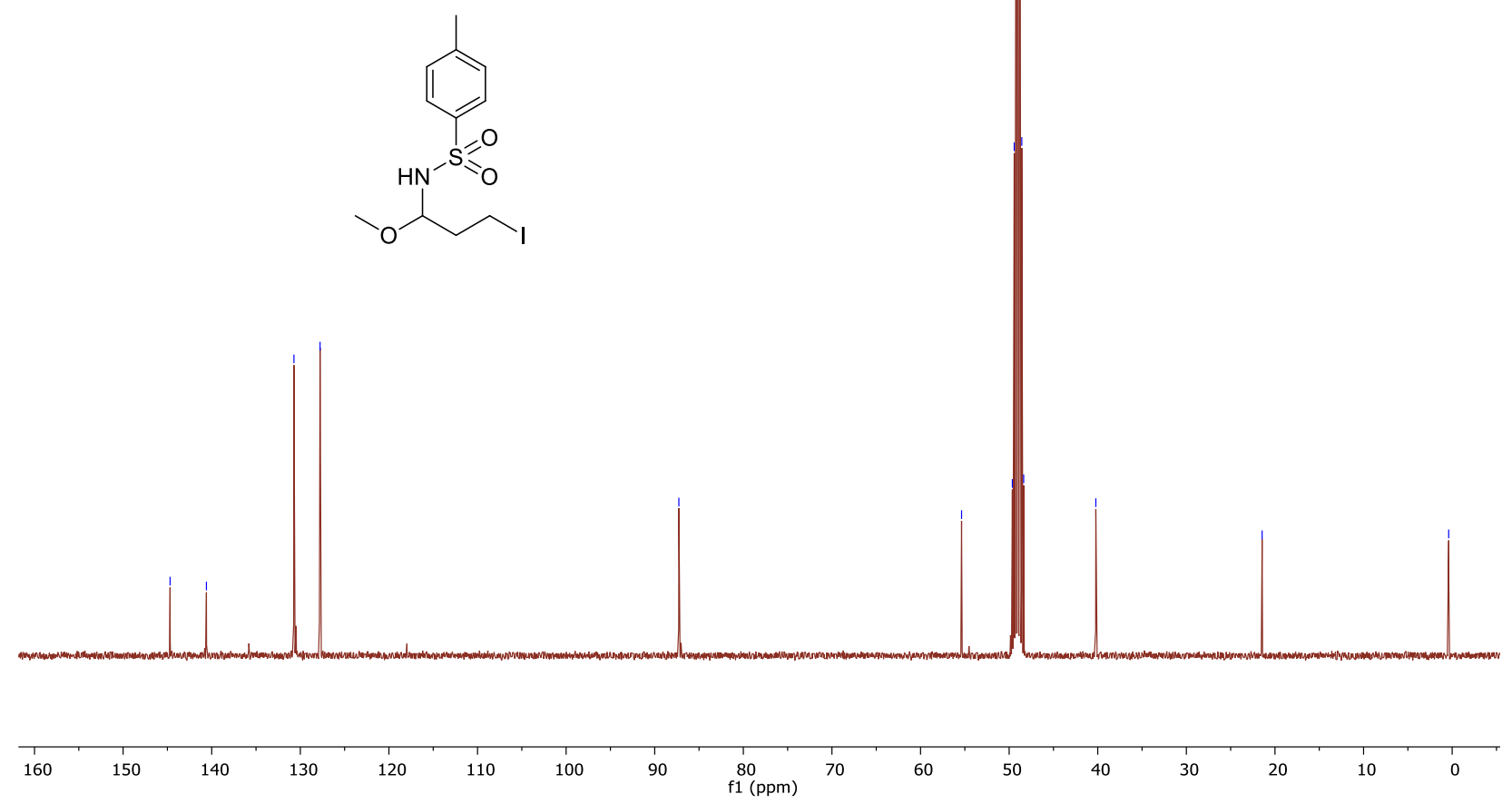

IR

$\stackrel{8}{\circ}$

ஃ

BRUKER

8

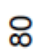

운

8

요

g

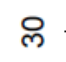
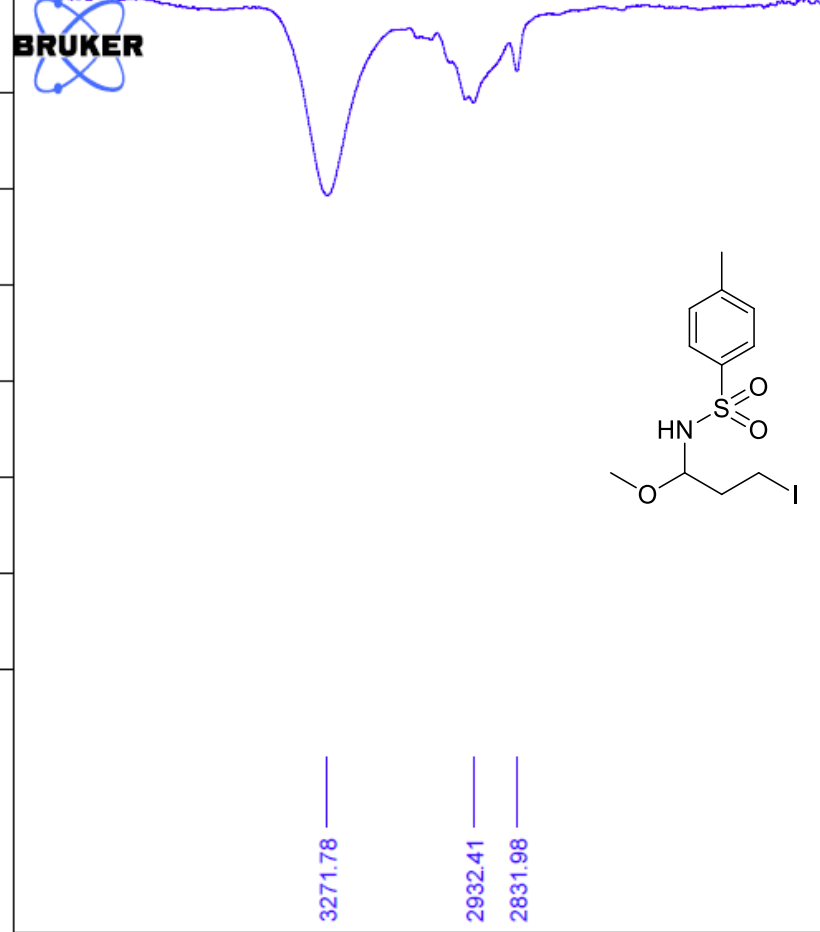

3500

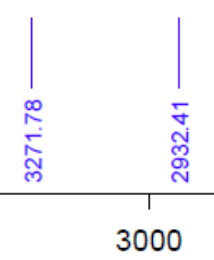

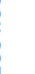
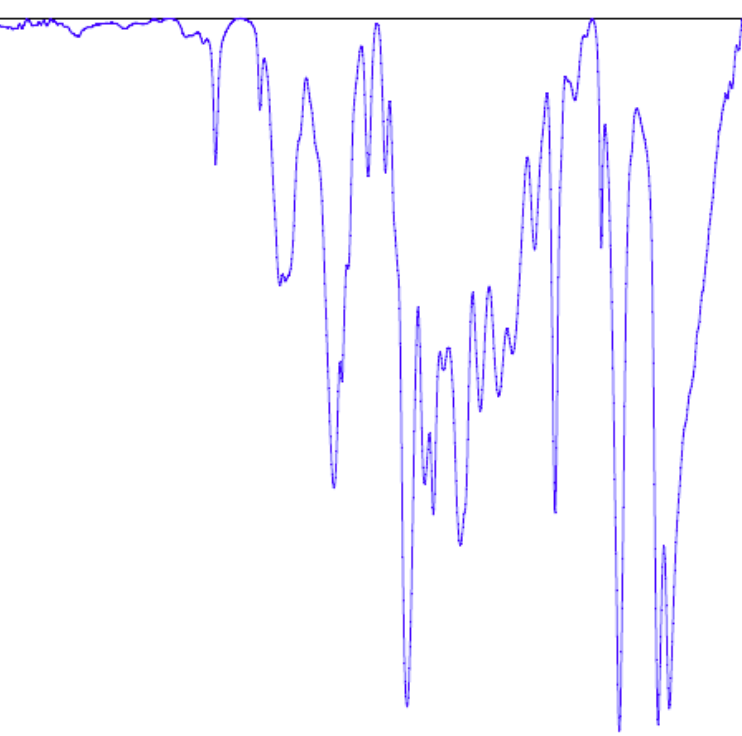

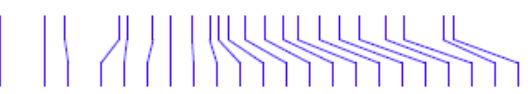

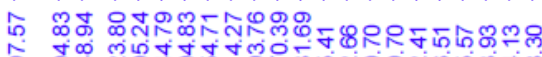

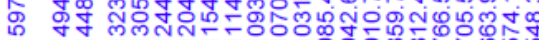

1500

500 
N-(3-Iodo-1-methoxypropyl)-N,4-dimethylbenzenesulfonamide (4r)

${ }^{1}$ H-NMR (400 MHz, Acetone-d6)

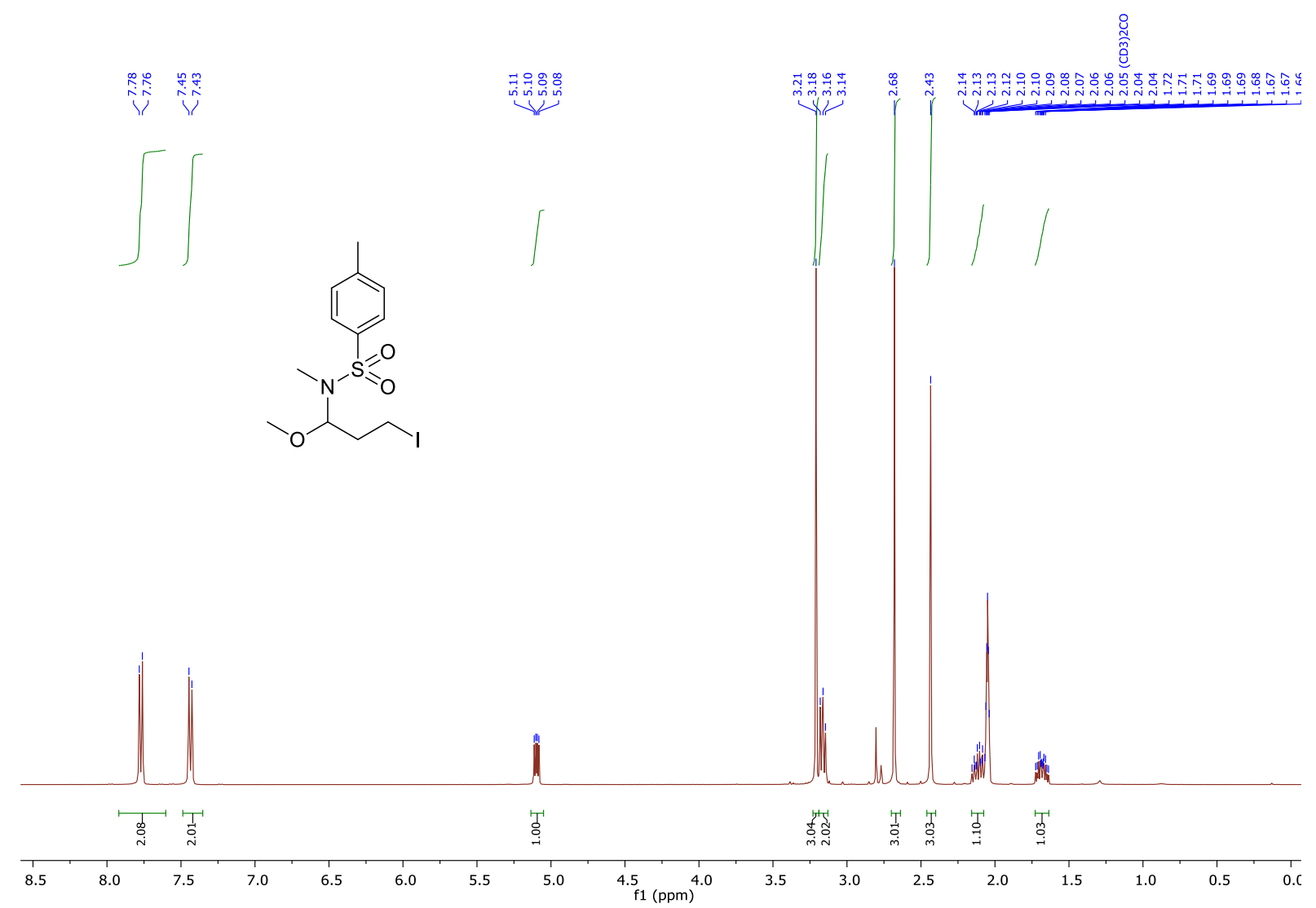

${ }^{13}$ C-NMR (101 MHz, Acetone-d6)

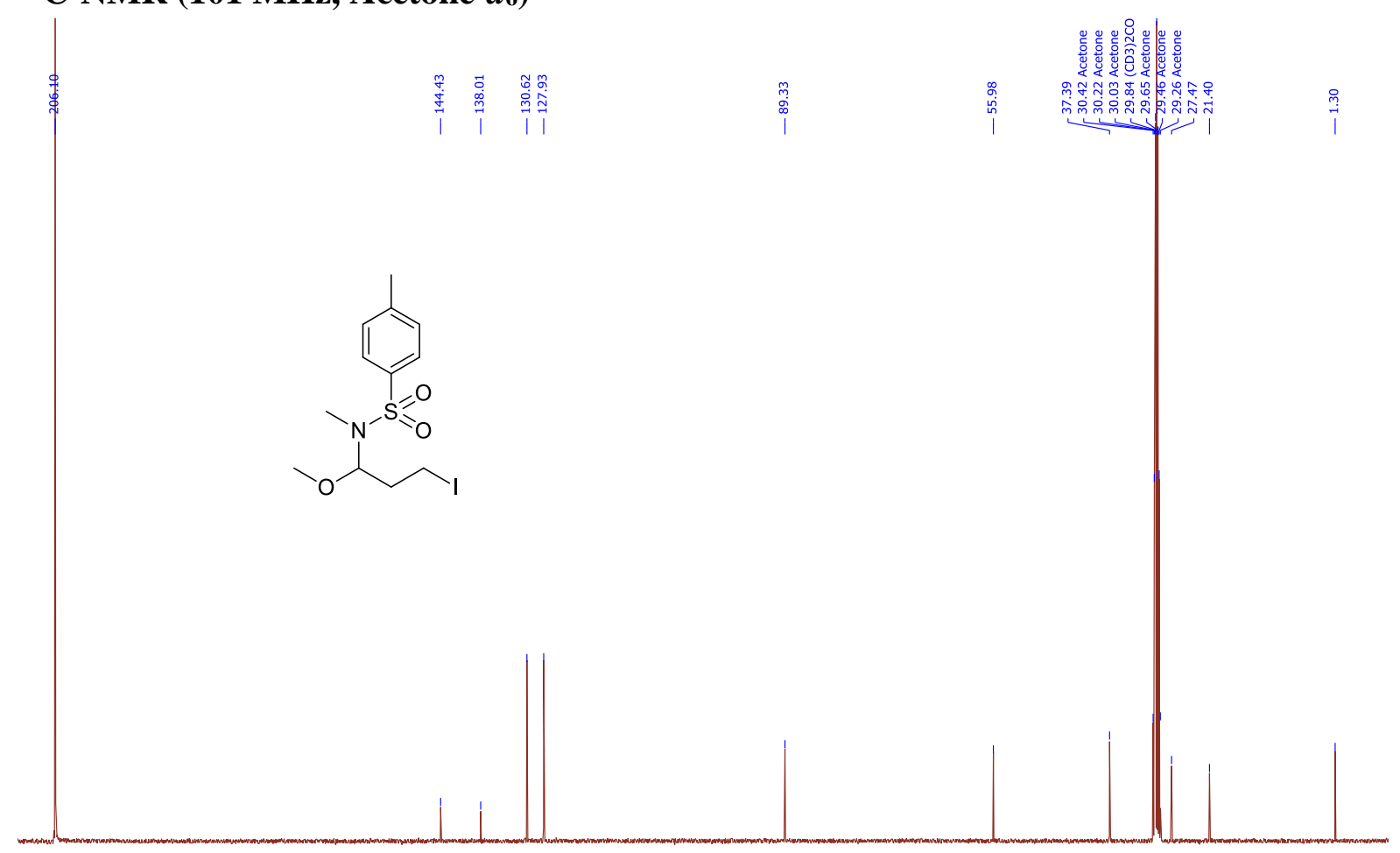

$\begin{array}{lllllllllll}210 & 200 & 190 & 180 & 170 & 160 & 150 & 140 & 130 & 120 & 110 \\ \mathrm{f} 1(\mathrm{ppm}) & 100\end{array}$ 
IR

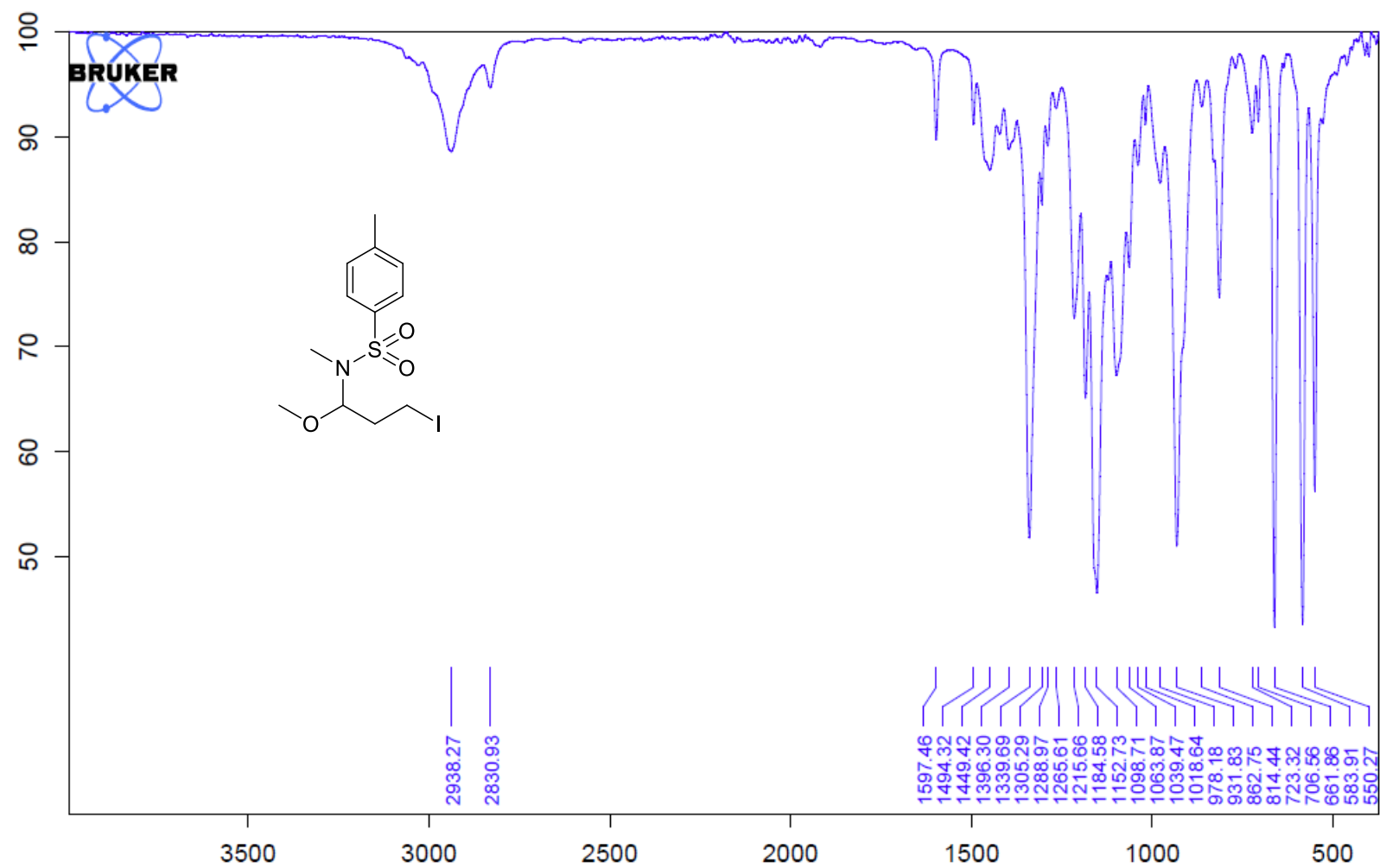

tert-Butyl benzyl(3-iodo-1-methoxypropyl)carbamate (4s)

${ }^{1}$ H-NMR (400 MHz, Acetone-d $\left.d_{6}\right)$

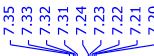

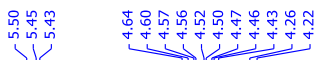

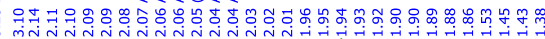

$\int$<smiles>COC(CCI)N(Cc1ccccc1)C(=O)OC(C)(C)C</smiles>
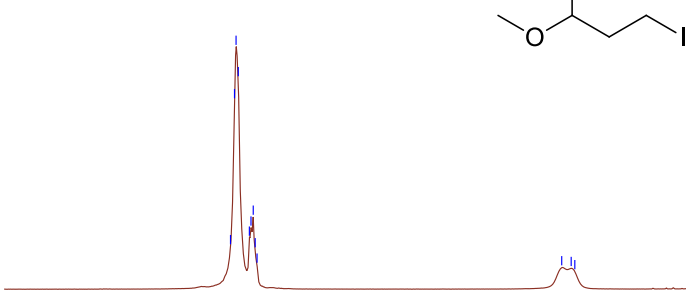

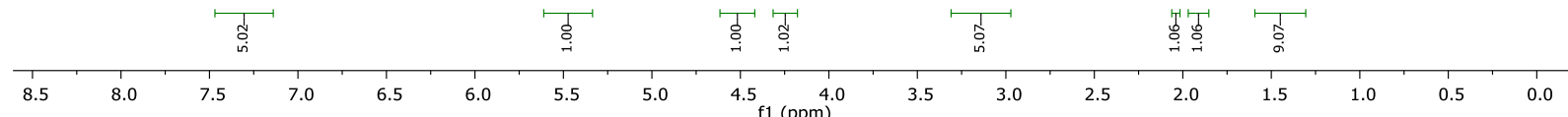


${ }^{13}$ C-NMR (101 MHz, Acetone-d6)
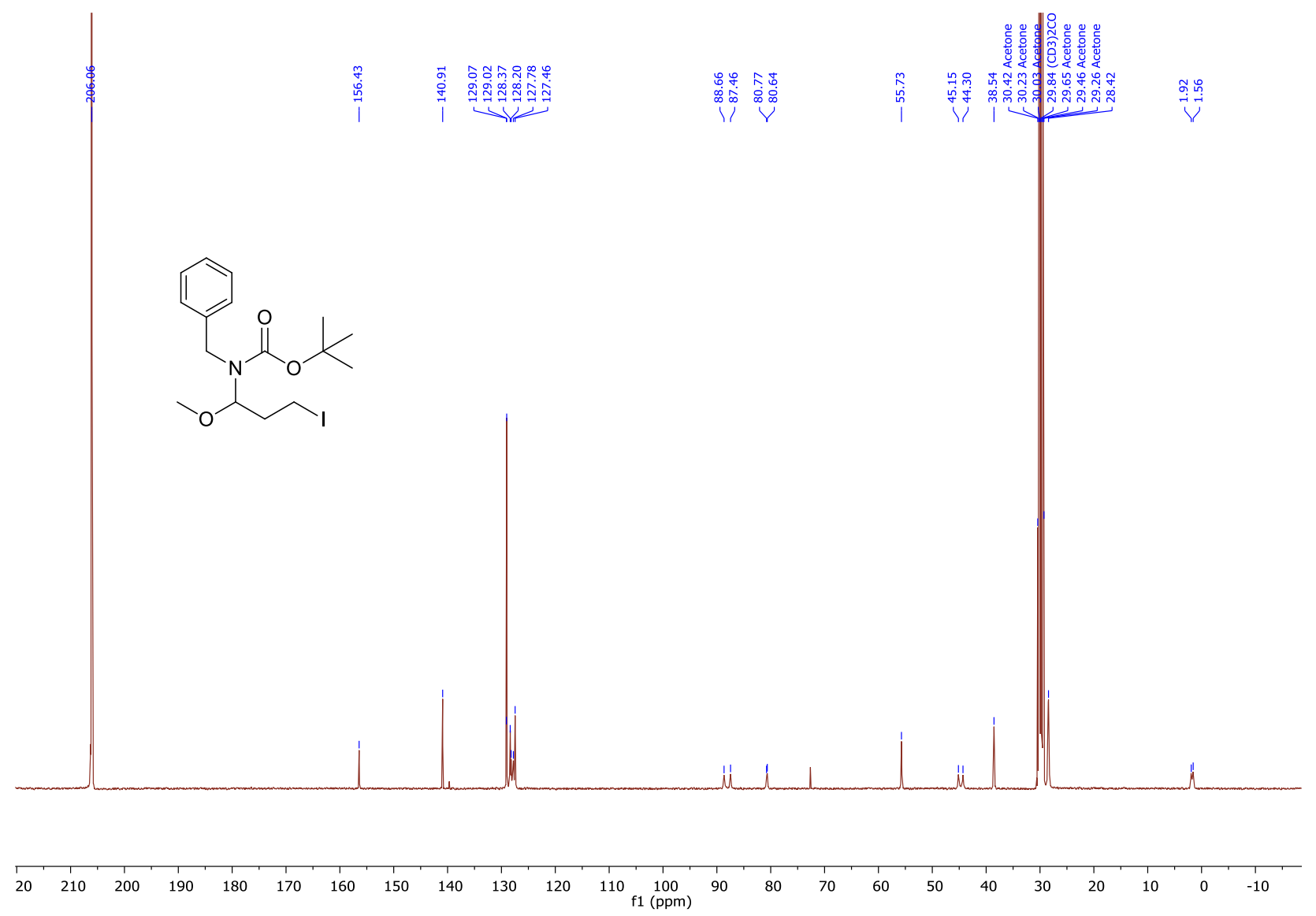

IR

음

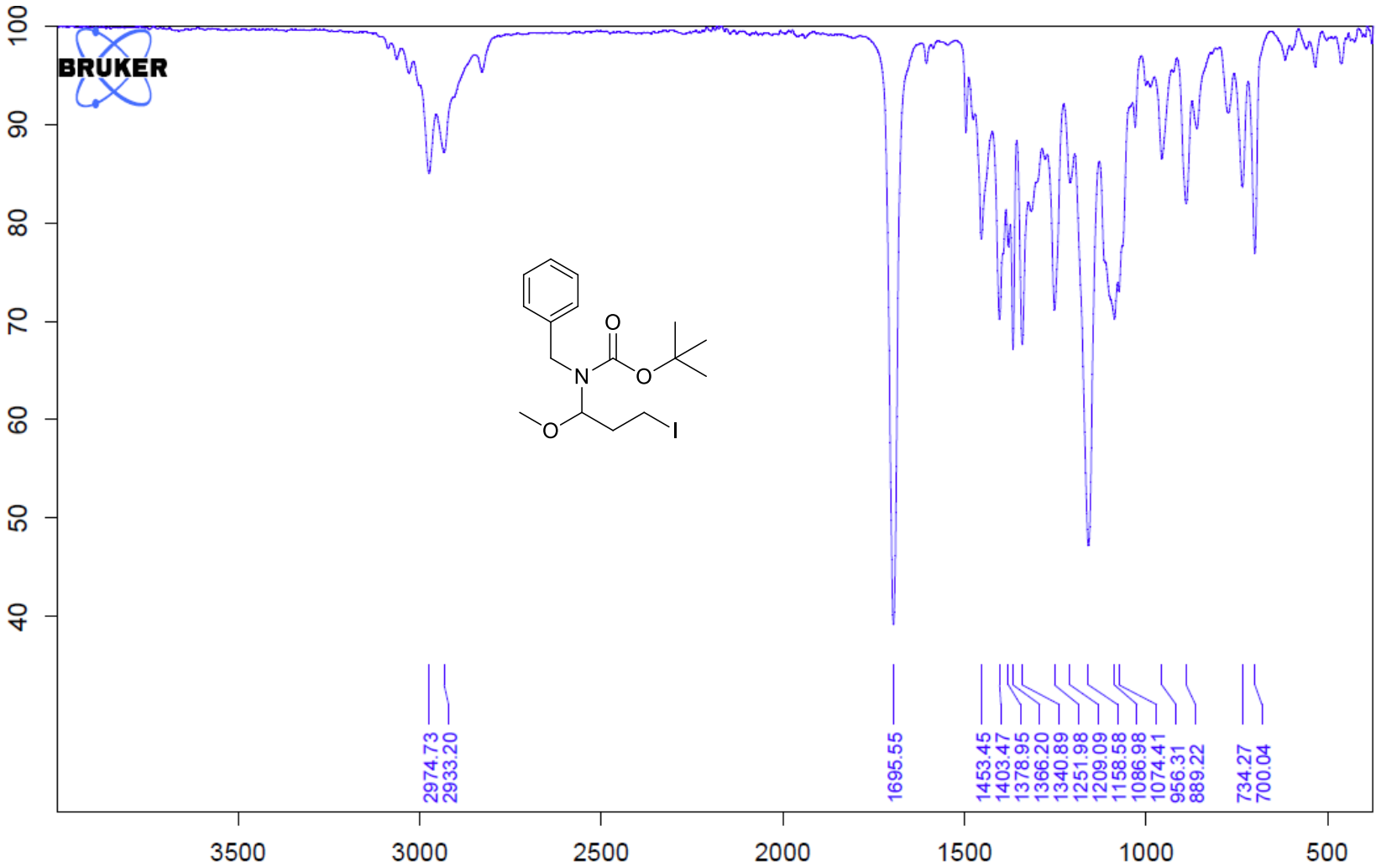


Benzyl ((2S)-1-((3-iodo-1-methoxypropyl)amino)-1-oxo-3-phenylpropan-2-yl)carbamate $(4 \mathrm{t})$ ${ }^{1}$ H-NMR (400 MHz, Acetone-d6)

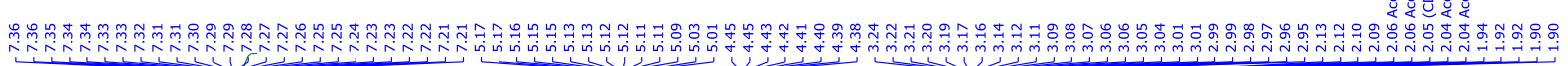

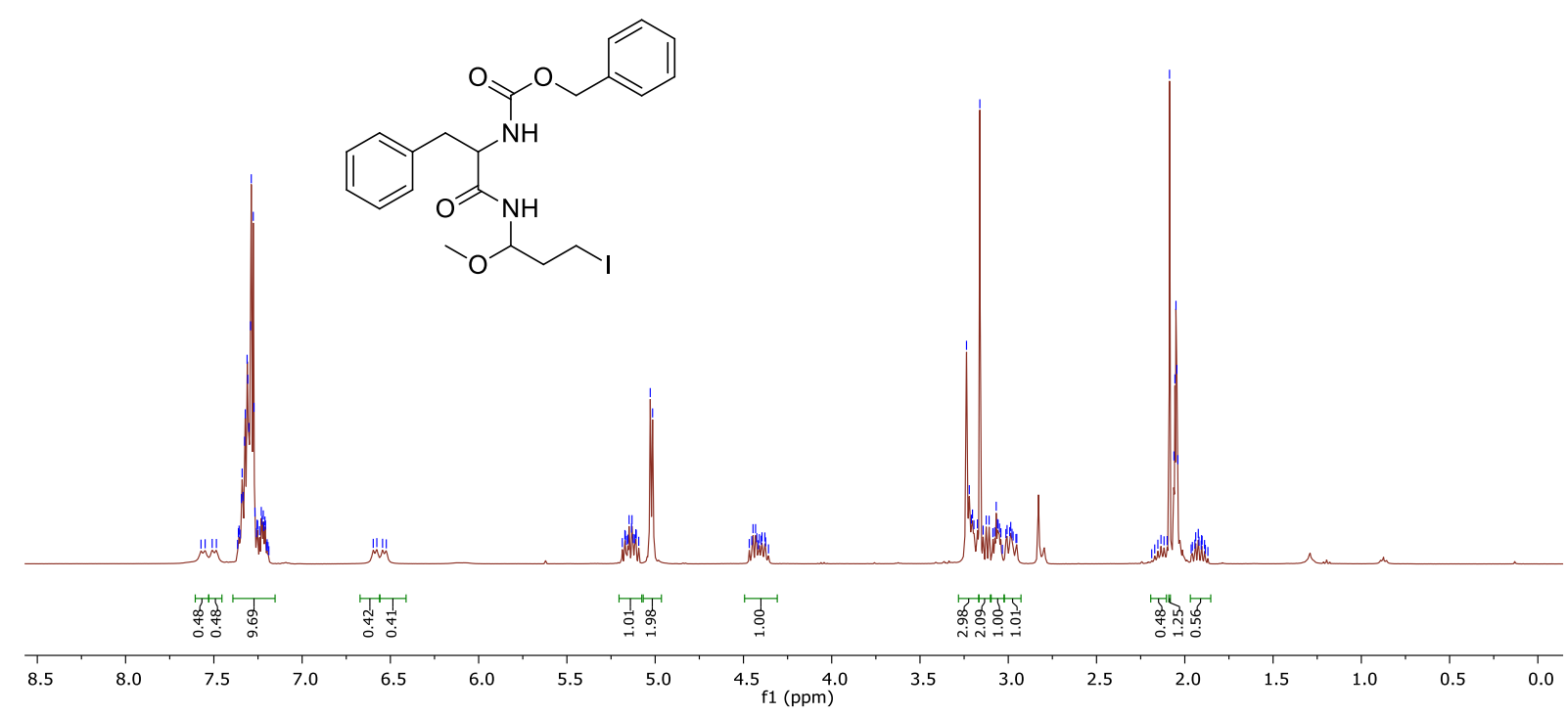

${ }^{13}$ C-NMR (101 MHz, Acetone-d6)

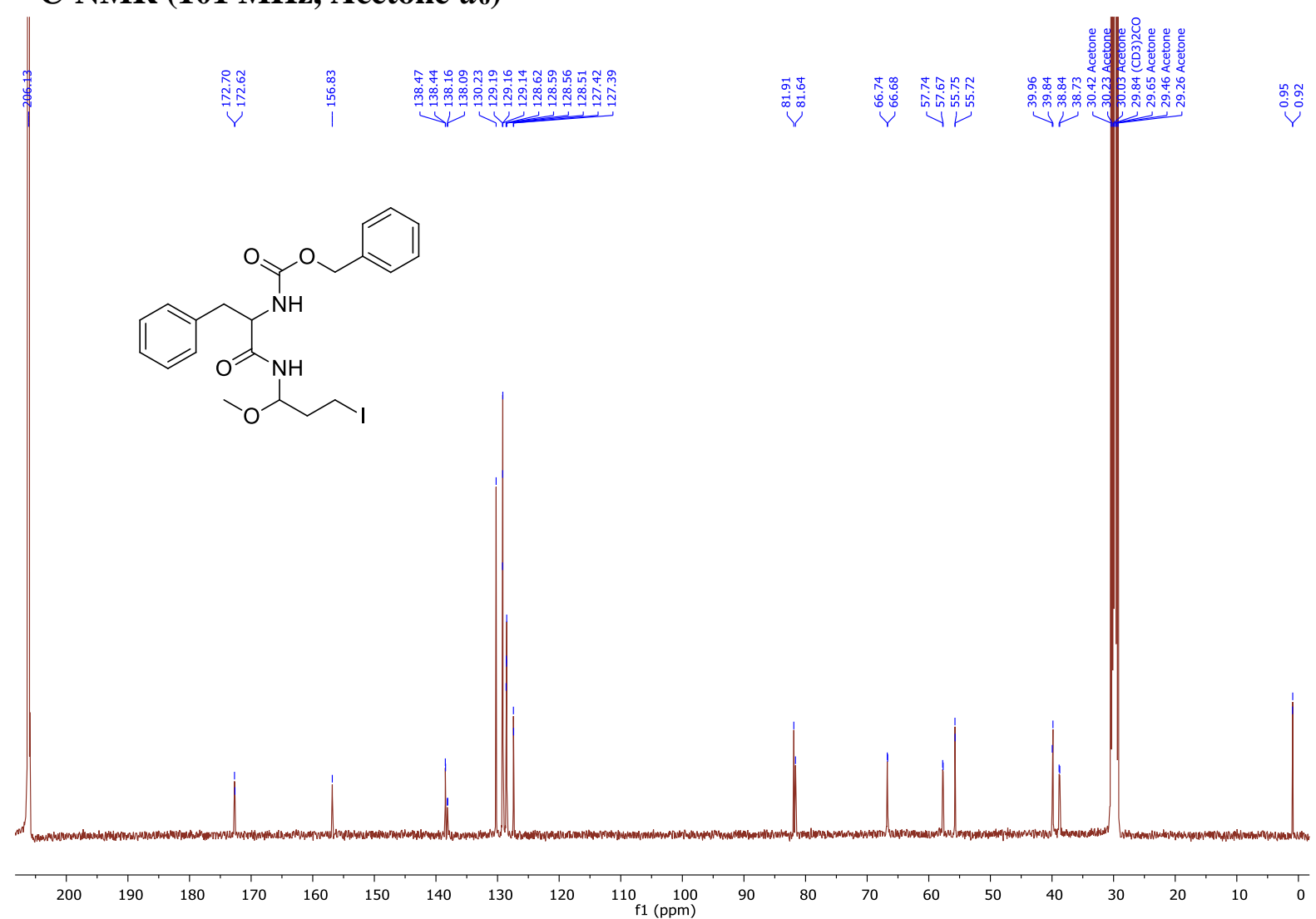


IR

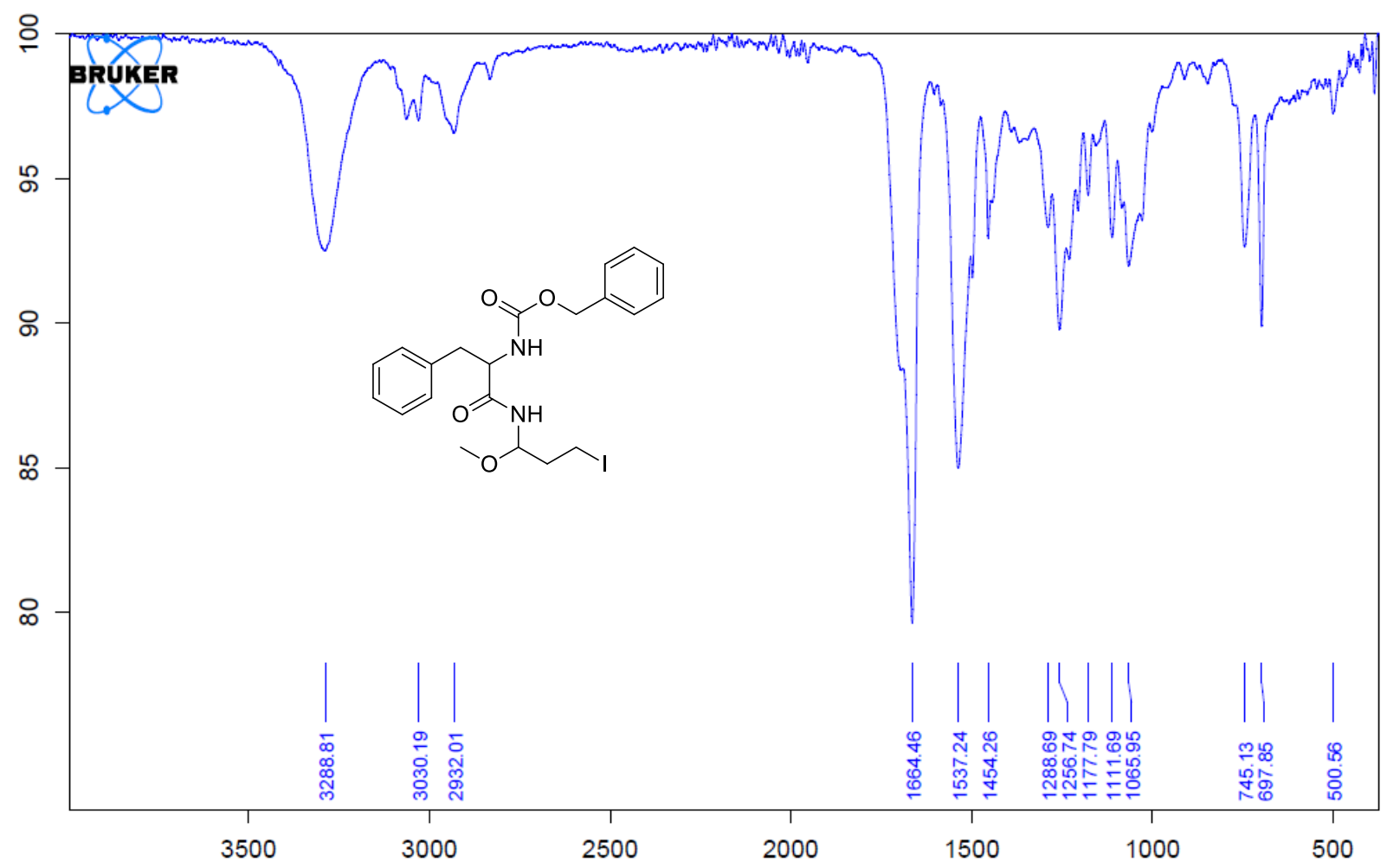

N-(3-Iodo-1-methoxypropyl)-2-(11-oxo-6,11-dihydrodibenzo[b,e]oxepin-2-yl)acetamide (4u)

${ }^{1}$ H-NMR (400 MHz, Acetone- $d_{6}$ )

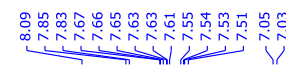

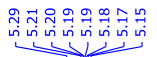

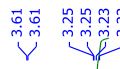

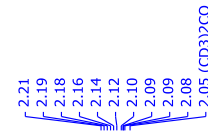
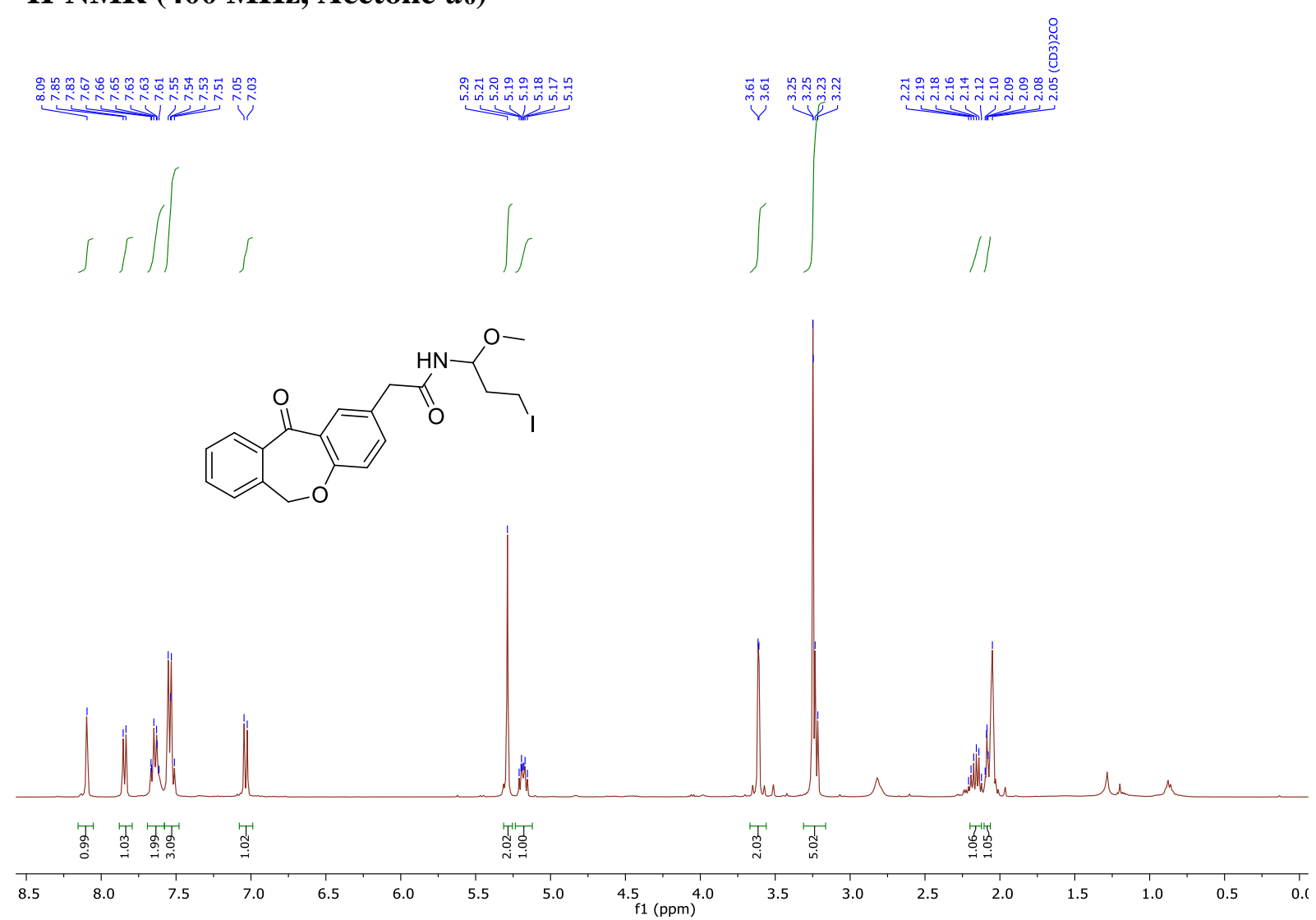


\section{${ }^{13}$ C-NMR (101 MHz, Acetone-d6)}
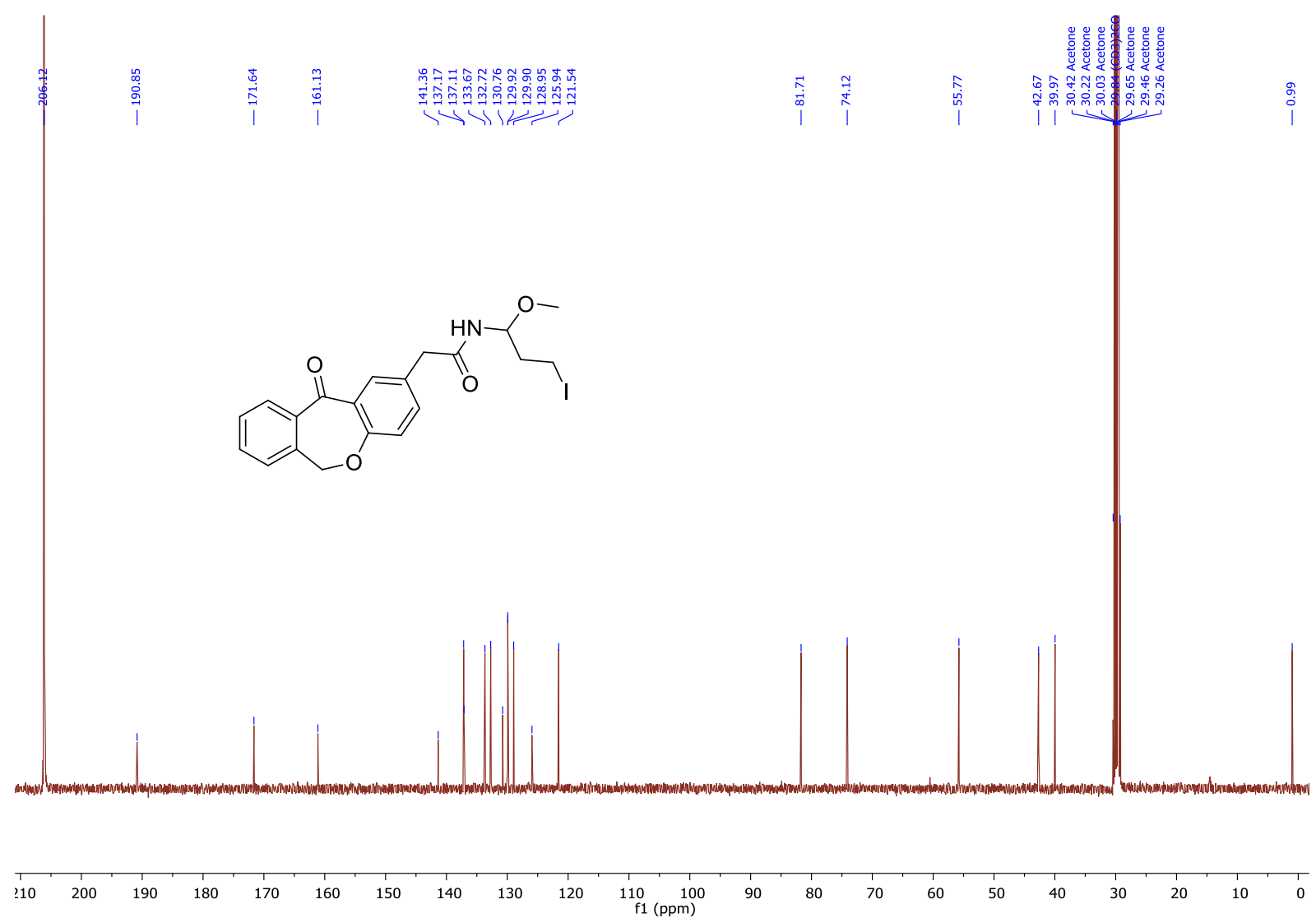

IR

음

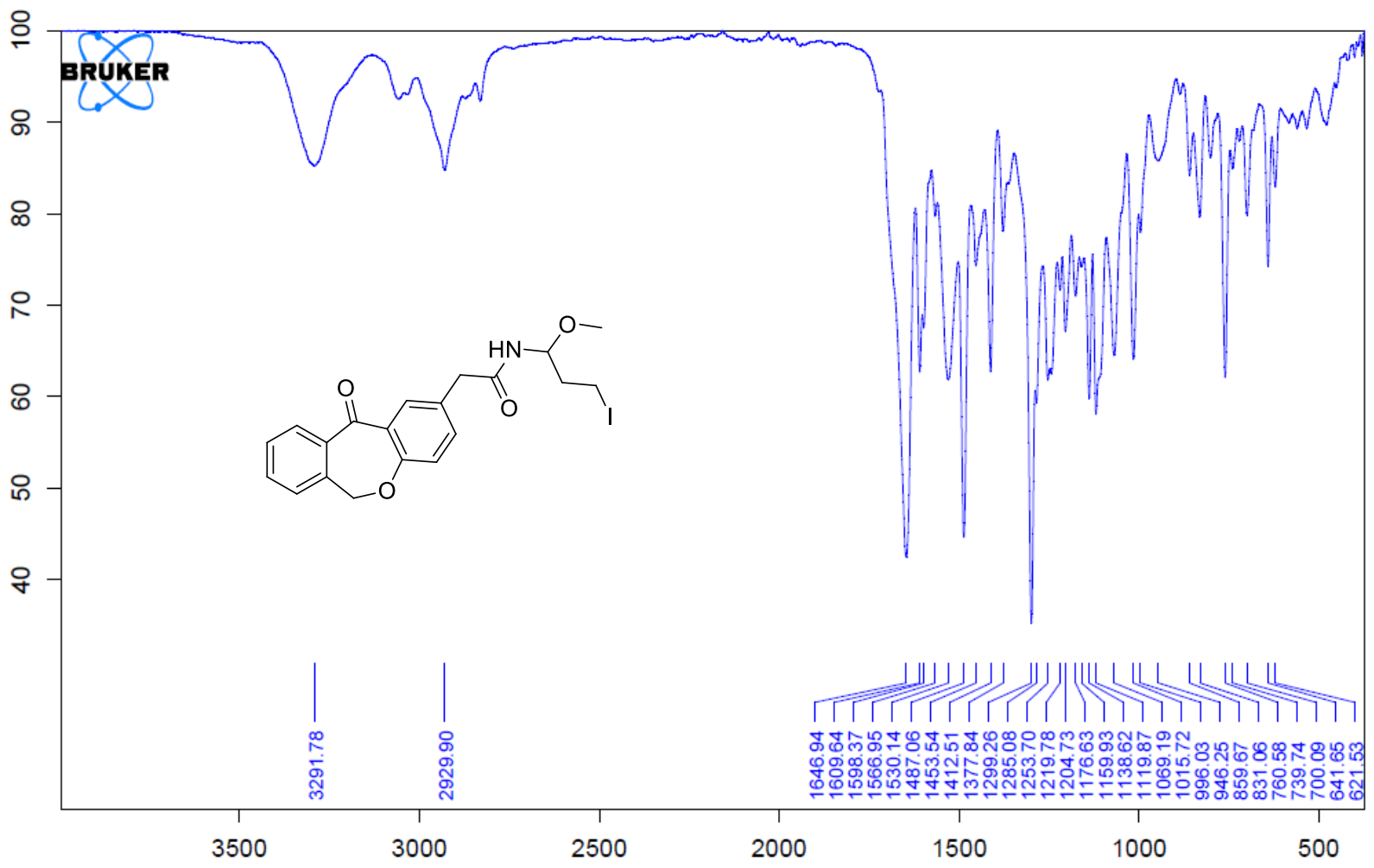


2-(1-(4-Chlorobenzoyl)-5-methoxy-2-methyl-1H-indol-3-yl)-N-(3-iodo-1methoxypropyl)acetamide (4v)

${ }^{1}$ H-NMR (400 MHz, Acetone- $d_{6}$ )
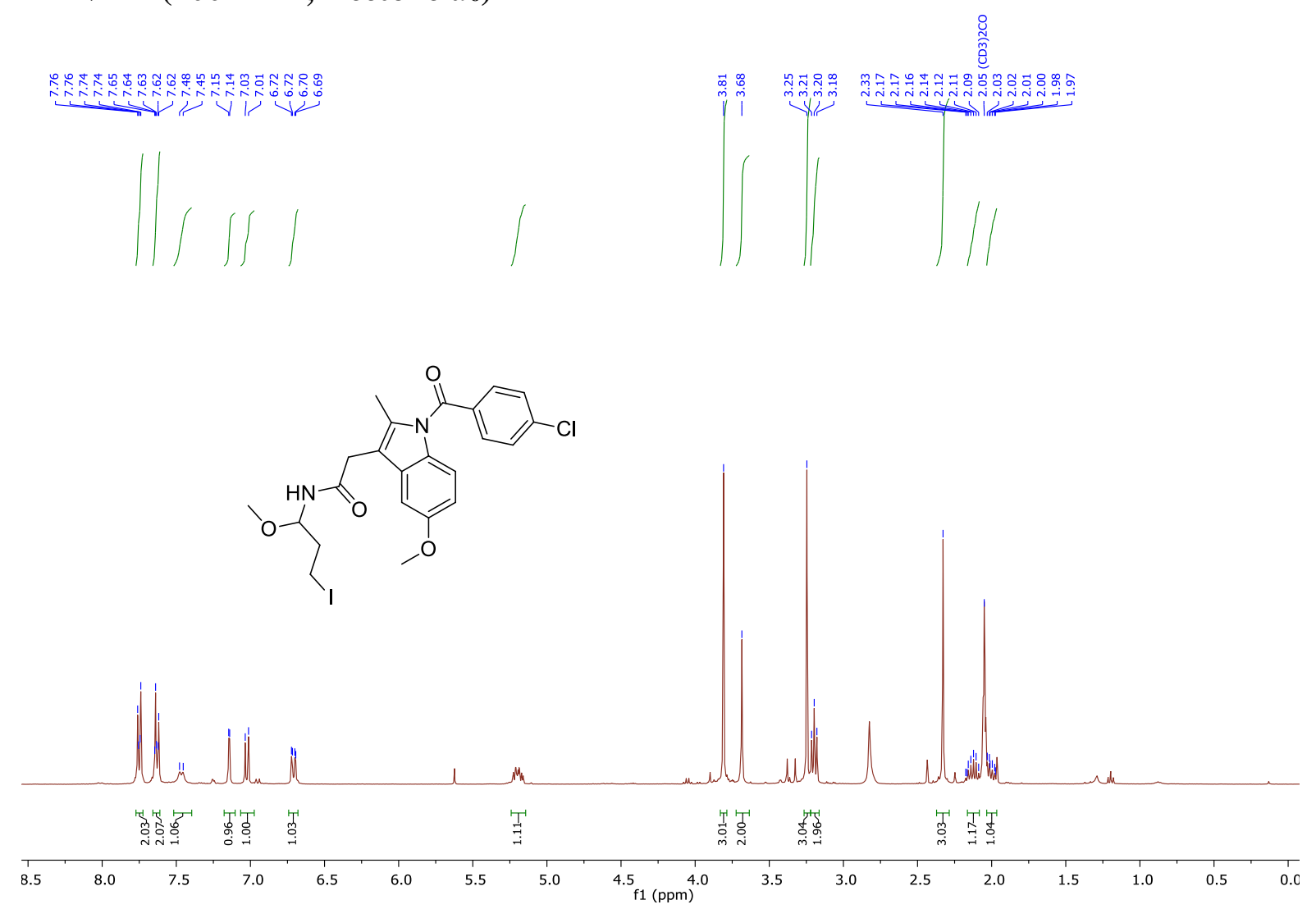

${ }^{13}$ C-NMR (101 MHz, Acetone-d6)

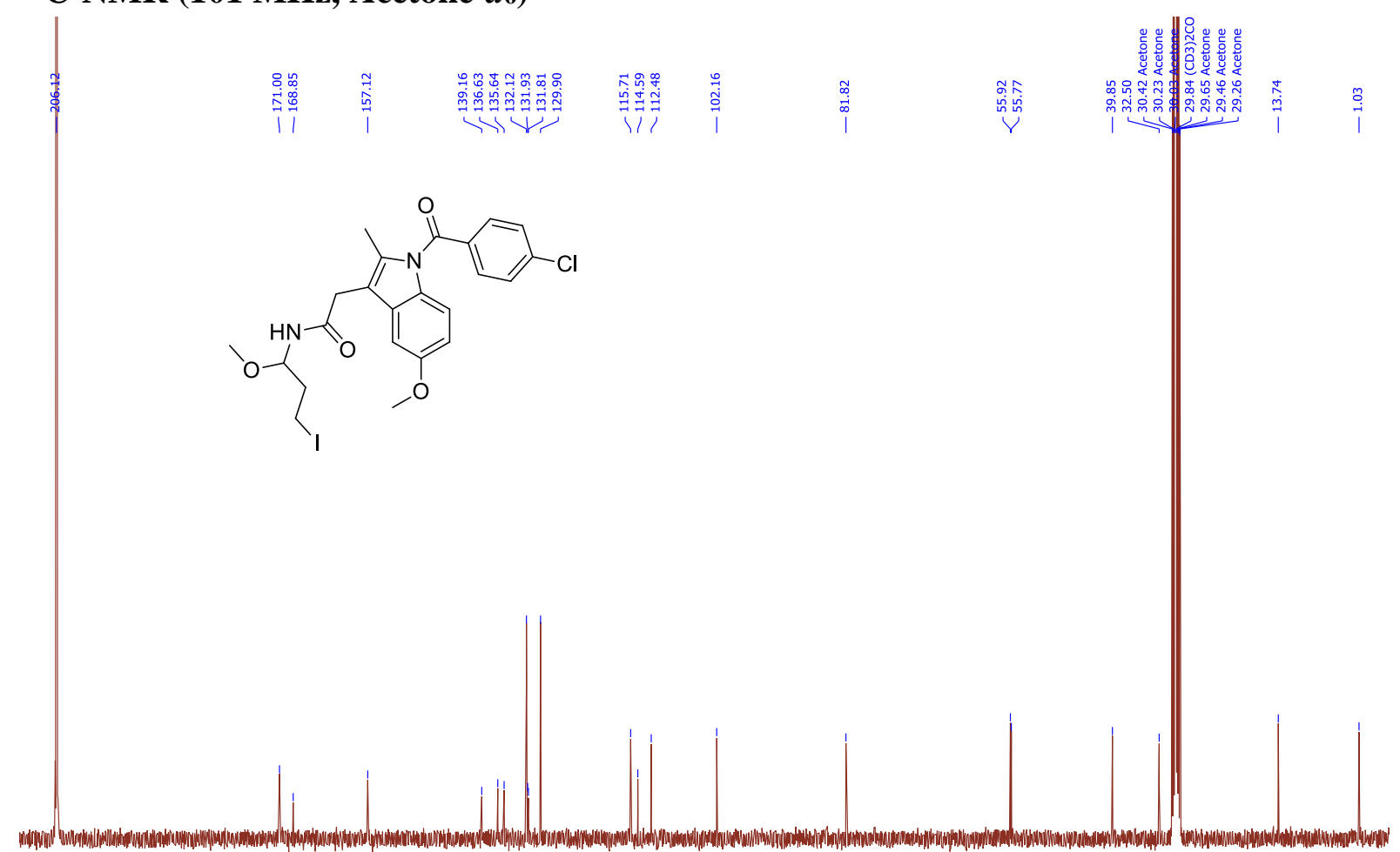

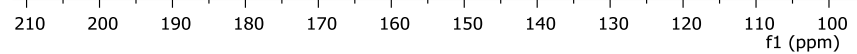




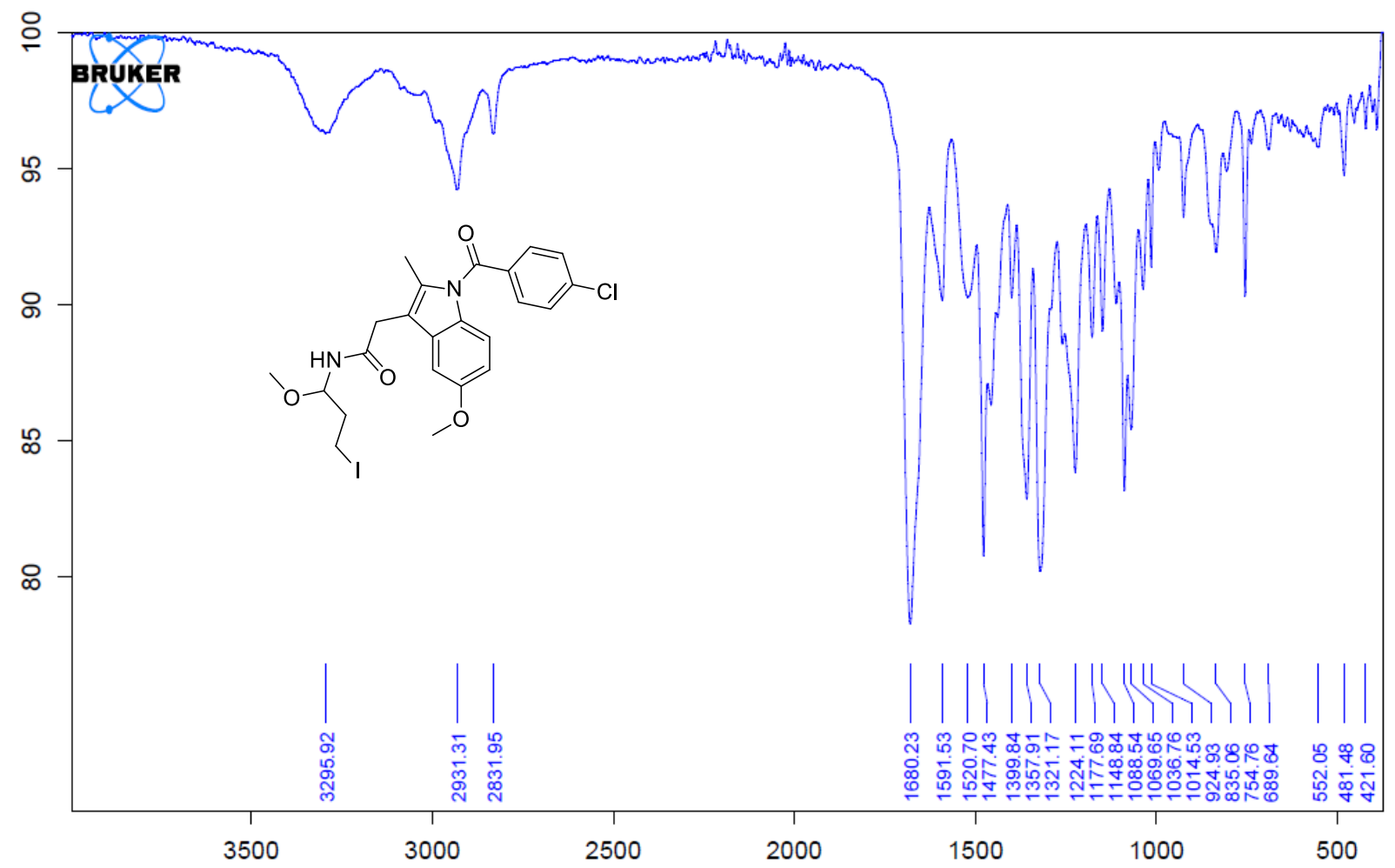

N-(3-Bromo-1-methoxypropyl)-4-methoxybenzamide (5)

${ }^{1} \mathrm{H}-\mathrm{NMR}$ (400 $\mathrm{MHz}^{\mathrm{CDCl}} 3$ )
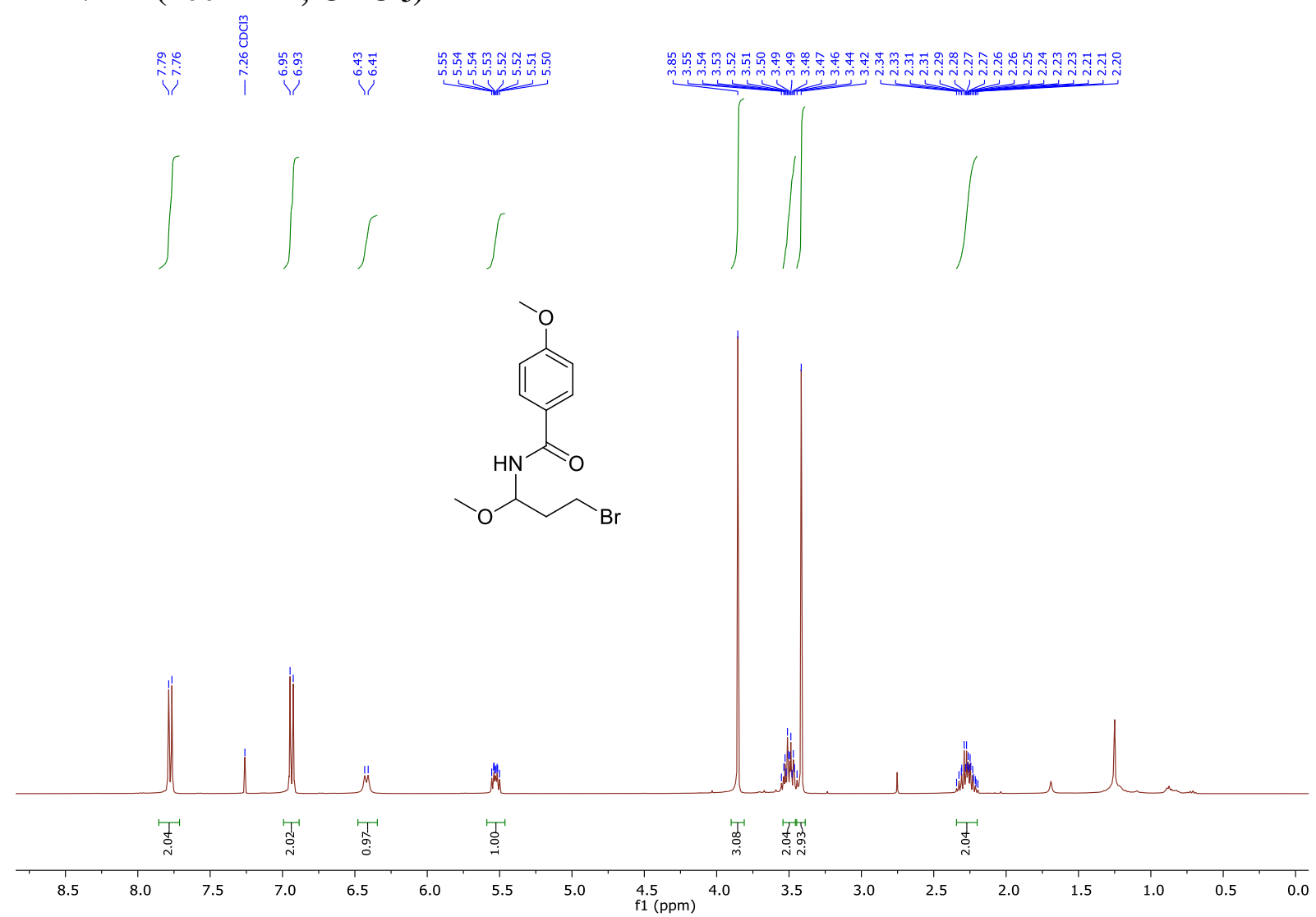

\% 
${ }^{13}$ C-NMR (101 MHz, CDCl 3$)$

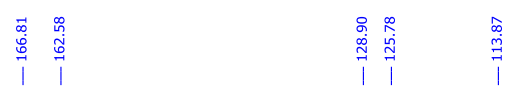
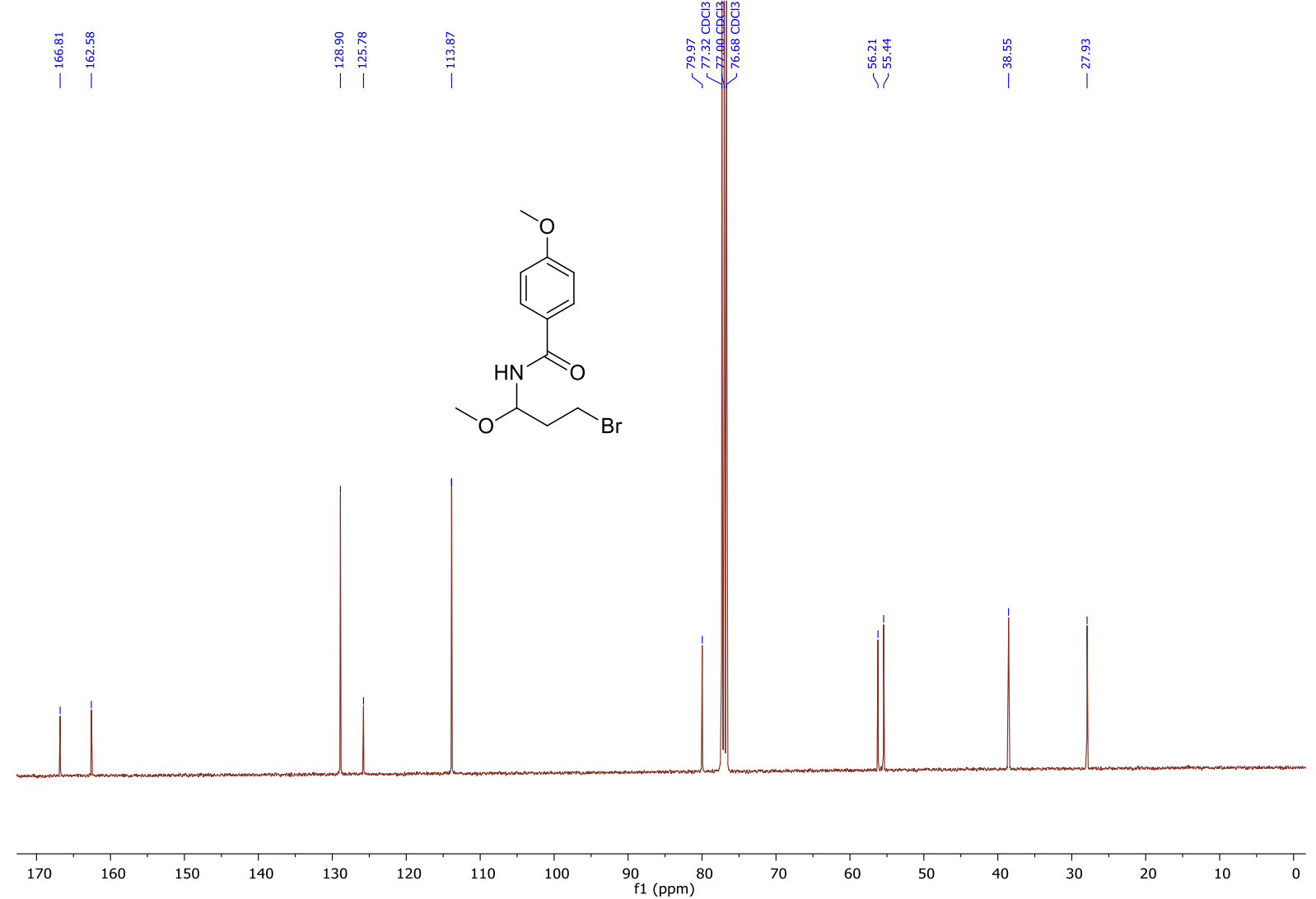

IR

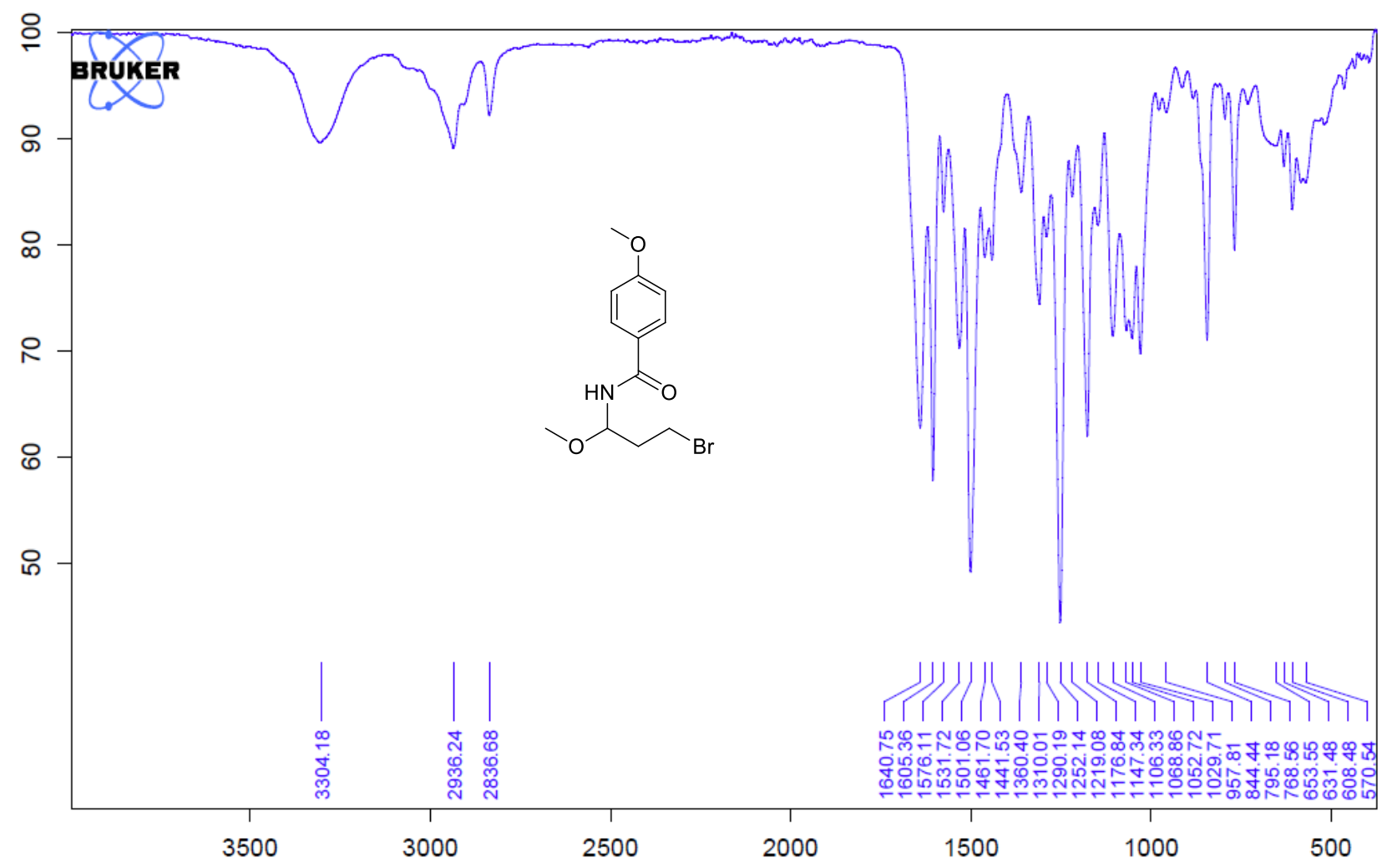


3-Iodopropanenitrile (9)

${ }^{1} \mathrm{H}-\mathrm{NMR}$ (400 MHz, $\mathrm{CDCl}_{3}$ )

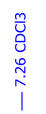

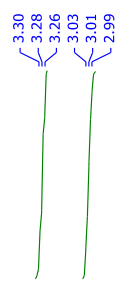

$\mathrm{NC} \sim$

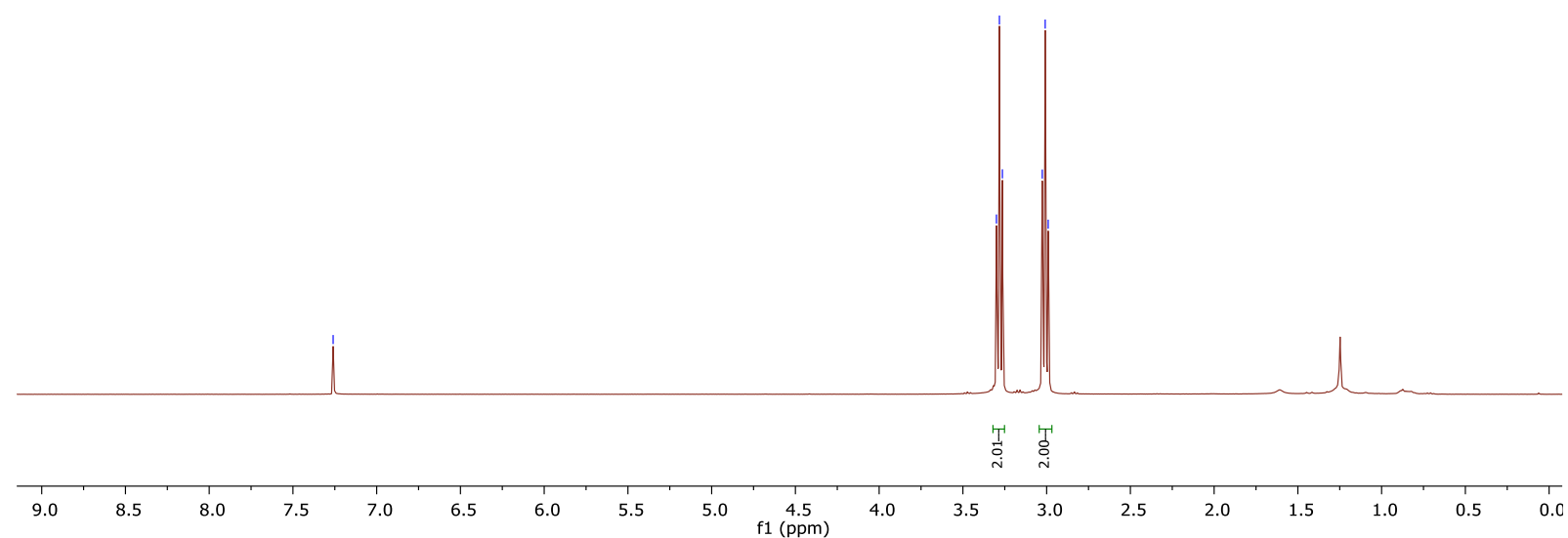

${ }^{13} \mathrm{C}-\mathrm{NMR}$ (101 MHz, CDCl3)

\begin{tabular}{l}
7 \\
$\infty$ \\
\multirow{1}{*}{} \\
1
\end{tabular}

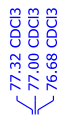

$\mathrm{NC}^{\sim}$
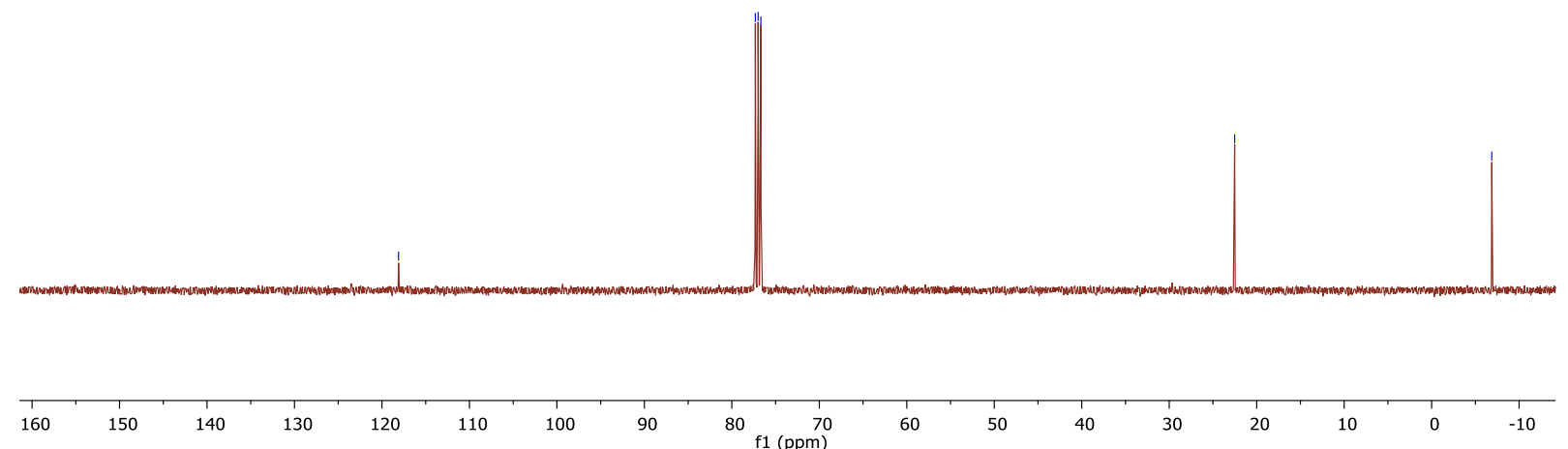


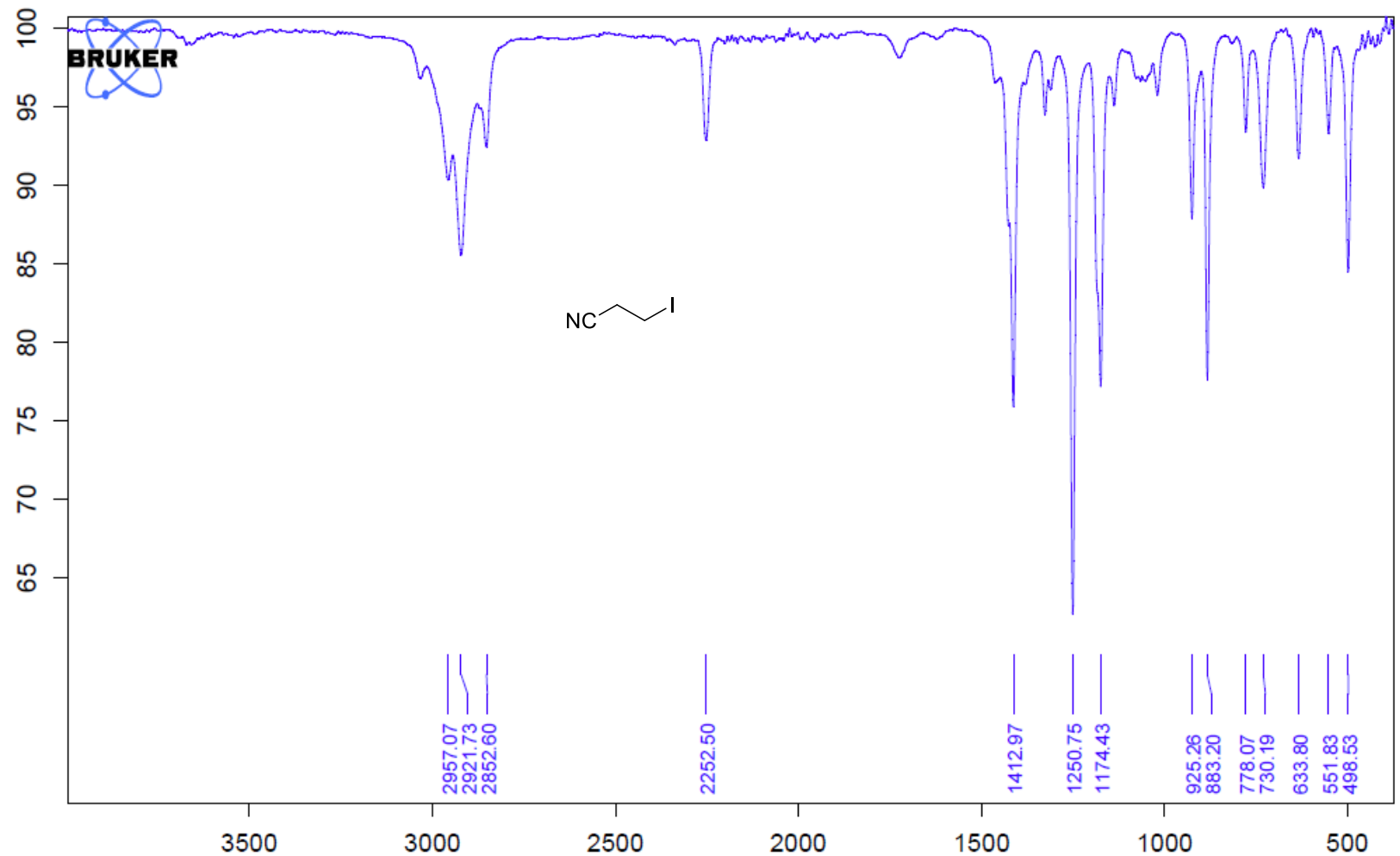

N-(1-(Benzyloxy)-3-iodopropyl)-4-methoxybenzamide (10a)

${ }^{1} \mathrm{H}-\mathrm{NMR}$ (400 MHz, CDCl3)

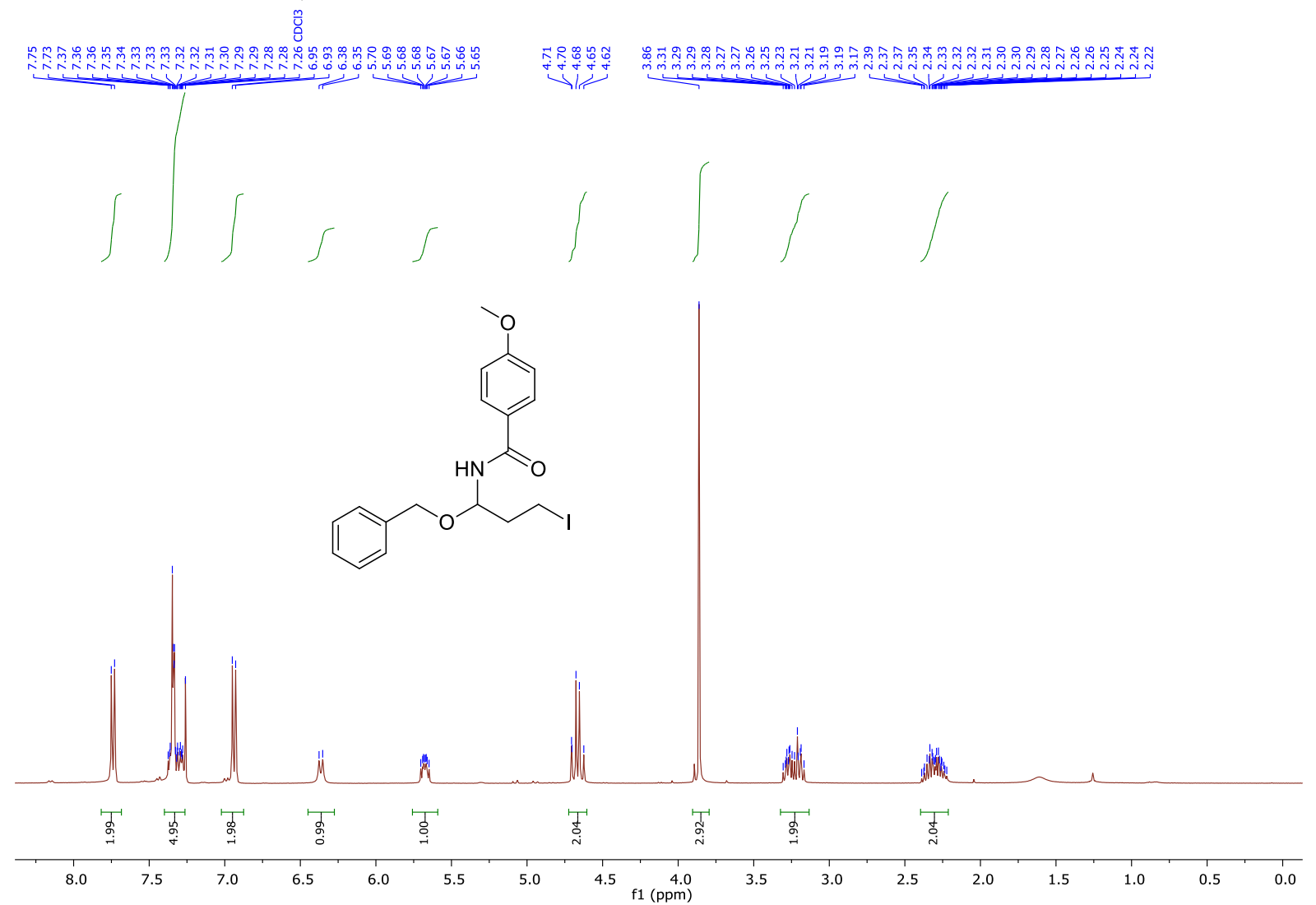


${ }^{13} \mathrm{C}-\mathrm{NMR}$ (101 MHz, CDCl 3$)$

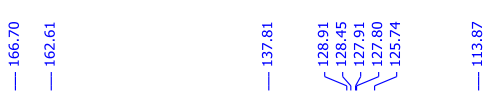
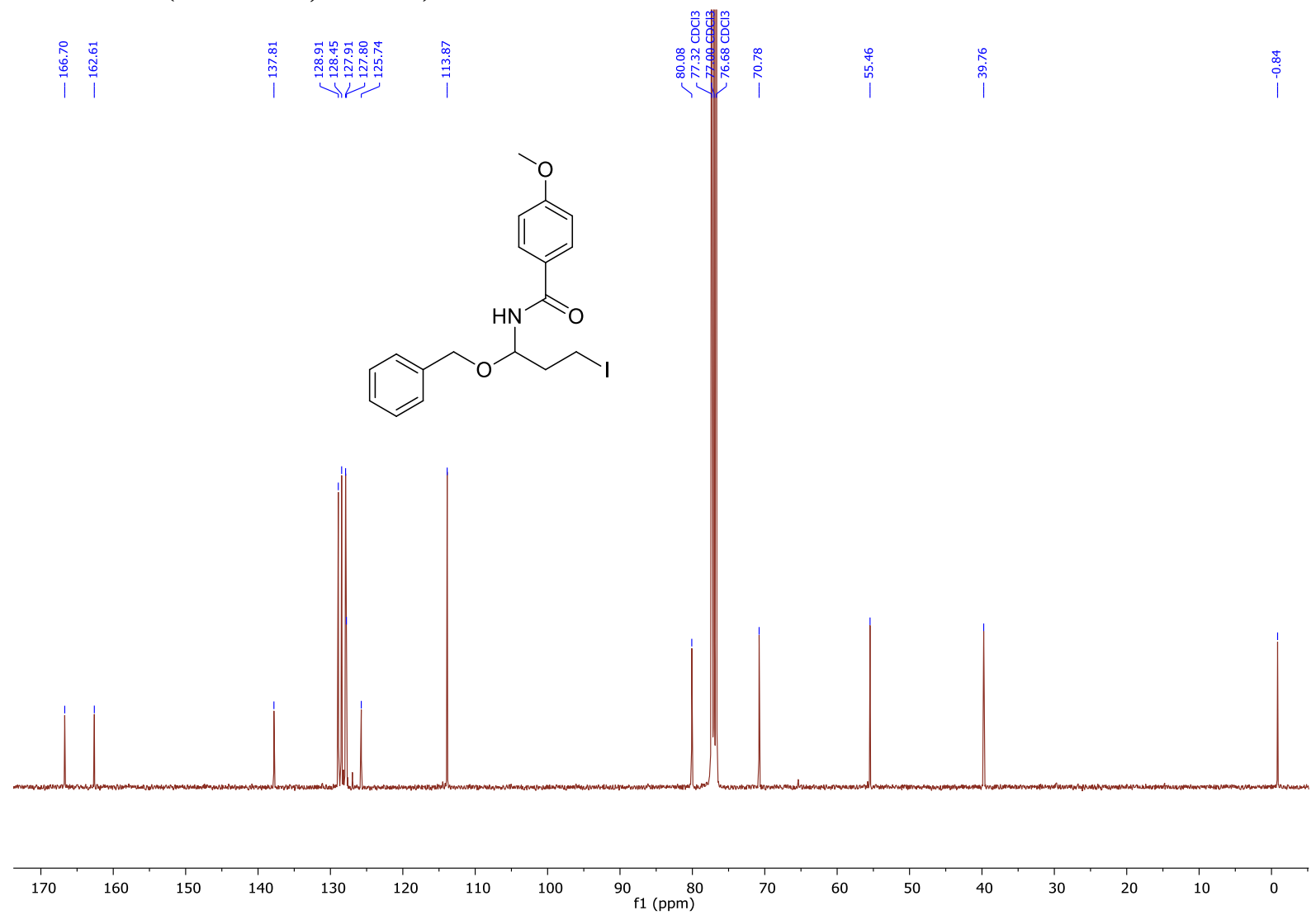

IR

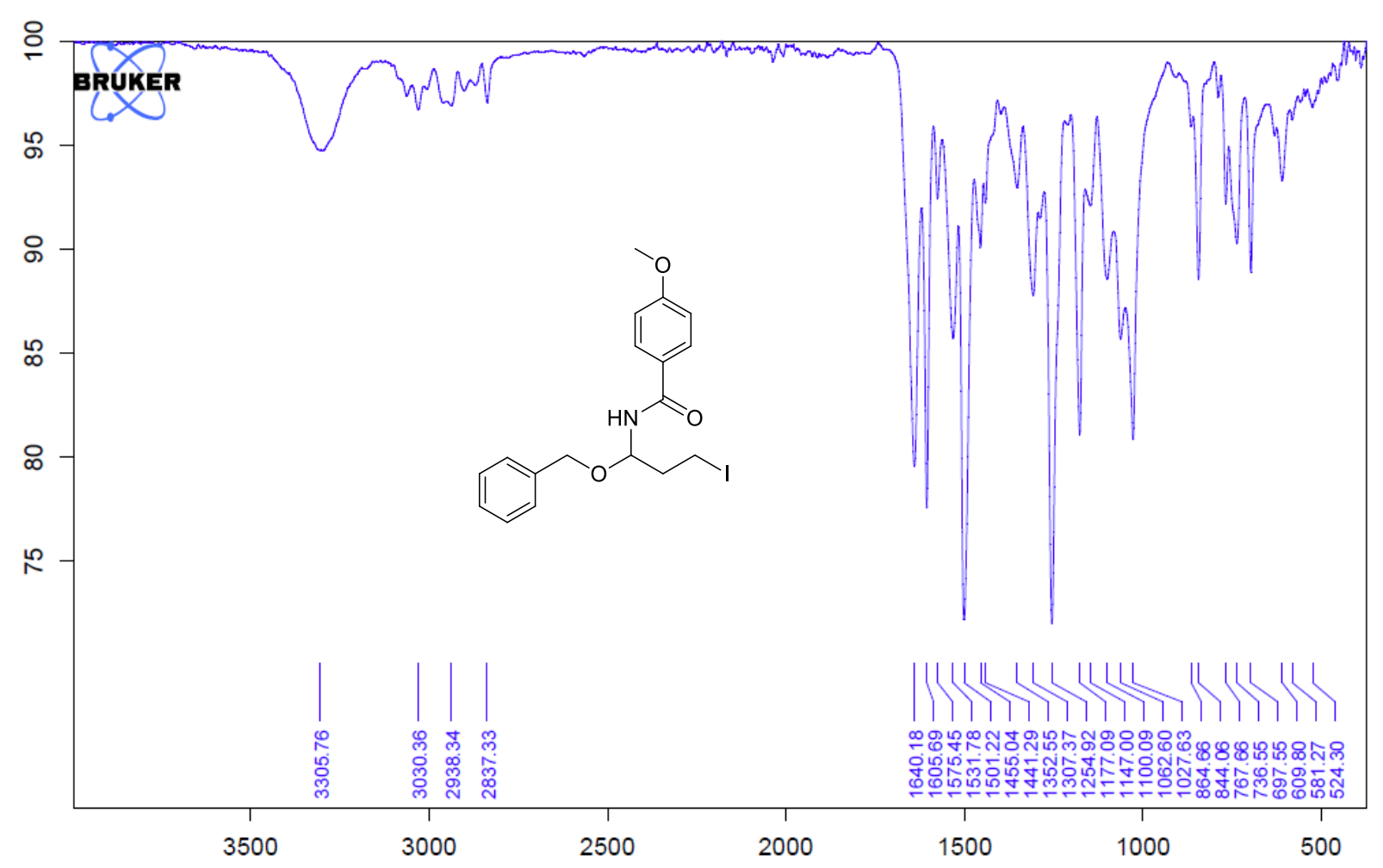


N-(3-Iodo-1-(prop-2-yn-1-yloxy)propyl)-4-methoxybenzamide (10b)

${ }^{1} \mathrm{H}-\mathrm{NMR}$ (400 MHz, CDCl 3$)$
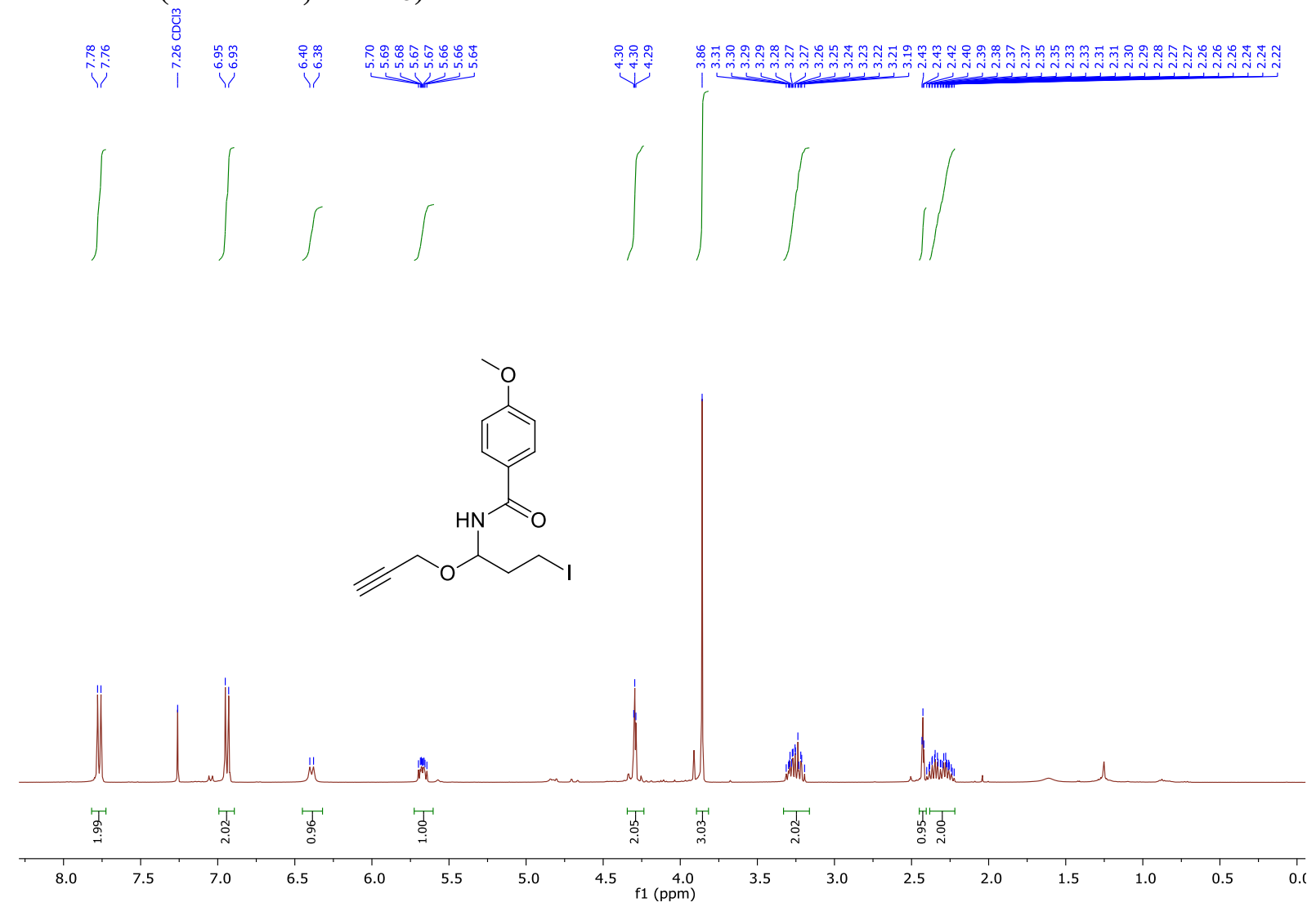

${ }^{13} \mathrm{C}-\mathrm{NMR}\left(101 \mathrm{MHz}, \mathrm{CDCl}_{3}\right)$

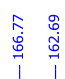
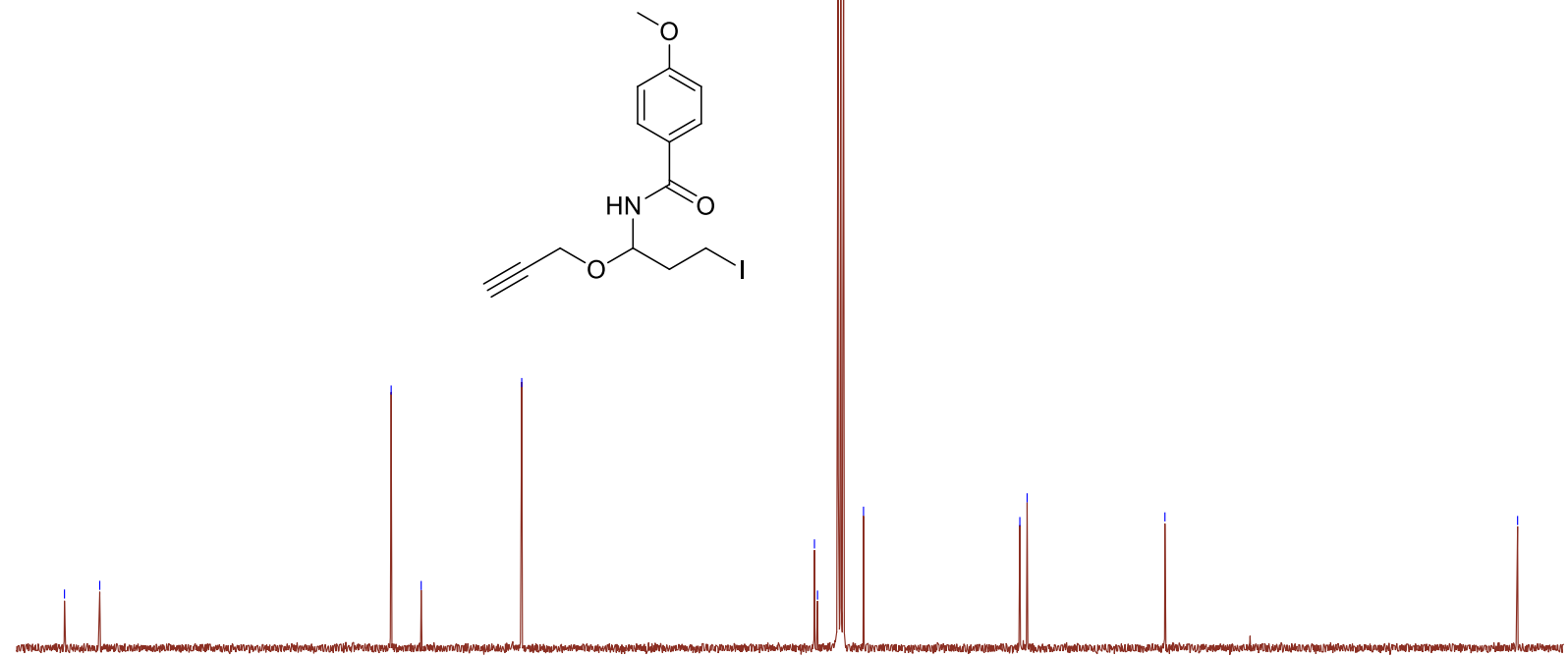

$\begin{array}{lllllllll}170 & 160 & 150 & 140 & 130 & 120 & 110 & 100 & 90 \\ \mathrm{f} 1(\mathrm{ppm})\end{array}$ 
IR

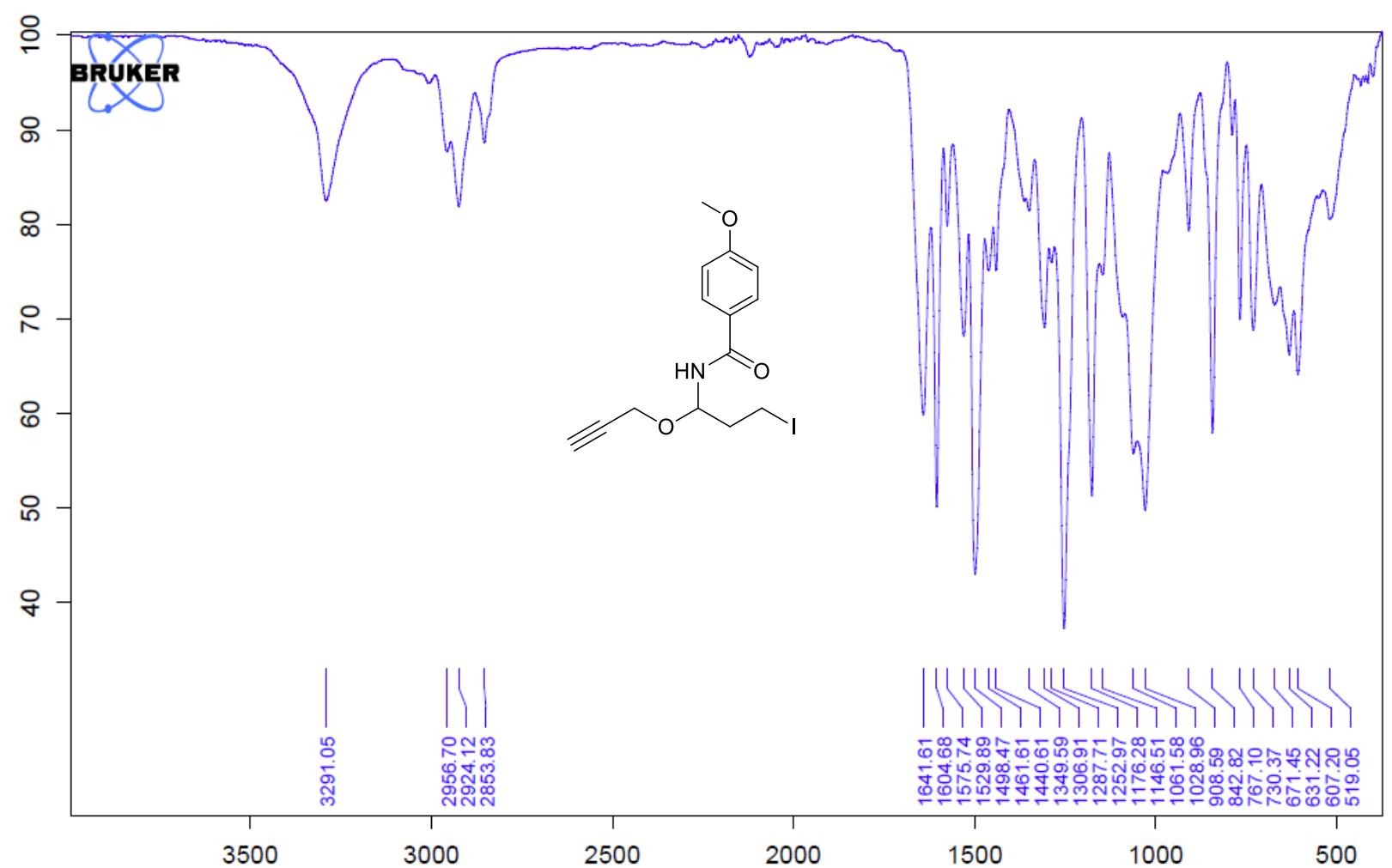

N-(1-(Allyloxy)-3-iodopropyl)-4-methoxybenzamide (10c)

${ }^{1}$ H-NMR (400 MHz, CDCl3)

象

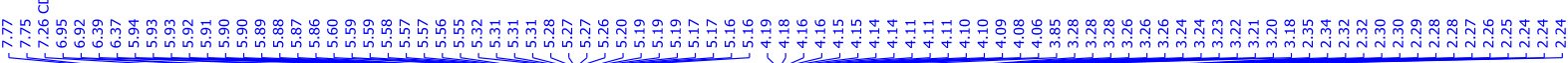

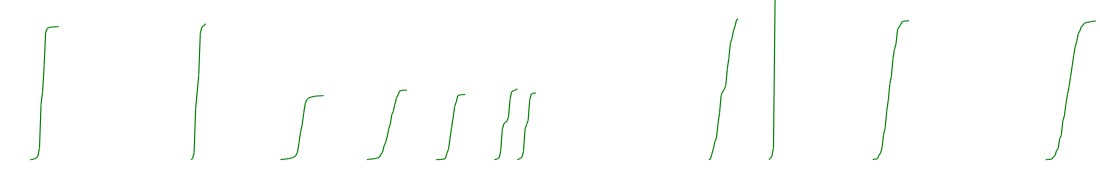<smiles>C=CCOC(CCI)NC(=O)c1ccc(OC)cc1</smiles>

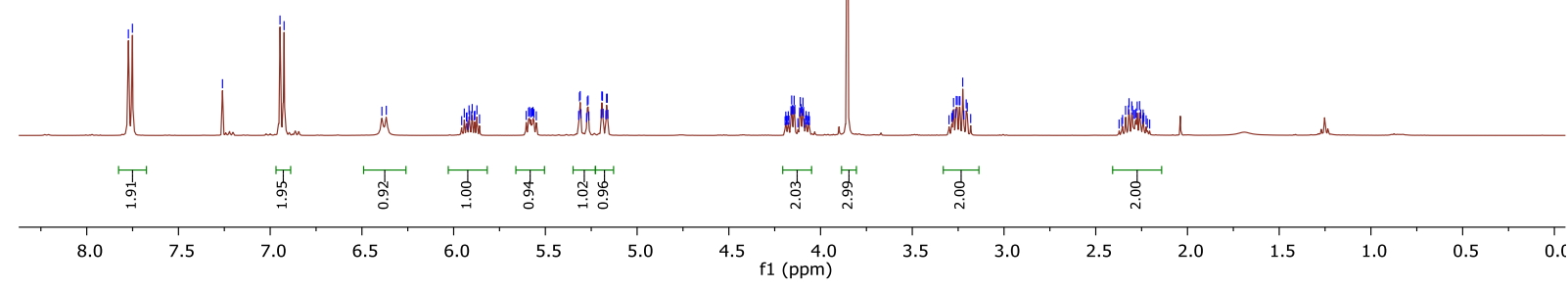


${ }^{13} \mathrm{C}$-NMR (101 MHz, $\left.\mathrm{CDCl}_{3}\right)$

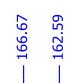

告

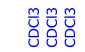

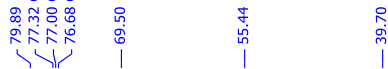
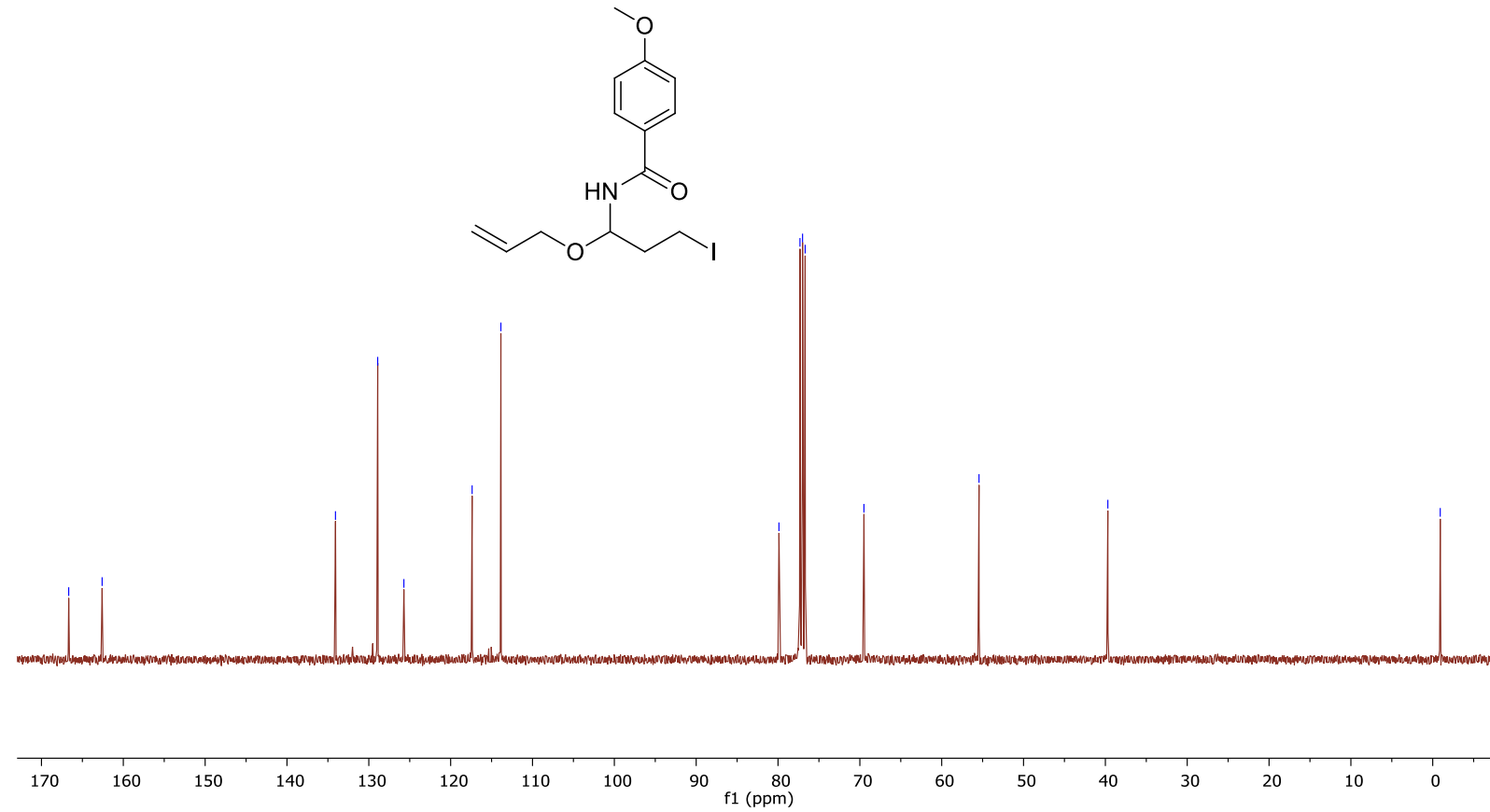

IR

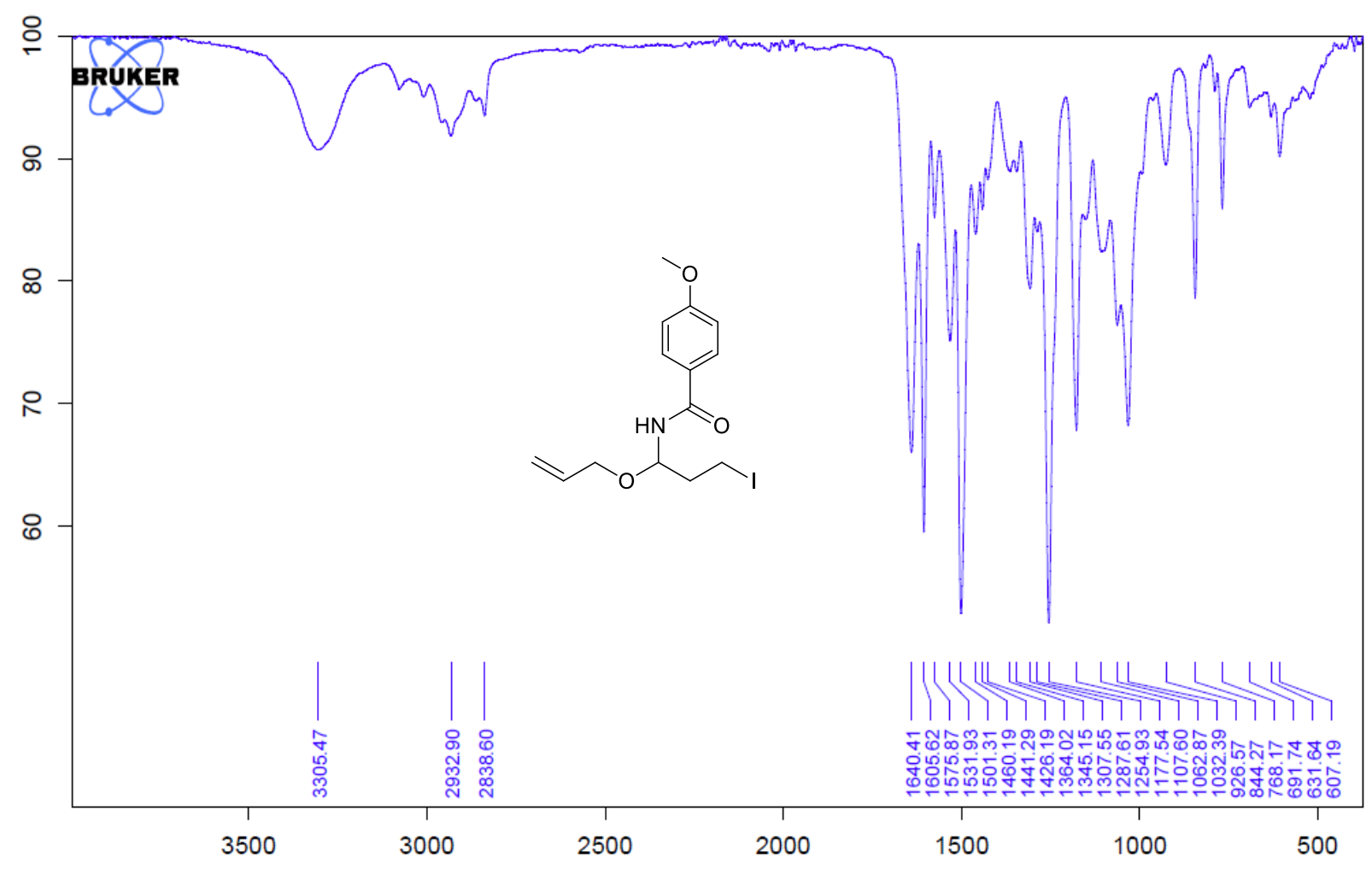


N-(3-Iodo-1-isopropoxypropyl)-4-methoxybenzamide (10d)

${ }^{1} \mathrm{H}-\mathrm{NMR}$ (400 MHz, CDCl 3$)$
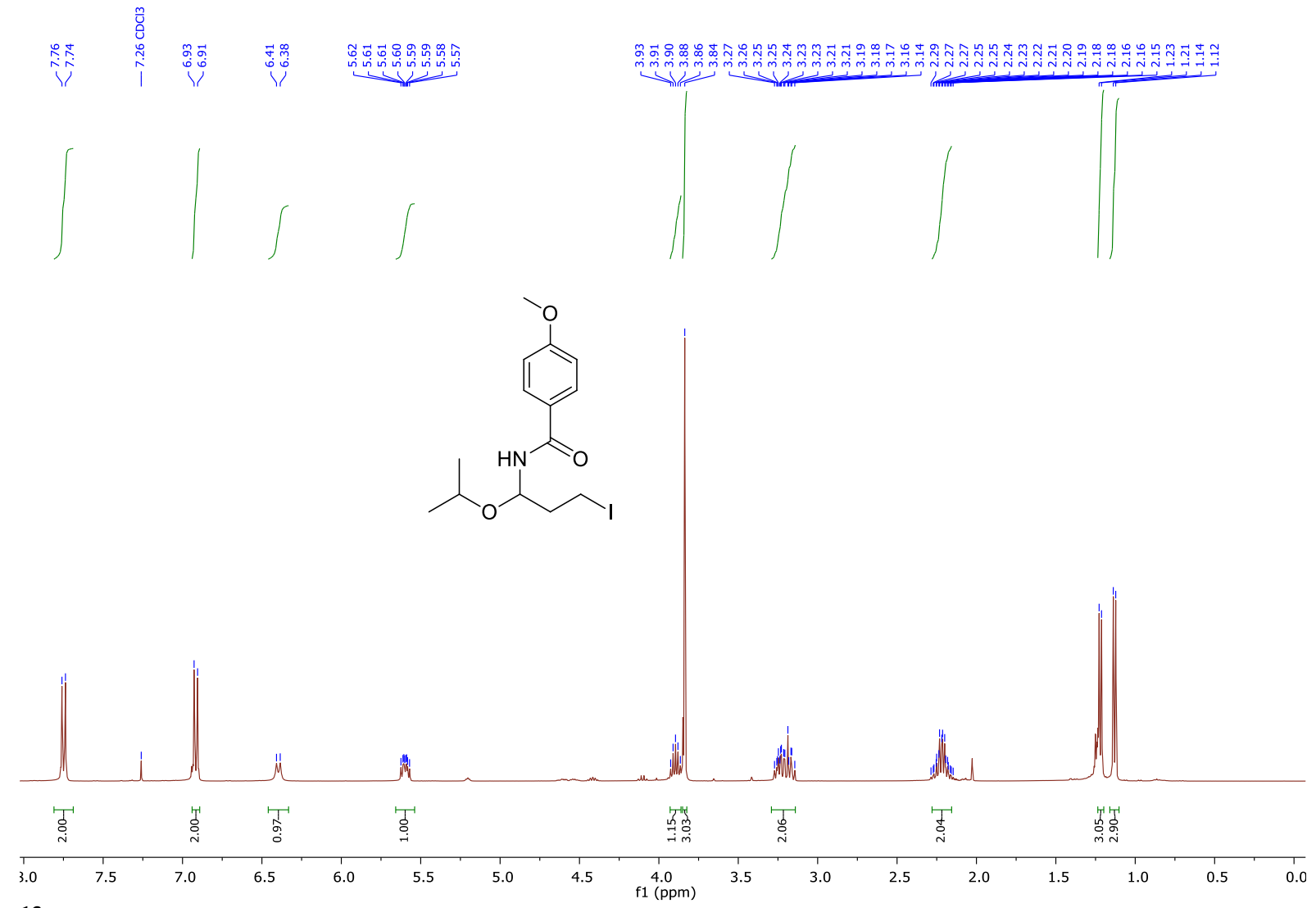

${ }^{13} \mathrm{C}-\mathrm{NMR}\left(101 \mathrm{MHz}, \mathrm{CDCl}_{3}\right)$
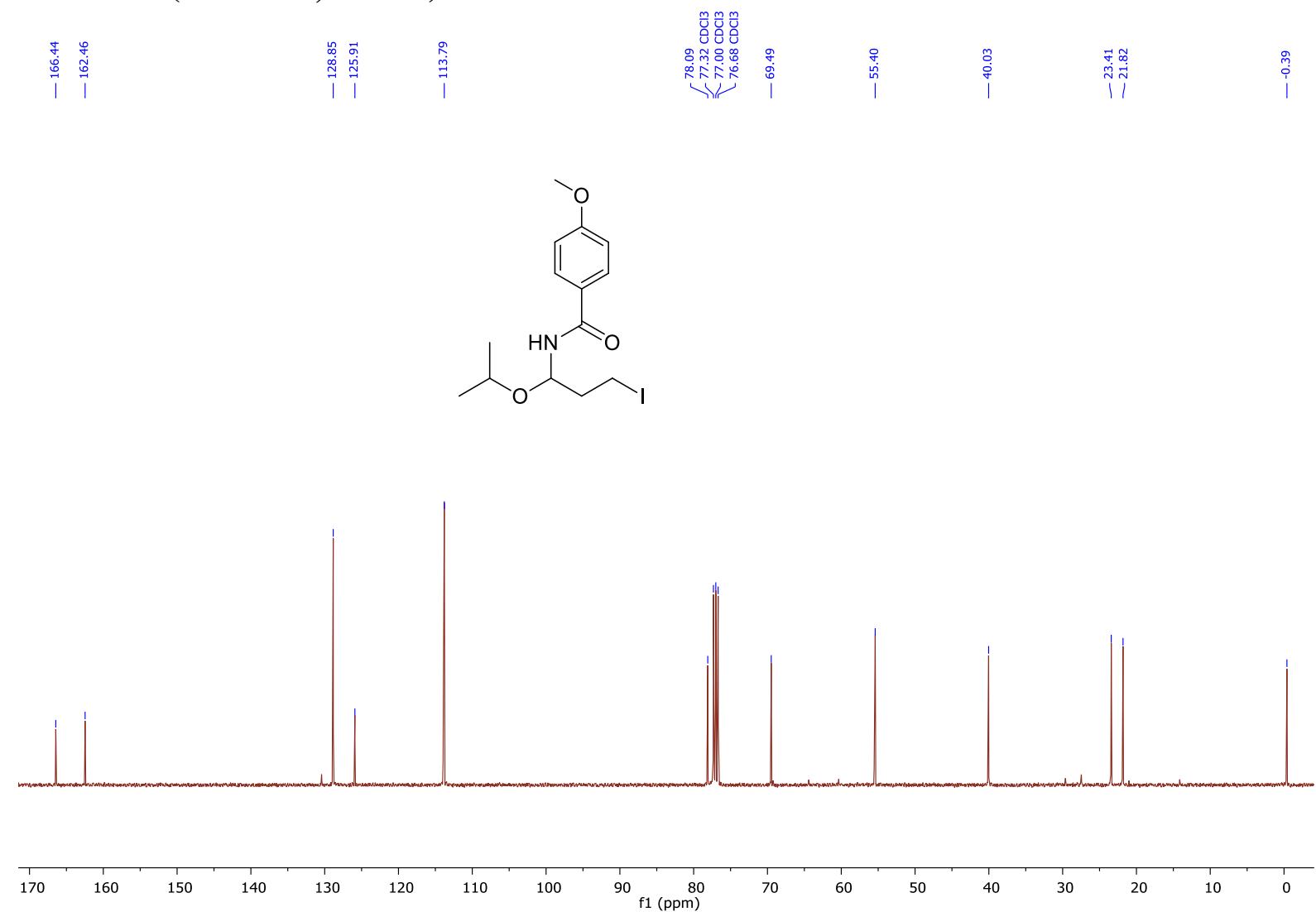
IR

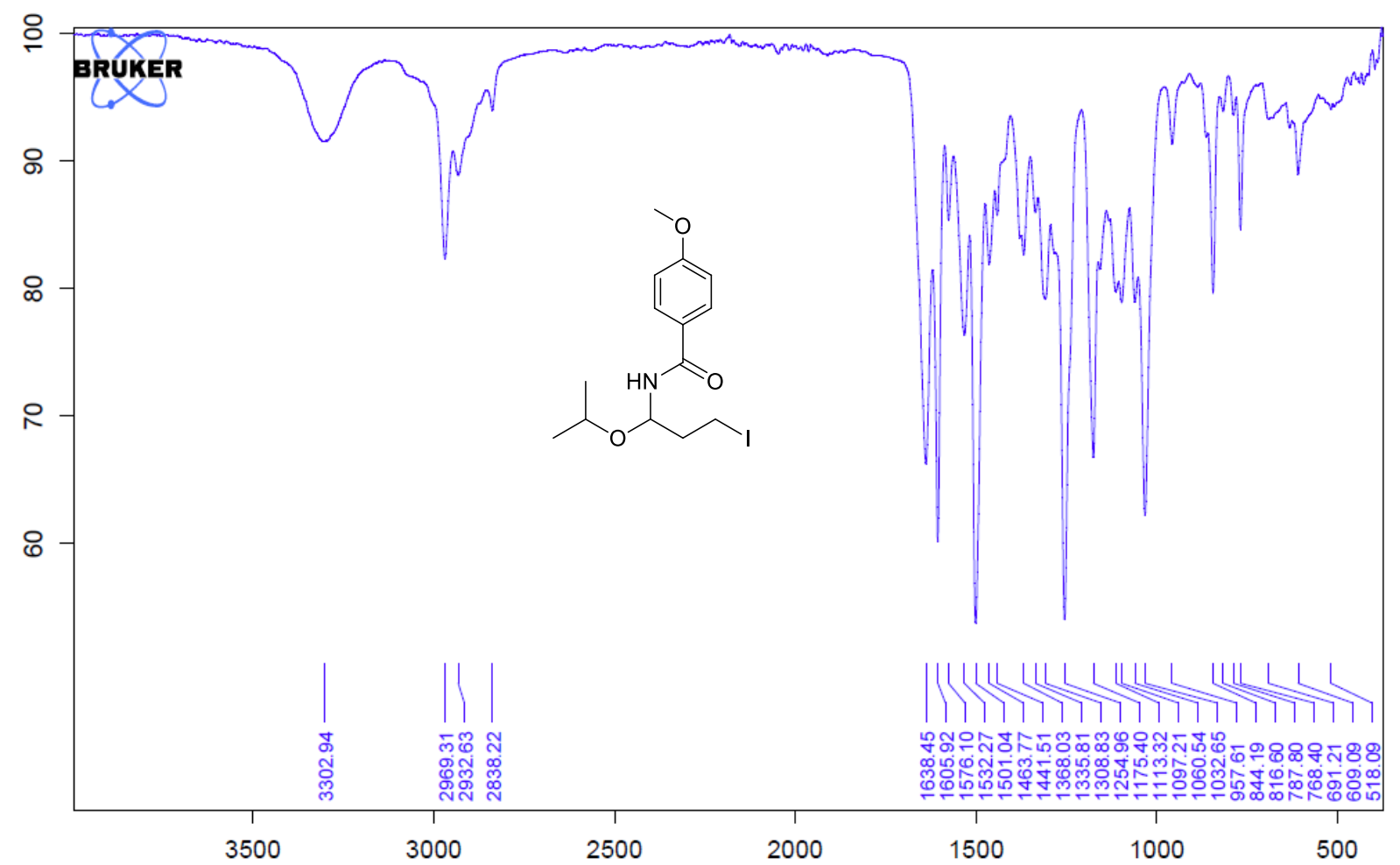

N-(3-Iodo-1-(((1R,2S,5R)-2-isopropyl-5-methylcyclohexyl)oxy)propyl)-4-

methoxybenzamide (10e)

${ }^{1} \mathrm{H}-\mathrm{NMR}\left(400 \mathrm{MHz}, \mathrm{CDCl}_{3}\right)$

范

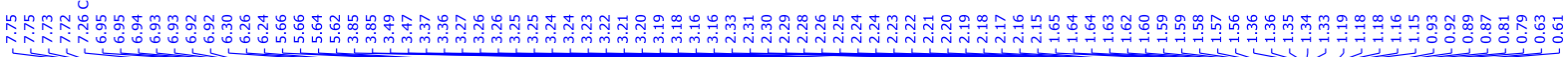<smiles>CCCCC</smiles>

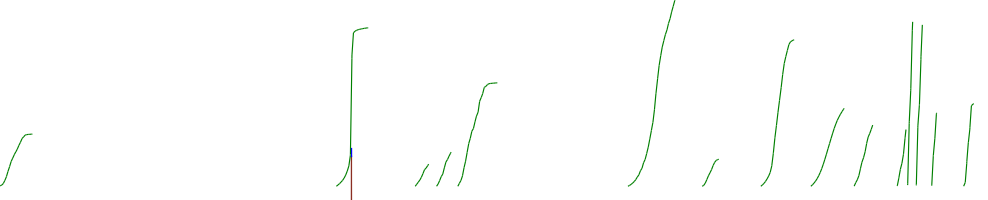<smiles>COc1ccc(C(=O)NC(CCI)OC2CC(C)CCC2C(C)C)cc1</smiles>

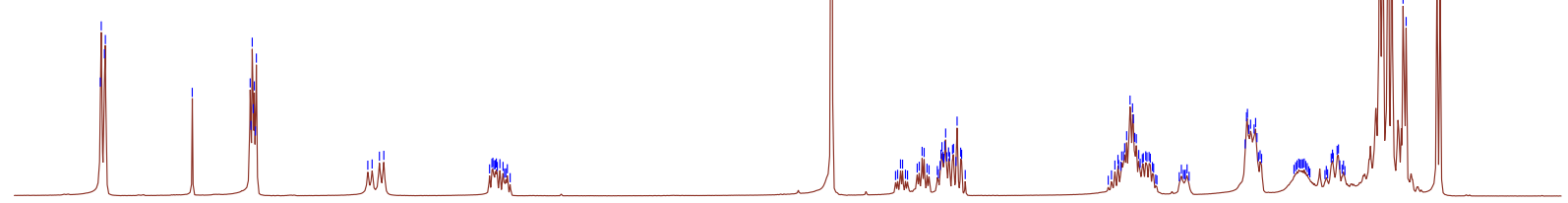

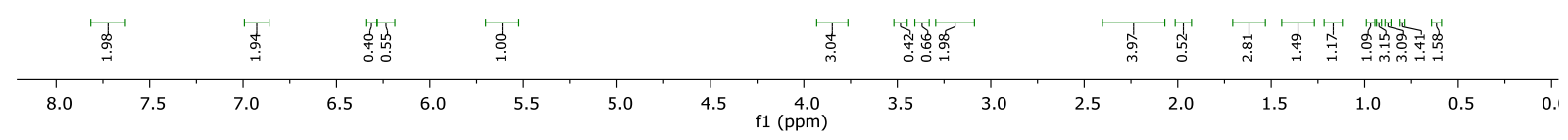


${ }^{13} \mathrm{C}$-NMR (101 MHz, CDCl3)
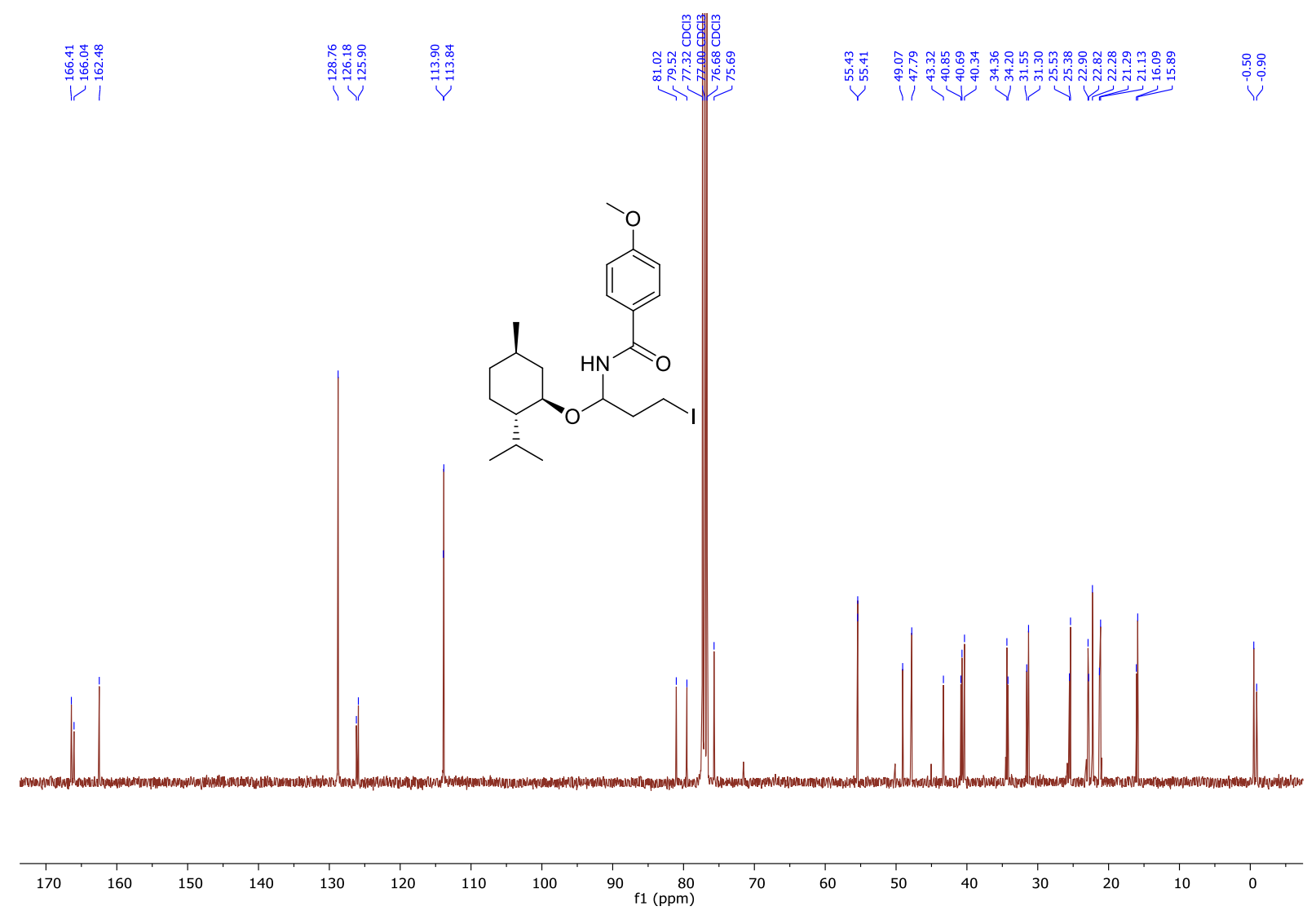

IR

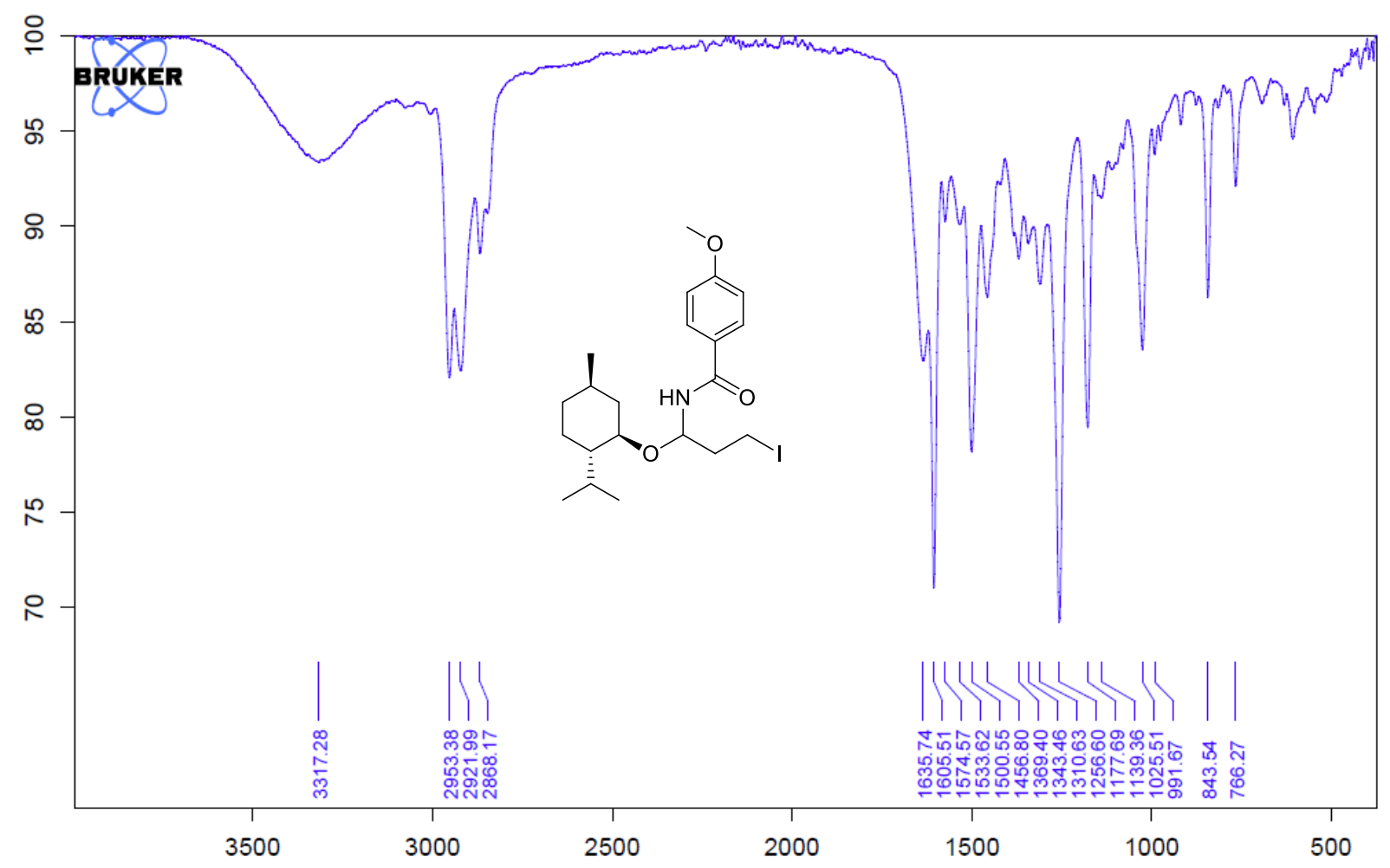


N-(1-(2-Hydroxyethoxy)-3-iodopropyl)-4-methoxybenzamide (10f)

${ }^{1}$ H-NMR (400 MHz, Acetone-d6)

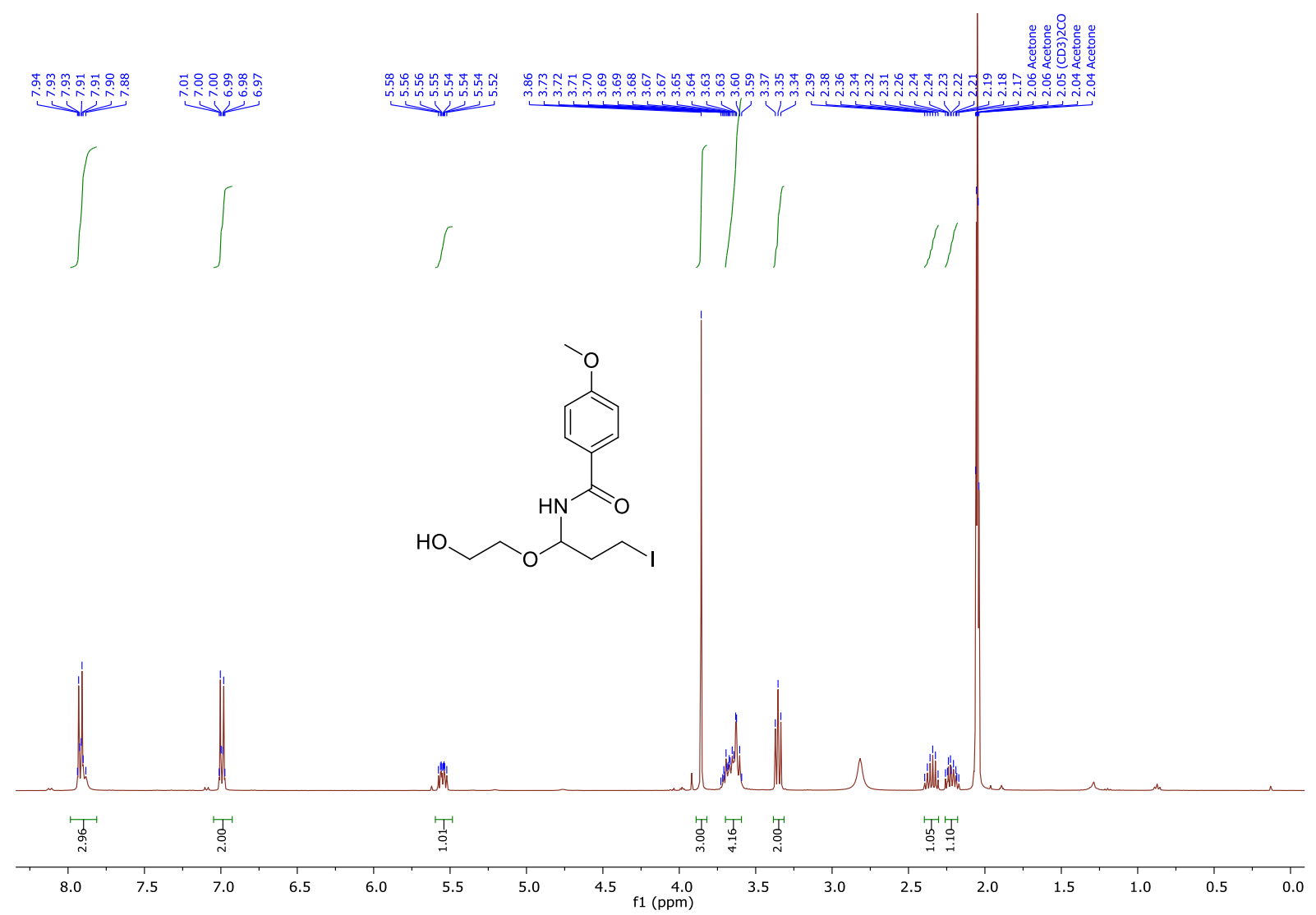

${ }^{13}$ C-NMR (101 MHz, Acetone-d6)

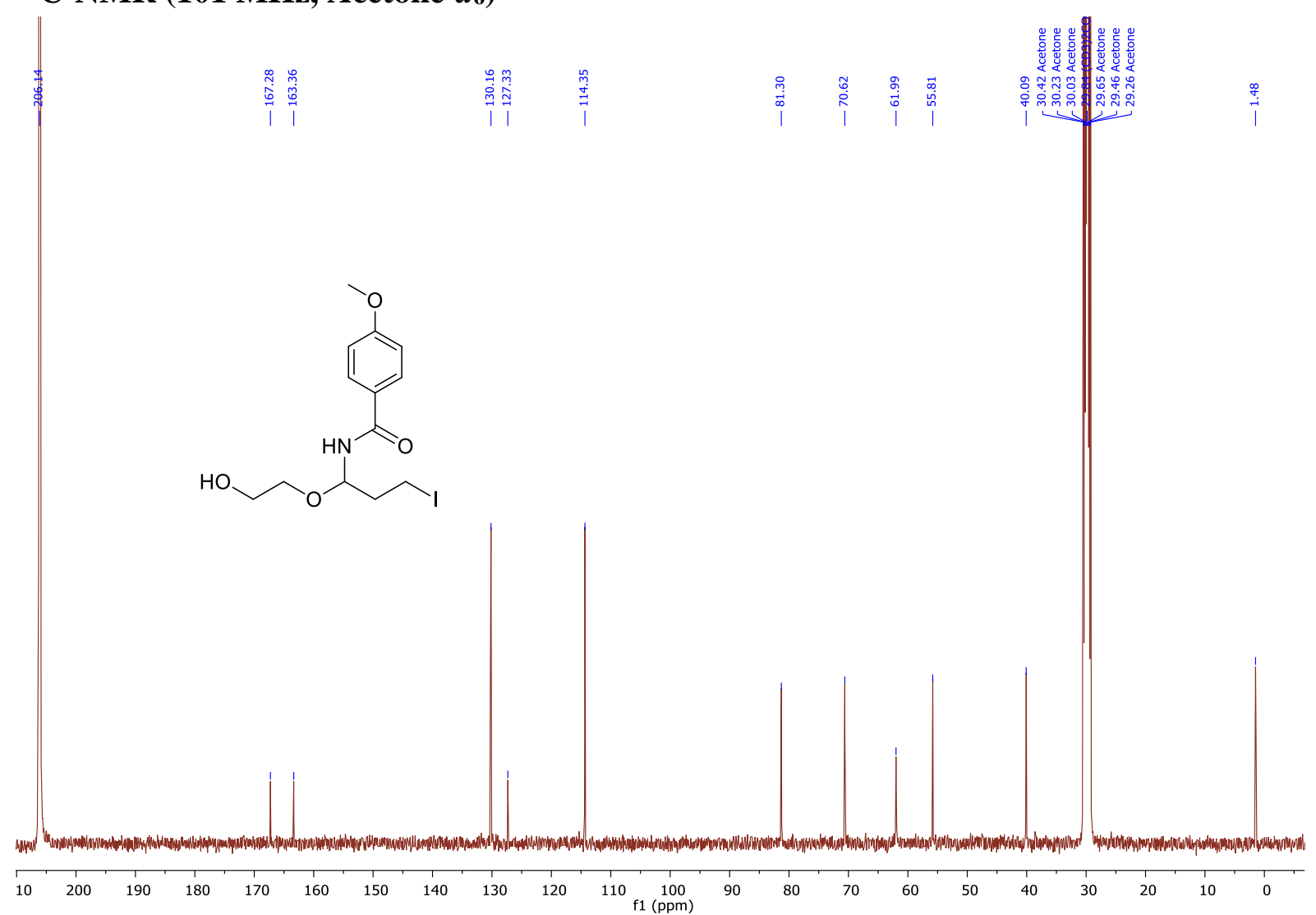


IR

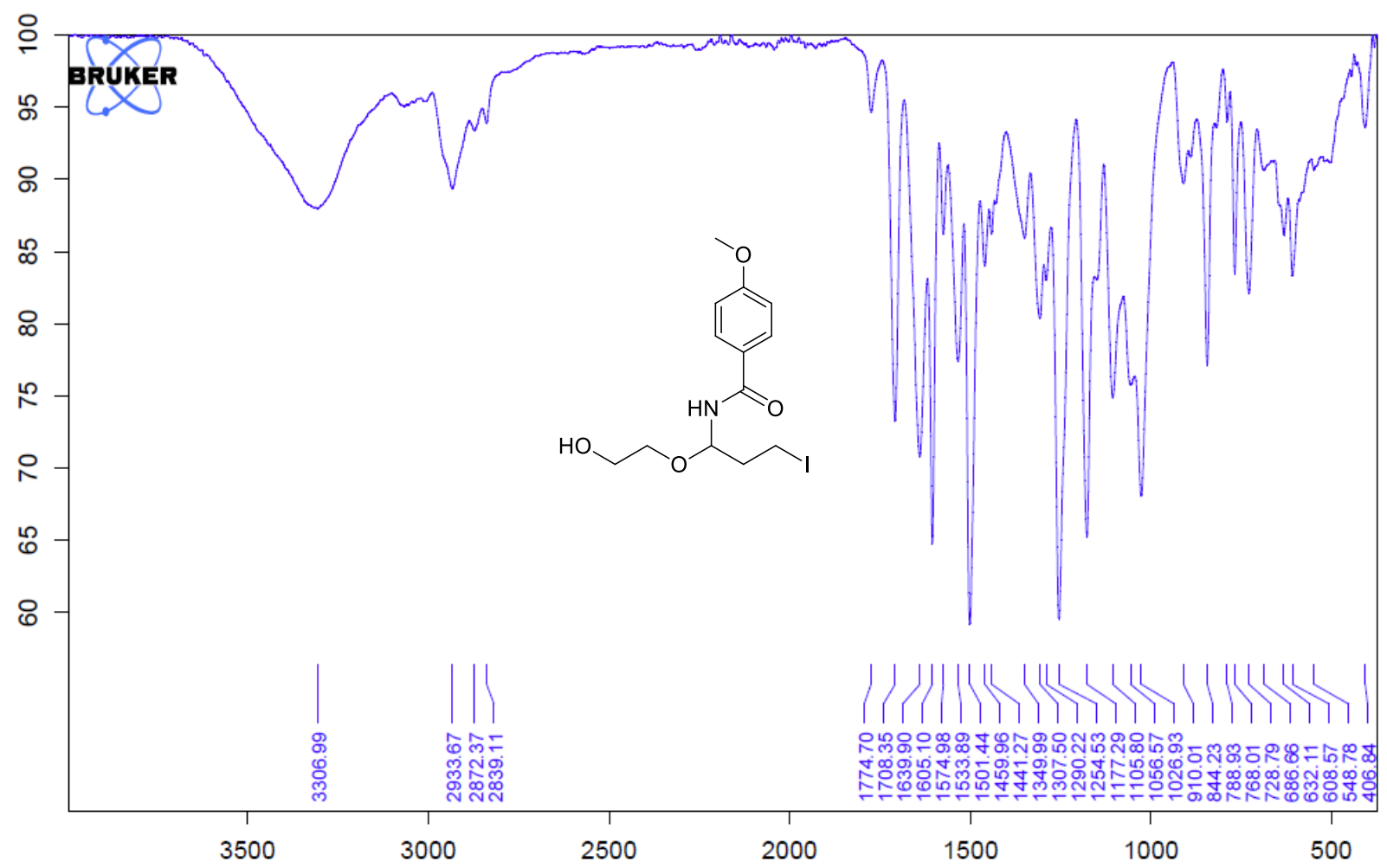

N-(1-(tert-Butylperoxy)-3-iodopropyl)-4-methoxybenzamide (10g)

${ }^{1} \mathrm{H}-\mathrm{NMR}$ (400 MHz, CDCl3)

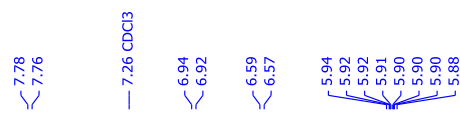

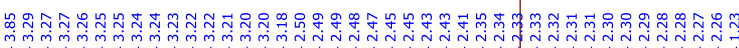

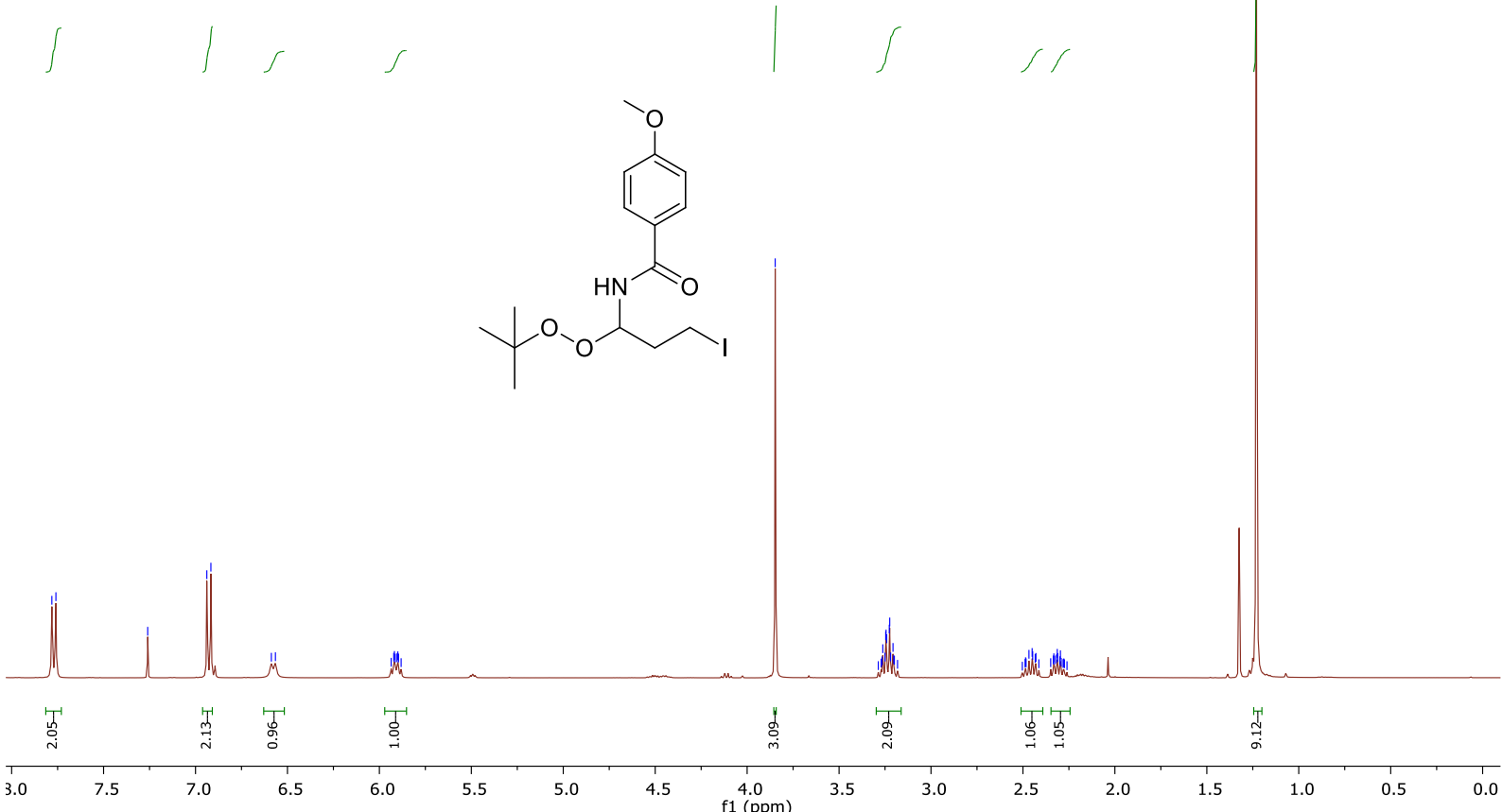


${ }^{13}$ C-NMR (101 MHz, CDCl3)

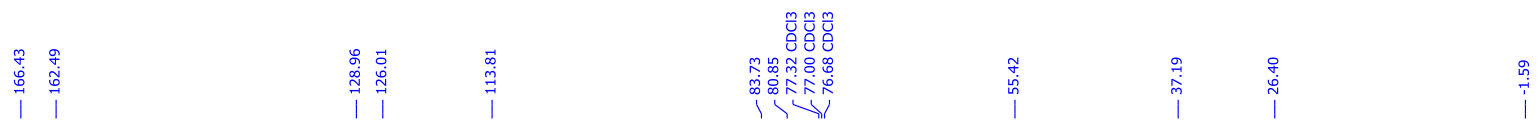<smiles>COc1ccc(C(=O)NC(CCI)OOC(C)(C)C)cc1</smiles>
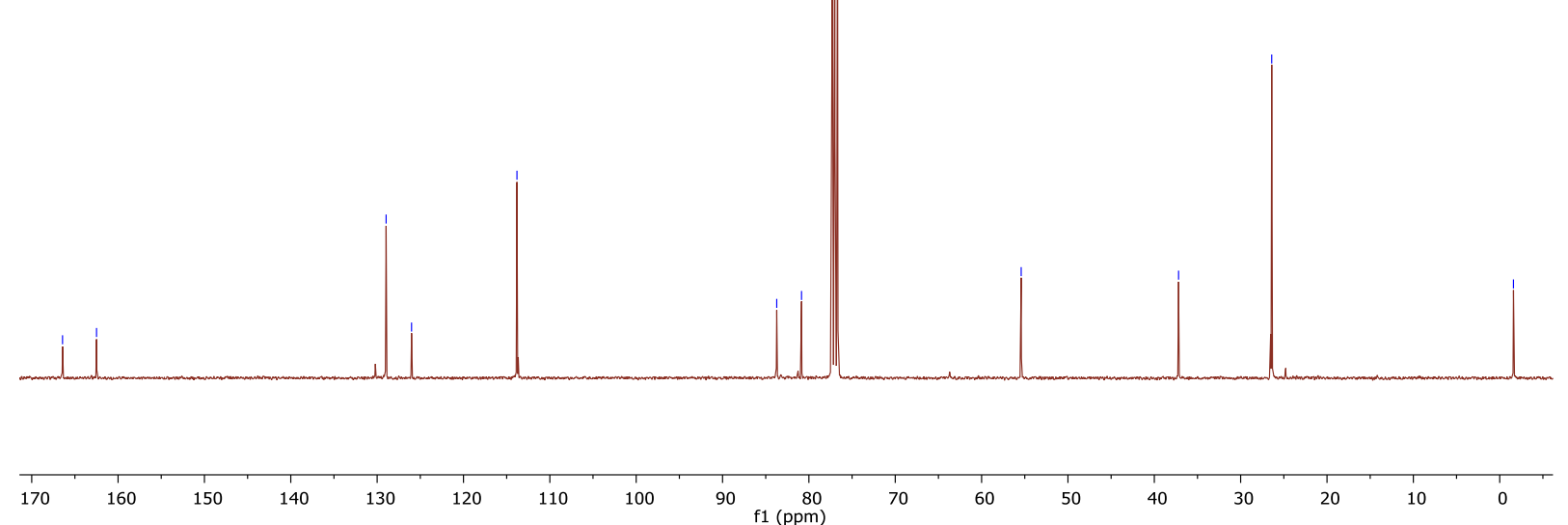

IR

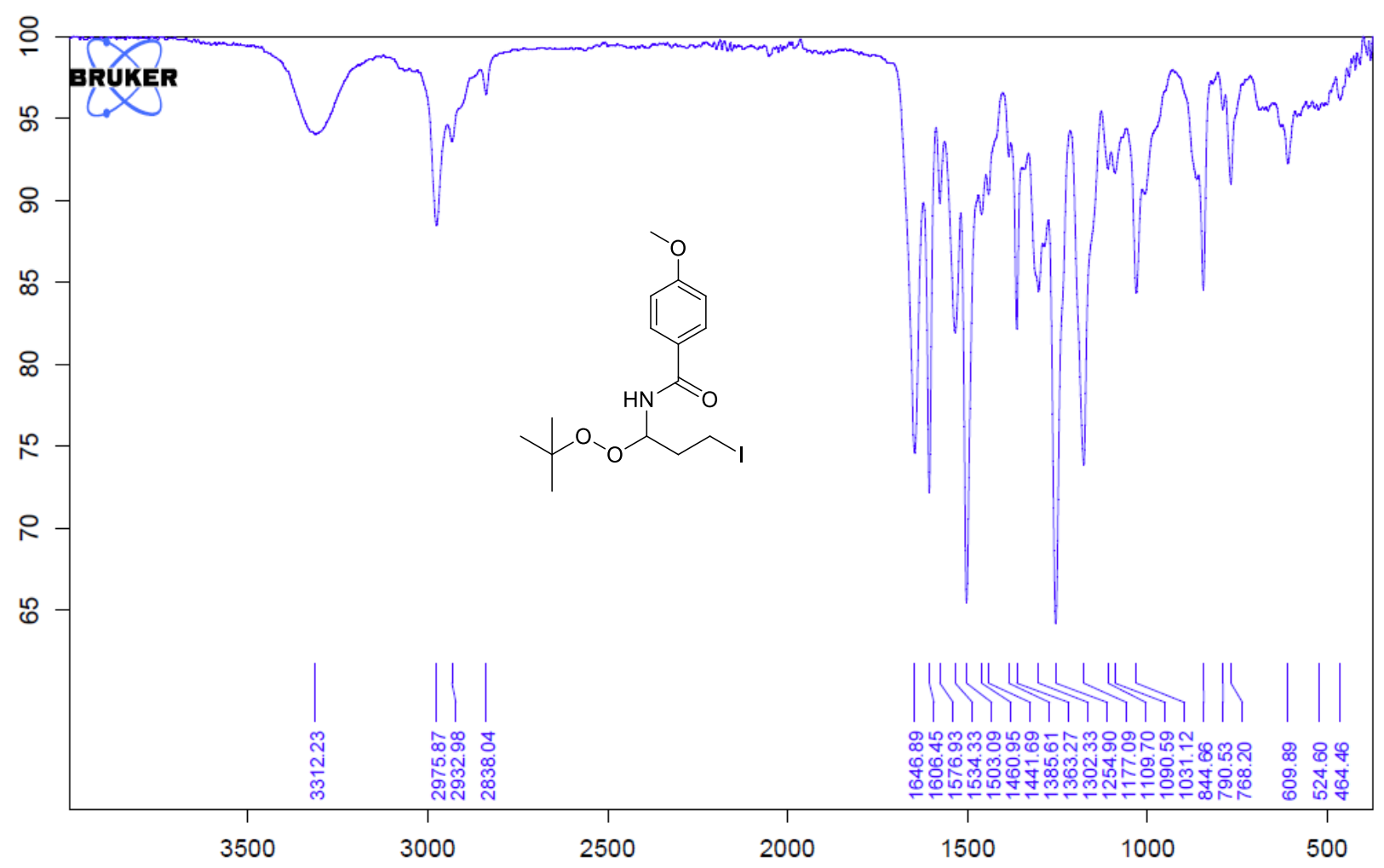


N-(1-Hydroperoxy-3-iodopropyl)-4-methoxybenzamide (10h)

${ }^{1}$ H-NMR (400 MHz, Acetone-d6)

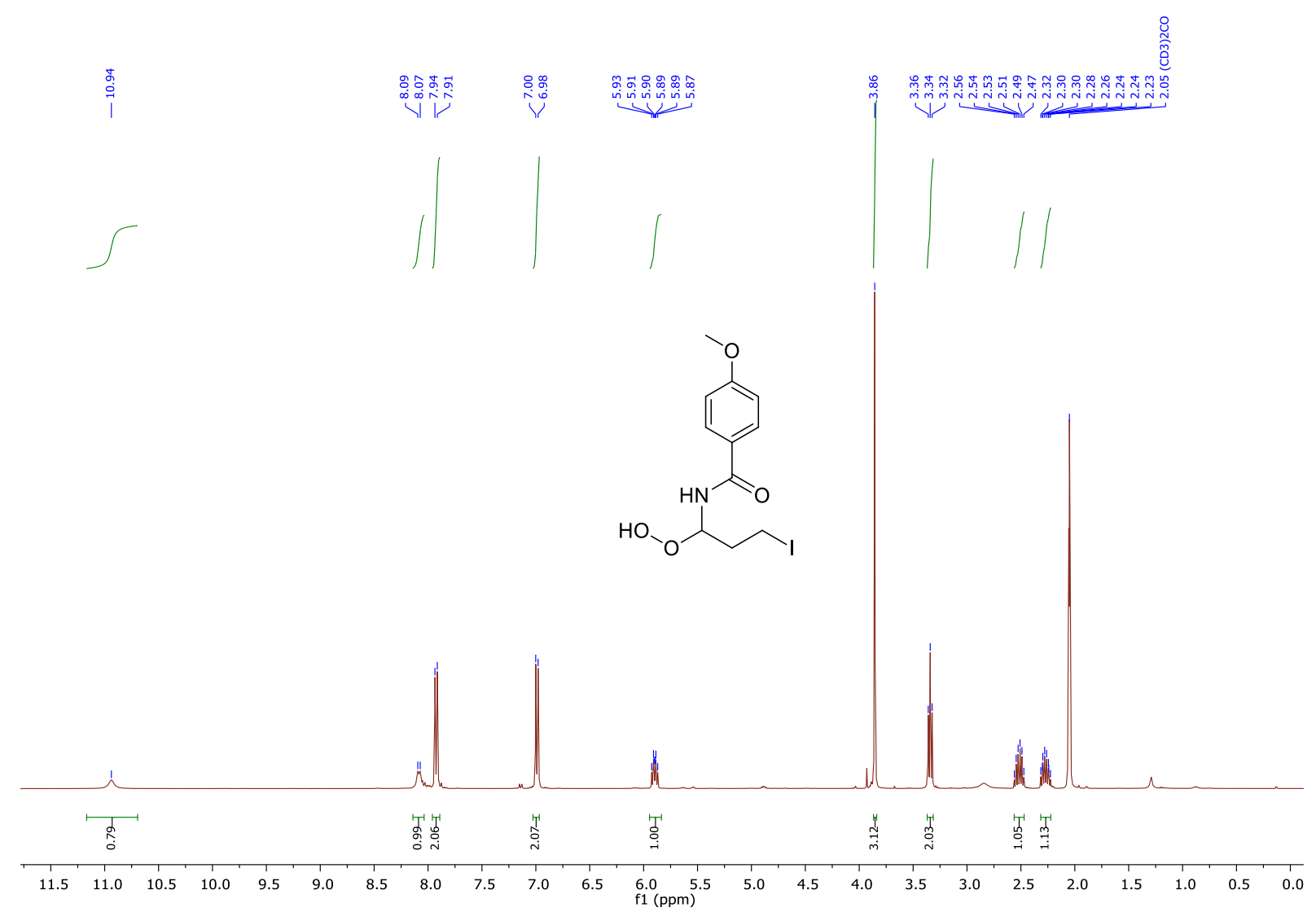

${ }^{13}$ C-NMR (101 MHz, Acetone- $\left.d_{6}\right)$

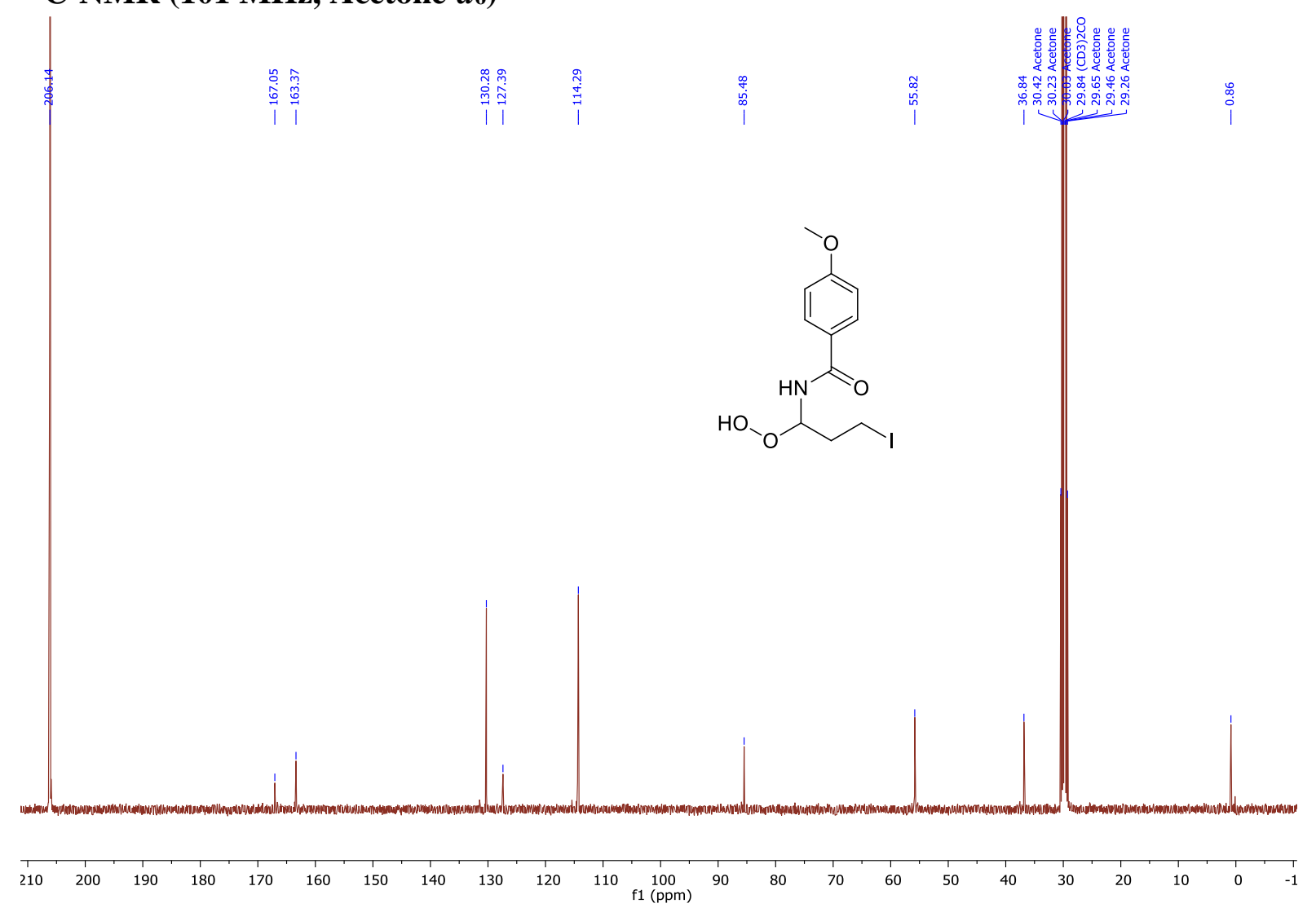


IR

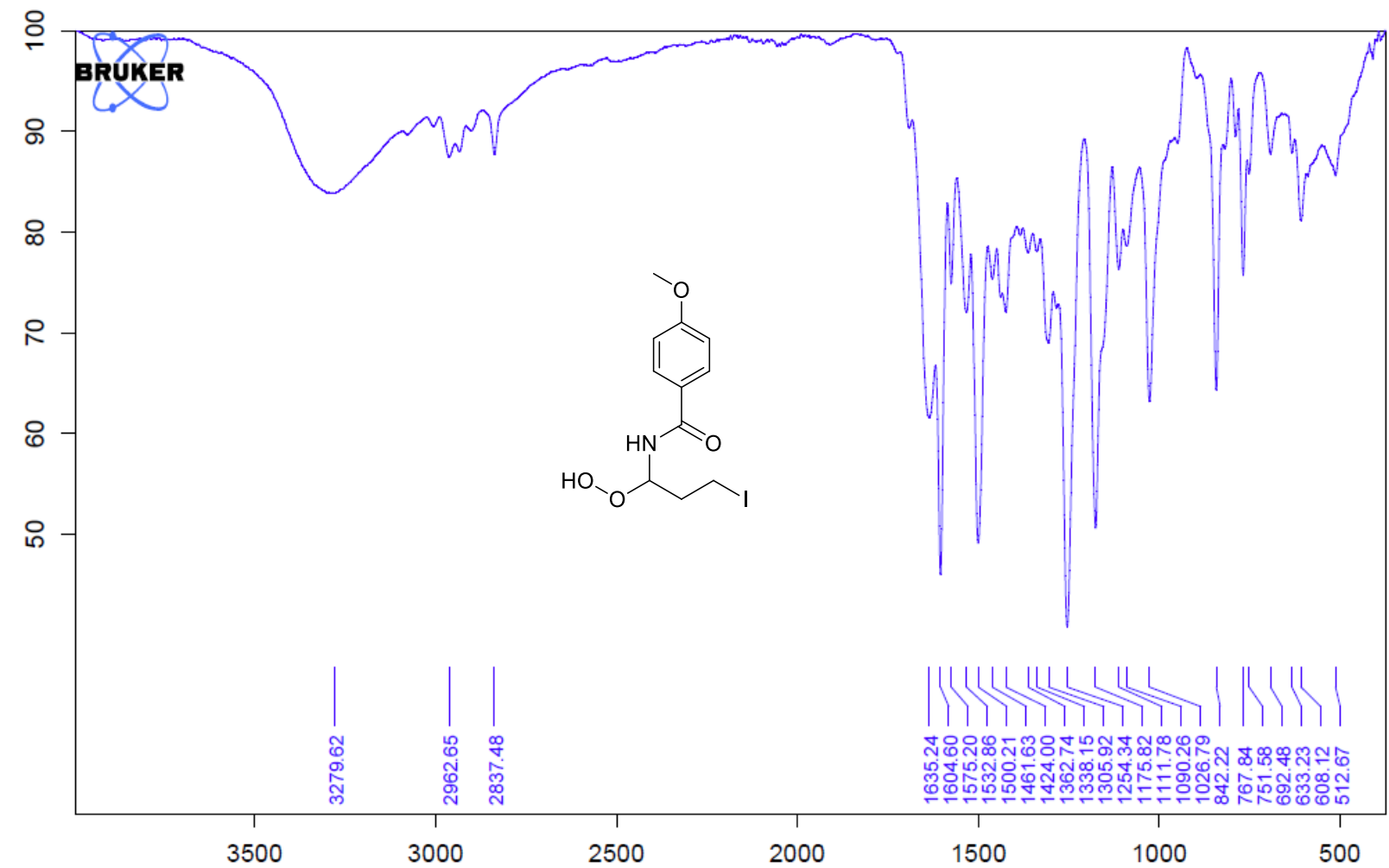

N-(3-Iodo-1-(methylsulfonamido)propyl)-4-methoxybenzamide (11)

${ }^{1} \mathrm{H}-\mathrm{NMR}$ (400 MHz, $\mathrm{CDCl}_{3}$ )

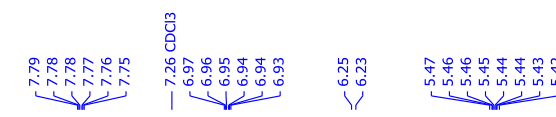

$\underbrace{\circ}$
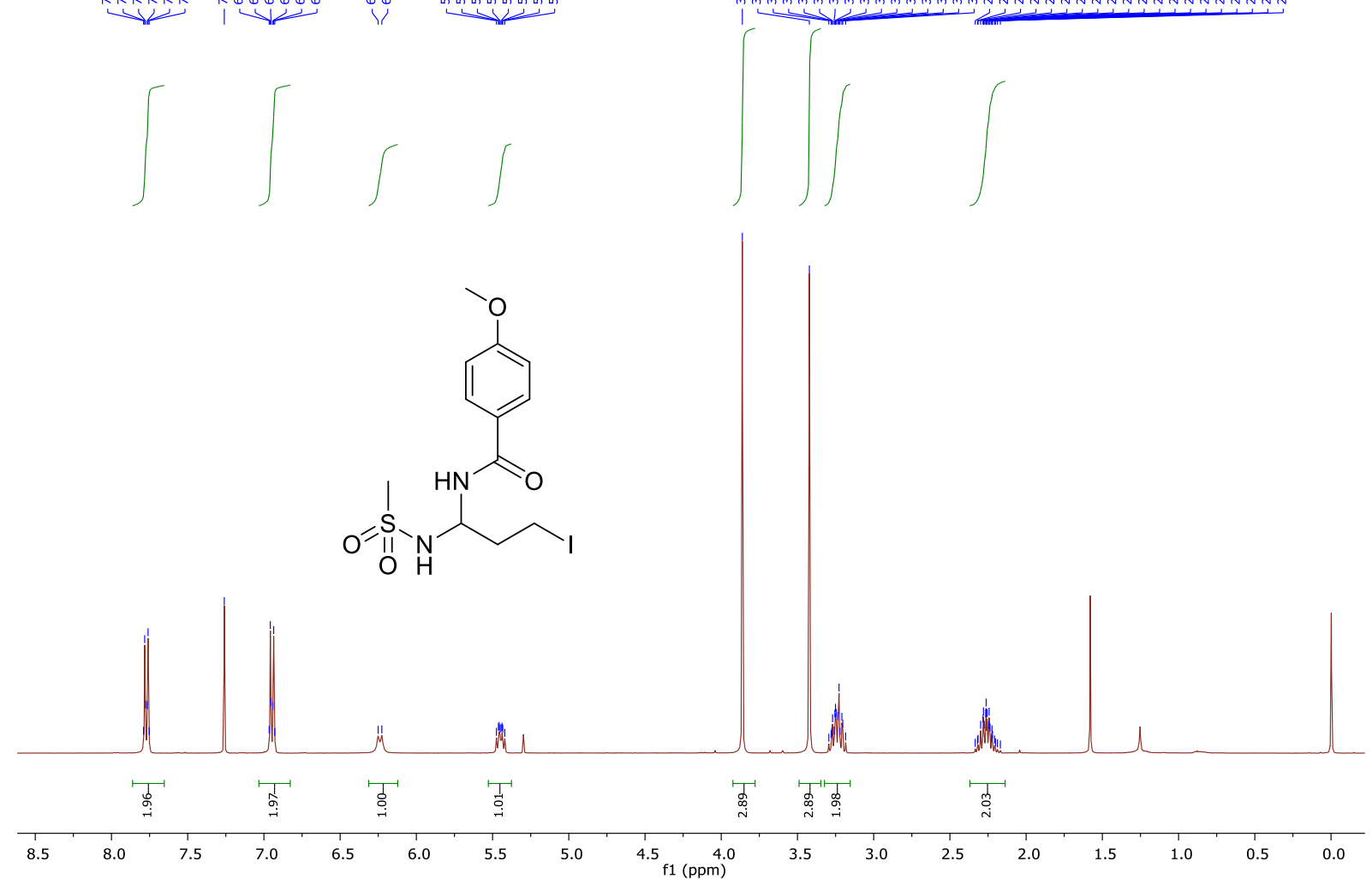
${ }^{13}$ C-NMR (101 MHz, CDCl3)

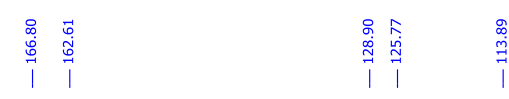<smiles>COc1ccc(C(=O)NC(CCI)NS(C)(=O)=O)cc1</smiles>
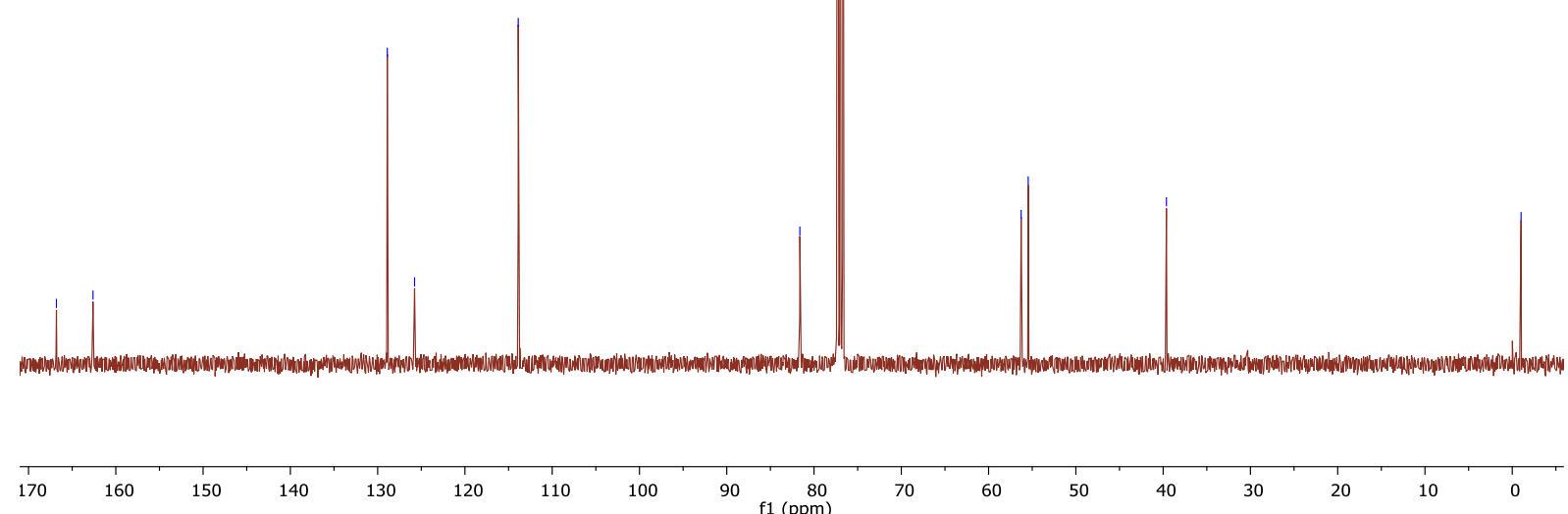

IR

은

ณ

BRUKER

ஃ

$\infty$

$\infty$

$\stackrel{\infty}{\sim}$

o

๑ึ

8
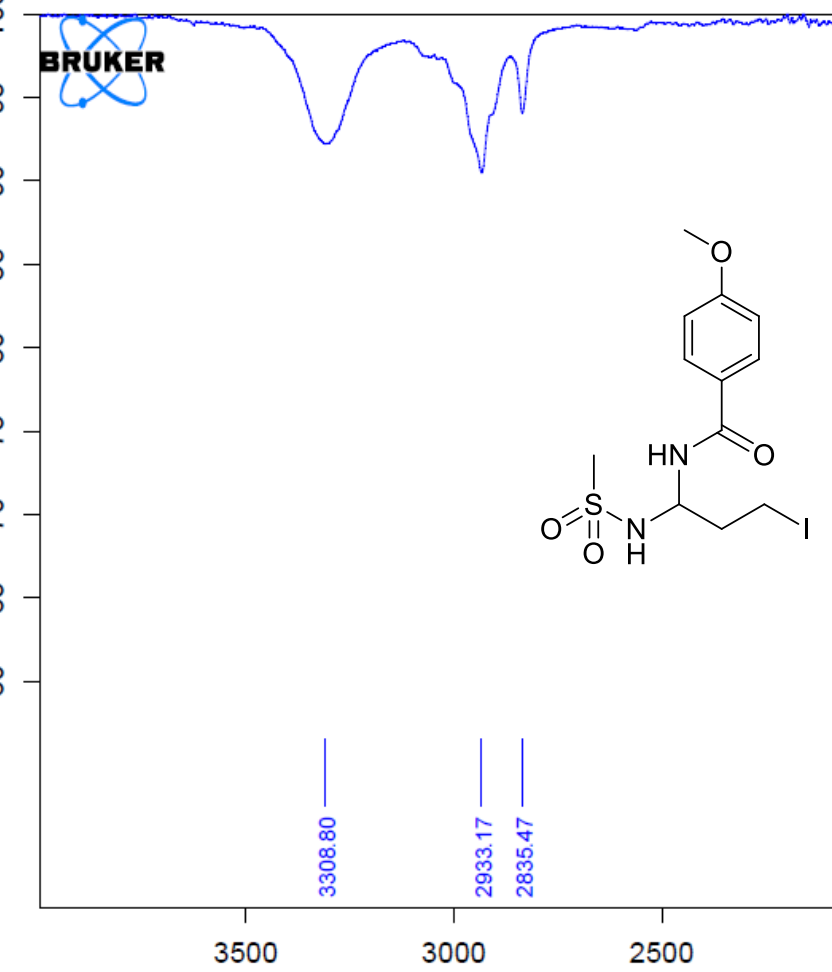
N-(1-Cyano-3-iodopropyl)-4-methylbenzenesulfonamide (12)

${ }^{1}$ H-NMR (400 MHz, CDCl3)
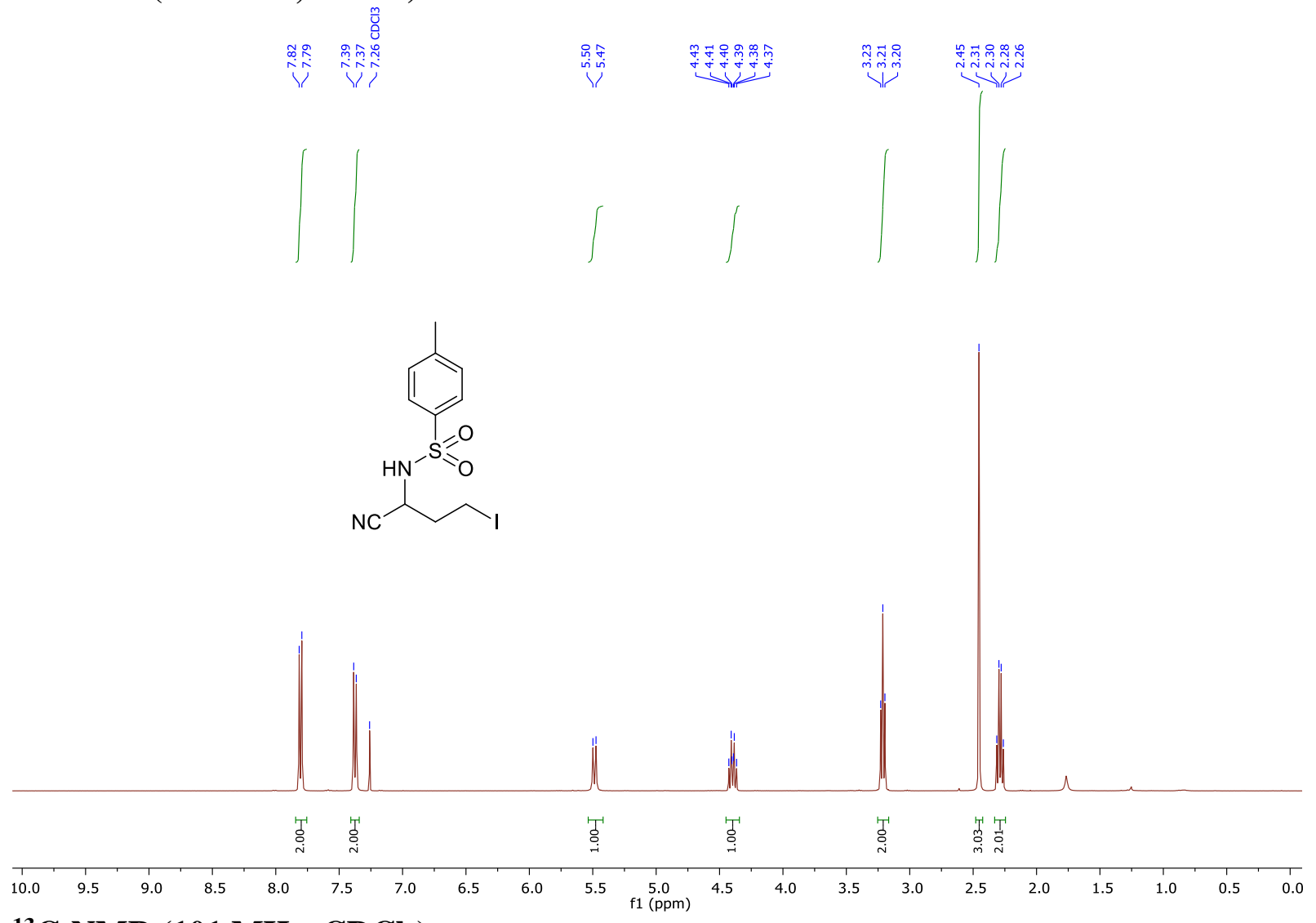

${ }^{13} \mathrm{C}$-NMR (101 MHz, CDCl 3$)$

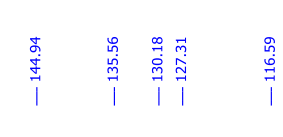

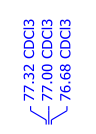

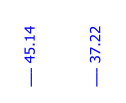

$\stackrel{\widehat{̣}}{1}$

i্j

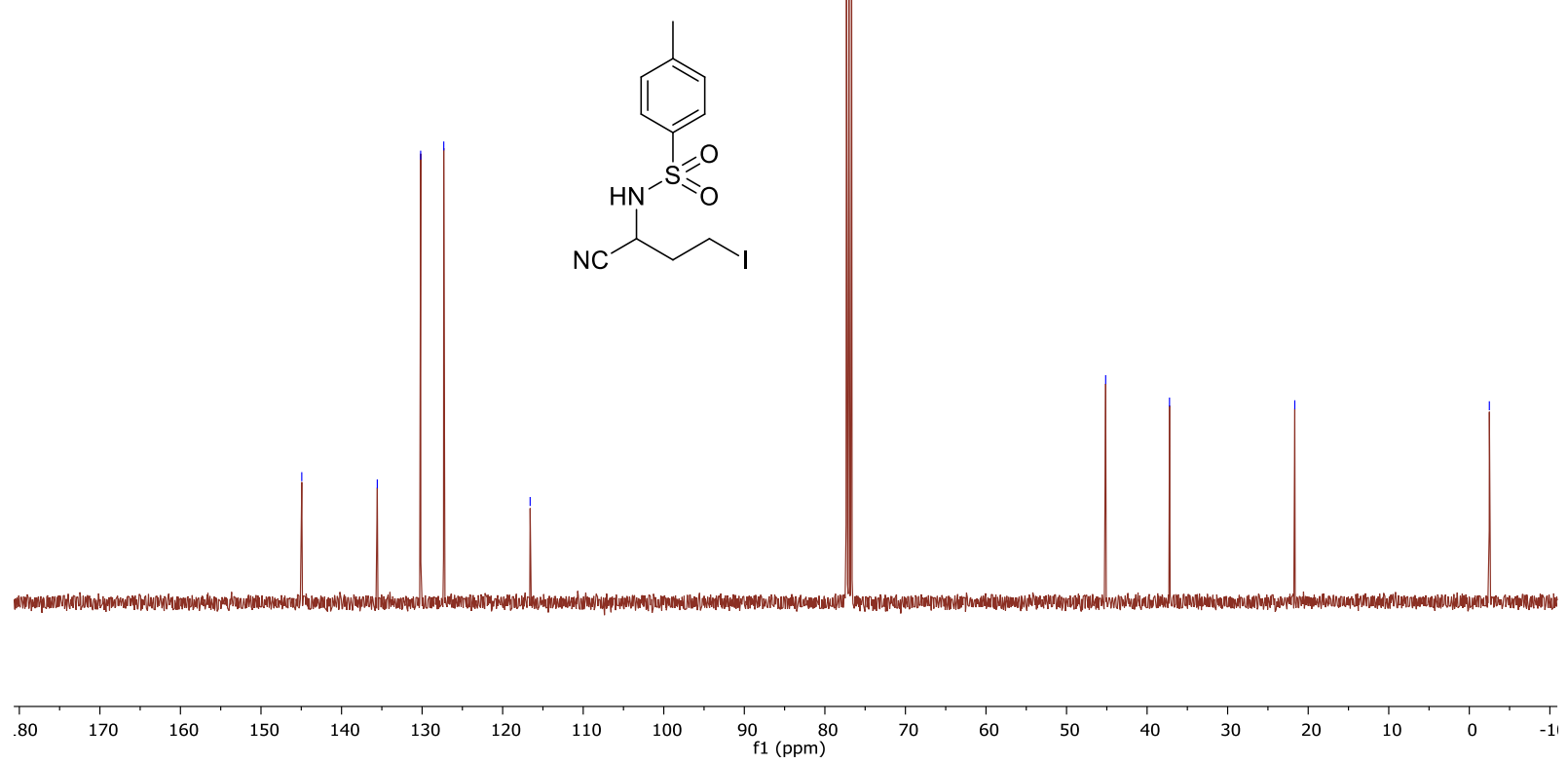

S119 


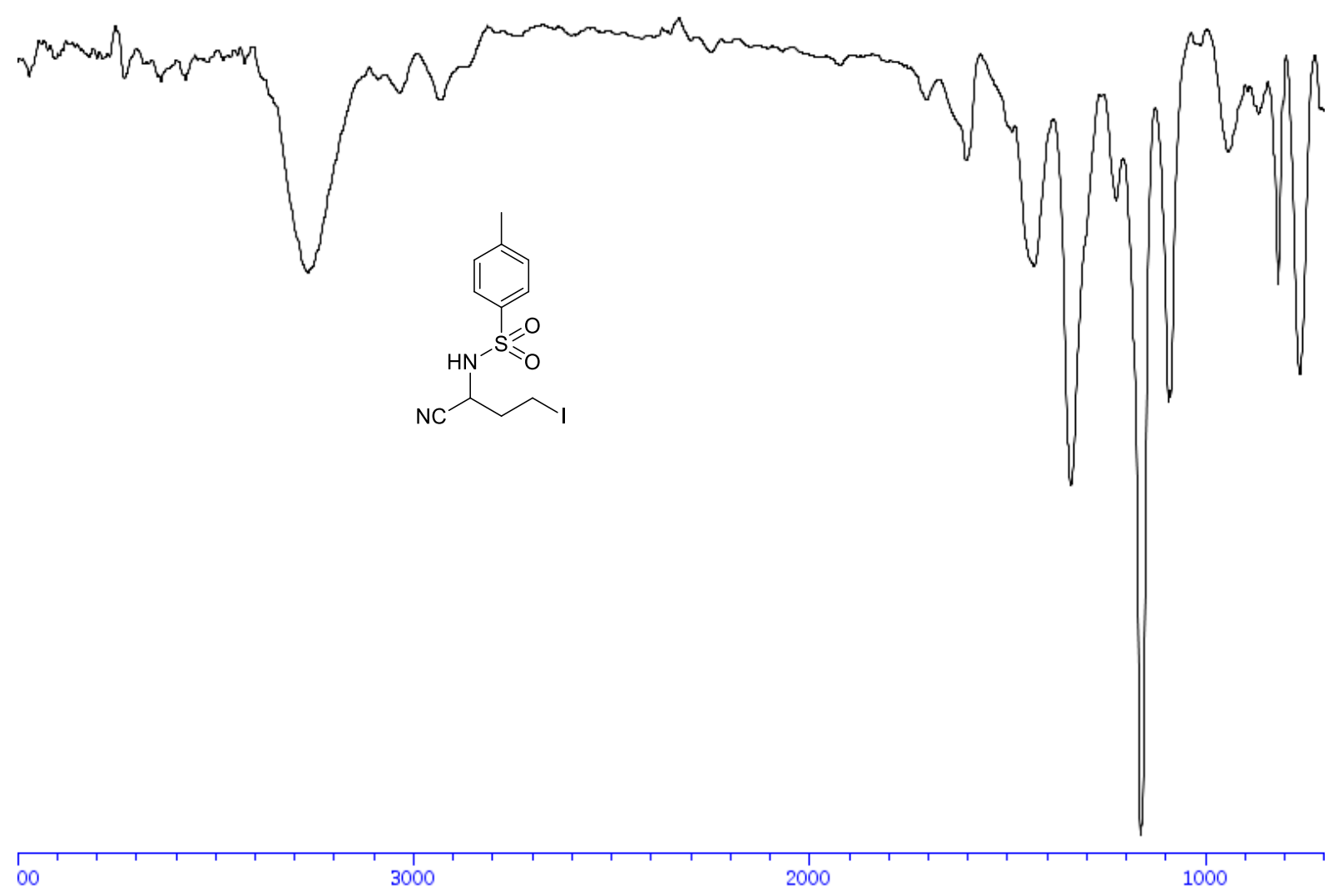

N-(1-(1-Hydroxynaphthalen-2-yl)-3-iodopropyl)-4-methoxybenzamide (13)

${ }^{1}$ H-NMR (400 MHz, Acetone-d6)

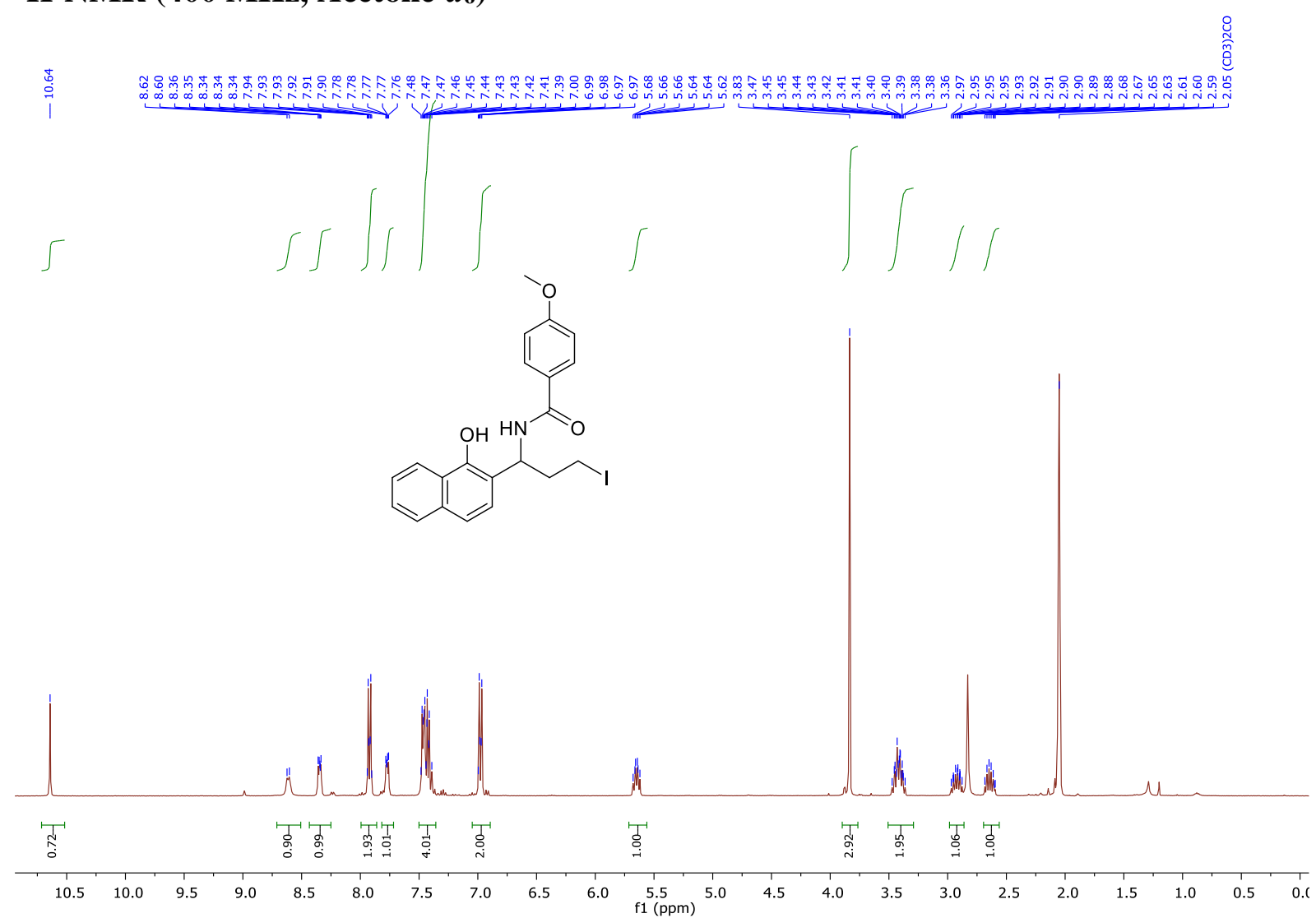


${ }^{13}$ C-NMR (101 MHz, Acetone-d6)

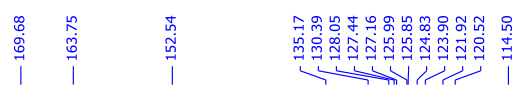
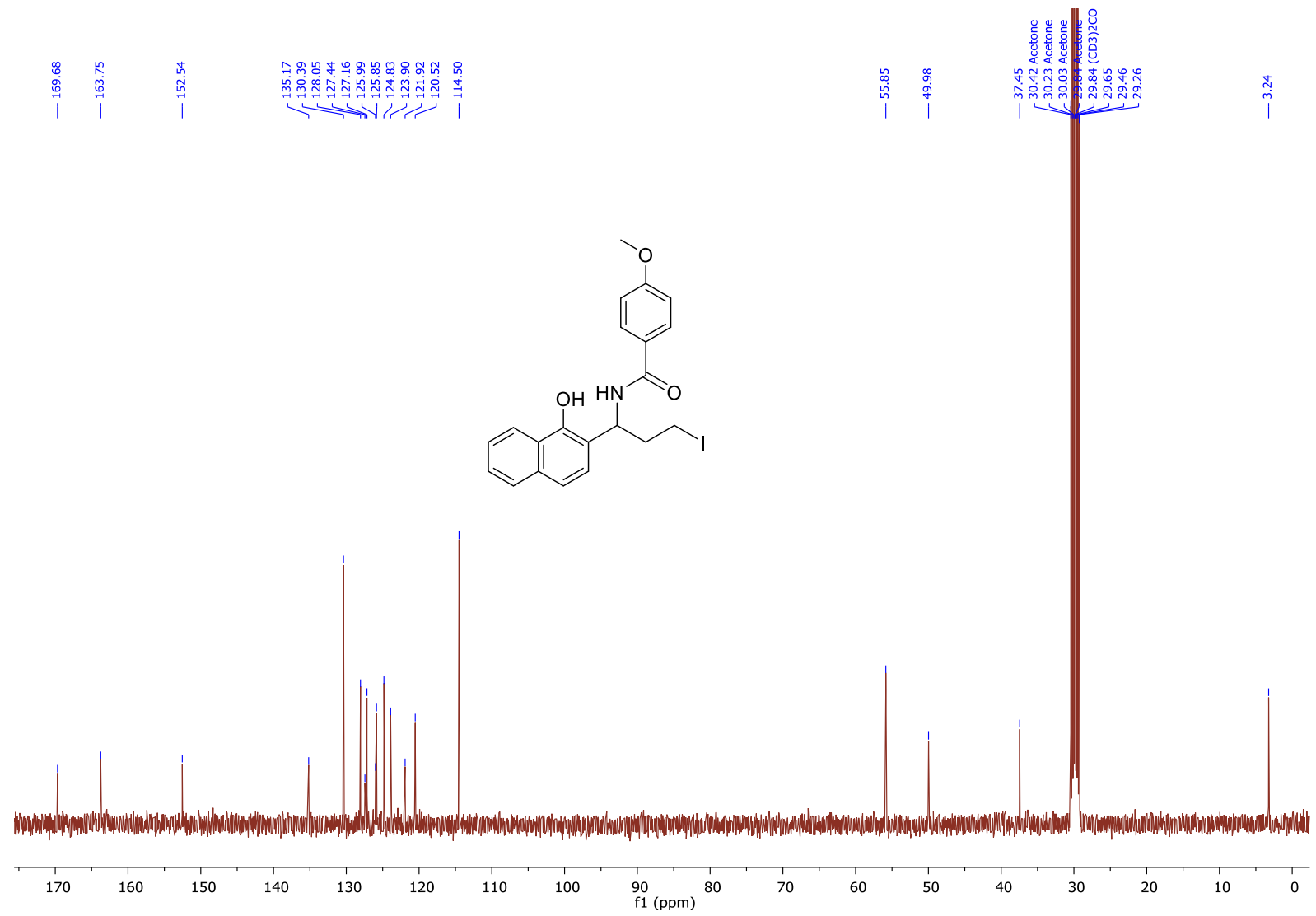

IR

우

๕

RिR

BRUKER

ㅇ

$\infty$

$\infty$

온

ㅇ

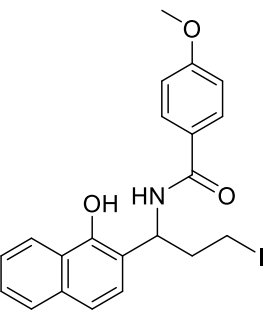

r

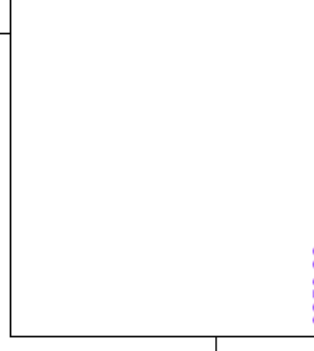

3500

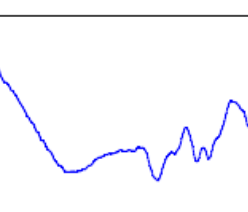


N-(3-Iodo-1-(3-methyl-1H-indol-2-yl)propyl)-4-methoxybenzamide (14)

${ }^{1}$ H-NMR (400 MHz, Acetone-d6)

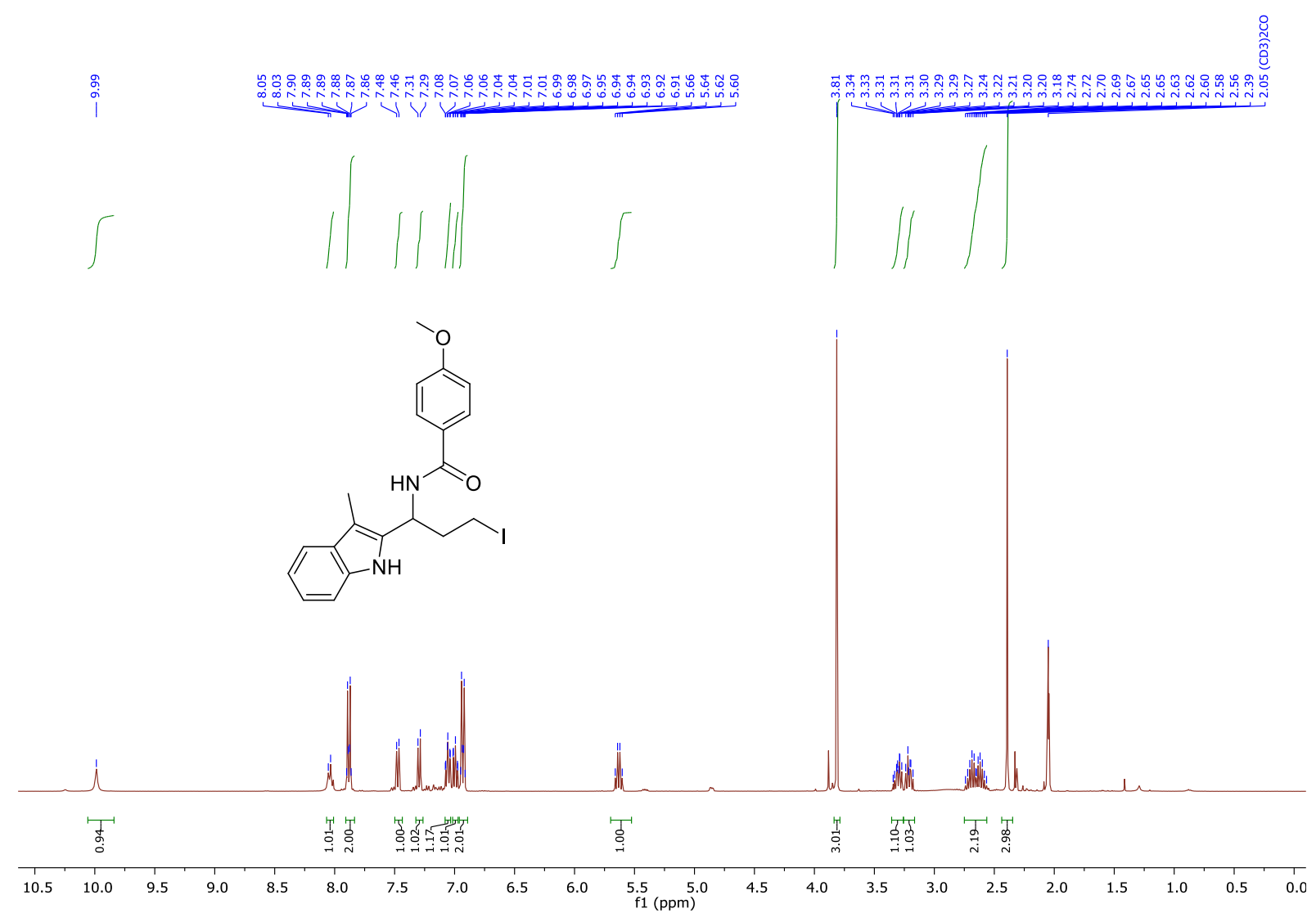

${ }^{13}$ C-NMR (101 MHz, Acetone-d6)

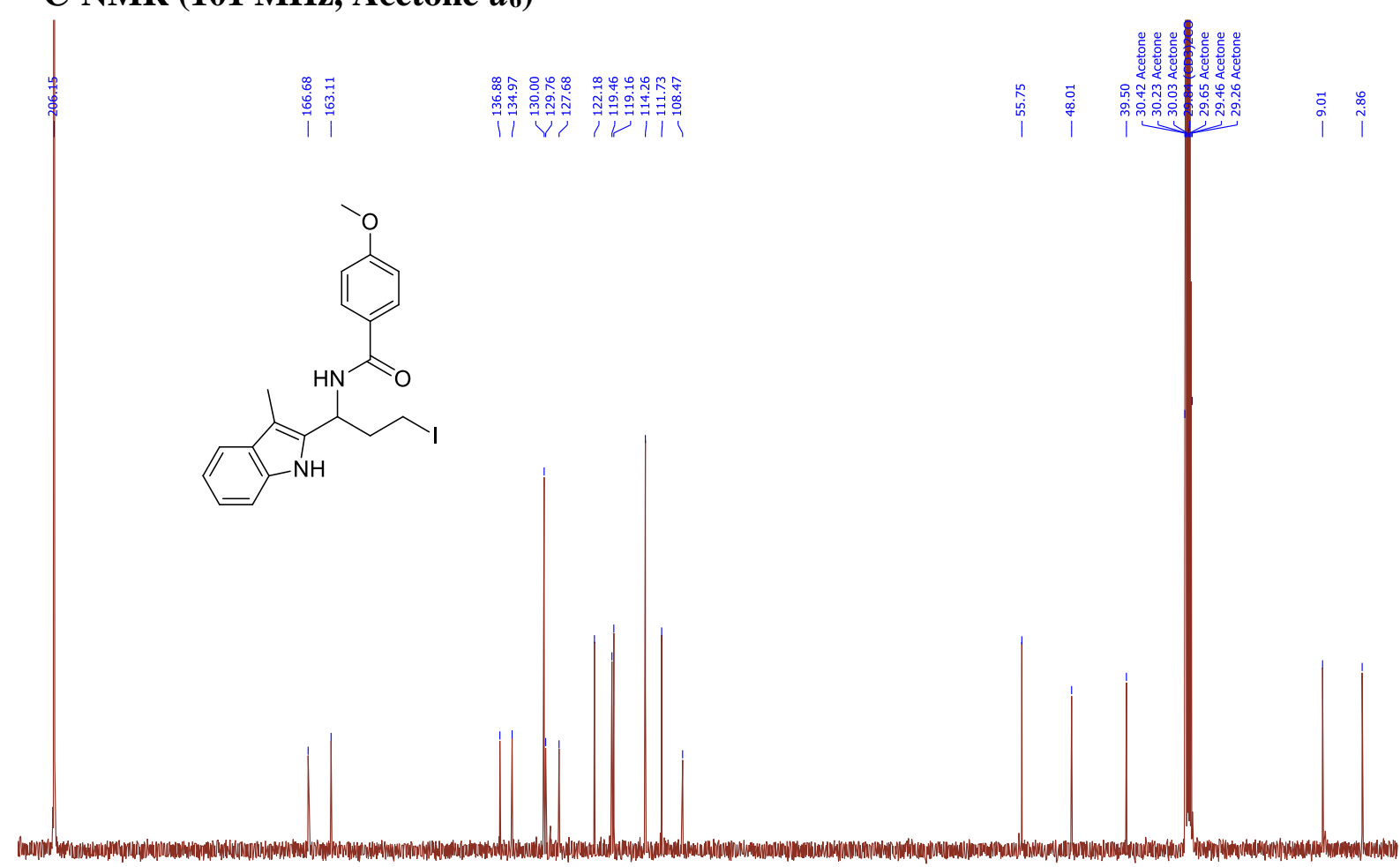

$\begin{array}{lllllllllll}210 & 200 & 190 & 180 & 170 & 160 & 150 & 140 & 130 & 120 & 110 \\ \mathrm{f} 1(\mathrm{ppm}) & 100\end{array}$ 
IR

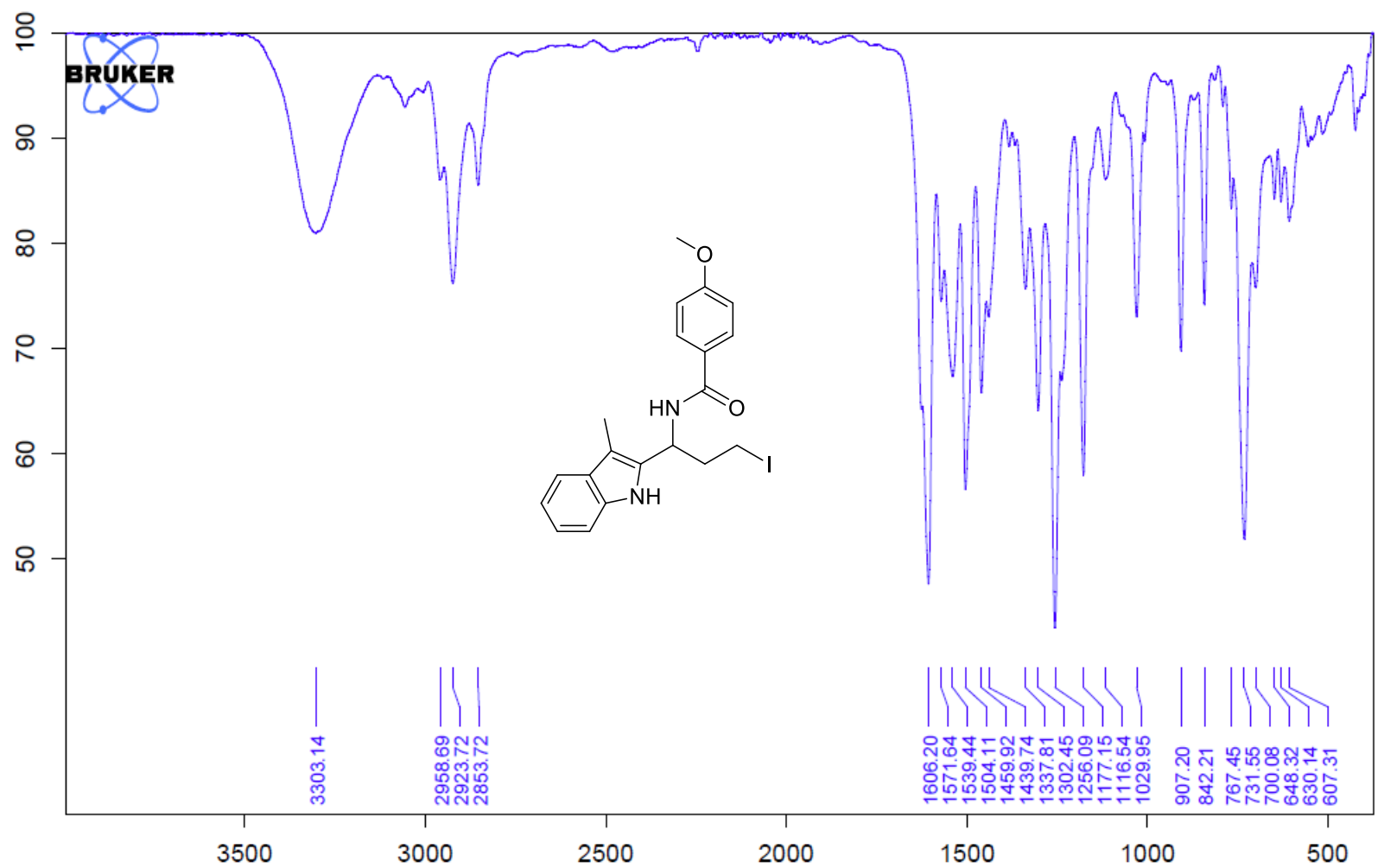

N-(3-Iodopropyl)-4-methoxybenzamide (15)

${ }^{1} \mathrm{H}-\mathrm{NMR}$ (400 MHz, $\mathrm{CDCl}_{3}$ )
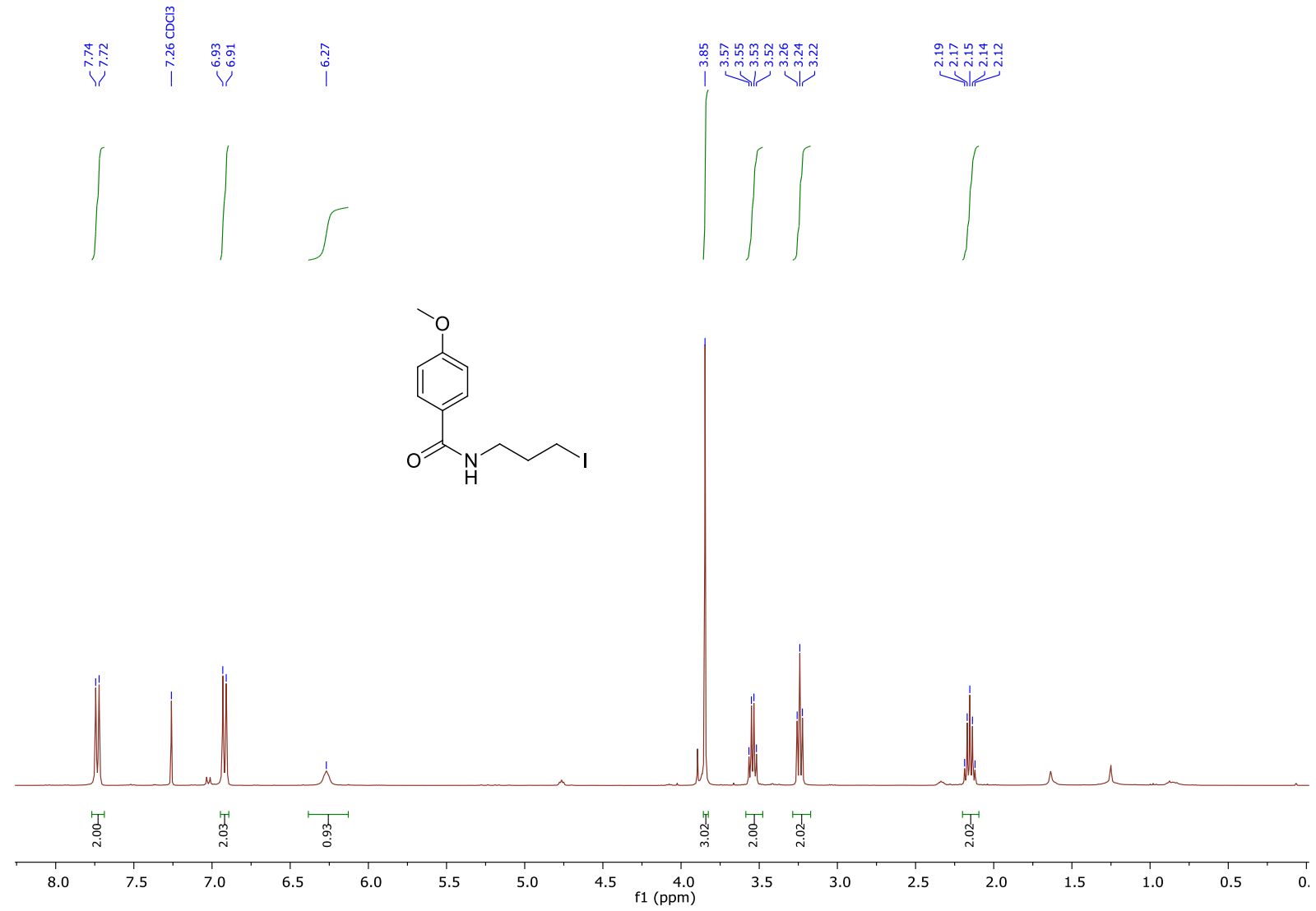
${ }^{13} \mathrm{C}$-NMR (101 MHz, CDCl3)

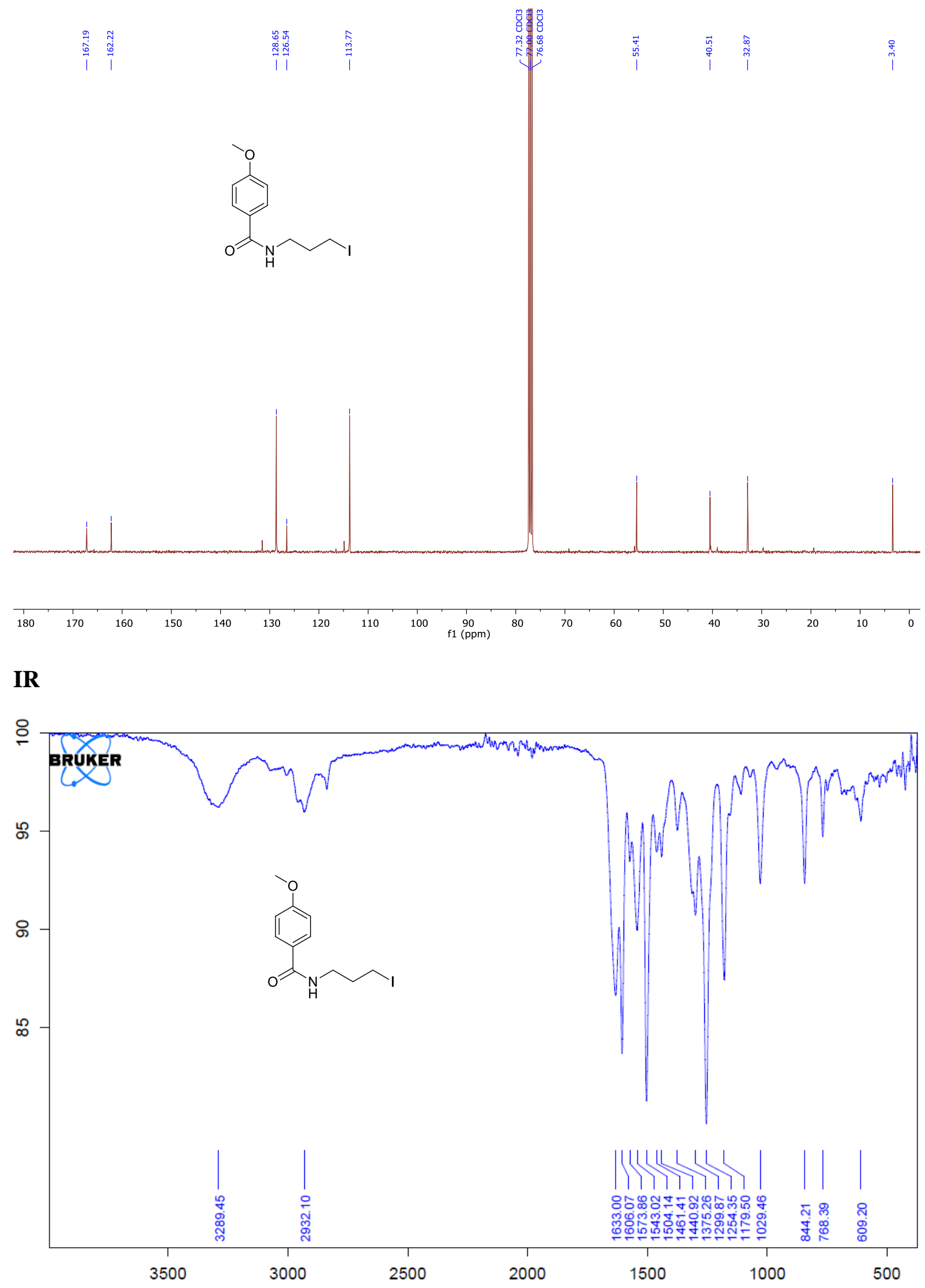


3-((tert-Butoxycarbonyl)amino)-3-methoxypropyl benzoate (16)

${ }^{1} \mathrm{H}-\mathrm{NMR}$ (400 MHz, $\mathrm{CDCl}_{3}$ )

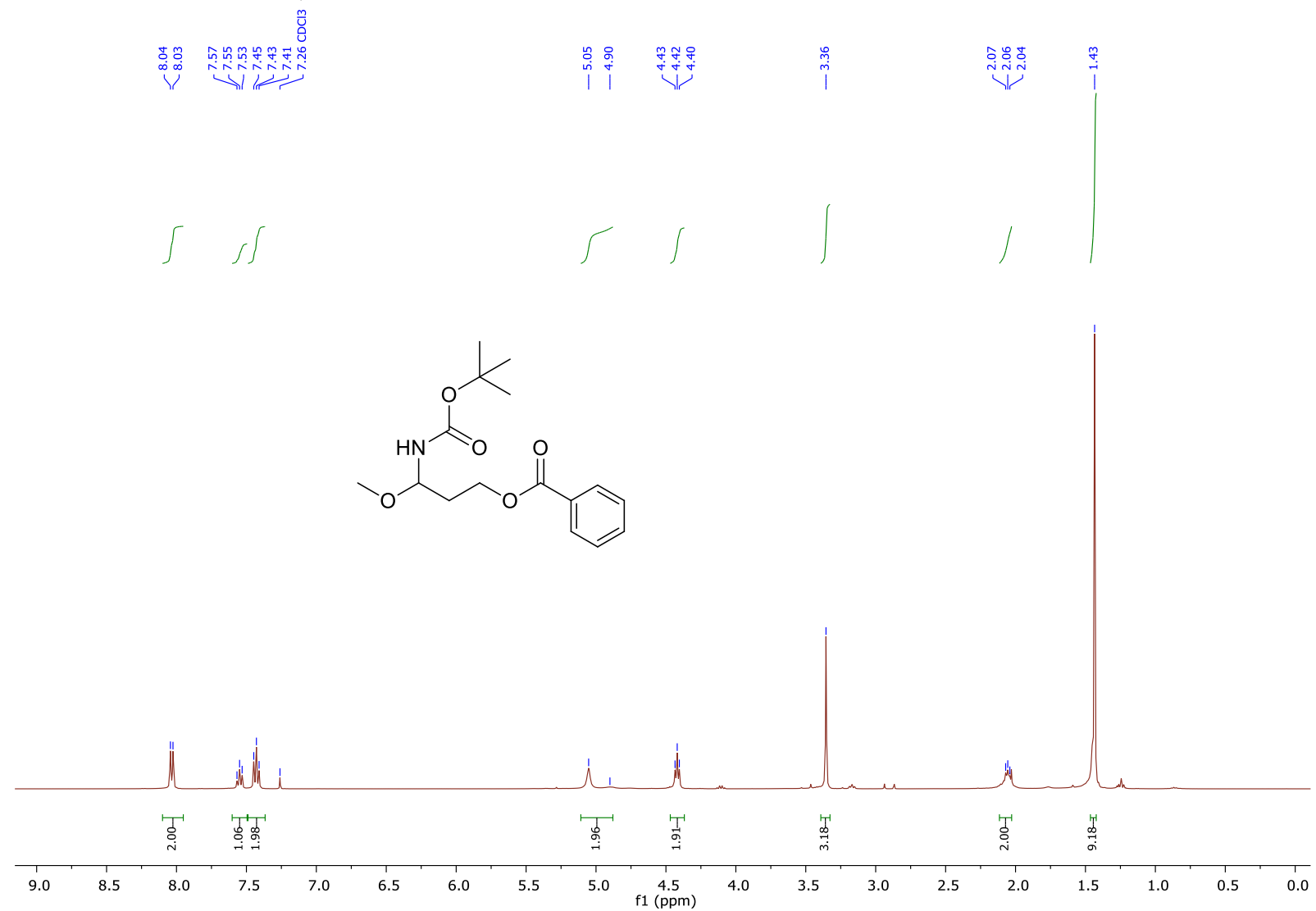

${ }^{13} \mathrm{C}-\mathrm{NMR}$ (101 MHz, CDCl3)
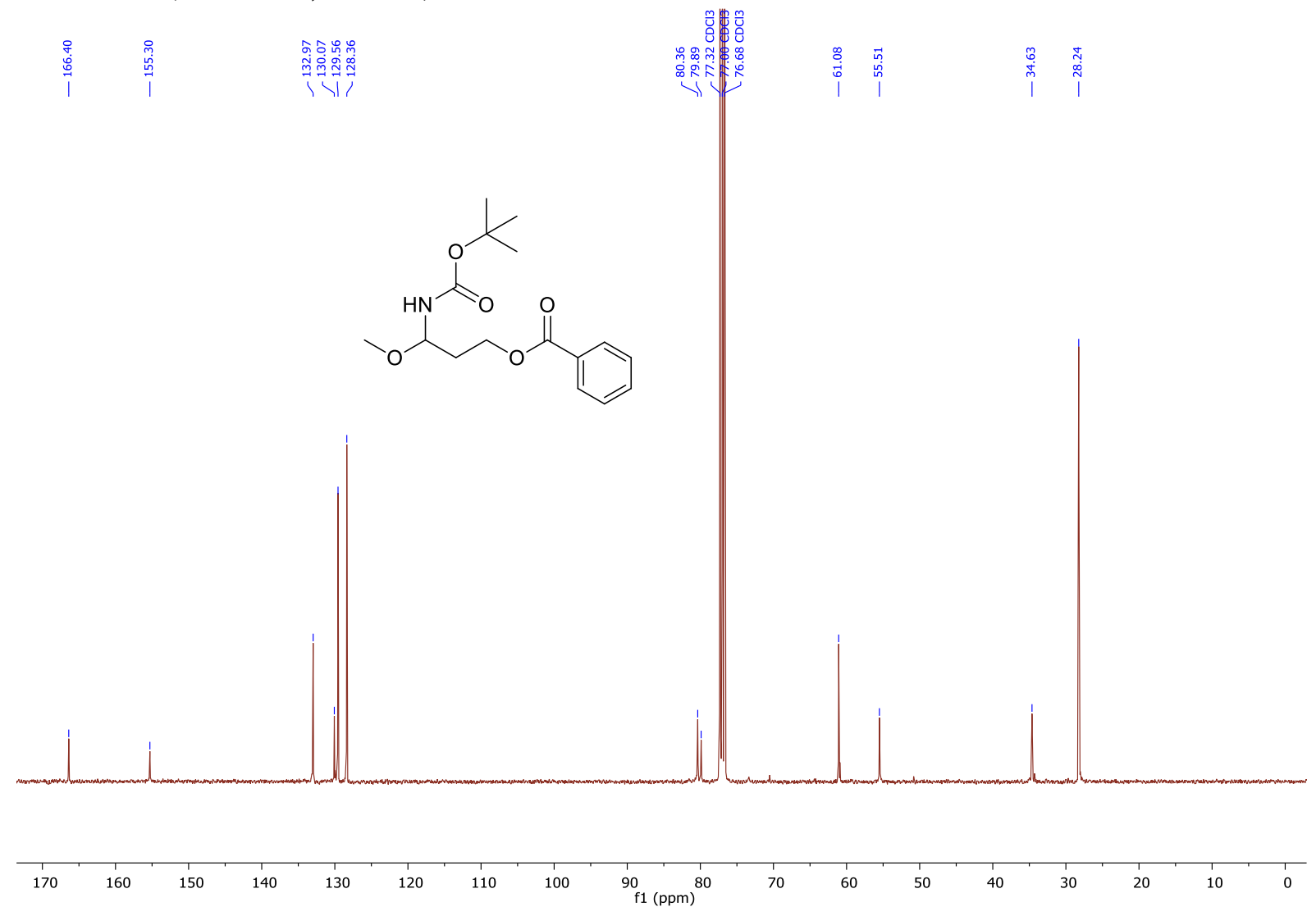
IR

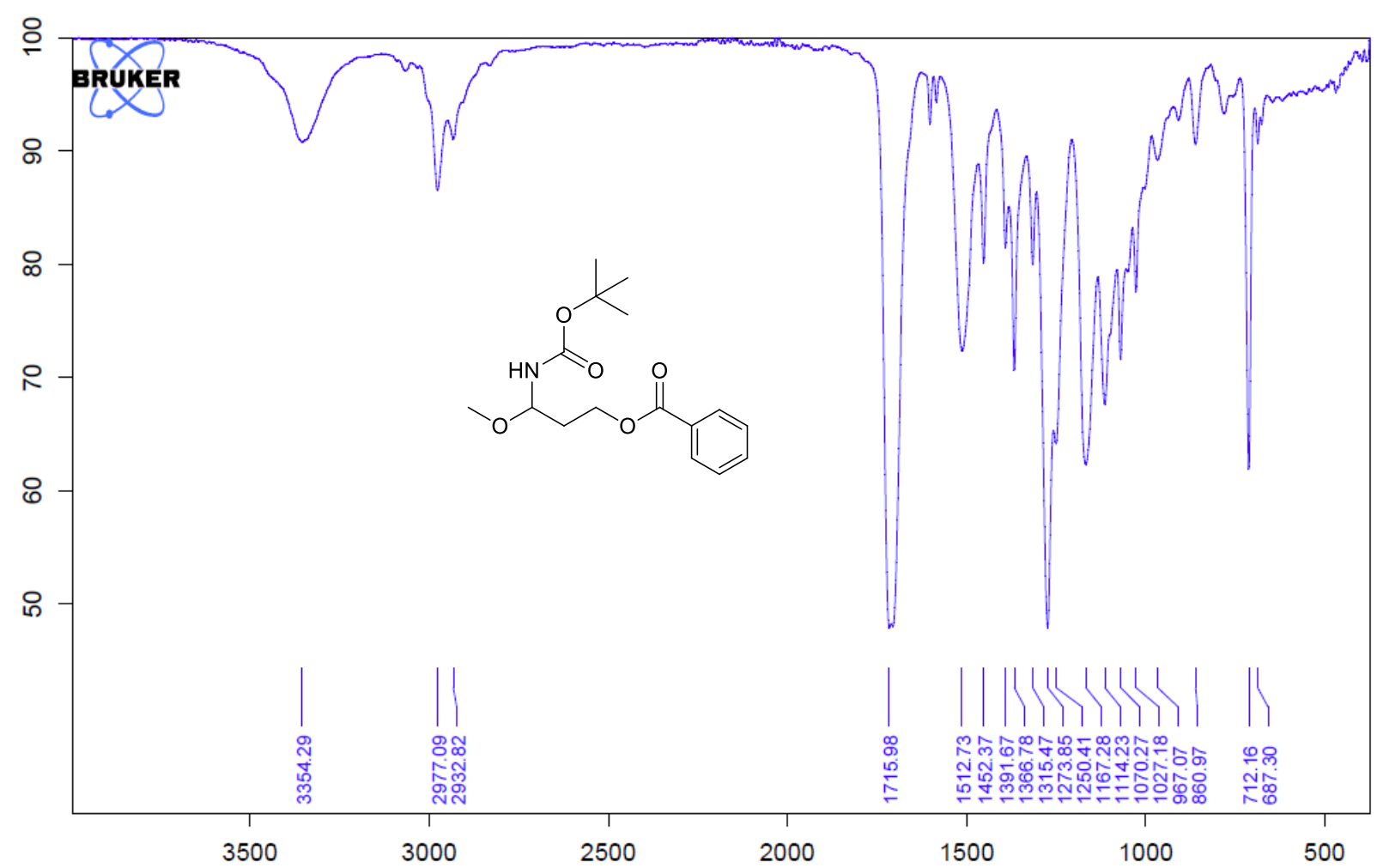

tert-Butyl (3-(benzylthio)-1-methoxypropyl)carbamate (17)

${ }^{1} \mathrm{H}-\mathrm{NMR}$ (400 MHz, CD $3 \mathrm{CN}$ )

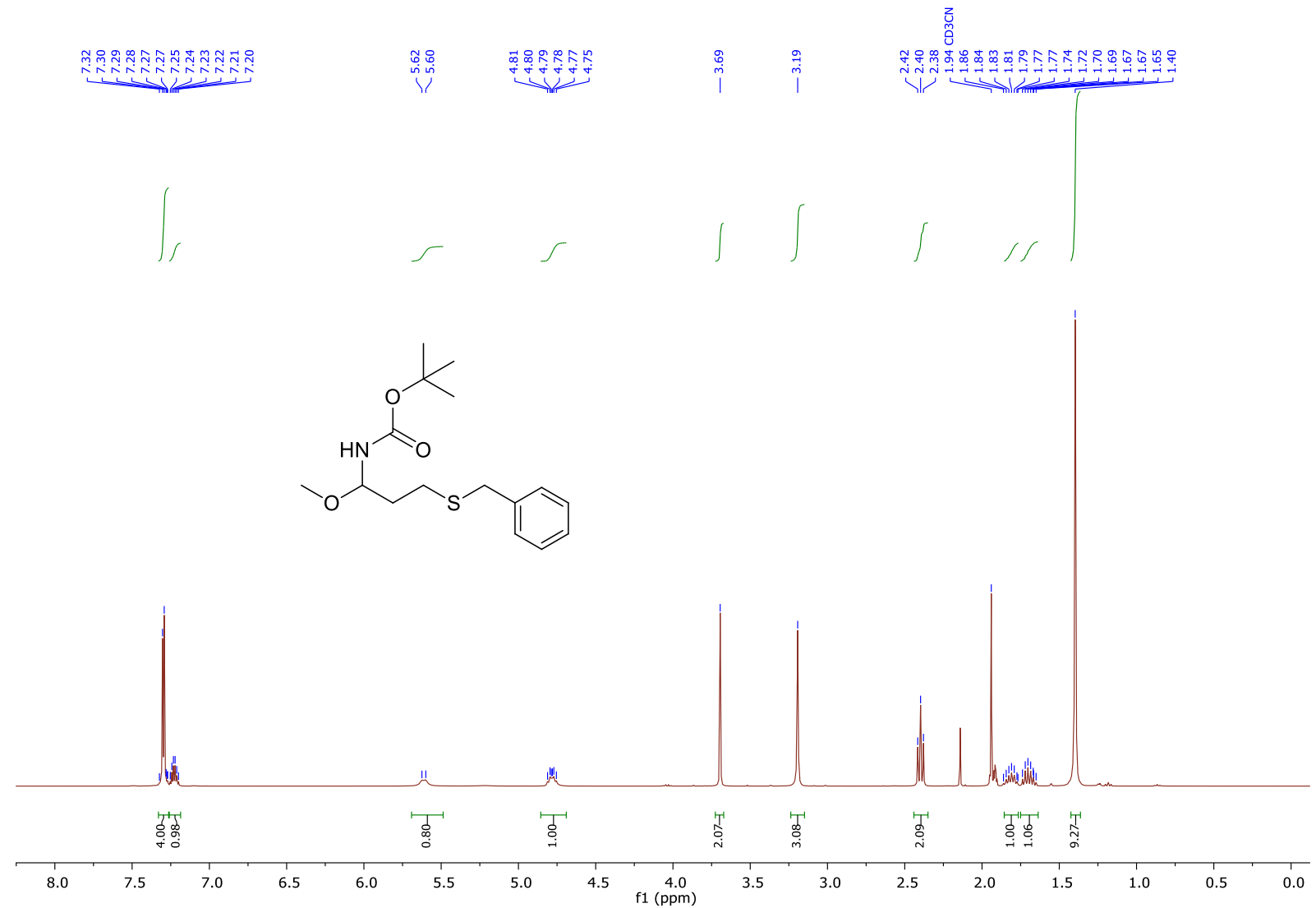


${ }^{13} \mathrm{C}$-NMR (101 MHz, CD3CN)
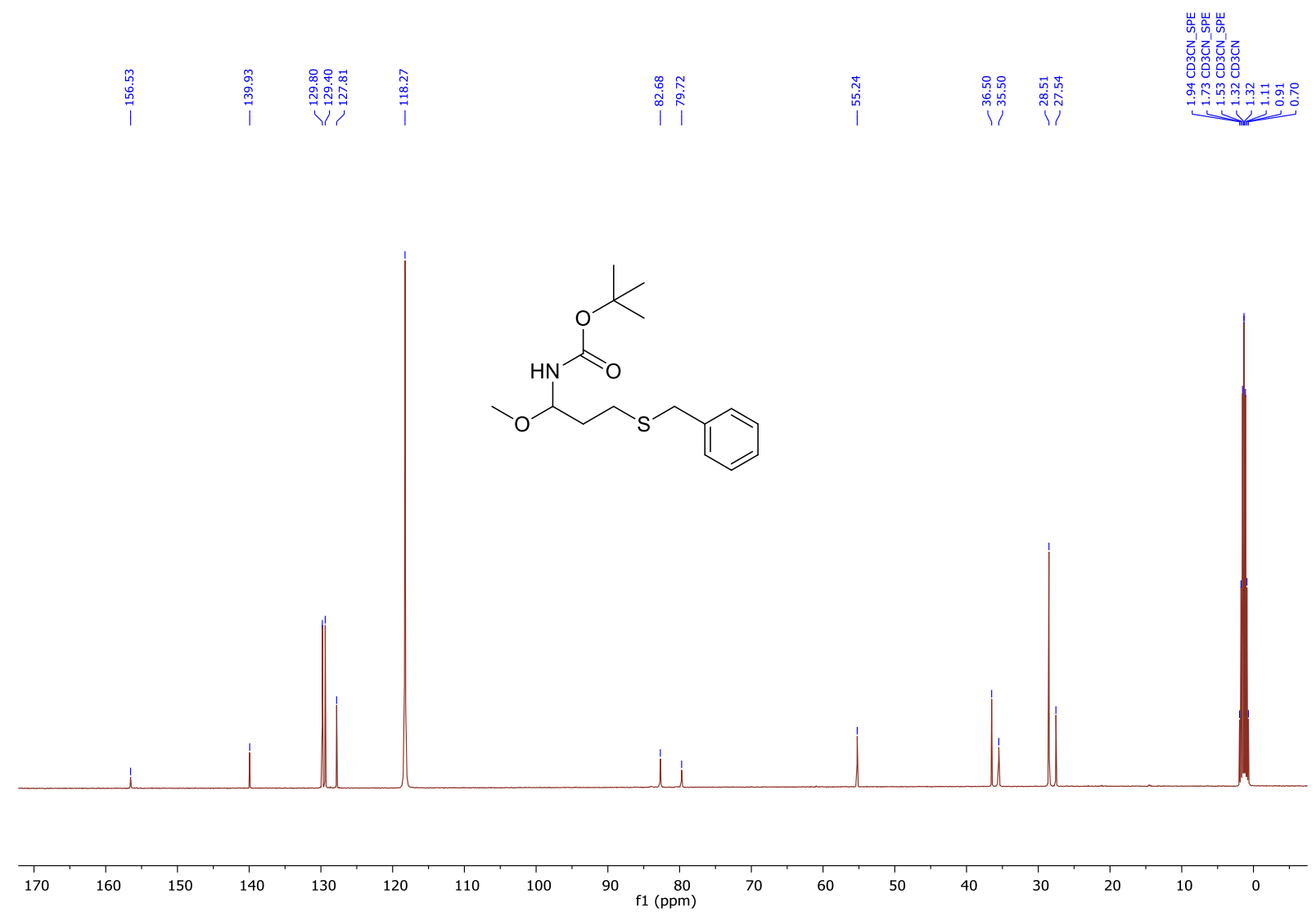

IR

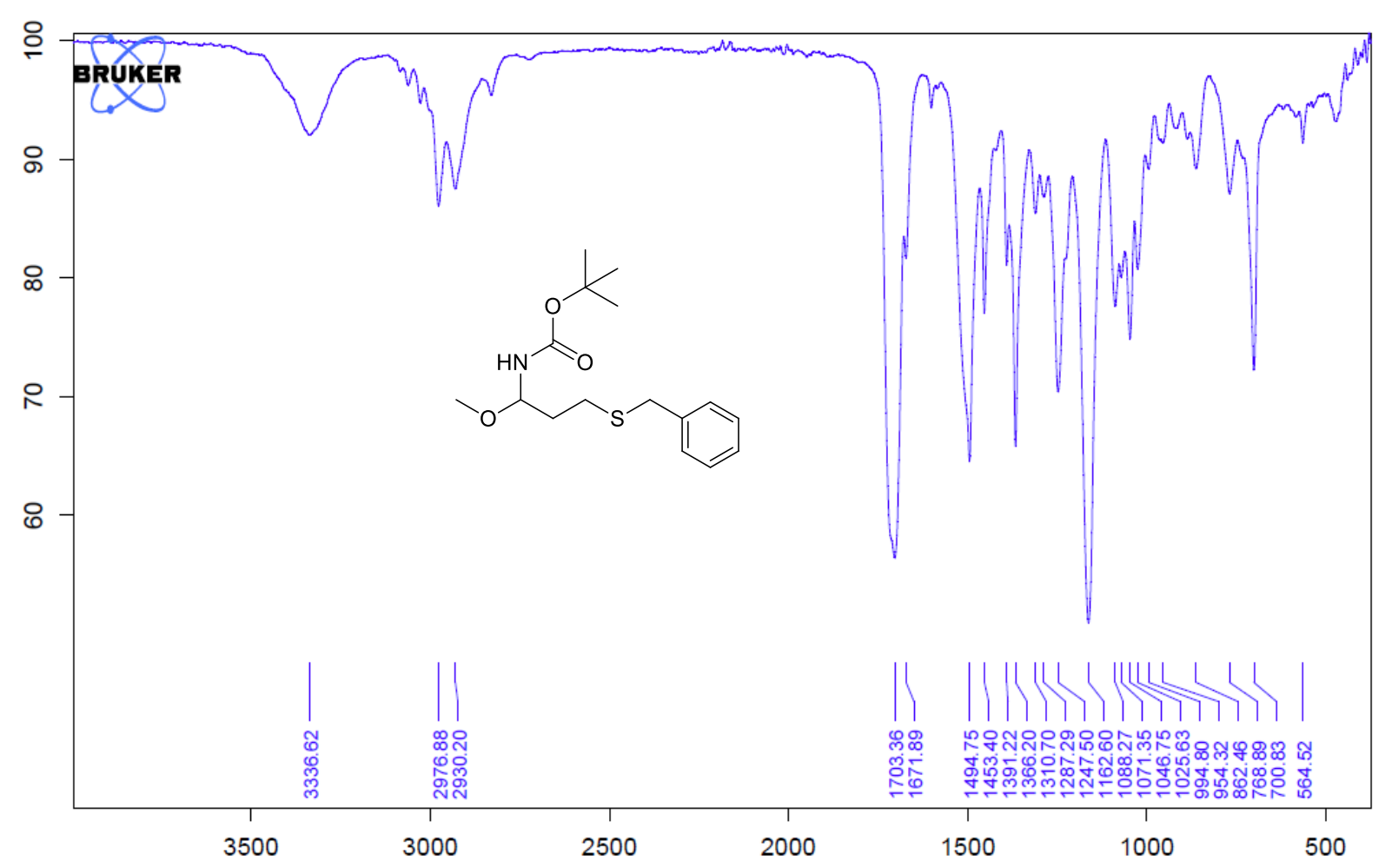


tert-Butyl (1-methoxy-3-(phenylamino)propyl)carbamate (18)

${ }^{1} \mathrm{H}-\mathrm{NMR}$ (400 MHz, $\mathrm{CDCl}_{3}$ )

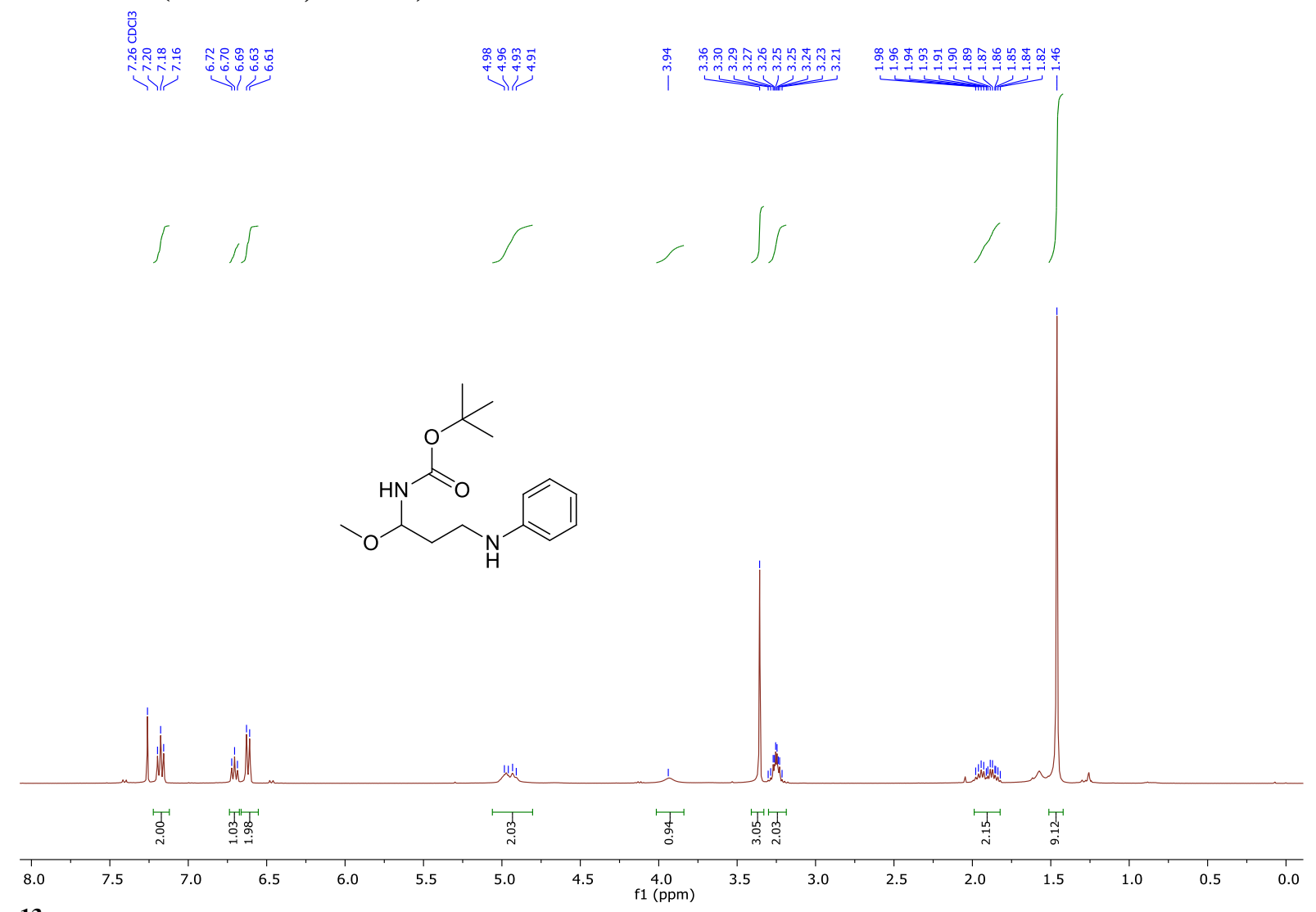

${ }^{13}$ C-NMR (101 MHz, CDCl3)
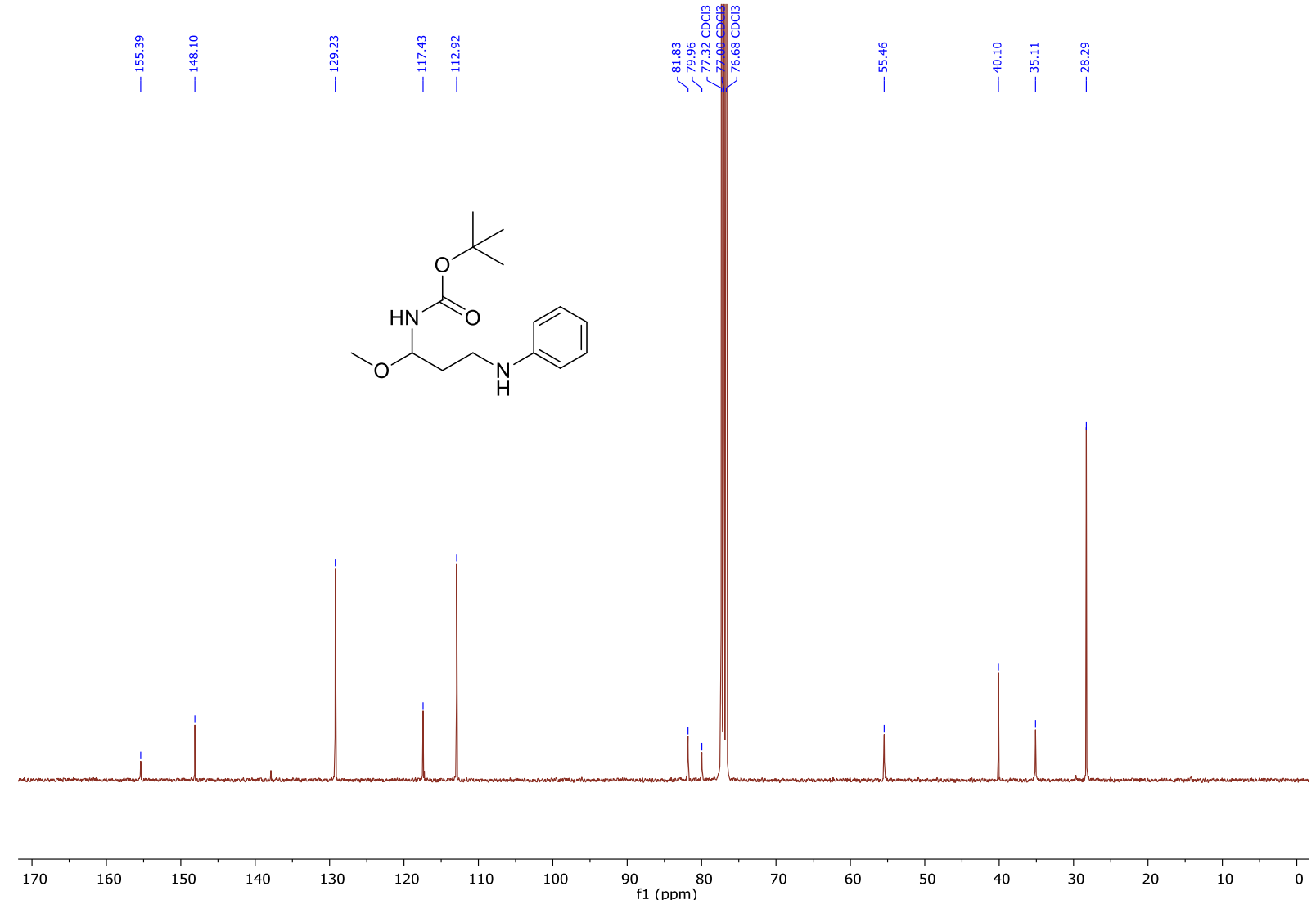
IR

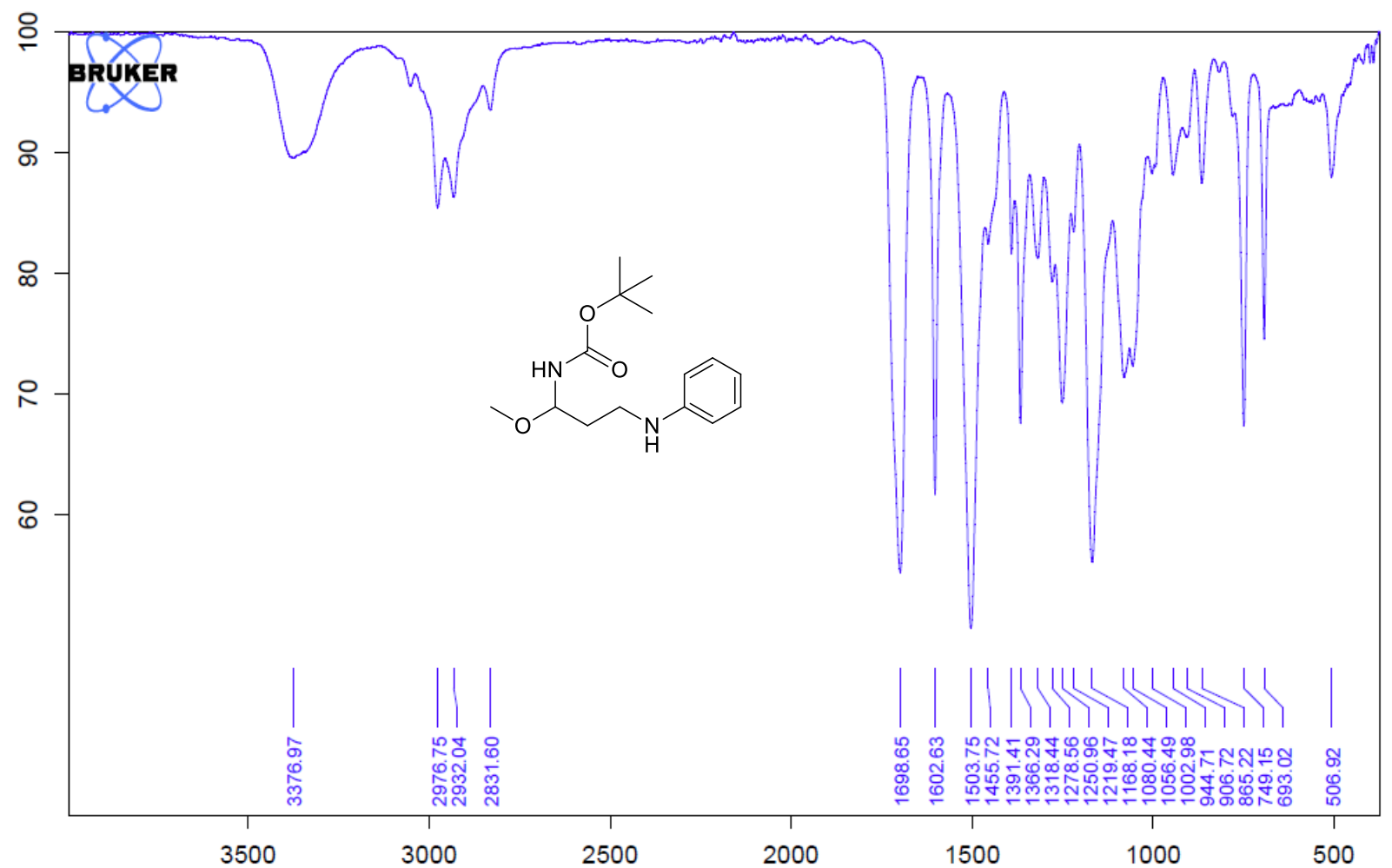

Dimethyl 2-(3-((tert-butoxycarbonyl)amino)-3-methoxypropyl)malonate (19) ${ }^{1}$ H-NMR (400 MHz, Acetone- $d_{6}$ )

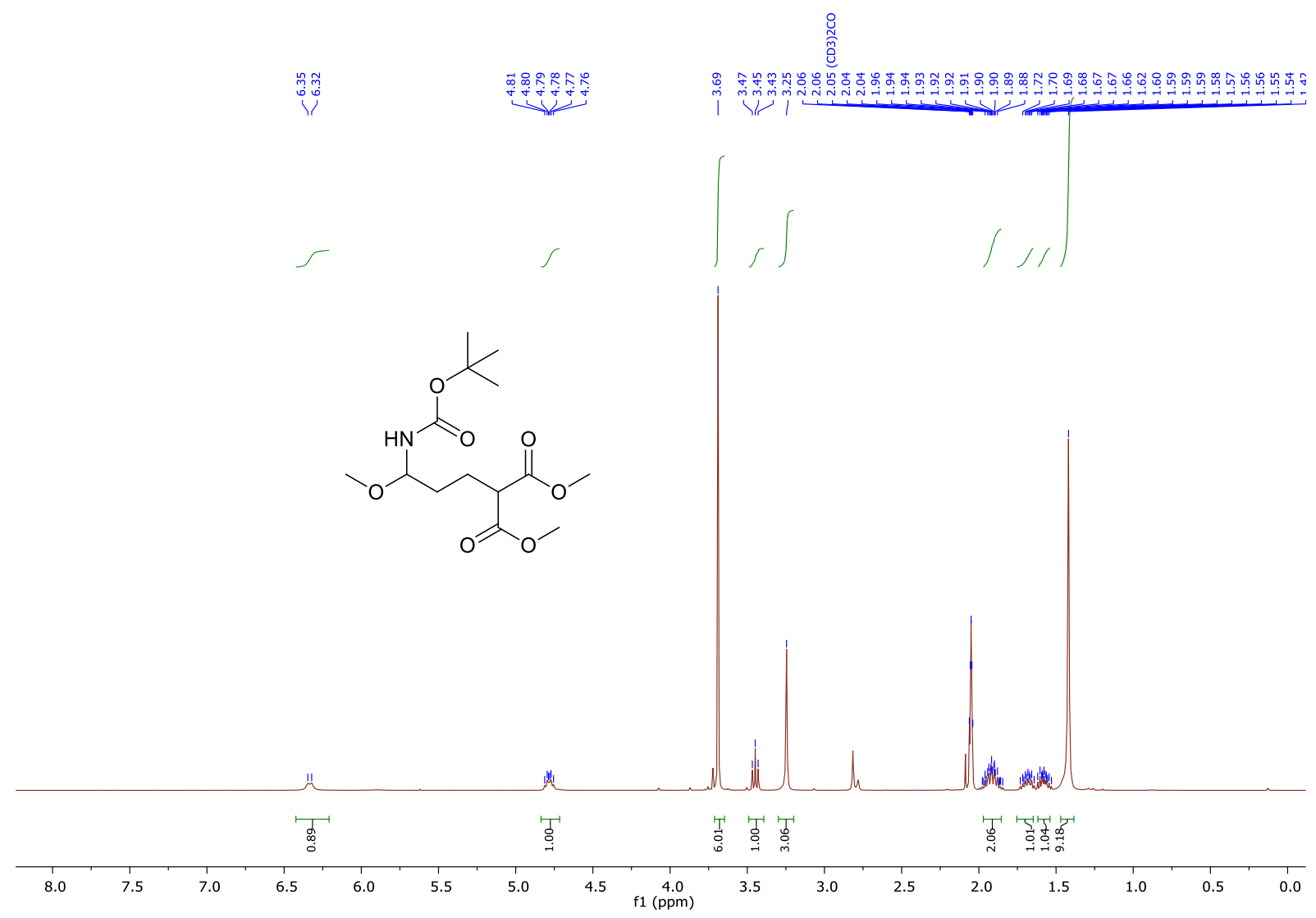


${ }^{13}$ C-NMR (101 MHz, Acetone-d6)
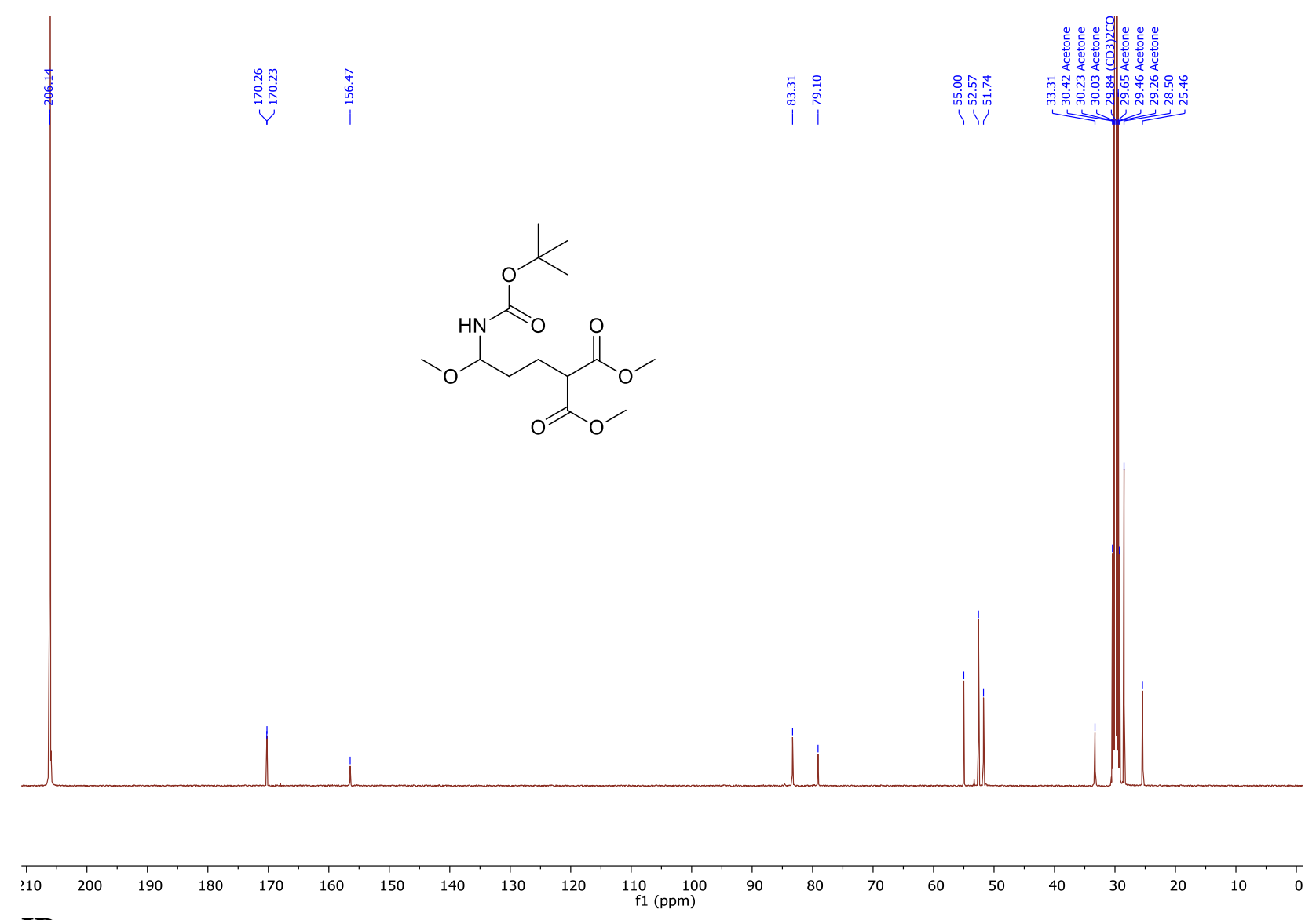

IR

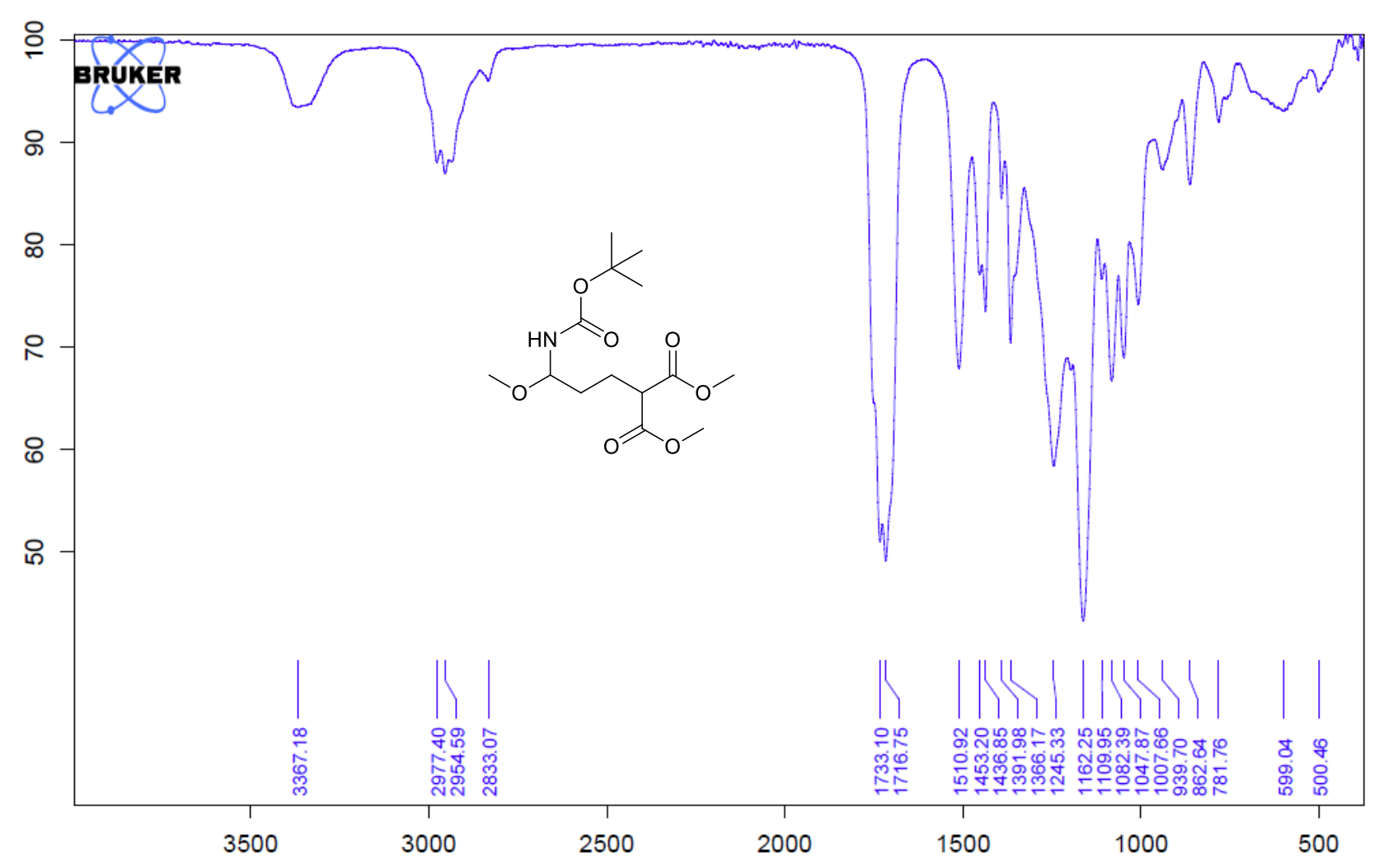


tert-Butyl (3-cyano-1-methoxypropyl)carbamate (20)

${ }^{1} \mathrm{H}-\mathrm{NMR}$ (400 MHz, $\mathrm{CDCl}_{3}$ )

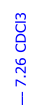

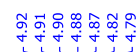

I

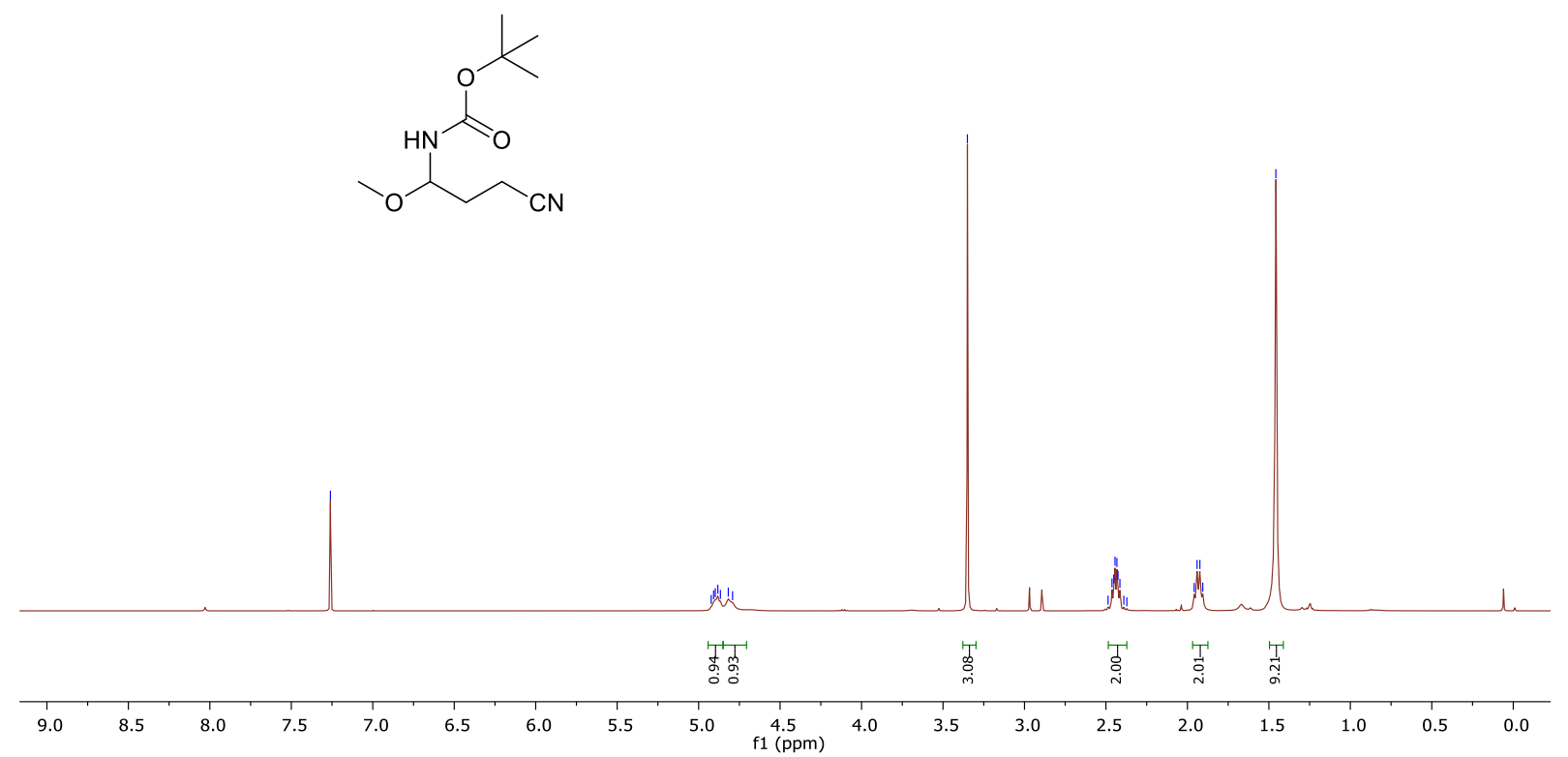

Jr<smiles>C1CCC1</smiles>

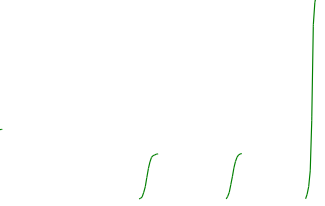

${ }^{13} \mathrm{C}-\mathrm{NMR}$ (101 MHz, $\left.\mathrm{CDCl}_{3}\right)$

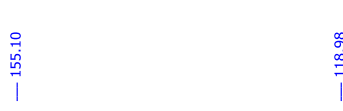

$\stackrel{\substack{\infty \\ \infty}}{\stackrel{\infty}{I}}$

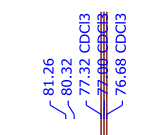

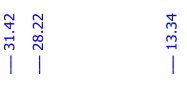
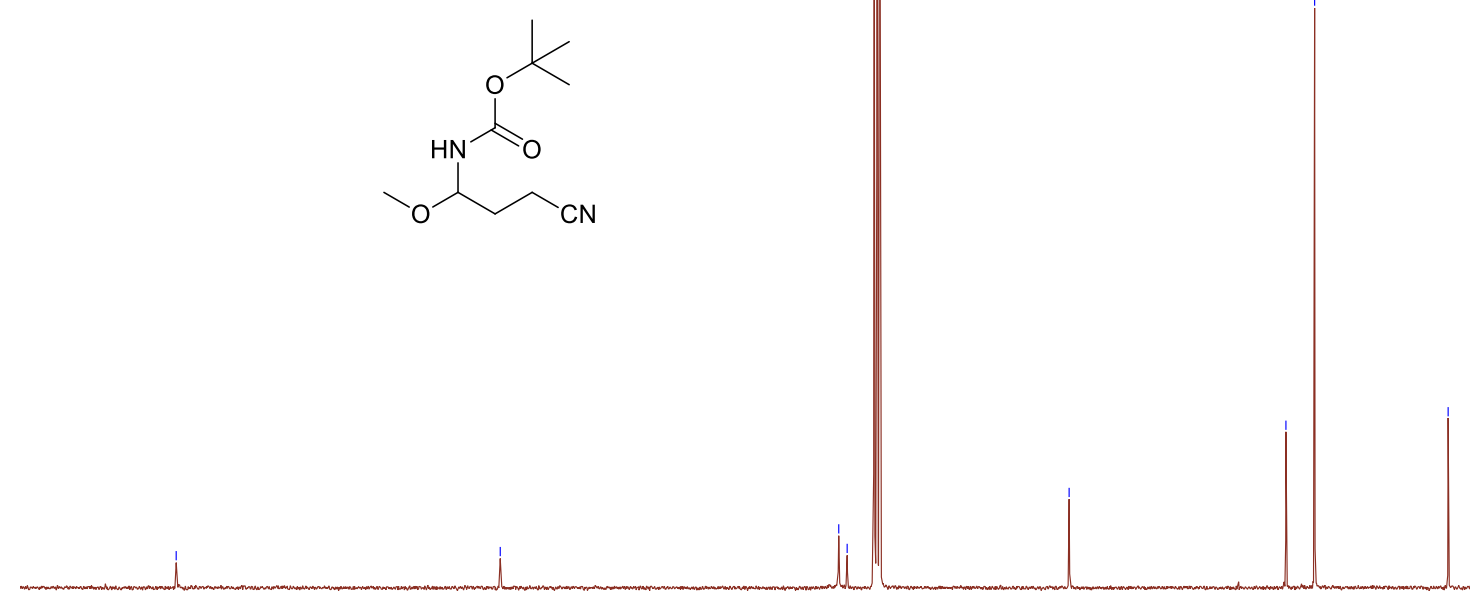

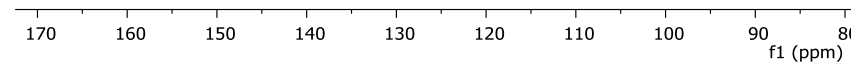


IR

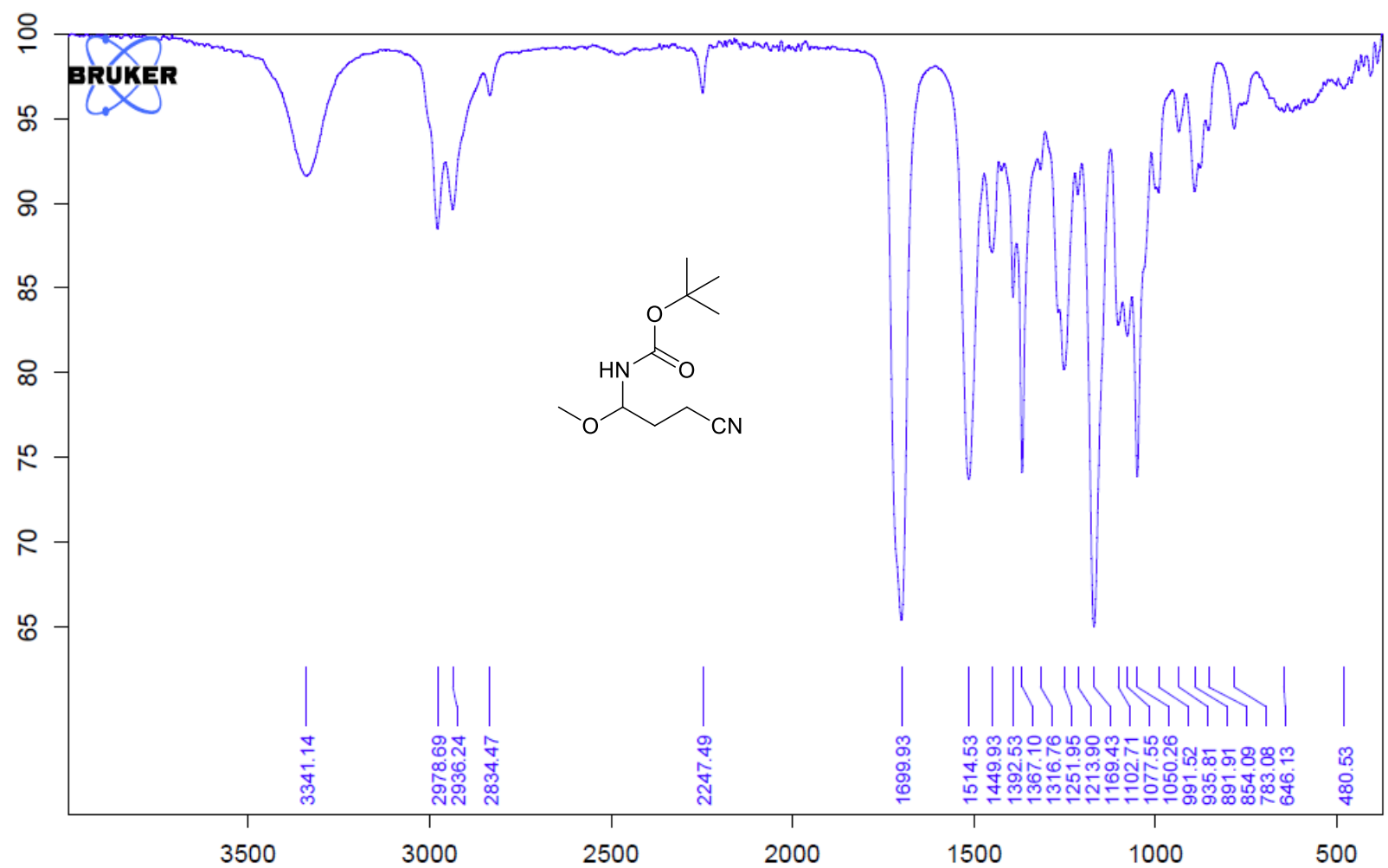

tert-Butyl 2-methoxyazetidine-1-carboxylate (20)

${ }^{1} \mathrm{H}-\mathrm{NMR}$ (400 MHz, $\mathrm{CDCl}_{3}$ )

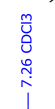

$\underbrace{\circ \rightarrow 0}$

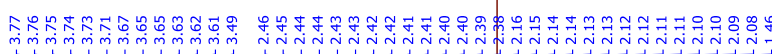<smiles>COC1CCN1C(=O)OC(C)(C)C</smiles>

$1 /$
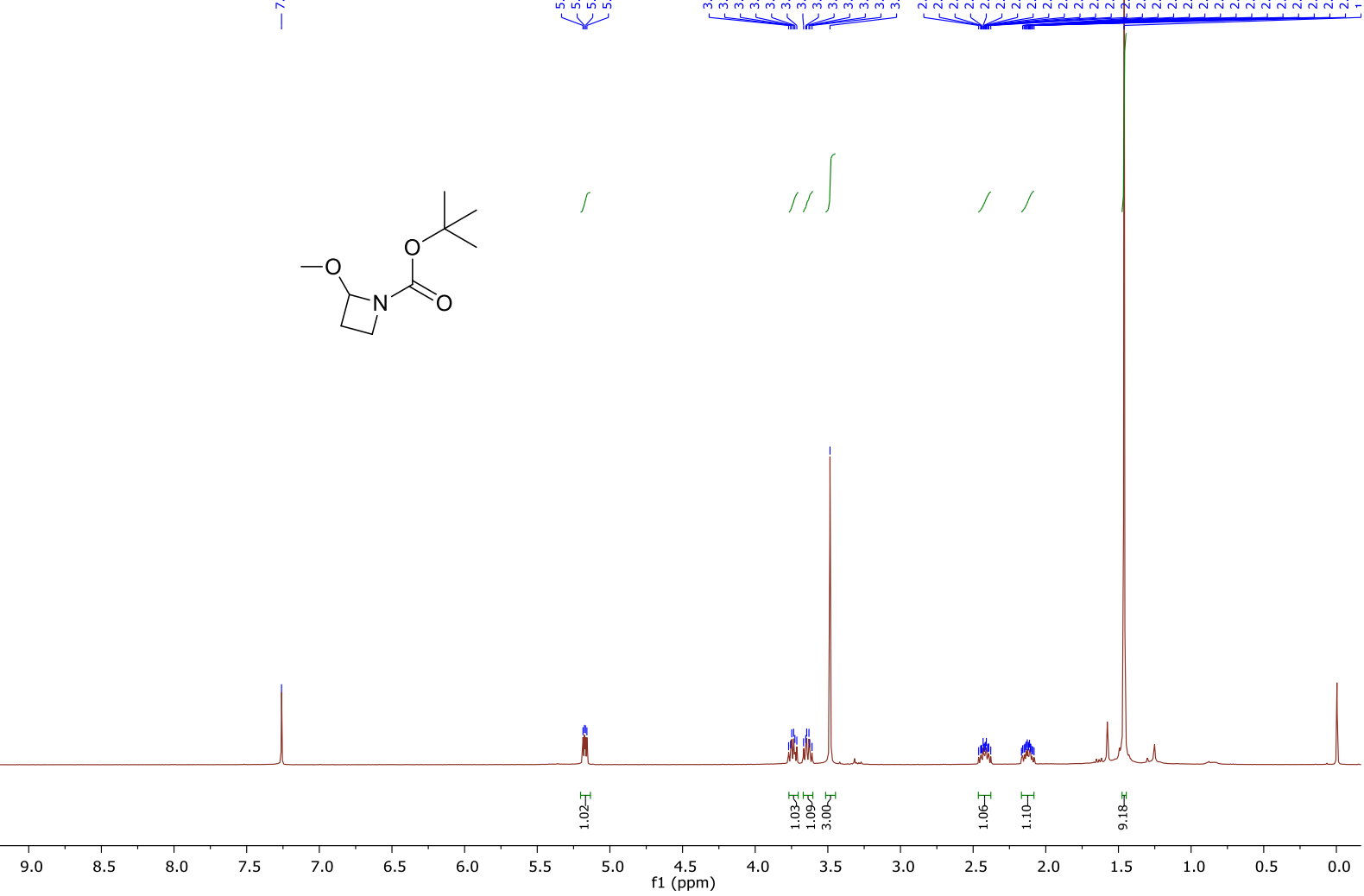
${ }^{13} \mathrm{C}$-NMR (101 MHz, CDCl3)

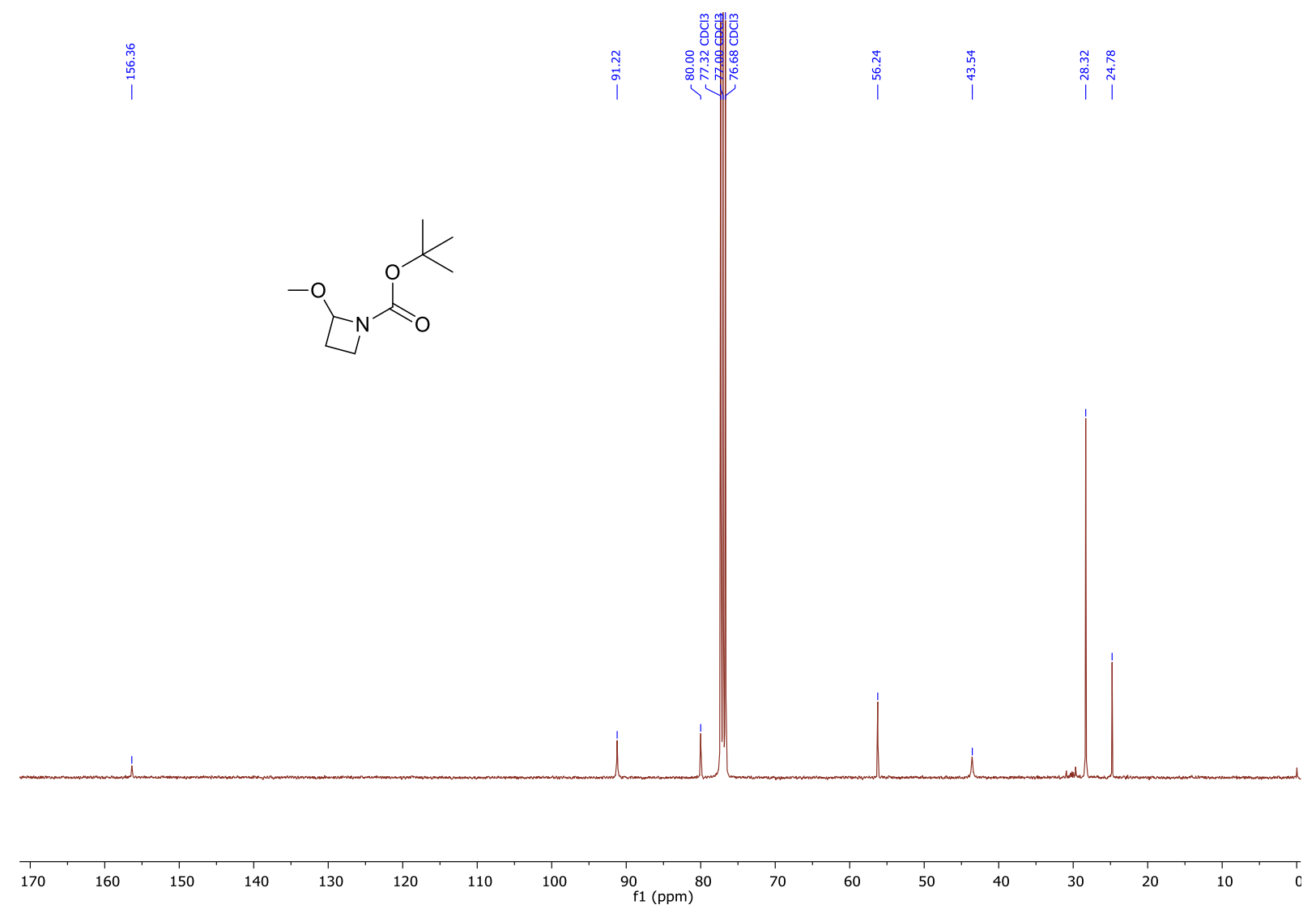

IR

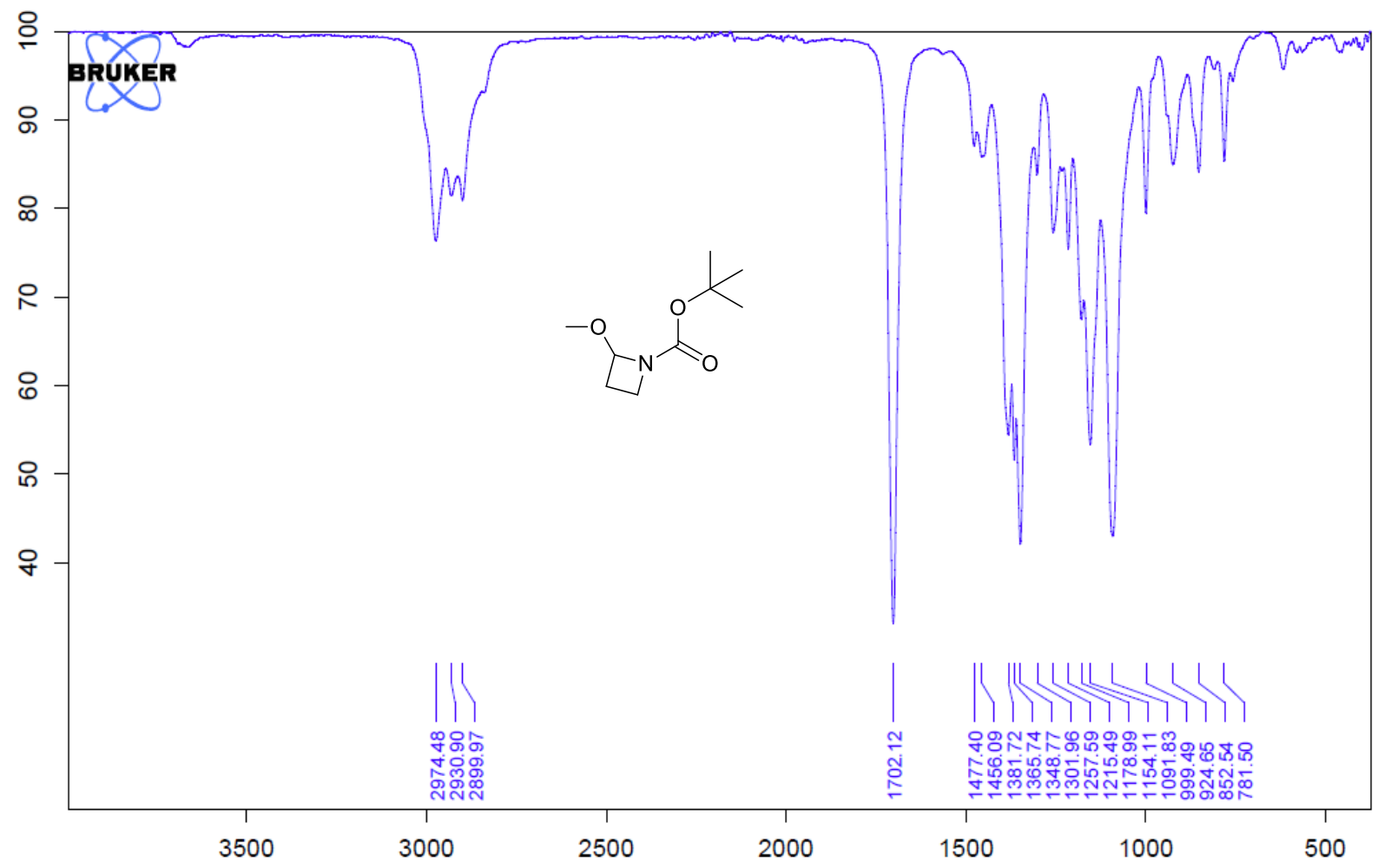


4-Methoxy-1,3-oxazinan-2-one (21')

${ }^{1} \mathrm{H}-\mathrm{NMR}$ (400 MHz, CDCl3)

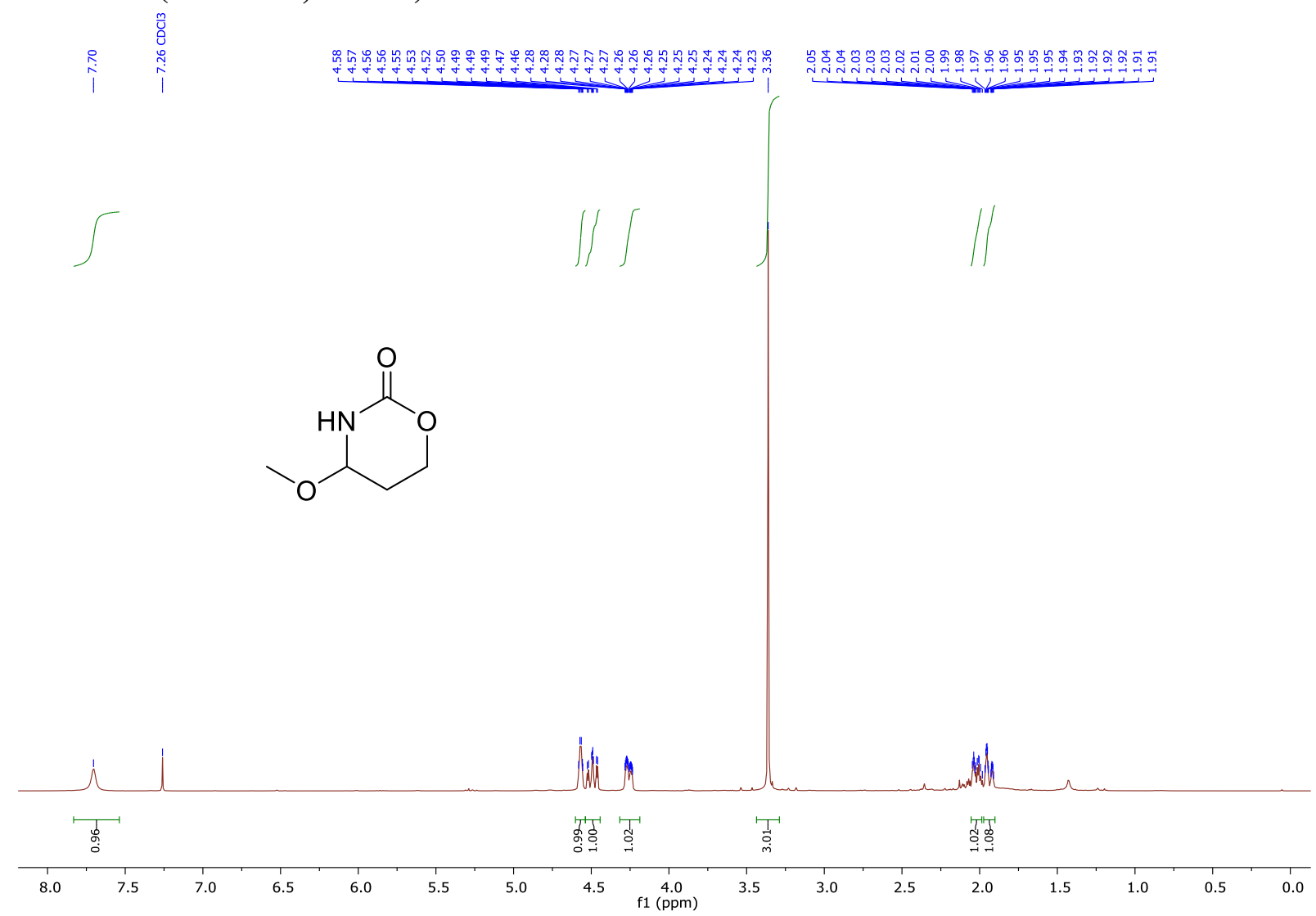

${ }^{13}$ C-NMR (101 MHz, CDCl3)

|
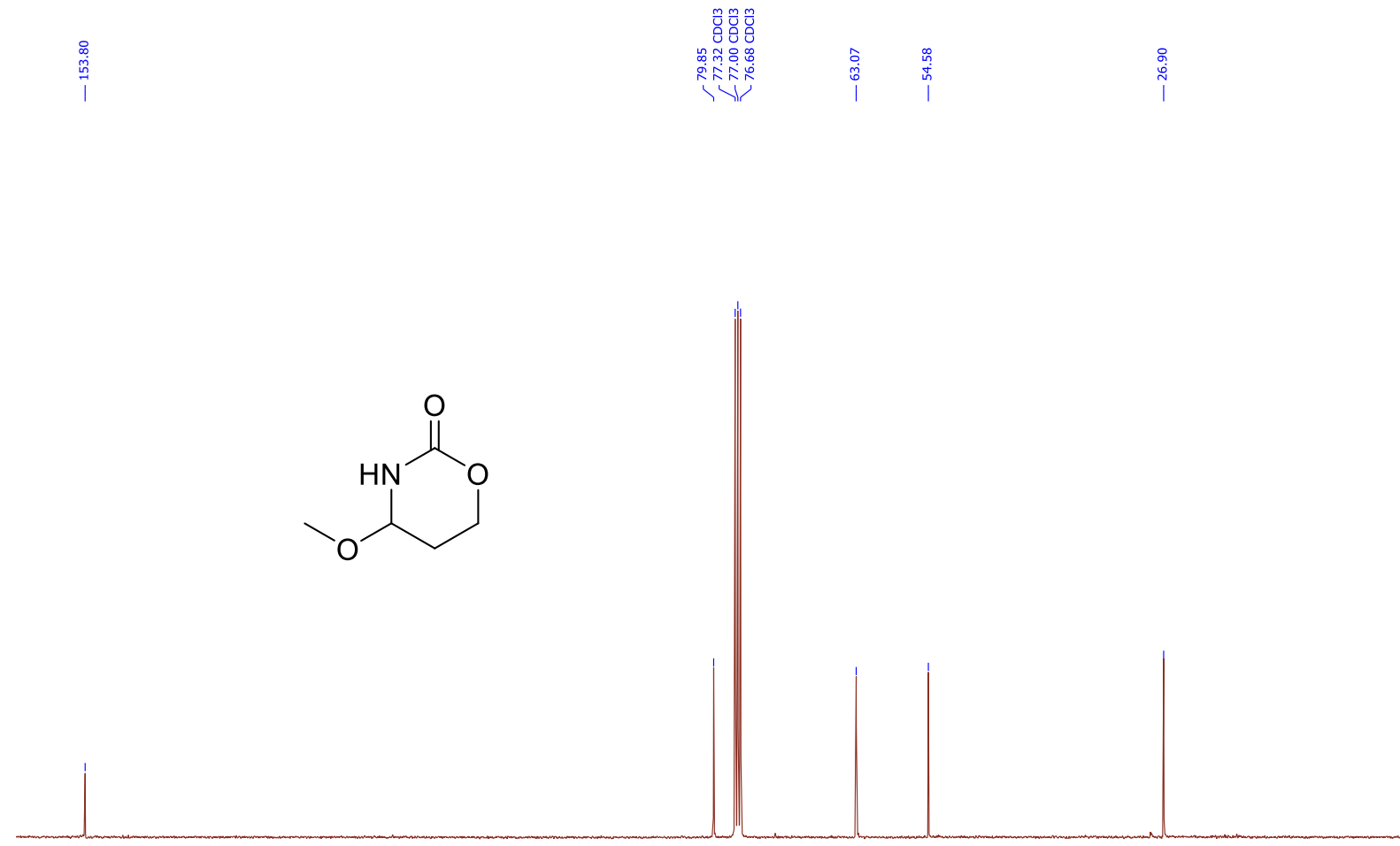
IR

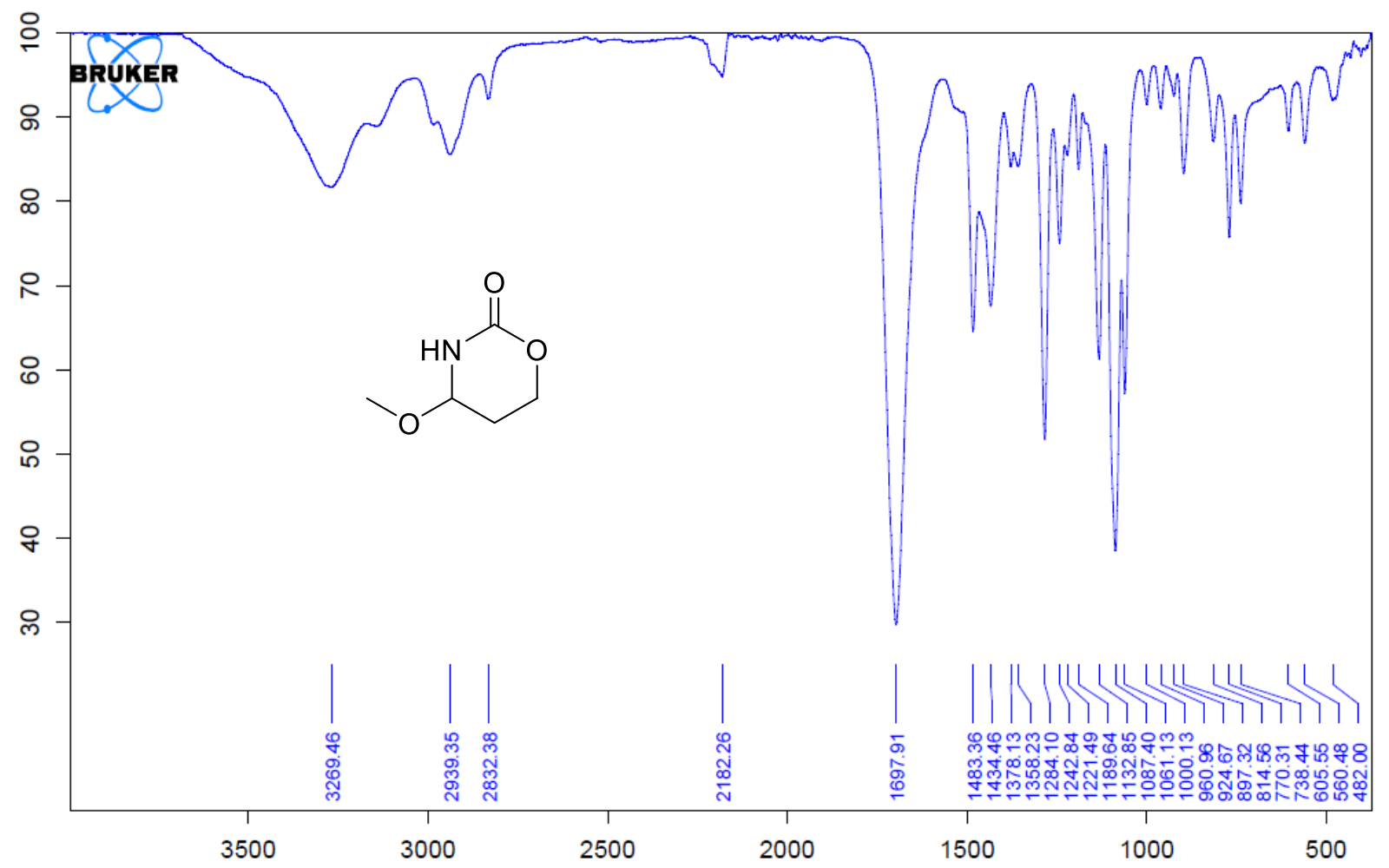

N-(3-((4-Fluorophenyl)thio)-1-(3-methyl-1H-indol-2-yl)propyl)-4-methoxybenzamide (22) ${ }^{1} \mathrm{H}-\mathrm{NMR}$ (400 MHz, $\left.\mathrm{CDCl}_{3}\right)$

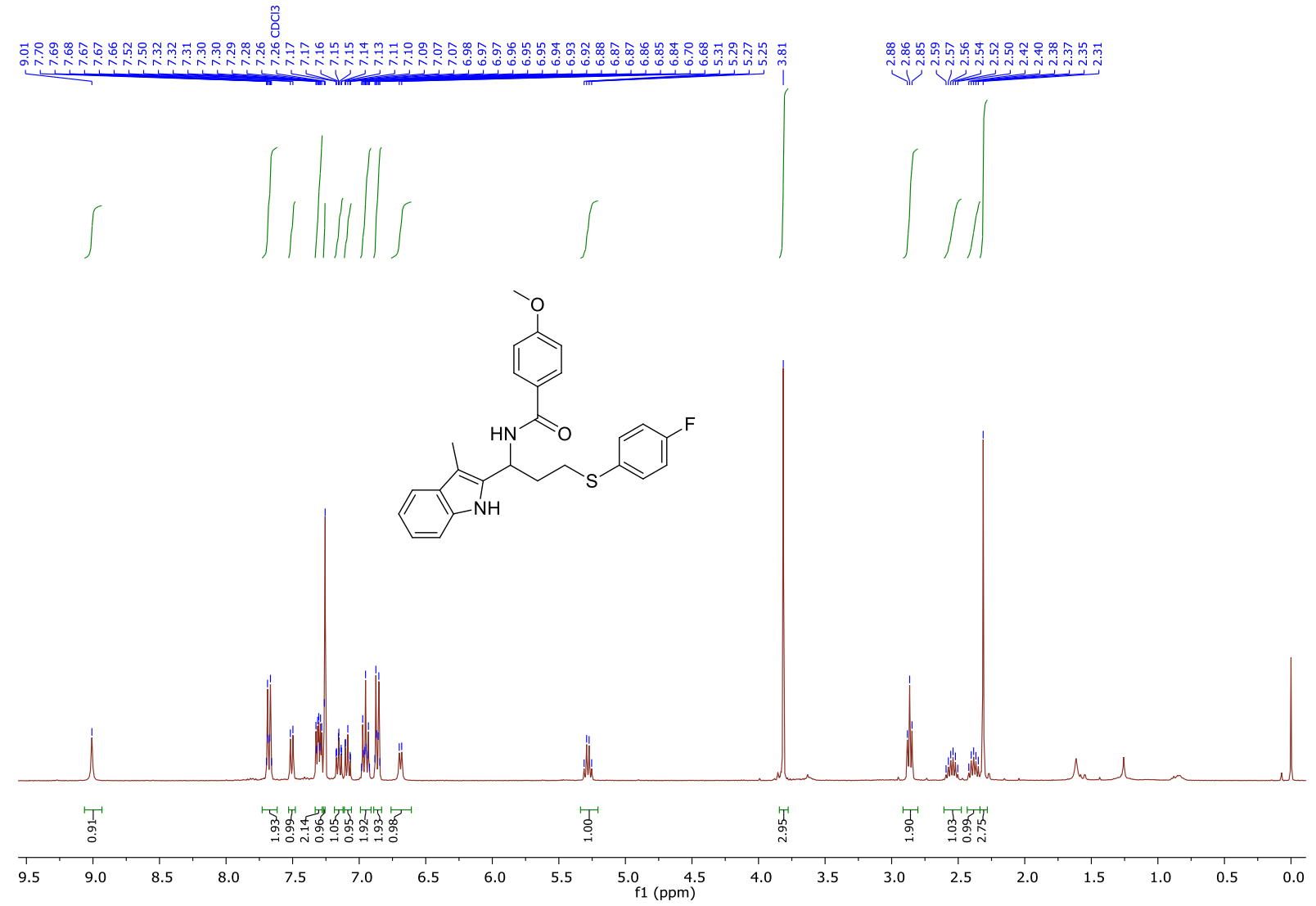


${ }^{13}$ C-NMR (101 MHz, CDCl3)
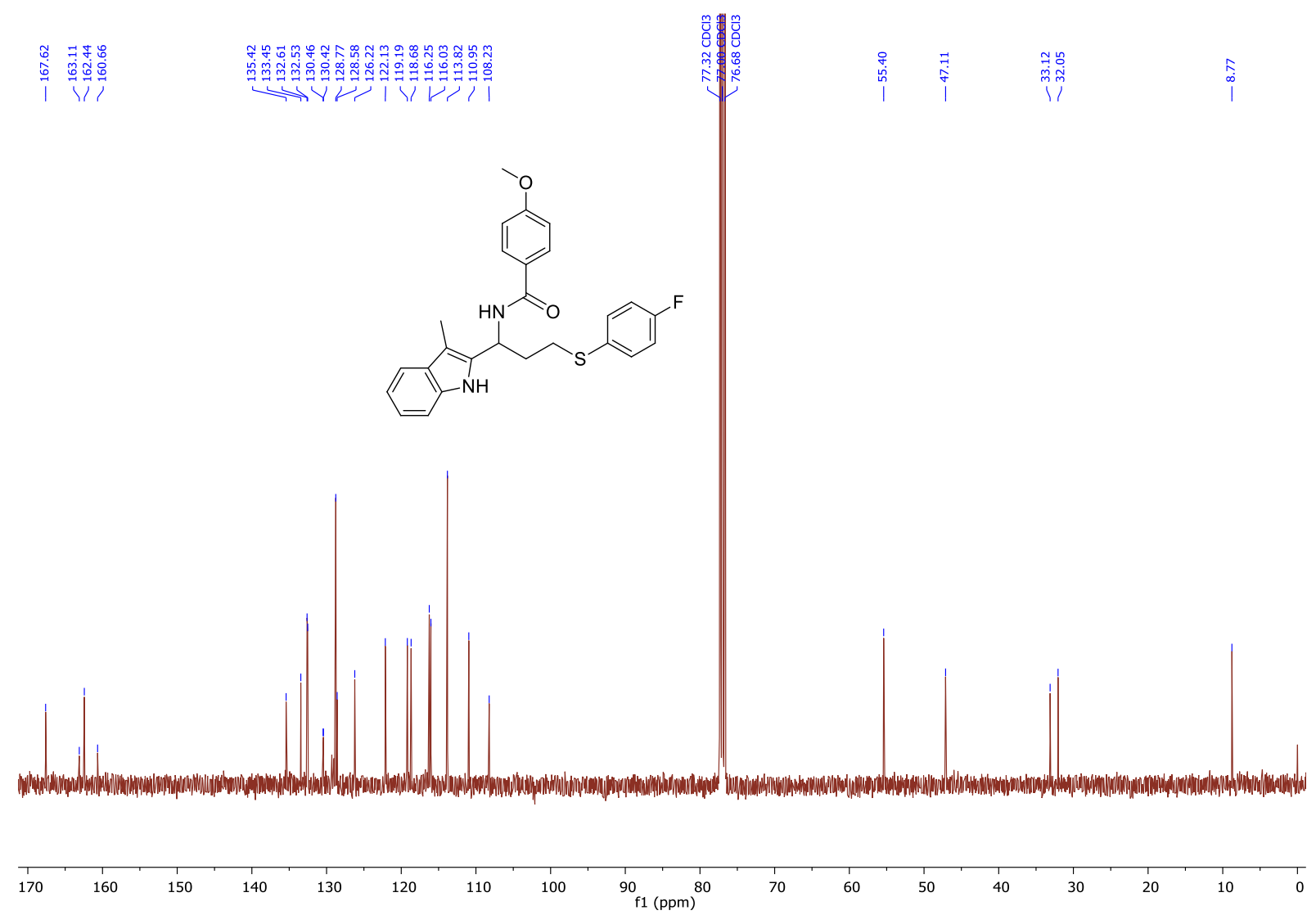

IR

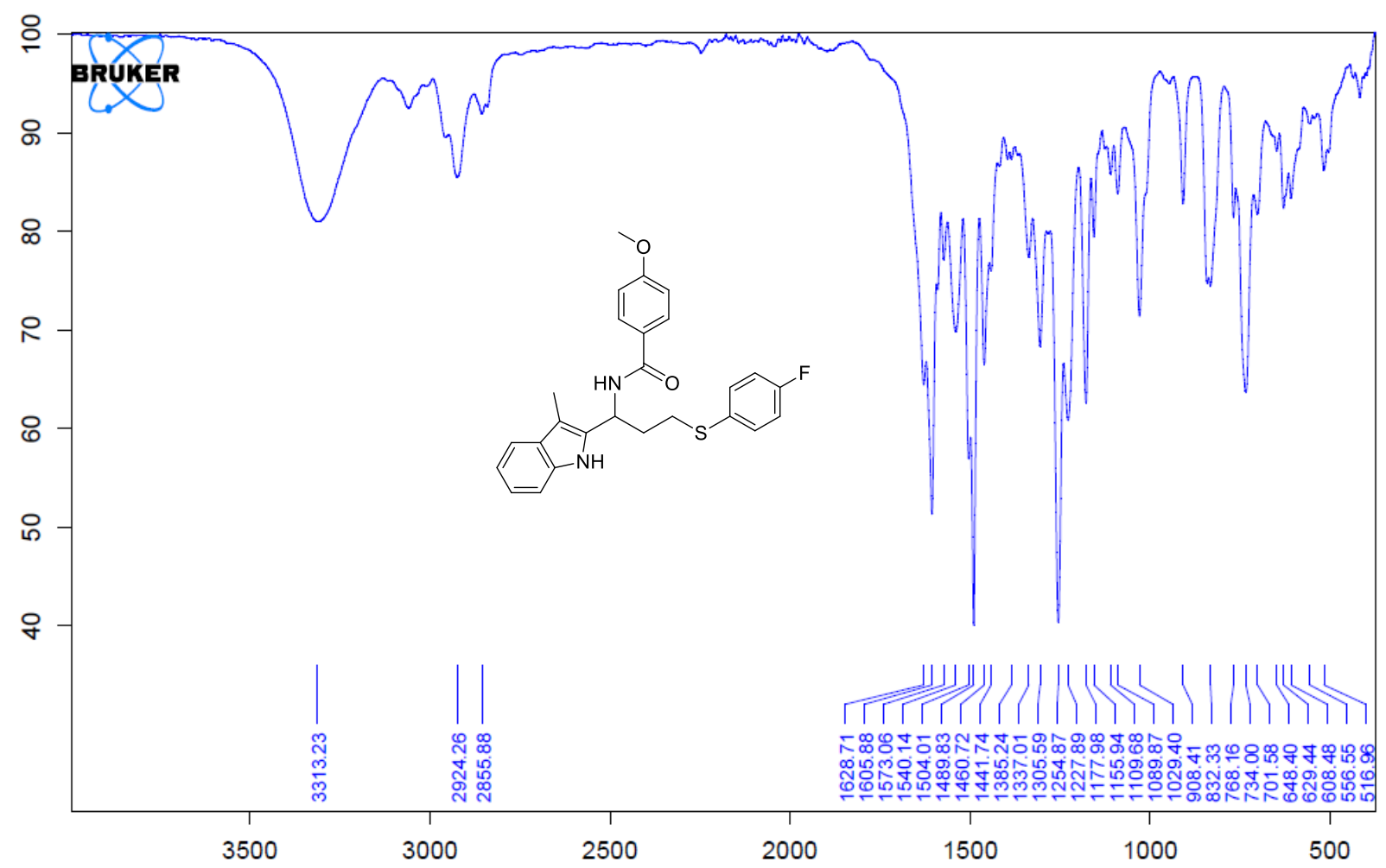


N-(3-(Cyclopropylamino)-1-(3-methyl-1H-indol-2-yl)propyl)-4-methoxybenzamide (23) ${ }^{1}$ H-NMR (400 MHz, Acetone-d6)

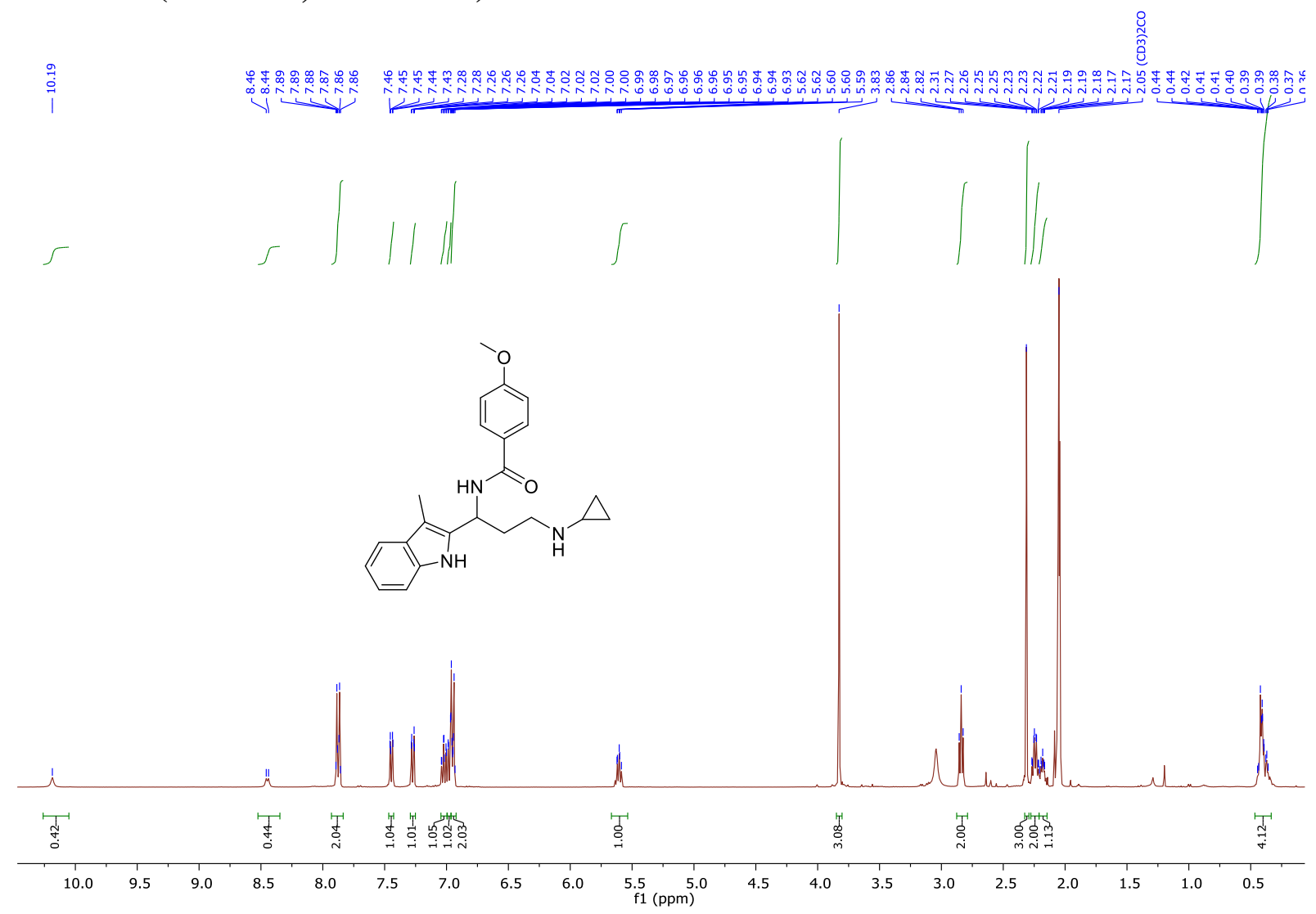

${ }^{13}$ C-NMR (101 MHz, Acetone-d6)
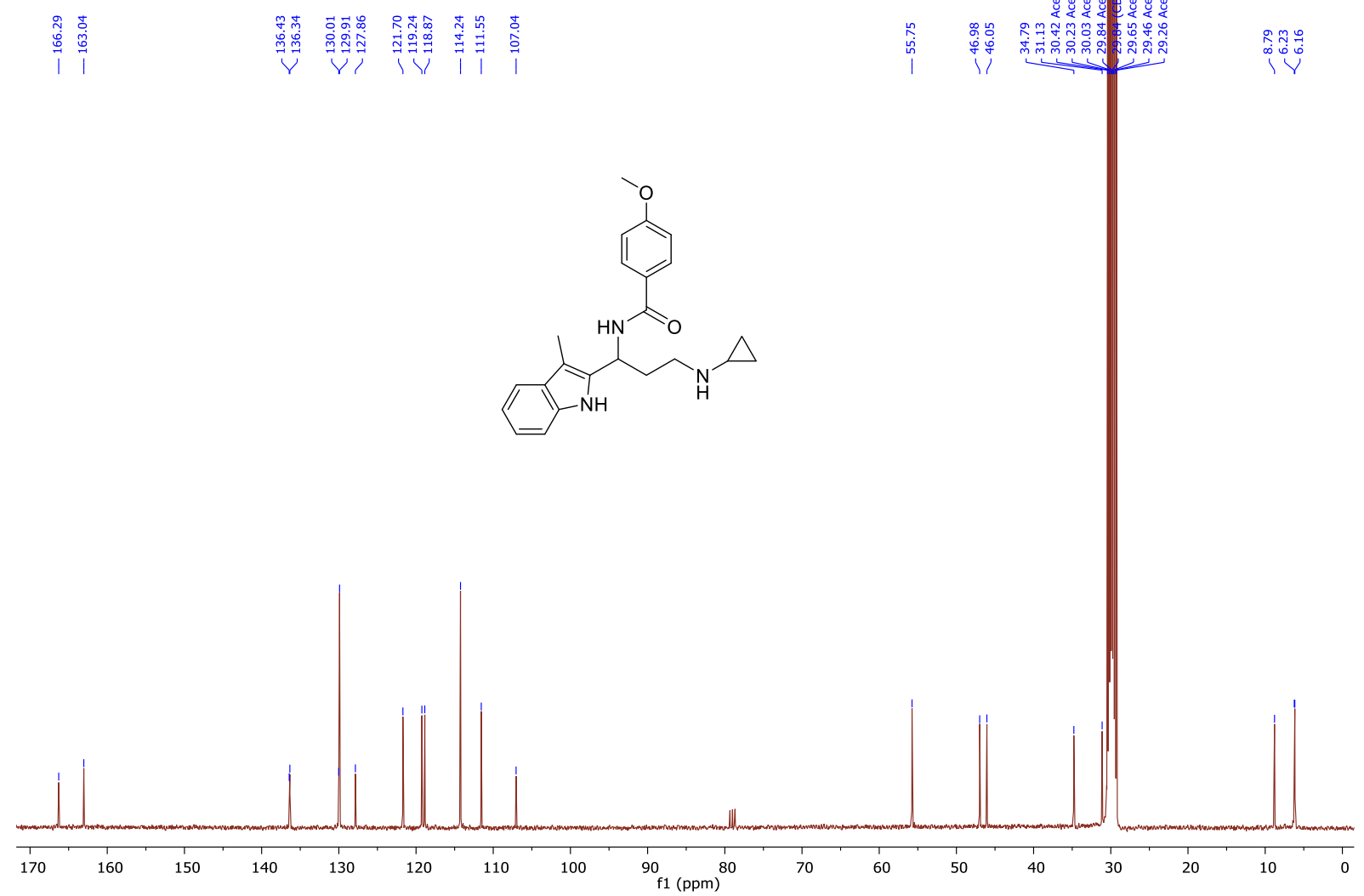
IR

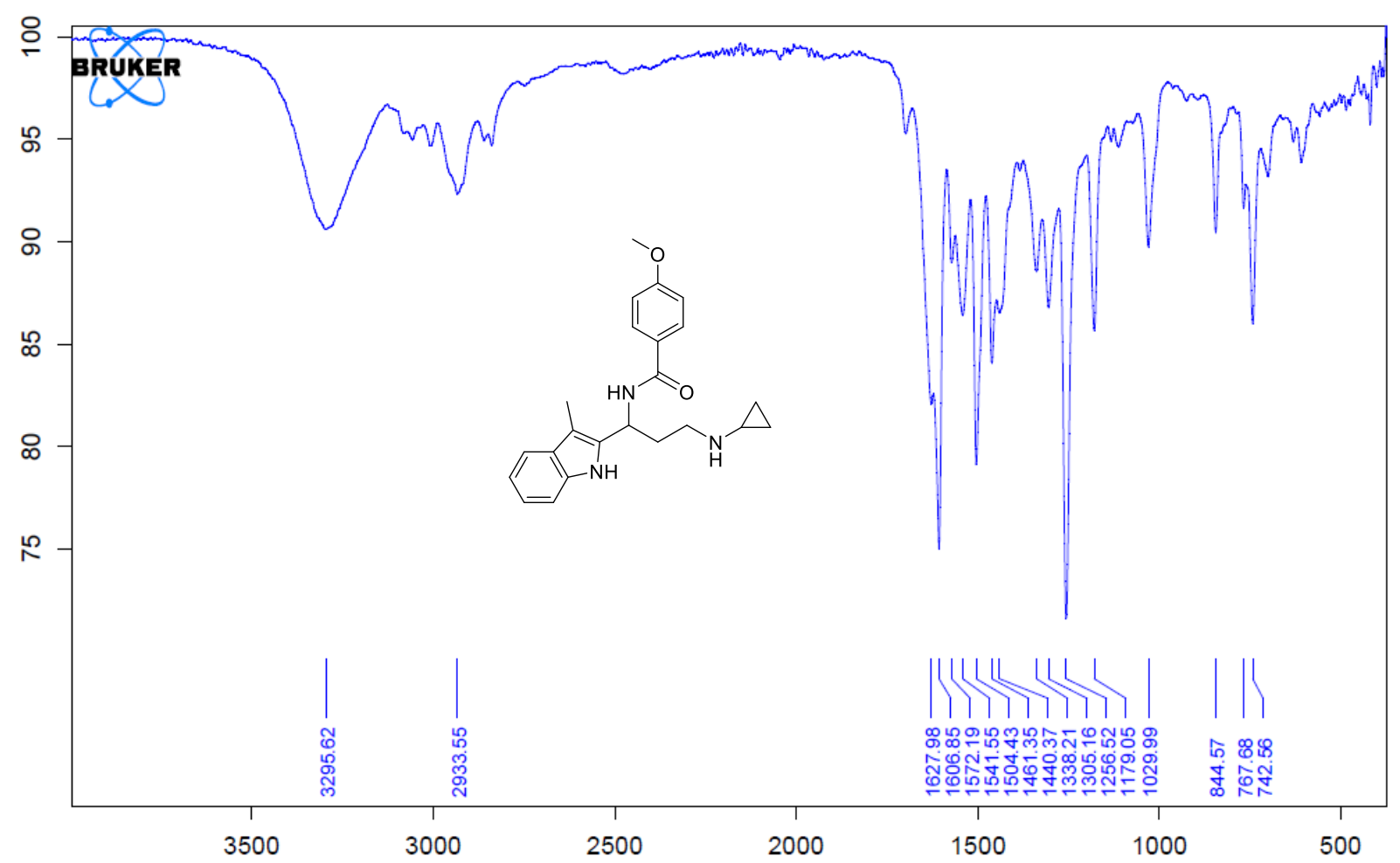

3-((tert-Butoxycarbonyl)amino)-3-methoxy-2-methylpropyl benzoate (29) ${ }^{1} \mathrm{H}-\mathrm{NMR}$ (400 MHz, CDCl3)

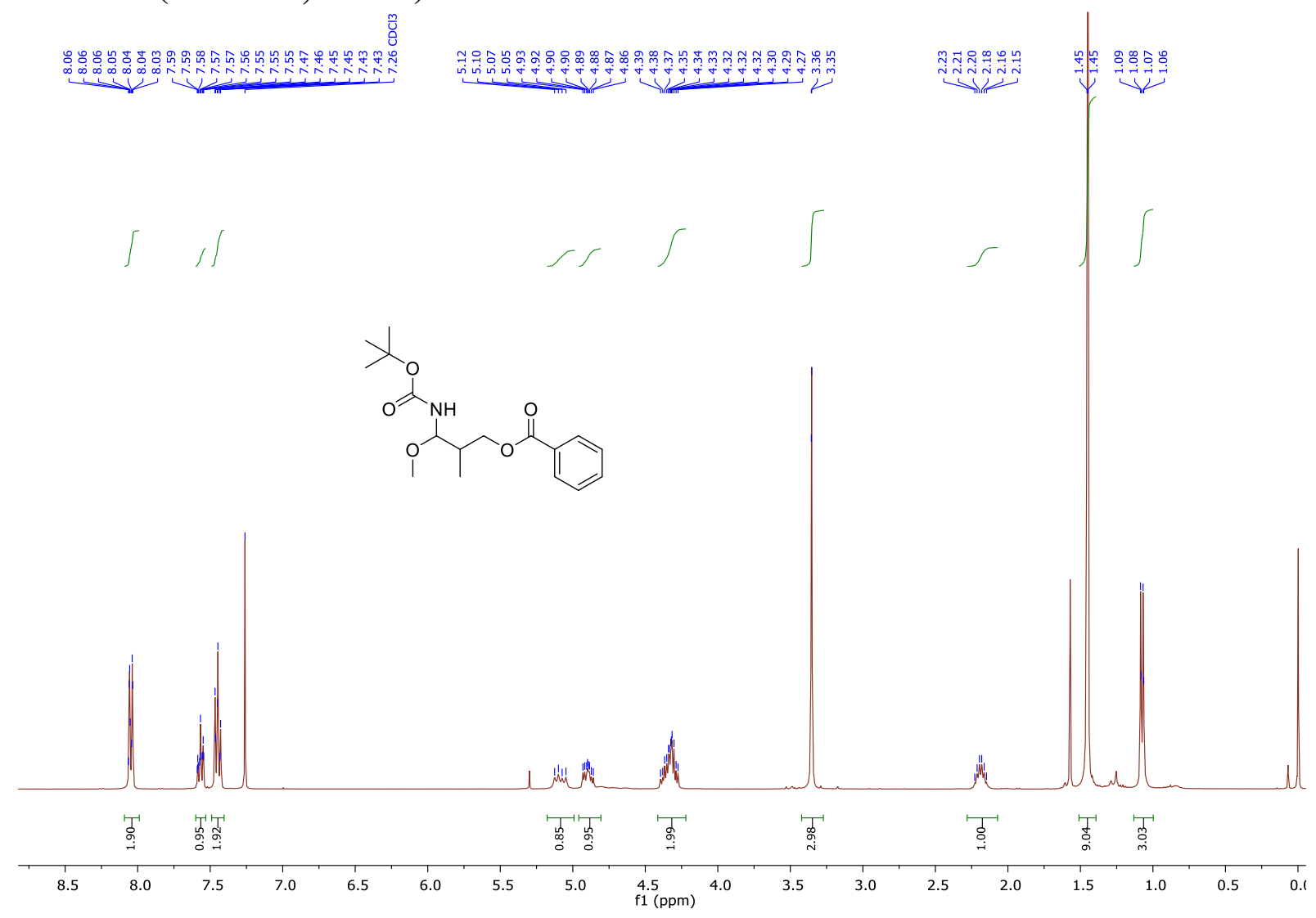


${ }^{13} \mathrm{C}$-NMR (101 MHz, CDCl3)
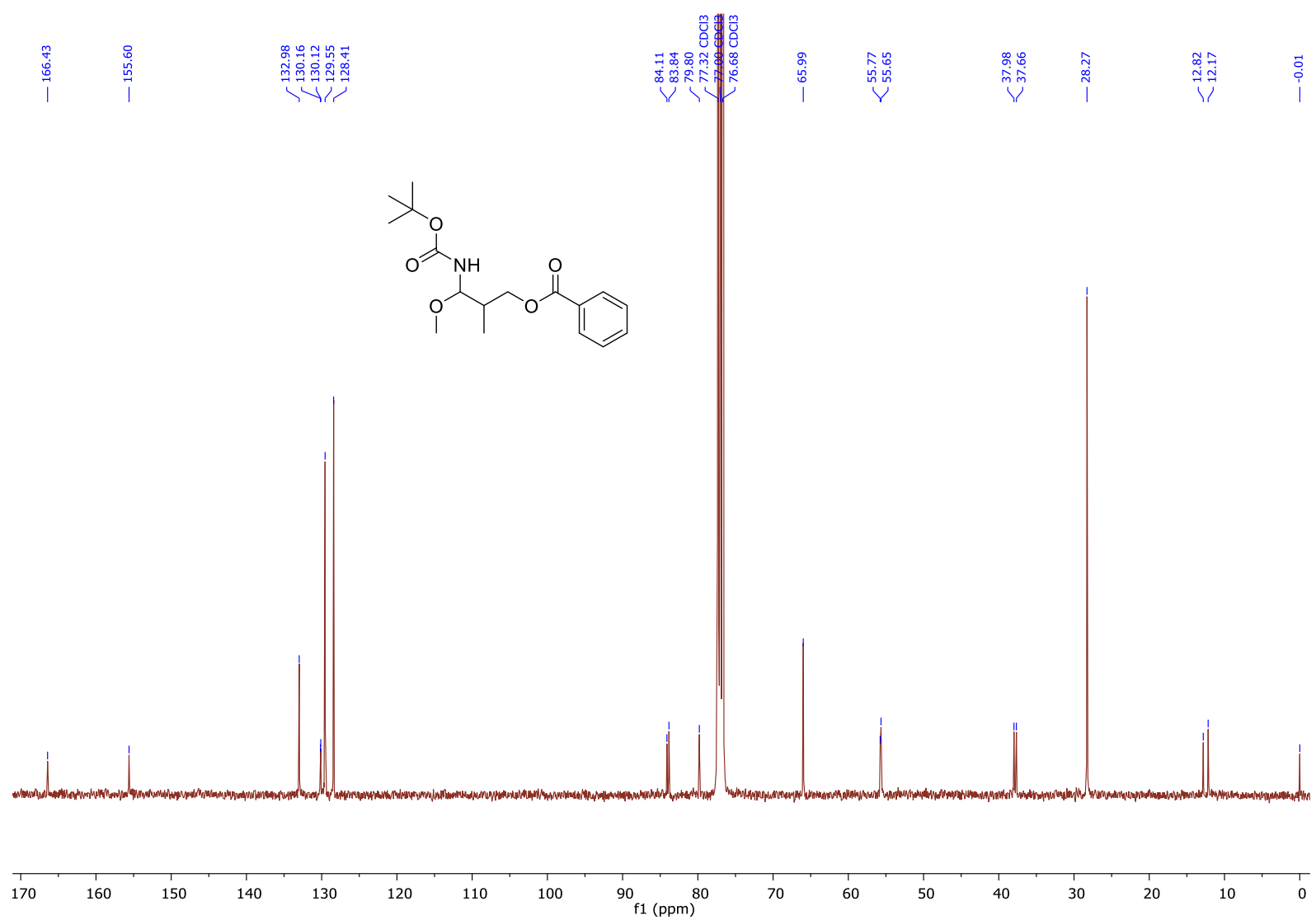

IR

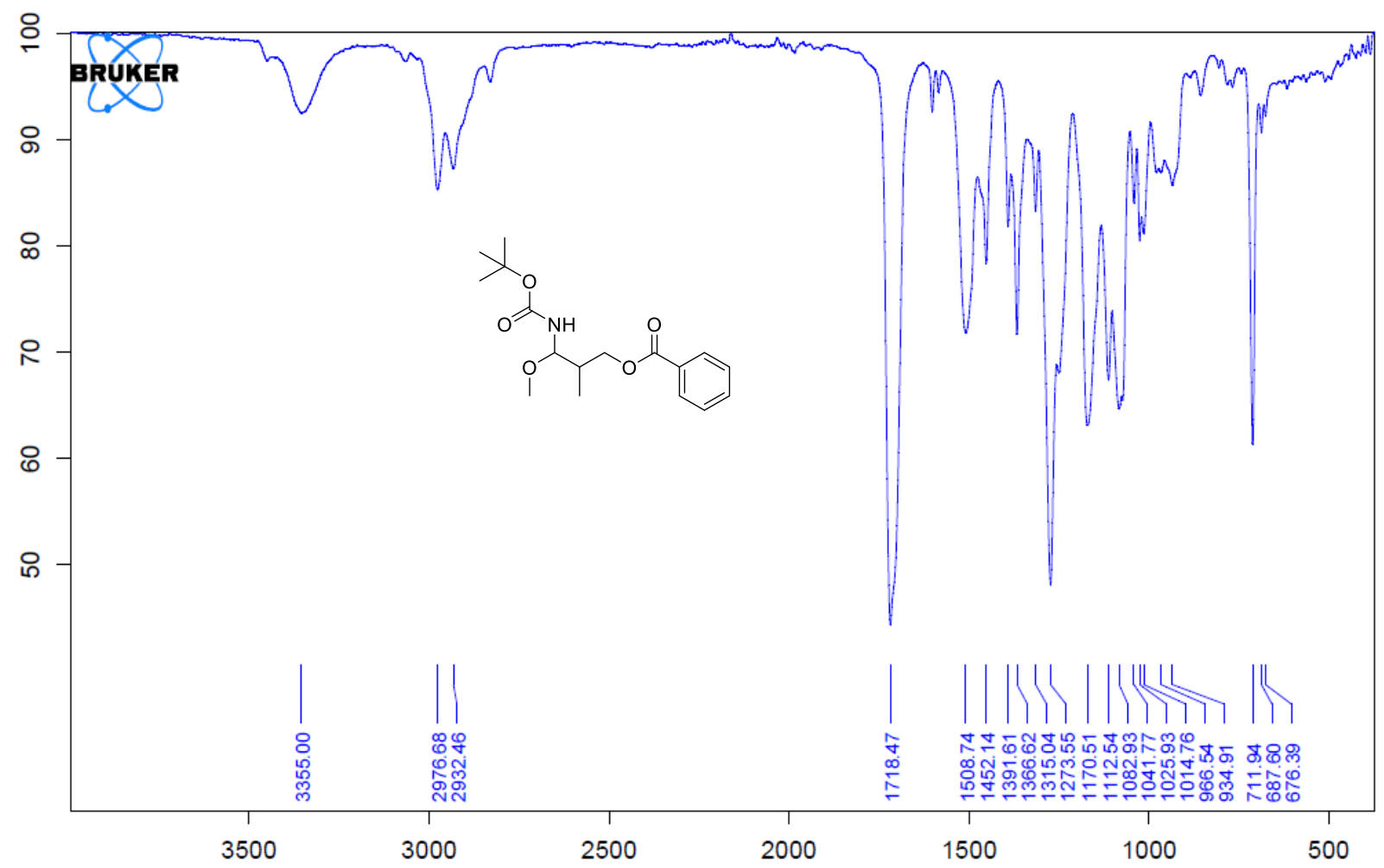

